University of Rhode Island

DigitalCommons@URI

Open Access Dissertations

2004

\title{
A Meta-Analytic Examination of Decisional Balance Across Stage Transitions: A Cross-Sectional Analysis and Cross-Sequential Cross-Validation
}

Kara L. Hall

University of Rhode Island

Follow this and additional works at: https://digitalcommons.uri.edu/oa_diss

\section{Recommended Citation}

Hall, Kara L., "A Meta-Analytic Examination of Decisional Balance Across Stage Transitions: A CrossSectional Analysis and Cross-Sequential Cross-Validation" (2004). Open Access Dissertations. Paper 905. https://digitalcommons.uri.edu/oa_diss/905

This Dissertation is brought to you for free and open access by DigitalCommons@URI. It has been accepted for inclusion in Open Access Dissertations by an authorized administrator of DigitalCommons@URI. For more information, please contact digitalcommons-group@uri.edu. 
A META-ANALYTIC EXAMINATION OF DECISIONAL BALANCE

ACROSS STAGE TRANSITIONS:

A CROSS-SECTIONAL ANALYSIS AND CROSS-SEQUENTIAL CROSS-VALIDATION

BY

KARA L. HALL

A DISSERTATION SUBMITTED IN PARTIAL FULFILLMENT OF THE REQUIREMENTS FOR THE DEGREE OF

DOCTOR OF PHILOSOPHY

IN

PSYCHOLOGY

UNIVERSITY OF RHODE ISLAND 


\section{Abstract}

Interventions to decrease unhealthy behaviors and to increase healthy behaviors are crucial for health promotion and disease prevention. Computerized tailored interventions provide a promising approach for creating positive health behavior change. The Transtheoretical Model (TTM) delineates a way to conceptualize behavior change, provides the foundation for developing assessments of an individual's readiness to change, and is utilized in tailoring interventions for actualizing behavior change. To produce optimally tailored interventions: (1) theory that guides interventions needs to be comprehensively tested; and (2) empirical data that drive the tailored interventions needs to be systematically generated. This program of research tests theoretical assumptions of the model and begins to outline necessary tailoring data, and does so in three main phases: (1) comparison of effect size procedures; (2) cross-sectional meta-analytic investigation of the Stages of Change and Decisional Balance; and (3) a cross-sequential cross-validation study of the cross-sectional study.

In meta-analyses, essential information for calculating effect size is often missing in published studies. Such missing data precludes the inclusion of studies in the meta-analysis thereby introducing bias to the review. Methods to utilize as much data as possible are invaluable to meta-analytic work. A comparison of effect size procedures was conducted in order to identify a bias correction for studies otherwise unusable. The study included 38 studies, with a total of 46 datasets for each of the Decisional Balance measures. Hedge's $g$ provided a $10 \%$ larger effect size than a standard score method of effect size estimation for both the Pros and Cons measures. 
A single correction equation was developed to allow the use of standard score estimation in future meta-analytic work with these constructs.

The second phase of this program of research utilized meta-analytic procedures with longitudinal data to examine 146 datasets across 55 behaviors spanning 18 countries and including 85,272 participants. This study identified a $2-$ factor structure for the Pros and Cons in $96 \%$ of the studies, with a crossover occurring before the Action Stage in the majority of studies. Overall magnitudes of effect were larger in the earlier Stages for Pros and in the later Stages for Cons. Heterogeneity of effect size distribution was found and moderators assessed. Generalizability of these constructs were supported, especially across behaviors and populations. Moderators of effect were found to be differentially related across particular Stage transitions but in no readily apparent pattern.

Thirdly, longitudinal changes in Decisional Balance across the Stages of Change were assessed across the Stage transitions using a cross-sequential approach. Overall magnitudes of effect were somewhat larger for the longitudinal data. Similar magnitudes of effects for cross-sectional adjacent stage transitions and longitudinal stage movements in the early stages were found between cross-sectional and crosssequential profile for the two behaviors examined.

Overall, the use of preexisting studies can lead to groundbreaking empirical data for the development of more comprehensive and more precisely tailored health prevention and disease prevention interventions and further research will continue to delineate important patterns of relationships between these variables. 


\section{Acknowledgements}

There are certainly so many folks to thank for making this project

possible. I would like to start by thanking my mother for her love and support through this long endeavor. Her patience and her great cooking kept me going. I would like to thank all the researchers around the world for the data that made this work possible, especially to those who took the time to provide me with data that was otherwise unavailable. In particular I would like to thank Stefan Keller for his extensive contributions. From across the country, Scott C. Roesch went above and beyond to answer my questions as time ran short - I owe him many thanks. With certainty, the breadth of this project would not have been possible without the help of undergraduate research assistants, most notably Aimee Martel.

During the past 6 years, Dom Valentino has given me endless unwavering unconditional support -- always there full of kindness and sincerity. I am so grateful to have such an amazing person in my life. A special thanks to Jim Campbell for being there at the right time, offering up the right help at the right moment. Thanks to my friends and colleagues for those things big and small that make a difference, especially to Leanne Mauriello, Andrea Paiva, Paul Krebs, Bettina Hoepner, Milena Anachakova, Colleen Redding, and Kathy Meier -- I thank them for being there.

Thanks to my dissertation committee for helping me through the project. Thanks to Bryan Blissmer for sticking with me through all the milestones and the countless pages that came with them. I thank Mark Robbins 
for, among many things, keeping the laughing going day after day after day after long day. Jim Prochaska offered me an intellectual forum in which to thrive, I am grateful to him for that - and I look forward to continuing my intellectual growth with all the great minds at the Cancer Prevention Research Center.

This project and so many of my achievements could not have been possible without Joe Rossi. His dedication and mentorship has been a great gift. I thank him for the countless emails, lunches, and of course sushi that were essential components to this intense process. My sincerest gratitude goes to him for all his efforts and his generosity. 


\section{Dedication}

To my husband Seth - with all my love.

I look forward to sharing the rest of our lives together. 


\section{Preface}

This dissertation document combines the standard format and the manuscript format in order to most comprehensively cover the relevant material while minimizing redundancy across studies. The document begins with an introductory chapter (Chapter 1) that sets the stage for the program of research as a whole. Chapters 2,3 , and 4 provide theoretical, methodological and practical background information. Chapters 5,6, and 7 are written in manuscript format and detail the three main phases of the program of research. This program of research was conducted in 3 main phases: (1) comparison of effect size procedures (Chapter 5); (2) cross-sectional meta-analytic investigation of the Stages of Change and Decisional Balance (Chapter 6); and

(3) a cross-sequential cross-validation of the cross-sectional study (Chapter 7).

The first phase, Determining Effect Size for Meta-Analysis: A

Comparison of Alternative Procedures, provides an important methodological building block for the subsequent meta-analytic study. The $T$-score is a standardized metric frequently used in the body of literature explored in this program of research. This metric, similar to a z-score, has a mean of fifty and a standard deviation of ten. Unfortunately, $T$-scores means when reported are often unaccompanied by the specific standard deviation of the study groups. Since standard deviations are an essential element for effect size calculation, the potential for the loss of a large portion of data is present. This study examined alternative ways to calculate effect sizes based on available data and 
compares effects size methods with other procedures and develops adjustment for bias in the resulting effect size calculation.

In the second phase, A Meta-Analytic Examination of Decisional Balance across Stage Transitions: Cross-sectional Analysis, nearly 150 studies are combined to assess theoretical prescriptions of two primary constructs central to a commonly used behavior change model. The Transtheoretical Model of behavior change has been used around the world and across more than 50 behaviors to help elucidate the behavior change process and provide a framework for interventions for guiding that change process. This study examines a variety of relationships among the two behavior change factors, most notably the magnitude of effect for the relationship between the Decisional Balance measures and adjacent Stage of Change transitions.

The third phase, A Cross-sequential Analysis and Cross-Validation of Decisional Balance across Stage Transitions, provides an investigation of the primary relationships examined in phase two but does so with longitudinal datasets. Instead of examining the magnitude of the relationship of Decisional Balance across adjacent Stage transitions, Decisional Balance will be examined for group of individuals actually moving from one Stage to another. The results of the longitudinal data will then be compared to those of the crosssectional data.

Overall, the main aims of this program of research are to: 1) quantitatively investigate the relationship of Decisional Balance and Stages of Change by identifying the magnitudes of effect for Decisional Balance in 
relation to the transition between each adjacent Stage of Change; 2) more

clearly delineate the patterns of change across and within behaviors; and 3)

provide information that will help inform intervention development in order to create more efficient and effective health behavior change. 


\section{Table of Contents}

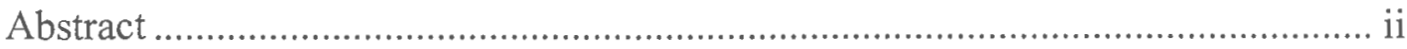

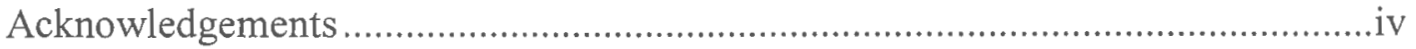

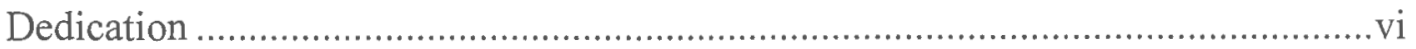

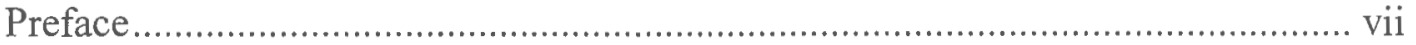

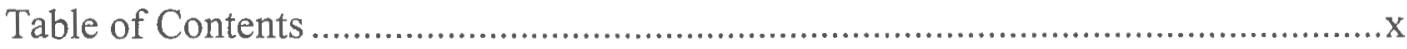

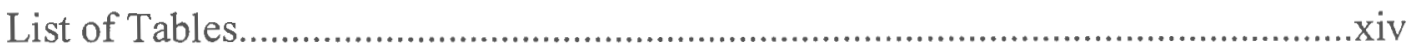

List of Figures ..................................................................................... Xvii

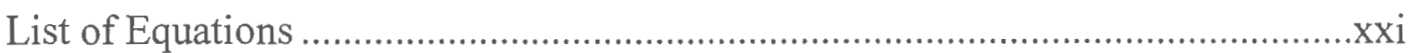

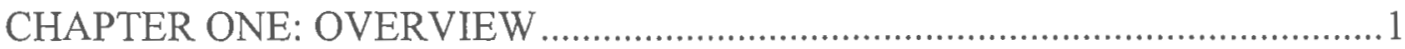

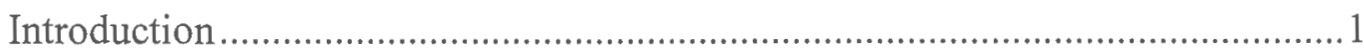

Health Promotion and Disease Prevention........................................................ 2

Computerized Tailored Intervention Systems..................................................

Importance of Well-Tested Theory for Interventions (TTM) ............................

Empirical Data for Optimally Tailoring Interventions .....................................4

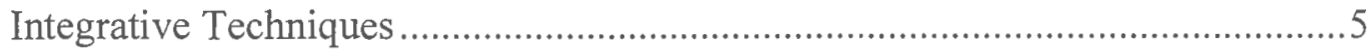

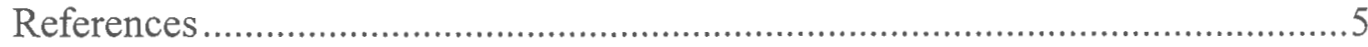

CHAPTER TWO: THE TRANSTHEORETICAL MODEL OF BEHAVIOR

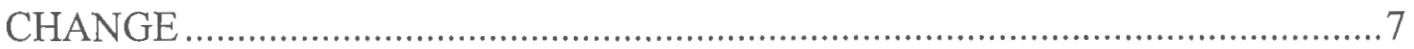

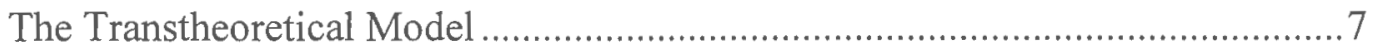

Stage of Change and Decisional Balance ........................................................11

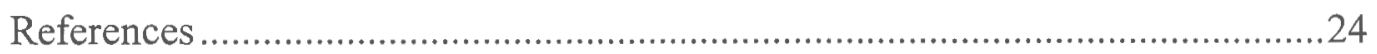

CHAPTER THREE: INTEGRATIVE APPROACH TO THEORY TESTING .....30 
Introduction

Integrative Approaches

Meta-Analysis for Theory Testing

Importance of Comprehensive and Systematic Review Strategies.

Integrative and Meta-analytic Approaches to Testing the TTM. 36

Integrative Analyses: Current Project .............................................................50

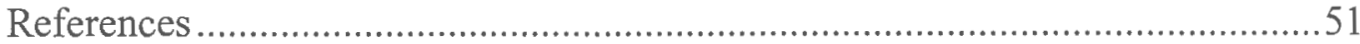

CHAPTER FOUR: META-ANALYTIC APPROACH …...................................58

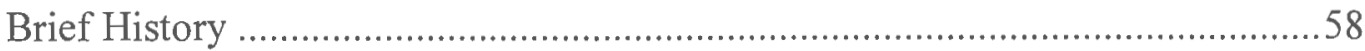

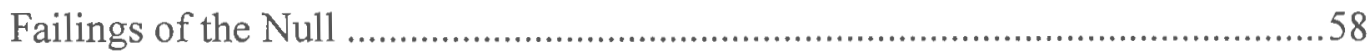

The Role of Meta-analysis in the Significance Testing Debate.........................59

When to Use Meta-Analytic Techniques .........................................................6 60

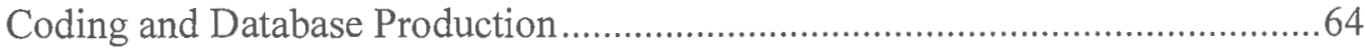

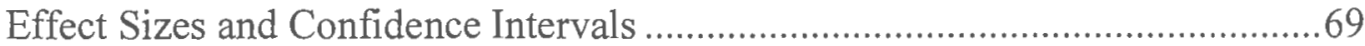

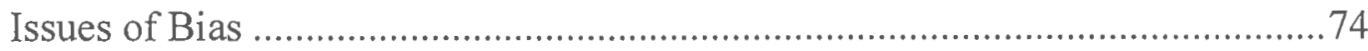

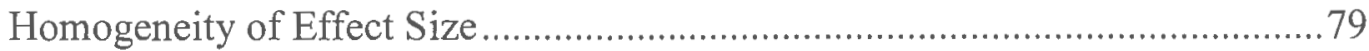

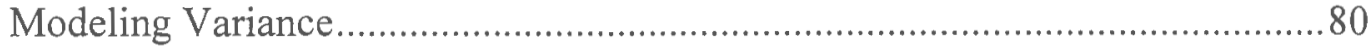

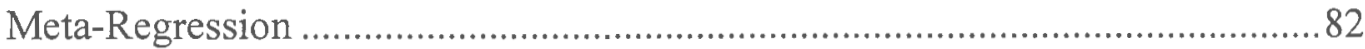

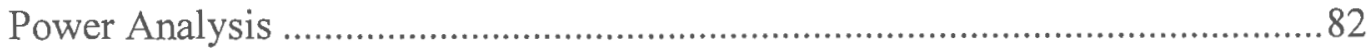

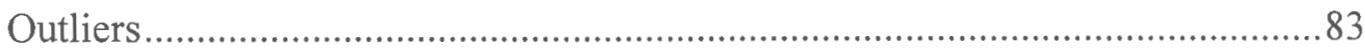

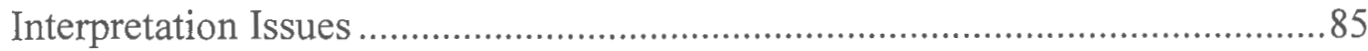

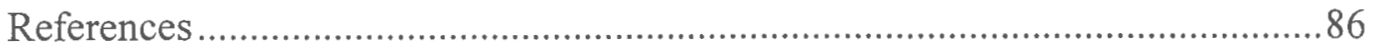


CHAPTER FIVE: DETERMINING EFFECT SIZE FOR META-ANALYSIS:

A COMPARISON OF ALTERNATIVE PROCEDURES ..................................90

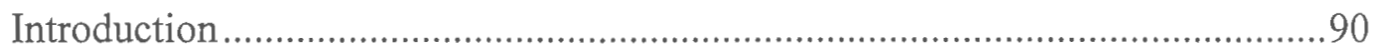

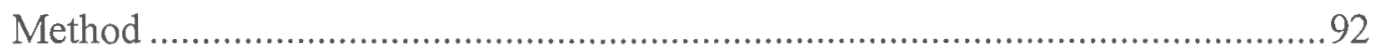

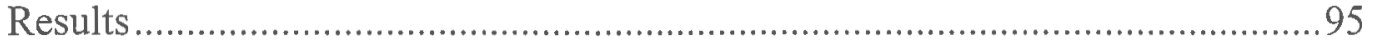

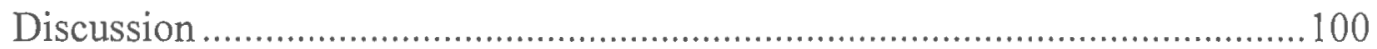

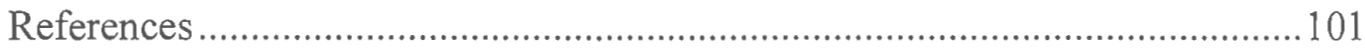

CHAPTER SIX: A META-ANALYTIC EXAMINATION OF DECISIONAL

BALANCE ACROSS STAGE TRANSITIONS: CROSS-SECTIONAL

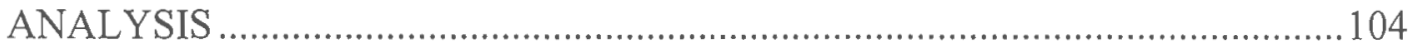

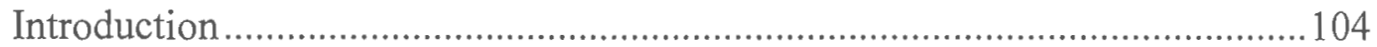

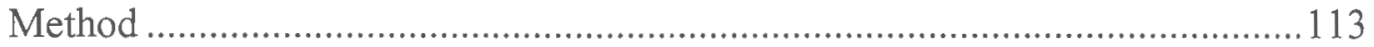

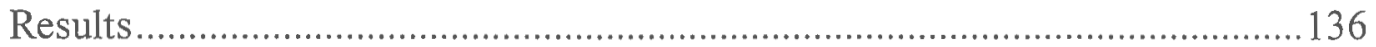

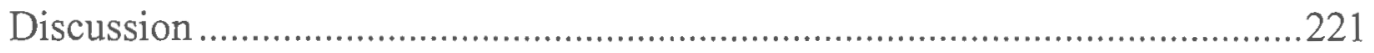

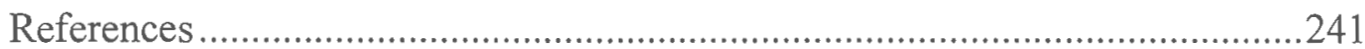

CHAPTER SEVEN: A CROSS-SEQUENTIAL ANALYSIS AND CROSS-

VALIDATION OF DECISIONAL BALANCE ACROSS STAGE

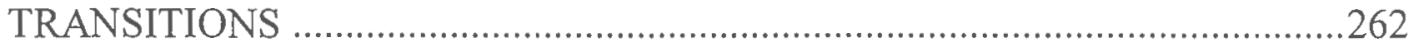

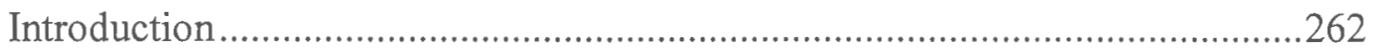

Stages of Change and Decisional Balance ....................................................263

Research Hypotheses and Predictions...........................................................2270

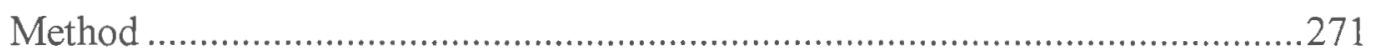

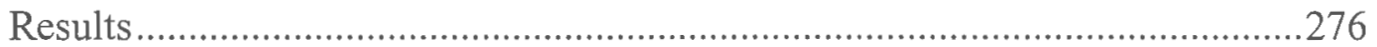


Discussion

References

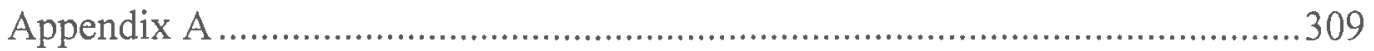

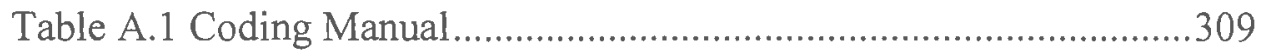

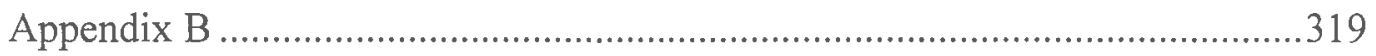

Table B.1 Stage Descriptions for Each Study ....................................319

Table B.2 Supplemental Matrix of Coded Variables .............................350

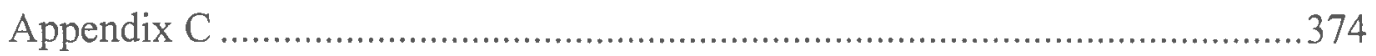

Figure C.1. Composite Pros and Cons Graphs for 33 Target

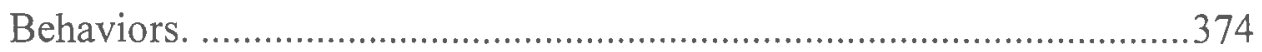

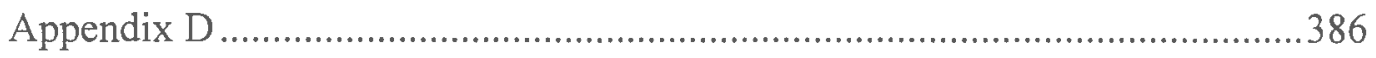

Table D.1 Behavior Categories with Associated Target Behaviors.........386

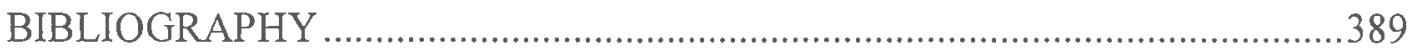




\section{List of Tables}

Table 1. Comparison of Effect Size Calculation Methods for the Pros and

Cons of Change 96

Table 2. Examples of Data Categories 117

Table 3. List of Target Behaviors

Table 4. Summary of Country and Language

Table 5. Summary of Sampling Methods across Studies...................................140

Table 6. Summary of Publication Status across the Studies ...............................140

Table 7. Study Characteristics.......................................................................... 143

Table 8. Mean Samples Sizes by Stage for Each Crossover Transitions............167

Table 9. Adjusted Effect Sizes of the Pros and Cons of Change for All

Studies across Stage Transitions ...........................................................169

Table 10. Summary of Outliers 3 SD from the Mean ...........................................177

Table 11. Comparison of Fixed and Random Effect Models for Effect Sizes of Pros and Cons across Stage Transitions 180

Table 12. Overall Test of Homogeneity and Random Effects Variance for Mean Effect Sizes of the Pros and Cons across Stage Transitions ......182

Table 13. Between Groups Test of Homogeneity of Calculation Method for the Pros and Cons across Stage Transitions.

Table 14. Comparison of Calculation Methods ....................................................184

Table 15. Comparison of Behavior Categories for Pros of Change.......................185

Table 16. Comparison of Behavior Categories for Cons of Change......................186 
Table 17. Within Group Homogeneity Tests of the Pros and Cons across

Stage Transitions for Behavior Categories

Table 18. Comparison of Smoking Acquisition and Smoking Cessation

Table 19. Comparison of Pros and Cons of Quitting Versus Smoking for

Smoking Cessation Studies

Table 20. Comparison of Condom Use Sub-Groups

Table 21. Effect Size Comparison of Dietary Fat Reduction and Fruit and

Vegetable Consumption 200

Table 22. Comparison of Age Groups 203

Table 23. Means, Standard Error and Confidence Intervals for Cessation and Acquisition Behaviors 206

Table 24. Comparison of Healthy and Unhealthy Behaviors. 208

Table 25. Comparison of Response Formats 210

Table 26. Comparison of Country. 212

Table 27. Comparison of Language 214

Table 28. Comparison of Publication Status. 216

Table 29. Comparison of Direction of Pros and Cons 218

Table 30. Overview of Effect Sizes by Moderator Variable 220

Table 31. Patterns of Fixed and Random Effect Results across Moderator

Variables for Pros. 225

Table 32. Patterns of Fixed and Random Effect Results across Moderator

Variables for Cons. .226

Table 33. Between Groups Heterogeneity for Primary Moderators 239 
Table 34. Magnitude of Stage Transitions for Pos and Cons of Change

Table 35. Mean Effect Sizes for Stage Transitions in the Random Digit Dial

Sample across Time Points for Smoking Cessation..............................22

Table 36. Mean Effect Sizes for Stage Transitions in the Random Digit Dial

Sample with All Time Points Combined

Table 37. Mean Effect Sizes for Stage Transitions in the Parent Sample across

Time Points for Smoking Cessation.

Table 38. Mean Effect Sizes for Stage Transitions in the Parent Sample for

Smoking with All Time Points Combined.

Table 39. Mean Effect Sizes for Stage Transitions across Time Points in the

Beach Study for Sun Protection

Table 40. Mean Effect Sizes for Stage Transitions in the Beach Sample with

All Time Points Combined.

Table 41. Mean Effect Sizes for Stage Transitions in the Parent Sample across

Time Points for Sun Protection

Table 42. Mean Effect Sizes for Stage Transitions in the Parent Sample for

Sun Protection with All Time Points Combined 


\section{List of Figures}

Figure 1. Relationship between Hedges' $g$ and Standard Score for Pros of

Change with Outliers Removed.

.98

Figure 2. Relationship between Hedges' $g$ and Standard Score for Cons of

Change with Outliers Removed.

Figure 3. Relationship between Hedges' $g$ and Standard Score for Pros and

Cons of Change Combined.

Figure 4. Power for Q Test of Effect Size Homogeneity for Small, Medium, and Large Between- to Within-Study Variance Ratios (alpha $=.05) . .136$

Figure 5. Composite Decisional Balance Graphic Using Random Effects

Model.

Figure 6. Forest Plots of Behavior Categories for Pros Stage Transitions

(Means and Confidence Intervals)

Figure 7. Forest Plots of Behavior Categories for Cons Stage Transitions

(Means and Confidence Intervals)

Figure 8. Composite Graphic for Smoking Acquisition .194

Figure 9. Composite Graphic for Smoking Cessation. 194

Figure 10. Composite Graphic for Pros and Cons of Quitting Smoking..... 196

Figure 11. Composite Graphic for Pros and Cons of Smoking. 196

Figure 12. Composite Graphic for Condom Use - General. 198

Figure 13. Composite Graphic for Condom Use - Main Partner. 198

Figure 14. Composite Graphic for Condom Use - Other Partner. 199

Figure 15. Composite Graphic for Dietary Fat Reduction. .201 
Figure 16. Composite Graphic for Fruit and Vegetable Consumption...................201

Figure 17. Composite Graphic for Adolescents. ...................................................204

Figure 18. Composite Graphic for College Students............................................204

Figure 19. Composite Graphic for Adults..........................................................205

Figure 20. Composite Graphic for Cessation Behaviors......................................207

Figure 21. Composite Graphic for Acquisition Behaviors....................................207

Figure 22. Composite Graphic for Studies Examining Healthy Behaviors...........209

Figure 23. Composite Graphic for Studies Examining Unhealthy Behaviors.......209

Figure 24. Composite Graphic for "How Important" Format, .................................211

Figure 25. Composite Graphic for "Agree/Disagree" Format................................211

Figure 26. Composite Graphic for Studies Conducted in the US...........................213

Figure 27. Composite Graphic for Studies Conducted Outside the US.................213

Figure 28. Composite Graphic for Studies Conducted in English. ........................215

Figure 29. Composite Graphic for Studies Conducted in Languages Other than

English.

Figure 30. Composite Graphic for Peer-Reviewed Publications............................217

Figure 31. Composite Graphic for Non-Peer-Reviewed Datasets..........................217

Figure 32. Composite Graphic for "Actual" ......................................................219

Figure 33. Composite Graphic for "Reversed". .................................................219

Figure 34. Composite Profile for Smoking Acquisition.........................................233

Figure 35. Composite Profile for Prevention Behaviors. ........................................234

Figure 36. Composite Profile of "Yearly" behaviors. ............................................234

Figure 37. Composite Decisional Balance Profile across 55 Behaviors. ...............269 
Figure 38. Stage Transition Membership.

Figure 39. Graphic for Pros and Cons of Smoking from Baseline to 6 Months for the Random Digit Dial Sample. 280

Figure 40. Graphic for Pros and Cons of Smoking from 6 to 12 Months for the Random Digit Dial Sample. 280

Figure 41. Graphic for Pros and Cons of Smoking from 12 to 18 Months for the Random Digit Dial Sample.

Figure 42. Graphic for Pros and Cons of Smoking from 18 to 24 Months for the Random Digit Dial Sample.

Figure 43. Graphic for Pros and Cons of Smoking for the Random Digit Dial Sample with all Time Points Combined. 283

Figure 44. Graphic for Pros and Cons of Smoking from Baseline to 6 Months for the Parent Sample. 286

Figure 45. Graphic for Pros and Cons of Smoking from 6 to 12 Months for the Parent Sample. 286

Figure 46. Graphic for Pros and Cons of Smoking Smoking from 12 to 24 Months for the Parent Sample. 287

Figure 47. Graphic for Pros and Cons of Smoking for the Parent Sample with all Time Points Combined. 289

Figure 48. Graphic of Sun Protection for Baseline to 2 Months for the Beach Study. .292

Figure 49. Graphic of Sun Protection for 2 to 12 Months for the Beach Study....292 
Figure 50. Composite Graphic for Sun Protection in the Beach Sample across
All Time Points

Figure 51. Graphic of Sun Protection for Baseline to 6 Months for the Parent

Study

Figure 52. Graphic of Sun Protection for 6 to 12 Months for the Parent Study....297

Figure 53. Graphic of Sun Protection for 12 to 24 Months for the Parent Study..298

Figure 54. Composite Graphic for Sun Protection in the Parent Sample across

All Time Points 300

Figure 55. Cross-sectional Smoking Cessation Profile $(n=25)$.

Figure 56. Cross-sectional Profile for Unhealthy Behaviors $(n=45) \ldots \ldots \ldots \ldots \ldots \ldots \ldots . . . .303$

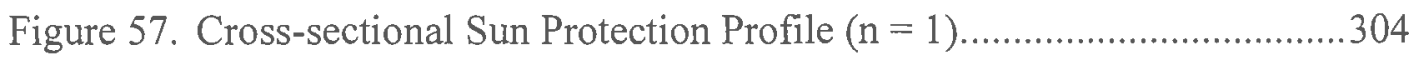




\section{List of Equations}

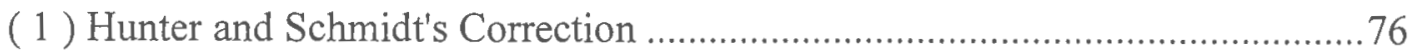

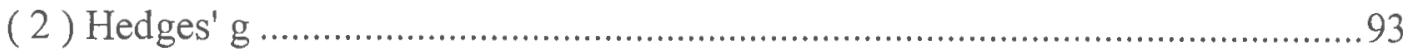

( 3 ) Standard Graphical Effect Size ............................................................ 93

(4) Pros Bias Correction for Standard Score Method ........................................96

( 5 ) Cons Bias Correction for Standard Score Method .........................................96

(6 ) Pros and Cons Correction for Standard Score Method ….............................100

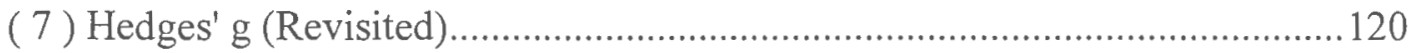

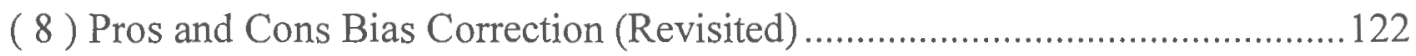

(9) Standardized Mean Difference with Sample Size Correction........................ 122

( 10 ) Standard Error for Standardized Mean Difference......................................123

( 11 ) Inverse Variance for Standardized Mean Difference ................................. 123

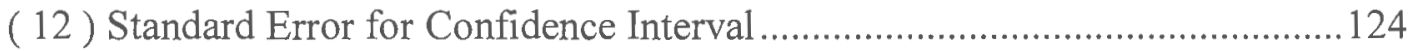

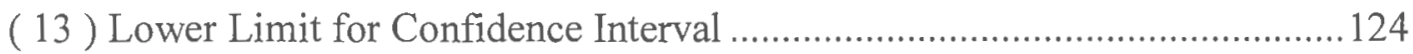

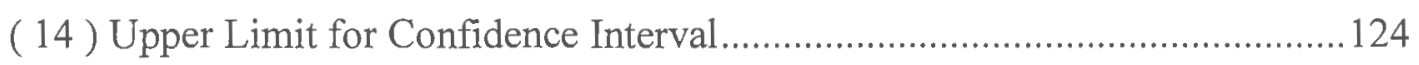

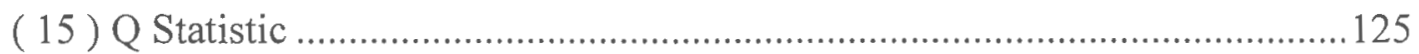

( 16 ) ANOVA Analog for Fixed Effects Model - Between Groups .................... 128

( 17 ) ANOVA Analog for Fixed Effects - Within Groups................................ 128

( 18 ) Random Effects Variance Components..................................................... 129

( 19 ) Random Variance Component - Method of Moments ...............................129

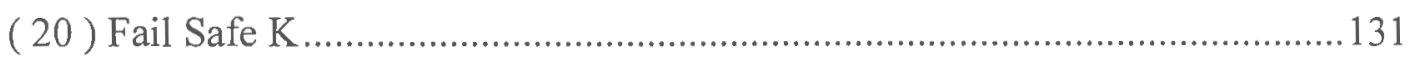

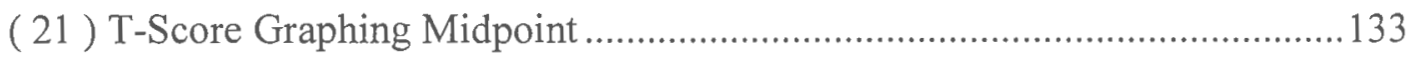

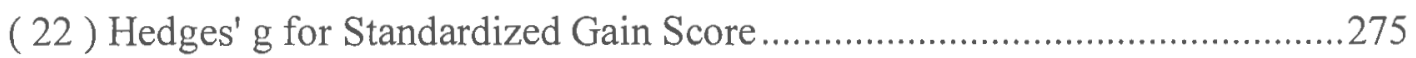


( 23 ) Pooled Variance for Standardized Gain Score .........................................275

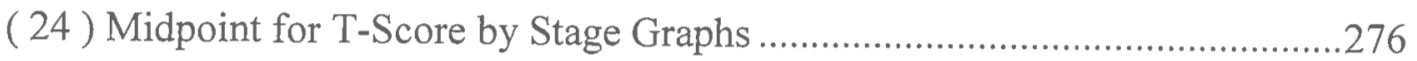




\section{CHAPTER ONE}

\section{OVERVIEW}

\section{Introduction}

Interventions to decrease unhealthy behaviors (e.g., smoking and sun exposure) and to increase healthy behaviors (e.g., exercise and mammography screening) are crucial for health promotion and disease prevention. Tailored interventions provide a promising approach for creating positive health behavior change. In order to produce optimal tailored interventions: (1) the theory that guides interventions must be comprehensively tested; and (2) empirical data designed to drive the tailored interventions must be systematically gathered and analyzed.

Many theories have been developed to explain and to assist in behavior change. One theory, the Transtheoretical Model (TTM), has drawn from some of the most powerful processes and principles of change from across multiple theories of behavior and behavior change to form one comprehensive model. The model delineates a way to conceptualize behavior change, provides the foundation for developing assessments of an individual's readiness to change, and is utilized in tailoring interventions for actualizing behavior change. Since the development of the

TTM, hundreds of health promotion and disease prevention studies around the world have used the model, yet many theoretical assumptions of the relationships between TTM constructs have not been thoroughly tested.

To date there has been no comprehensive examination of TTM constructs across behaviors. The common measures utilized in hundreds of studies, across the 
many different content areas, research designs and populations, provide a unique opportunity for comparative and integrative analyses of important behavior change factors. Unique meta-analytic techniques will be used to analyze and compare crosssectional and longitudinal datasets between and within multiple behaviors on two behavior change factors across five Stages of Change. This quantitative review aims to: (1) comprehensively identify if and when theoretical prescriptions of the TTM are maintained or if modifications of the model need to be made; and (2) update and expand the data that drives intervention development of tailored behavior change systems. Overall, this study will help bridge the gap in knowledge between theoretical prescriptions and intervention applications to create more efficient and effective population-based health promotion and disease prevention interventions ultimately reducing the incidence of disease, such as cancer.

\section{Health Promotion and Disease Prevention}

The development of many diseases is often rooted in unhealthy behaviors.

For instance, many behaviors that can lead to cancer, such as smoking and alcohol abuse, are preventable and many behaviors that reduce the risk of cancer, such as exercise and healthy diet, are readily attainable. Unfortunately, it is often difficult for individuals to change well-established but unhealthy behaviors, even when the need to do so is apparent and acknowledged by the individual, thus emphasizing the need for behavior change interventions. 


\section{Computerized Tailored Intervention Systems}

Computerized systems that deliver population-based health promotion and disease prevention interventions have proven particularly effective when systems provide tailored feedback to each participant. The combination of advances in both the behavioral sciences and in computer technology has enabled the development of expert system interventions that guide and motivate individuals to change. Expert systems are broadly defined as computer programs that mimic the reasoning and problem solving of a human 'expert'. Early programs implemented the advice of an individual without the inclusion of empirical data (Negotia, 1985). In contrast, empirically based systems generate interventions driven more by science than opinion. Expert systems start with basic principles and theoretical decision rules and are then enriched with heuristics and empirical data.

Current expert systems provide tailored print feedback or interactive and immediate tailored feedback to each individual (Redding et al., 1999; Velicer et al., 1993; Velicer \& Prochaska, 1999). Feedback includes both normative feedback, which compares the individual to other successful and unsuccessful individuals, as well as ipsative feedback, which compares the individual to his or her own previous responses.

\section{Importance of Well-Tested Theory for Interventions (TTM)}

To ensure maximum effectiveness of tailored interventions for behavior change, the theory that guides interventions must be comprehensively tested. Since many theoretical assumptions of the TTM have not been thoroughly tested, developing interventions based primarily on theory rather than empirical data may 
prove problematic. This program of research will explore theoretical prescriptions and determine what, if any, modifications are appropriate to enhance this behavior change model.

\section{Empirical Data for Optimally Tailoring Interventions}

To date, no study has produced a set of empirical data to drive complex behavior change intervention systems; the paucity of available data results in interventions primarily guided by the theoretical principles alone. This study provides an empirical review of several TTM variables across a wide range of behaviors. Traditionally, the empirical basis for expert systems has been based on small-sample pilot data. In order to approach truly optimal tailoring, data that currently drive expert systems need updating. Additionally, a more precise understanding of where, when and on what to tailor need to be generated. An optimal tailoring approach can reduce demands on participants and costs to providers while increasing intervention effectiveness. Optimal tailoring can be achieved in part by systematically and quantitatively analyzing key relationships between various populations and behaviors across hundreds of available studies, both crosssectionally and longitudinally.

Several studies have shown differences in intervention outcomes for adolescence vs. adults (Spencer et al., 2002; Weinstock et al., 2002), and males vs. females (Laforge et al., 2003; Weinstock et al., 2002) that suggest tailoring on particular demographics may be warranted. For example, in reexamining the work of Spencer et al. (2002), it was found that two of the three negative studies using partial or full tailoring involved adolescent smokers, while all but one of the positive results 
were with adult smokers. Laforge et al. (2003) found differential effects for males and females in a tailored expert system intervention to reduce binge drinking in a college population. Weinstock et al. (2002) found differential outcomes for both gender and age in a randomized controlled trial of a tailored expert system intervention to reduce unprotected sun exposure. Meta-analytic procedures provide a unique opportunity to assess these variables across multiple data sets and behaviors.

\section{Integrative Techniques}

This program of research will utilize established meta-analytic procedures and employ new and innovative meta-analytic techniques and integrative analyses across more than one hundred and fifty cross-sectional and longitudinal data sets. The overall goal of this program of research is to produce a preliminary set of cumulative data specifically designed to guide the creation of "next generation" optimally tailored expert system interventions for behavior change.

\section{References}

Laforge, R.G. et al. (2003). Enhancing brief tailored college alcohol interventions.

Unpublished NIAAA grant proposal.

Negotia, U. N. (1985). Expert systems and fuzzy systems. Menlo Park, CA:

Benjamin. 
Prochaska, J.O., DiClemente, C.C., Velicer, W.F., \& Rossi, J.S. (1993).

Standardized, individualized, interactive, and personalized self-help programs for smoking cessation. Health Psychology, 12, 399-405.

Spencer, L., Pagell, F., Hellion, M. E., \& Abrams, D. B. (2002). Applying the

Transtheoretical model to tobacco cessation and prevention: A review of the literature. American Journal of Health Promotion, 17(1), 7-71.

Redding, C.A., Prochaska, J.O., Pallonen, U.E., Rossi, J.S., Velicer, W.F., Rossi, S.R., Greene, G.W., Meier, K.S., Evers, K.E., Plummer, B.A., \& Maddock, J.E. (1999). Transtheoretical individualized multimedia expert systems targeting adolescents' health behaviors. Cognitive \& Behavioral Practice, 6(2), 144-153.

Velicer, W. F., \& Prochaska, J. O. (1999). An expert system intervention for smoking cessation. Patient Education and Counseling, 36, 119-129.

Velicer, W. F., Prochaska, J. O., Bellis, J. M., DiClemente, C. C., Rossi, J. S., Fava, J. L., \& Steiger, J. H. (1993). An expert system intervention for smoking cessation. Addictive Behaviors, 18, 269-290.

Weinstock, M. A., Rossi, J. S., Redding, C. A., \& Maddock, J. E. (2002). Randomized controlled community trial of the efficacy of a multi-component stage-matched intervention to increase sun protection among beachgoers. Preventive Medicine, 35, 584-592. 


\section{CHAPTER TWO}

\section{THE TRANSTHEORETICAL MODEL OF BEHAVIOR CHANGE}

\section{The Transtheoretical Model}

Many different theories are utilized in behavior change interventions and serve as the basis for tailored interventions. One theory, the Transtheoretical Model (TTM) of behavior change, is particularly suited to provide the framework for individualized interventions using expert system approaches. The TTM involves three dimensions: the temporal dimension, the independent variable dimension, and the intermediate variable dimension (Velicer et al., 2000). The central organizing construct of the TTM characterizes behavior change over time through five distinct Stages of change: Precontemplation, Contemplation, Preparation, Action, and Maintenance. The independent variable dimension of the TTM identifies behavior change strategies through ten primary Processes of Change that are grouped into two higher order factors - the behavioral and experiential Processes. Two intermediate indicators of when the Stage of Change will occur are Decisional Balance (weighing of Pros and Cons) and Self-efficacy (Situational Confidence or Temptation).

\section{Stages of Change}

In health psychology, the concept of change occurring in a series of stages has been examined in efforts to understand the temporal aspects of change in human nature or behavior. An example of the stage concept can be seen in Horn's (1976; Horn \& Waingrow, 1966) work with smoking behavior and cessation. Horn developed a four stage process of change investigating smoking behavior, which 
consisted of 1) contemplation of change; 2) the decision to change; 3) short-term change; and 4) long-term change. Although arising independently and in a different context, the Stages of change as conceptualized by the TTM are similar to Horn's stages. Over the years, through its own evolution, the TTM ultimately identified five Stages (DiClemente, Prochaska, Fairhurst, Velicer, Velasquez, et al., 1991).

Historically, Prochaska and DiClemente (1982) identified the five Stages (Precontemplation, Contemplation, Action, Maintenance, and Relapse). Around that same time, (1983) developed the URICA (University of Rhode Island Change Assessment) Stage of change assessment instrument identifying five Stages (Precontemplation, Contemplation, Decision Making, Action, and Maintenance). Due to what appeared at the time to be lack of empirical support, Decision Making was dropped (McConnaughy, 1989) and four Stages became the primary focus of subsequent study. In 1991 the Preparation Stage, similar to the Decision Making Stage, was reinstituted into the Stage of Change construct and in general has remained a part of the model (DiClemente et. al., 1991).

The algorithms for the five Stages of Change are specific for each behavior, but usually follow these general Stage concepts. Participants are considered to be in the Precontemplation Stage if they report an undesired status, that is, the presence of a problem behavior or the lack of a healthy one, and express no intention of changing in the next six months. Participants are considered the Contemplation Stage if they intend to change in the next six months. Participants in the Preparation Stage plan to change in the next month and have begun to engage in target behaviors, but have not yet met particular criteria. Participants reach the Action Stage once they have met the 
given behavioral criteria. Lastly, if the participant has met the specified behavioral criteria for more than six months, they have reached the Maintenance Stage.

\section{Processes}

The TTM also includes a series of independent variables, the Processes of

Change (Prochaska, Velicer, DiClemente, \& Fava, 1988). The Processes represent strategies for changing one's behavior. The Processes of Change instruments that measure this construct assess affective, cognitive, evaluative, experiential, and behavioral activities as one moves through the Stages of Change. The Processes have been found to have a correlated higher order factor structure and measure change Processes that represent two primary dimensions, experiential and behavioral (Prochaska et al., 1988). In general, the experiential Processes include consciousness raising, dramatic relief, environmental reevaluation, self-reevaluation, and social liberation. The behavioral Processes include stimulus control, counter conditioning, reinforcement management, self-liberation, and helping relationships. Experiential Processes generally are considered to be more salient in the earlier Stages of Change whereas the behavioral Processes are considered to be more salient in the later Stages.

\section{Self-efficacy/Temptations}

Situational Self-efficacy (or Situational Confidence) and Temptations are variations of Bandura's self-efficacy construct (Bandura, 1977; 1982; DiClemente, 1986; Velicer, DiClemente, Rossi \& Prochaska, 1990) and describe one's perceived confidence or temptation to engage in a behavior in particular challenging situations. 
Most typically, when measuring Self-efficacy, a general Self-efficacy scale is

reported. If subscales are found or reported, they most commonly are: Positive/Social and Negative Affect. Additional examples of subscales representing different types of situations include Emotional Situations, Skill Applications, Relapse Recovery (Dijkstra \& deVries, 2000), Excuse Making, Resistance from Others (Rossi et al., 2000) and Habit/Addictive (Velicer et al., 1990). For Temptations, in addition to the most common subscales - Positive/Social and Negative Affect, other examples include Curiosity (Pallonen et al., 1998) and Habit/Addictive (Velicer et al., 1990).

\section{Decisional Balance}

The TTM originated by integrating theories of psychotherapy as well as incorporating constructs from alternative models. One of the most important and reliable TTM constructs, Decisional Balance, was inspired by Janis and Mann's (1977) conflict model of decision-making. Janis (1959) proposed a descriptive schema called a "balance sheet" of incentives. The four main categories of consideration for decisional conflicts are: a) utilitarian gains and loses for self; b) utilitarian gains and losses for significant others; c) self-approval or-disapproval; d) approval or disapproval by significant others. These four comparative categories of potential positive and negative incentives involve both instrumental effects of utilitarian objectives and nonutilitarian considerations such as issues of self-esteem and value-based determinations.

The development of the TTM Decisional Balance measure (Velicer, DiClemente, Prochaska, \& Brandenburg, 1985) was based on the 8 factors (4 gains 
and 4 losses) of Janis and Mann (1977). The researchers constructed the scale to study the decision-making process across the Stages for smoking cessation. Instead of achieving an 8 factor-structure as anticipated, principal components analysis identified two orthogonal components. These two components were called the Pros and Cons of Smoking.

\section{Stage of Change and Decisional Balance}

The Stages of Change and Decisional Balance will be the focus of the subsequent studies in this program of study, therefore special emphasis is given to these constructs.

\section{Stages: The Central Organizing Construct}

The Stages of Change are considered the central organizing construct because many of the theoretical assumptions and construct relationships are framed around this temporal process of change. Therefore, the role of the Stages of Change in the context of the Transtheoretical Model is important theoretically and practically.

A variety of theoretical prescriptions of the model center on the relationships between the Stages of Change and the intermediate (Self-efficacy and Decisional Balance) and independent variables (Processes of Change) that serve as guides for the development of health behavior change interventions. The TTM is particularly suited to provide the framework for individualized tailored interventions. The Stage of Change construct delineates the first hierarchical tier in the tailoring process. At each time point, an individual's "readiness to change" or Stage of Change is assessed. Feedback is then provided on an individual's Stage and all subsequent 
feedback is framed within the context of that Stage. The independent dimension includes the ten Processes of Change, which delineate strategies to bring about behavioral change. The intermediate dimension includes Decisional Balance, which represents the weighting of the Pros and Cons, and Self-efficacy, which incorporates confidence and temptation to engage in particular behaviors across challenging situations. These two dimensions form the second tier of the algorithms that drive expert systems. Thus, individuals receive normative feedback on each independent and intermediate dimension with respect to others in the same Stage of Change and ipsative feedback based on how they previously performed on these measures.

\section{Measuring Stage of Change}

The Stages of Change can be measured in a variety of ways. These measures can be divided into two broad categories: continuous and discrete. Most typically, though not exclusively, continuous measures utilize clustering procedures to create Stage profiles. These approaches usually include more lengthy questionnaires than the discrete or algorithm procedures and include "clusters" of statements that represent different Stages that are subsequently analyzed based on profiles using cluster analysis. Discrete Stage measures appear to be more frequently used by researchers, since they are more prominent in the literature. These procedures are guided by decision rules and typically examine behavioral intentions as well as actions regarding behavior change.

\section{Discrete Stage Approaches}

Discrete Stage measures are designed to unambiguously classify individuals into one of the defined Stages. The algorithms for the five Stages of Change are 
specific for each targeted behavior. In general they adhere to the following Stage concepts, though particular aspects of the staging (e.g., time frame, behavioral criteria) vary among studies. Participants are considered to be in the Precontemplation Stage if they report an undesired status, that is, the presence of a problem behavior or the lack of a healthy one, and express no intention of changing in the next six months. The Contemplation Stage indicates that the participant intends to change in the next six months. Participants in the Preparation Stage plan to change in the next month and have begun to engage in target behaviors, but have not yet met particular criteria. For well studied behaviors these criteria are set according to gold standards in their given field. The Action Stage is attained once the participant reaches the given behavioral criteria. Lastly, if the participant has met the specified behavioral criteria for more than six months, they have reached the Maintenance Stage.

The stage concepts guide the staging algorithms but typically vary in time frame, behavior criteria, action criteria, and response format. Staging also can vary in clarity of behavioral operationalization, in complexity of target behavior and in consistency in stage description.

Time Frame. Most, but not all, Stage algorithms include a time frame. The most typical time frames used in staging algorithms are: Precontemplation $=$ no intention in the next 6 months; Contemplation = seriously considering in the next 6 months; Preparation = considering in the next 30 days; Action = changed behavior within the past 6 months; Maintenance $=$ changed behavior for more than 6 months. The use of the time frame across studies is inconsistent, however, and variations 
include: (1) time frame is not used at all (e.g., Never having had a prior mammogram nor having high intentions to have one; Lauver, et al., 2003); (2) time frame is only used in some Stages (e.g., Wyse, 1995); (3) staging includes additional time frame criteria (e.g., Smokers who planned to quit in the next 30 days and had made an attempt to quit in the past 12 months; Etter et al., 1997); (4) staging includes alternative placements of time frame (e.g., Action $=$ Reported regular vigorous exercise for at least one month; Armstrong, 1993); (5) staging includes "unstandard" time frames (e.g., Intending to use CPAP in the next two weeks; Stepnowsky, 2002; and no screening mammogram 2-4 years ago, with one in the past 2 years, intends to have mammogram in future; Chamot et al., 2001). Further exploration is needed for such issues as impact of wording of time frame (one month versus 30 days), possible patterns of the use of time frame, relevance of particular time frames with respect to particular behavior (e.g., 2 weeks $=\mathrm{CPAP}$ and 2 years $=$ mammography).

Critics of the TTM consider the use of time frames (e.g., 6 months) "arbitrary" (Bandura, 1997; Sutton, 1996; Weinstein et al., 1998) and indicate that this is a contradiction to the idea that Stages differ based on qualitatively different attributes (Bandura, 1997; Weinstein et al., 1998). It is also thought that altering the time frame may alter the Stage distribution (Weinstein et al., 1998).

Behovioral Criteria. In some staging algorithms, Contemplation and Preparation are differentiated by the time frame criteria (seriously considering changing in the next six months versus the next month). In order to create a greater distinction between these two Stages a behavioral criterion is sometimes used. If a behavioral criterion is used, it is typically included in the Preparation Stage. 
Behavioral criteria demonstrate an attempt by the individual to take action. For instance, in a recent study, Keller et al. (2000) defined the Preparation Stage with the typical intention statement, time frame and then set behavioral criteria. The Preparation statement reads: I intend to change my behavior in the next 30 days and in the last 6 months, have made steps to actively deal with the topic of good body posture, like reading a book or watching a TV program about it.

Action Criteria. "Action criteria" typically indicates the particular behavioral criteria necessary for a participant to reach the Action Stage. This seemingly simple criterion can be the source of much debate when developing or evaluating Stage measures. When a behavior is clear, discrete, and agreed upon by a given field then setting a behavior criterion can be simple, for instance "quitting smoking". An action criteria of "reduction of cigarettes smoked" complicates the measurement of the behavior and behavior change. There is not a clear consensus among professionals or in the field how much reducing the amount of cigarettes per day is helpful to an individual. Choosing this criterion could minimize the value of the overall study outcomes or could introduce ethical concerns if in fact this strategy proves unhelpful or more harmful (e.g., as in the example menthol and light cigarettes). Additional complications arise concerning the need for tracking or recall for participants. For instance, it is much easier to remember if one did or did not smoke versus an individual remembering precisely the number of cigarettes smoked over the course of a day, a week or a month.

Utilizing gold standards in the field is often a good approach for setting an action criterion. But not all behaviors have established gold standards, for instance 
areas such as diet (healthy eating) / weight loss face much debate regarding the best strategies. For example, in the area of weight loss, it is unclear what the best strategy is - should one eat a low fat diet or the Atkins diet? In the area of nutrition, should individuals eat $5,7,9$, or 11 fruits and vegetable a day? Another example, treatment for sleep apnea - it is unclear what treatment is best, how often should the treatment be given or how long should the treatment last?

Response Format. Perhaps one of the most variable aspects of staging (aside from behavior) is the response format of the algorithms. Response formats include various combinations of characteristics such as: (1) length of definition; (2) number of questions; (3) true/false questions; (4) yes/no questions; (5) total number of items; and (6) single item vs. multiple item format. The multitude of combinations introduces a significant concern for comparability of measures. Studies have shown that different response formats can differentially impact Stage distributions; for example, algorithms using longer, more complete definitions of exercise produced larger number of participants in the earlier Stages, whereas Likert scale format resulted in lower percentage of participants in Precontemplation and Maintenance than fixed format. Additionally, in one study (Reed et al., 1997), the algorithm that provided a long definition and measured vigorous exercise using a 5-choice format revealed a pattern that most closely matched the theoretical prescription of the Pros and Cons across Stages as well as for the pattern for hours of exercise across Stages (i.e., hours increasing across Stages). In some cases similar results were found between response formats. For example, effect size comparisons found both long definitions measuring vigorous exercise using a 5-choice and true/false format 
equally effective across Pros, Cons, confidence, and hours of exercise. And lastly, true/false and choice formats were found to be comparable.

\section{Continuous Stage Approaches}

A variety of continuous measures have been developed to assess Stages of Change. The primary continuous Stage measures are considered clustering approaches (e.g., URICA, Socrates). These staging procedures do not offer consistent methods and staging (as described below) and therefore will not be included in the dissertation project analyses.

The most common continuous measure is the URICA (University of Rhode Island Change Assessment). The URICA scale is considered a more subtle approach to staging than the discrete Stage measures. This subtle approach has made it particularly appealing for clinical contexts. The URICA includes a lengthy set of statements that try to tap into the Stage of Change of an individual rather than relying on the individual to identify their readiness to change. The original scale consists of 32 items, 8 items for each of the four identified Stages at the time (Precontemplation, Contemplation, Action, and Maintenance). The URICA measure is thought to accurately measure Stage but unfortunately the many drawbacks of this approach have raised questions regarding the comparability of the URICA to the Stage algorithm approaches.

Stages Variations. The URICA (and similar procedures) often does not yield "traditional" Stage categories. Sometimes, the URICA approach Stages participants into four categories (Precontemplation, Contemplation, Action, and Maintenance). Other times the URICA (and similar approaches) includes alternative categories 
called "non-reflective action", "taking steps", "recognition" "ambivalent",

"uninvolved/discouraged" or "participation". The numbers of categories or clusters also vary, including 5- (DiClemente \& Hughes, 1990), 8- (McConnaughy et al., 1983 ) and 18-cluster solutions (McConnaughy et al., 1989) found in some studies.

Scale Construction Problems. A review by Littell and Girvin (2002) summarizes a variety of problems seen in cluster-type staging. Examples of scale construction issues include: (1) items in each scale are scored in the same direction increasing the likelihood of response sets; (2) many double barreled items are included in the scales; (3) the scales use many double negative items; (4) some items are awkwardly worded (e.g., "I'm not following through with what I had already changed as well as I had hoped, and I'm here to prevent a relapse of a problem"; Jefferson, 1991); (5) uncommonly used phases are included in items; (6) overlap among items cause possible inflation of internal consistency measure; and (7) generalizability is potentially limited since the scales were normed on middle-class, Caucasian participants.

Scoring issues. The cluster-type measures utilize a variety of methods for "scoring" or identifying Stages. The most simplistic method involves identifying the highest raw score or standardized score of a given scale and classifying an individual into a stage based on that scale. One complication of this method is that more than one scale can be tied. Strategies include: (1) placing the participant in the more advanced stage; (2) identifying ties as a new stage; or (3) a combination of approaches (Heather, Rollnick \& Bell, 1993). These strategies are rather arbitrary and clearly result in different stage distributions (Littell \& Girvin, 2002). 
Prochaska and DiClemente (1998) indicate that regardless of an individual's Stage of Change, participants may experiences attitudes described across the stage designated items, therefore more complex cluster approaches are likely to be the best method for interpreting the continuous instruments. Though currently, there is no definitive answer as to which of these procedures is best.

Stage of Change: Continuous or Discrete?

In the current studies only discrete measures of Stage of Change will be used. This will eliminate the methodological issues discussed above regarding the continuous clustering measures of Stage of Change as well as increase consistency across Stages as much as possible. Additionally, although there has been an on-going debate regarding the theoretical conceptualization for stage as continuous versus discrete, regardless of the outcome of this debate and regardless of when or if stage is measured on a continuous scale, practically speaking discrete stages are needed in order to tailor interventions.

\section{Decisional Balance: Pros and Cons of Change}

Following the original study by Velicer et al. (1985), the use of the construct began to expand, encompassing an array of behaviors such as exercise, condom use, and mammography screening. This early work culminated in a paper by Prochaska et al. (1994), which looked at patterns in Decisional Balance across Stages in 12 behaviors. This integrative study investigated: 1) the generalizability of the TTM for Stage and Decisional Balance across behaviors; 2) the generalizability of the TTM for a variety of populations; 3 ) the number of components in Decisional Balance and 
their respective internal consistencies; 4) patterns of Pros and Cons across Stages;

and 5) the Stage of crossover between standardized Pros and Cons scores. More recently, a meta-analysis examining 37 behaviors utilizing 81 datasets including nearly 40,000 participants was conducted re-examining these relationships and exploring additional ones (Hall \& Rossi, 2004).

\section{Generalizability}

Prochaska et al. (1994) and Hall \& Rossi (2004) found clear support for the generalizability of the Stages of Change, the Pros and Cons, and the integration between them. The researchers additionally found that these constructs generalized across a variety of populations and behaviors.

\section{Structure of Decisional Balance}

Consistent with the two-factor structure identified by Velicer et al. (1985), Prochaska et al. (1994) and Hall \& Rossi (2004) respectively found 92\% and 94\% of the studies reported a two-component solution for Decisional Balance with average internal consistency coefficients ranging from .72 to .95 and from .64 to .95 for Pros and Cons, respectively.

\section{Functional Aspects of Decisional Balance}

Decisional Balance Crossovers. In Kurt Lewin's (1948) expectancy theory, it is postulated that behavior changes as a function of the increases and decreases in motivation to contemplate gains and losses. The TTM builds on this notion by suggesting a clear directionality to the function as well as a characteristic way of examining it. This function is based on the relationship of when and how much the Pros increase and the Cons decrease (Prochaska et al., 1994) and is examined by 
identifying a graphical crossover of the Pros and Cons as they change relative to each other. It is believed that interaction of the Pros and Cons may increase or decrease the likelihood of a cognitive conflict between the benefits and disadvantages of a particular behavior. One possible interaction occurs when a person believes there are greater personal gains and less disadvantages associated with a behavior. It is hypothesized that at this time, the Pros increase and the Cons decrease, making it more likely that an individual will change their behavior. The crossover indicates an equal weighting in a person's cognitive framing of the gains and losses of engaging in a particular behavior. This equal weighting can create a state of ambivalence towards change.

This crossover therefore is a theoretically important marker for the success of the functional relationship between the Stages and Decisional Balance. Prochaska et al. (1994) and Hall \& Rossi (2004) found that the Decisional Balance crossovers occurred during the Contemplation Stage for $58 \%$ and $53 \%$ of the studies, respectively. Based on the timing of the crossover, the researchers suggest that progress from Precontemplation to Contemplation involves an increase in Pros whereas progress from Contemplation to Action involves a decrease in Cons.

Patterns across Stage. Several additional patterns in the relationship between Stage of Change and Decisional Balance were found in the two studies (Hall \& Rossi, 2004, Prochaska et al, 1994). For example, the Cons of Changing were higher than the Pros of Changing in the Precontemplation Stage for all datasets in both studies. Prochaska found the opposite true for 11 out of 12 behaviors in the Action Stage. 
Decisional balance (Pros and Cons) serves as an intermediate indicator of

when change will occur and has generally been thought to be especially salient in the earlier Stages of Change (Redding et al., 1999; Velicer et al., 2000). Velicer et al. (2000) also illustrated that the relationship between the Stages and Decisional Balance for an unhealthy behavior is different than for a healthy behavior. Specifically, the pattern for an unhealthy behavior was such that the Cons decreased across the Stages whereas the Pros displayed a curvilinear pattern, which paralleled the decline of the Cons in the later Stages. In contrast, the healthy behavior showed more of an X configuration, with the Pros continuing to increase across the Stages whereas the Cons decrease across the Stages.

Strong and Weak Principles.

Across twelve studies (Prochaska, 1994), mathematical relationships were found between the Pros and Cons of Changing and progress across the early Stages into Action (Prochaska, 1994). The Strong Principle of Change states that PC $\rightarrow \mathrm{A}$ $\cong 1 S D \uparrow$ PROS: progress from Precontemplation to Action involves approximately one standard deviation increase in the Pros of Changing. The Weak Principle of Change states that $\mathrm{PC} \rightarrow \mathrm{A} \cong 0.5 S D \downarrow$ CONS: progress from Precontemplation to Action involves approximately $0.5 S D$ decrease in the Cons of Changing.

In re-examination of the Strong and Weak Principles, the magnitude of the maximum increase in the Pros of change was again found to be greater than the maximum decrease in the Cons of change from Precontemplation to Action across 37 different health behaviors (Hall \& Rossi, 2004). Consistent with Prochaska's 
(1994) Strong principle, the average effect size for the Pros was approximately one standard deviation $(d=1.05, S D=0.45)$, almost identical to Prochaska's (1994) original finding ( $d=1.06, S D=0.26)$. Hall and Rossi's (2004) findings also revealed that Prochaska's Weak Principle may not be so weak. That is, the average effect size for Cons was stronger $(d=0.62, S D=0.38)$ than was found in the previous study $(d$ $=0.45, S D=0.22$ ) by Prochaska (1994), though clearly the Cons remains weak relative to the Pros. Practical implications of these principles are that the Pros of Changing must increase twice as much as the Cons must decrease, suggesting that an intervention place twice as much emphasis on raising the benefits as on reducing the costs or barriers.

\section{Stage Transitions}

The Strong and Weak Principles are useful for understanding the amount of work generally needed to move from Precontemplation to Action. Although theoretically the principles have been important in conceptualizing and understanding the relationship between Decisional Balance and Stages of Change, these principles are essentially action-oriented when applied to interventions. That is, by examining characteristics of the transition only from Precontemplation to Action, and using these to tailor interventions, one is potentially neglecting three Stages: Contemplation, Preparation and Maintenance. In addition, adjacent Stage transitions should be examined. Adjacent Stage transitions include Precontemplation to Contemplation (PC-C), Contemplation to Preparation (C-PR), Preparation to Action (PR-A), and Action to Maintenance (A-M). Therefore more practically, the 
examination of each Stage transition can help identify the most effective strategy for tailoring behavior change interventions.

\section{References}

Armstrong, C.A., Sallis, J.F., Hovell, M.F., and Hofstetter, C.R. (1993). Stages of change, self-efficacy, and the adoption of vigorous exercise: A prospective analysis. Journal of Sport and Exercise Psychology, 15, 390-402.

Bandura, A. (1977). Self-efficacy: Toward a unifying theory of behavior change.

Bandura, A. (1982). Self-efficacy mechanism in human agency. American Psychologist, 37, 122-147.

Chamot, E., Charvet, A.I., \& Perneger, T.V. (2001). Predicting stages of adoption of mammography screening in a general population. European Journal of Cancer, 37, 1869-1877.

DiClemente, C. C. (1986). Self-efficacy and the addictive behaviors. Journal of Social \& Clinical Psychology, 4(3), 302-315.

DiClemente C.C. \& Hughs, S.O. (1990). Stages of change profiles in outpatient alcoholism treatment. Journal of Substance Abuse, 2, 217-235.

DiClemente, C.C., Prochaska, J.O., Fairhurst, S.K., Velicer, W.F., Velasquez, M.M., \& Rossi, J.S. (1991). The process of smoking cessation: An analysis of precontemplation, contemplation and preparation stages of change. Journal of Consulting and Clinical Psychology, 59, 295-304.

Dijkstra, A. \& De Vries, H. (2000). Clusters of precontempating smokers defined by the perception of the pros, cons, and self-efficacy. Addictive Behaviors, 25, 373385. 
Etter, J.F., Perneger, T.V., \& Ronchi, A. (1997). Distributions of smokers by stage: international comparison and association with smoking prevalence. Preventive Medicine, 26, 580-85.

Hall, K.L., \& Rossi, J.S. (2004). Structure, function, and effect size of decisional balance across the stages of change for 37 health behaviors: A meta-analytic study. Unpublished Manuscript.

Heather, N., Rollnick, S., \& Bell, A. (1993). Predictive validity of the Readiness to Change Questionnaire. Addiction, 88(12), 1667-77.

Horn, D. \&Waingrow S. (1966). Some dimensions of a model for smoking behavior change. American Journal of Public Health, 56, 21.

Horn, D. (1976). A model for the study of personal choice health behavior. International Journal of the Addictions, 19, 89-98.

Janis, I. L., \& Mann, L. (1977). Decision making: A psychological analysis of conflict, choice and commitment. New York: Free Press.

Janis, I.L. (1959). Decisional conflicts: A theoretical analysis. Journal of Personality and Social Psychology, 31, 8-33-839.

Keller, S., Herda, C., Ridder, K., \& Basler, H. (2001). Readiness to adopt adequate postural habits: An application of the transtheoretical model in the context of back pain prevention. Patient Education and Counseling, 42, 175-184.

Lauver, D.R., Settersten, L., Kane, J.H., \& Henriques, J.B.(2003). Tailored messages, external barriers, and women's utilization of professional breast cancer screening over time. Cancer, 97(11), 2724-35. 
Lewin, K. (1948). Resolving social conflicts: Selected papers on group dynamics.

Gertrude W. Lewin (Ed.). New York: Harper.

Littell, J.H., \& Girvin, H. (2002). Stages of change: A critique. Behavior

Modification, 26(2), 223-273.

McConnaughy, E.A., DiClemente, C.C., Prochaska, J.O., Velicer, W.F.(1983).

Stages of change in psychotherapy: Measurement and sample profiles,

Psychotherapy: Theory, Research and Practice, 20, 368-375.

McConnaughy, E.A., DiClemente, C.C., Proschaska, J.O., Velicer, W.F.(1989).

Stages of change in psychotherapy: a follow up report. Psychotherapy: Theory, Research and Practice, 26, 494-503.

Pallonen, U. E., Velicer, W. F., Prochaska, J. O., Rossi, J. S., Bellis, J. M., Tsoh, J. Y., Migneault, J. P., Smith, N. F., \& Prokhorov, A. V. (1998). Computer-based smoking cessation interventions in adolescents: Description, feasibility, and sixmonth follow-up findings. Substance Use and Misuse, 33, 935-965.

Prochaska, J. O., Velicer, W. F., DiClemente, C. C., \& Fava, J. L. (1988). Measuring

Processes of Change: Applications to the cessation of smoking. Journal of Consulting \& Clinical Psychology, 56, 520-528.

Prochaska, J. O., Velicer, W. F., Rossi, J. S., Goldstein, M. G., Marcus, B. H., Rakowski, W., Fiore, C., Harlow, L. L., Redding, C. A., Rosenbloom, D., \& Rossi, S. R. (1994). Stages of change and decisional balance for 12 problem behaviors. Health Psychology, 13, 39-46. 
Prochaska, J.O. (1994). Strong and weak principles for progressing from precontemplation to action on the basis of twelve problem behaviors. Health Psychology, 13(1), 47-51.

Prochaska, J.O., \& DiClemente, C.C. (1982). Transtheoretical therapy: Toward a more integrative model of change. Psychotherapy, Theory, Research and Practice, 19, 276-288.

Prochaska, J.O., \&DiClemente, C.C. (1998). Comments, criteria, and creating better models: In response to Davidson. In W.R. Miller \& N. Heather (Eds.), Treating addictive behaviors ( $2^{\text {nd }}$ ed., pp 39-45). New York: Plenum.

Prochaska, J.O., Velicer, W.F., Guadagnoli, E., Rossi, J.S. \& DiClemente, C.C. (1991). Patterns of change: A dynamic typology applied to smoking cessation. Multivariate Behavioral Research, 26, 83-107.

Prochaska, J.O., Velicer, W.F., Rossi, J.S., Goldstein, M.G., Marcus, B.H., Rakowski, W., Fiore, C., Harlow, L.L., Redding, C.A., Rosenbloom, D., et al. (1994). Stages of change and decisional balance for 12 problem behaviors. Health Psychology, 13(1), 39-46.

Redding, C.A., Rossi, J.S., Rossi, S.R., Prochaska, J.O., \& Velicer, W.F. (1999). Health Behavior Models. In G.C. Hyner, K.W. Peterson, J.W. Travis, J.E. Dewey, J.J. Foerster, \& E.M. Framer (Eds.), SPM Handbook of Health Assessment Tools. Pittsburg, PA: The Society of Prospective Medicine \& The Institute for Health and Productivity Management. p. 83-93. 
Reed, G.R., Velicer, W.F., Prochaska, J.O., Rossi, J.R , \& Marcus, B.F. (1997).

What makes a good staging algorithm: Examples from regular exercise.

American Journal of Health Promotion, 12, 57-66.

Rossi, J.S., Benisovich, S.V., Norman, G.J., \& Nigg, C.R. (2004). Development of a hierarchical multidimensional measure of exercise self-efficacy. Manuscript submitted for publication.

Sutton, S. (1996). Can 'stages of change' provide guidance in the treatment of addictions? A critical examination of Prochaska and DiClemente's model. In Edward, G. \& Dare, C. (Eds.), Psychotherapy, Psychological Treatments and the Addictions, 189-205. Cambridge: Cambridge University Press.

Velicer, W. F., DiClemente, C. C., Prochaska, J. O., \& Brandenberg, N. (1985). A decisional balance measure for assessing and predicting smoking status. Journal of Personality and Social Psychology, 48, 1279-1289.

Velicer, W. F., DiClemente, C. C., Prochaska, J. O., \& Brandenberg, N. (1985). A decisional balance measure for assessing and predicting smoking status. Journal of Personality and Social Psychology, 48, 1279-1289.

Velicer, W. F., DiClemente, C. C., Rossi, J. S., \& Prochaska, J. O. (1990). Relapse situations and self-efficacy: An integrative model. Addictive Behaviors, 15, 271-283.

Velicer, W. F., Fava, J. L., Prochaska, J. O., Abrams, D. B., Emmons, K. M., \& Pierce, J. P. (1995). Distribution of smokers by stage in three representative samples. Preventive Medicine, 24, 401-411. 
Velicer, W.F., DiClemente, C.C., Rossi, J.S., \& Prochaska, J.O. (1990). Relapse situations and self-efficacy: An integrative model. Addictive Behaviors, 15, 271283.

Velicer, W.F., Prochaska, J.O., Fava, J.L., Rossi, J.S., Redding, C.A., Laforge, R.G., \& Robbins, M.L. (2000). Using the transtheoretical model for population-based approaches to health promotion and disease prevention. Homeostasis, 40, 174 195.

Weinstein, N. D., Rothman, A. J., \& Sutton, S. R. (1998). Stage theories of health behavior: Conceptual and methodological issues. Health Psychology, 17(3), 290299.

Wyse, J., Mercer, T., Ashford, B., Buxton, K., \& Gleeson, N. (1995). Evidence for the validity and utility of the stages of exercise behavior change scale in young adults. Health Education Research, 10, 365-377. 


\section{CHAPTER THREE}

\section{INTEGRATIVE APPROACH TO THEORY TESTING}

\section{Introduction}

In Sante Fe, New Mexico May of 2002, the National Cancer Institute's

Division of Cancer Control and Population Sciences Behavioral Research Program brought together a group of esteemed researchers for a workshop entitled, "Toward Better Theories of Health Behavior". The workshop was designed to help move the field forward by identifying and discussing gaps in health communication and health behaviors theory with the primary goal of "accelerating progress in the testing, validation, and development, and revision of health behavior theories" (National Cancer Institute: Division of Cancer Control and Population Sciences, 2002). This program of research directly answers to this important goal by testing several theoretical assumptions from a health behavior theory using innovative and unique methods in order to make recommendations for revising or rejecting parts of the theory. Furthermore, at this workshop, Weinstein indicated that "all current theories of health behavior have significant limitations" and that, "progress in improving theories that explain or encourage health behavior has been slow" (National Cancer Institute: Division of Cancer Control and Population Sciences, 2002). This program of research seeks to speed that process. Weinstein continues by explaining, "The scientific progress, in which theories are tested, weaknesses exposed, inadequate theories rejected, and better theories arise to take their place has not been taking place." This program of research intends to advance this scientific progress. 


\section{Integrative Approaches}

There are a variety of approaches that can be employed to test theory. In fact, most empirical studies attempt to test some theory at least in part. Powerful approaches to assess theoretical models involve integrative approaches. These are approaches which attempt to combine the data of previously conducted studies, such as combining longitudinal secondary data or employing meta-analytic strategies.

\section{Meta-Analysis for Theory Testing}

One particularly effective integrative tool is meta-analysis. Researchers encourage the use of meta-analysis for informing theory (Cook et al., 1992) as well as testing and advancing theory. This is in part because meta-analyses have the power to test large sets of explanatory mechanisms by more broadly examining moderators (Marsh et al., 2001) than traditional qualitative reviews as well as individual empirical studies. Therefore, meta-analyses have the potential to more comprehensively examine aspects of theories that are oftentimes not testable due to practical constraints. Researchers have begun utilizing traditional meta-analytic techniques as a basis of theory testing (e.g., Marsh et al., 2001).

Meta-analyses can be used for theory testing in varying degrees. A simple meta-analysis combines the results of previous studies, examining a single question the same question, asked by previous researchers. This type of meta-analysis is considered the lowest level and provides the least theoretical advance. That is, the simple meta-analysis can be an essential tool for clarifying inconsistencies in the literature, but typically introduces no new ideas and offers little additional information to the field. A more sophisticated method of employing meta-analysis 
for theory testing is to build on the aforementioned method by aggregating the effect sizes from the literature and subsequently assessing homogeneity. If heterogeneity is discovered, searches for moderators become possible. (It is possible to establish a priori moderator tests precluding the need for heterogeneity to be found). The introduction of moderator analyses enables the possibility of generating new theoretical evidence. Lastly, the most important method of conducting meta-analysis for theory testing involves testing theoretical relationships or concepts not previously conducted in primary-level studies. This creative use of previously conducted studies offers the highest potential for theoretical advance. This dissertation seeks to conduct this highest level meta-analysis.

Additionally, meta-analysis can be used to clarify conflicting results due to particular failings of null hypothesis testing, especially with single studies. The use of effect sizes can provide important information about effects that hypothesis testing does not offer, namely, the magnitude of an effect. Confidence intervals can provide a means to test statistical significance in meta-analysis and increase precision with each study added to a meta-analysis. Moreover, meta-analysis has typically been used as a method to simply combine results of studies based on null hypothesis testing. More sophisticated uses of meta-analysis can go beyond the simplicity of null hypothesis testing to identify results not previously reported in the literature, explore moderators of effect that may not be able to be examined in a single study, and even provide a tool for advancing theory. This program of research aims to provide results that overcome many of these hypothesis testing failings by synthesizing data from nearly 150 studies utilizing effect sizes and confidence 
intervals. Additionally, this meta-analysis aims to go beyond the simplicity of hypothesis testing exploring new relationships in the data and exploring moderators with the goal of advancing theory.

\section{Importance of Comprehensive and Systematic Review Strategies}

Although seemingly obvious, careful implementation of integration strategies is essential to the success of qualitative and quantitative reviews. Comprehensive and systematic review strategies have not often been found in previous reviews examining the TTM. For instance, hundreds of studies related to behavior change, and more specifically health-related behaviors have been conducted using the TTM, yet no study has integrated this vast body of literature. A small number of studies have attempted to review the TTM literature, but these studies have either: (1) limited their review to one behavior (e.g., Horowitz, 2003; Horwath, 1999; Marshall \& Biddle, 2001; Spencer, Pagell, Hellion \& Abrams, 2002); (2) qualitatively compiled studies (e.g., Horowitz, 2003; Horwath, 1999; Spencer et al., 2002; Riemsma et al., 2003); or (3) failed to systematically or comprehensively gather and analyze all available data (Riemsma et al., 2003; Spencer et al., 2002

Many meaningful relationships are lost in reviews that limit analysis to one behavior. Efforts in health promotion and disease prevention can best be served by understanding the similarities and differences in behavior change factors across behaviors. The advent of recent efforts to focus on multiple behavior change, where constellations of related (or unrelated) behaviors are treated simultaneously, make understanding the inter- and intra-relationships of behavior change factors increasingly critical for developing effective interventions. 
Most literature reviews examining the TTM have focused on overall

intervention effects or study design and methodology rather than quantitatively examining process-to-outcome relationships that can aid in improving intervention development. Additionally, the role of sample size and power has not always been fully appreciated as a factor in these reviews. Sample size is often a source of discrepancy in the results of outcome studies, especially as the effect size for most such interventions tends to be fairly small, particularly for population- or community-based interventions. A meta-analytic review of the literature is especially well suited to evaluate the role of sample size, effect size, and power in the generation of discrepant study outcomes (Rossi, 1990, 1997).

Inadequate review strategies can produce misleading results. For example, TTM tailoring is usually incorporated into interventions in one of three ways: 1) stage only - providing feedback specific to only an individual's SOC; 2) partial tailoring - providing individual feedback on SOC, Decisional Balance, and/or Selfefficacy; and 3) full tailoring - providing individual feedback on all TTM constructs, including SOC, Decisional Balance, Self-efficacy, and processes of change. In a qualitative review of smoking, Spencer et al. (2002) identified 22 studies evaluating TTM tailored or stage-matched interventions. The researchers concluded that the body of literature on staged-matched interventions was "acceptable" but not "conclusive." Upon closer examination, it appears that the authors did not perform the review in a careful systematic way. New and informative patterns were revealed by simply re-grouping the studies by the type of tailoring: 13 of the studies used stage only tailoring, 4 of which had positive results (Canga et al., 2000; Leed-Kelly, 
Russell, Bobo, 1996; Valanis et al., 2001; Wang, 1994), five of which had negative results (Lancaster et al., 1999; Lenox et al., 1998; Quinlan \& McCaul, 2000; Steptoe et al., 2001; Velicer et al., 1999), and 4 of which were unclear (Bernstein \& Stoduto, 1999; Goldberg et al., 1994; Pallonen et al., 1994; Rohren et al.., 1994). In contrast, the five partial tailoring studies produced positive results on a 3 to 2 ratio. The three positive studies were Coleman-Wallace et al. (1999), Dijkstra et al. $(1998,1999)$ and the two negative studies were Dijkstra et al. (1998) and Pallonen et al. (1998). Lastly, the five studies using full TTM tailoring produced positive results on a 4 to 1 ratio. The four positive studies were Prochaska et al. (1993, 2001a, 2001b), Velicer et al. (1999) and the negative study was Aveyard et al. (1999). In sum, partial tailoring studies were 2 times more likely and full tailoring studies were 2.7 times more likely to find significant treatment effects than stage only intervention studies. Additionally, in Spencer et al.'s (2002) review, length of follow-up was not accounted for when comparing studies. The five partial tailoring studies were limited to shorter-term effects with follow-ups ranging from only 10 weeks to 6 months, while the five full tailoring studies demonstrated more sustained effects with followups ranging from 18 to 24 months. Also, none of the partial tailoring studies involved population cessation, while four of the full tailoring studies were population-based. Hence, it appears the more demanding studies (e.g., full tailoring, long follow-ups and population-based) produced the highest rates of significant results and had the highest impacts on smoking. This example suggests that the variations in treatment effectiveness are possibly due to systematic differences in study and intervention designs. More specifically, variation in use, application, and 
measurement of the TTM may significantly contribute to the discrepancies in assessment of the overall effectiveness of TTM-based tailoring. In a meta-analytic review, these factors would be coded and analyzed to preclude such vague, contradictory, and misleading results. It is clear that more care and rigor is needed when compiling such rich data and that systematic analysis is critical to discovering such essential findings regarding key factors of successful interventions.

The use of existing datasets for secondary data analysis is often thought of as a simple procedure, but this is not necessarily the case. For instance, both Spencer et al. (2002) and Riemsma et al. (2003) performed a "comprehensive" review of smoking studies utilizing the TTM. Spencer et al. (2002) identified 22 stage-matched intervention studies and Riemsma et al. (2003) identified 23 such studies over similar time frames. Amazingly, only 7 of the studies were the same. Inadequate or selective data collection inherently leads to bias. Therefore, it is clear that sufficient time and resources need to be devoted to systematic data collection in order to attain a minimally biased and maximally complete set of datasets for a meta-analytic review.

\section{Integrative and Meta-analytic Approaches to Testing the TTM}

In addition to reviews by Spencer et al. (2002) and Riemsma et al. (2003) a variety of of studies have used integrative approaches to examine the TTM; some of these are highlighted below.

\section{Predicting Behavior Change}

A powerful question that drives psychology is: can we predict behavior? And if so, how can we predict behavior? The Transtheoretical Model explains that 
individuals move through a series of Stages as an individual prepares to and eventually succeeds in changing a given behavior. In this way the model can predict behavior. Two ways to explore these predictions are to examine relationship of the constructs to stage movements and stage effects.

Stage Movement. In the context of the Transtheoretical Model, the concept that primarily is being predicted is stage movement. That is, can the model help predict the movement of individuals from one stage to the next? As described above, the model also predicts that particular constructs serve as indicators for change and play a part in predicting stage movement. For instance, one may ask - do the processes of change predict movement from Action to Maintenance? More specifically, since experiential processes have been shown to be more salient in the earlier Stages and the behavioral processes more salient in the later Stages, we might refine that question to - do the behavioral processes predict stage movement from Action to Maintenance? These predictive questions have been at the heart of recent debates. Overall, the model's ability to make these predictions has been demonstrated cross-sectionally, cross-sequentially, and longitudinally. Although it is beyond the scope of this essay to review the body of literature on the predictive nature of the model one particular integrative method, examining stage effects, will be highlighted. In sum, it is clear that the Stages of Change play the key role in understanding this key aspect of the model.

Stage Effects. Examining stage effects is another means to explore the predictive nature of the model, and more importantly the role of stage in the context of the model. Stage effects involve the prediction of a person's behavior over time 
depending on which stage the individual is in at baseline. More specifically, a person further along in the Stages is predicted to be more likely to move to Action or Maintenance than a person in the earlier Stages. Overall, stage effects across behaviors such as sun, smoking, and diet have been supported (Krebs, 2003; Prochaska et al., 2003). This suggests that persons in Precontemplation were less likely to take Action than those in Contemplation, who were, in turn, less likely to take Action than those in Preparation. An important implication of these findings is that moving forward one stage can increase the adoption of the target behavior. For instance, recent research has demonstrated that stage movement from Precontemplation to Contemplation increased the chances that smokers quit by $75 \%$ (Prochaska et al., 2003). Again, we see the importance of the Stages of Change in the context of the model.

\section{Moderators of Stage Distributions}

In a preliminary review of the literature a variety of moderators of stage distributions have been found. Examples include study characteristics and sample characteristics as well as behavior specific variables. More specifically, these moderators have included variables such as behavior, age, gender, setting, income, education, race, ethnicity, country, recruitment method, response format and activity criteria. For sake of brevity, several of the main moderating variables will be discussed in more detail below.

Behavior. Overall, stage distribution is typically examined across behaviors in the context of other potentially moderating variables and therefore will be touched 
upon in most other sections. The only review identified in this preliminary

examination of stage distributions that specifically compared stage distributions across behaviors was a study conducted by Nigg, Burbank, Padula, Dufresne, Rossi, Veleicer, LaForge, \& Prochaska (1999). This study examined Stage distributions across ten health behaviors in older adults (and many findings will be highlighted in the age section). In the broadest scope, the researchers discovered that highest percentages of individuals across the age groups were in either the Precontemplation or Maintenance Stages across all behaviors. This finding indicates that for all behaviors getting people to think about change may be the most important place to start in population-based interventions.

When examining across behaviors we can assess trends in readiness to change, adoption/cessation of a behavior, and maintenance and then use these trends can help determine where the greatest emphasis should be placed for heath interventions. One example might be utilizing stage distribution information to determine which order behaviors will be intervened upon in a multiple behavior intervention. An intervention targeting a large population may want to begin with a behavior where the most individuals are ready to change. Successful change in one behavior may increase participant confidence and potentially lead to greater success for subsequent behaviors. If this idea is valid, accomplishing this may best be done by tailoring the order of behaviors at the individual level. But individual tailoring to this extent may not be possible or practical. For instance, many multi-level multibehavioral interventions include group wide (e.g., school or workplace) intervention 
activities or campaigns for a given behavior. In this case, it likely would be best to simultaneously work on the same behavior at the group level and individual level.

Age. In a meta-analytic study examining exercise, Marshall and Biddle (2001), found that the youngest group $(<25)$ had the lowest percentage of individuals in Precontemplation and the highest proportion in Preparation. In contrast, the oldest group $(55+)$ had the highest proportion in extreme Stages, Precontemplation and Maintenance. Velicer, Fava, Prochaska, Abrams, Emmons, and Pierce (1995) found stable patterns in stage distributions across three samples of smokers for three of four age categories. The age categories consisted of 18-24 years, 24-44 years, 45-64 years, and $64+$ years. Of these, the oldest category, $64+$ years, was consistently different from the other three categories.

Nigg et al. (1999) looked specifically at stage distributions in various older samples. Differential distributions were identified for these age groups and especially in comparison to distributions in younger adults. Some interesting patterns were identified across behaviors for age, for instance the prevalence of maintaining a lowfat and high fiber diet increased as age increased whereas for exercise distributions increasingly stratified to the extremes as age increased (consistent with findings by Marshall \& Biddle, 2001). Overall, of the 10 behaviors all but two showed higher prevalence of individuals maintaining behaviors in one of the two oldest age groups (65-74 and $75+)$. This may be due to the fact that individuals who maintain healthy behaviors in fact live longer. Therefore, it seems this level of specificity is important for guiding age-related interventions, especially with regards to the elder end of the spectrum. For instance, an intervention developed for an assisted living facility 
would best be informed with data specific to this age group rather than with data from the general population.

Study Characteristics. Marshall and Biddle (2001) conducted a study examining stage distributions for exercise in a variety of different samples. Their results indicate that stage distributions were influenced by factors such as sampling method, with random / nonrandom recruitment, and active / passive recruitment strategies. More specifically, passive recruitment and nonrandom sampling strategies resulted in a higher percentage of participants in the later Stages where as active recruitment and random sampling strategies resulted in higher percentages of participants in the earlier Stages. Likert scale format for staging resulted in lower percentage of participants in Precontemplation and Maintenance than fixed format. Ultimately, it is important to be able to know how a particular study design impacts stage distributions to enable researchers to more accurately interpret findings of a particular study or more adequately design a new study.

Gaps. Overall, more studies examining stage distributions are warranted. Specifically, a comprehensive review examining multiple moderators across a variety of behaviors would be appropriate. A variety of limitations in previously conducted studies have left gaps in the exploration of stage distributions. For example, these studies have either: (1) limited their review to one behavior (e.g., Marshall \& Biddle, 2001; Velicer et al. 1995); one age group (Nigg et al., 1999) or one specific staging algorithm (Nigg et al.,1999; Velicer et al., 1995); (2) did not include all Stages (Velicer et al., 1995); (3) were unable to explore the influence of study design (Nigg et al, 1999; Rossi, 1992; Velicer et al., 1995); (4) examined only 
US samples (Nigg et al., 1999; Velicer et al., 1995); and (5) failed account for multiple operationism (Marshall \& Biddle, 2001).

\section{Strong and Weak Principles}

Hall and Rossi (2004a) re-examined Prochaska's (1994) Strong and Weak Principles for Decisional Balance by updating the number of studies and expanding the types of analyses conducted. Hall and Rossi (2004a) investigated the crossover profile patterns and effect sizes for Decisional Balance across the Stages of Change for 37 different health behaviors in 81 independent datasets including nearly 40,000 participants. Behaviors such as exercise, smoking, and condom use were represented by more than 10 datasets each, enabling preliminary exploration of each of these behaviors in more detail.

Patterns consistent with those reported by Prochaska et al. (1994) were found for Pros and Cons; for instance, in all studies the Cons of Changing outweighed the Pros of Changing during the Precontemplation stage. The "crossover" pattern of the Pros and Cons occurs when the standardized Decisional Balance scores are graphed by stage. Conceptually, the crossover point is thought to represent the ambivalence individuals feel when they begin to seriously consider changing their behavior. Prochaska et al. (1994) and Hall and Rossi (2004a) found that the Decisional Balance crossovers occurred during the Contemplation stage for $58 \%$ and $53 \%$ of the studies, respectively. However, although often thought of as a simple function, Hall and Rossi (2004a) discovered complexities in the crossover patterns. For example, $16 \%$ of the datasets revealed multiple crossovers of the Pros and Cons, suggesting 
further examinations are needed. One methodological consideration of the crossover function is that many of the studies found very unequal numbers of participants across the Stages of Change. Unequal sample size distributions affect the standardized score estimates of the Pros and Cons and tends to distort the crossover profile pattern, typically by "pulling" the crossover point towards either Precontemplation or Maintenance, since it is usually one of these two Stages that contains disproportionately greater numbers of participants. Recent work by Hall and Rossi (2004b) has begun the development of strategies to standardize these unequal sample size distributions which will allow a "truer" pattern to be revealed, enabling more accurate testing of theory and shedding more light on the meaning of the crossovers and what influences them.

In re-examining the strong and weak principles (Prochaska, 1994), the magnitude of the maximum increase in the Pros of Change was again found to be greater than the maximum decrease in the Cons of Change from Precontemplation to Action (Hall \& Rossi, 2004a). Consistent with Prochaska's (1994) strong principle, the average effect size for the pros was approximately one standard deviation $(d=$ $1.05, S D=0.45)$, almost identical to Prochaska's (1994) original finding $(d=1.06$, $S D=0.26$ ). But our findings also revealed that Prochaska's weak principle may not be so weak. That is, the average effect size for cons was stronger $(d=0.62, S D=$ $0.38)$ than was found in the previous study $(d=0.45, S D=0.22)$ by Prochaska (1994), though clearly the cons remains weak relative to the pros.

Heterogeneity of the distribution of effect size across all datasets was found, which signifies the presence of modifiers of effect size. Preliminary exploration of 
the moderators was conducted, beginning with behavior. Behaviors represented by more than 10 datasets were compared; four behaviors met this criterion (smoking, exercise, diet, condom use). Significant differences between behaviors were found for both Pros and Cons of change. The magnitude of effect for pros of exercise was significantly greater than for smoking, condom use, and diet. The effect size for cons of smoking was significantly greater than for diet and condom use. Examination of heterogeneity within each behavior was also significant, therefore additional moderators were explored.

Studies were characterized as either focusing on the cessation or acquisition of a behavior. Analyses revealed that cessation and acquisition behaviors did not differ significantly. One explanation could be that, conceptually, the distinction between cessation and acquisition may not be as straightforward as it initially appears. For instance, with a behavior like exercise, the emphasis may be on increasing or encouraging a person to exercise regularly, but this also means the person ultimately must decrease their sedentary behavior. Therefore, although measures of Decisional Balance and other TTM constructs may focus on the acquisition of a particular behavior, achieving acquisition may involve the cessation of other behaviors as well. More elaborate coding methodology may be necessary in order to differentiate more accurately the cessation and acquisition characteristics of the behaviors.

"Framing" Decisional Balance in the context of healthy versus an unhealthy behavior also showed no significant relationship to effect size (e.g., pros of quitting smoking versus the pros of smoking). The number of items in the Decisional Balance 
scale was also unrelated to effect size. Effect size was related to gender for cons but not pros. One implication of this finding is that expert system scoring algorithms might need to be different for men and women, at least for the Cons of Change.

Experimenter bias was also examined as a potential moderator of effect size. All 81 datasets were divided into three groups: studies performed by investigators at the Cancer Prevention Research Center (CPRC) at the University of Rhode Island (the originators of the Stages of Change and Decisional Balance measures); studies performed by CPRC trained researchers but conducted outside the center; and studies conducted by researchers with no formal CPRC affiliation. Bias was assessed by examining effect sizes rather than significance levels ( $p$-values). This method provides a more sensitive context within which to investigate bias since, unlike significance tests, these indices are independent of sample size. So far as we know, this was the first time experimenter bias has been evaluated in this way. Analyses showed that there were no significant differences in effect sizes between the three groups. These findings suggest that the effect size results are not biased towards the researchers who originally developed the Decisional Balance measure and that the measures can be modified and applied by a variety of researchers with equal effectiveness.

The main limitation of this study was that all of the datasets were crosssectional. We can not assume the same relationships will necessarily occur when examining the movement of participants from Precontemplation to Action with longitudinal data, therefore it is important these results be replicated using longitudinal data. Additionally longitudinal data allows for more elaborate 
exploration of moderators and subsamples, which can increase the richness of the results. The analyses of potential moderators in this study were conducted using correlational and univariate statistics. More sensitive tools such as random effects modeling need to be utilize to advance such research.

Only 4 of the 37 behaviors had sufficient numbers of datasets to perform subanalyses by behaviors. More comprehensive data collection and updated searches of published and unpublished research will increase these numbers, greatly enhancing the depth of the results. Since average effect size significantly varied by behavior, it is important to look carefully at the effect sizes for individual behaviors rather than just across all behaviors as more studies are conducted and identified for each behavior.

\section{Self-efficacy Across the Stages}

Both Self-efficacy and the Stages of Change serve as important aspects of the design and evaluation of clinic-based and population-based health promotion interventions. Rossi and Redding (2001) examined the relationship between Selfefficacy and the Stages in 28 independent studies across 14 behaviors (total $\mathrm{N}=$ $21,244)$, including cancer-related behaviors such as smoking cessation and prevention, exercise adoption, dietary fat reduction, fruit and vegetable consumption, sun exposure, cocaine use, binge drinking, weight control, contraceptive use, highrisk sex and condom use.

The functional relationship between Self-efficacy and the Stages of Change varied across behaviors but typically was monotonically increasing and linear across 
Stages. Studies that assessed both situational confidence and temptations displayed a consistent profile across Stages with a characteristic "crossover" towards the Action stage.

The magnitude of effect was examined in a similar fashion as the strong and weak principles for Decisional Balance (described above). The magnitude of the maximum increase from Precontemplation to Maintenance was strong and fairly consistent across behaviors, about 1.5 standard deviations, suggesting that Stage of Change accounts for about $36 \%$ of the variance in Self-efficacy.

This preliminary study again demonstrates the use of effect sizes to quantify theoretical relationships. Unfortunately this study is insufficiently powered and limited in scope.

\section{Longitudinal Analyses of the TTM}

Rossi (2001) conducted a very preliminary study of stage transition effect sizes for the processes of change in a single longitudinal dataset. In general, sample sizes in population-based health promotion research are often so large that statistical power can be quite high even for fairly small effect sizes. Consequently, all or most statistical tests may be significant. This can lead to difficulty in distinguishing between important effects and trivial ones. The distinction often cannot be made simply on the basis of the magnitude of effect sizes but must be grounded in theory. For example, the transtheoretical model posits that behavior change occurs in a series of Stages and includes a number of independent and intervening variables underlying the change process. The model makes explicit predictions about which variables are 
most important across each stage transition. For the transition from Precontemplation to Contemplation, the experiential processes of change are expected to be more salient. For the transition from Contemplation to Preparation, the behavioral processes of change are expected to be more important. These transitions were examined using data from a smoking cessation study (DiClemente et al., 1991; Prochaska et al., 1993). Because the sample size for this study was large $(N=1466)$, nearly all statistical tests across stage transitions were significant. However, calculation of contrast effect sizes for each stage transition showed a clear pattern that was consistent with transtheoretical model predictions: for the early stage transition, effect sizes were larger for experiential than for behavioral processes; for the later stage transition, effect sizes were larger for behavioral than for experiential processes. The utility of effect sizes for the analysis of population-based research is evident, especially as compared to standard significance testing approaches. The use of such procedures within the context of theory-based research promises to be of even greater utility in the development of more quantitative models of health behavior change.

\section{Stage Transitions}

The strong and weak principles are useful for understanding the amount of work generally needed to move from Precontemplation to Action. Therefore, although the strong and weak principles have been important in conceptualizing and understanding the relationship between Decisional Balance and Stages of Change, these two principles have an action-oriented influence when applied to interventions. 
That is, by virtue of examining characteristics of the transition from

Precontemplation to Action and using these to tailor interventions, one is gathering information which potentially neglects three Stages: Contemplation, Preparation and Maintenance. If the ultimate goal is to advance the quantitative resources for development of stage matched tailored interventions in order to move people from Precontemplation to Contemplation to Preparation to Action and then finally to Maintenance, more specific transitions should be examined. It then becomes imperative to examine the magnitude of effect for each stage transition in order to explore the possibility of more complex impact patterns. For instance, some behaviors maintain a classic crossover profile, but that does not necessarily mean that their patterns of effect are straightforward; the patterns of effect may be curvilinear rather than linear. In order to identify curvilinear patterns in the relationship between Decisional Balance and Stages of Change one would need to examine the magnitude of adjacent stage transitions.

Stage transition analyses could identify the most effective strategy for tailoring behavior change interventions. For example, for a given behavior if the effect size from Precontemplation to Contemplation for pros is large but very small for cons, in this instance pros could be intervened on whereas cons could be eliminated for precontemplators in an intervention. From Contemplation to Preparation, if both the Pros and Cons effect sizes were large, both might be emphasized at this transition in an intervention. Following this strategy across each stage transition would create the most efficacious and efficient application of the measure, maximizing the impact and minimizing the resource expenditure. 
Ultimately, careful and systematic investigation of the changes in these measures across stage transitions by behavior can provide even more detailed evidence for exactly how to use these measures most efficiently in future stage-matched interventions.

\section{Integrative Analyses: Current Project}

The current project was conducted in three main phases: (1) comparison of effect size procedures and development of correction formulas through the use of meta-analytic data; (2) comprehensive cross-sectional meta-analytic investigation of stage and Decisional Balance; (3) exploratory integrative analysis of longitudinal investigation Stage and Decisional Balance and cross-sectional and longitudinal comparison of stage and Decisional Balance. Each phase utilized integrative techniques building on each other to create a comprehensive program of studies aimed at testing and advancing theory and informing intervention development.

The first phase is a preliminary study to help inform and facilitate a more comprehensive exploration of the Stages of Change and Decisional Balance in phases two and three. The second and third phases will each contribute a set of results, which will uniquely contribute to the aims of the project. Additionally, phase three integrates the results of the cross-sectional and longitudinal studies by comparing similarities and differences between the two. That is, in phase three, the longitudinal data will serve as a cross-validation of the cross-sectional data among common relationships between the two types of data. 


\section{References}

Aveyard, P., Cheng, K. K., Almond, J., et al. (1999). Cluster randomized controlled trial of an expert system based on the Transtheoretical ("stages of change") Model for smoking prevention and cessation in schools. British Medical Journal, 319, 948-953.

Bernstein, S. M., Stoduto, G. (1999). Adding a choice-based program for tobacco smoking to an abstinence-based addiction treatment program. Journal of Substance Abuse Treatment, 17, 167-173.

Canga, N., DeIrala, J., Vara, E. et al. (2000). Intervention study for smoking cessation in diabetic patients. Diabetes Care, 23, 1455-1460.

Coleman-Wallace, D., Lee, J. W., Montgomery, et al. (1999). Evaluation of developmentally appropriate programs for adolescent tobacco cessation. Journal of Social Health, 69, 314-319.

Cook, T.D., Cooper, H., Cordray, D.S., Hartmann, H., Hedges, L.V., Light, R.J., Louis, T.A., Mostseller, F. (1992). Meta-Analysis for Explanation. New York: Russell Sage Foundation.

DiClemente, C.C., Prochaska, J.O., Fairhurst, S.K., Velicer, W.F., Velasquez, M.M., \& Rossi, J.S. (1991). The process of smoking cessation: An analysis of precontemplation, contemplation and preparation stages of change. Journal of Consulting and Clinical Psychology, 59, 295-304.

Dijkstra, A., De Vries, H. \& Roijackers, M. A. (1999). Targeting smokers with low readiness to change with tailored and nontailored self-help materials. Preventive Medicine, 28, 203-211. 
Dijkstra, A., De Vries, H., Roijackers, J. \& Van Breukelen, G. (1998). Tailored interventions to communicate stage-matched information to smokers in different motivational stages. Journal of Consulting and Clinical Psychology, 3, 549-557.

Goldberg, D.N., Hoffman, A.M., Farinha, M.F., Marder, D.C., Tinson-Mitchem, L. Burton, D., \& Smith, E.G. (1994). Physician delivery of smoking-cessation advice based on the stages-of-change model. American Journal of Preventive Medicine, 10(5), 267-74.

Hall, K.L., \& Rossi, J.S. (2004a). Structure, function, and effect size of decisional balance across the stages of change for 37 health behaviors: A meta-analytic study. Manuscript in preparation.

Hall, K.L., \& Rossi, J.S. (2004b). Determining effect size for meta-analysis: A comparison of alternative procedures. Manuscript in preparation.

Horowitz, S. M. (2003). Applying the transtheoretical model to pregnancy and STD prevention: a review of the literature. American Journal of Health Promotion, 17(5), 304-328.

Horwath, C. C. (1999). Applying the transtheoretical model to eating behaviour change: Challenges and opportunities. Nutrition Research Reviews, 12, 281-317. Krebs, P. (2003). Composition and comparison of stage effects for smoking cessation, dietary fat reduction, and sun protection. Unpublished Masters Thesis, University of Rhode Island, Kingston.

Lancaster, T., Dobbie, W., Vos, K., et al. (1999). Randomized trial of nurse-assisted strategies for smoking cessation in primary care. British Journal General Practice, 49, 267-274. 
Leed-Kelly, A., Russell, K. S., Bobo, J. K. (1996). Feasibility of smoking cessation counseling by phone with alcohol treatment center graduates. Journal of Substance Abuse Treatment, 13, 203-210.

Lennox, A. S., Bain, N., Groves, J., et al. (1998). Stages of Change training for opportunistic smoking intervention by the primary health care team. Part I: randomized controlled trial of the effect of training on patient smoking outcomes and health professional behaviour as recalled by patients. Health Education Journal, 57, 140-149.

Marsh, K.L., Johnson, B.T., Carey, M.P. (2001). Conducting meta-analyses of HIV prevention literatures from a theory-testing perspective. Evaluation \& The Health Professions, 24(3):255-76.

Marshall, S. J., \& Biddle, S. J. (2001). The transtheoretical model of behavior change: a meta-analysis of applications to physical activity and exercise. Annals of Behavioral Medicine, 23(4), 229-246.

National Cancer Institute: Division of Cancer Control and Population Sciences Behavioral Research Program. (2002). Toward Better Theories of Health Behavior, Theories Projects Workshop 2002, Meeting Summary. Retrieved December 5, 2003. http://dccps.nci.nih.gov/brp/santafe_workshop_summaries. pdf

Nigg, C.R., Burbank, P., Padula, C., Dufresne, R., Rossi, J. S., Velicer, W. F., Laforge, R. G. \& Prochaska, J. O. (1999). Stages of change across ten health risk behaviors for older adults. The Gerontologist, 39, 473-482. 
Pallonen, U. E., Leskinen, L., Prochaska, J. O., Willey, C. J., Kaariainen, R., \&

Salonen, J. T. (1994). A 2-year self-help smoking cessation manual intervention among middle-aged Finnish men: An application of the Transtheoretical Model. Preventive Medicine, 23, 507-514.

Pallonen, U. E., Velicer, W. F., Prochaska, J. O., Rossi, J. S., Bellis, J. M., Tsoh, J. Y., Migneault, J. P., Smith, N. F., \& Prokhorov, A. V. (1998). Computer-based smoking cessation interventions in adolescents: Description, feasibility, and sixmonth follow-up findings. Substance Use and Misuse, 33, 935-965.

Prochaska, J.J., Rossi, J.S., Redding, C.A. Hall, S.M., Rosen, A.B., Tsoh, J.Y., Humfleet, G.L., \& Eisendrath, S.J. (in press). Depressed Smokers and Stage of Change: Implications for Cessation Interventions. Drug and Alcohol Dependence.

Prochaska, J. O. (1994). Strong and weak principles for progressing from precontemplation to action based on twelve problem behaviors. Health Psychology, 13, 47-51.

Prochaska, J. O., DiClemente, C. C., Velicer, W. F., \& Rossi, J. S. (1993). Standardized, individualized, interactive, and personalized self-help programs for smoking cessation. Health Psychology, 12, 399-405.

Prochaska, J.O., Velicer, W.F., Fava, J.L., Rossi, J.S., \& Tsoh, J.Y. (2001a).

Evaluating a population-based recruitment approach and a stage-based expert system intervention for smoking cessation. Addictive Behaviors, 26, 583-602.

Prochaska, J. O., Velicer, W. F., Fava, J. L., Ruggiero, L., Laforge, R. G., Rossi, J. S., Johnson, S. S., \& Lee, P. A. (2001b). Counselor and stimulus control 
enhancements of a stage-matched expert system intervention for smokers in a managed care setting. Preventive Medicine, 32, 23-32.

Prochaska, J. O., Velicer, W. F., Rossi, J. S., Goldstein, M. G., Marcus, B. H., Rakowski, W., Fiore, C., Harlow, L. L., Redding, C. A., Rosenbloom, D., \& Rossi, S. R. (1994). Stages of change and decisional balance for 12 problem behaviors. Health Psychology, 13, 39-46.

Quinlan, K. B., McCaul, K. D. (2000). Matched and mis-matched interventions with young adult smokers: testing a stage theory. Health Psychology, 9, 165-171.

Riemsma, R. P., Pattenden, J., Bridle., C, Sowden, A. J., Mather, L., Watt, I. S., \& Walker, A. (2003). Systematic review of the effectiveness of stage based interventions to promote smoking cessation. British Medical Journal, 326, 11751177.

Rohren, C. L., Croghan, I. T., Hurt, R. D. et al. (1994). Predicting smoking cessation outcome in a medical center from stage of readiness: Contemplation vs. action. Preventive Medicine, 23, 335-344.

Rossi, J. S. (1990). Statistical power of psychological research: What have we gained in 20 years? Journal of Consulting and Clinical Psychology, 58, 646-656.

Rossi, J.S. (1992, August). Common processes of change across nine problem behaviors. Paper presented at the $100^{\text {th }}$ annual convention of the American Psychological Association, Washington, DC.

Rossi, J. S. (1997). A case study in the failure of psychology as a cumulative science: The spontaneous recovery of verbal learning. In L. L. Harlow, S. A. Mulaik, \& J. 
H. Steiger (Eds.), What if there were no significance tests? (pp. 175-197).

Hillsdale, NJ: Lawrence Erlbaum.

Rossi, J. S. (2001). Contrast effect size analysis and theory testing: An example using the transtheoretical model. Annals of Behavioral Medicine, 23 (Suppl.), S156.

Rossi, J. S., \& Redding, C. A. (2001). Structure and function of self-efficacy across the stages of change for 10 health behaviors. Annals of Behavioral Medicine, 23, S094.

Spencer, L., Pagell, F., Hellion, M. E., \& Abrams, D. B. (2002). Applying the Transtheoretical model to tobacco cessation and prevention: A review of the literature. American Journal of Health Promotion, 17(1), 7-71.

Steptoe, A., Kerry, S. Rink, E. et al. (2001). The impact of behavioral counseling on stage of change in fat intake, physical activity, and cigarette smoking in adults at increased risk of coronary heart disease. American Journal of Public Health, 91, 265-269.

Valanis, B., Lichtenstein, E., Mullooly, J. P., Labuhn, K., Brody, K., Severson, H. H., \& Stevens, N. (2001). Maternal smoking cessation and relapse prevention during health care visits. American Journal of Preventive Medicine, 20(1), 1-8 Velicer, W. F., Fava, J. L., Prochaska, J. O., Abrams, D. B., Emmons, K. M., \& Pierce, J. P. (1995). Distribution of smokers by stage in three representative samples. Preventive Medicine, 24, 401-411.

Velicer, W. F, Prochaska, J. O., Fava, J. L., Laforge, R. G., \& Rossi, J. S. (1999). Interactive versus non-interactive interventions and dose-response relationships 
for stage matched smoking cessation programs in a managed care setting.

Health Psychology, 18, 21-28.

Wang, W. D. (1994). Feasibility and effectiveness of stages-of-change model in cigarette smoking cessation counseling. Journal of Formosa Medical Association, 93, 752-757. 


\section{CHAPTER FOUR}

\section{META-ANALYTIC APPROACH}

\section{Brief History}

Methods of combining data were being developed as early as 1904 when Karl Pearson combined average correlations across five independent samples (Cooper \& Hedges, 1994), and furthermore into the 1930's (Fisher, 1932; Pearson 1933), but these integration techniques were rarely applied (Cooper, 1989). In 1976, Gene Glass coined the term "meta-analysis". Together with the increasing need for integrating research findings, Glass's fitting term seemed to finally launch this methodology into the field. Since then, meta-analytic techniques have continued to grow more complex and have slowly been expanding to meet the needs of the growing applications across the social and medical sciences.

\section{Failings of the Null}

In the behavioral and social sciences, empirical questions have been explored and theories guiding those questions have been tested primarily using null hypothesis procedures. As the field progresses, it is becoming clearer that this indirect procedure is an inadequate tool for efficiently answering research questions or for testing, modifying or rejecting theories. The focus on rejection of the lack of a relationship among variables as a means to clarify a research question not only obfuscates the objective, but also can ultimately be misleading.

The failure to reject the null hypothesis can occur for a variety of reasons, for example, insufficient power due to small sample sizes. Additionally, sample sizes 
can be so large that statistical power essentially becomes "too high", which can lead to all or most statistical tests being statistically significant and thereby make it difficult to distinguish between important effects and trivial ones. Furthermore, null hypothesis testing can only provide ordinal or directional characterization of a relationship between variables not the magnitude of particular relationships.

\section{The Role of Meta-analysis in the Significance Testing Debate}

Meta-analytic techniques can be used to help overcome some of the limitations of null hypothesis testing as well as to move beyond null hypothesis testing. Meta-analysis can help resolve conflicting results, reduce variability in results, and increase the precision of reported results. Meta-analysis can best achieve this by synthesizing studies using point estimates and confidence intervals.

Meta-analysis can be used to synthesize the results of a body of conflicting studies and avoid some of the pitfalls of relying on traditional hypothesis testing. For example, low power can result for a variety of reasons such as not considering issues of power when planning a study, low retention during the course of a study and insufficient resources to achieve adequate power. Low power typically will create conflicting results within a body of literature due to statistical probability in null hypothesis testing. In fact, the effect size of two studies can be equal even when their level of significance may differ (i.e., one significant, the other not) due to variations in sample size. Meta-analyses can combine the results of multiple studies to overcome issues of low power. By placing confidence intervals around effect sizes the overall significance of a body of research can be determined and thereby clarify discrepant results found among individual studies. 
Additionally, meta-analysis can be seen as overcoming failings of traditional hypothesis testing by increasing the precision of results by accounting for sampling error and other sources of variance. Meta-analytic techniques, by controlling for sampling error, can reduce variability in results. Likewise, corrections for study artifacts such as measurement error can be implemented using meta-analytic techniques. Various methods for modeling these types of error (i.e., fixed, random, and mixed effects modeling) can further increase precision in meta-analyses.

In sum, meta-analysis can be used to clarify conflicting results due to particular failings of null hypothesis testing, especially with single studies. The use of effect sizes can provide important information about effects that hypothesis testing does not offer, namely, the magnitude of an effect. Confidence intervals can provide a means to test statistical significance in meta-analysis and increase precision with each study added to a meta-analysis. Moreover, meta-analysis has typically been used as a method to simply combine results of studies based on null hypothesis testing. More sophisticated uses of meta-analysis can go beyond the simplicity of the null hypothesis testing to identify results not previously reported in the literature, explore moderators of effect that may not be able to be examined in a single study, and even provide a tool for advancing theory.

\section{When to Use Meta-Analytic Techniques}

Many literature reviews can and should consider the inclusion of quantitatively synthesizing results so long as they meet certain basic criteria. First, the review must include empirical studies. And secondly, these studies must include at least in part quantitative data. Beyond these minimum requirements, several 
additional issues should be considered. First, the research question(s) driving the research synthesis should be clearly defined. The question should be narrow enough to provide meaningful results from the literature, but broad enough to advance theory. A question too broadly defined risks creating the "apples and oranges" problem, in other words, summarizing studies that are not really dealing with the same constructs or relationships (Lipsey \& Wilson, 2001). One must use keen judgment to differentiate an "apples and oranges" study from a study aptly examining "fruit". In addition to these considerations of the research question, ultimately, data must be available in order to transform data or summary statistics into comparable statistical forms for the successful integration of research findings. Although Rosenthal (1995) stated that "The level of quantitative skill and training required to use basic meta-analytic procedures is so modest that researchers capable of readily learning the small number of calculations required to answer standard meta-analytic questions", this idea is quickly changing. Increased attention to meta-analysis and in meta-analytic techniques has led to many advances and refinements. These strategies are more statistically demanding and these statistically demanding techniques are becoming more and more standard in practice. This does mean that more sophisticated and user-friendly software is being developed to aid the conduct of meta-analyses, though clearly this does not eliminate the need to be able to conceptually understand many complex issues. 


\section{Getting Started}

\section{Literature searches}

The advent of computerized reference databases has made searching for available studies, more "up-to-date" and much easier in many respects, but it does have its caveats. For instance, the seeming ease of searching computerized reference databases may provide researchers with a false security regarding their success in collecting available studies. Researchers should be aware that there are currently many available reference databases (e.g., some health related databases: MEDLINE, PsychInfo, Cancerlit, Cinahl, Health and Wellness Resource Center, Pubmed). It is important for researchers to determine which of these are relevant to their particular review and since some databases are more inclusive than others, researchers should not rely on just one or two. Each field of study has a handful of primary databases (e.g., MEDLINE, PsychINFO) that are typically used. Unfortunately, the quality of these databases can vary from institution to institution. That is, a literature. search conducted on a reference database at one institution can yield different results than at another institution since institutions can purchase different versions or levels of a particular database. Therefore, discrepancies will exist in the level of inclusiveness yielded by searches. Despite these cautions, the use of reference databases is an essential tool for literature reviews.

At the outset of the computerized search, keywords that represent the area of interest should be identified and entered into the database. These keywords may need to be modified as the search progresses in order to include important keywords that were overlooked or unknown to the reviewer at the onset. Documentation of 
keywords, reference databases, and other search strategies should be kept during the conduct of the meta-analysis.

In addition to computerized reference databases, search strategies include (1) manual searches of relevant (and typically the most prominent) journals; (2) careful examination of reference lists from review articles and acquired articles; (3) conference programs and proceeding; and (4) contacting authors and experts. Thorough use of each of these procedures is important to minimize bias in the data collection phase.

\section{Identifying the Studies}

Several strategies can be used for determining which studies will be included in the meta-analysis. Usually preliminary screening of studies occurs at the literature search level. Oftentimes abstracts are used to give a gross assessment of applicability of the study to the meta-analytic review. Any study that is considered potentially relevant is then collected and should be carefully reviewed to further assess if each study meets the complete inclusionary criteria.

Inclusionary criteria should be explicit and directly related to the overall study objectives. Researchers should consider criteria such as: (1) Does the study include particular research participant groups (based on demographics or other characteristics) relevant to the research questions? (2) Are the key variables present?

(3) Is there adequate information regarding the variables to calculate the needed effects? (4) Does the study use a design (e.g., RCT) that is acceptable to examine the question at hand? And (5) Are there particular time frames that the studies are 
limited to? These types of issues should be considered in terms of the specific research question for the meta-analytic study. Decisions regarding the precise research question should be made as early in the processes as possible to avoid the need to redo the literature search.

\title{
Coding and Database Production
}

\author{
Software.
}

Coding of study results, constructs, characteristics, treatment, samples and design are the essence of meta-analysis. To most efficiently and effectively create a database, several software options should be considered, including software for reference management, database production, meta-analysis computation and graphical display.

Firstly, literature searches and bibliographic information form the core of data collection. In order to manage the oftentimes unwieldy number of studies that are gathered during the collection phase, a reference management software program is recommended. Several different programs are available, such as Reference Manager and Refworks, which aid in cataloging each study (that was included or excluded) and save considerable time when referencing the studies during the write- . up phase of the meta-analysis.

Next, deciding whether a meta-analytic software package is needed. The size of the meta-analysis, the type of effect sizes, and the type of analyses can impact this decision. A variety of meta-analytic software programs are now available, such as Comprehensive Meta-Analysis, Review Manager, MetaGraphs, SAS Macros, 
STATA, ARCUS, DSTAT, Meta-Analyst, Easy MA, Fast Pro, True Epistat, and DESCARTES and macros for SPSS. Each of these software packages or macros has advantages and disadvantages and should be carefully reviewed before purchasing. Types of features to consider include: (1) Does the software package include the appropriate statistical model (e.g., fixed, random, mixed effects models) (2) How is data entered into the program (e.g., forms, spreadsheets)? (3) What effect size measures are available? (4) Can the software perform advanced procedures? (5) What types of graphics, if any, does it offer? (6) Can the graphics be easily imported into another program (e.g., Word or PowerPoint) so they can be shared with other researchers? (7) Does the program have a test of homogeneity? and (8) Is the software flexible enough to allow adaptations of the algorithms? Typically, a given software package will not have all of the features needed. One must assess the particular needs of the individual meta-analysis and base decisions on the most important needs.

A viable option for many researchers may be to develop their own software using a program such as EXCEL. Algorithms for the calculation of effect sizes and bias corrections can be programmed into the spreadsheet. The advantage of this option is that data entry and preliminary computations analyses can be formatted in a way that is most intuitive or useful for the individual researcher. But more importantly, many programs are not sufficiently flexible to allow for specialized calculations not included in the software to be added or algorithms adapted to the specific needs of the researchers. Building your own program can give you that flexibility. 
Depending whether you decided to create your own program or which metaanalysis software package you choose will play a part in determining how data entry will take place. For instance, Version 1 of Comprehensive Meta-analysis is created to have data entered directly into the program using a form template. In this case, data should be entered directly into the program at the start of the meta-analysis in order to maximize the features of the program. Other programs allow for data to be entered in spreadsheets, such as EXCEL, and can then be later imported into the software program.

No matter which way the researcher chooses, data entry should be conducted directly into a computerized spreadsheet or form rather than on pencil and paper forms. Using paper and pencil forms that are subsequently keyed into a computer database merely introduces additional error. It is also likely that this would require additional data entry procedures in order to verify the accuracy of the keyed data. This will increase the overall amount of work.

\section{Coding Manuals and Forms.}

A coding manual outlines the items that should be extracted from each study, gives detailed definitions of each item, and specifies codes for each item. Coding forms traditionally were paper and pencil and each study was summarized by filling in the form. The contemporary method is to develop a spreadsheet that contains places for each item to be entered.

The coding manual is typically a work in progress. That is, the researcher first carefully considers the data that should be extracted from the studies. 
Subsequently, as the coders are trained and as the coding manuals are being used,

modifications and refinements will be made. For instance, a researcher may too broadly or too narrowly define a variable in the coding manual. This may result in inconsistent data extraction or an unusually high rate of missing data. The researcher must then reassess the item in question and modify the manual accordingly. Many of the coding manual refinements will occur during the training of the coders.

\section{Training of Coders}

Lipsey and Wilson (2001) emphasize that the coding of studies is "one of the most technically demanding aspects of meta-analysis". Therefore the training of coders should be give careful attention. At the onset of the project several instructional seminars, including readings, lectures, and discussions, should be provided. The extent of the instructional seminars will depend largely on the knowledge and sophistication of the coders. Issues that should be covered include:

(1) general and theoretical knowledge about the body of literature; (2)

methodological and statistical procedures common in the body of literature and relevant to the research question; and (3) meta-analytic procedures and strategies. Subsequently, coding manuals should be reviewed line-by line and discussions of various aspects of the coding manual discussed. Coders should practice using the manuals until all researchers are comfortable with the coding procedures. Periodic meetings should be conducted to review progress and discuss any difficulties encountered. 
Choosing variables to extract and code in a meta-analysis should be done with careful thought and planning. The coded variables provide a description of the set of studies included in the meta-analysis and serve as potential moderating variables for analysis. In general, coding is done at the study level. Oftentimes a given study will offer more than one type of result, relationship, sample, construct, or time point. Each of these can result in more than one effect size that can be included in the meta-analysis. Care needs to be taken to provide study characteristics specific to each effect size included. Variables related to each effect size include characteristics such as, measure or construct, effect size specific sample size, calculation procedures, and scale reliability.

Study characteristics such as date of publication, form of publication (e.g., dissertation, peer-reviewed, conference presentation), sample size, demographics (e.g., age, gender, education), population description and setting should be included. Variables related to methods and procedures such as, sampling procedure, attrition and survey design are also important. Additionally, variables specific to the research question or particular body of literature that may be theoretically relevant should be identified.

Studies are sometimes coded for methodological soundness of the study. This type of coding is particularly relevant for comparisons between groups (e.g. treatment and control) across studies that may be directly impacted by study design. Setting explicit criteria for assessing methodological soundness is essential for avoiding the pitfalls of subjectivity. Using guidelines for methodological soundness 
set by the field as much as possible can help increase objectivity. Two independent coders should rate the quality of studies and the coders should be blinded to names of authors and journals if possible.

\section{Effect Sizes and Confidence Intervals}

Effect sizes and confidence intervals are the most central elements of metaanalysis. Effect sizes provide valuable measures of magnitude of an effect, where as confidence intervals illustrate the precision of the given parameter estimate.

\section{Effect sizes}

Effect sizes are the primary index for reporting results in a meta-analysis. Effect sizes provide an estimate of an effect of an independent variable on a dependent variable (Rosenthal, 1991). This relationship between variables most often in meta-analysis is describing a study outcome or treatment effect, though this is by no means the only relationship examined in meta-analytic studies. Although an effect size can represent the direction or magnitude, the most typical (and rich) effect size measure both direction and magnitude. As the central statistic in meta-analysis, understanding effect sizes and the possible variations of effect sizes that exist prove essential for conceptualizing and starting a meta-analysis. Knowing the precise information needed to calculate effect sizes at the outset enables the researcher to more accurately identify which studies can be included in the meta-analysis.

Although authors can be contacted for some missing information, this can prove to be unsuccessful for a variety of reasons and decisions about what type of information will be gathered directly from authors should be carefully selected. 
Effect size measures can include the follow broad categories: (1) one-variable

relationships (e.g., frequencies, proportions, sums); (2) two-variable relationships

(e.g., pre-post contrasts, group contrasts; (3) association between variables (e.g.,

Pearson product moment correlation coefficients); and (4) multi-variate relationships

(e.g., use of SEM, FA).

\section{One-variable relationships}

One-variable relationships are the least used measure of effect size and metaanalyses focusing on these relationships are not typical. In general this refers to the pattern of observation across a variable reported as a central tendency distribution (mean, median or mode) or as a distribution of values (frequencies, proportions or sums). An example of a one-variable relationship is the comparison of scores between two types of measures that represent the same construct (Lipsey \& Wilson, 2001). These scores would need to be reported or converted in the same metric (e.g., percentiles) and associated standard errors calculated in order to be compared as an effect size.

Two-variable relationships

Two-variable relationships are the most common in meta-analyses. One common type of two-variable relationships is the pre-post contrasts, which involves the comparison of two central tendencies. These comparisons can be made on unstandarized means when the metric is the same for all measures. Otherwise comparisons can be made with standardized means scores.

Another common two-variable relationship is the group contrasts. Group contrasts involve a variable that is measured in two or more groups. Means, standard 
deviations, and sample sizes for each group on each variable or proportions based on the characteristic of interest can be used for comparisons. There are a variety of effect size measures that can be used for group comparisons, such as, the unstandardized mean difference, the standardized mean difference, the proportion difference and the odds ratio.

\section{Association Between Variables}

The association between any two variables can serve as the basis of the metaanalysis. These bivariate relationships involve the covariation between two variables. The primary effect size in this context is the Pearson product-moment correlation coefficient. In some situations one could include the odds-ratio and the standardized mean difference. The standard Pearson product-moment correlation coefficient is used as an effect size index for two continuous variables. In contrast, a biserial point correlation coefficient is used for dichotomous-continuous variable relationships. Another possibility includes the two-dichotomous variable relationships assessed with the odds-ratio and phi coefficient. The odds-ratio is generally used to describe the comparison between two subgroups whereas the phi coefficient describes the relationship or predictive strength in a group.

When mixing continuous and dichotomous variables one must first decide if the dichotomous variables are inherently dichotomous or artificially dichotomous. If one variable is inherently continuous and the other is artificially dichotomous then product-moment correlation is used but it should be corrected for the artificial dichotomization or a $z$-transformed $r$ can be used as the effect size index. On the other hand, if one variable is inherently continuous and the other is inherently 
dichotomous either a biserial coefficient with a correction for dichotomy or a standardized mean difference with probit, logit or arcsine correction is used.

\section{Multivariate Relationships}

Multivariate relationships are the least common relationships examined. The methods for examining multivariate relationships are few and the applications limited. Various types of multivariate relationships, such as multiple regression, structural equation modeling and factor analysis have been discussed in the literature. The most challenging problem with exploring multivariate relationships is the lack of data needed to calculate standard errors. Researchers have begun to explore strategies such as synthesizing correlations statistics and meta-analyzing them by doing multivariate analyses on synthesized matrices. Ultimately, the most feasible approach identified so far is to actually conduct meta-analyses on individual studies that have conducted multivariate analyses and examining the multivariate relationships by combining correlations of reported predictors. The limited proportion of studies that use multivariate statistics to examine particular relationships in a body of literature poses a significant limitation. Additionally, multivariate meta-analyses are limited due to the lack of full correlation matrices reported in studies. Much more research is needed in this area to identify methods for working with available data. And more importantly, standards for reporting needed information such as correlation matrices, should be set by journal editors to help facilitate the use of these advanced procedures, which will no doubt have important impacts on the field. 
In sum. There are advantages and disadvantages to each of effect size indices

discussed. When conducting a meta-analysis, the relevant indices need to be identified. Potential effect size indices should be examined to determine which is most relevant, which requires information most attainable from the particular body of literature in question, and which will provide the most reliable measure possible.

\section{Confidence Intervals}

Confidence intervals illustrate the precision of a parameter estimate. The confidence interval provides an additional dimension to reporting effect sizes by indicating how "confident" one can be of the measure of magnitude obtained. The researcher has the flexibility to choose the level of probability, and this is done by setting the probability percentage for the confidence interval. For instance, a $95 \%$ confidence interval indicates that the confidence interval has a $95 \%$ probability of containing the population parameter.

The width of the confidence interval is directly related to: (1) the amount of data used to generate a given effect size; (2) the level of confidence interval chosen by the researcher; and (3) the computational model used. The larger the amount of data used the more precise the measure of effect size and therefore the narrower the confidence interval. Inversely, the greater the confidence level chosen by the researcher, the wider the confidence interval will be. Lastly, variance across studies attributed to entirely random influences are modeled with a fixed effects model. This model allows for confidence intervals that can actually reach zero. On the other hand, if the random variance and between-studies variance is assumed then a random 
effects model is typically employed. This model tends to produce wider confidence intervals and limits the ability of the confidence intervals to approach zero.

In a traditional meta-analysis, interpretation of confidence intervals can be guided by clinical utility. In the medical field for instance, some research questions may be focused on reduction of mortality. These studies will hold different criteria than a treatment effect for less "critical" outcomes. Such practical issues illustrate the need for flexibility when setting particular confidence interval levels or for which results are considered "significant" or "not significant". The most important consideration is whether the differences are clinically important, not important or yet unresolved. In general, caution should be used in interpreting point estimates. An emphasis is placed on the use of confidence intervals to increase the meaningfulness of interpretation.

Additionally, in meta-analytic reviews, confidence intervals should be reported for the individual studies as well as for the combined effects. Graphical representations of these confidence intervals provide an important tool for interpretations of the overall effects and the influences of individual studies on the overall effects. These simple but informative schematics, such as Forest Plots, provide the first step in the exploration of moderating variables of the effects.

\section{Issues of Bias}

It is sometimes necessary to correct the meta-analysis for bias. This can happen at two levels, either by correcting effect sizes with study-level information or by correcting more globally with meta-analysis level bias corrections. Most typically bias is corrected using study level information. Various types of biases that are 
important to consider include, sample size bias, artifact biases (e.g., measurement bias), publication bias, and the 'file drawer' problem.

\section{Sample Size Bias}

Simple pooling of data occurs when each set of data from a particular study is pooled together without regard to differences in sample size. Therefore, studies which include 10,000 participants provide the same weight in the analysis as a study with 10 participants. To avoid this problem, effect sizes can be corrected for sample size bias by weighting each data point by its respective sample size. The inverse variance weight reflects the precision of the effect size estimate, which varies as a function of sample size. This method is used to weight the contribution of each study effect size so that larger studies are given more weight in the calculation of the overall meta-analysis effect size.

\section{Artifact Adjustments}

Artifacts are imperfections that arise when conducting studies. Adjustments can be made to account for study artifacts such as sampling error, variable reliability, restriction for range of variables, dichotomization of continuous variables, and imperfect construct validity. Most often the information needed to make such corrections is not available in published reports and even when the information is available for many studies, likely it is not available for all. Efforts should be made to identify alternative sources of information for conducting bias corrections. For instance, one may turn to manuals for a given measure (if available) in order to identify the overall reliability coefficient. Another way to deal with the lack of 
consistent data is by conducting artifact distribution analyses to correct for biases using distributions. This method allows for bias correction at the meta-analysis level rather than at the study level. Ultimately, bias corrections can increase accuracy in some areas but may decrease accuracy in others. For instance, sampling error is larger for effect sizes with individually corrected effect size (e.g., measurement error correction; Hunter \& Schmidt, 1990). Additionally, the researcher must decide if he or she is comfortable making interpretations using adjustments or basing interpretations on unadjusted data. If the researcher chooses to correct for bias, comparisons of effect sizes can always be made by reporting effect sizes both with and without the corrections.

\section{Measurement bias}

An example of an artifact bias is measurement or scale reliability bias. Effect sizes can be corrected for attenuation due to scale unreliability using Hunter and Schmidt's (1990) correction:

$$
\delta_{E S^{\prime}}=\frac{\delta_{E S}}{\sqrt{r_{y y}}}
$$

where, $\mathrm{ES}=$ the observed (attenuated) effect size estimate, $\mathrm{ES}$ ' $=$ the disattenuated effect size estimate, and, $r_{y y}=$ the reliability of the dependent variable measure, which was estimated using the reported value of the scale internal consistency coefficient Alpha. 
When data is missing at the study level due to non-publication, it is called the "file drawer" problem and represents a form of publication bias. The most common procedure to estimate the impact of this sampling bias is computing the "fail-safe K" (Orwin, 1983; Rosenthal, 1979). This is an estimate of the number of undiscovered (presumably mostly unpublished) studies that, if known, would in aggregate reduce the overall meta-analysis effect size so that it was not statistically significant. Additional methods of assessing the robustness of the meta-analytic results in efforts to account for unpublished or missing studies include: (1) file drawer estimated by Iyengar and Greenhouse (1988); (2) missing studies estimate method by Gleser and Olkin (1996); and (3) trim and fill (Duval \& Tweedie, 2000).

Although the "fail-safe $\mathrm{K}$ " has been a widely used procedure, Pham et al. (2001) found that the Iyengar and Greenhouse (1988) method provide a better estimate of missing studies. Pham et al. (2001) also indicate that the trim and fill method provides an adequate estimate of the number of missing studies. Again more research and consensus is warranted.

\section{Publication bias.}

Historically there has been a tendency to published studies with statistically positive results as well as larger effects. Including published studies, "grey literature" and/or unpublished studies can result in an attenuated effect size estimate. McAuley, Pham, Tugwell, and Muher (2000) suggest that the inclusion of grey literature can 
reduce the effect size estimates by $12-50 \%$. Publication bias is a form of sampling bias at the study-level.

There are a variety of methods for assessing publication bias. A simple procedure for assessing this type of bias is to compare effect sizes between published and unpublished studies. Additionally, studies can be coded to differentiate between different types of sources of study results (e.g., dissertation, conference presentation). Pham, Platt, McAuley, Klassen and Moher (2001) conducted a study examining some additional procedures including (1) funnel plots; (2) the rank correlation test; (3) the graphical test assessing funnel plot asymmetry; and (4) the graphical test assessing funnel plot asymmetry allowing for between-study heterogeneity. Appropriate use of these methods is important for increasing the reliability and accuracy of meta-analytic reviews. For instance, funnel plots can be difficult to interpret and asymmetry found in the funnel plots may be due to the presence of heterogeneity of the studies rather than publication bias (Eggar, Davey, Schneider \& Minder, 1997; Sterne, Gavaghan, \& Egger, 2000) or may be due to both heterogeneity and publication bias (Pham, et al., 2001). Therefore, these tests should be conducted in conjunction with other tests to increase the accuracy of interpretation. One additional solution is to perform a full artifact distribution interim meta-analysis to determine a meta-analysis level correction rather than study-level corrections.

In sum. Ultimately, a meta-analyst must take into account a variety of information before deciding when to correct for bias, how many different bias corrections are needed (if any), or with which methods will then correct the bias. 
Researchers need to consider that corrections of certain biases can increase others.

For instance, overall sample size (i.e., number of studies) can decrease the sampling error, therefore the researcher may feel more inclined to correct for measurement error in these more "robust" situations. On the other hand, although the sample size may be large, if subgroup analyses are intended, this may in fact increase the sampling error within subgroup analyses and the researcher may then decide against correcting for measurement error. Another example may be that the range of reliability measures is quite small and the measures on the whole quite reliable, in this case the research may chose to not correct for measurement error in order to avoid the draw backs associated with individually correcting effect sizes.

Ideally, if bias corrections are needed, researchers of "primary" studies will report all information needed to perform comprehensive meta-analyses. This can enable meta-analysts to individually correct for bias rather than need to rely on interim meta-analyses to correct for artifacts at the meta-analytic level rather than the individual study level. If bias corrections are used it important to know that metaanalytic formulas used for corrected effect sizes typically are different than those used for uncorrected effect sizes.

\section{Homogeneity of Effect Size}

In meta-analytic studies, variation among effect sizes sometimes occurs due to random error, though oftentimes this variation becomes statistically larger than one would expect (due to random or sampling error). Tests of homogeneity attempt to identify the difference between variation due to random error and variation due to systematic differences between study design and participant characteristics or other 
theoretically relevant variables. Ultimately, assessing heterogeneity of effect size is important because the lack of a homogeneous distribution typically suggests the presence of possible predictors or moderators of the effect size magnitude. A variety of methods for testing homogeneity are available including tests of homogeneity designed to assess variation in treatment effects with odds ratios, percent of variance accounted for measure of effect size, and standardized effect size measures.

\section{Modeling Variance}

There are three primary methods of modeling variance: fixed, random and mixed effects modeling. The fixed effects model assumes the only source of variance is subject-level sampling error, whereas the random effects model assumes that the source of variance includes subject-level sampling error and study-level sampling error (i.e., random error). The fixed effects model can also include systematic variance due to identifiable moderating variables. A mixed model assumes all three sources of error, sampling error, random error and systematic error.

In a fixed effects model, it is assumed that the variance in the effect size distribution is only due to subject-level sampling error. So it is assumed that an effect size from an individual study represents the population effect with only random sampling error associated with chance factors. Tests of homogeneity examine the assumption that error is only due to sampling error and thereby serves as an assessment for whether the fixed effects model holds, though as discussed below, evidence of heterogeneity does not necessarily rule out a fixed effects model.

If the fixed effects model does not hold then another model is sought. The random effects model assumes that sampling error is accompanied by other sources 
of variability randomly distributed. The sampling error is considered to be from the subject-level whereas the random error is an estimate of between-studies variance. This between-studies error is thought to be analogous to study-level sampling error. Identifying the random effects variance component is the most difficult of the two components and can be assessed using noniterative methods based on method of moments and iterative methods based on maximum likelihood.

If one assumes that the fixed effects model holds but additional error is due to a systematic difference in the coded variables then one can attempt to partition the effect size variance. This can be tested by performing an analog to analysis of variance. This ANOVA will be used to explore variables by partitioning the total variance Q (Total) into Q (Between) and Q (Within). Q (Between) values will be used to test between group differences by using a Chi-square with $\mathrm{df}=\mathrm{p}-1$, where $\mathrm{p}$ $=$ the number of studies.

If the variance is assumed to be due to subject-level sampling error, studylevel sampling error (or random error) and some systematic variance then the model would be a mixed effects one. This means that the component of error not accounted by sampling or systematic variance must be incorporated into the effect size analysis by creating a weighting function. In this case, the residual Q (Between) or Q (Within) would still be heterogeneous. Fitting the mixed effects model is similar to the random effects model.

The mixed effects model is used less often than fixed and random effects models due to the complexity of the modeling procedures. Ultimately, fixed effects are more likely to identify systematic variance than the mixed effects model since it 
has more power to do so. On the other hand, mixed effects models have more accuracy in terms of type I error. On the whole these models require much more research to understand how best to account for and assess variance, especially in relation to accounting for and calculating the study-level sampling or random error.

\section{Meta-Regression}

In addition to ANOVA analogs using fixed and random effects models, one can employ the use of meta-regression to examine the association of effect with study characteristics. Meta-regression is a more sophisticated approach to assessing moderators when heterogeneity is observed (Song, Sheldon, Sutton, Abrams, Jones, 2001). As with other regression analyses care should be taken to assess colinearity by assessing correlations between variables. Overall fit of the meta-regression can be calculated with a $Q_{R}$ for the regression and a $Q_{E}$ for the residual error, which are distributed as a chi-square (Lipsey \& Wilson, 2001).

\section{Power Analysis}

When conducting a meta-analysis that is primarily aimed at resolving conflicting studies in the literature, namely those using null hypothesis testing, metaanalysts should perform power analyses. In this case, using the obtained overall effect size and assuming it is equivalent to the population effect size, the use of Cohen's power tables are appropriate. In other situations, the principal question of power for meta-analysis involves not so much whether or not the overall effect size is statistically significant but rather whether there is sufficient power to determine if the effect size distribution is heterogeneous. Power for the $\mathrm{Q}$ test depends on the 
ratio of between- to within-study variance (Hedges \& Pigott, 2001). Unfortunately, it is difficult to know what this ratio might be a priori. Schmidt (1992) reports a range of 0.33 to 1.0 across a large number of meta-analyses that he examined. Hedges and Pigott (2001) suggest variance ratios of $0.33,0.67$, and 1.00 be considered small, medium, and large degrees of heterogeneity, respectively.

\section{Outliers}

Effect size distributions should be examined for extreme high or low scores in order to identify outliers. Outliers should be examined carefully in efforts to identify any potential errors (e.g., transcription errors) on the part of meta-analyst that can be corrected. In some cases summary data in published studies may appear to be causing the outlier and the meta-analyst can attempt to contact authors for verification.

In order to identify the outliers several methods can be employed. For instance, standardized residuals can be used to identify outliers when using models (e.g., the standardized difference from the mean, using fixed or random effects), though the guidelines for cutoffs are most appropriate when the underlying distribution is homogeneous. Another method is to test the suspected outliers by eliminating one effect size at a time and assessing the change in Q (homogeneity statistic). If underlying heterogeneity exists, Forest plots or other subgroup analyses can be employed to assess the outlier within the context of a variety of moderators and to identify the outlier with respect to potential associated clusters of effect sizes. Another method includes identifying effect sizes that are 2 to 3 standard deviations from the mean to be considered for removal or recoding. 
Once identified outliers are most typically removed from further analyses. If a researcher does not wish to remove studies from the meta-analysis, a more inclusive approach can be employed. This involves identifying a break in the effect size distribution and coding outliers back to the next largest cluster of effect sizes (Lipsey \& Wilson, 2001). The advantage to this method is that it ensures as much data as possible is maintained and allows for the extreme values to be retained but not to distort other effect sizes in the distribution.

Each of these methods requires a degree of researcher judgment or subjectivity when determining which values are outliers and when those outliers should be removed. Furthermore, these methods have typically not been developed specifically for meta-analysis. Huffcutt and Arthur (1995) developed an outlier statistic for meta-analytic data, called SAMD (sample-adjusted meta-analytic deviancy statistic). Unfortunately it requires a high number of computations and is typically not appropriate for coefficients that are individually corrected for bias (e.g., measurement error; though in some instances with additional adjustments these can be accounted for). Additionally, recent research has raised critical questions about its potential utility. In particular, when SAMD was used for removing outliers with correlational data, small correlation outliers are more likely to be removed than larger correlation outliers (Beal, Corey \& Dunlap, 2002) which introduces yet another bias problem. It is unclear if this problem persists in the examination of outliers for standardized mean difference scores. To date, recommendations by Beal et al. (2002) to resolve the asymmetric outlier identification have not yet been explored; therefore more research should be done before this procedure is used. 


\section{Interpretation Issues}

The interpretation of results in a meta-analysis largely depends on the research question that the meta-analysis was designed to examine. Simple metaanalysis will often seek to determine if a body of literature, when combined, produces significant results. Overall assessments of significance allow interpretations that parallel the individual studies on which the meta-analysis was based. More sophisticated meta-analysis will examine moderating variables and explore aspects of theories and interpretations will be couched in underlying theories or new discoveries.

At a more basic level, the index of effect size is the central measure in the meta-analysis. Effect sizes have general rules of thumb for interpretation. Although important, these should merely serve as guidelines and ultimately need to be interpreted within the context of the measured relationships. For instance, Cohen (1977) suggests that effect size magnitudes have range of small $\left(d=.20 ; r=.10 ; \eta^{2}\right.$ $=.010)$, medium $\left(d=.50 ; r=.25 ; \eta^{2}=.059\right)$, and large $\left(d=.80 ; r=.40 ; \eta^{2}=\right.$ .138). These can also be interpreted as $1 \%, 6 \%$, and $14 \%$ of the variance is accounted for respectively for small, medium, and large effects. In a recent study, Rossi (2003) found that typical effects for public health interventions were closer to $0.5 \%, 1.0 \%$, and $1.5 \%$ of the variance for small, medium, and large effects. These findings are dramatically smaller than rules of thumbs set by Cohen due to differences in the context of their development. Similarly, when analyses are dealing with clinically and practically significant results it is up to the researcher to determine the significance of the findings within its particular context. 
Currently, meta-analysis has much growing to do and the field has much to

do to embrace and foster that growth. In such an environment, meta-analysts should be particularly thorough in reporting methods used to conduct meta-analyses, in providing data in a variety of forms (e.g., with and without bias corrections or fixed versus random effects modeling), and avoid interpreting results with unfounded confidence (e.g., without sufficient consideration to limitations). These strategies enable other researchers to replicate meta-analytic findings, and help provide the data needed to replicate findings in the face of new, refined, or more advanced techniques while minimizing the risk of unwarranted controversy over a technique that, as it matures, will be more and more important to the field.

\section{References}

Beal, D. J., Corey, D. M., \& Dunlap, W. P. (2002). On the bias of Huffcutt and Arthur's (1995) procedure for identifying outliers in meta-analysis of correlations Journal of Applied Psychology, 87, 583-589.

Cohen, J. (1977). Statistical power analysis for the behavioral sciences, $\left(2^{\text {nd }}\right.$ ed.). New York: Academic Press.

Cooper, H.M. (1989). Integrating research: A guide for literature reviews (2nd ed.). Newbury Park, CA: Sage.

Cooper, H.M., \& Hedges, L.V. (1994). Research synthesis as a scientific enterprise. New York: Russell Sage Foundation. 
Duval, S.J. \& Tweedie, R.L. (2000). Trim and fill: A simple funnel plot-based method of testing and adjusting for publication bias in meta-analysis. Biometrics, $56,276-284$.

Egger, M., Davey Smith G., Schneider, M., \& Minder, C. (1997), Bias in metaanalysis detected by a simple graphical test. British Medical Journal, 315, 62934.

Fisher, R.A. (1932). Statistical methods for research workers ( $4^{\text {th }}$ ed.). London: Oliver and Boyd.

Glass, G.V. (1976). Primary, secondary and meta-analysis of research. Educational Researcher 10, 3-8.

Glesser, L.J., \& Olkin, I. (1994). Stochastically dependent effect sizes. In: Cooper H, Hedges LV, eds. The handbook of research synthesis. New York: Russell Sage Foundation, 339-56.

Glesser, L.J., \& Olkin, I. (1996). Models for estimating the number of unpublished studies. Statistics in Medicine, 15, 2493-2507.

Hedges, L. V. \& Pigott, T. D. (2001). The power of statistical tests in meta-analysis. Psychological Methods, 6, 203-217.

Huffcutt, A.I. \& Arthur W. (1995). Development of a new outlier statistic for metaanalytic data. Journal of Applied Psychology, 80, 327-334.

Hunter, J. and Schmidt, F. (1990). Methods of meta-analysis: Correcting error and bias in research findings. Beverly Hills CA: Sage.

Iyengar, S., Greenhouse, J.B. (1988). Selection models and the file drawer problem. Statistical Sciences, 3, 109-35. 
Lipsey, M. W., \& Wilson, D. B. (2001). Practical meta-analysis. Thousand Oaks, CA: Sage.

McAuley L., Pham, B., Tugwell, P., Moher, D. (2000). Does the inclusion of grey literature influence estimates of intervention effectiveness reported in metaanalyses? Lancet, 7 (356), 1228-31.

Orwin, R. G. (1983). A fail-safe $N$ for effect size in meta-analysis. Journal of Educational Statistics, 8, 157-159.

Pearson, K. (1933). On a method of determining whether a sample of given size $n$ supposed to have been drawn from a parent population having a known probability integral has probably been drawn at random. Biometrika 25, 370-410.

Pham, B., Platt, R., McAuley, L., Sampson, M., Klassen, T.P., Moher, D. (2001). Detecting and Minimizing the Impact of Publication Bias: An Empirical study of Methods. Evaluation and the Health Professions, 24, 109-125.

Rosental, R. (1991). Meta-anlytic procedures for social research. Thousand Oaks, CA: Sage.

Rosenthal, R. (1979). The "file drawer problem" and tolerance for null results. Psychological Bulletin, 86, 638-641.

Rosenthal, R. (1995). Writing meta-analytic reviews. Psychological Bulletin, 118, 183-192.

Rossi, J.S. (2003, September). Comparison of the use of significance testing and effect sizes in theory-based health promotion research. Paper presented at the 43rd annual meeting of the Society for Multivariate Experimental Psychology, Keystone, CO. 
Schmidt (1992). What do data really mean? Research findings, meta-analysis, and cumulative knowledge in psychology. American Psychologist, 47, 1173-1181.

Song, F., Sheldon, T.A., Sutton, A.J., Abrams, K.R., \& Jones, D.R. (2001). Methods for exploring heterogeneity in meta-analysis. Evaluation \& The Health Professions, 24(2), 126-151.

Sterne JAC, Gavaghan D, Egger ME. (2000). Publication and related bias in metaanalysis: power of statistical tests and prevalence in the literature. Journal of Clinical Epidemiology, 53, 1119-1129.

Velicer, W.F., Cumming, G., Rossi, J.S., Fava, J.L., Prochaska, J.O., \& Johnson, J. (2003). Theory testing using quantitative predictions of effect size. Manuscript submitted for publication.

Zwick, W. R. \& Velicer, W. F. (1986). Comparison of five rules for determining the number of components to retain. Psychological Bulletin, 99, 432-442. 


\section{CHAPTER FIVE}

\section{DETERMINING EFFECT SIZE FOR META-ANALYSIS: A COMPARISON OF ALTERNATIVE PROCEDURES}

\section{Introduction}

Effect sizes are the primary index for reporting results in a meta-analysis. Effect sizes provide an estimate of an effect of an independent variable on a dependent variable (Rosenthal, 1991). This relationship between variables most often in meta-analysis is describing a study outcome or treatment effect, though this is by no means the only relationship examined in meta-analytic studies. As the central statistic in meta-analysis, determining effect size is essential for meta-analysis. Although relatively little information is typically needed to compute the most common measures of effect size, the necessary data are not always reported in research articles. Rosenthal (1994) and others (Lipsey \& Wilson, 2001) have provided useful summaries of methods for computing effect sizes under a variety of situations. For example, to compute Cohen's $d$ or Hedges' $g$ it is necessary to know the means, standard deviations $(S D)$, and sample sizes of the groups that are being compared. If some or all of these data are unavailable, one could estimate effect size from $t$ or $F$ test results as long as the degrees of freedom of the test are known.

A different type of problem occurs when reported data appear to support the calculation of effect size but instead lead to inappropriate or misleading results. This might happen when data are reported as standard scores, such as $z(M=0, S D=1)$ or $T$-scores $(M=50, S D=10)$. For example, a meta-analysis by Prochaska (1994) 
included a study by Velicer et al. (1985) that used $T$-scores to describe the benefits of smoking. The effect size comparing smokers and ex-smokers was determined by subtracting $T$-scores and dividing by 10 , resulting in an effect size of 0.43 . This procedure appears correct because it is known that the $S D$ of $T$-scores equals 10 . Therefore, dividing the $T$-score difference by 10 should result in the standardized difference between the means. However, this procedure is sub-optimal because 10 is the total group $S D$, not the pooled within-group $S D$ used to compute Hedges' $g$ or Cohen's $d$ (Rosenthal, 1994). Since Velicer et al. (1985) provided group n's and $S D$ 's, we were able to compute an effect size based on the pooled within-group $S D$. This resulted in an effect size of 0.55 , nearly $30 \%$ larger than the value reported by Prochaska (1994).

In most cases the within-group variance will be less than the total group variance whenever the grouping variable has a non-zero relationship with the dependent variable, since this will reduce within-group variance relative to total variance (i.e., $S S_{\text {error }}=S S_{\text {total }}-S S_{\text {between }}$ ). Thus, computing effect size by subtracting $T$-scores should underestimate Cohen's $d$ or Hedges' $g$. This study examined this possibility using two meta-analyses that were being conducting (Hall \& Rossi, 2002, 2004), including only studies that provided enough information to compute effect size using both methods. It was predicted that the method of subtracting $T$-scores would underestimate the effect size based on the pooled within-group $S D$.

Conceptual Framework for Effect Size. The effect sizes computed for this study represent the cross-sectional "change" in Pros and Cons scores across the stages from Precontemplation to Action. Prochaska (1994) found that the effect size 
for Pros and Cons across the stages from Precontemplation to Action was approximately $1 S D$ for Pros and $0.5 S D$ for Cons. He coined this relationship the Strong and Weak Principles. The Strong and Weak Principles were re-examined in this study using three different methods of effect size calculation that served as the basis of the effect size comparison. Additionally, correction factors to account for differences between the calculation methods for each of the constructs were considered and a final bias correction was developed.

\section{Method}

The current study compiles two sets of data from an on-going study examining Transtheoretical Model Decisional Balance measures - both the Pros and Cons of Change.

\section{Procedure}

Literature Searches. The datasets for this study were acquired through literature searches on two computerized databases, MEDLINE, and PsycLIT from 1983 (the year of initial study using the TTM by Prochaska \& DiClemente) to February 2002. Additional search procedures included personal communications with authors and reviews of reference lists from acquired articles. Datasets for this meta-analysis included published articles, conference presentations, and papers in progress.

Inclusion Criteria. As part of a larger study, datasets involving any type of behavior, which at least in part examined Stages of Change and Decisional Balance were considered for analysis. The primary inclusion criteria for the current study 
required that articles must provide: 1) enough information to compute the pooled $S D$; 2) mean $T$-scores for each group (either in the text, in a table, or in a graph depicting $T$-scores across groups).

\section{Analyses}

Effect Size Calculations. Three methods of determining effect size were used in the analysis: Hedges' $g$, standard score, and graphical standard score estimation. Hedges' $g$ requires means, $S D$ 's, and n's for each group (either raw scores or $T$ scores). Hedges' $g$ is the difference between the sample means divided by the average pooled sample standard deviation (Rosenthal, 1994).

$$
\text { Hedges' } g=\frac{M_{1}-M_{2}}{\text { Spooled }}
$$

Both the standard score and the graphical standard score method utilize the same equation to compute effect size. This equation simply requires standard $T$ scores $(M=50, S D=10)$ for each group, and is defined as the difference between the sample means divided by the standard deviation.

$$
\text { Standard/Graphical Effect Size }=\frac{M_{1}-M_{2}}{10}
$$

The only difference between the standard score and the graphical method is that group means for the standard score method was obtained from the text or a table, whereas, the graphical method used scores estimated from a graph.

Estimations of $T$-scores from graphs were made by using a ruler to measure the point against the y-axis where the $T$-scores are graphed. The ruler measurements were then used to interpolate $T$-scores between adjacent tic marks on the y-axis. The 
scores were interpolated to the nearest hundredth. It should be noted that the graph method was used only when Hedges' $g$ could not be calculated and the actual standardized scores were not available.

Effect Size Differences. Paired-samples $t$-tests were conducted between the $T$ score and graphical methods for each of the constructs. Additionally, paired-samples $t$-tests were conducted between the standard score approach and the Hedges' $g$ for each of the constructs.

Effect Size Correlations. Correlations were performed between the effect size methods for each of the constructs.

Regression Analyses. Regression analyses were conducted with the Pros and Cons data. Regression analyses were performed including the variables: sample size, standard score approaches ( $T$-score and graphical standard score estimation), Hedges' $g$ and standard score, to assess the best fit. Mean differences in effect sizes between the Hedges' $g$ and the standard score calculations for each study was assessed.

A second set of analyses were run following the removal of outliers. Differences in scores between the Hedges' g method and scores estimated via the regression formula of 0.5 or greater were considered outliers and were removed from the analyses.

Corrections Formulas. Regression analyses and trendlines for each set of data were compared to identify the best fit for subsequent use as regression correction formulas. 


\section{Results}

Thirty-eight studies investigating Decisional Balance (Pros and Cons), satisfying all inclusionary criteria were identified. Some studies examined multiple health behaviors; therefore the total number of datasets was 46 for each of the constructs.

Prior to comparing Hedges' $g$ to the standard score method, the comparability of the two different techniques for obtaining the standard score was examined. This comparison was conducted for the subset of studies that provided information on $T$ scores both in text and in graphs. Paired-samples $t$-tests were conducted to test the equivalency of these two approaches. There was no significant difference in using reported $T$-scores versus estimating scores from a graph for Pros, $t(24)=1.201$, Cons, $t(24)=-1.160$, with correlations of 0.965 and 0.981 respectively. Therefore, these two methods were collapsed. $T$-scores were estimated from graphs only when the actual standardized scores were not available.

The standard score approach was then compared to the Hedges' $g$ method using paired-samples $t$-tests. As expected, a significant difference was found for Pros, $t(45)=3.896, p<.05, \mathrm{~d}=0.193$, Cons, $t(45)=3.807, p<.05, \mathrm{~d}=0.105$. Table 1 presents the means, $S D$ 's, and n's for Hedges' $g$ and standard score effect size's for the two constructs. The correlation between the two effect size methods was nearly the same for the two variables: 0.924 for Pros, 0.944 for Cons. 
Table 1

Comparison of Effect Size Calculation Methods for the Pros and Cons of Change

\begin{tabular}{|c|c|c|c|c|}
\hline \multirow[b]{3}{*}{ Mean } & \multicolumn{2}{|c|}{ Hedges' g } & \multicolumn{2}{|c|}{ Standard Score } \\
\hline & Pros & Cons & Pros & Cons \\
\hline & 1.17 & .64 & 1.04 & .58 \\
\hline$S D$ & .54 & .31 & .42 & .26 \\
\hline$N$ & 46 & 46 & 46 & 46 \\
\hline
\end{tabular}

Regression Analyses. The data were first examined including all available data and using regressors: 1) standard score effect sizes; 2) sample sizes of each mean score used to calculate the effect sizes; and 3 ) calculation type ( $T$-score or graphical method). For Pros, the regression was a good fit $\left(\mathrm{R}^{2}{ }_{\text {adj }}=.843\right)$, with an overall significant relationship $\left(\mathrm{F}_{4,40}=59.998, \mathrm{p}<.01\right)$. For Cons, the regression was also a good fit $\left(\mathrm{R}^{2}{ }_{\text {adj }}=.905\right)$, with an overall significant relationship $\left(F_{4,40}=95.033\right.$, $\mathrm{p}<.01)$.

Since, sample size is not always available, and for parsimonious efforts the regression were run again including the standard score effect size as the only regressor. This resulted in a good fit for the Pros data $\left(\mathrm{R}^{2}{ }_{\mathrm{adj}}=.851\right)$, with an overall significant relationship $\left(\mathrm{F}_{1,44}=258.028, \mathrm{p}<.01\right)$, as well as a good fit for the Cons data $\left(\mathrm{R}^{2}{ }_{\mathrm{adj}}=.888\right)$, with an overall significant relationship $\left(\mathrm{F}_{1,44}=359.116, \mathrm{p}<.01\right)$.

Regression correction equations were developed based on the parsimonious regressions stated above yielding one correction for each construct:

$$
\begin{aligned}
& \text { Pros correction }=(E S)(1.829)-0.065 \\
& \text { Cons correction }=(E S)(1.130)-0.014
\end{aligned}
$$


where $E S=$ standard score effect size. Diagnostics were then assessed and difference scores between the Hedges' $g$ effect size and the estimated effect size based on the regressions reported above. Four sets of data showed differences of 0.5 or greater and therefore were considered outliers. These data were removed from the analyses, including one data set for Cons (Marcus et al., 1994, exercise) and three for Pros (King et al., 1996, smoking; Jordon, 2002, bulimia; Grimley et al., 1995, condom use - main).

Regressions were then run on the data with the outliers removed. This resulted in an even better fit for the Pros data $\left(\mathrm{R}^{2}{ }_{\mathrm{adj}}=.946\right)$, with an overall significant relationship $\left(\mathrm{F}_{4,41}=713.848, \mathrm{p}<.01\right)$, as well as a very good fit for the Cons data $\left(\mathrm{R}^{2}{ }_{\text {adj }}=.925\right)$, with an overall significant relationship $\left(\mathrm{F}_{4,41}=527.631, \mathrm{p}<\right.$ $.01)$. Figure 1 and Figure 2 illustrate the relationships between the effect sizes calculated with Hedges' g and Standard Score methods. 


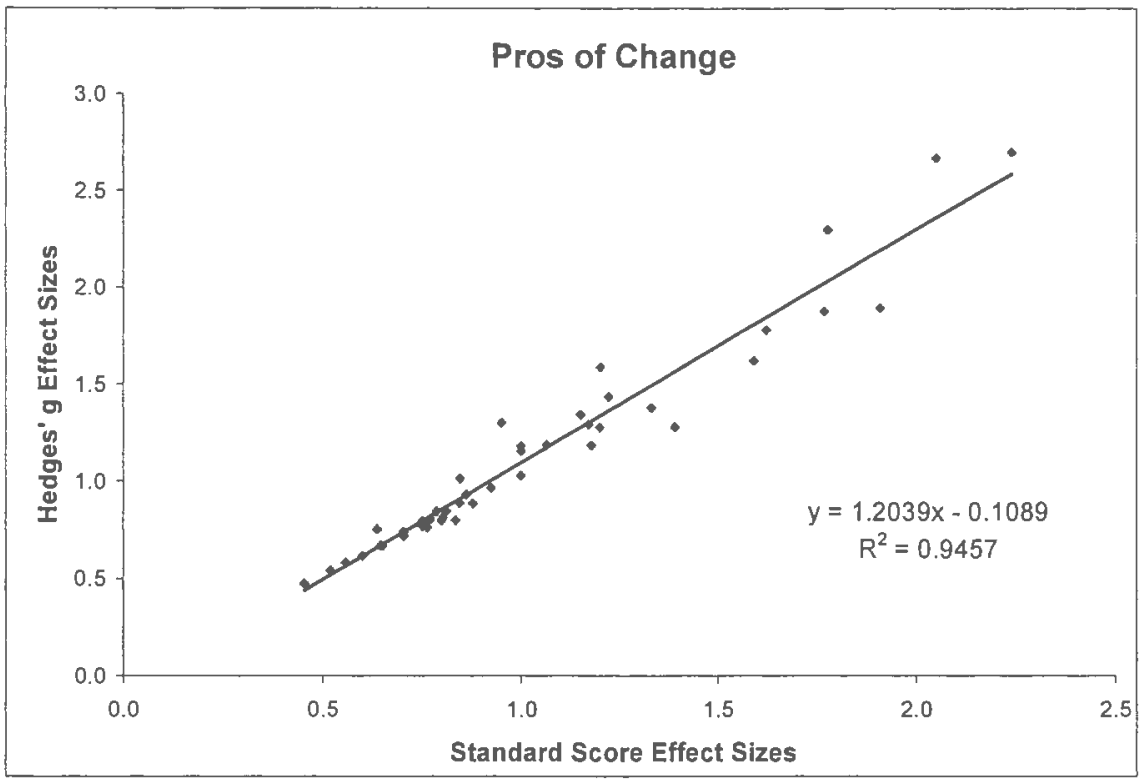

Figure 1. Relationship between Hedges' g and Standard Score for Pros of Change with Outliers Removed.

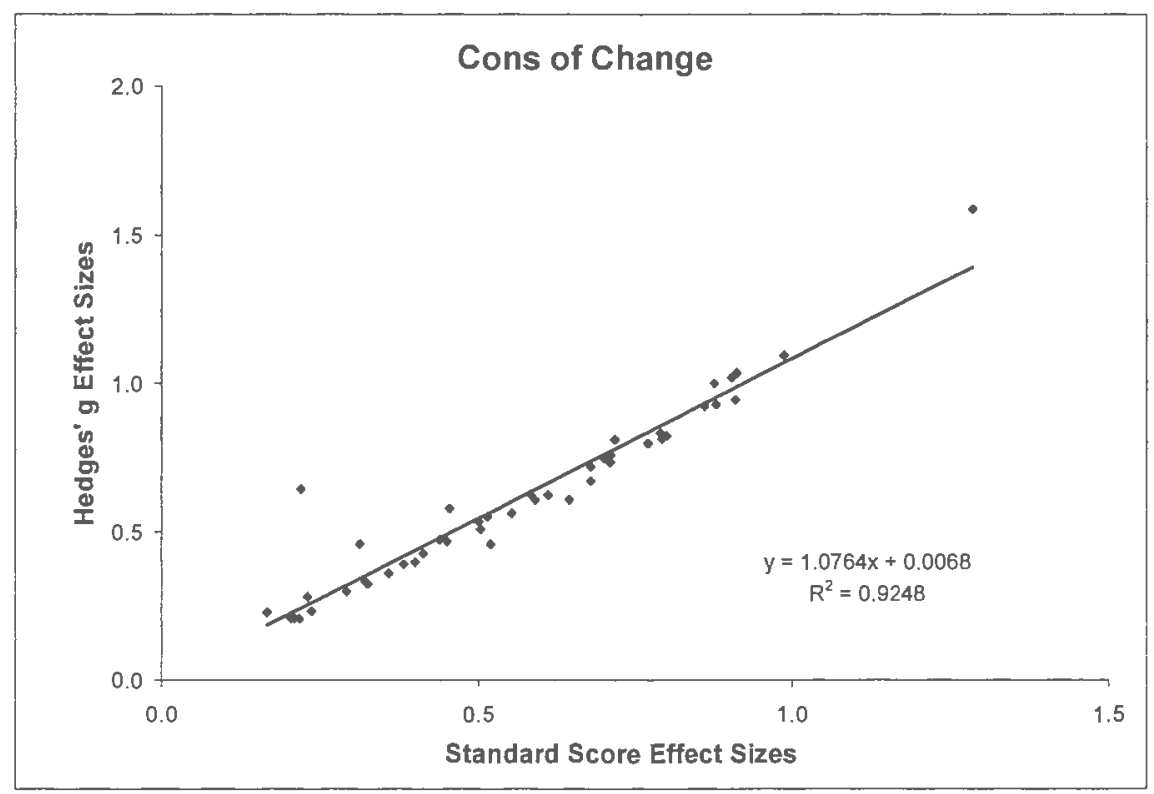

Figure 2. Relationship between Hedges' $g$ and Standard Score for Cons of Change with Outliers Removed. 
Lastly, since the relationships for the Pros and Cons between the Hedges' $g$ effect sizes and the standard score effect sizes revealed similar patterns the two sets of data (see Figure 1 and Figure 2) for these constructs were combined (see Figure 3) and regression analyses were performed. This resulted in the best fit $\left(\mathrm{R}_{\text {adj }}^{2}=95.5 \%\right)$, with an overall significant relationship $\left(\mathrm{F}_{4,41}=1826.575, \mathrm{p}<.01\right)$.

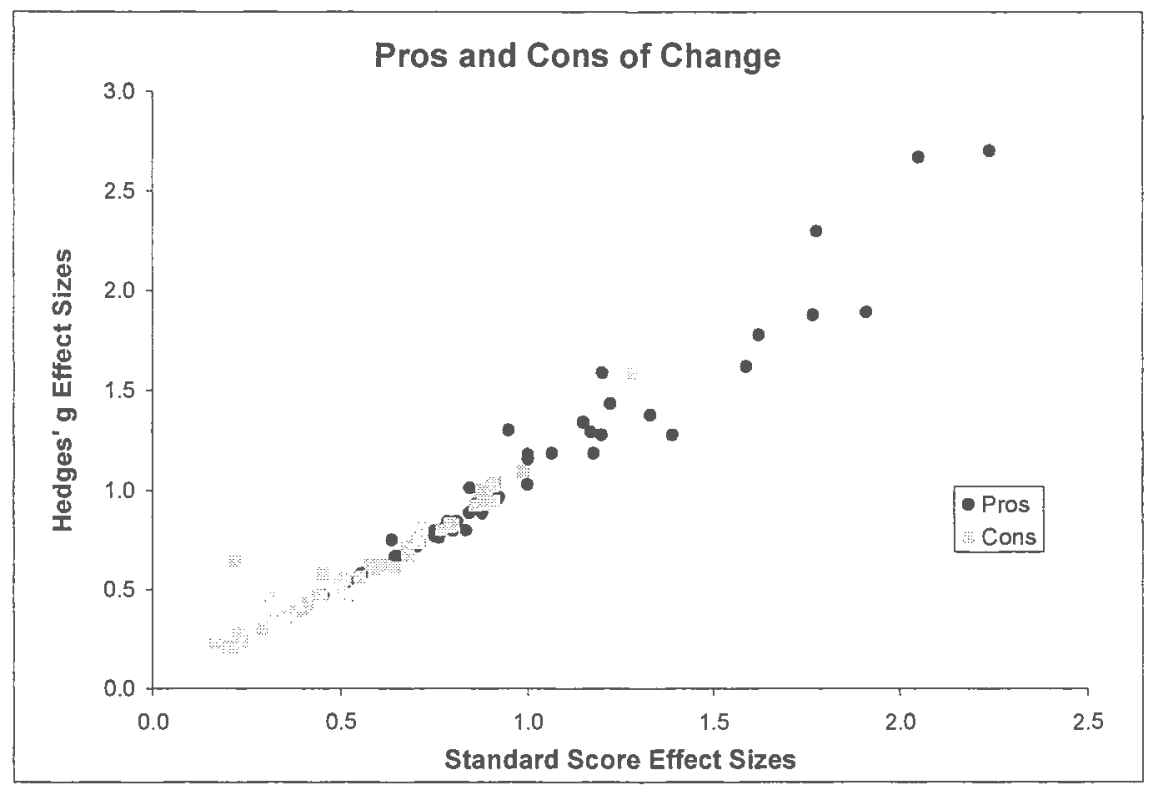

Figure 3. Relationship between Hedges' g and Standard Score for Pros and Cons of Change Combined.

Correction Formula. Regression formulas were specifically designed to correct for this bias for the Pros and Cons of change. Using the best fit regression, the combined Pros and Cons data with outliers removed, a regression correction formula was created. Effect sizes based on either the standard score or the graphical standard score methods can subsequently be corrected for bias using the following equation: 
where, $\mathrm{ES}=$ standard score effect size estimate.

\section{Discussion}

Our results showed that Hedges' $g$ consistently provided a larger effect size estimate than the standard score method for both constructs. For Pros and Cons, Hedges' $g$ was approximately $10 \%$ greater than the standard score effect size. In fact, not only were there overall differences but the standard score method underestimated effect size for $87 \%$ of the data included in the analysis. An implication of this finding is that one might consider using a correction for standard score estimates to better approximate the effect size.

A single correction equation was achieved by combining the data from both constructs that provides a good effect size adjustment. Unfortunately, it is unclear if the same correction will suffice for other constructs. Thus, meta-analysts may need to determine their own correction factor specific to their given measures.

It is clear from the data that the regression line fit small effect sizes better than larger effect sizes; therefore the correction formula is likely to be more accurate for smaller effect sizes. More exploration is needed to understand this relationship.

An important limitation of our study is the use of only two variables. Our preliminary findings suggest that a more extensive examination is warranted in order to identify possible systematic variation in effect size procedures. Simulation studies may provide an effective way to examine more complex patterns and relationships in the data to clarify what factors affect the magnitude of the discrepancy between these two approaches of estimating effect size. 
In sum, a reasonably accurate correction factor was achieved by creating a single regression equation for both constructs. Effect sizes based on either the standard score or the graphical standard score methods can subsequently be corrected for bias using the regression equation. In addition to reducing the underestimation in the magnitude of effect sizes based on standard scores, these procedures will also help reduce the number of studies that must be discarded from the study due to insufficient reporting of data on which to compute effect sizes, thus eliminating a potential source of bias.

\section{References}

Cohen, J. (1977). Statistical power analysis for the behavioral sciences, $\left(2^{\text {nd }}\right.$ ed.). New York: Academic Press.

Grimley, D.M., Prochaska, J.O., Velicer, W.F., \& Prochaska, G.E. (1995). Contraceptive and condom use adoption and maintenance: A stage paradigm approach. Health Education Quarterly, 22(1), 20-35.

Hall, K.L., \& Rossi, J.S. (2002). Meta-analysis of the structure, function, and effect size of decisional balance across the stages of change for 25 health behaviors [Abstract]. Annals of Behavioral Medicine, 24, S051.

Hall, K.L., \& Rossi, J.S. (2004). A meta-analysis of the magnitude of effect in selfefficacy across adjacent stage transitions for 24 health behaviors: informing interventions [Abstract]. Annals of Behavioral Medicine, 26, S123.

Jordan, P.J., Nigg, C.R., Norman, G.J., Rossi, J.S., \& Benisovich, S.V. (2002). Does the Transtheoretical Model need an additude adjustment? Integrating Attitude 
with Decisional Balance as predictors of Stage of Change for Exercise.

Psychology of Sports and Exercise, 3, 65-83.

King, T.K., Marcus, B.H., Pinto, B.M., Emmons, K..M., \& Abrams, D.B. (1996).

Cognitive-behavioral mediators of changing multiple behaviors: smoking and a sedentary lifestyle. Preventative Medicine, 25, 684-691.

Lipsey, M.W., \& Wilson, D.B. (2001). Practical meta-analysis. Thousand Oaks, CA: Sage.

Marcus, B.H., Pinto, B.M., Simkin, L.R., Audrain, J.E., \& Taylor, E.R. (1994). Application of theoretical models to exercise behavior among employed women. American Journal Health Promotion, 9(1), 49-55.

Prochaska, J.O. (1994). Strong and weak principles for progressing from precontemplation to action on the basis of twelve problem behaviors. Health Psychology, 13, 47-51.

Rosenthal, R. (1984). Meta-analytic procedures for social research. Beverly Hilss, CA: Sage.

Rosenthal, R. (1991). Meta-analytic procedures for social research. Thousand Oaks, CA: Sage.

Rosenthal, R. (1994). Parametric measures of effect size. In H. Cooper \& L.V. Hedges (Eds.), The handbook of research synthesis (pp. 231-244). New York: Russell Sage.

Schmidt (1992). What do data really mean? Research findings, meta-analysis, and cumulative knowledge in psychology. American Psychologist, 47, 1173-1181. 
Song, F., Sheldon, T.A., Sutton, A.J., Abrams, K.R., \& Jones, D.R. (2001). Methods for exploring heterogeneity in meta-analysis. Evaluation \& The Health Professions, 24(2), 126-151.

Velicer, W. F., DiClemente, C. C., Prochaska, J. O., \& Brandenberg, N. (1985). A decisional balance measure for assessing and predicting smoking status. Journal of Personality and Social Psychology, 48, 1279-1289. 


\section{CHAPTER SIX}

\section{A META-ANALYTIC EXAMINATION OF DECISIONAL BALANCE ACROSS STAGE TRANSITIONS: CROSS-SECTIONAL ANALYSIS}

\section{Introduction}

Interventions to decrease unhealthy behaviors and to increase healthy behaviors are crucial for health promotion and disease prevention. Tailored interventions provide a promising approach for creating positive health behavior change. To produce optimally tailored interventions: (1) theory that guides interventions needs to be comprehensively tested; and (2) empirical data that drive the tailored interventions needs to be systematically generated.

One important model of behavior change, the Transtheoretical Model (TTM), is particularly suited for serving as a framework for interventions. The TTM delineates a way to conceptualize behavior change, provides the foundation for developing assessments of an individual's readiness to change, and is utilized in tailoring interventions for actualizing behavior change. Since the development of the TTM, hundreds of studies have used the model, yet, to date there has been no comprehensive examination of the TTM across behaviors. Furthermore, many of model's theoretical assumptions have not been thoroughly tested. As a result, developing interventions based primarily on theory rather than on a firmly established empirical database may prove problematic.

The current investigation aimed to: 1) quantitatively investigate the relationship of Decisional Balance and Stages of Change by identifying the 
magnitudes of effect for Decisional Balance in relation to the transition between each adjacent Stage of Change; 2) begin to more clearly delineate the patterns of change across and within behaviors; 3 ) provide information that will help inform intervention development in order to create more efficient and effective health behavior change.

\section{Transtheoretical Model of Behavior Change}

The Transtheoretical Model (TTM) of behavior change has been utilized around the world and across a large variety of behaviors. The TTM originated by integrating theories of psychotherapy as well as incorporating constructs from alternative models. The central organizing construct of the TTM characterizes behavior change over time through five distinct Stages of Change: Precontemplation, Contemplation, Preparation, Action, and Maintenance. Two intermediate indicators of when these changes will occur are Decisional Balance (weighing of Pros and Cons) and Self-efficacy (Situational Confidence or Temptation). Additionally, the TTM explains behavior change strategies through ten Processes of Change.

The algorithms for the five Stages of Change are specific for each behavior, but usually follow these general Stage concepts: participants are considered to be in the Precontemplation Stage if they report an undesired status, that is, the presence of a problem behavior or the lack of a healthy one, and express no intention of changing in the next six months; participants are considered the Contemplation Stage if they intend to change in the next six months; participants in the Preparation Stage plan to change in the next month and have begun to engage in target behaviors, but have not 
yet met particular criteria; participants reach the Action Stage once they have met the given behavioral criteria. Lastly, if the participant has met the specified behavioral criteria for greater than six months, they have reached the Maintenance Stage.

One of the most important and reliable TTM constructs, Decisional Balance, was inspired by Janis and Mann's (1977) conflict model of decision-making. Janis (1959) proposed a descriptive schema called a "balance sheet" of incentives. The four main categories of consideration for decisional conflicts are: a) utilitarian gains and loses for self; b) utilitarian gains and losses for significant others; c) selfapproval or-disapproval; d) approval or disapproval by significant others. These four comparative categories of potential positive and negative incentives involve both instrumental effects of utilitarian objectives and nonutilitarian considerations such as issues of self-esteem and value-based determinations.

The development of the TTM Decisional Balance measure (Velicer, DiClemente, Prochaska, and Brandenburg, 1985) was based on the 8 factors (4 gains and 4 losses) of Janis and Mann (1977). The researchers constructed the scale to study the decision-making process across the Stages for smoking cessation. Instead of achieving an 8 factor-structure as anticipated, principal components analysis identified two orthogonal components. These two components were called the Pros and Cons of Smoking.

Following the original study by Velicer, the use of the construct began to expand, encompassing an array of behaviors such as exercise, condom use, and mammography screening. This early work culminated in a paper by Prochaska et al. (1994), which looked at patterns in Decisional Balance across Stages in 12 
behaviors. This integrative study investigated: 1) the generalizability of the TTM for

Stage and Decisional Balance across behaviors; 2) the generalizability of the TTM

for a variety of populations; 3 ) the number of components in Decisional Balance and their respective internal consistencies; 4) patterns of Pros and Cons across Stages; and 5) the Stage of crossover between standardized Pros and Cons scores. More recently, a meta-analysis examining 37 behaviors utilizing 81 datasets including nearly 40,000 participants was conducted re-examining these relationships and exploring additional ones (Hall \& Rossi, 2004).

\section{Generalizability of Stage and Decisional Balance}

Prochaska et al. (1994) and Hall \&Rossi (2004) found clear support for the generalizability of the Stages of Change, the Pros and Cons, and the integration between them. The researchers additionally found that these constructs generalized across a variety of populations and behaviors.

\section{Structure of Decisional Balance}

Consistent with the two-factor structure identified by Velicer et al. (1985), Prochaska et al. (1994) and Hall and Rossi (2004) respectively found 92\% and 94\% of the studies reported a two-component solution for Decisional Balance with an average internal consistency coefficients ranging from .72 to .95 for Pros and from .64 to .95 for Cons. 
In Kurt Lewin's (1948) expectancy theory, it is postulated that behavior changes as a function of the increases and decreases in motivation to contemplate gains and losses. The TTM builds on this notion by suggesting a clear directionality to the function as well as a characteristic way of examining it. The function is based on the relationship of when and how much the Pros increase and the Cons decrease (Prochaska et al., 1994) and is examined by identifying a graphical crossover of the Pros and Cons as they change relative to each other. More specifically, the crossover occurs when the standardized Decisional Balance scores are graphed by Stage. Prochaska et al. (1994) and Hall and Rossi (2004) found that the Decisional Balance crossovers occurred during the Contemplation Stage for 58\% and 53\% of the studies, respectively. Based on the timing of the crossover, the researchers suggest that progress from Precontemplation to Contemplation involves an increase in Pros whereas progress from Contemplation to Action involves a decrease in Cons.

Several Decisional Balance patterns were found in the two studies (Hall \& Rossi, 2004, Prochaska et al, 1994). For example, the Cons of Changing were higher than the Pros of Changing in the Precontemplation Stage for all datasets in both studies. Prochaska found the opposite true for 11 out of 12 behaviors in the Action Stage.

\section{Patterns across Stage}

Several additional patterns in the relationship between Stage of Change and Decisional Balance were found in the two studies (Hall \& Rossi, 2004, Prochaska et 
al, 1994). For example, the Cons of Changing were higher than the Pros of Changing in the Precontemplation Stage for all datasets in both studies. Prochaska found the opposite true for 11 out of 12 behaviors in the Action Stage.

Decisional balance (Pros and Cons) serves as an intermediate indicator of when change will occur and has generally been thought to be especially salient in the earlier Stages of Change (Redding et al., 1999; Velicer et al., 2000). Velicer et al. (2000) also illustrated that the relationship between the Stages and Decisional Balance for an unhealthy behavior is different than for a healthy behavior. That is the pattern for an unhealthy behavior was such that the Cons decreased across the Stages whereas the Pros displayed a curvilinear pattern which paralleled the decline of the Cons in the later Stages. In contrast, the healthy behavior showed more of an X configuration, with the Pros continuing to increase across the Stages whereas the Cons decrease across the Stages.

\section{Strong and Weak Principles}

Across twelve studies (Prochaska, 1994), mathematical relationships were found between the Pros and Cons of Changing and progress across the early Stages into Action (Prochaska, 1994). The Strong Principle of Change states that PC $\rightarrow \mathrm{A}$

$\cong 1 S D \uparrow$ PROS: progress from Precontemplation to Action involves approximately one standard deviation increase in the Pros of Changing. The Weak Principle of Change states that $\mathrm{PC} \rightarrow \mathrm{A} \cong .5 S D \downarrow$ CONS: progress from Precontemplation to Action involves approximately $.5 S D$ decrease in the Cons of Changing. 
In re-examination of the strong and weak principles, the magnitude of the maximum increase in the Pros of change was again found to be greater than the maximum decrease in the Cons of change from Precontemplation to Action across 37 difference health behaviors (Hall \& Rossi, 2004). Consistent with Prochaska's (1994) strong principle, the average effect size for the Pros was approximately one standard deviation $(d=1.05, S D=.45)$, almost identical to Prochaska's (1994) original finding ( $d=1.06, S D=.26)$. Though, Hall and Rossi's (2004) findings also revealed that Prochaska's weak principle may not be so weak. That is, the average effect size for Cons was stronger $(d=.62, S D=.38)$ than was found in the previous study $(d=.45, S D=.22)$ by Prochaska (1994), though clearly the Cons remains weak relative to the Pros. Practical implications of these principles are that the Pros of Changing must increase twice as much as the Cons must decrease, suggesting that an intervention place twice as much emphasis on raising the benefits as on reducing the costs or barriers.

\section{Stage Transitions}

The Strong and Weak Principles are useful for understanding the amount of work generally needed to move from Precontemplation to Action. Although theoretically the principles have been important in conceptualizing and understanding the relationship between Decisional Balance and Stages of Change, these principles are essentially action-oriented when applied to interventions. That is, by examining characteristics of the transition only from Precontemplation to Action, and using these to tailor interventions, one is potentially neglecting three Stages: 
Contemplation, Preparation and Maintenance. Therefore more practically, the examination of each Stage transition can help identify the most effective strategy for tailoring behavior change interventions.

\section{Research Hypotheses and Predictions}

The aims of this study are focused on the two constructs, Stages of Change and Decisional Balance. The research hypotheses and predictions are as follows:

Hypothesis 1. Decisional Balance for behavior change will show dimensional structure.

Prediction 1. Decisional Balance for behavior change is composed of two separate, distinguishable dimensions, the Pros and Cons (Hall \& Rossi, 2004;

Prochaska et al., 1994).

Prediction 2. The two dimensions, Pros and Cons, are not correlated (Hall \& Rossi, 2004).

Hypothesis 2. There is a functional relationship between the Stages of Change and the two Decisional Balance measures, Pros and Cons.

Prediction 1 . The majority of the crossovers will occur during the Contemplation Stage as seen in previous studies (Hall \& Rossi, 2004; Prochaska et al., 1994)

Hypothesis 3. There is a relationship between the Stage transitions and the two Decisional Balance measures, Pros and Cons.

Prediction 1. The Strong and Weak Principles of Change have shown approximately 1 standard deviation increase in the Pros of Change as one progresses 
from Precontemplation to Action, whereas the Weak principle is approximately a $1 / 2$ standard deviation decrease in the Cons of Change as one progresses from Precontemplation to Action (Hall \& Rossi, 2004; Prochaska, 1994). Since the Strong and Weak Principles measure the maximum increase and decrease of the Pros and Cons from Precontemplation to Action, rather than an absolute difference, these two principles are "biased". That is, the principles result in a potential over-estimation of the cumulative effect size across the three transitions PC-C, C-PR, and PR-A.

Therefore it is predicted that $(\mathrm{PC}-\mathrm{C})+(\mathrm{C}-\mathrm{PR})+(\mathrm{PR}-\mathrm{A})<1 S D$ (standard deviation) for Pros and $(\mathrm{PC}-\mathrm{C})+(\mathrm{C}-\mathrm{PR})+(\mathrm{PR}-\mathrm{A})<.62 \mathrm{SD}$ for Cons.

Prediction 2. The Pros and Cons appear to be most salient in the earlier Stages of Change (Redding et al., 1999; Velicer et al., 2000), therefore it is predicted that the greatest magnitude of effect will be seen in the transition from PC-C for both the Pros and Cons. The transition from PC-C is anticipated to be approximately .5 $S D$ with the transitions C-PR and PR-A each approximately $.2 S D$. Finally, since it is believed that the earlier Stages are most salient and since one would anticipate more "work" would be needed to move from pre-Action Stages towards Action than from Action to continuing to maintain that Action, the transition from A-M for Pros and Cons is predicted to have the smallest effect size.

Prediction 3. In an examination of the Strong and Weak Principles (Hall \& Rossi, 2004), the distribution of effect sizes were found to be heterogeneous for both Pros and Cons, therefore it is predicted that the distribution of effect sizes for each Stage transition will also be heterogeneous and that there will be several moderators of the effect size distributions. 
3a. Although it is clear that the model generalizes to a variety of behaviors, there are many factors within the characteristic of the behaviors and the studies that may contribute to variation (e.g. similarity of measures within some behaviors but not across behaviors). Therefore, as seen in previous studies (Hall \& Rossi, 2004), it is anticipated that behavior category will be a moderator variable and that there will be heterogeneity within behaviors, indicating additional moderators.

3b. Studies have shown that patterns of TTM variables vary by age group (Hall \& Rossi, 2004, Weinstock et al., 2002), therefore it is predicted that mean age of study participants will be a moderator of effect sizes.

3c. A recent study found significant effect size differences between response formats (Hall \& Rossi, 2004) for the Pros and Cons, therefore it is predicted that response format will be a moderating variable.

3d. Publication status has not previously been examined as a potential moderator of effect size distribution. Datasets will be gathered from sources such as dissertation and conference presentations, since these types of publications are "refereed", although perhaps not as rigorously as peer-reviewed publications, it is anticipated that publication status will not be a moderating variable.

\section{Method}

Procedure

\section{Literature Searches}

The datasets for this study were identified through literature searches on computerized databases, PUBMED, Cancerlit, Cinahl, Health and Wellness 
Resource Center and PsycLIT, starting in 1985 (the year of the initial Decisional Balance study). Keywords to identify appropriate studies included, Transtheoretical Model, Stages of Change Model, Stages of Change, Decisional Balance, decision making, and Pros and Cons. Additional search procedures included personal communications with authors and reviews of reference lists from acquired articles.

Datasets include published articles, conference presentations, papers in progress, and raw data.

\section{Inclusion Criteria}

Studies involving any behavior, which examined Stages of Change and Decisional Balance, were considered for analysis. Next, the studies needed to include: 1) means, $S D$ 's, and $n$ 's of the Decisional Balance measures for each given Stage; or 2) mean $T$-scores of the Decisional Balance measures for each given Stage (in either text, table, or graph). In addition, the studies needed to report at least two consecutive (e.g., Precontemplation and Contemplation or Contemplation and Preparation) Stages of Change.

There are a variety of ways in which researchers assess Stage of Change. Two main categories of Stage assessment are algorithm type staging and clustering type staging. For inclusion in the current study, the Stages must have been assessed by an algorithm or Likert procedure and not cluster procedures (e.g. URICA, Socrates, or cluster analysis approach). Firstly, procedures such as the URICA, often don't yield traditional Stage categories (e.g., they often include categories called "immotive" and "non-reflective action"). Attempts at consistent interpretation of the profiles to ensure consistent interpretation would be a complex procedure and would 
require sufficient reporting of profiles to "re-categorize" them and link them to the

Stages or order them from "least ready" to "most ready" to change. Additionally, profiles across studies are inconsistent which would mean interpretation would have to be done on a case-by-case basis. Lastly, scoring for cluster approaches, specifically URICA, are complicated and are sometimes improperly or inconsistently scored, therefore raw data and reanalysis would likely be necessary (D.A. Levesque, personal communication, December, 17, 2003).

\section{Training of Coders}

Lipsey and Wilson (2001) emphasize that the coding of studies is "one of the most technically demanding aspects of meta-analysis". Therefore the training of coders should be given careful attention. At the onset of the project several instructional seminars, including readings, lectures, and discussions, were provided. The extent of the instructional seminars depended largely on the knowledge and sophistication of the coders. Issues that were covered included, for example: (1) general and theoretical knowledge about the Transtheoretical Model; (2) methodological and statistical procedures common in the body of literature and relevant to the research question; and (3) meta-analytic procedures and strategies. Subsequently, coding of variables were reviewed and procedures for the coding process discussed. Coders practiced coding variables until researchers were comfortable with the coding procedures. Periodic meetings were conducted to review progress and discussion any difficulties encountered followed. In some cases significant discrepancies occurred between researchers for particular variables. These variables were then redefined and recoded for all studies. 
A coding manual (see Appendix A) was developed during the course of the project to delineate the coding for study design, participant, measurement, publication, and research characteristic variables. Rosenthal (1984) found that the typical rate of recording errors is approximately $1 \%$, though this rate can be as high as $48 \%$. In order to minimize transcription error, data was entered directly into an excel spreadsheet by the researcher and by trained research assistants. To minimize clerical error and reduce subjective bias in coding, all data was checked at least twice. A third researcher reviewed any discrepancies and consensus was reached. Table 2 provides a summary of the primary variables extracted and coded. The coding manual found in Appendix A provides more detailed descriptions of coded variables. Additionally key variables are expanded upon below. 
Table 2

Examples of Data Categories

\begin{tabular}{|l|l|}
\hline Data Category & Description/Example \\
\hline Target Behavior & Specific behavior measured in study \\
\hline Population description & Brief description of sample \\
\hline Setting & Place where study was conducted \\
\hline Sample Size(s) & $\begin{array}{l}\text { Number of participants for the overall study, for each } \\
\text { dataset, and for each Stage }\end{array}$ \\
\hline Sampling Method & Random, convenience, or mixed \\
\hline Age & Mean age of the participants \\
\hline Age Group & Adolescents, College, Adults or Mixed \\
\hline Gender & Percentage of males and females in the study \\
\hline Country & Country where the study is conducted \\
\hline Language & Language of participants and/or measures \\
\hline Stage Descriptions & Descriptions of Stages used in each study \\
\hline Stage Algorithm Type & e.g., one item staging; multiple item skip pattern \\
\hline Response Format & e.g., How important or Agree/disagree \\
\hline Coefficient Alphas & $\begin{array}{l}\text { Reported scale reliability values calculated specifically on } \\
\text { study data }\end{array}$ \\
\hline Correlation Coefficients & Scale and/or latent construct values \\
\hline Number of Items & Number of items per measure \\
\hline $\begin{array}{l}\text { Decisional Balance } \\
\text { Description }\end{array}$ & e.g., Pros and Cons of Weight Bearing Exercise \\
\hline $\begin{array}{l}\text { Effect Size Calculation } \\
\text { Method }\end{array}$ & $\begin{array}{l}\text { e.g., Hedges g, standard score method or graphical standard } \\
\text { score method }\end{array}$ \\
\hline Publication status & $\begin{array}{l}\text { e.g., peer-reviewed publication, dissertation, conference } \\
\text { presentation }\end{array}$ \\
\hline Healthy vs Unhealthy & e.g., Exercise verse Smoking \\
\hline Frequency of Behavior & i.e., Daily, Situational, Yearly \\
\hline Reversal / Direction & Direction of the Pros and Cons in relation to Staging \\
\hline
\end{tabular}

\section{Target Behaviors}

Target behaviors were defined based on the researcher's treatment of the behavior. A particular behavior was considered a target behavior if the researcher used separate Staging or Decisional Balance Measures for the named behavior. For example, the cessation and acquisition of a behavior such as smoking were considered separate behaviors since specific staging measures were developed for 
smokers and nonsmokers (e.g., Plummer et al., 2001). Likewise, when researchers developed Decisional Balance measures specifically for particular behaviors, such as for condom use with main partner or condom use with other partners (e.g., Lauby et al., 1998), the given behaviors were considered separate target behaviors.

\section{Data Reversal}

In general researchers consistently define the Pros and Cons. In some cases, the Pros and Cons are inversely defined, that is, the measures focus on the positive aspects of an unhealthy rather than of a healthy behavior (e.g., Borland \& Segan, 2000; Rossi et al., 2001; Velicer et al., 1985). For example, a study may examine the Pros of smoking (Velicer et al., 1985) rather than the Pros of quitting smoking (Keller et al., 1999). In this type of study, the Pros and Cons will be reversed for analytic purposes.

\section{Acquisition versus Cessation}

Behavior change can happen in two main ways, acquiring new behaviors (e.g., begin to exercise regularly) or ending an existing behavior (e.g., quit smoking). Behaviors were coded accordingly.

\section{Analysis}

Five main aspects of the Stage of Change and Decisional Balance measures were examined across behaviors and within behaviors (when there were five or more datasets of a particular behaviors): (1) the measurement structure of Decisional Balance; (2) the nature of the functional relationship between the Stages and Decisional Balance; (3) the magnitude of the effect of Decisional Balance across 
Stage transitions; (4) the homogeneity of the effect size distributions; and (5) moderators of effect.

\section{Structure}

It was predicted that the majority of the studies identified in the meta-analysis would identify or utilize a two-factor structure for Decisional Balance. The dimensional structure of Decisional Balance was determined by identifying results of analyses such as principal components analyses (PCA), or structural equation modeling. The factor structures of the studies are described descriptively. Additionally, it was predicted that the two dimensions, Pros and Cons, would not be correlated. The strength of association between the Pros and Cons measures was extracted when either the scale or construct correlation coefficient was reported in the studies.

The internal consistency of the Decisional Balance measures was also examined; for those studies that provided alpha coefficients, the alphas were averaged and their range reported.

\section{Function}

The functional relationship between the Stages and Decisional Balance were explored by examining the given graphs or constructing new graphs with Stage plotted on the $\mathrm{x}$-axis and the mean $T$-scores plotted on the $\mathrm{y}$-axis. It was predicted that the majority of the crossovers would occur during the Contemplation and Preparation Stage. The functional relationships in Decisional Balance were examined descriptively and tabulations of crossovers conducted. 
Effect size magnitude was investigated for each Stage transition: 1)

Precontemplation to Contemplation; 2) Contemplation to Preparation; 3) Preparation to Action; 4) Action to Maintenance. Stage transition effect size were calculated using Hedges' $g$, standard score, or graphical standard score estimation.

\section{Calculation Method}

The preferred and most accurate method of estimating effect size is the Hedges' $g$ method. Hedges' $g$ requires means, $S D$ 's, and n's for each group (either raw scores or $T$-scores), and is defined as the difference between the sample means divided by the average pooled sample standard deviation (Rosenthal, 1994).

$$
\text { Hedges' } g=\frac{M_{1}-M_{2}}{S_{\text {pooled }}}
$$

If sufficient data were not available to compute Hedges' $g$, one of the standard score methods was used to compute effect size. Both the standard score and the graphical standard score methods require the standard (T) scores $(M=50, S D=$ 10) for each group, and were calculated by subtracting the sample means and dividing by the standard deviation. The only difference between the standard score and the graphical method was that the sample means for the standard score method were obtained from the text or a table, whereas, the graphical method used scores estimated from a graph.

\section{Graphical Estimation Procedure}

Estimations of $T$-scores from graphs were made by using a ruler to measure the point against the $y$-axis where the $T$-scores are graphed. The ruler measurements 
were then used to interpolate $T$-scores between adjacent tic marks on the $y$-axis. Chapter Five illustrated that the $T$-scores method and $T$-scores estimated from a graph for Pros and Cons measures were very highly correlated and no significant differences were found between the two methods, thereby demonstrating that the graphical method provides a good estimation of $T$-scores. It should be noted that the graphical method was used only when Hedges' $g$ could not be calculated and the actual standardized scores were not available in text or table.

\section{Calculation Method Adjustment}

When data are reported as standard scores, such as z $(M=0, S D=1)$ or $T$ scores $(M=50, S D=10)$, effect sizes are determined by subtracting $T$-scores and dividing by 10 . This procedure appears correct because it is known that the $S D$ of $T$ scores equals 10 . Therefore, dividing the $T$-score difference by 10 should result in the standardized difference between the means. However, this procedure is suboptimal because 10 is the total group $S D$, not the pooled within-group $S D$ used to compute Hedges' $g$ or Cohen's $d$ (Rosenthal, 1994). In most cases the within-group variance will be less than the total group variance (i.e., whenever the grouping variable has a non-zero relationship with the dependent variable), since this will reduce within-group variance relative to total variance (i.e., $S S_{\text {error }}=S S_{\text {total }}$ $\left.S S_{\text {between }}\right)$, computing effect size by subtracting $T$-scores underestimates Hedges' $g$. As described in Chapter 5, regression equations specifically designed to correct for this bias in the Pros and Cons of Change were developed. Therefore, effect sizes based on either the standard score or the graphical standard score methods were subsequently corrected for bias using the following equation: 
where, $E S=$ standard score effect size estimate.

Additional analyses were conducted to verify the effectiveness of this bias correction, including analog ANOVA's. In the event that calculation method appears to moderate the effect size distribution, all cross-sectional data analyses including effect size calculations would be conducted separately for the effect size methods. Based on previous work (Hall \& Rossi, 2004), it was anticipated that $70 \%$ of the effect sizes would be calculated using Hedges' $g$, whereas $30 \%$ would be calculated using standard scores.

Sample Size Bias. Simple pooling of data occurs when each set of data from a particular study is pooled together without regard to differences in sample size. Therefore, studies that include 10,000 participants provide the same weight in the analysis as a study with 10 participants. To avoid this problem, all effect sizes were corrected for sample size bias by weighting each data point by its respective sample size (see Equations 9-11). Another concern regarding sample size is that Hedges' $g$ has been shown to be upwardly biased with small sample sizes, especially those less than 20 (Hedges, 1981). Although the overall n's for the datasets were quite large, since the effect size for the Stage transitions were based on the sample size of a given Stage, these $n$ 's could be reasonably small. Therefore, the obtained effect size was corrected for sample size bias. This calculation was computed using Hedges' formula:

$$
E S^{\prime}=\left[1-\left(\frac{3}{4 N-9}\right)\right] E S
$$




$$
\begin{aligned}
& S E=\sqrt{\frac{n_{1}+n_{2}}{n_{1} n_{2}}+\frac{\left(E S^{\prime}\right)^{2}}{2\left(n_{1}+n_{2}\right)}} \\
& \omega=\frac{1}{S E^{2}}=\frac{2 n_{1} n_{2}\left(n_{1}+n_{2}\right)}{2\left(n_{1}+n_{2}\right)^{2}+n_{1} n_{2}\left(E S^{\prime}\right)^{2}}
\end{aligned}
$$

where, $E S=$ the observed (uncorrected) effect size estimate, $E S^{\prime}=$ the corrected effect size estimate, $N=$ the total sample size, $S E=$ the standard error of the corrected effect size estimate, $n_{1}$ and $n_{2}=$ the sample sizes of the two groups (adjacent Stages) being compared, and $\omega=$ the inverse variance weight. The inverse variance weight reflects the precision of the effect size estimate, which varies as a function of sample size. This method was used to weight the contribution of each study effect size so that larger studies were given more weight in the calculation of the overall meta-analysis effect size (Hedges \& Olkin, 1985; Lipsey \& Wilson, 2001).

Confidence Intervals

Confidence intervals illustrate the precision of a parameter estimate. The confidence interval provides an additional dimension to reporting effect sizes by indicating how "confident" one can be in the measure of magnitude obtained. The researcher has the flexibility to choose the level of probability, and this is done by setting the probability percentage for the confidence interval. For instance, a $95 \%$ confidence interval indicates that the confidence interval has a $95 \%$ probability of containing the population parameter.

The width of the confidence interval is directly related to: (1) the amount of data used to generate a given effect size; (2) the level of confidence interval chosen 
by the researcher; and (3) the computational model used (Borenstein \& Rothstein, 1999). The larger the amount of data used the more precise the measure of effect size and therefore the narrower the confidence interval. Inversely, the greater the confidence level chosen by the researcher, the wider the confidence interval will be. Lastly, variance across studies attributed to entirely random influences are modeled with a fixed effects model. This model allows for confidence intervals that can actually reach zero. On the other hand, if the random variance and between-studies variance is assumed then a random effects model is typically employed. This model tends to produce wider confidence intervals and limits the ability of the confidence intervals to approach zero (i.e., no confidence interval but rather a point "estimate").

Ninety-five percent confidence intervals were calculated around the mean effect sizes. This indicates that the confidence interval has a $95 \%$ probability of containing the population parameter. Based on the standard error for the mean and a critical value from the $z$-distribution the confidence intervals were calculated as follows:

$$
\begin{aligned}
& S E_{E S}=\sqrt{\frac{1}{\sum \omega_{i}}} \\
& E S_{L}=E S-z_{(1-\alpha)}\left(S E_{E S}\right) \\
& E S_{U}=E S+z_{(1-\alpha)}\left(S E_{E S}\right)
\end{aligned}
$$

where $S E_{E S}$ is the standard error of the effect size mean, $\omega_{i}$ is the inverse weight associated with the effect size $i$ with $i=1$ to $k$ effect sizes included in the mean and $E S$ is the mean effect size, $z_{(1-\alpha)}$ is the critical value for the $\mathrm{z}$-distribution (1.96 for $\alpha=.05$ ). Confidence intervals were reported for combined effect sizes. 
In meta-analytic studies, variation among effect sizes sometimes occurs due to random error, though oftentimes this variation becomes statistically larger than one would expect (due to random or sampling error). Tests of homogeneity attempt to identify the difference between variation due to random error and variation due to systematic differences between study design and participant characteristics.

Ultimately, assessing heterogeneity of effect size is important because the lack of a homogeneous distribution suggests the presence of possible predictors or moderators of the effect size magnitude.

A variety of methods of heterogeneity are available, though the majority are designed for assessing variation in treatment effects with odds ratios and percent of variance accounted for measure of effect size. Since this study utilized standardized effect size measures, the test of homogeneity employed in this study was based on the $Q$ statistic.

The $Q$ statistic is distributed as a chi-square with $k$ - 1 degrees of freedom $(k=$ number of effect sizes) (Lipsey \& Wilson, 2000). The homogeneity analysis will be calculated using the equation:

$$
\mathrm{Q}=\left(\sum \omega_{i} E S_{i}{ }^{2}\right)-\frac{\left(\sum \omega_{i} E S_{i}\right)^{2}}{\sum \omega_{i}}
$$

where $E S_{i}$ is the individual effect size for $i=1$ to $k$ (the number of effect sizes), and $\omega_{i}$ is the individual weight for $E S_{i}$ calculated using Equation 11 defined above.

The $Q$ statistic is most accurate for sample sizes greater than 10 (Hedges \& Olkin, 1985) or 20 (Takkouche, Cadarso-Suarez \& Speigelman, 1999) and increases 
in accuracy as sample size increases. Low power can result in statistical tests that fail to detect heterogeneity. Since many of the sub-groups included less than 20 (e.g., many of the behavior categories have less than 20), a priori subgroup analyses were established to ensure primary theoretically-based hypotheses were thoroughly tested. Firstly, subgroup analyses between behavior categories with greater than 5 studies were conducted as well as additional behavioral subgroup analyses on each of these behavior categories (i.e., smoking cessation vs. smoking acquisition). Additionally, regardless of identified heterogeneity, subgroup analyses were performed for categorical moderators. Additionally, other methods of assessing heterogeneity were also employed. In particular, graphical methods for testing heterogeneity were used. Forest plots displaying point estimates and confidence intervals for individual studies and summary estimates were created. Excel macros to create forest plots were developed. Forest plots were examined for systematic patterns in study or construct characteristics.

Tests of homogeneity of the distribution of effect size were conducted on all effect sizes for each transition for both the Pros and the Cons. Fixed effects and Random effects models were tested (as described below). Additionally, tests of homogeneity were conducted on all subgroup and moderator analyses.

Due to the variability in the dimensions of each study, it was anticipated that the effect sizes would be heterogeneous. Upon the discovery of heterogeneity, the follow-up tests were conducted initially for calculation method and then subsequently for the remaining potential moderator variables. 


\section{Modeling Variance}

Two methods of modeling variance, fixed and random effects modeling, were used in this study. The fixed effects model assumes the only source of variance is subject-level sampling error, whereas the random effects model assumes that the source of variance includes subject-level sampling error and study-level sampling error (i.e., random error). The fixed effects model can also include systematic variance due to identifiable moderating variables.

Although a third model, the mixed effects model, exists - the mixed model is not employed in this study. Theoretically, a mixed model assumes all three sources of error, sampling error, random error and systematic error. The mixed effects model is typically used less often than fixed and random effects models due to the complexity of the modeling procedures and lack of consensus in the field regarding these procedures.

In a fixed effects model, it is assumed that the variance in the effect size distribution is only due to subject-level sampling error. So it is assumed that an effect size from an individual study represents the population effect with only random sampling error associated with chance factors. Tests of homogeneity examine the assumption that error is only due to sampling error and thereby serve as an assessment for whether the fixed effects model holds, though as discussed below, evidence of heterogeneity does not necessarily rule out a fixed effects model.

If one assumes that the fixed effects model holds but additional error is due to a systematic difference in the coded variables then one can attempt to partition the effect size variance. This assumption can be tested by performing an analog to 
analysis of variance. This analog ANOVA was used to explore variables by partitioning the total variance $Q$ (Total) into $Q$ (Between) and $Q$ (Within). $Q$

(Between) values were used to test between group differences by using a Chi-square with $d f=p-1$.

The meta-analytic analog to the analysis of variance for the fixed effects model is calculated as follows (Lipsey \& Wilson, 2001):

$$
\mathrm{Q}_{\mathrm{B}}=\left(\sum \omega_{j} \overline{E S}_{j}^{2}\right)-\frac{\left(\sum \omega_{j} \overline{E S}_{j}\right)^{2}}{\sum \omega_{j}}
$$

where $\mathrm{Q}_{\mathrm{B}}$ is the between groups, $\overline{E S}_{j}$ is the weighted mean effect size for each group, $\omega_{j}$ is the sum of the weights within each group, and $j$ is the number of groups.

$$
\mathrm{Q}_{\mathrm{w}}=\sum \omega_{i}\left(E S_{i}-\overline{E S}_{j}\right)^{2}
$$

where $\mathrm{Q}_{w}$ is the pooled $Q$ within groups, $E S_{i}$ is the individual effect size, $\overline{E S}_{j}$ is the weighted mean effect size for each group, $\omega_{j}$ is the sum of the weights within each group, $i$ is the number of effect sizes, and $j$ is the number of groups.

If the fixed effects model does not hold then another model is sought. The random effects model assumes that sampling error is accompanied by other sources of variability randomly distributed. The sampling error is considered to be from the subject-level whereas the random error is an estimate of between-studies variance. This between-studies error is thought to be analogous to study-level sampling error. The variance components can be illustrated by the following equation (Lipsey \& Wilson, 2001): 


$$
v_{i}^{*}=v_{\theta}+v_{i}
$$

where, $v_{\theta}$ is the random or between-subjects component, and $v_{i}$ is the subject-level sampling error.

Identifying the random effects variance component is the more difficult of the two components and can be assessed using a noniterative methods based on method of moment or iterative methods based on maximum likelihood. The method of moments estimate is calculated using the following formula (Lipsey \& Wilson, 2001):

$$
v_{\theta}=\frac{Q-(k-1)}{\sum \omega_{i}-\left(\frac{\sum \omega_{i}^{2}}{\sum \omega_{i}}\right)}
$$

where, $Q$ is the value of the homogeneity test, $k$ is the number of effect sizes and $\omega_{i}$

is the inverse weight for each effect size. The iterative maximum likelihood random effects variance estimate is a more accurate measure and was calculated using SPSS Macros written by David Wilson (Lipsey \& Wilson, 2001).

Fixed and random effects models were fit to the effect size data for the Pros and Cons for each of the four Stage transitions (PC-C, C-PR, PR-A, A-M). The random effects variance component $(v)$ for each transition was determined for each transition for each construct. A $Q$-test was then conducted on the random effects variance component in order determine if the null $(v=0)$ was rejected. A significant $Q$-test for the random effects variance component indicates that the random effects component is significantly greater than zero. If significantly greater than zero, the variance in the sample of effect sizes is greater than would be expected from 
sampling error alone (Lipsey \& Wilson, 2001). In this case, statistically the random

effects model is appropriate. A non-significant $Q$-test for the random effects

component would indicate statistical support for the fixed effects model.

Additionally, it is suggested that a random effects model is appropriate for studies pooled across populations with pre-existing differences (Hedges \& Olkin, 1985).

Additional justification for the random effects model where the random effects variance component is found to be significant due to the wide range of age groups and recruitment settings the populations are drawn from in the studies combined in this meta-analysis.

\section{Follow-up Comparisons}

When conducting follow-up comparisons for the ANOVA analog, a Bonferoni test is sometimes recommended since typical follow-up tests for ANOVA such as Tukey are not available. A Bonferoni correction would be used for data given a fixed effects model. Since the random effects model is already a conservative approach to examining relationships among the effect sizes, Bonferoni corrections were not used for follow-ups to random effects comparisons.

\section{Publication Bias or The 'File Drawer" Problem}

Publication bias is always a potential problem when conducting metaanalyses. One of the most concerning problems occurs when a meta-analysis is examining intervention effects, since historically there has been a propensity to published studies with statistically positive results. One common method of testing publication bias is the "fail-safe $K$ " method. The impact of any remaining sampling bias was estimated by computing the "fail-safe $K$ ". This is an estimate of the number 
of undiscovered studies that, if known, would in aggregate reduce the overall metaanalysis effect size so that it was not statistically significant. Fail-safe $K$ is computed using the following equation:

$$
K_{0}=K\left[\frac{E S}{E S_{c}}-1\right]
$$

where $K_{0}=$ the number of studies with an effect size of zero required to reduce the overall effect size to nonsignificance, $K=$ the observed number of studies, $E S=$ the observed mean effect size, and $E S_{c}=$ the minimum effect size deemed significant. In the current study it is not of theoretical importance to test if the effect size estimates calculated are significantly different from zero, in fact some effect sizes are anticipated to approach zero. In the context of this study $E S_{c}$ is not relevant and therefore the fail-safe $\mathrm{K}$ is not an appropriate measure of publication bias.

Since the current study is not directly examining overall treatment effects a more important bias measure in this context will be the examination of the publication characteristics as potential moderator variables. Therefore, effect sizes for published and unpublished studies will be compared to assess the potential impact of publication bias on effect sizes. Additionally, publication status will be treated as a potential moderator variable and will be subjected to the moderator analyses described above.

Missing Data Procedures. A variety of missing data strategies have been developed to statistically deal with missing data, unfortunately very few have been specifically designed to deal with missing data in meta-analytic studies. Three types of missing data are found when conducting meta-analyses, these are: 1) entire 
studies; 2) essential information for computing effect sizes; and 3) missing characteristic variables.

When data is missing at the study level due to non-publication, typically this is considered publication bias and is often called the "file drawer" problem and is typically assessed using the fail-safe $K$. Unfortunately the fail-safe $K$ is not relevant to the current study; therefore the impact of the missing studies can not be assessed.

Some studies were missing information for calculating effect sizes. When possible, information was extracted from alternative sources. For instance, in one study sample sizes by stage were not reported precluding the ability to calculate the pooled variance (Hulton, 2001). In this case, alternative sources of data (i.e., mean squared error term) were used instead. Additionally, for some studies alternative calculation methods were used to compute effect sizes (as described above) and adjustments were made to minimize potential bias. Otherwise, studies missing essential information to compute effect sizes were excluded from the study.

Data missing from characteristic variables, that is, potential moderator variables can be handled in a variety of ways: complete case analysis, mean substitution, and available case analysis (Pigott, 2001). Available case analysis is the most common procedure used in meta-analytic studies and allows for the most data to be retained thereby increasing power. Available case analysis was used in this study.

\section{Outliers}

Since such a wide range of potential moderating variables were likely to be examined and large variations in effect sizes across the transitions were anticipated, 
only the most extreme effect sizes ( 2 to 3 standard deviations from the mean) were considered for removal or recoding. First, any identified outliers were scrutinized to assess possible sources of error. Only variables with clear sources of error (that can not be corrected) were considered for removal. Next, effects sizes were compared to the mean effect sizes two and three standard deviations from the mean were identified. Studies were then coded as containing outliers or not and crosstabs were conducted with potential moderating variables.

\section{Graphing Techniques}

In order to visually compare results between moderator variables graphically several techniques were employed, including $T$-score by Stage graphs and forest plots.

\section{T-score by Stage Graphs}

In order to create graphical representations in the "classic" $T$-score by Stage format, as typically seen in the TTM body of literature effect sizes were converted into " $T$-scores" and graphed by Stage. In order to illustrate the results to show the effect size differences, a starting point of 0 was chosen for the PC Stage for each set of data. Effect sizes were then incrementally added to this starting point for each Stage transition. The data was then recentered by computing the mid-point of the effect sizes by subtracting the difference between the lowest and highest effect sizes, dividing by 2 and adding this to the the lowest effect size.

$$
\text { Midpoint }=\frac{T_{\text {high }}-T_{\text {low }}}{2}+T_{\text {low }}
$$


This midpoint then functions as the $T$-score of 50 (once the score is converted as described below) and is subtracted from all Stage scores. This gives the number of standard deviations above and below the midpoint for each Stage. The scores are then converted to $T$-scores by multiplying by 10 and adding 50 . Finally, these $T$ scores are plotted by Stage.

\section{Forest Plots}

Forest plots provide a visual tool for assessing differences between studies and study characteristics. The primary data provided in a forest plot is the effect size estimate and the confidence interval around that estimate. Typically the means and confidence intervals are plotted around an axis point that is set at zero; this provides a visual aid for assessing significance of the mean. That is, if the confidence interval around the mean does not cross the zero axis then the mean is significantly different from zero. For treatment effects, a confidence interval that is greater than zero would mean the mean is significant with respect to the null hypothesis. In this study, the point of interest is the comparative value of an individual mean to the overall mean of each effect size estimate for each transition since there is no null hypothesis that is being tested. Therefore, forest plots will utilize the mean effect sizes estimate of the given transition for its axis rather than the zero (i.e., the null).

\section{Power Analysis}

The principal question of power for meta-analysis involves not so much whether or not the overall effect size is statistically significant but rather whether there is sufficient power to determine if the effect size distribution is heterogeneous. 
Power for the $Q$ test depends on the ratio of between- to within-study variance (Hedges \& Pigott, 2001). It is difficult to know what this ratio might be a priori. As an estimate, Schmidt (1992) reports a range of .33 to 1.0 across a large number of meta-analyses that he examined. Hedges and Pigott (2001) suggest variance ratios of $.33, .67$, and 1.00 be considered small, medium, and large degrees of heterogeneity, respectively. The figure below gives power for this range of variance ratios for sample sizes of 10 to 150 studies and alpha $=.05$. These results suggest that for the overall meta-analysis (across behaviors), power would be excellent for medium and large degrees of heterogeneity and would be very good (at least .80) even for a small degree of heterogeneity, since the sample size is nearly 150 . For $Q$ tests within behaviors, the degree of heterogeneity would have to be in the medium to large range for power to be good for the expected sample sizes. Based on the results of a previous study on Decisional Balance (Hall \& Rossi, 2004), the degree of heterogeneity within behaviors was anticipated to be fairly large, since significant $Q$ test statistics were obtained for each of the five behaviors that were tested individually, with sample sizes ranging from only 9 to 15 . 


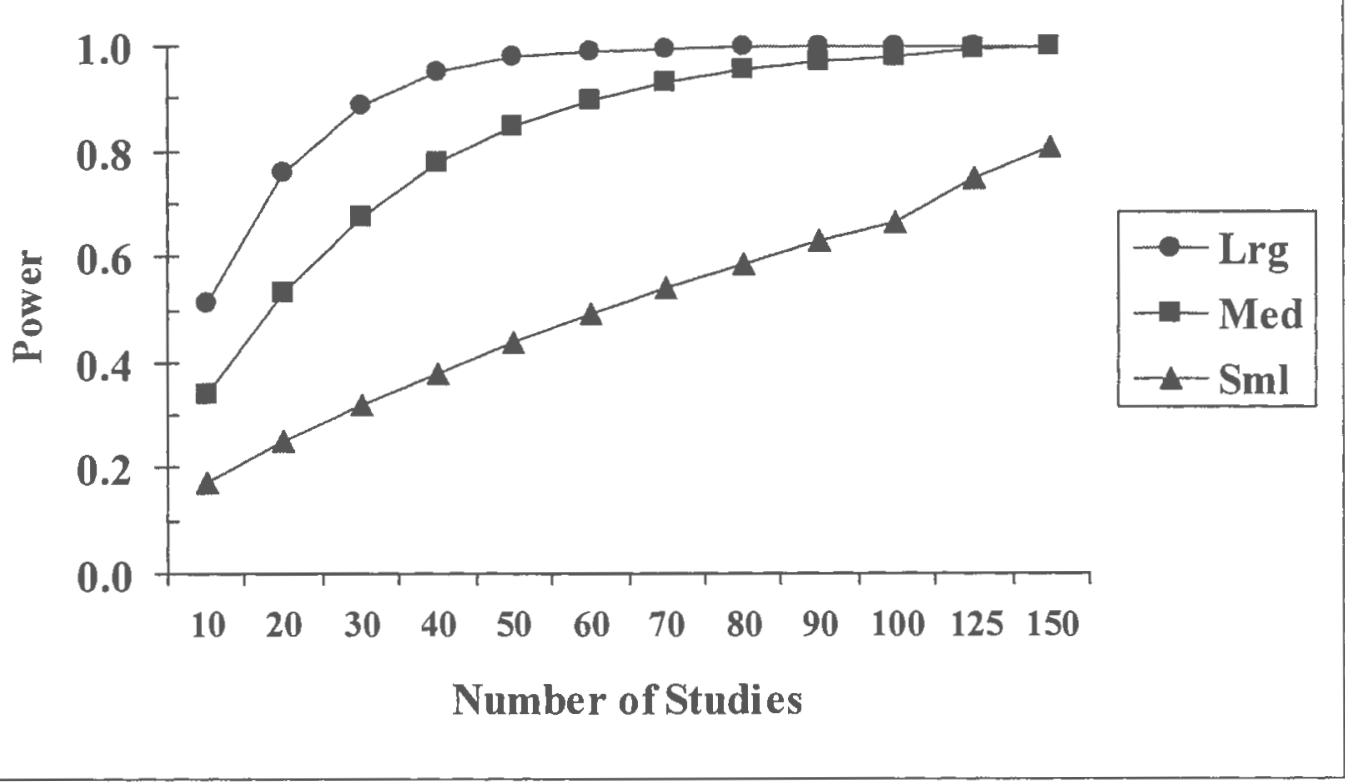

Figure 4. Power for Q Test of Effect Size Homogeneity for Small, Medium, and Large Between- to Within-Study Variance Ratios (alpha $=.05)$

\section{Results}

General Characteristics of the Studies

One hundred sixteen studies encompassing 55 different target behaviors (Table 3) were examined in this meta-analysis. Some studies reported multiple studies (e.g., Nigg et al., 1998) or multiple behaviors (e.g., Herrick et al, 1997), therefore a total of 146 cross-sectional datasets were included. One hundred forty four reported sufficient data for the Pros of behavior change and 144 reported 
sufficient data for the Cons of behavior change. Approximately 32,000 pieces of data

were extracted, coded or computed to serve as the basis for subsequent analyses.

Table 3

List of Target Behaviors

\begin{tabular}{|ll|}
\hline Administrative Change & Anorexia - Recovery From \\
Asthma - Medication & Back Pain /Keeping Body Posture \\
Management & \\
Binge Drinking Acquisition & Binge Drinking Cessation \\
Bone Density Testing & Breast Self-Examination \\
Bulimia - Recovery From & Bullying Prevention \\
Calcium Intake & Cocaine Use \\
Colorectal Cancer Screening & Condom Use - General \\
Condom Use with Main Partner & Condom Use with Other Partners \\
Condom Use - Anal Sex & Condom Use - Vaginal Sex \\
Contraception - General & Daily Use of Dental Floss \\
Decreasing Drug Use & Delinquent Behavior \\
Depression Prevention & Diabetes - Medication \\
& Management \\
Dietary Fat Reduction & Domestic Violence \\
Drug Treatment & Exercise \\
Fruit \& Vegetable Consumption & Fruit Intake \\
Glucose Testing & Healthy Diet \\
Mammography & Oral Hygiene \\
Organ Donation - Family Consent & Organ Donation - Intentions \\
Participating in Decision Making & Physicians Assisting Smokers \\
for Medication & \\
Quitting Drugs & Radon Testing \\
Safer Sex Decision Making & Sedentary Behavior \\
Sex Decision Making/ Abstinence & Sex Decision Making/ Abstinence \\
- Non-Virgins & - Virgins \\
Smokeless Tobacco Use & Smoking Acquisition \\
Smoking Cessation & STD Screening \\
Stress Management & Sun Protection \\
Sunscreen Use & Tooth-Friendly Diet \\
Vegetable Intake & Weight Bearing Exercise \\
Weight Loss & \\
\hline
\end{tabular}


The studies were conducted in 18 different countries including Australia,

Canada, China, Denmark, England, Finland, Germany, Israel, Japan, The

Netherlands, Northern Ireland, Norway, Portugal, Scotland, Spain, Switzerland, United States and Wales, with each study conducted in the native language of the given country. Seventy-five percent of the studies were conducted in the United States and two percent of the studies included multiple countries (Table 4). It should be noted that not all countries and languages are listed separately in the table since data from some countries were combined in the studies. 
Table 4

Summary of Country and Language

\begin{tabular}{|c|c|c|c|c|}
\hline \multirow{2}{*}{$\frac{\text { Study Characteristic }}{\text { Country }}$} & \multicolumn{2}{|r|}{ Categories } & \multirow{2}{*}{$\begin{array}{c}\text { Sample } \\
\text { Size }\end{array}$} & $\begin{array}{l}\% \text { Across } \\
\text { Studies }\end{array}$ \\
\hline & & Australia & & .68 \\
\hline & & Canada & 2 & 1.37 \\
\hline & & China & 2 & 1.37 \\
\hline & & England & 2 & 1.37 \\
\hline & & Germany & 20 & 13.70 \\
\hline & & Japan & 3 & 2.05 \\
\hline & & Netherlands & 2 & 1.37 \\
\hline & & Norway & 1 & .68 \\
\hline & & Switzerland & 1 & .68 \\
\hline & & United States & 109 & 74.66 \\
\hline & & Multiple & 3 & 2.05 \\
\hline & $\overline{T \text { Total }}$ & & 146 & $100 \%$ \\
\hline \multirow[t]{4}{*}{ US vs Non-US } & & US & 109 & 74.66 \\
\hline & & Non-US & 34 & 23.29 \\
\hline & & Multiple & 3 & 2.05 \\
\hline & Total & & 146 & $100 \%$ \\
\hline \multirow{9}{*}{ Language } & & Chinese & 2 & 1.37 \\
\hline & & Dutch & 2 & 1.37 \\
\hline & & English & 115 & 78.77 \\
\hline & & French & 1 & .68 \\
\hline & & German & 20 & 13.70 \\
\hline & & Japanese & 3 & 2.05 \\
\hline & & Norwegian & 1 & .68 \\
\hline & & Multiple & 2 & 1.37 \\
\hline & Total & & 146 & $100 \%$ \\
\hline \multirow[t]{3}{*}{ English vs Non-English } & & English & 115 & 64.97 \\
\hline & & Non-English & 31 & $35 / 03$ \\
\hline & Total & & 146 & $100 \%$ \\
\hline
\end{tabular}

The studies utilized three types of sampling procedures, convenience samples (85\%), randomly sampled participants (14\%), mixed studies $(1 \%)$, that is, they used a combination of the first two procedures. One study did not provide sufficient information to determine sampling method. The total number of participants for the 
meta-analysis was 85,272 . The sample sizes for the studies ranged from 19 to 21,535 participants.

Table 5

Summary of Sampling Methods across Studies

\begin{tabular}{|c|c|c|c|}
\hline Study Characteristic & Categories & $\begin{array}{c}\text { Sample } \\
\text { Size }\end{array}$ & $\begin{array}{c}\% \text { Across } \\
\text { Studies }\end{array}$ \\
\hline \multirow{5}{*}{ Sampling Method } & Convenience & 122 & 83.56 \\
\hline & Random & 21 & 14.38 \\
\hline & Mixed & 1 & 68 \\
\hline & Not Reported & 2 & 1.37 \\
\hline & & 146 & $100 \%$ \\
\hline
\end{tabular}

The datasets came from a variety of sources, including peer-reviewed journals (55\%), dissertations or theses (21\%), manuscripts in Preparation (9\%), conference presentations (8\%), unpublished data (5\%), and technical manuals (3\%).

Table 6

Summary of Publication Status across the Studies

\begin{tabular}{|c|c|c|c|}
\hline Study Characteristic & Categories & $\begin{array}{c}\text { Sample } \\
\text { Size }\end{array}$ & $\begin{array}{c}\% \text { Across } \\
\text { Studies }\end{array}$ \\
\hline \multirow{7}{*}{ Publication Status } & Peer-Reviewed Journal & 80 & 54.79 \\
\hline & Dissertation / Thesis & 30 & 20.55 \\
\hline & Manuscript in Preparation & 13 & 8.90 \\
\hline & Conference Presentation & 11 & 7.53 \\
\hline & Unpublished Data & 8 & 5.48 \\
\hline & Technical Manual & 4 & 2.74 \\
\hline & Total & 146 & $100 \%$ \\
\hline
\end{tabular}

The author, year of study, target behavior, sample size, and description of participants, mean age, age group, sampling method and country for each study can 
be found in Table 7. Additionally, information regarding each of the studies can be found in Appendix B. Table B.1 provides detailed information on the Stages of Change for each study and Table B.2 provides detailed matrix of the coded variables used in the moderator analyses. 
THERE IS NO PAGE 142 
Table 7

Study Characteristics

\begin{tabular}{|c|c|c|c|c|c|c|c|c|c|}
\hline Source & Year & $\begin{array}{l}\text { Target } \\
\text { Behavior }\end{array}$ & $\begin{array}{l}\text { N/ } \\
\text { Data } \\
\text { Set }\end{array}$ & Sample Description & Setting & $\begin{array}{l}\text { Age } \\
\text { Group }\end{array}$ & $\begin{array}{l}\text { Mean } \\
\text { Age }\end{array}$ & $\begin{array}{l}\text { Sampl- } \\
\text { ing } \\
\text { Method }\end{array}$ & Country \\
\hline $\begin{array}{l}\text { Acton } \\
\text { et al. }\end{array}$ & 2001 & $\begin{array}{l}\text { Smoking } \\
\text { Cessation }\end{array}$ & 205 & Psychiatric outpatients & $\begin{array}{l}\text { Treatment } \\
\text { centers }\end{array}$ & Adults & 41.2 & Conven. & United States \\
\hline $\begin{array}{l}\text { Ahijevych } \\
\text { et al. }\end{array}$ & 1999 & $\begin{array}{l}\text { Smoking } \\
\text { Cessation }\end{array}$ & 95 & $\begin{array}{l}\text { Black and White women } \\
\text { smokers }\end{array}$ & $\begin{array}{l}\text { Community } \\
\text { centers; } \\
\text { Worksites }\end{array}$ & dults & 34.6 & Conven. & United States \\
\hline $\begin{array}{l}\text { Bane } \\
\text { et al. }\end{array}$ & 1999 & $\begin{array}{l}\text { Smoking } \\
\text { Cessation }\end{array}$ & 281 & $\begin{array}{l}\text { Pregnant women; low } \\
\text { SES }\end{array}$ & Clinic & Adults & 23.8 & Conven. & States \\
\hline \multirow[t]{2}{*}{$\begin{array}{l}\text { Banikarim } \\
\text { et al. }\end{array}$} & \multirow[t]{2}{*}{2003} & $\begin{array}{l}\text { STD } \\
\text { Screening- } \\
\text { Change in } \\
\text { Partners }\end{array}$ & 135 & $\begin{array}{l}\text { English-speaking single } \\
\text { females seeking services } \\
\text { at an urban reproductive } \\
\text { health clinic }\end{array}$ & Clinic & Adults & $\begin{array}{l}\text { Not } \\
\text { reported }\end{array}$ & Conven. & United States \\
\hline & & $\begin{array}{l}\text { STD } \\
\text { Screening- } \\
\text { Main }\end{array}$ & 201 & $\begin{array}{l}\text { English-speaking single } \\
\text { females seeking services } \\
\text { at an urban reproductive } \\
\text { health clinic }\end{array}$ & Clinic & Adults & $\begin{array}{l}\text { Not } \\
\text { reported }\end{array}$ & Conven. & United States \\
\hline $\begin{array}{l}\text { Basler } \\
\text { et al. }\end{array}$ & 1999 & Exercise & 451 & $\begin{array}{l}\text { Patients of German } \\
\text { general practitioners }\end{array}$ & Physician's 0 & Adults & 48.0 & Random & Germany \\
\hline $\begin{array}{l}\text { Block } \\
\text { et al. }\end{array}$ & 1998 & $\begin{array}{l}\text { Condom } \\
\text { Use }\end{array}$ & 127 & $\begin{array}{l}\text { Graduate and } \\
\text { undergraduate women }\end{array}$ & $\begin{array}{l}\text { College / } \\
\text { University }\end{array}$ & College & $\begin{array}{l}\text { Not } \\
\text { reported }\end{array}$ & Conven. & United States \\
\hline $\begin{array}{l}\text { Borland } \\
\text { et al. }\end{array}$ & 2000 & $\begin{array}{l}\text { Smoking } \\
\text { Cessation }\end{array}$ & 304 & RDD & Community & Adults & $\begin{array}{l}\text { Not } \\
\text { reported }\end{array}$ & Random & Australia \\
\hline
\end{tabular}


Table 7 (continued)

\begin{tabular}{|c|c|c|c|c|c|c|c|c|c|}
\hline Source & Year & $\begin{array}{l}\text { Target } \\
\text { Behavior }\end{array}$ & $\begin{array}{l}\text { N/ } \\
\text { Data } \\
\text { Set }\end{array}$ & Sample Description & Setting & $\begin{array}{l}\text { Age } \\
\text { Group }\end{array}$ & $\begin{array}{l}\text { Mean } \\
\text { Age }\end{array}$ & $\begin{array}{l}\text { Sampl- } \\
\text { ing } \\
\text { Method }\end{array}$ & Country \\
\hline $\begin{array}{l}\text { Boudreaux } \\
\text { et al. }\end{array}$ & 1998 & $\begin{array}{l}\text { Smoking } \\
\text { Cessation }\end{array}$ & 554 & $\begin{array}{l}\text { Outpatient primary care; } \\
\text { low SES }\end{array}$ & Clinic & Adults & 47.2 & Random & United States \\
\hline $\begin{array}{l}\text { Buckworth } \\
\text { et al. }\end{array}$ & 2002 & Exercise & 57 & $\begin{array}{l}\text { Young adult males and } \\
\text { females in elective college } \\
\text { level conditioning activity } \\
\text { classes }\end{array}$ & $\begin{array}{l}\text { College / } \\
\text { University }\end{array}$ & College & 21.2 & Conven. & United States \\
\hline $\begin{array}{l}\text { Callaghan } \\
\text { et al. }\end{array}$ & 2002 & Exercise & 298 & $\begin{array}{l}\text { Chinese undergraduate } \\
\text { students attending classes } \\
\text { in the nursing and } \\
\text { psychology departments at } \\
\text { the Chinese University of } \\
\text { Hong Kong }\end{array}$ & $\begin{array}{l}\text { College / } \\
\text { University }\end{array}$ & College & 20.0 & Conven. & China \\
\hline $\begin{array}{l}\text { Chamot } \\
\text { et al. }\end{array}$ & 2001 & $\begin{array}{l}\text { Mammo- } \\
\text { graphy }\end{array}$ & 885 & Random Swiss women & Community & Adults & 56.4 & Random & Switzerland \\
\hline $\begin{array}{l}\text { DiClemente } \\
\text { et al. }\end{array}$ & 1991 & $\begin{array}{l}\text { Smoking } \\
\text { Cessation }\end{array}$ & 1466 & $\begin{array}{l}\text { Radio and newspaper ads; } \\
\text { Texas }\end{array}$ & Community & Adults & 41.0 & Conven. & United States \\
\hline $\begin{array}{l}\text { Dijkstra } \\
\text { et al. }\end{array}$ & 1996 & $\begin{array}{l}\text { Smoking } \\
\text { Cessation }\end{array}$ & 275 & $\begin{array}{l}\text { Local ad for smokers } \\
\text { (Dutch population) }\end{array}$ & Community & Adults & 41.4 & Conven. & Netherlands \\
\hline $\begin{array}{l}\text { Dijkstra } \\
\text { et al. }\end{array}$ & 1998 & $\begin{array}{l}\text { Smoking } \\
\text { Cessation }\end{array}$ & 1540 & $\begin{array}{l}\text { Smokers recruited by } \\
\text { advertisements in local } \\
\text { newspapers }\end{array}$ & Community & Adults & $\begin{array}{l}\text { Not } \\
\text { reported }\end{array}$ & Conven. & Netherlands \\
\hline Dryfoos & 1996 & $\begin{array}{l}\text { Glucose } \\
\text { Testing }\end{array}$ & 338 & $\begin{array}{l}\text { Representative Diabetes } \\
\text { Sample }\end{array}$ & Community & Adults & 59.1 & Random & United States \\
\hline
\end{tabular}


Table 7 (continued)

\begin{tabular}{|c|c|c|c|c|c|c|c|c|c|}
\hline Source & Year & $\begin{array}{l}\text { Target } \\
\text { Behavior }\end{array}$ & $\begin{array}{l}\text { N/ } \\
\text { Data } \\
\text { Set }\end{array}$ & Sample Description & Setting & $\begin{array}{l}\text { Age } \\
\text { Group }\end{array}$ & $\begin{array}{l}\text { Mean } \\
\text { Age }\end{array}$ & $\begin{array}{l}\text { Sampl- } \\
\text { ing } \\
\text { Method }\end{array}$ & Country \\
\hline & & $\begin{array}{l}\text { Diabetes: } \\
\text { Medication } \\
\text { Manage- } \\
\text { ment }\end{array}$ & 1048 & $\begin{array}{l}\text { Representative Diabetes } \\
\text { Sample }\end{array}$ & Community & Adults & 59.1 & Random & United States \\
\hline $\begin{array}{l}\text { Eaton } \\
\text { et al. }\end{array}$ & 1992 & $\begin{array}{l}\text { Physicians } \\
\text { Assisting } \\
\text { Smoker }\end{array}$ & 165 & Physicians & Community & Adults & 43.0 & Conven. & United States \\
\hline $\begin{array}{l}\text { Evers } \\
\text { et al. }\end{array}$ & 2001 & $\begin{array}{l}\text { Decreasing } \\
\text { Drug Use }\end{array}$ & 293 & $\begin{array}{l}\text { Adolescents in } \\
\text { participating schools }\end{array}$ & School & Adol. & $\begin{array}{l}\text { Not } \\
\text { reported }\end{array}$ & Conven. & $\begin{array}{l}\text { England/ } \\
\text { Israel }\end{array}$ \\
\hline $\begin{array}{l}\text { Evers } \\
\text { et al. }\end{array}$ & 2003 & $\begin{array}{l}\text { Stress } \\
\text { Manage- } \\
\text { ment }\end{array}$ & 241 & $\begin{array}{l}\text { Undergraduate students, } \\
\text { graduate students, faculty } \\
\text { and staff at a university }\end{array}$ & $\begin{array}{l}\text { College / } \\
\text { University }\end{array}$ & $\begin{array}{l}\text { College/ } \\
\text { Adults }\end{array}$ & 21.6 & Conven. & United States \\
\hline $\begin{array}{l}\text { Fahrenwald } \\
\text { et al. }\end{array}$ & 2003 & Exercise & 30 & $\begin{array}{l}\text { WIC mothers from a WIC } \\
\text { program in a small } \\
\text { Midwest city }\end{array}$ & WIC Program & $\begin{array}{l}\text { College/ } \\
\text { Adults }\end{array}$ & 24.3 & Conven. & United States \\
\hline $\begin{array}{l}\text { Fava } \\
\text { et al. }\end{array}$ & 1995 & $\begin{array}{l}\text { Smoking } \\
\text { Cessation }\end{array}$ & 4144 & Rhode Island sample & Community & Adults & 40.7 & Random & United States \\
\hline $\begin{array}{l}\text { Frenn } \\
\text { et al. }\end{array}$ & 2003 & $\begin{array}{l}\text { Dietary Fat } \\
\text { Reduction }\end{array}$ & 74 & $\begin{array}{l}\text { Sixth, Seventh, and Eighth } \\
\text { grade students who } \\
\text { attended a central city } \\
\text { middle school serving } \\
\text { low-income, culturally } \\
\text { diverse students }\end{array}$ & & Adol. & 13.8 & Conven. & United States \\
\hline
\end{tabular}


Table 7 (continued)

\begin{tabular}{|c|c|c|c|c|c|c|c|c|c|}
\hline Source & Year & $\begin{array}{l}\text { Target } \\
\text { Behavior }\end{array}$ & $\begin{array}{l}\text { / } \\
\text { Data } \\
\text { Set }\end{array}$ & Sample Description & Setting & $\begin{array}{l}\text { Age } \\
\text { Group }\end{array}$ & $\begin{array}{l}\text { Mean } \\
\text { Age }\end{array}$ & $\begin{array}{l}\text { Sampl- } \\
\text { ing } \\
\text { Method }\end{array}$ & Country \\
\hline \multirow[t]{3}{*}{$\begin{array}{l}\text { Galavotti } \\
\text { et al. }\end{array}$} & \multirow[t]{3}{*}{1995} & $\begin{array}{l}\text { Contracep- } \\
\text { tion- } \\
\text { General }\end{array}$ & 233 & $\begin{array}{l}\text { Women high risk for HIV; } \\
\text { low SES }\end{array}$ & $\begin{array}{l}\text { Homeless } \\
\text { shelters; Drug } \\
\text { treatment } \\
\text { facilities; Hi- } \\
\text { drug use / } \\
\text { prostitution } \\
\text { neighborhoods }\end{array}$ & Adults & 28.0 & Conven. & United States \\
\hline & & $\begin{array}{l}\text { Condom } \\
\text { Use - Main }\end{array}$ & 233 & $\begin{array}{l}\text { Women high risk for HIV; } \\
\text { low SES }\end{array}$ & $\begin{array}{l}\text { Homeless } \\
\text { shelters; Drug } \\
\text { treatment } \\
\text { facilities; Hi- } \\
\text { drug use / } \\
\text { prostitution } \\
\text { neighborhoods }\end{array}$ & Adults & 28.0 & Conven. & United States \\
\hline & & $\begin{array}{l}\text { Condom } \\
\text { Use - Other }\end{array}$ & 122 & $\begin{array}{l}\text { Women high risk for HIV; } \\
\text { low SES }\end{array}$ & $\begin{array}{l}\text { Homeless } \\
\text { shelters; Drug } \\
\text { treatment } \\
\text { facilities; Hi- } \\
\text { drug use / } \\
\text { prostitution } \\
\text { neighborhoods }\end{array}$ & Adults & 28.0 & Conven. & United States \\
\hline Gazabon & 2000 & $\begin{array}{l}\text { Condom } \\
\text { Use }\end{array}$ & 340 & $\begin{array}{l}\text { Women sexually at risk } \\
\text { for HIV }\end{array}$ & Community & Adults & 31.0 & Conven. & United States \\
\hline
\end{tabular}


Table 7 (continued)

\begin{tabular}{|c|c|c|c|c|c|c|c|c|c|}
\hline Source & Year & $\begin{array}{l}\text { Target } \\
\text { Behavior }\end{array}$ & $\begin{array}{l}\text { N/ } \\
\text { Data } \\
\text { Set } \\
\end{array}$ & Sample Description & Setting & $\begin{array}{l}\text { Age } \\
\text { Group }\end{array}$ & $\begin{array}{l}\text { Mean } \\
\text { Age }\end{array}$ & $\begin{array}{l}\text { Sampl- } \\
\text { ing } \\
\text { Method }\end{array}$ & Country \\
\hline \multirow[t]{4}{*}{ Giebel } & \multirow[t]{4}{*}{1999} & $\begin{array}{l}\text { Smoking } \\
\text { Cessation }\end{array}$ & 235 & Military sample & Military & Adults & 26.0 & Conven. & Germany \\
\hline & & $\begin{array}{l}\text { Dietary Fat } \\
\text { Reduction }\end{array}$ & 361 & Military sample & Military & Adults & 27.0 & Conven. & Germany \\
\hline & & Exercise & 361 & Military sample & Military & Adults & 28.0 & Conven. & Germany \\
\hline & & $\begin{array}{l}\text { Stress } \\
\text { Manage- } \\
\text { ment }\end{array}$ & 244 & Military sample & Military & Adults & 29.0 & Conven. & Germany \\
\hline \multirow[t]{3}{*}{$\begin{array}{l}\text { Grimley } \\
\text { et al. }\end{array}$} & \multirow[t]{3}{*}{1995} & $\begin{array}{l}\text { Contracep- } \\
\text { tion- } \\
\text { General }\end{array}$ & 244 & $\begin{array}{l}\text { Undergraduate } \\
\text { psychology; New England }\end{array}$ & $\begin{array}{l}\text { College / } \\
\text { University }\end{array}$ & College & 18.9 & Conven. & United States \\
\hline & & $\begin{array}{l}\text { Condom } \\
\text { Use - Main }\end{array}$ & 78 & $\begin{array}{l}\text { Undergraduate } \\
\text { psychology; New England }\end{array}$ & $\begin{array}{l}\text { College / } \\
\text { University }\end{array}$ & College & 18.9 & Conven. & United States \\
\hline & & $\begin{array}{l}\text { Condom } \\
\text { Use - Other }\end{array}$ & 134 & $\begin{array}{l}\text { Undergraduate } \\
\text { psychology; New England }\end{array}$ & $\begin{array}{l}\text { College / } \\
\text { University }\end{array}$ & College & 18.9 & Conven. & United States \\
\hline Happel & 2002 & $\begin{array}{l}\text { Fruit \& } \\
\text { Vegetable } \\
\text { Consump- } \\
\text { tion }\end{array}$ & 255 & University students & $\begin{array}{l}\text { College / } \\
\text { University }\end{array}$ & College & 21.4 & Conven. & Germany \\
\hline Hempelman & 2003 & $\begin{array}{l}\text { Tooth- } \\
\text { friendly } \\
\text { Diet }\end{array}$ & 448 & $\begin{array}{l}\text { Employees of three } \\
\text { different companies }\end{array}$ & Worksites & Adults & $\begin{array}{l}\text { Not } \\
\text { reported }\end{array}$ & Conven. & Germany \\
\hline
\end{tabular}


Table 7 (continued)

\begin{tabular}{|c|c|c|c|c|c|c|c|c|c|}
\hline Source & Year & $\begin{array}{l}\text { Target } \\
\text { Behavior }\end{array}$ & $\begin{array}{l}\text { N/ } \\
\text { Data } \\
\text { Set } \\
\end{array}$ & Sample Description & Setting & $\begin{array}{l}\text { Age } \\
\text { Group }\end{array}$ & $\begin{array}{l}\text { Mean } \\
\text { Age }\end{array}$ & $\begin{array}{l}\text { Sampl- } \\
\text { ing } \\
\text { Method }\end{array}$ & Country \\
\hline Henderson & 1999 & $\begin{array}{l}\text { Breast Self- } \\
\text { Examina- } \\
\text { tion }\end{array}$ & 214 & $\begin{array}{l}\text { Female employees from a } \\
\text { large, Midwestern } \\
\text { university }\end{array}$ & $\begin{array}{l}\text { College / } \\
\text { University }\end{array}$ & Adults & 39.8 & Random & United States \\
\hline \multirow[t]{4}{*}{$\begin{array}{l}\text { Herrick } \\
\text { et al. }\end{array}$} & \multirow[t]{4}{*}{1997} & $\begin{array}{l}\text { Dietary Fat } \\
\text { Reduction }\end{array}$ & 392 & Worksite employees & Worksite & Adults & 42.2 & Conven. & United States \\
\hline & & Exercise & 389 & Worksite employees & Worksite & Adults & 42.2 & Conven. & United States \\
\hline & & $\begin{array}{l}\text { Smoking } \\
\text { Cessation }\end{array}$ & 393 & Worksite employees & Worksite & Adults & 42.2 & Conven. & United States \\
\hline & & $\begin{array}{l}\text { Sun } \\
\text { Protection/ } \\
\text { Reduction }\end{array}$ & 392 & Worksite employees & Worksite & Adults & 42.2 & Conven. & United States \\
\hline $\begin{array}{l}\text { Herzog } \\
\text { et al. }\end{array}$ & 1999 & $\begin{array}{l}\text { Smoking } \\
\text { Cessation }\end{array}$ & 1390 & Worksite employees & $\begin{array}{l}\text { Study centers; } \\
\text { Worksites }\end{array}$ & Adults & 39.3 & Random & United States \\
\hline \multirow[t]{2}{*}{ Hulton } & \multirow[t]{2}{*}{2001} & $\begin{array}{l}\text { Sex } \\
\text { Decision } \\
\text { Making / } \\
\text { Abstinence } \\
\text { (Non } \\
\text { Virgins) }\end{array}$ & 103 & 7th graders; Virginia & School & Adol. & $\begin{array}{l}\text { Not } \\
\text { reported }\end{array}$ & Conven. & United States \\
\hline & & $\begin{array}{l}\text { Sex } \\
\text { Decision } \\
\text { Making / } \\
\text { Abstinence } \\
\text { (Virgins) }\end{array}$ & 622 & 7th graders; Virginia & School & Adol. & $\begin{array}{l}\text { Not } \\
\text { reported }\end{array}$ & Conven. & United States \\
\hline
\end{tabular}


Table 7 (continued)

\begin{tabular}{|c|c|c|c|c|c|c|c|c|c|}
\hline Source & Year & $\begin{array}{l}\text { Target } \\
\text { Behavior }\end{array}$ & $\begin{array}{l}\text { N/ } \\
\text { Data } \\
\text { Set }\end{array}$ & Sample Description & Setting & $\begin{array}{l}\text { Age } \\
\text { Group }\end{array}$ & $\begin{array}{l}\text { Mean } \\
\text { Age }\end{array}$ & $\begin{array}{l}\text { Sampl- } \\
\text { ing } \\
\text { Method }\end{array}$ & Country \\
\hline Jeffries & 2001 & Exercise & 537 & $\begin{array}{l}\text { Predominantly low- } \\
\text { income patients over the } \\
\text { age of } 18 \text { attending } \\
\text { primary care clinics from } \\
\text { four separate sites; } \\
\text { Louisiana }\end{array}$ & $\begin{array}{l}\text { Primary care } \\
\text { clinics }\end{array}$ & Adults & 45.3 & Random & United States \\
\hline \multirow[t]{2}{*}{$\begin{array}{l}\text { Johnson, } \\
\text { J.L. } \\
\text { et al. }\end{array}$} & 2002 & $\begin{array}{l}\text { Bone } \\
\text { Density } \\
\text { Testing }\end{array}$ & 465 & $\begin{array}{l}\text { UnitedHeathcare; Women } \\
\text { ages } 45+\text {; New England }\end{array}$ & Community & Adults & 62.4 & Conven. & United States \\
\hline & & $\begin{array}{l}\text { Calcium } \\
\text { Intake }\end{array}$ & 419 & $\begin{array}{l}\text { UnitedHeathcare; Women } \\
\text { ages } 45+\text {; New England }\end{array}$ & Community & Adults & 62.4 & Conven. & United States \\
\hline \begin{tabular}{|l} 
Johnson, \\
S.S. \\
et al.
\end{tabular} & 2002 & $\begin{array}{l}\text { Participat- } \\
\text { ing in } \\
\text { Decision } \\
\text { Making for } \\
\text { Medication }\end{array}$ & 185 & $\begin{array}{l}\text { UnitedHeathcare; Women } \\
\text { ages } 45+\text {; New England }\end{array}$ & Community & Adults & 62.4 & Conven. & United States \\
\hline $\begin{array}{l}\text { Johnson } \\
\text { et al. }\end{array}$ & 2002 & $\begin{array}{l}\text { Smoking } \\
\text { Cessation }\end{array}$ & 296 & $\begin{array}{l}\text { Ethnically diverse Parents } \\
\text { with young children }\end{array}$ & $\begin{array}{l}\text { Community } \\
\text { health centers } \\
\text { that serve } \\
\text { diverse, low- } \\
\text { income } \\
\text { populations }\end{array}$ & Adults & 28.4 & Conven. & United States \\
\hline
\end{tabular}


Table 7 (continued)

\begin{tabular}{|c|c|c|c|c|c|c|c|c|c|}
\hline Source & Year & $\begin{array}{l}\text { Target } \\
\text { Behavior }\end{array}$ & $\begin{array}{l}\text { N/ } \\
\text { Data } \\
\text { Set }\end{array}$ & Sample Description & Setting & $\begin{array}{l}\text { Age } \\
\text { Group }\end{array}$ & $\begin{array}{l}\text { Mean } \\
\text { Age }\end{array}$ & $\begin{array}{l}\text { Sampl- } \\
\text { ing } \\
\text { Method }\end{array}$ & Country \\
\hline \multirow[t]{2}{*}{ Jordan } & \multirow[t]{2}{*}{2002} & $\begin{array}{l}\text { Anorexia- } \\
\text { Recovery } \\
\text { From }\end{array}$ & 276 & Recruited internationally & $\begin{array}{l}\text { Internet / } \\
\text { Treatment } \\
\text { centers / } \\
\text { University }\end{array}$ & Adults & 30.5 & Conven. & United States \\
\hline & & $\begin{array}{l}\text { Bulimia - } \\
\text { Recovery } \\
\text { From }\end{array}$ & 148 & Recruited internationally & $\begin{array}{l}\text { Internet / } \\
\text { Treatment } \\
\text { centers / } \\
\text { University }\end{array}$ & Adults & 30.0 & Conven. & $\begin{array}{l}\text { United } \\
\text { States/ } \\
\text { England }\end{array}$ \\
\hline $\begin{array}{l}\text { Jordon } \\
\text { et al. }\end{array}$ & 2002 & Exercise & 223 & $\begin{array}{l}\text { New England } \\
\text { undergraduate psychology }\end{array}$ & $\begin{array}{l}\text { College / } \\
\text { University }\end{array}$ & College & 19.8 & Conven. & United States \\
\hline Katlin & 2001 & $\begin{array}{l}\text { Drug } \\
\text { Treatment }\end{array}$ & 114 & $\begin{array}{l}\text { Chemical abusers in out- } \\
\text { patient treatment }\end{array}$ & $\begin{array}{l}\text { Treatment } \\
\text { centers }\end{array}$ & Adults & 37.2 & Conven. & United States \\
\hline \multirow[t]{2}{*}{ Keller } & 1998 & $\begin{array}{l}\text { Eating } \\
\text { behavior- } \\
\text { MEN }\end{array}$ & 405 & $\begin{array}{l}\text { Participants in a health } \\
\text { check-up for } \\
\text { cardiovascular risk factors } \\
\text { at their general } \\
\text { practitioner's office. }\end{array}$ & $\begin{array}{l}\text { Physician's } \\
\text { office }\end{array}$ & Adults & 49.2 & Conven. & Germany \\
\hline & 1998 & $\begin{array}{l}\text { Eating } \\
\text { behavior- } \\
\text { WOMEN }\end{array}$ & 407 & $\begin{array}{l}\text { Participants in a health } \\
\text { check-up for } \\
\text { cardiovascular risk factors } \\
\text { at their general } \\
\text { practitioner's office. }\end{array}$ & $\begin{array}{l}\text { Physician's } \\
\text { office }\end{array}$ & Adults & 49.2 & Conven. & Germany \\
\hline
\end{tabular}


Table 7 (continued)

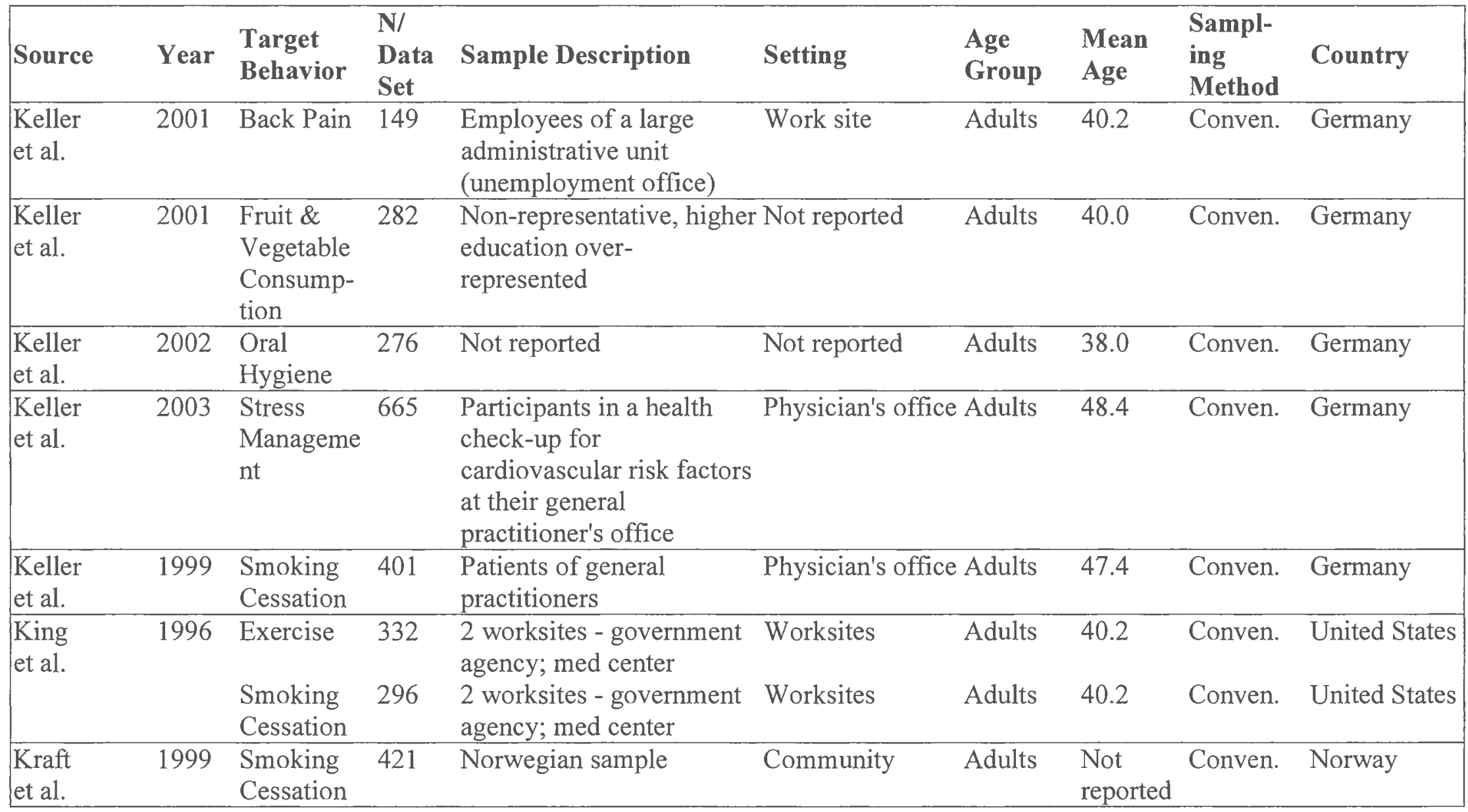


Table 7 (continued)

\begin{tabular}{|c|c|c|c|c|c|c|c|c|c|}
\hline Source & Year & $\begin{array}{l}\text { Target } \\
\text { Behavior }\end{array}$ & $\begin{array}{l}\text { N/ } \\
\text { Data } \\
\text { Set }\end{array}$ & Sample Description & Setting & $\begin{array}{l}\text { Age } \\
\text { Group }\end{array}$ & $\begin{array}{l}\text { Mean } \\
\text { Age }\end{array}$ & $\begin{array}{l}\text { Sampl- } \\
\text { ing } \\
\text { Method }\end{array}$ & Country \\
\hline $\begin{array}{l}\text { Kremers } \\
\text { et al. }\end{array}$ & 2001 & $\begin{array}{l}\text { Smoking } \\
\text { Acquisi- } \\
\text { tion }\end{array}$ & 21535 & $\begin{array}{l}\text { Seventh graders in a large } \\
\text { international sample of } \\
\text { European adolescents }\end{array}$ & School & Adol. & 13.2 & Conven. & $\begin{array}{l}\text { Denmark; } \\
\text { Finland; } \\
\text { Netherlands; } \\
\text { Portugal; } \\
\text { Spain; UK }\end{array}$ \\
\hline \multirow[t]{3}{*}{$\begin{array}{l}\text { Lauby } \\
\text { et al. }\end{array}$} & \multirow[t]{3}{*}{1998} & $\begin{array}{l}\text { Contracep- } \\
\text { tion - } \\
\text { General }\end{array}$ & 2361 & $\begin{array}{l}\text { Women- HIV; High-risk } \\
\text { HIV, unintended } \\
\text { pregnancy }\end{array}$ & $\begin{array}{l}\text { Community- } \\
\text { based sites; } \\
\text { Facility-based } \\
\text { sites }\end{array}$ & Adults & $\begin{array}{l}\text { Not } \\
\text { reported }\end{array}$ & Conven. & United States \\
\hline & & $\begin{array}{l}\text { Condom } \\
\text { Use - Main }\end{array}$ & 2925 & $\begin{array}{l}\text { Women- HIV; High-risk } \\
\text { HIV, unintended } \\
\text { pregnancy }\end{array}$ & $\begin{array}{l}\text { Community- } \\
\text { based sites; } \\
\text { Facility-based } \\
\text { sites }\end{array}$ & Adults & $\begin{array}{l}\text { Not } \\
\text { reported }\end{array}$ & Conven. & United States \\
\hline & & $\begin{array}{l}\text { Condom } \\
\text { Use - Other }\end{array}$ & 1213 & $\begin{array}{l}\text { Women- HIV, High-risk } \\
\text { HIV, unintended } \\
\text { pregnancy; U.S. }\end{array}$ & $\begin{array}{l}\text { Community- } \\
\text { based sites; } \\
\text { Facility-based } \\
\text { sites }\end{array}$ & Adults & $\begin{array}{l}\text { Not } \\
\text { reported }\end{array}$ & Conven. & United States \\
\hline Lerner & 1990 & $\begin{array}{l}\text { Delinquent } \\
\text { Behavior }\end{array}$ & 159 & $\begin{array}{l}\text { At-risk adolescents at } \\
\text { juvenile court schools, } \\
\text { training schools, and } \\
\text { Urban Collaborative } \\
\text { Accelerated }\end{array}$ & $\begin{array}{l}\text { Juvenile court } \\
\text { schools; Training } \\
\text { schools; Urban } \\
\text { Collaborative } \\
\text { Accelerated } \\
\text { Program }\end{array}$ & Adol. & 15.5 & Conven. & United States \\
\hline
\end{tabular}


Table 7 (continued)

\begin{tabular}{|c|c|c|c|c|c|c|c|c|c|}
\hline Source & Year & $\begin{array}{l}\text { Target } \\
\text { Behavior }\end{array}$ & $\begin{array}{l}\text { N/ } \\
\text { Data } \\
\text { Set }\end{array}$ & Sample Description & Setting & $\begin{array}{l}\text { Age } \\
\text { Group }\end{array}$ & $\begin{array}{l}\text { Mean } \\
\text { Age }\end{array}$ & $\begin{array}{l}\text { Sampl- } \\
\text { ing } \\
\text { Method }\end{array}$ & Country \\
\hline $\begin{array}{l}\text { Levesque } \\
\text { et al. }\end{array}$ & 2000 & $\begin{array}{l}\text { Administra } \\
\text {-tive } \\
\text { Change }\end{array}$ & 134 & University employees & $\begin{array}{l}\text { College / } \\
\text { University }\end{array}$ & Adults & $\begin{array}{l}\text { Not } \\
\text { reported }\end{array}$ & Conven. & United States \\
\hline Levesque & 1999 & $\begin{array}{l}\text { Domestic } \\
\text { Violence }\end{array}$ & 256 & $\begin{array}{l}\text { Men in group counseling } \\
\text { for partner violence }\end{array}$ & $\begin{array}{l}\text { Treatment } \\
\text { Centers }\end{array}$ & Adults & $\begin{array}{l}\text { Not } \\
\text { reported }\end{array}$ & Conven. & United States \\
\hline $\begin{array}{l}\text { Levesque } \\
\text { et al. }\end{array}$ & 1999 & $\begin{array}{l}\text { Collabora- } \\
\text { tive } \\
\text { Delivery } \\
\text { Service }\end{array}$ & 31 & $\begin{array}{l}\text { University staff/ } \\
\text { administration }\end{array}$ & $\begin{array}{l}\text { College / } \\
\text { University }\end{array}$ & Adults & $\begin{array}{l}\text { Not } \\
\text { reported }\end{array}$ & Conven. & United States \\
\hline $\begin{array}{l}\text { Levesque } \\
\text { et al. }\end{array}$ & 1999 & $\begin{array}{l}\text { Depression } \\
\text { Prevention }\end{array}$ & 181 & $\begin{array}{l}\text { Undergraduate students/ } \\
\text { Mental health agency } \\
\text { staff, family, and friends }\end{array}$ & $\begin{array}{l}\text { University/ } \\
\text { Community }\end{array}$ & $\begin{array}{l}\text { College/ } \\
\text { Adults }\end{array}$ & $\begin{array}{l}\text { Not } \\
\text { reported }\end{array}$ & Conven. & United States \\
\hline Lowry & 2000 & $\begin{array}{l}\text { Smokeless } \\
\text { Tobacco } \\
\text { Use }\end{array}$ & 99 & $\begin{array}{l}\text { Young adult male college } \\
\text { students who had used } \\
\text { SLT in the past or who } \\
\text { were currently using SLT } \\
\text { and were between the ages } \\
\text { of } 18 \text { and } 25 \text { attending a } 2 \\
\text { or } 4 \text { year college }\end{array}$ & $\begin{array}{l}\text { College/Universi } \\
\text { ty }\end{array}$ & College & 20.5 & Conven. & United States \\
\hline Maier & 2002 & Exercise & 251 & $\begin{array}{l}\text { Employees of } \\
\text { unemployment office, } \\
\text { police personnel and } \\
\text { others }\end{array}$ & Not reported & Adults & 40.7 & Conven. & Germany \\
\hline
\end{tabular}


Table 7 (continued)

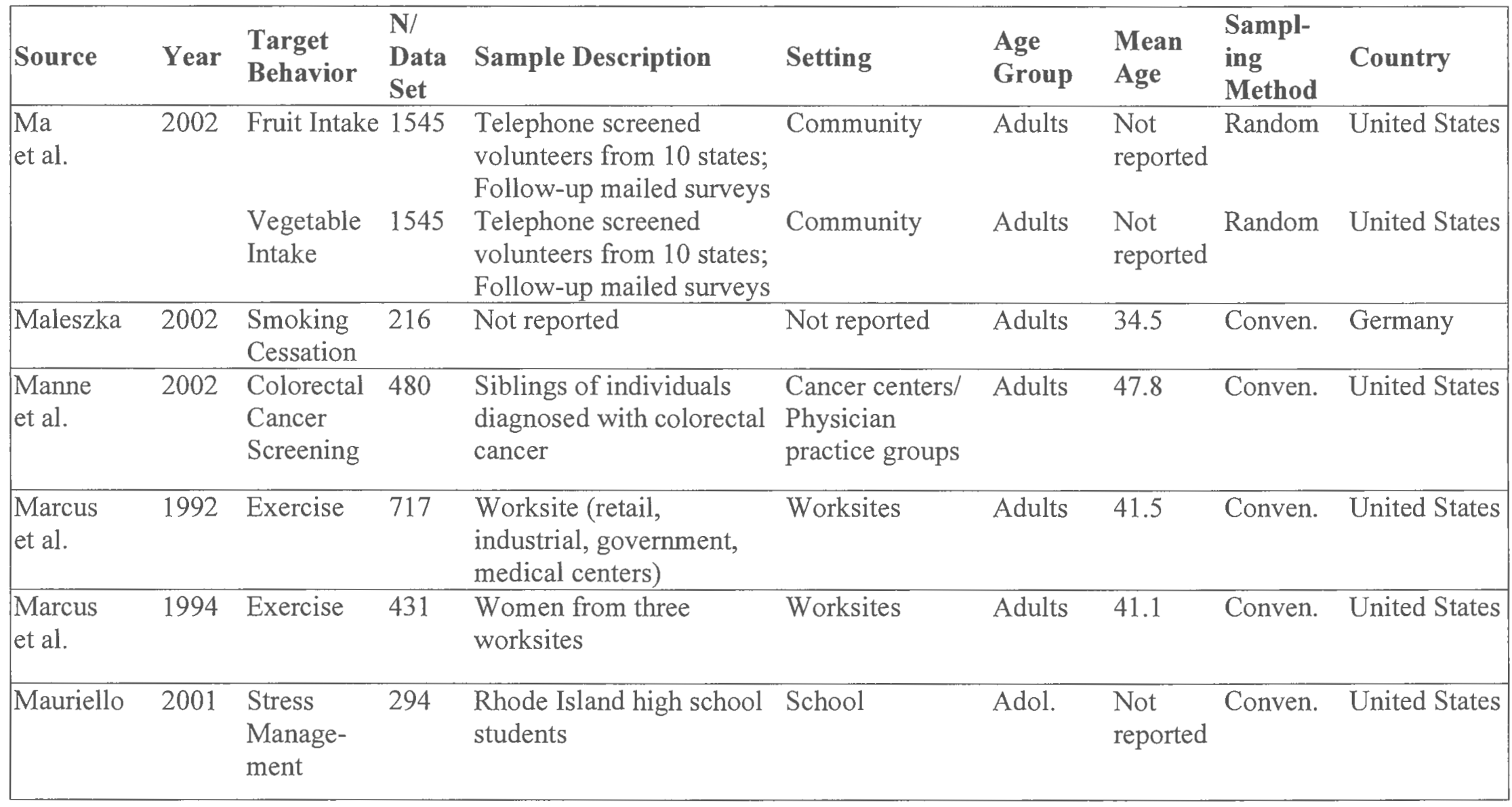


Table 7 (continued)

\begin{tabular}{|c|c|c|c|c|c|c|c|c|c|}
\hline Source & Year & $\begin{array}{l}\text { Target } \\
\text { Behavior }\end{array}$ & $\begin{array}{l}\text { N/ } \\
\text { Data } \\
\text { Set }\end{array}$ & Sample Description & Setting & $\begin{array}{l}\text { Age } \\
\text { Group }\end{array}$ & $\begin{array}{l}\text { Mean } \\
\text { Age }\end{array}$ & $\begin{array}{l}\text { Sampl- } \\
\text { ing } \\
\text { Method }\end{array}$ & Country \\
\hline $\begin{array}{l}\text { Mettler } \\
\text { et al. }\end{array}$ & 2000 & Exercise & 690 & $\begin{array}{l}\text { Volunteers from the } \\
\text { community intervention } \\
\text { program }\end{array}$ & $\begin{array}{l}\text { Community } \\
\text { Intervention } \\
\text { Program }\end{array}$ & Adults & 40.2 & Random & United States \\
\hline \multirow[t]{2}{*}{$\begin{array}{l}\text { Migneault } \\
\text { et al. }\end{array}$} & \multirow[t]{2}{*}{1997} & $\begin{array}{l}\text { Binge } \\
\text { Drinking } \\
\text { Acquisi- } \\
\text { tion }\end{array}$ & 421 & $\begin{array}{l}\text { 10th/11th grade vocational } \\
\text { students; Rhode Island }\end{array}$ & School & Adol. & 16.2 & Conven. & United States \\
\hline & & $\begin{array}{l}\text { Binge } \\
\text { Drinking } \\
\text { Cessation }\end{array}$ & 255 & $\begin{array}{l}\text { 10th/11th vocational } \\
\text { students; Rhode Island }\end{array}$ & School & Adol. & 16.2 & Conven. & United States \\
\hline Moll & 2001 & $\begin{array}{l}\text { Physical } \\
\text { activity }\end{array}$ & 287 & $\begin{array}{l}\text { University students; } 14 \% \\
\text { medical, } 14 \% \text { law } \\
\text { students, rest: other }\end{array}$ & University & College & 25.0 & Conven. & Germany \\
\hline \multirow[t]{2}{*}{$\begin{array}{l}\text { Morrison- } \\
\text { Beedy } \\
\text { et al. }\end{array}$} & \multirow[t]{2}{*}{2001} & $\begin{array}{l}\text { Condom } \\
\text { Use - Main }\end{array}$ & 254 & $\begin{array}{l}\text { Sexually active, single } \\
\text { urban women from two } \\
\text { urban health centers in a } \\
\text { city in western New York } \\
\text { state }\end{array}$ & Clinic & Adults & 27.0 & Conven. & United States \\
\hline & & $\begin{array}{l}\text { Condom } \\
\text { Use - Other }\end{array}$ & 110 & $\begin{array}{l}\text { Sexually active, single } \\
\text { urban women from two } \\
\text { urban health centers in a } \\
\text { city in western New York } \\
\text { state }\end{array}$ & Clinic & Adults & 27.0 & Conven. & United States \\
\hline
\end{tabular}


Table 7 (continued)

\begin{tabular}{|c|c|c|c|c|c|c|c|c|c|}
\hline Source & Year & $\begin{array}{l}\text { Target } \\
\text { Behavior }\end{array}$ & $\begin{array}{l}\text { N/ } \\
\text { Data } \\
\text { Set }\end{array}$ & Sample Description & Setting & $\begin{array}{l}\text { Age } \\
\text { Group }\end{array}$ & $\begin{array}{l}\text { Mean } \\
\text { Age }\end{array}$ & $\begin{array}{l}\text { Sampl- } \\
\text { ing } \\
\text { Method }\end{array}$ & Country \\
\hline $\begin{array}{l}\text { Nigg } \\
\text { et al. }\end{array}$ & 1998 & Exercise & 240 & $\begin{array}{l}\text { Undergraduate psych } \\
\text { students }\end{array}$ & $\begin{array}{l}\text { College / } \\
\text { University }\end{array}$ & College & 19.1 & Conven. & United States \\
\hline $\begin{array}{l}\text { Nigg } \\
\text { et al. }\end{array}$ & 1998 & Exercise & 346 & RDD; Rhode Island & Community & Adults & 43.0 & Conven. & United States \\
\hline $\begin{array}{l}\text { Nigg \& } \\
\text { Courneya }\end{array}$ & 1998 & Exercise & 819 & 5 community high schools & School & Adol. & 15.0 & Conven. & Canada \\
\hline Noar et al & 1998 & $\begin{array}{l}\text { Condom } \\
\text { Use - } \\
\text { Vaginal } \\
\text { Sex }\end{array}$ & 168 & $\begin{array}{l}\text { Undergraduate } \\
\text { psych/soc/fraternity male } \\
\text { students }\end{array}$ & $\begin{array}{l}\text { College / } \\
\text { University }\end{array}$ & College & 19.53 & Conven. & United States \\
\hline \multirow[t]{2}{*}{$\begin{array}{l}\text { Norman } \\
\text { et al. }\end{array}$} & \multirow[t]{2}{*}{2002} & $\begin{array}{l}\text { Sedentary } \\
\text { Behavior }\end{array}$ & 178 & $\begin{array}{l}\text { Adolescents recruited } \\
\text { through primary care } \\
\text { providers }\end{array}$ & Community & Adol. & 13.1 & Conven. & United States \\
\hline & & $\begin{array}{l}\text { Sedentary } \\
\text { Behavior }\end{array}$ & 254 & $\begin{array}{l}\text { Adolescents recruited } \\
\text { through primary care } \\
\text { providers }\end{array}$ & Community & Adol. & 13.2 & Conven. & United States \\
\hline $\begin{array}{l}\text { O'Connell } \\
\& \text { Velicer }\end{array}$ & 1988 & $\begin{array}{l}\text { Weight } \\
\text { Loss }\end{array}$ & 264 & $\begin{array}{l}\text { Undergraduate/ graduate } \\
\text { students in psychology } \\
\text { courses }\end{array}$ & $\begin{array}{l}\text { College / } \\
\text { University }\end{array}$ & College & $\begin{array}{l}\text { Not } \\
\text { reported }\end{array}$ & Conven. & United States \\
\hline $\begin{array}{l}\text { Otake \& } \\
\text { Shimai }\end{array}$ & 2001 & $\begin{array}{l}\text { Smoking } \\
\text { Acquisi- } \\
\text { tion }\end{array}$ & 556 & Senior high students & School & Adol. & $\begin{array}{l}\text { Not } \\
\text { reported }\end{array}$ & Conven. & Japan \\
\hline
\end{tabular}


Table 7 (continued)

\begin{tabular}{|c|c|c|c|c|c|c|c|c|c|}
\hline Source & Year & $\begin{array}{l}\text { Target } \\
\text { Behavior }\end{array}$ & $\begin{array}{l}\text { N/ } \\
\text { Data } \\
\text { Set }\end{array}$ & Sample Description & Setting & $\begin{array}{l}\text { Age } \\
\text { Group }\end{array}$ & $\begin{array}{l}\text { Mean } \\
\text { Age }\end{array}$ & $\begin{array}{l}\text { Sampl- } \\
\text { ing } \\
\text { Method }\end{array}$ & Country \\
\hline & & $\begin{array}{l}\text { Smoking } \\
\text { Acquisi- } \\
\text { tion }\end{array}$ & 1002 & Junior high students & School & Adol. & $\begin{array}{l}\text { Not } \\
\text { reported }\end{array}$ & Conven. & Japan \\
\hline \multirow[t]{2}{*}{$\begin{array}{l}\text { Pallonen } \\
\text { et al. }\end{array}$} & \multirow[t]{2}{*}{1998} & $\begin{array}{l}\text { Smoking } \\
\text { Acquisi- } \\
\text { tion }\end{array}$ & 505 & $\begin{array}{l}3 \text { Rhode Island } \\
\text { vocational-tech high } \\
\text { schools (never smokers) }\end{array}$ & School & Adol. & $\begin{array}{l}\text { Not } \\
\text { reported }\end{array}$ & Conven. & United States \\
\hline & & $\begin{array}{l}\text { Smoking } \\
\text { Cessation }\end{array}$ & 254 & $\begin{array}{l}4 \text { Rhode Island } \\
\text { vocational-tech high } \\
\text { schools (current smokers) }\end{array}$ & School & Adol. & 16.6 & Conven. & United States \\
\hline $\begin{array}{l}\text { Park } \\
\text { et al. }\end{array}$ & 2003 & $\begin{array}{l}\text { Physicians } \\
\text { Counseling } \\
\text { Smokers }\end{array}$ & 259 & $\begin{array}{l}\text { Primary care physicians, } \\
\text { Rhode Island }\end{array}$ & Physician's office & Adults & 44.0 & Conven. & United States \\
\hline Perez & 2001 & $\begin{array}{l}\text { Condom } \\
\text { Use }\end{array}$ & 828 & $\begin{array}{l}\text { Non-pregnant female } \\
\text { youth obtaining } \\
\text { contraceptive services at } \\
\text { one of four federally } \\
\text { funded family planning } \\
\text { clinics }\end{array}$ & Clinic & Adol. & $\begin{array}{l}\text { Not } \\
\text { reported }\end{array}$ & Conven. & United States \\
\hline \multirow[t]{2}{*}{$\begin{array}{l}\text { Plummer } \\
\text { et al. }\end{array}$} & \multirow[t]{2}{*}{2001} & $\begin{array}{l}\text { Smoking } \\
\text { Acquisi- } \\
\text { tion }\end{array}$ & 2010 & $\begin{array}{l}\text { High school students; } \\
\text { Rhode Island }\end{array}$ & School & Adol. & 15.2 & Conven. & United States \\
\hline & & $\begin{array}{l}\text { Smoking } \\
\text { Cessation }\end{array}$ & 798 & $\begin{array}{l}\text { High school students; } \\
\text { Rhode Island }\end{array}$ & School & Adol. & 15.2 & Conven. & United States \\
\hline
\end{tabular}




\begin{tabular}{|c|c|c|c|c|c|c|c|c|c|}
\hline Source & Year & $\begin{array}{l}\text { Target } \\
\text { Behavior }\end{array}$ & $\begin{array}{l}\text { N/ } \\
\text { Data } \\
\text { Set }\end{array}$ & Sample Description & Setting & $\begin{array}{l}\text { Age } \\
\text { Group }\end{array}$ & $\begin{array}{l}\text { Mean } \\
\text { Age }\end{array}$ & $\begin{array}{l}\text { Sampl- } \\
\text { ing } \\
\text { Method }\end{array}$ & Country \\
\hline \multirow[t]{2}{*}{ Evers et al. } & 2001 & $\begin{array}{l}\text { Condom } \\
\text { Use }\end{array}$ & 270 & $\begin{array}{l}\text { Students from years 9-11 } \\
\text { in } 7 \text { different schools }\end{array}$ & School & Adol. & $\begin{array}{l}\text { Not } \\
\text { reported }\end{array}$ & Conven. & England \\
\hline & & $\begin{array}{l}\text { Waiting to } \\
\text { have sex }\end{array}$ & 602 & $\begin{array}{l}\text { Students from years 9-11 } \\
\text { in } 7 \text { different schools }\end{array}$ & School & Adol. & $\begin{array}{l}\text { Not } \\
\text { reported }\end{array}$ & Conven. & England \\
\hline $\begin{array}{l}\text { Prochaska, } \\
\text { J.J. } \\
\text { et al. }\end{array}$ & 2003 & $\begin{array}{l}\text { Smoking } \\
\text { Cessation }\end{array}$ & 322 & $\begin{array}{l}\text { Smokers from four } \\
\text { psychiatric outpatient } \\
\text { clinics; San Francisco }\end{array}$ & Clinic & Adults & 42.0 & Conven. & United States \\
\hline $\begin{array}{l}\text { Prochaska, } \\
\text { J.O. } \\
\text { et al. }\end{array}$ & 1994 & $\begin{array}{l}\text { Condom } \\
\text { Use -Anal } \\
\text { Main }\end{array}$ & 325 & $\begin{array}{l}\text { Community sample } \mathrm{w} / \mathrm{Hx} \\
\text { of high-risk sex }\end{array}$ & Comn & Adults & 31.9 & Conven. & United States \\
\hline $\begin{array}{l}\text { Rakowski } \\
\text { et al. }\end{array}$ & 1992 & $\begin{array}{l}\text { Mammo- } \\
\text { graphy }\end{array}$ & 141 & Worksite volunteers & Worksites & Adults & 52.6 & Conven. & United States \\
\hline Redding & 1990 & $\begin{array}{l}\text { Safer Sex } \\
\text { Decision } \\
\text { Making }\end{array}$ & 213 & $\begin{array}{l}\text { University undergraduates } \\
\text { (psychology and } \\
\text { chemistry) }\end{array}$ & $\begin{array}{l}\text { College / } \\
\text { University }\end{array}$ & College & 20.2 & Conven. & United States \\
\hline Redding & 1993 & $\begin{array}{l}\text { Safer Sex } \\
\text { Decision } \\
\text { Making }\end{array}$ & 305 & $\begin{array}{l}\text { Sexually active students } \\
\text { attending a mid-size, } \\
\text { northeastern state } \\
\text { university }\end{array}$ & $\begin{array}{l}\text { College / } \\
\text { University }\end{array}$ & College & 19.0 & Conven. & United States \\
\hline Reed & 1996 & Exercise & 271 & $\begin{array}{l}\text { phone book ( } 6 \text { states }) ; 3 \\
\text { weddings in New } \\
\text { England; }\end{array}$ & $\begin{array}{l}\text { Community / } \\
\text { Weddings }\end{array}$ & Adults & 47.8 & Mixed & United States \\
\hline
\end{tabular}


Table 7 (continued)

\begin{tabular}{|c|c|c|c|c|c|c|c|c|c|}
\hline Source & Year & $\begin{array}{l}\text { Target } \\
\text { Behavior }\end{array}$ & $\begin{array}{l}\text { N/ } \\
\text { Data } \\
\text { Set }\end{array}$ & Sample Description & Setting & $\begin{array}{l}\text { Age } \\
\text { Group }\end{array}$ & $\begin{array}{l}\text { Mean } \\
\text { Age }\end{array}$ & $\begin{array}{l}\text { Sampl- } \\
\text { ing } \\
\text { Method }\end{array}$ & Country \\
\hline $\begin{array}{l}\text { Riley } \\
\text { et al. }\end{array}$ & 2000 & $\begin{array}{l}\text { Stress } \\
\text { Manage- } \\
\text { ment }\end{array}$ & 19 & $\begin{array}{l}\text { HIV-negative women } \\
\text { from an HIV high-risk } \\
\text { community w/a high } \\
\text { incidence of STDs and } \\
\text { drug use; HIV-positive } \\
\text { women from an HIV } \\
\text { community health center }\end{array}$ & $\begin{array}{l}\text { Community } \\
\text { health center }\end{array}$ & Adults & 32.9 & Conven. & United States \\
\hline $\begin{array}{l}\text { Riley } \\
\text { et al. }\end{array}$ & 2003 & $\begin{array}{l}\text { Stress } \\
\text { Manage- } \\
\text { ment }\end{array}$ & 126 & $\begin{array}{l}\text { HIV-positive women } \\
\text { recruited as part of a } \\
\text { larger study from three } \\
\text { HIV primary care clinics } \\
\text { in northeast Ohio }\end{array}$ & Clinic & Adults & 34.7 & Conven. & United States \\
\hline $\begin{array}{l}\text { Riley } \\
\text { et al. }\end{array}$ & 2003 & $\begin{array}{l}\text { Stress } \\
\text { Manage- } \\
\text { ment }\end{array}$ & 42 & $\begin{array}{l}\text { Impoverished HIV- } \\
\text { positive women }\end{array}$ & Not Reported & Adults & 38.4 & $\begin{array}{l}\text { Not } \\
\text { Reported }\end{array}$ & United States \\
\hline $\begin{array}{l}\text { Robbins } \\
\text { et al. }\end{array}$ & 2001 & $\begin{array}{l}\text { Organ } \\
\text { Donation - } \\
\text { Family } \\
\text { Consent } \\
\end{array}$ & 163 & $\begin{array}{l}\text { Legal next of kin from } 19 \\
\text { organ procurement } \\
\text { organizations }\end{array}$ & $\begin{array}{l}\text { Organ } \\
\text { Procurement } \\
\text { Organizations }\end{array}$ & Adults & 46.5 & Conven. & United States \\
\hline Robbins & 1999 & $\begin{array}{l}\text { Organ } \\
\text { Donation - } \\
\text { Intentions }\end{array}$ & 278 & $\begin{array}{l}\text { Undergraduate students } \\
\text { age } 18 \text { and older enrolled } \\
\text { in psychology and nursing } \\
\text { courses at URI; Rhode } \\
\text { Island }\end{array}$ & $\begin{array}{l}\text { College / } \\
\text { University }\end{array}$ & College & 20.8 & Conven. & United States \\
\hline
\end{tabular}


Table 7 (continued)

\begin{tabular}{|c|c|c|c|c|c|c|c|c|c|}
\hline Source & Year & $\begin{array}{l}\text { Target } \\
\text { Behavior }\end{array}$ & $\begin{array}{l}\text { N/ } \\
\text { Data } \\
\text { Set }\end{array}$ & Sample Description & Setting & $\begin{array}{l}\text { Age } \\
\text { Group }\end{array}$ & $\begin{array}{l}\text { Mean } \\
\text { Age }\end{array}$ & $\begin{array}{l}\text { Sampl- } \\
\text { ing } \\
\text { Method }\end{array}$ & Country \\
\hline Robbins & 2002 & $\begin{array}{l}\text { Organ } \\
\text { Donation - } \\
\text { Intentions }\end{array}$ & 333 & $\begin{array}{l}\text { College students with } \\
\text { sophomore and junior } \\
\text { standing from four } \\
\text { historically African } \\
\text { American colleges or } \\
\text { universities in North } \\
\text { Carolina }\end{array}$ & $\begin{array}{l}\text { College / } \\
\text { University }\end{array}$ & College & 18.0 & Random & United States \\
\hline Robbins & 1999 & $\begin{array}{l}\text { Organ } \\
\text { Donation - } \\
\text { Intentions }\end{array}$ & 475 & Adults in Louisiana & Community & Adults & 42.8 & Random & United States \\
\hline Robbins & 2002 & $\begin{array}{l}\text { Organ } \\
\text { Donation - } \\
\text { Intentions }\end{array}$ & 2233 & $\begin{array}{l}\text { College students at URI; } \\
\text { Rhode Island }\end{array}$ & $\begin{array}{l}\text { College / } \\
\text { University }\end{array}$ & College & 19.0 & Conven. & United States \\
\hline Rossi & 1990 & $\begin{array}{l}\text { Radon } \\
\text { Testing }\end{array}$ & 698 & Worksite employees & Work site & Adults & 42.0 & Conven. & United States \\
\hline Rossi & 1990 & $\begin{array}{l}\text { Sunscreen } \\
\text { Use }\end{array}$ & 227 & $\begin{array}{l}\text { Smoking study } \\
\text { participants }\end{array}$ & Not reported & Adults & 44.9 & Conven. & United States \\
\hline $\begin{array}{l}\text { Rossi } \\
\text { et al. }\end{array}$ & 2001 & $\begin{array}{l}\text { Cocaine } \\
\text { Use }\end{array}$ & 298 & $\begin{array}{l}\text { In/ outpatient drug } \\
\text { treatment }\end{array}$ & $\begin{array}{l}\text { Treatment } \\
\text { centers }\end{array}$ & Adults & 29.6 & Conven. & United States \\
\hline $\begin{array}{l}\text { Rossi. SR } \\
\text { et al. }\end{array}$ & 2001 & $\begin{array}{l}\text { Dietary Fat } \\
\text { Reduction }\end{array}$ & 2639 & $\begin{array}{l}\text { 9th grade students from } 22 \\
\text { Rhode Island high schools }\end{array}$ & School & Adol. & 15.2 & Conven. & United States \\
\hline
\end{tabular}


Table 7 (continued)

\begin{tabular}{|c|c|c|c|c|c|c|c|c|c|}
\hline Source & Year & $\begin{array}{l}\text { Target } \\
\text { Behavior }\end{array}$ & $\begin{array}{l}\text { N/ } \\
\text { Data } \\
\text { Set }\end{array}$ & Sample Description & Setting & $\begin{array}{l}\text { Age } \\
\text { Group }\end{array}$ & $\begin{array}{l}\text { Mean } \\
\text { Age }\end{array}$ & $\begin{array}{l}\text { Sampl- } \\
\text { ing } \\
\text { Method }\end{array}$ & Country \\
\hline $\begin{array}{l}\text { Rossi, SR } \\
\text { et al. }\end{array}$ & 2001 & $\begin{array}{l}\text { Fruit \& } \\
\text { Vegetable } \\
\text { Consump- } \\
\text { tion }\end{array}$ & 353 & Older adults & $\begin{array}{l}\text { Community (for } \\
\text { seniors) }\end{array}$ & Adults & $\begin{array}{l}\text { Not } \\
\text { reported }\end{array}$ & Conven. & United States \\
\hline $\begin{array}{l}\text { Rossi, SR } \\
\text { et al. }\end{array}$ & 2001 & $\begin{array}{l}\text { Dietary Fat } \\
\text { Reduction }\end{array}$ & 1204 & RDD; Rhode Island & Community & Adults & 42.0 & Random & United States \\
\hline $\begin{array}{l}\text { Rossi, SR } \\
\text { et al. }\end{array}$ & 1993 & $\begin{array}{l}\text { Dietary Fat } \\
\text { Reduction }\end{array}$ & 196 & $\begin{array}{l}\text { Non-smoking Rhode } \\
\text { Island residents (RDD) }\end{array}$ & Community & Adults & 43.5 & Random & United States \\
\hline $\begin{array}{l}\text { Ruggiero } \\
\text { et al. }\end{array}$ & 2000 & $\begin{array}{l}\text { Smoking } \\
\text { Cessation }\end{array}$ & 206 & $\begin{array}{l}\text { Pregnant smoking women } \\
\text { from community health } \\
\text { clinics in New England }\end{array}$ & Clinic & Adults & 25.4 & Conven. & United States \\
\hline $\begin{array}{l}\text { Ruggiero } \\
\text { et al. }\end{array}$ & 2003 & $\begin{array}{l}\text { Glucose } \\
\text { Testing }\end{array}$ & 668 & $\begin{array}{l}\text { Canadian sample recruited } \\
\text { for intervention }\end{array}$ & Community & Adults & 55.8 & Conven. & Canada \\
\hline $\begin{array}{l}\text { Sarkin } \\
\text { et al. }\end{array}$ & 2001 & Exercise & 670 & $\begin{array}{l}\text { List provided by market } \\
\text { research company } \\
\text { including apparently } \\
\text { healthy adults (no signs of } \\
\text { chronic disease) }\end{array}$ & Community & Adults & $\overline{50.9}$ & Conven. & United States \\
\hline $\begin{array}{l}\text { Schmaling } \\
\text { et al. }\end{array}$ & 2000 & $\begin{array}{l}\text { Asthma: } \\
\text { Medication } \\
\text { Manage- } \\
\text { ment }\end{array}$ & 53 & Adult asthma patients & $\begin{array}{l}\text { Private Practice } \\
\text { Asthma Clinic; } \\
\text { Emergency } \\
\text { Department; } \\
\text { Hospital Clinic }\end{array}$ & Adults & 36.1 & Conven. & United States \\
\hline
\end{tabular}


Table 7 (continued)

\begin{tabular}{|c|c|c|c|c|c|c|c|c|c|}
\hline Source & Year & $\begin{array}{l}\text { Target } \\
\text { Behavior }\end{array}$ & $\begin{array}{l}\text { N/ } \\
\text { Data } \\
\text { Set }\end{array}$ & Sample Description & Setting & $\begin{array}{l}\text { Age } \\
\text { Group }\end{array}$ & $\begin{array}{l}\text { Mean } \\
\text { Age }\end{array}$ & $\begin{array}{l}\text { Sampl- } \\
\text { ing } \\
\text { Method }\end{array}$ & Country \\
\hline \multirow[t]{2}{*}{ Schorling } & \multirow[t]{2}{*}{1995} & $\begin{array}{l}\text { Smoking } \\
\text { Cessation } \\
\text { (Women) }\end{array}$ & 243 & $\begin{array}{l}\text { Rural African-American } \\
\text { female smokers }\end{array}$ & Community & Adults & 40.1 & Random & United States \\
\hline & & $\begin{array}{l}\text { Smoking } \\
\text { Cessation } \\
\text { (Men) }\end{array}$ & 292 & $\begin{array}{l}\text { Rural African-American } \\
\text { male smokers }\end{array}$ & Community & Adults & 41.9 & Random & United States \\
\hline $\begin{array}{l}\text { Schumann } \\
\text { et al. }\end{array}$ & 2003 & $\begin{array}{l}\text { Smoking } \\
\text { Cessation }\end{array}$ & 877 & $\begin{array}{l}\text { General population } \\
\text { sample }\end{array}$ & Community & Adults & 41.6 & Random & Germany \\
\hline Shulze & 2003 & $\begin{array}{l}\text { Daily Use } \\
\text { of Dental } \\
\text { Floss }\end{array}$ & 453 & $\begin{array}{l}\text { Employees of three } \\
\text { different companies }\end{array}$ & Worksites & Adults & $\begin{array}{l}\text { Not } \\
\text { reported }\end{array}$ & Conven. & Germany \\
\hline \multirow[t]{2}{*}{ Silverman } & \multirow[t]{2}{*}{1995} & $\begin{array}{l}\text { Condom } \\
\text { Use }\end{array}$ & 223 & $\begin{array}{l}\text { University students; } \\
\text { Hong Kong }\end{array}$ & $\begin{array}{l}\text { College/ } \\
\text { University }\end{array}$ & College & 20.4 & Conven. & China \\
\hline & & $\begin{array}{l}\text { Condom } \\
\text { Use } \\
\end{array}$ & 230 & University students & $\begin{array}{l}\text { College/ } \\
\text { University }\end{array}$ & College & 19.1 & Conven. & United States \\
\hline $\begin{array}{l}\text { Snow } \\
\text { et al. }\end{array}$ & 1992 & $\begin{array}{l}\text { Smoking } \\
\text { Cessation }\end{array}$ & 191 & $\begin{array}{l}\text { Newspaper ad for } \\
\text { smoking and alcohol }\end{array}$ & Community & Adults & 44.1 & Conven. & United States \\
\hline \multirow[t]{2}{*}{$\begin{array}{l}\text { Stark } \\
\text { et al. }\end{array}$} & \multirow[t]{2}{*}{1998} & $\begin{array}{l}\text { Condom } \\
\text { Use - Main }\end{array}$ & 822 & $\begin{array}{l}\text { Inner city females at high } \\
\text { risk for HIV or STD's }\end{array}$ & Community & Adults & 25.6 & & \\
\hline & & $\begin{array}{l}\text { Condom } \\
\text { Use - Other }\end{array}$ & 754 & $\begin{array}{l}\text { Inner city females at high } \\
\text { risk for HIV or STD's }\end{array}$ & Community & Adults & 26.6 & Conven. & United States \\
\hline Susenbeth & 2000 & $\begin{array}{l}\text { Condom } \\
\text { Use }\end{array}$ & 96 & Bisexual men & Not reported & Adults & 29.0 & Conven. & Germany \\
\hline
\end{tabular}


Table 7 (continued)

\begin{tabular}{|c|c|c|c|c|c|c|c|c|c|}
\hline Source & Year & $\begin{array}{l}\text { Target } \\
\text { Behavior }\end{array}$ & $\begin{array}{l}\text { N/ } \\
\text { Data } \\
\text { Set }\end{array}$ & Sample Description & Setting & $\begin{array}{l}\text { Age } \\
\text { Group }\end{array}$ & $\begin{array}{l}\text { Mean } \\
\text { Age }\end{array}$ & $\begin{array}{l}\text { Sampl- } \\
\text { ing } \\
\text { Method }\end{array}$ & Country \\
\hline Tseng & 2001 & Exercise & 154 & $\begin{array}{l}\text { Elders residing in six } \\
\text { assisted living facilities } \\
\text { located in two large } \\
\text { adjacent cities in an upper } \\
\text { Midwest state }\end{array}$ & $\begin{array}{l}\text { Assisted Living } \\
\text { Facilities }\end{array}$ & Adults & $\begin{array}{l}\text { Not } \\
\text { reported }\end{array}$ & Conven. & United States \\
\hline Tsoh & 1993 & $\begin{array}{l}\text { Quitting } \\
\text { Drugs }\end{array}$ & 169 & $\begin{array}{l}\text { Adult drug addicts/ Mass } \\
\text { treatment programs; } \\
\text { Lo SES; }\end{array}$ & $\begin{array}{l}\text { Treatment } \\
\text { centers }\end{array}$ & Adults & 34.6 & Conven. & United States \\
\hline $\begin{array}{l}\text { Van Marter } \\
\text { et al. }\end{array}$ & 2003 & $\begin{array}{l}\text { Bullying } \\
\text { Prevention }\end{array}$ & 473 & Middle school students & School & Adol. & $\begin{array}{l}\text { Not } \\
\text { reported }\end{array}$ & Conven. & United States \\
\hline $\begin{array}{l}\text { Velicer } \\
\text { et al. }\end{array}$ & 1985 & $\begin{array}{l}\text { Smoking } \\
\text { Cessation }\end{array}$ & 930 & $\begin{array}{l}\text { Volunteers from } \\
\text { newspaper article/ ads; } \\
\text { RI and TX }\end{array}$ & Community & Adults & $\begin{array}{l}\text { Not } \\
\text { reported }\end{array}$ & Conven. & United States \\
\hline $\begin{array}{l}\text { Wakui } \\
\text { et al. }\end{array}$ & 2002 & Exercise & 450 & $\begin{array}{l}\text { Female students from two } \\
\text { universities in Tokyo }\end{array}$ & $\begin{array}{l}\text { College/ } \\
\text { University }\end{array}$ & College & 18.4 & Conven. & Japan \\
\hline Whyte & 2003 & $\begin{array}{l}\text { Dietary Fat } \\
\text { Reduction }\end{array}$ & 416 & $\begin{array}{l}\text { Male and female adults } \\
\text { aged } 18 \text { to } 60 \text { recruited via } \\
\text { the internet }\end{array}$ & Internet & Adults & 30.0 & Conven. & United States \\
\hline $\begin{array}{l}\text { Wright } \\
\text { et al. }\end{array}$ & 2002 & $\begin{array}{l}\text { Weight } \\
\text { Bearing } \\
\text { Exercise }\end{array}$ & 250 & $\begin{array}{l}\text { Female member of } \\
\text { UnitedHeathcare of New } \\
\text { England, ages } 45+\end{array}$ & Community & Adults & 62.4 & Conven. & United States \\
\hline
\end{tabular}

Note: Conven. $=$ convenience sampling. 
Factor Structure. The internal validity of the two-factor structure of Pros and Cons of Change identified by Velicer et al. (1985) was maintained for $96 \%$ of the datasets. One dataset (Bane et al., 1999) reported 2 Pros and 2 Cons; three others (Pallonen et al., 1997, Studies 1 and 2; Plummer et al., 2001) reported 2 Pros and 1 con, and lastly Susenbeth (2000) reported 1 pro and 2 Cons.

Alpha Coefficients. One hundred twelve datasets for Pros and 114 datasets for Cons included alpha coefficients as a measure of internal consistency. For Pros the average coefficient alpha was $.82(S D=.09)$, with a range from .58 to .96 . The average coefficient alpha for Cons was a bit lower at .76 $(S D=.10)$, and ranged from .41 to .94 .

Scale and Construct Correlations. Thirty-two datasets reported scale correlation coefficients for the Pros and Cons measures, with an average of .04 (SD= .20 ), and a range from -.54 to .38 . Twenty-seven datasets reported latent variable (construct) correlation coefficients based on structural equation modeling, with an average of $.08(S D=.27)$, and a range from -.29 to .63 .

\section{Function: Patterns and Crossovers}

Stages. Not all studies used all five Stages of Change. As mentioned previous, historically the Preparation Stage did not always exist as part of the model therefore a handful of studies did not include the Preparation Stage. During the conduct of some studies, occasionally particular Stages included very few participants or a disproportionately small number of participants in a given Stage. 
Researchers sometimes chose to combine Stages in order to compensate for a small sample size. Overall, $91 \%$ of the datasets included the Preparation Stage, and $8 \%$ of the studies including the Preparation Stage combined the Preparation Stage with the Contemplation Stage when reporting results. Eighty-four percent of the studies included the Maintenance Stage, and 11\% of those combined Action and Maintenance.

Crossovers. Crossovers occur when the standardized Pros and Cons scores change position relative to each other. If, at one Stage Cons are higher than Pros and at the next Stage Pros are higher than Cons, then on a graph a literal crossing of lines occur, hence the name crossover. Therefore, it can be said that the crossover is a function of how much and when the Pros increase and the Cons decrease (Prochaska et al., 1994). In this study, crossovers were determined for all datasets that reported $T$-scores or graphs based on $T$-scores; crossovers could be determined for $86 \%$ of the datasets.

Seventeen percent of the crossovers revealed multiple intersections, or crossovers, of the Pros and Cons. Twenty-five percent of the crossovers occurred in the transition from Precontemplation to Contemplation. The greatest number of crossovers, $32 \%$, occurred during the Stage transition from Contemplation to Preparation. Eighteen percent of the crossovers occurred in the transition from Preparation to Action. A handful of studies did not include Preparation as a Stage of Change. This resulted in possible crossovers in the transition from Contemplation to Action. Four percent of the studies had crossovers in the Contemplation to Action 
transition. The fewest number of crossovers, $2 \%$, occurred in Action to Maintenance.

Finally, there was one dataset in which no crossover occurred at all.

Sample size can impact the graphical representations of the crossovers based on the standardized $T$-scores. Large sample sizes can "pull" the cross-over towards a given Stage. Sample sizes across the crossover transitions were examined to provide a gross assessment of the relationship between sample size and Stage transition. Interestingly, for the crossovers found between PC-C, the largest sample sizes average was in the Precontemplation Stage $(n=275)$ with the second largest in Contemplation ( $n=146$ ). For the crossovers found between C-PR, although the largest mean sample size was in the Maintenance Stage $(n=129)$, the Precontemplation, Contemplation and Preparations Stages were also reasonably large and of similar magnitude ( $n=120,111,98$, respectively). The crossovers found between PR-A had the largest mean sample size in the Maintenance Stage $(n=136)$, with the rest of the Stages somewhat evenly distributed. The crossovers found between A-M also had the largest mean sample size in Maintenance Stage $(n=415)$ with quite small mean sample sizes for Precontemplation, Contemplation and Preparation ( $n=15,25,9$, respectively). Following a similar trend the C-A transition had the largest sample size in Maintenance $(n=83)$, with notably smaller averages for the previous three Stages $(n=23,29,28)$. Lastly, the studies that displayed multiple crossovers displayed a somewhat unique pattern of mean sample sizes, with the largest mean sample sizes on the polar ends of the Stage distribution (Precontemplation, $n=112$ and Maintenance, $n=131$ ) and similar mean sample 
sizes for the middle three Stages $(n=73,67,65)$. Table 8 displays the mean sample sizes across the Stages of Change for each of the primary cross-over transitions.

Table 8

Mean Samples Sizes by Stage for Each Crossover Transitions

\begin{tabular}{|c|c|c|c|c|c|c|}
\hline \multirow{2}{*}{$\begin{array}{l}\text { Crossover } \\
\text { Transition }\end{array}$} & & \multicolumn{5}{|c|}{ Stage of Change } \\
\hline & & $\mathbf{P}$ & $\mathrm{C}$ & PR & $\mathbf{A}$ & $\mathbf{M}$ \\
\hline \multirow[t]{3}{*}{ PC-C } & Mean $\mathbf{N}$ & 275 & 146 & 97 & 52 & 109 \\
\hline & $\mathrm{N}$ & 30 & 30 & 24 & 23 & 16 \\
\hline & $S D$ & 427 & 293 & 156 & 38 & 97 \\
\hline \multirow[t]{3}{*}{ C-PR } & Mean $\mathbf{N}$ & 120 & 111 & 98 & 59 & 129 \\
\hline & $\mathrm{N}$ & 40 & 40 & 39 & 34 & 33 \\
\hline & $S D$ & 159 & 150 & 139 & 78 & 191 \\
\hline \multirow[t]{3}{*}{ PR-A } & Mean N & 69 & 50 & 71 & 43 & 136 \\
\hline & $\mathrm{N}$ & 22 & 21 & 21 & 22 & 22 \\
\hline & $S D$ & 69 & 37 & 80 & 36 & 116 \\
\hline \multirow[t]{3}{*}{ A-M } & Mean $\mathbf{N}$ & 15 & 25 & 9 & 46 & 415 \\
\hline & $\mathrm{N}$ & 3 & 2 & 2 & 3 & 3 \\
\hline & $S D$ & 10 & 34 & 9 & 11 & 483 \\
\hline \multirow[t]{3}{*}{$\mathrm{C}-\mathrm{A}$} & Mean N & 23 & 29 & - & 28 & 83 \\
\hline & $\mathrm{N}$ & 5 & 5 & - & 5 & 5 \\
\hline & $S D$ & 10 & 21 & - & 18 & 64 \\
\hline \multirow[t]{3}{*}{ Multiple } & Mean $\mathbf{N}$ & 112 & 73 & 67 & 65 & 131 \\
\hline & $\mathrm{N}$ & 21 & 20 & 19 & 20 & 19 \\
\hline & $S D$ & 150 & 100 & 76 & 69 & 233 \\
\hline
\end{tabular}

\section{Magnitude of Transitions}

Overview. A total of 2144 primary effect sizes were calculated across 4 Stage transitions for the Decisional Balance measures. Nine hundred forty-seven unadjusted Hedges' $g$ or Standard Score effect sizes were calculated for decisional balance. These 947 effect sizes were then adjusted for sample size, resulting in 947 adjusted effect sizes. Finally, 250 effect sizes based on the standard score method were corrected using the Pros and Cons regression correction (see Equation 119). 
Table 9 displays the sample size and calculation adjusted effect sizes for the Pros and

Cons across the Stage transitions for all datasets. 
Table 9

Adjusted Effect Sizes of the Pros and Cons of Change for All Studies across Stage

\section{Transitions}

\begin{tabular}{|c|c|c|c|c|c|c|c|c|c|c|c|}
\hline \multirow[b]{3}{*}{ Study/ Author } & \multirow[b]{3}{*}{ r Year } & \multirow[b]{3}{*}{$\begin{array}{c}\text { Target } \\
\text { Behavior }\end{array}$} & \multirow{3}{*}{$\begin{array}{c}\mathrm{N} / \\
\text { data- } \\
\text { set }\end{array}$} & \multicolumn{8}{|c|}{ Effect Sizes for Stage Transitions } \\
\hline & & & & \multicolumn{4}{|c|}{ Pros } & \multicolumn{4}{|c|}{ Cons } \\
\hline & & & & PC-C & C-PR & PRA & A-M & PC-C & C-PR & PR-A & A-M \\
\hline Acton et al. & 2001 & $\begin{array}{l}\text { Smoking } \\
\text { Cessation }\end{array}$ & 205 & .76 & -.15 & -.52 & .33 & -.20 & .00 & -.31 & -.17 \\
\hline $\begin{array}{l}\text { Ahijevych et } \\
\text { al. }\end{array}$ & 1999 & $\begin{array}{l}\text { Smoking } \\
\text { Cessation }\end{array}$ & 95 & .66 & .27 & n.a. & n.a. & -.11 & -.33 & n.a. & n.a. \\
\hline Bane et al. & 1999 & $\begin{array}{l}\text { Smoking } \\
\text { Cessation }\end{array}$ & 281 & .58 & .19 & -.68 & -.19 & $\mid-.11$ & -.15 & -.12 & -.28 \\
\hline $\begin{array}{l}\text { Banikarim et } \\
\text { al. }\end{array}$ & 2003 & $\begin{array}{l}\text { STD Screening- } \\
\text { Change in } \\
\text { Partners }\end{array}$ & 135 & .28 & .62 & .18 & .06 & -1.42 & -.18 & -.37 & .09 \\
\hline $\begin{array}{l}\text { Banikarim et } \\
\text { al. }\end{array}$ & 2003 & $\begin{array}{l}\text { STD Screening- } \\
\text { Main }\end{array}$ & 201 & -.32 & .10 & -.15 & .76 & .25 & .07 & .07 & -.42 \\
\hline Basler et al. & 1999 & Exercise & 451 & .63 & .39 & -.08 & .07 & .18 & -.28 & -.51 & .14 \\
\hline Block et al. & 1998 & Condom Use & 127 & .26 & n.a. & n.a. & n.a. & -.08 & n.a. & n.a. & n.a. \\
\hline Borland et al. & 2000 & $\begin{array}{l}\text { Smoking } \\
\text { Cessation }\end{array}$ & 304 & .54 & .32 & .18 & n.a. & .16 & -.17 & -.23 & n.a. \\
\hline $\begin{array}{l}\text { Boudreaux et } \\
\text { al. }\end{array}$ & 1998 & $\begin{array}{l}\text { Smoking } \\
\text { Cessation }\end{array}$ & 554 & 1.28 & -.22 & -.56 & -1.64 & -.16 & -.59 & -.58 & -.78 \\
\hline $\begin{array}{l}\text { Buckworth et } \\
\text { al. }\end{array}$ & 2002 & Exercise & 57 & n.a. & n.a. & n.a. & .98 & n.a. & n.a. & n.a. & -1.26 \\
\hline Callaghan et al. & 2002 & Exercis & 298 & 1.68 & .39 & .36 & .36 & .28 & -.42 & .06 & -.20 \\
\hline Chamot et al. & 2001 & Mammography & 885 & .97 & n.a. & n.a. & .27 & -.77 & n.a. & n.a. & -.06 \\
\hline DiClemente & 1991 & $\begin{array}{l}\text { Smoking } \\
\text { Cessation }\end{array}$ & 1466 & .82 & .18 & n.a. & n.a. & -.31 & -.25 & n.a. & n.a. \\
\hline Dijkstra et al. & 1996 & $\begin{array}{l}\text { Smoking } \\
\text { Cessation }\end{array}$ & 275 & .73 & .56 & -.48 & .12 & .23 & -.14 & -.32 & -.39 \\
\hline Dijkstra et al. & 1998 & $\begin{array}{l}\text { Smoking } \\
\text { Cessation }\end{array}$ & 1540 & n.a. & n.a. & n.a. & n.a. & .02 & -.01 & n.a. & n.a. \\
\hline Dryfoos & 1996 & $\begin{array}{l}\text { Glucose Testing } \\
\text { Diabetes: }\end{array}$ & 338 & 1.01 & .08 & .79 & .28 & .44 & -.17 & -.58 & -.49 \\
\hline Dryfoos & 1996 & $\begin{array}{l}\text { Medication } \\
\text { Mangt } \\
\text { Physicians }\end{array}$ & 1048 & n.a. & n.a. & n.a. & .11 & n.a. & n.a. & n.a. & -.69 \\
\hline Eaton et al. & 1992 & $\begin{array}{l}\text { Assisting } \\
\text { Smokers }\end{array}$ & 165 & .50 & .58 & .06 & -.42 & $\mid-.46$ & .08 & -.78 & .75 \\
\hline Evers et al. & 2001 & Condom Use & 270 & -1.07 & 1.02 & .44 & -.26 & .00 & .37 & -.48 & .16 \\
\hline Evers et al. & 2001 & $\begin{array}{l}\text { Decreasing Drug } \\
\text { Use }\end{array}$ & 293 & .07 & .49 & .26 & -.06 & -.44 & -.06 & -.05 & -.43 \\
\hline Evers et al. & 2001 & $\begin{array}{l}\text { Waiting to have } \\
\text { sex }\end{array}$ & 602 & .10 & -.05 & n.a. & n.a. & -.55 & -.42 & n.a. & n.a. \\
\hline
\end{tabular}


Table 9 (continued)

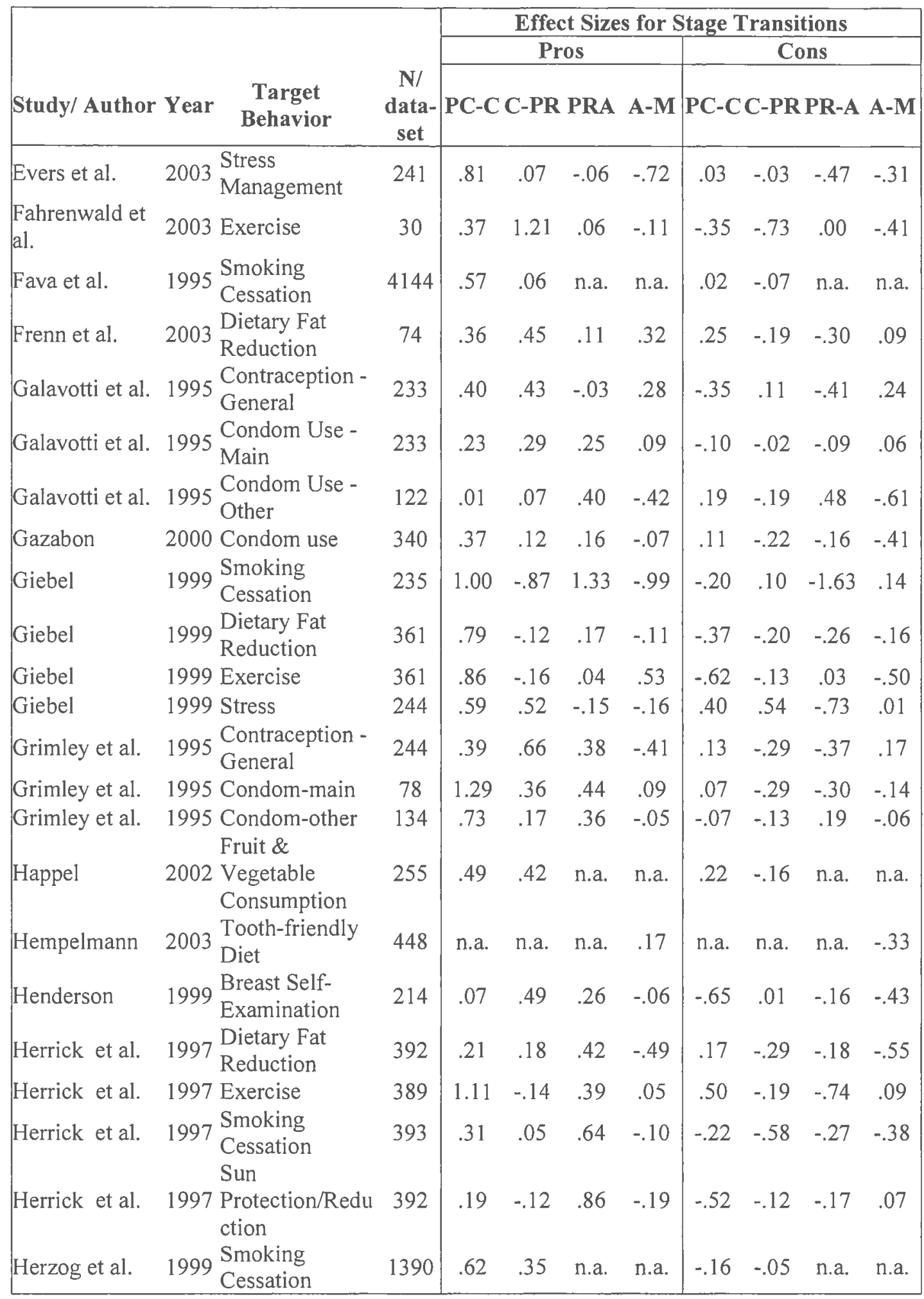


Table 9 (continued)

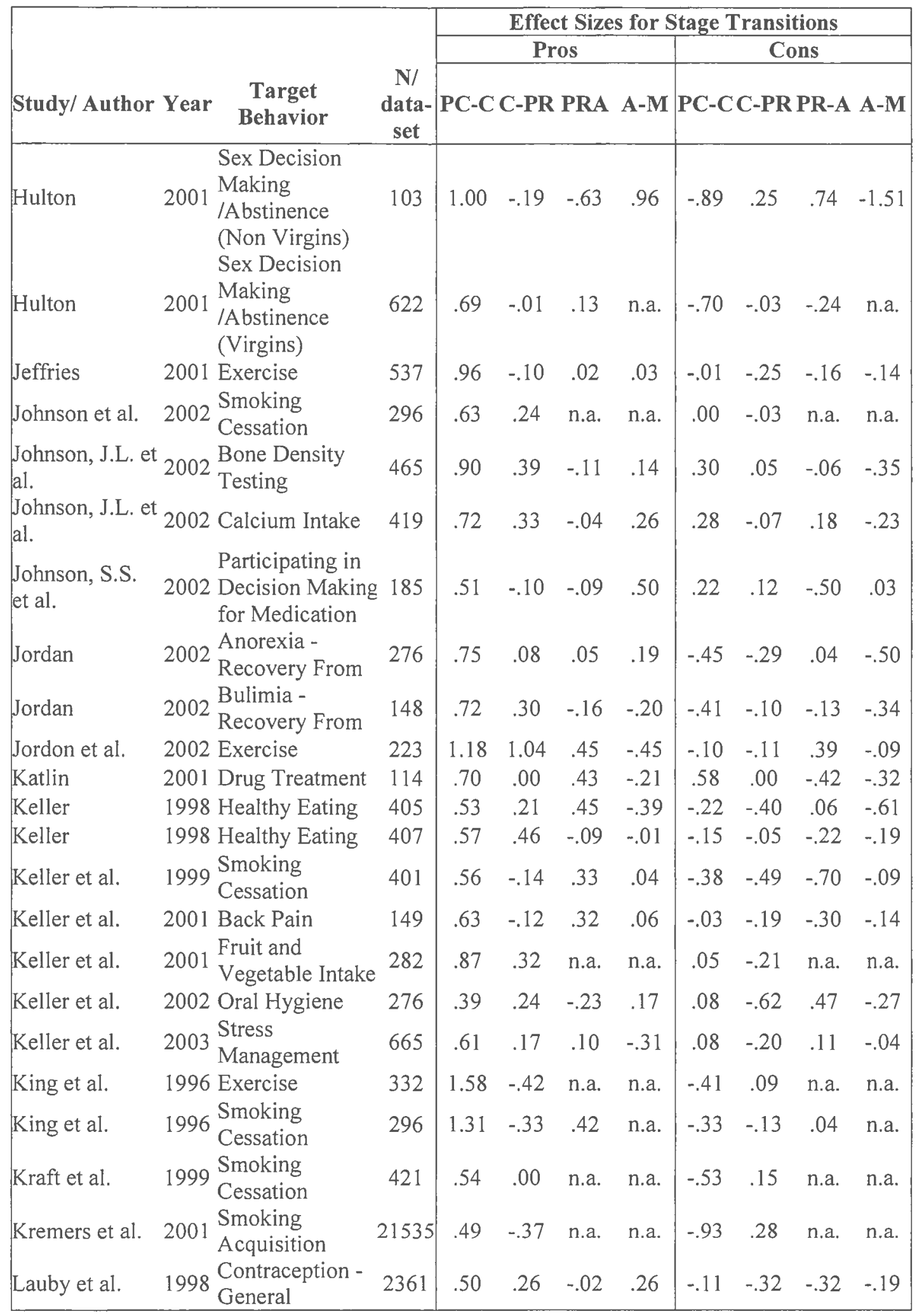




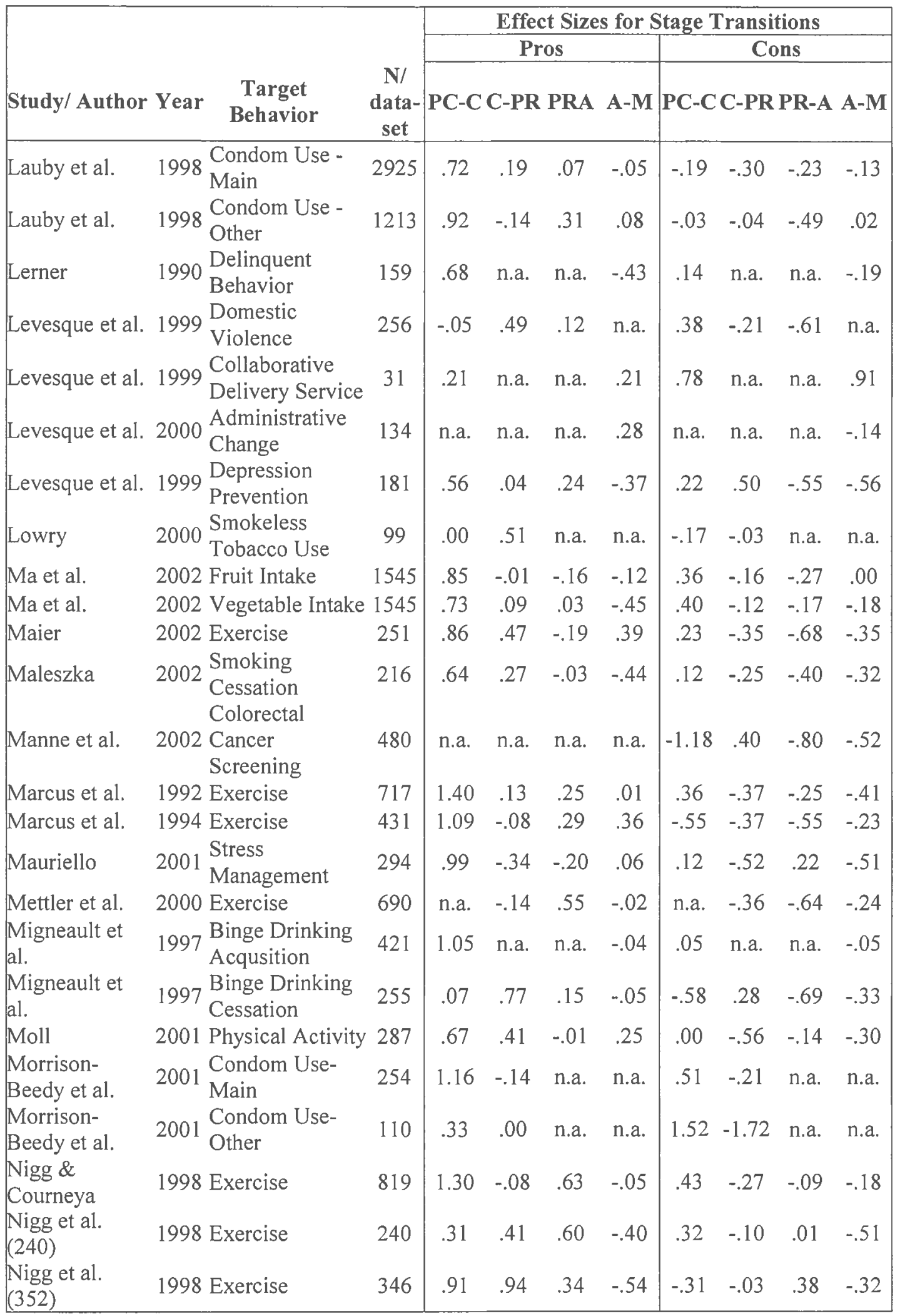




\begin{tabular}{|c|c|c|c|c|c|c|c|c|c|c|c|}
\hline \multirow[b]{3}{*}{ Study/ Author } & \multirow[b]{3}{*}{ Year } & \multirow[b]{3}{*}{$\begin{array}{c}\text { Target } \\
\text { Behavior }\end{array}$} & \multirow[b]{3}{*}{$\begin{array}{c}\mathrm{N} / \\
\text { data- } \\
\text { set }\end{array}$} & \multicolumn{8}{|c|}{ Effect Sizes for Stage Transitions } \\
\hline & & & & \multicolumn{4}{|c|}{ Pros } & \multicolumn{4}{|c|}{ Cons } \\
\hline & & & & PC-C & C-PR & PRA & A-M & PC-C & C-PR & PR-A & A-M \\
\hline Noar et al & 1998 & $\begin{array}{l}\text { Condom Use - } \\
\text { Vaginal Sex }\end{array}$ & 168 & .49 & -.04 & -.01 & .52 & .19 & -.74 & .01 & -.01 \\
\hline Norman et al. & 2002 & $\begin{array}{l}\text { Sedentary } \\
\text { Behavior }\end{array}$ & 178 & .36 & .17 & .07 & -.31 & -.96 & .01 & -.45 & .18 \\
\hline Norman et al. & 2002 & $\begin{array}{l}\text { Sedentary } \\
\text { Behavior }\end{array}$ & 254 & .57 & .10 & .18 & -.19 & -.41 & -.58 & -.26 & -.12 \\
\hline $\begin{array}{l}\text { O'Connell \& } \\
\text { Velicer }\end{array}$ & 1988 & Weight Loss & 123 & 1.00 & n.a. & n.a. & -.48 & .37 & n.a. & n.a. & .39 \\
\hline $\begin{array}{l}\text { Otake \& } \\
\text { Shimai }\end{array}$ & $\begin{array}{l}2001 \\
(J R)\end{array}$ & $\begin{array}{l}\text { Smoking } \\
\text { Acquisition }\end{array}$ & 556 & .73 & .18 & .35 & n.a. & -.29 & -.24 & -.78 & n.a. \\
\hline $\begin{array}{l}\text { Otake \& } \\
\text { Shimai }\end{array}$ & $\begin{array}{l}2001 \\
\text { (SR) }\end{array}$ & $\begin{array}{l}\text { Smoking } \\
\text { Acquisition }\end{array}$ & 1002 & .44 & .30 & -.03 & n.a. & -.43 & .08 & -.69 & n.a. \\
\hline Pallonen et al. & 1997 & $\begin{array}{l}\text { Smoking } \\
\text { Acquisition }\end{array}$ & 505 & .89 & -1.05 & 2.60 & n.a. & -.33 & -.06 & -.84 & n.a. \\
\hline Pallonen et al. & 1997 & $\begin{array}{l}\text { Smoking } \\
\text { Cessation } \\
\text { Physicians }\end{array}$ & 254 & .97 & .11 & .36 & .22 & -.09 & -.34 & -.09 & -.49 \\
\hline Park et al. & 2003 & $\begin{array}{l}\text { sunseling } \\
\text { nokers }\end{array}$ & 259 & .70 & .45 & -.07 & .34 & .21 & -.58 & .87 & -1.16 \\
\hline Perez & 2001 & Condom Use & 828 & .70 & .24 & .03 & .12 & .09 & .04 & -.02 & -.24 \\
\hline Plummer et al. & 2001 & $\begin{array}{l}\text { Smoking } \\
\text { Acquisition }\end{array}$ & 2010 & .74 & .54 & n.a. & n.a. & -.49 & -.60 & n.a. & n.a. \\
\hline Plummer et al. & 2001 & $\begin{array}{l}\text { Smoking } \\
\text { Cessation }\end{array}$ & 798 & .81 & .32 & .02 & .37 & -.36 & -.42 & -.18 & -.63 \\
\hline $\begin{array}{l}\text { Prochaska et } \\
\text { al. }\end{array}$ & 1990 & $\begin{array}{l}\text { Condom Use - } \\
\text { Anal Main }\end{array}$ & 325 & 1.33 & n.a. & n.a. & 27 & -.21 & n.a. & n.a. & -.21 \\
\hline $\begin{array}{l}\text { Prochaska, JJ } \\
\text { et al. }\end{array}$ & 2003 & $\begin{array}{l}\text { Smoking } \\
\text { Cessation }\end{array}$ & 322 & .93 & .25 & n.a. & n.a. & -.31 & -.03 & n.a. & n.a. \\
\hline Rakowski et al. & 1992 & Mammography & 141 & .59 & n.a. & n.a. & .34 & -.36 & n.a. & n.a. & -.33 \\
\hline Redding & 1990 & ex & 213 & 1.27 & n.a. & n.a. & n.a. & -.03 & n.a. & n.a. & n.a. \\
\hline Redding & 1993 & $\begin{array}{l}\text { Safer Sex } \\
\text { Decision Making }\end{array}$ & 305 & 1.26 & n.a. & n.a. & -.05 & -.11 & n.a. & n.a. & -.28 \\
\hline Reed & 1996 & Exercise & 271 & 1.75 & .63 & .26 & -.42 & -.38 & -.09 & -.34 & -.10 \\
\hline Riley et al. & 2000 & $\begin{array}{l}\text { Stress } \\
\text { Management }\end{array}$ & 19 & .22 & .25 & -.40 & -.10 & .19 & -.75 & .77 & -.30 \\
\hline Riley et al & 2003 & $\begin{array}{l}\text { Stress } \\
\text { Management }\end{array}$ & 42 & -.32 & -.08 & -.26 & .32 & -.20 & .39 & -.36 & .04 \\
\hline Rile & 2003 & $\begin{array}{l}\text { Stress } \\
\text { Managem }\end{array}$ & 126 & .79 & -.09 & -.13 & -.10 & 01 & .47 & -.32 & -.05 \\
\hline Robbi & 2002 & Organ Donation & 2233 & .74 & -.17 & .17 & .10 & -.33 & .11 & -.17 & -.22 \\
\hline Robbins et al. & 2002 & Organ Donation & 333 & .33 & .17 & .26 & -.47 & -.08 & .27 & -.61 & .29 \\
\hline Robbins et al. & 1999 & Organ Donation & 475 & .60 & -.05 & n.a. & n.a. & -.32 & -.37 & n.a. & n.a. \\
\hline Robbins et al. & 1999 & Organ Donation & 280 & .48 & .20 & n.a. & n.a. & -.49 & -.04 & n.a. & n.a. \\
\hline
\end{tabular}




\begin{tabular}{|c|c|c|c|c|c|c|c|c|c|c|c|}
\hline \multirow[b]{3}{*}{ Study/ Author } & \multirow[b]{3}{*}{ Year } & \multirow[b]{3}{*}{$\begin{array}{c}\text { Target } \\
\text { Behavior }\end{array}$} & \multirow[b]{3}{*}{$\begin{array}{c}\mathrm{N} / \\
\text { data- } \\
\text { set }\end{array}$} & \multicolumn{8}{|c|}{ Effect Sizes for Stage Transitions } \\
\hline & & & & \multicolumn{4}{|c|}{ Pros } & \multicolumn{4}{|c|}{ Cons } \\
\hline & & & & PC-C & C-PR & PRA & $\mathbf{A}-\mathbf{M}$ & PC-C & C-PR & PR-A & $\mathbf{A}-\mathbf{M}$ \\
\hline Robbins et al. & 2001 & Organ Donation & 163 & .59 & .04 & .21 & n.a. & -.18 & -.35 & -.19 & n.a. \\
\hline Rossi & 1990 & Sunscreen Use & 136 & .86 & n.a. & n.a. & 1.05 & .11 & n.a. & n.a. & -.25 \\
\hline Rossi & 1990 & Radon Testing & 698 & 1.00 & n.a. & n.a. & n.a. & -.27 & n.a. & n.a. & n.a. \\
\hline Rossi et al. & 2001 & Cocaine Use & 298 & 1.15 & n.a. & n.a. & .00 & .50 & n.a. & n.a. & -.21 \\
\hline Rossi, SR et al. & 1993 & $\begin{array}{l}\text { Dietary Fat } \\
\text { Reduction }\end{array}$ & 196 & .78 & n.a. & n.a. & -.47 & .56 & n.a. & n.a. & -.27 \\
\hline Rossi, SR et al. & 2001 & $\begin{array}{l}\text { Dietary Fat } \\
\text { Reduction } \\
\text { Fruit \& }\end{array}$ & 1204 & .21 & .08 & -.25 & -.06 & .49 & .05 & -.10 & -.02 \\
\hline Rossi, SR et al. & 2001 & $\begin{array}{l}\text { Vegetable } \\
\text { Consumption }\end{array}$ & 353 & .47 & .33 & -.04 & -.11 & -.12 & -.06 & -.30 & .46 \\
\hline Rossi. SR et al. & 2001 & $\begin{array}{l}\text { Dietary Fat } \\
\text { Reduction }\end{array}$ & 2639 & .73 & .12 & .20 & .12 & -.06 & -.09 & -.20 & -.26 \\
\hline Ruggiero et al. & 2000 & $\begin{array}{l}\text { Smoking } \\
\text { Cessation }\end{array}$ & 206 & .52 & .08 & n.a. & n.a. & -.02 & -.24 & n.a. & n.a. \\
\hline Ruggiero et al. & 2003 & Glucose Testing & 668 & .29 & .02 & .29 & .28 & .30 & -.04 & -.46 & .01 \\
\hline Sarkin, et al. & 2001 & Exercise & 670 & 1.01 & .54 & -.16 & -.42 & .25 & -.11 & -.46 & -.23 \\
\hline Schmaling & 2000 & $\begin{array}{l}\text { Medication } \\
\text { Management }\end{array}$ & 53 & 2.21 & -.19 & .26 & -.09 & 1.14 & -.73 & -.85 & -.40 \\
\hline Schorling & 1995 & $\begin{array}{l}\text { Smoking } \\
\text { Cessation- }\end{array}$ & 243 & .51 & -.06 & n.a. & n.a. & -.47 & .05 & n.a. & n.a. \\
\hline Schorling & 1995 & $\begin{array}{l}\text { Smoking } \\
\text { Cessation- }\end{array}$ & 292 & .36 & .38 & n.a. & n.a. & -.10 & -.13 & n.a. & n.a. \\
\hline Schulze & 2003 & $\begin{array}{l}\text { Daily Use of } \\
\text { Dental Floss }\end{array}$ & 877 & .76 & .33 & -.24 & .38 & .31 & -.23 & .20 & -.71 \\
\hline $\begin{array}{l}\text { Schumann et } \\
\text { al. }\end{array}$ & & $\begin{array}{l}\text { Smoking } \\
\text { Cessation }\end{array}$ & 453 & -.32 & -.10 & -.75 & -.07 & .66 & .12 & -.85 & .49 \\
\hline Silverman & 1995 & Condom Use & 223 & .20 & -.15 & .42 & -.32 & -.16 & -.01 & -.38 & -.08 \\
\hline Silverman & 1995 & Condom Use & 230 & .19 & .38 & -.02 & .32 & .18 & -.61 & .20 & -.36 \\
\hline Snow et al. & 1992 & $\begin{array}{l}\text { Smoking } \\
\text { Cessation }\end{array}$ & 191 & .47 & .95 & n.a. & n.a. & -.27 & -.41 & n.a. & n.a. \\
\hline Stark et al. & 1998 & $\begin{array}{l}\text { Condom Use - } \\
\text { Other }\end{array}$ & 754 & .78 & .09 & .45 & .04 & n.a. & n.a. & n.a. & n.a. \\
\hline Stark et al. & 1998 & $\begin{array}{l}\text { Condom Use - } \\
\text { Main }\end{array}$ & 822 & .73 & .09 & .22 & -.16 & n.a. & n.a. & n.a. & n.a. \\
\hline Susenbeth & 2000 & Condom Use & 96 & n.a. & n.a. & n.a. & .12 & n.a. & n.a. & n.a. & n.a. \\
\hline Tseng & 2001 & Exercise & 154 & .93 & .85 & .49 & .29 & -.03 & .19 & -.30 & -.46 \\
\hline Tsoh & 1993 & Quitting Drugs & 169 & .66 & -.22 & n.a. & n.a. & .47 & -.66 & n.a. & n.a. \\
\hline $\begin{array}{l}\text { Van Marter et } \\
\text { al. }\end{array}$ & 2003 & Bullying & 473 & .57 & .61 & n.a. & n.a. & .02 & -.10 & n.a. & n.a. \\
\hline Velicer et al. & 1985 & $\begin{array}{l}\text { Smoking } \\
\text { Cessation }\end{array}$ & 930 & .75 & .00 & -.29 & -.40 & .10 & .06 & -.59 & -.38 \\
\hline Wakui et al. & 2002 & Exercise & 450 & .67 & -.19 & -.01 & .09 & -.06 & -.07 & -.27 & .23 \\
\hline
\end{tabular}


Table 9 (continued)

\begin{tabular}{|c|c|c|c|c|c|c|c|c|c|c|c|}
\hline \multirow[b]{3}{*}{ Study/ Author } & \multirow[b]{3}{*}{ - Year } & \multirow[b]{3}{*}{$\begin{array}{c}\text { Target } \\
\text { Behavior }\end{array}$} & \multirow[b]{3}{*}{$\begin{array}{c}\mathrm{N} / \\
\text { data- } \\
\text { set }\end{array}$} & \multicolumn{8}{|c|}{ Effect Sizes for Stage Transitions } \\
\hline & & & & \multicolumn{4}{|c|}{ Pros } & \multicolumn{4}{|c|}{ Cons } \\
\hline & & & & PC-C & C-PR & PRA & A-M & PC-C & C-PF & PR-A & A-M \\
\hline Whyte & 2003 & $\begin{array}{l}\text { Dietary Fat } \\
\text { Reduction }\end{array}$ & 416 & .36 & .26 & -.18 & .93 & .51 & -.45 & .07 & -.57 \\
\hline Wright et al. & 2002 & $\begin{array}{l}\text { Weight Bearing } \\
\text { Exercise }\end{array}$ & 250 & .86 & .18 & .23 & .15 & .27 & -.21 & -.15 & -.56 \\
\hline
\end{tabular}

Outliers. Outliers were examined for each Stage transition of both the Pros and Cons measures. Potential outliers identified by calculating the standard deviation of the mean for each effect size across the four Stage transitions. Effect sizes that were $2 S D$ or $3 S D$ from the mean were carefully examined. Once outliers were corrected for clerical or calculation error, preliminary analyses of the outliers were conducted. Between 4 and 9 effect sizes were identified as 2 or $3 S D$ above the mean for each of the transitions, with a total of 27 for Pros and 21 for Cons. The 20 effect sizes for Pros and 14 for Cons that were greater than $2 S D$ above the mean (but less than $3 S D$ above the mean) were examined by Stage transition. Essentially, all these effect sizes showed clear clusters. For example, 7 effect sizes were identified as $2 S D$ above the mean for Pros transition PC-C; four of these effect sizes (Evers et al., 2001; Schumann et al., 2003; Banikarim et al., 2003; Riley et al., 2003) were clustered between -.3 and -.4 and three of the studies (King et al., 1996; Callaghan et al., 2002; Reed, 1996) were clustered between 1.6 and 1.8. Interestingly, the three studies clustered around 1.6 and 1.8 examined exercise, which is a behavior previously found to have, overall, the largest effect sizes across the Stages (Hall \& Rossi., 2004) and in particular for the PC-C transition (Hall \& Rossi., 2003). 
Therefore, it is clear since essentially all effect sizes clustered together and often did so in theoretically predictable manner, for this study - the standard of $2 S D$ above the mean was considered insufficient for identifying outliers. Further analysis was then conducted.

Effect sizes found to be $3 S D$ or more above the mean were examined for possible removal. In total 14 effect sizes were identified, 7 for Pros and 7 for Cons. These outliers were examined to assess possible relationships with various potential moderator variables. No significant relationships were found between the outliers and each of the moderating variables, including behavior category, cessation versus acquisition behaviors, age, calculation method, and publication status. Significant differences were found for recruitment setting $\left(\chi^{2}=15.002, d f=6, p<.020\right)$. In particular a large portion of the outliers were studies that recruited participants from clinic settings. Analysis revealed that these datasets typically were small in sample size. Although all datasets included in this study had a sample size of 19 or greater, since the total sample size for each study is divided across the Stages of Change in a given dataset, some of these studies were found to have remarkably small sample sizes per Stage.

In the end, the outlier analyses provided the basis for three criteria to be used to determine the removal of the outliers (1) effect sizes must be at least $3 S D$ from the mean for a given Stage; and (2) effect sizes must be based on sample sizes with Stage sample sizes less than 5; and/or (3) effect sizes must be greater than .2 from the next closest effect size cluster. Therefore, if the effect size was $3 S D$ above the mean and met one of the other two criteria the effect size was removed. Of the 14 
outliers identified as $3 S D$ above the mean, six Pros and six Cons were removed and one Pros and one Cons was retained (see Table 10 below).

Table 10

Summary of Outliers $3 \mathrm{SD}$ from the Mean

\begin{tabular}{|c|c|c|c|c|}
\hline \multicolumn{5}{|l|}{ PROS } \\
\hline \multirow[t]{6}{*}{ Removed } & Evers et al., 2001 & Condom Use & $\mathrm{PC}-\mathrm{C}$ & -1.07 \\
\hline & Schmaling et al., 2000 & Medication Management & PC-C & 2.212 \\
\hline & Pallonen et al., 1997 & Smoking Acquisition & C-PR & $-1.05^{*}$ \\
\hline & Geibel, 1999 & Smoking Cessation & C-PR & -.87 \\
\hline & Pallonen et al., 1997 & Smoking Acquisition & PR-A & 2.60 \\
\hline & Boudreaux et al., 1998 & Smoking Cessation & $A-M$ & $-1.64^{*}$ \\
\hline Retained & Fahrenwald et al, 2003 & Exercise & $C-D$ & 1.21 \\
\hline \multicolumn{5}{|l|}{ CONS } \\
\hline \multirow[t]{6}{*}{ Removed } & Banikarim et al., 2003 & STD Screening & PC-C & $-1,42^{*}$ \\
\hline & Morrison-Beedy et al., 2001 & Condom Use & $\mathrm{PC}-\mathrm{C}$ & $1.52^{*}$ \\
\hline & Morrison-Beedy et al., 2001 & Condom Use & C-PR & $-1.72^{*}$ \\
\hline & Geibel, 1999 & Smoking Cessation & PR-A & $-1.63^{*}$ \\
\hline & $\overline{\text { Hulton, } 2001}$ & Sex Decision Making & $\overline{\mathrm{A}-\mathrm{M}}$ & -1.51 \\
\hline & Levesque et al., 1999 & Administrative Change & A-M & .91 \\
\hline Retained & Buckworth et al., 2002 & Exercise & $A-M$ & -1.26 \\
\hline
\end{tabular}

Note. Asterisks indicate effect sizes based on Stage sample sizes less than five.

Magnitudes across all Datasets. Fixed and random effects models were fit to the effect size data for the Pros and Cons for each of the four Stage transitions (PCC, C-PR, PR-A, A-M). The random effects variance component (v) was determined for each Stage transition for each construct. A $Q$-test was then conducted on the random effects variance component in order determine if there is statistical support for the random effects model.

The largest ES average was found for the Pros of Change, specifically in the transition between the Precontemplation and Contemplation Stages. This effect size 
was found to be significantly greater than zero for both the fixed and random effects model, with a mean of $.63(p<.01 ; C I=.61$ to .66$)$ for the fixed effects and mean of $.65(p<.01 ; C I=.60$ to .70$)$ for the random effects model. The random effects variance component and overall test for heterogeneity was found to be significant ( $v$ $=.06 ; Q(d f=135)=504.60, p<.01)$.

A smaller increase was seen in the transition from Contemplation to Preparation for the Pros of Change with significant effects for the fixed effects $(M=$ $.14, p<.01 ; C I=.11$ to .17$)$ and random effects model $(M=.17, p<.01 ; C I=.12$ to .21). The random effects variance component and overall test for heterogeneity for this transition was found to be significant $(v=.03 ; Q(d f=121)=275.01, p<.01)$ Again, significant $(p<.01)$ but smaller increases were seen for the Pros of Change for the transition Preparation to Action for both models. A mean of .12 was found for both models, though with a smaller confidence interval for the fixed effects $(C I=.08$ to .16$)$ than for the random effects $(C I=.06$ to .18$)$ model. The random effects variance component and overall test for heterogeneity was found to be significant $(v=.04 ; Q(d f=99)=206.65, p<.01)$.

Essentially no effect was found from Action to Maintenance. The fixed effects $(M=.02, p=.17 ; C I=-.01$ to .06$)$ and random effects $(M=.02, p=.60 ; C I=$ -.05 to .08 ) means were not found to be significantly different from zero. As with the other transitions, the random effects variance component and overall test for heterogeneity was found to be significant $(v=.06 ; Q(d f=107)=290.01, p<.01)$.

The Cons of Change showed somewhat of an inverse pattern across transitions, though with overall smaller effect sizes. The smallest effect for the Cons 
of Change was found for the transition from Precontemplation to Contemplation.

Although small the fixed effects $(M=-.07, p<.01 ; C I=-.10$ to -.05$)$ and random effects $(M=-.08, p=.01 ; C I=-.13$ to -.02$)$ models revealed effect sizes significantly different from zero. As with the Pros of Change transitions, the random effects variance component and overall test for heterogeneity was found to be significant $(v$ $=.07 ; Q(d f=135)=620.55, p<.01)$.

Small, but relatively larger decreases were found for the Cons from Contemplation to Preparation for the fixed effects $(M=-.14, p<.01 ; C I=-.17$ to $.11)$ and random effects $(M=-.15, p<.01 ; C I=-.19$ to -.11$)$ models. The random effects variance component and overall test for heterogeneity was found to be significant $(v=.01 ; Q(d f=123)=174.44, p<.01)$.

Significant, and even larger, effect sizes were found for Preparation to Action for the fixed effects $(M=-.26, p<.01 ; C I=-.30$ to -.22$)$ and random effects $(M=$ $.24, p<.01 ; C I=-.30$ to -.18$)$. The random effects variance component and overall test for heterogeneity was found to be significant $(v=.04 ; Q(d f=98)=212.57, p<$ $.01)$.

Lastly, the Action to Maintenance transition showed significant effects for fixed $(M=-.22, p<.01 ; C I=-.26$ to -.19$)$ and random $(M=-.23, p<.01 ; C I=-.28$ to -.18) effects models. And as with all other transitions, the random effects variance component and overall test for heterogeneity was found to be significant $(v=.02 ; Q$ $(d f=104)=167.87, p<.01)$. A summary of all transitions for Pros and Cons of Change for both fixed and random effects models is found in Table 11. 
Comparison of Fixed and Random Effect Models for Effect Sizes of Pros and Cons across Stage Transitions

\begin{tabular}{|c|c|c|c|c|c|c|c|c|c|c|c|}
\hline \multirow{4}{*}{ PROS } & \multirow[b]{3}{*}{$N$} & \multicolumn{5}{|c|}{ FIXED EFFECTS } & \multicolumn{5}{|c|}{ RANDOM EFFECTS } \\
\hline & & \multirow[b]{2}{*}{$E S$} & \multirow[b]{2}{*}{$S E$} & \multicolumn{2}{|c|}{$95 \% \mathrm{CI}$} & \multirow[b]{2}{*}{$p$} & \multirow[b]{2}{*}{$E S$} & \multirow[b]{2}{*}{$S E$} & \multicolumn{2}{|c|}{$95 \% C I$} & \multirow[b]{2}{*}{$p$} \\
\hline & & & & $\mathrm{L}$ & $\mathrm{U}$ & & & & $\mathrm{L}$ & $\mathrm{U}$ & \\
\hline & & & & & & & & & & & \\
\hline $\mathrm{PC}-\mathrm{C}$ & 136 & .63 & .01 & .61 & .66 & $<.01$ & .65 & .02 & .60 & .70 & $<.01$ \\
\hline C-PR & 122 & .14 & .01 & .11 & .17 & $<.01$ & .17 & .02 & .12 & .22 & $<.01$ \\
\hline PR-A & 100 & .12 & .02 & .08 & .16 & $<.01$ & .12 & .03 & .06 & .18 & $<.01$ \\
\hline A-M & 108 & .02 & .02 & -.01 & .06 & .17 & .02 & .03 & -.05 & .08 & .60 \\
\hline CONS & & & & & & & & & & & \\
\hline PC-C & 136 & -.07 & .01 & -.10 & -.05 & $<.01$ & -.08 & .02 & -.13 & -.04 & .01 \\
\hline C-PR & 124 & -.14 & .01 & -.17 & -.11 & $<.01$ & -.15 & .02 & -.19 & -.11 & $<.01$ \\
\hline PR-A & 100 & -.26 & .02 & -.30 & -.22 & $<.01$ & -.24 & .03 & -.30 & -.18 & $<.01$ \\
\hline A-M & 105 & -.22 & .02 & -.26 & -.19 & $<.01$ & -.23 & .02 & -.28 & -.18 & $<.01$ \\
\hline
\end{tabular}

Note. $N=$ sample size; $E S=$ mean effect size; $\mathrm{L}=$ lower bound; $\mathrm{U}=$ upper bound; $p=$ significance of effect as compared to zero; ${ }^{*}$ indicates overall significant differences between groups at $p=<.05$.

Due to the significant random effects variance component and overall test for heterogeneity for all transitions for both the Pros and Cons of Change, the random effects model was used for the remainder of effect size analyses. The graphical representation of the mean scores for all 146 datasets for Pros and Cons based on the random effects results is illustrated below (Figure 5). 


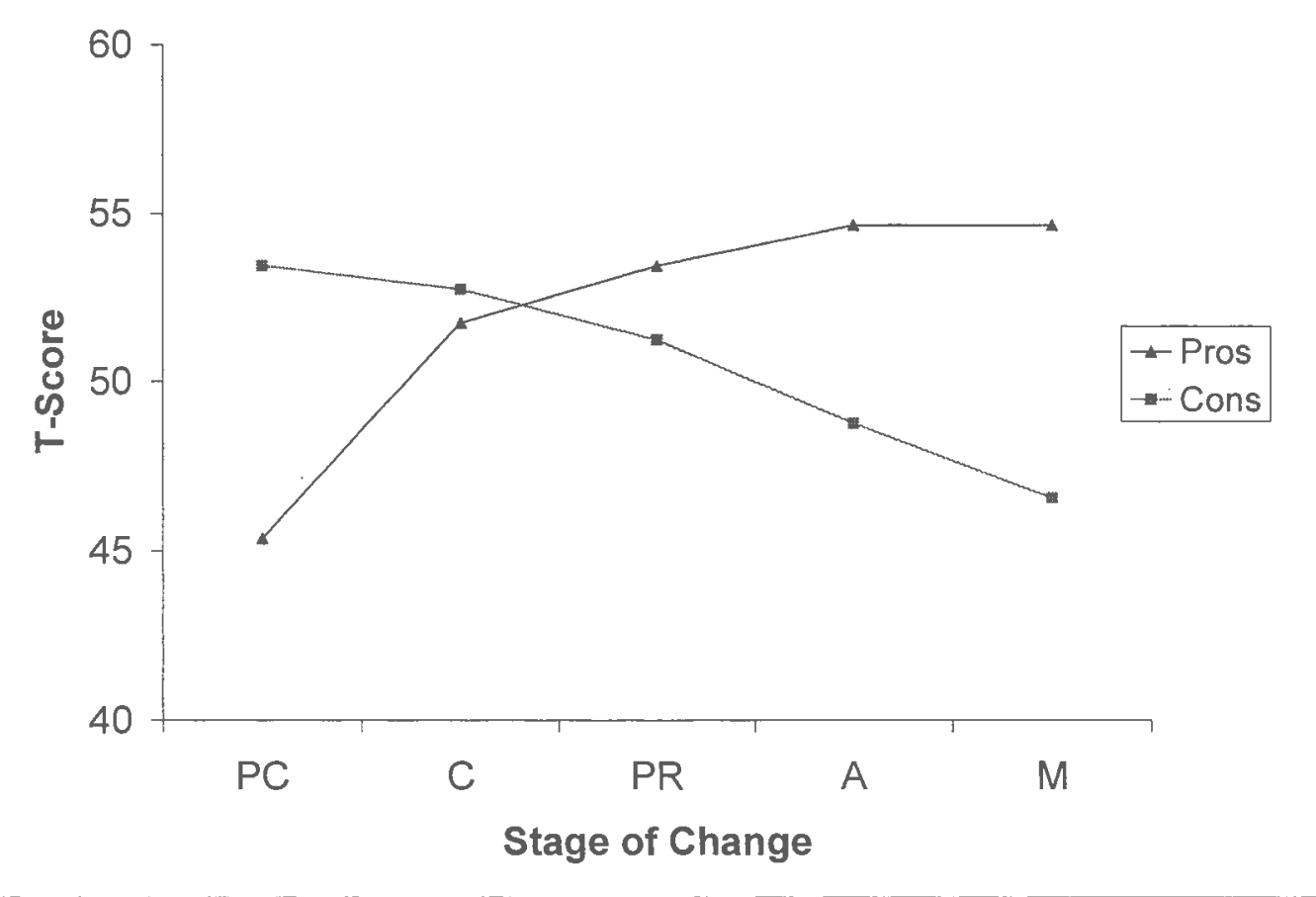

Figure 5. Composite Decisional Balance Graphic Using Random Effects Model.

Significant homogeneity $Q$-tests were found for all Stage transitions for both the Pros and Cons. This indicates that the effect size distributions are heterogeneous for both Decisional Balance measures; therefore, analyses to assess for possible moderators of the effect size distribution are warranted. A summary of the $Q$-tests can be found in Table 12 . 
Table 12

Overall Test of Homogeneity and Random Effects Variance for Mean Effect Sizes of the Pros and Cons across Stage Transitions

\begin{tabular}{|l|cccc|cccc|}
\hline & \multicolumn{4}{|c|}{ PROS } & \multicolumn{4}{c|}{ CONS } \\
\cline { 2 - 10 } & PC-C & C-PR & PR-A & A-M & PC-C & C-PR & PR-A & A-M \\
\cline { 2 - 9 } \\
$\boldsymbol{N}$ & .06 & .03 & .04 & .06 & .07 & .01 & .04 & .02 \\
$d \boldsymbol{f}$ & 504.60 & 275.01 & 206.65 & 290.01 & 620.55 & 174.44 & 203.13 & 167.87 \\
$\boldsymbol{p}$ & 135 & 121 & 99 & 107 & 135 & 123 & 98 & 104 \\
& $<.01$ & $<.01$ & $<.01$ & $<.01$ & $<.01$ & $<.01$ & $<.01$ & $<.01$ \\
\hline
\end{tabular}

Note. $v=$ random effects variance component; $Q=$ overall test of homogeneity; $d f=$ degrees of freedom; $p=p$-value; $Q / d f=$ normalized chi-squared index

Moderators of Effect Size Distribution.

Potential categorical moderators of effect sizes were explored by examining subgroups. Based on the findings above, random effects modeling was employed in each of the ANOVA analogs. Calculation Method was explored first to ensure bias was not introduced by the differing methods of effect size calculation and to assess the effectiveness of the regression correction developed in Chapter 5. Following the comparison of calculation methods, behavior categories were explored. A priori behavioral subgroup analyses were conducted. Additional potential moderators were then examined.

Calculation Method. In order to ensure the regression correction developed in Chapter 5 for calculation method was effective, the first potential moderator assessed was calculation method. As described previously, effect sizes were calculated by two primary methods: Hedges' $g$ and Standard Score. Seventy percent of the datasets 
were calculated using Hedges' $g$ and $30 \%$ were based on the Standard Score method, which was adjusted using the regression correction. No significant differences were found between the two methods using random effects modeling. The between-group tests of homogeneity for calculation methods for the Pros and Cons of Change across Stage transitions are reported in Table 13.

Table 13

Between Groups Test of Homogeneity of Calculation Method for the Pros and Cons across Stage Transitions

\begin{tabular}{|c|cccc|cccc|}
\hline \multirow{4}{*}{$Q$} & \multicolumn{4}{|c|}{ PROS } & \multicolumn{4}{c|}{ CONS } \\
\cline { 2 - 10 } & PC-C & C-PR & PR-A & A-M & PC-C & C-PR & PR-A & A-M \\
\cline { 2 - 9 } & .63 & .00 & .41 & .47 & .37 & .28 & .16 & 2.61 \\
$p$ & 1 & 1 & 1 & 1 & 1 & 1 & 1 & 1 \\
$p$ & .43 & $\mathbf{1 . 0 0}$ & .52 & .49 & .54 & .60 & .69 & .11 \\
\hline
\end{tabular}

Additionally, using random effects modeling, no within group tests of heterogeneity were found to be significant. The sample sizes, mean effect sizes and $95 \%$ confidence intervals are presented in Table 14 below. The $p$-values displayed in this table indicate whether or not the given effect sizes are significantly greater than zero. 


\begin{tabular}{|c|c|c|c|c|c|c|c|c|c|c|c|c|}
\hline \multirow[b]{5}{*}{ PROS } & \multicolumn{12}{|c|}{ CALCULATION METHOD } \\
\hline & \multicolumn{6}{|c|}{ Hedges'g } & \multicolumn{6}{|c|}{ Standard Score } \\
\hline & \multirow[b]{2}{*}{$N$} & \multirow[b]{2}{*}{$E S$} & \multirow[b]{2}{*}{$S E$} & \multicolumn{2}{|c|}{$95 \% C I$} & \multirow[b]{2}{*}{$p$} & \multirow[b]{2}{*}{$N$} & \multirow[b]{2}{*}{$E S$} & \multirow[b]{2}{*}{$S E$} & \multicolumn{2}{|c|}{$95 \% C I$} & \multirow[b]{2}{*}{$p$} \\
\hline & & & & $\mathrm{L}$ & U & & & & & $\mathrm{L}$ & $\mathrm{U}$ & \\
\hline & & & & & & & & & & & & \\
\hline PC-C & 97 & .67 & .04 & .60 & .74 & $<.01$ & 39 & .62 & .05 & .51 & .72 & $<.01$ \\
\hline C-PR & 87 & .17 & .03 & .11 & .23 & $<.01$ & 35 & .17 & .05 & .07 & .27 & $<.01$ \\
\hline PR-A & 72 & .13 & .04 & .06 & .21 & $<.01$ & 28 & .09 & .05 & -.01 & .20 & .09 \\
\hline A-M & 78 & .00 & .04 & -.07 & .08 & .96 & 30 & .05 & .06 & -.07 & .16 & .39 \\
\hline CONS & & & & & & & & & & & & \\
\hline $\mathrm{PC}-\mathrm{C}$ & 96 & -.06 & .04 & -.13 & .01 & .08 & 40 & -.10 & .06 & -.21 & .00 & .06 \\
\hline C-PR & 89 & -.16 & .02 & -.20 & -.12 & $<.01$ & 35 & -.13 & .04 & -.20 & -.06 & $<.01$ \\
\hline PR-A & 71 & -.25 & .04 & -.33 & -.18 & $<.01$ & 28 & -.23 & .06 & -.33 & -.12 & $<.01$ \\
\hline A-M & 76 & -.26 & .03 & -.31 & -.20 & $<.01$ & 29 & -.17 & .04 & -.26 & -.09 & $<.01$ \\
\hline
\end{tabular}

Note. $N=$ sample size; $E S=$ mean effect size; $\mathrm{L}=$ lower bound; $\mathrm{U}=$ upper bound; $p=$ significance of effect as compared to zero.

Magnitudes within Behaviors.

Since heterogeneity was found overall and no significant differences were found for calculation method, behavior categories were explored as possible moderator variables.

Many of the 55 target behaviors included in this study are represented by only one dataset, thereby precluding meaningful subgroup analyses. Thirty-three of the target behaviors had data for each of the transitions for both Pros and Cons. For descriptive purposes, the magnitudes of effects for each of the transitions for these target behaviors are depicted in composite graphics and displayed in Appendix C. Primary behavior categories were created (see Appendix D) in order to group together studies that examined similar behaviors. In total, 10 behavior categories included 3 or more datasets, including: contraception, condom use, diet, drugs, exercise, medical screening, organ donation, smoking, sex decisions, and stress. 
Mean effect sizes, standard errors, $95 \%$ confidence intervals, and $p$-values for

behavior categories with 3 or more datasets can be found in Table 15 and Table 16.

Table 15

Comparison of Behavior Categories for Pros of Change

\begin{tabular}{|c|c|c|c|c|c|c|c|c|c|c|}
\hline PROS & 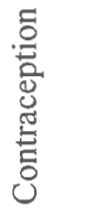 & 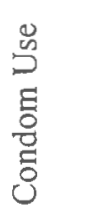 & 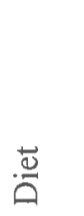 & 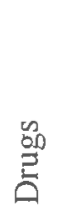 & 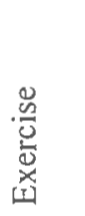 & 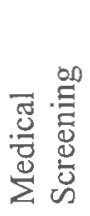 & 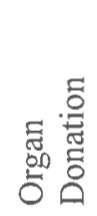 & 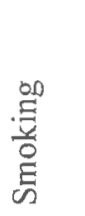 & 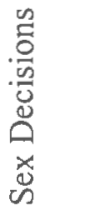 & 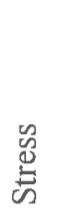 \\
\hline \multicolumn{11}{|l|}{ PC-C } \\
\hline$N$ & 3 & 17 & 15 & 4 & 20 & 5 & 5 & 33 & 5 & 7 \\
\hline$E S$ & .45 & .61 & .59 & .51 & .98 & .59 & .56 & .63 & .72 & .65 \\
\hline$S E$ & .18 & .08 & .08 & .21 & .07 & .15 & .13 & .05 & .14 & .15 \\
\hline $\mathrm{L}$ & .10 & .46 & .44 & .10 & .83 & .29 & .30 & .53 & .44 & .35 \\
\hline $\mathrm{U}$ & .80 & .77 & .74 & .91 & 1.12 & .89 & .82 & .73 & 1.0 & .95 \\
\hline$p$ & .01 & $<.01$ & $<.01$ & .01 & $<.01$ & $<.01$ & $<.01$ & $<.01$ & $<.01$ & $<.01$ \\
\hline \multicolumn{11}{|l|}{ C-PR } \\
\hline$N$ & 3 & 16 & 13 & 3 & 20 & 3 & 5 & 31 & 3 & 7 \\
\hline$E S$ & .40 & .13 & .18 & -.25 & .20 & .32 & -.00 & .17 & -.06 & .10 \\
\hline$S E$ & .14 & .07 & .07 & .22 & .06 & .18 & .12 & .05 & .15 & .16 \\
\hline $\mathrm{L}$ & .13 & -.01 & .05 & -.68 & .08 & -.04 & -.23 & .08 & -.35 & -.21 \\
\hline $\mathrm{U}$ & .68 & .27 & .12 & .17 & .30 & .68 & .22 & .26 & .24 & .40 \\
\hline$p$ & $<.01$ & .07 & .01 & .24 & $<.01$ & .08 & .97 & $<.01$ & .71 & .54 \\
\hline \multicolumn{11}{|l|}{ PR-A } \\
\hline$N$ & 3 & 14 & 11 & 2 & 20 & 3 & 3 & 18 & 2 & 7 \\
\hline$E S$ & .07 & .23 & .01 & .28 & .27 & -.01 & .21 & -.06 & -.04 & -.09 \\
\hline$S E$ & .13 & .07 & .07 & .28 & .06 & .18 & .15 & .07 & .17 & .14 \\
\hline $\mathrm{L}$ & -.19 & .10 & -.13 & -.27 & .15 & -.36 & -.08 & -.20 & -.38 & -.37 \\
\hline $\mathrm{U}$ & .33 & .37 & .16 & .82 & .38 & .34 & .50 & .08 & .29 & .18 \\
\hline$p$ & .58 & $<.01$ & .87 & .32 & $<.01$ & .94 & .15 & .41 & .80 & .50 \\
\hline \multicolumn{11}{|l|}{$\mathbf{A}-\mathbf{M}$} \\
\hline $\begin{array}{r}N \\
F \mathrm{~S}\end{array}$ & 3 & 16 & 13 & 3 & 21 & 5 & 2 & 13 & 2 & 7 \\
\hline $\begin{array}{l}E S \\
S E\end{array}$ & .08 & .02 & -.07 & -.06 & .03 & .30 & -.06 & -.08 & .29 & -.19 \\
\hline$S E$ & .17 & .08 & .09 & .18 & .07 & .15 & .25 & .10 & .24 & .13 \\
\hline $\mathrm{L}$ & -.26 & -.14 & -.24 & -.40 & -.11 & .01 & -.55 & -.28 & -.18 & -.45 \\
\hline $\mathrm{U}$ & .42 & .18 & .10 & .29 & .17 & .59 & .44 & .13 & .76 & .06 \\
\hline$p$ & .64 & .81 & .43 & .75 & .67 & .04 & .83 & .45 & .22 & .14 \\
\hline
\end{tabular}

Note. $N=$ sample size; $E S=$ mean effect size; $\mathrm{L}=$ Lower bound; $\mathrm{U}=$ Upper bound; $p=$ significance level of effect size as compared to zero. 
Table 16

Comparison of Behavior Categories for Cons of Change

\begin{tabular}{|c|c|c|c|c|c|c|c|c|c|c|}
\hline CONS & 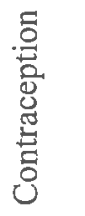 & 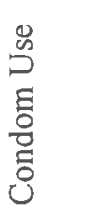 & 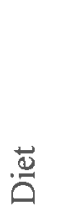 & $\begin{array}{l}\infty \\
\stackrel{\infty}{3} \\
\stackrel{2}{2}\end{array}$ & 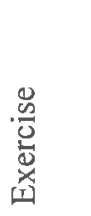 & 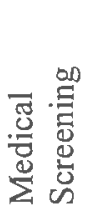 & 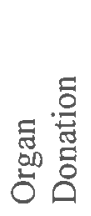 & $\frac{a 0}{\stackrel{\Xi}{\Xi}}$ & 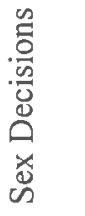 & 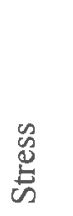 \\
\hline \multicolumn{11}{|l|}{ PC-C } \\
\hline$N$ & 3 & 15 & 15 & 4 & 20 & 5 & 5 & 34 & 5 & 7 \\
\hline$E S$ & -.14 & -.01 & .16 & .04 & .01 & -.34 & -.28 & -.17 & -.49 & .13 \\
\hline$S E$ & .17 & .08 & .07 & .20 & .07 & .14 & .13 & .05 & .14 & .14 \\
\hline $\mathrm{L}$ & -.47 & -.17 & .02 & -.35 & -.13 & -.61 & -.53 & -.26 & -.75 & -.15 \\
\hline $\mathrm{U}$ & .20 & .15 & .30 & .42 & .15 & -.08 & -.03 & -.07 & -.22 & .41 \\
\hline$p$ & .43 & .91 & .03 & .84 & .88 & .01 & .03 & $<.01$ & $<.01$ & .37 \\
\hline \multicolumn{11}{|l|}{ C-PR } \\
\hline$N$ & 3 & 13 & 13 & 3 & 21 & 4 & 5 & 34 & 3 & 7 \\
\hline$E S$ & -.23 & -.17 & -.14 & -.28 & -.21 & .11 & -.03 & -.15 & -.12 & -.01 \\
\hline$S E$ & .01 & .06 & .05 & .19 & .04 & .13 & .09 & .03 & .11 & .14 \\
\hline $\mathrm{L}$ & -.43 & -.30 & -.24 & -.65 & -.30 & -.15 & -.20 & -.21 & -.35 & -.28 \\
\hline $\mathrm{U}$ & -.03 & -.05 & -.05 & .09 & -.12 & .36 & .14 & -.08 & .10 & .26 \\
\hline$p$ & .03 & $<.01$ & $<.01$ & .14 & $<.01$ & .42 & .70 & $<.01$ & .28 & .94 \\
\hline \multicolumn{11}{|l|}{ PR-A } \\
\hline$N$ & 3 & 12 & 11 & 2 & 20 & 4 & 3 & 18 & 2 & 7 \\
\hline$E S$ & -.36 & -.15 & -.17 & -.11 & -.22 & -.42 & -.33 & -.38 & .01 & -.14 \\
\hline$S E$ & .14 & .08 & .08 & .29 & .06 & .14 & .16 & .07 & .19 & .15 \\
\hline $\mathrm{L}$ & -.64 & -.31 & -.33 & -.68 & -.35 & -.69 & -.64 & -.53 & -.36 & -.43 \\
\hline $\mathrm{U}$ & -.08 & .01 & -.01 & .46 & -.10 & -.14 & -.02 & -.24 & .37 & .15 \\
\hline$p$ & .01 & .06 & .04 & .71 & $<.01$ & $<.01$ & .04 & $<.01$ & .97 & .34 \\
\hline \multicolumn{11}{|l|}{ A-M } \\
\hline$N$ & 3 & 13 & 13 & 3 & 21 & 6 & 2 & 14 & 1 & 7 \\
\hline$E S$ & -.04 & -.12 & -.19 & -.30 & -.27 & -.24 & -.13 & -.37 & -.28 & -.18 \\
\hline$S E$ & .10 & .06 & .05 & .12 & .05 & .09 & .18 & .07 & .19 & .10 \\
\hline $\mathrm{L}$ & -.24 & -.23 & -.30 & -.54 & -.36 & -.42 & -.42 & -.51 & -.66 & -.36 \\
\hline $\mathrm{U}$ & .17 & -.00 & -.08 & -.06 & -.18 & -.06 & .23 & -.23 & .09 & .01 \\
\hline$p$ & .73 & .04 & $<.01$ & .02 & $<.01$ & .01 & .48 & $<.01$ & .14 & .06 \\
\hline
\end{tabular}

Note. $N=$ sample size; $E S=$ mean effect size; $\mathrm{L}=$ Lower bound; $\mathrm{U}=$ Upper bound; $p=$ significance level of effect size as compared to zero.

These same data are presented in forest plots in Figure 6 and Figure 7 below.

The reference line in each of the forest plots represents the overall random effects mean for all the behaviors combined (see Table 11). The mean and confidence intervals for each of the behaviors is then plotted in comparison to the overall mean. The forest plots show that for Pros for exercise are significantly greater than the 
overall mean in the PC-C and PR-A transitions. The Pros for smoking were found to be significantly less than the overall mean for PR-A and Pros of Medical Screening were found to be significantly less than the mean for the A-M transition. The Cons for diet were found to be significantly greater than the overall mean for the PC-C transition and the Cons for exercise were found to be significantly less than the C-PR transition. 


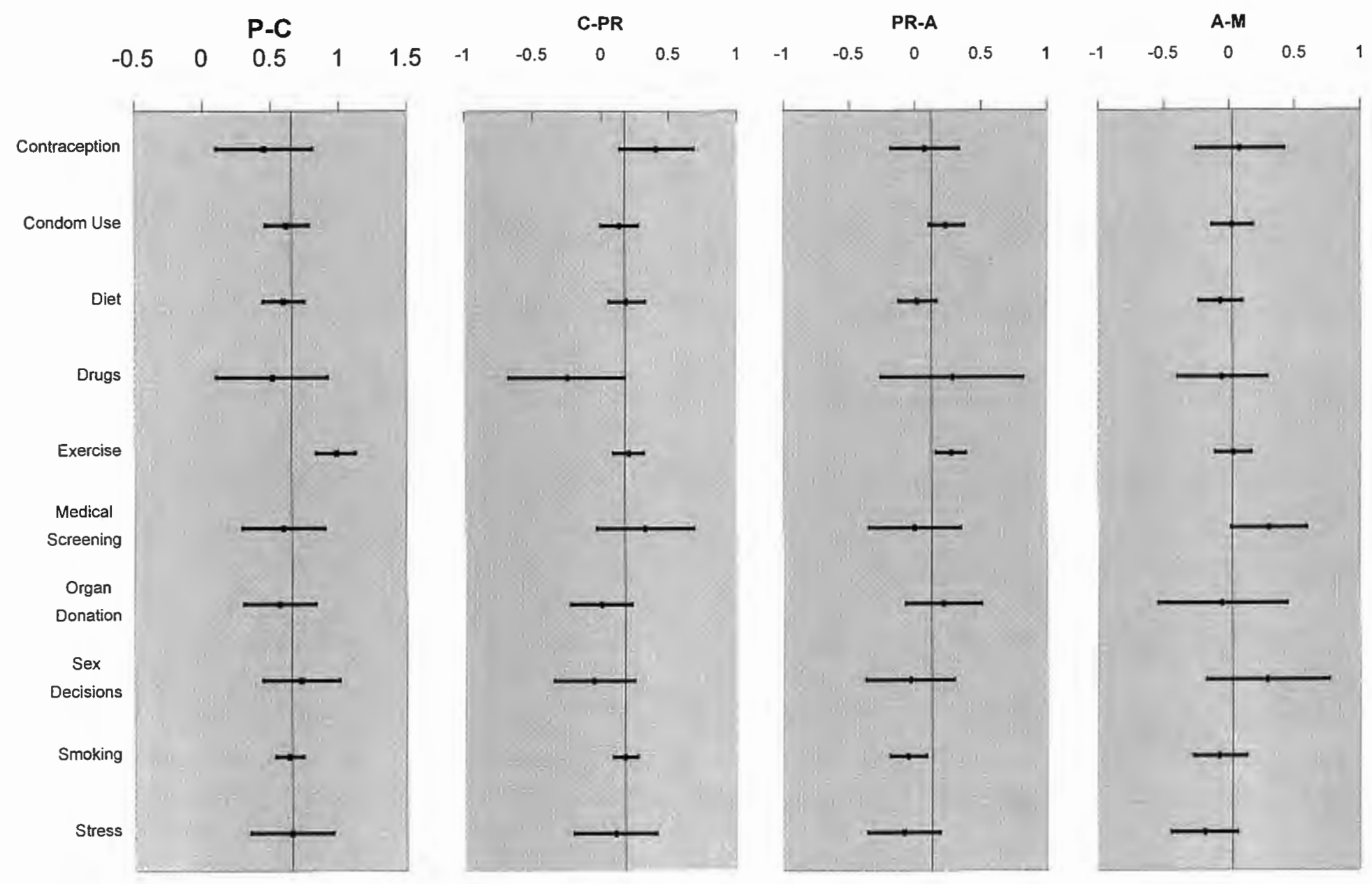

Figure 6. Forest Plots of Behavior Categories for Pros Stage Transitions (Means and Confidence Intervals) 


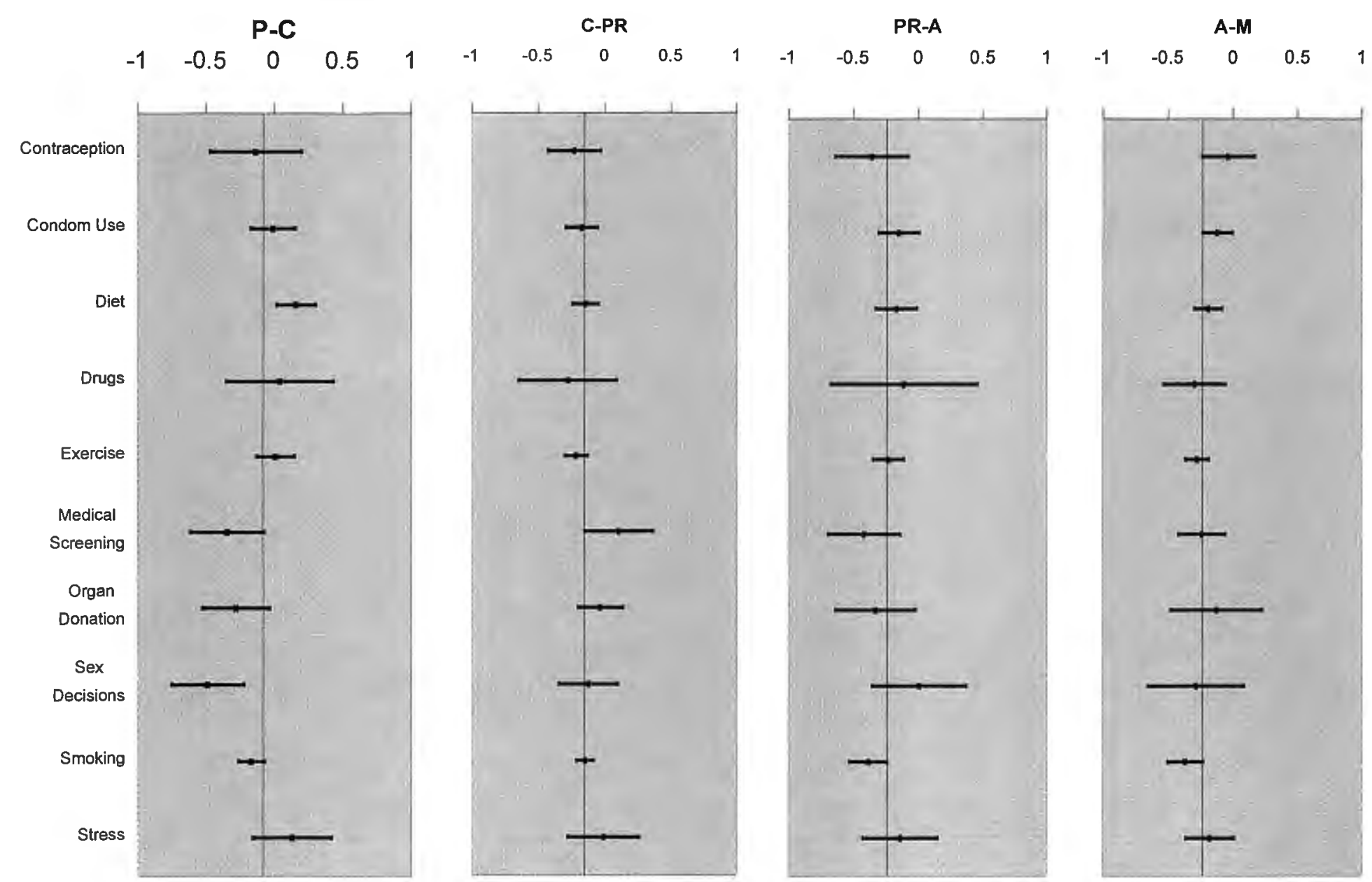

Figure 7. Forest Plots of Behavior Categories for Cons Stage Transitions (Means and Confidence Intervals) 
For further exploration between and within behavior categories, only

behavior categories with a sample size greater than five for all transitions were selected. These five behavior categories included - condom use, diet, exercise, smoking, and stress. ANOVA analogs were performed to identify possible effect size differences among the behavior categories for Pros and Cons of Change. Consistent with the overall results, significant differences were found between the five behaviors on Pros of Change for the PC-C transition $(Q(d f=4)=17.80, p=<.01)$ and for the PR-A transition $(Q(d f=4)=19.23, p=<.01)$. No significant differences were found between the groups for any of the Cons of Change transitions.

A follow-up comparison of effect sizes for the transition from PC-C showed the magnitude of Pros for exercise to be significantly greater than the Pros for smoking $(Q=6.48, p=.01)$, stress $(Q=5.36, p=.02)$, condom use $(Q=10.14, p<$ $.01)$, and $\operatorname{diet}(Q=10.13, p<.01)$. A follow-up comparison of effect sizes for the transition from PR-A showed the magnitude of Pros for condom use to be significantly greater than the Pros for smoking $(Q=6.48, p=.01)$, stress $(Q=5.36$, $p=.02)$, and the Pros of exercise to be significantly greater than smoking $(Q=6.48$, $p=.01)$, and $\operatorname{diet}(Q=5.36, p=.02)$.

Within group tests of homogeneity for each of the five major behavior categories were calculated (Table 17.). Using a random effect model, the effect sizes distributions for the majority of the behavior categories were found to be homogeneous for each of the Stage transition for Pros and Cons. Heterogeneity was only found for the Pros of Change for exercise in the transition from C-PR $(Q(d f=$ 
19) $=40.31, p<.01)$ and for Pros of Change for smoking in the transition from PR-A

$(Q(d f=15)=35.06, p<.01)$.

Table 17

Within Group Homogeneity Tests of the Pros and Cons across Stage Transitions for Behavior Categories

\begin{tabular}{|c|c|c|c|c|c|c|c|c|c|}
\hline \multirow{3}{*}{ Overall - Within } & & \multicolumn{4}{|c|}{ PROS } & \multicolumn{4}{|c|}{ CONS } \\
\hline & & PC-C & C-PR & PR-A & A-M & PC-C & C-PR & PR-A & A-M \\
\hline & 0 & 85.69 & 78.39 & 62.57 & 60.52 & 75.46 & 73.40 & 55.83 & 67.03 \\
\hline & $d t$ & 81 & 77 & 60 & 59 & 80 & 78 & 58 & 57 \\
\hline \multirow{4}{*}{ Condom Use } & $p$ & .34 & .43 & .39 & .42 & .62 & .56 & .56 & .17 \\
\hline & o & 19.52 & 6.88 & 5.91 & 6.95 & 3.72 & 13.89 & 10.54 & 9.1 \\
\hline & $d t$ & 16 & 15 & 13 & 15 & 14 & 12 & 11 & $\begin{array}{l}7.10 \\
12\end{array}$ \\
\hline & $p$ & .24 & .96 & .95 & .96 & 1.00 & .31 & .48 & .69 \\
\hline \multirow{3}{*}{ Diet } & $Q$ & 5.27 & 4.13 & 2.70 & 14.83 & 12.00 & 3.44 & 1.61 & 13.23 \\
\hline & $d t$ & 10 & 9 & 7 & 8 & 10 & 9 & 7 & 8 \\
\hline & $p$ & .87 & .90 & .91 & .06 & .28 & .94 & .98 & .10 \\
\hline \multirow{3}{*}{ Exercise } & 0 & 23.29 & $40.13^{*}$ & 18.03 & 23.82 & 21.72 & 15.58 & 24.77 & 23.04 \\
\hline & $d f$ & 19 & 19 & 19 & 20 & 19 & 20 & 19 & 20 \\
\hline & $p$ & .22 & .00 & .52 & .25 & .30 & .74 & .17 & .29 \\
\hline \multirow{3}{*}{ Stress } & 0 & 2.15 & 1.47 & .87 & 4.64 & 1.23 & 5.24 & 5.73 & 4.43 \\
\hline & $d f$ & 6 & 6 & 6 & 6 & 6 & 6 & 6 & 6 \\
\hline & $p$ & .91 & .96 & .99 & .59 & .98 & .51 & .45 & .62 \\
\hline \multirow{3}{*}{ Smoking } & $Q$ & 35.45 & 25.59 & $35.06^{*}$ & 10.27 & 36.77 & 35.25 & 13.17 & 17.22 \\
\hline & $d f$ & 30 & 28 & 15 & 10 & 31 & 31 & 15 & 11 \\
\hline & $p$ & .23 & .60 & .00 & .42 & .22 & .27 & .59 & .10 \\
\hline
\end{tabular}

A Priori Behavior Sub-Group Comparisons. Although the majority of the effect size distributions for the behavior categories across the Stage transitions for the Pros and Cons were not found to be heterogeneous, a priori sub-group comparisons were established for theoretical purposes. Therefore variety of subgroups comparisons were made, including behavioral comparisons (e.g., smoking 
cessation versus smoking acquisition) and descriptive comparisons (e.g., healthy versus unhealthy behaviors).

\section{Smoking Cessation versus Smoking Acquisition}

The behavior category of smoking was made up primarily of smoking cessation and smoking acquisition. Effect size means, sample sizes, standard error, $95 \%$ confidence intervals, and $p$-values for smoking cessation and acquisition were computed (Table 18) and composite graphics were created (Figure 8 and Figure 9). In order to determine potential differences between these behaviors, an ANOVA analog using random effects modeling was performed on the two groups. No significant differences were found for the Pros of Change. Significant differences were found between smoking cessation and smoking acquisition for Cons of Change in the transition from PC-C $(Q(d f=1)=8.50, p<.01)$ and in the transition from PR$\mathrm{A}(Q(d f=1)=7.18, p<.01)$. Due to lack of data for the A-M transition for smoking acquisition differences between smoking cessation and smoking acquisition could not be computed. 
Comparison of Smoking Acquisition and Smoking Cessation

\begin{tabular}{|c|c|c|c|c|c|c|c|c|c|c|c|c|}
\hline \multirow[b]{5}{*}{ PROS } & \multicolumn{12}{|c|}{ SMOKING } \\
\hline & \multicolumn{6}{|c|}{ Smoking Acquistion } & \multicolumn{6}{|c|}{ Smoking Cessation } \\
\hline & \multirow[b]{2}{*}{$N$} & \multirow[b]{2}{*}{$E S$} & \multirow[b]{2}{*}{$S E$} & \multicolumn{2}{|c|}{$95 \%$ CI } & \multirow[b]{2}{*}{$p$} & \multirow[b]{2}{*}{$N$} & \multirow[b]{2}{*}{$E S$} & \multirow[b]{2}{*}{$S E$} & \multicolumn{2}{|c|}{$95 \% C I$} & \multirow[b]{2}{*}{$p$} \\
\hline & & & & $\mathrm{L}$ & $\mathrm{U}$ & & & & & $\mathrm{L}$ & $\mathrm{U}$ & \\
\hline & & & & & & & & & & & & \\
\hline PC-C & 5 & .65 & .13 & .39 & .91 & $<.01$ & 25 & .64 & .06 & .53 & .76 & $<.01$ \\
\hline C-PR & 5 & .19 & .12 & -.04 & .42 & .11 & 25 & .15 & .05 & .04 & .25 & $<.01$ \\
\hline PR-A & 3 & .16 & .27 & -.36 & .69 & .54 & 14 & -.08 & .12 & -.30 & .15 & .52 \\
\hline A-M & 0 & - & - & - & - & - & 12 & -.18 & .06 & -.21 & -.04 & .19 \\
\hline \multicolumn{13}{|l|}{ CONS } \\
\hline PC-C* & 5 & -.48 & .12 & -.71 & -.25 & $<.01$ & 26 & -.11 & .05 & -.21 & -.01 & .03 \\
\hline C-PR & 5 & -.18 & .10 & -.37 & .01 & .06 & 26 & -.14 & .04 & -.22 & -.06 & $<.01$ \\
\hline PR-A* & 3 & -.75 & .14 & -1.03 & -.47 & $<.01$ & 14 & -.32 & .07 & -.46 & -.18 & .01 \\
\hline A-M & 0 & - & - & - & - & - & 12 & -.38 & .06 & -.50 & -.26 & $<.01$ \\
\hline
\end{tabular}

Note. $N=$ sample size; $E S=$ mean effect size; $\mathrm{L}=$ lower bound; $\mathrm{U}=$ upper bound; $p=$ significance of effect as compared to zero; * indicates overall significant differences between groups at $p=<.05$. 


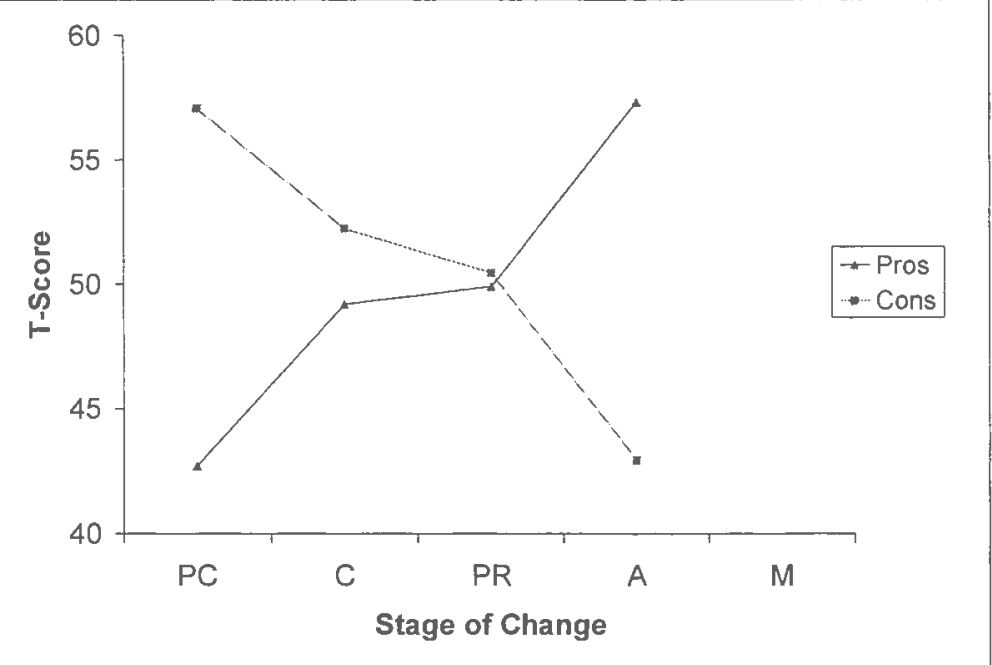

Figure 8. Composite Graphic for Smoking Acquisition.

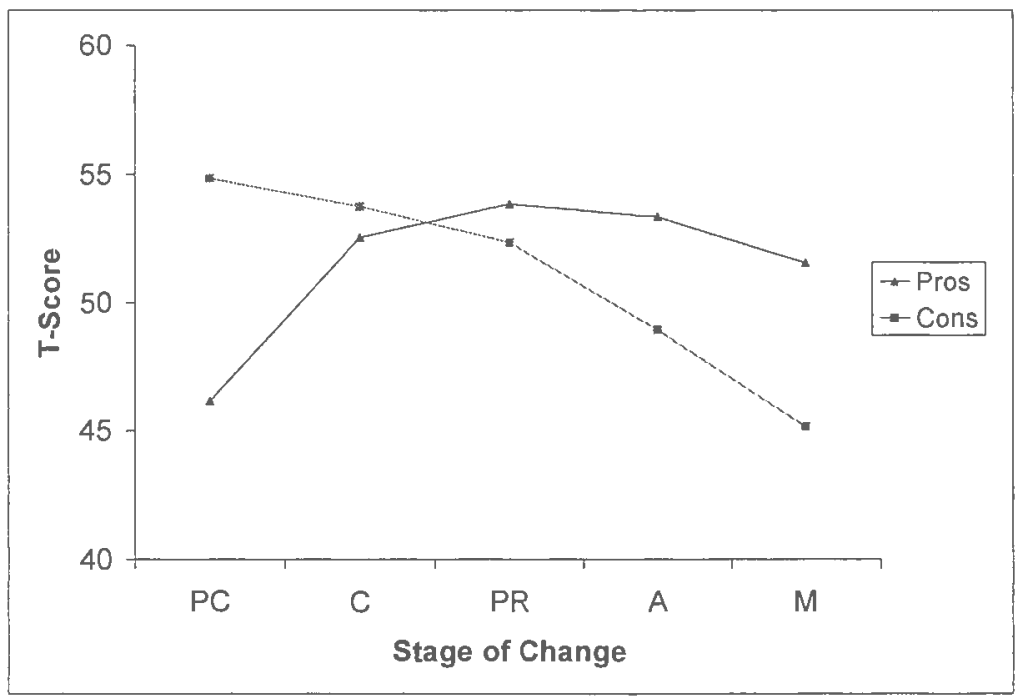

Figure 9. Composite Graphic for Smoking Cessation.

Smoking Cessation - Pros and Cons of Smoking versus Quitting 
The behavior category of smoking cessation was made up of studies that examined either the Pros and Cons of Quitting Smoking or the Pros and Cons of Smoking. Effect size means, sample sizes, standard error, 95\% confidence intervals, and $p$-values for Pros and Cons of Quitting Smoking and Pros and Cons of Smoking were computed (Table 19) and composite graphics were created (Figure 10 and Figure 11). In order to determine potential differences between these behaviors, an ANOVA analog using random effects modeling was performed on the two groups. No significant differences were found for the Pros of Change. Significant differences were found between the magnitudes of effect for the Cons of Change in the transition from $\mathrm{PC}-\mathrm{C}(Q=4.85, p<.028)$ and in the transition from A-M $(Q=7.32, p<.007)$.

\section{Table 19}

Comparison of Pros and Cons of Quitting Versus Smoking for Smoking Cessation

\section{Studies}

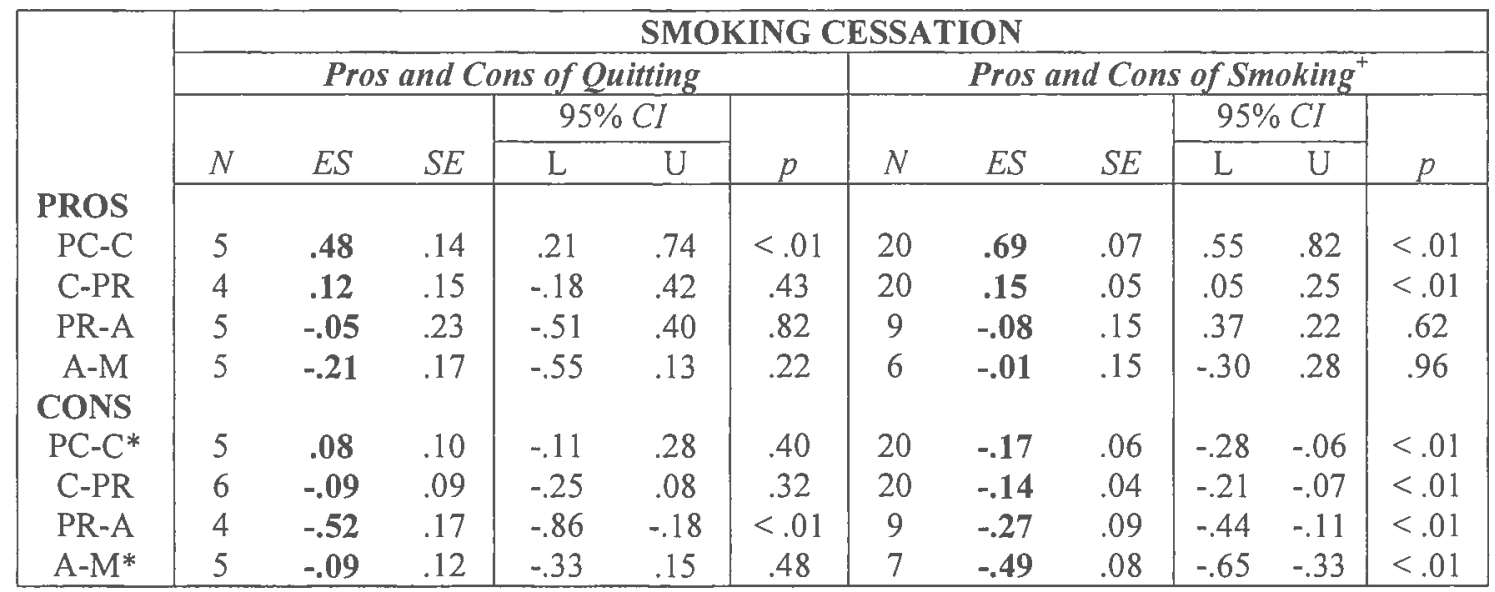

Note + indicates that the Pros and Cons of Smoking are reversed. $N=$ sample size; $E S=$ mean effect size; $\mathrm{L}=$ lower bound; $\mathrm{U}=$ upper bound; $p=$ significance of effect as compared to zero; * indicates overall significant differences between groups at $p=<.05$. 


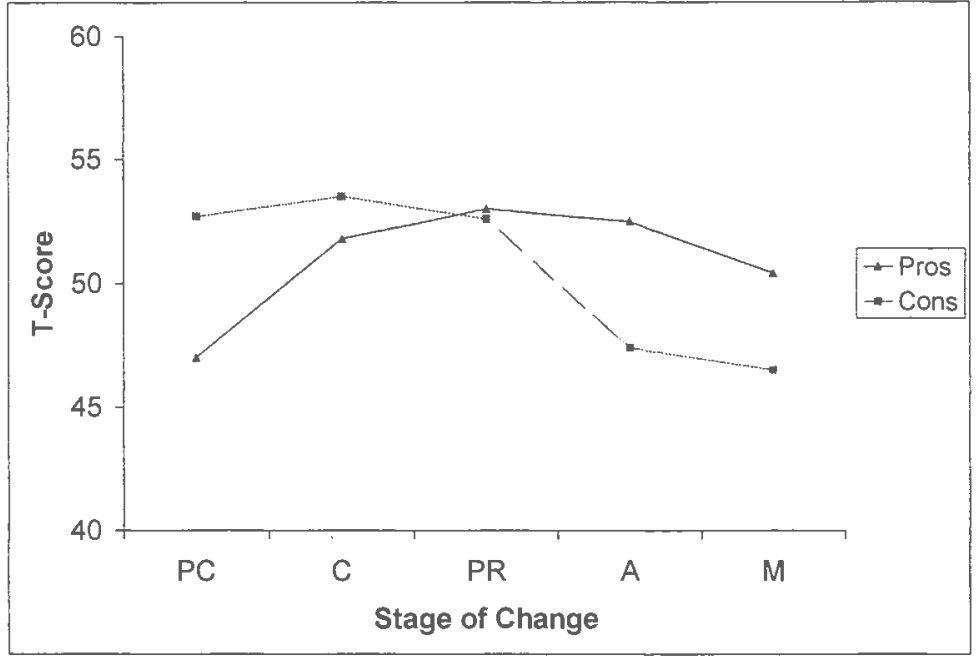

Figure 10. Composite Graphic for Pros and Cons of Quitting Smoking.

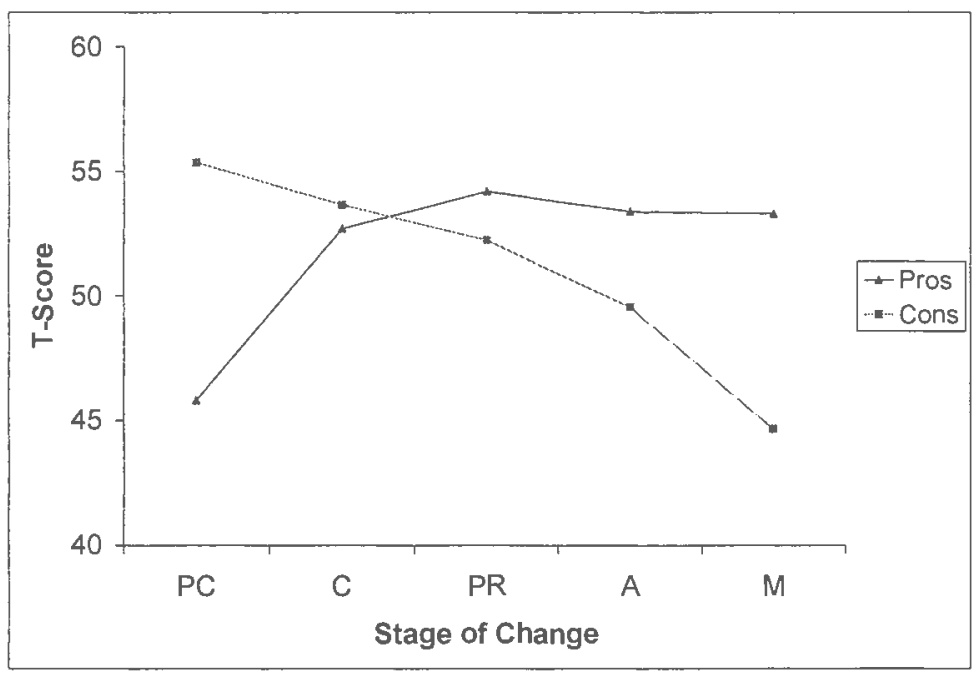

Figure 11. Composite Graphic for Pros and Cons of Smoking.

Condom Use-General, Main, Other

The behavior category of condom use was made up primarily of studies that examined either condom use in general or more specifically with a main partner or 
other partner. Effect size means, sample sizes, standard error, $95 \%$ confidence

intervals, and $p$-values for these three sub-sets of condom use were computed (Table 20) and composite graphics were created (Figure 12, Figure 13 and Figure 14). In order to determine potential differences between these behavioral subsets, an ANOVA analog using random effects modeling was performed on the three groups. Significant differences were only found for the Pros of Change in the transition from C-PR $(Q(d f=2)=6.22, p=.04)$. No significant differences were found for any of the transitions of the Cons of Change for these three groups.

Table 20

Comparison of Condom Use Sub-Groups

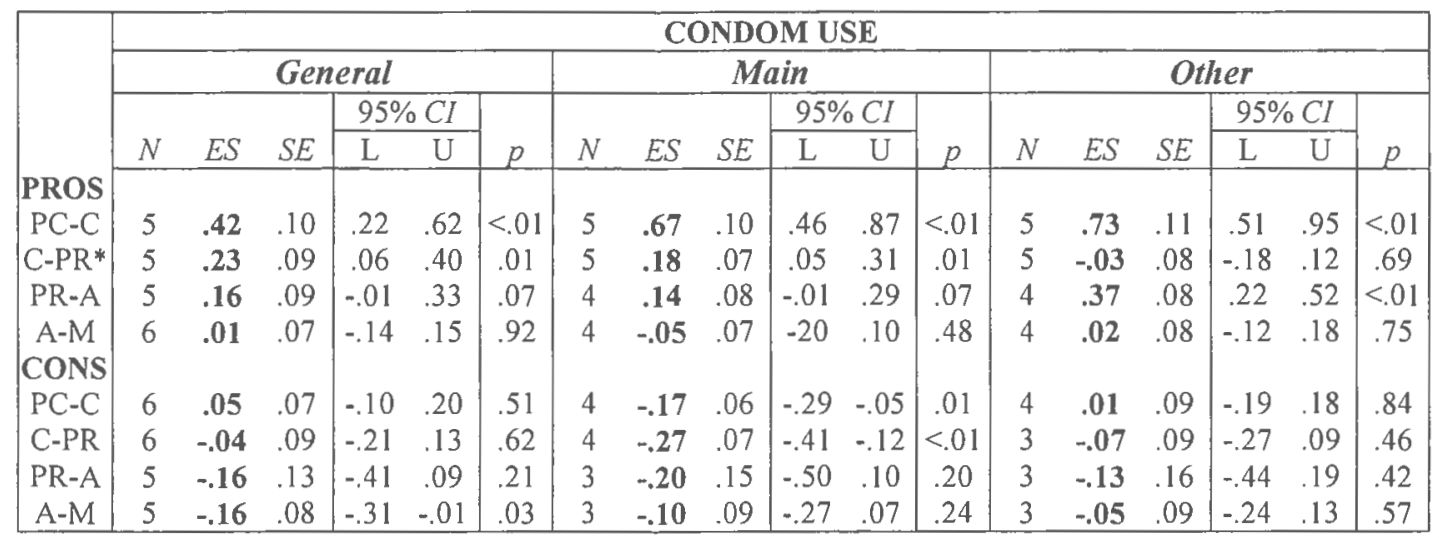

Note. $N=$ sample size; $E S=$ mean effect size; $\mathrm{L}=$ lower bound; $\mathrm{U}=$ upper bound; $p=$ significance of effect as compared to zero; * indicates overall significant differences between groups at $p=<.05$. 


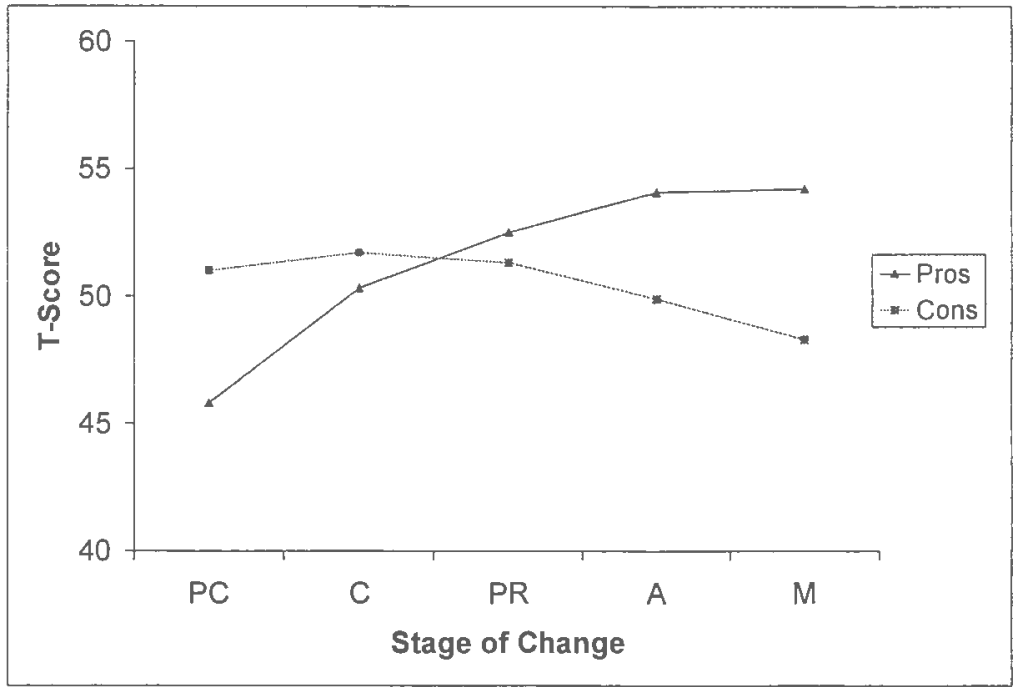

Figure 12. Composite Graphic for Condom Use - General.

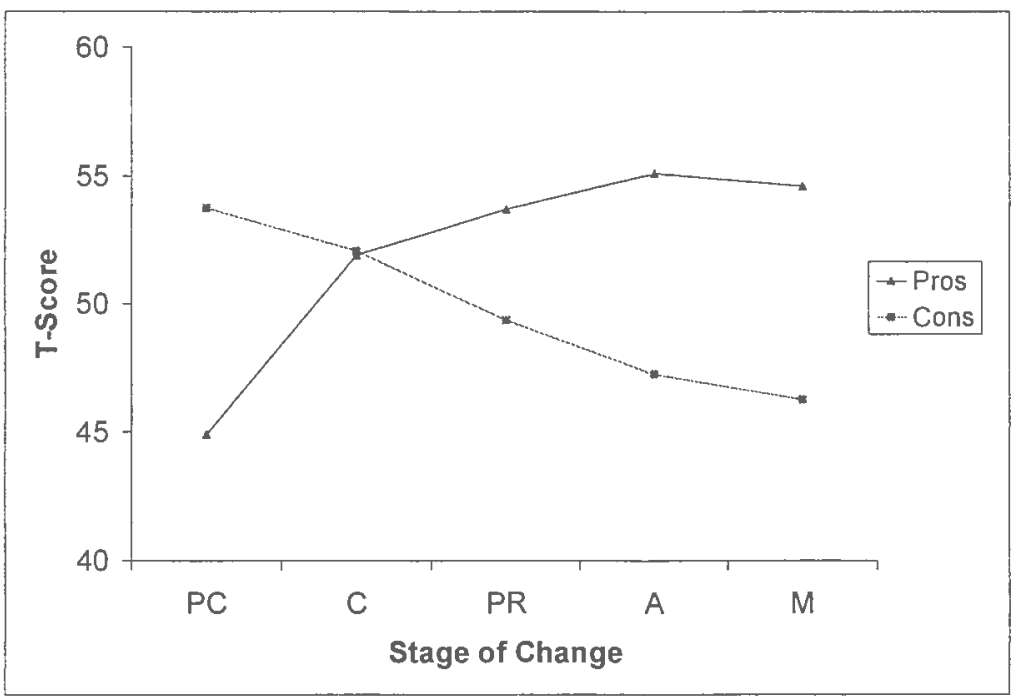

Figure 13. Composite Graphic for Condom Use - Main Partner. 


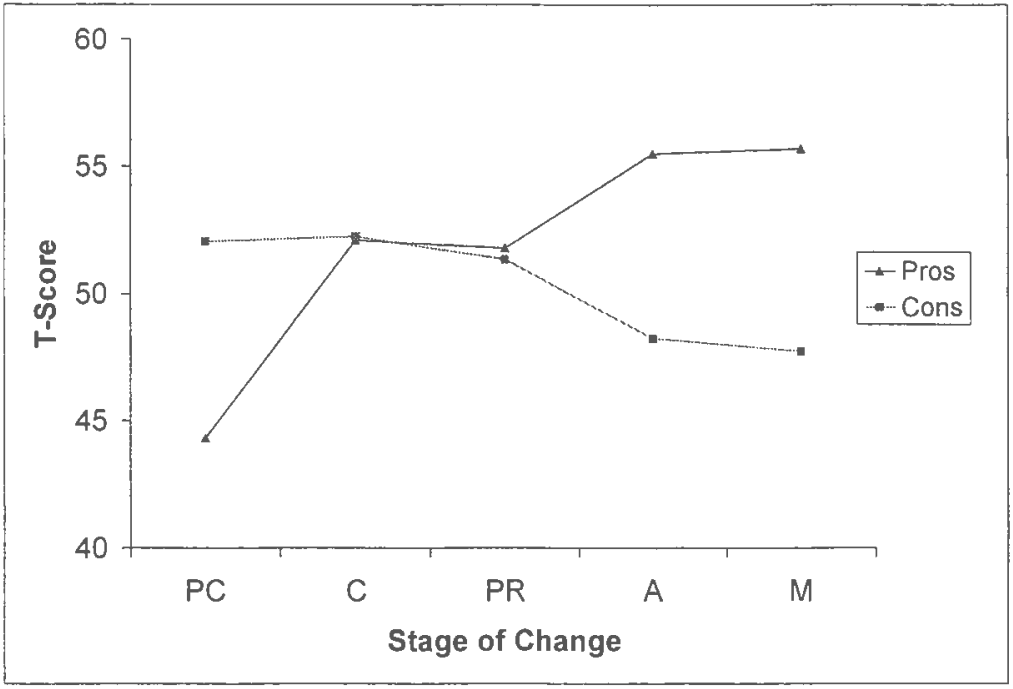

Figure 14. Composite Graphic for Condom Use - Other Partner.

Follow-up comparisons showed that condom use -general $(Q=4.95, p=$ $.026)$ and condom use - main $(Q=4.41, p=.035)$ were significantly greater than condom use - other.

\section{Dietary Fat Reduction versus Fruit and Vegetable Consumption}

The behavior category of diet was made up primarily of studies that examined either dietary fat reduction or fruit and vegetable consumption. Effect size means, sample sizes, standard error, $95 \%$ confidence intervals, and $p$-values for dietary fat reduction and fruit and vegetable consumption separately were computed (Table 21) and composite graphics were created (Figure 15 and Figure 16). In order to determine potential differences between these two behavioral subsets, an ANOVA analog using random effects modeling was performed. No significant differences were found for the Pros or Cons of Change for any of the transitions of for these two groups. 
Table 21

Effect Size Comparison of Dietary Fat Reduction and Fruit and Vegetable

Consumption

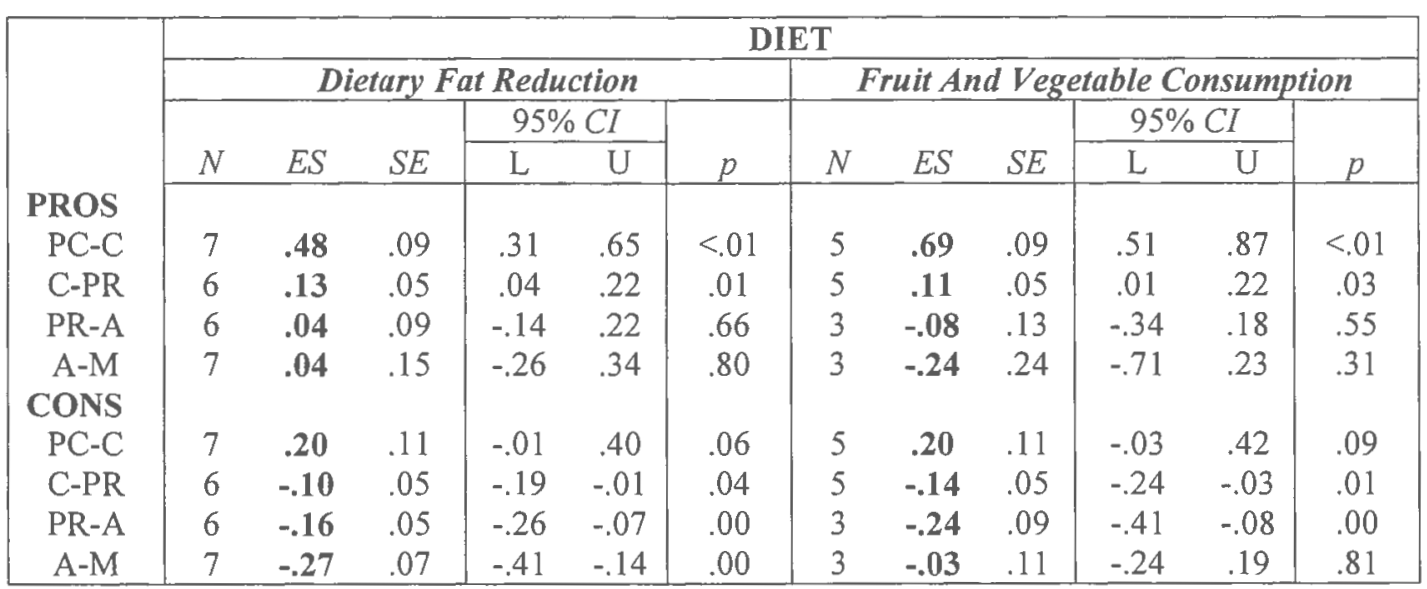

Note. $N=$ sample size; $E S=$ mean effect size; $\mathrm{L}=$ lower bound; $\mathrm{U}=$ upper bound; $p=$ significance of effect as compared to zero; ${ }^{*}$ indicates overall significant differences between groups at $p=<.05$. 


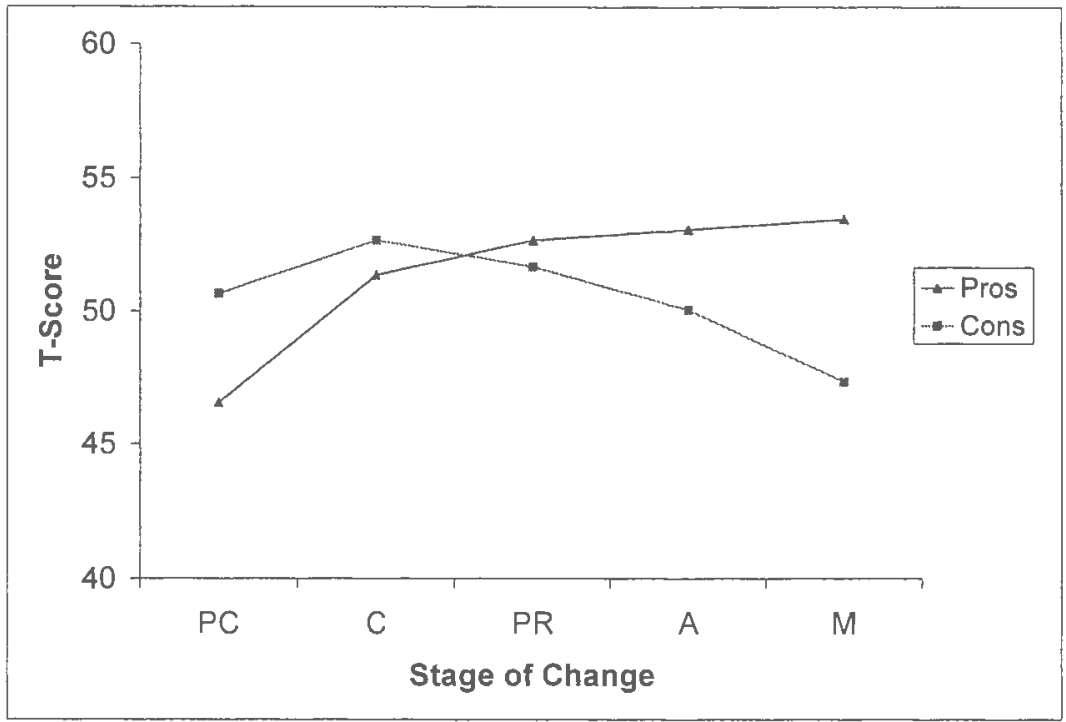

Figure 15. Composite Graphic for Dietary Fat Reduction.

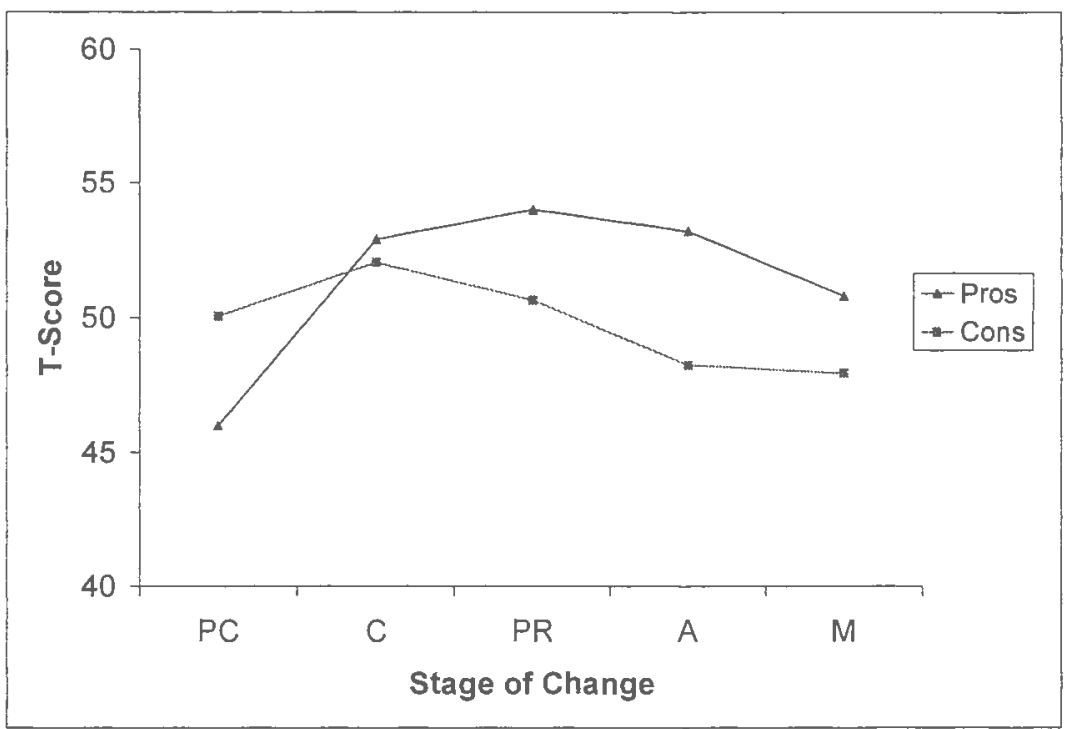

Figure 16. Composite Graphic for Fruit and Vegetable Consumption. 
Since overall effect sizes were heterogeneous and only a few instances of

heterogeneity were found for Stage transitions within behaviors, additional moderators of effect size distribution were explored.

Age Group - Adolescents, College, and Adults.

Effect size means, sample sizes, standard error, $95 \%$ confidence intervals, and $p$-values for age group were computed (Table 22) and composite graphics were created (Figure 17, Figure 18 and Figure 19). Differences among age groups were assessed for the Pros and Cons effect sizes across each of the four Stage transitions using random effects modeling. No significant differences were found between adolescents, college students and adults for any of the transitions for Pros.

Significant differences for Cons of Change were only found for the PC-C transition $(Q(d f=2)=13.54, p<.01)$. Follow-up analogs showed that Cons of Change were significantly greater for adolescents than college students $(p<.01)$ and adults $(p<$ $.01)$. 
Table 22

Comparison of Age Groups

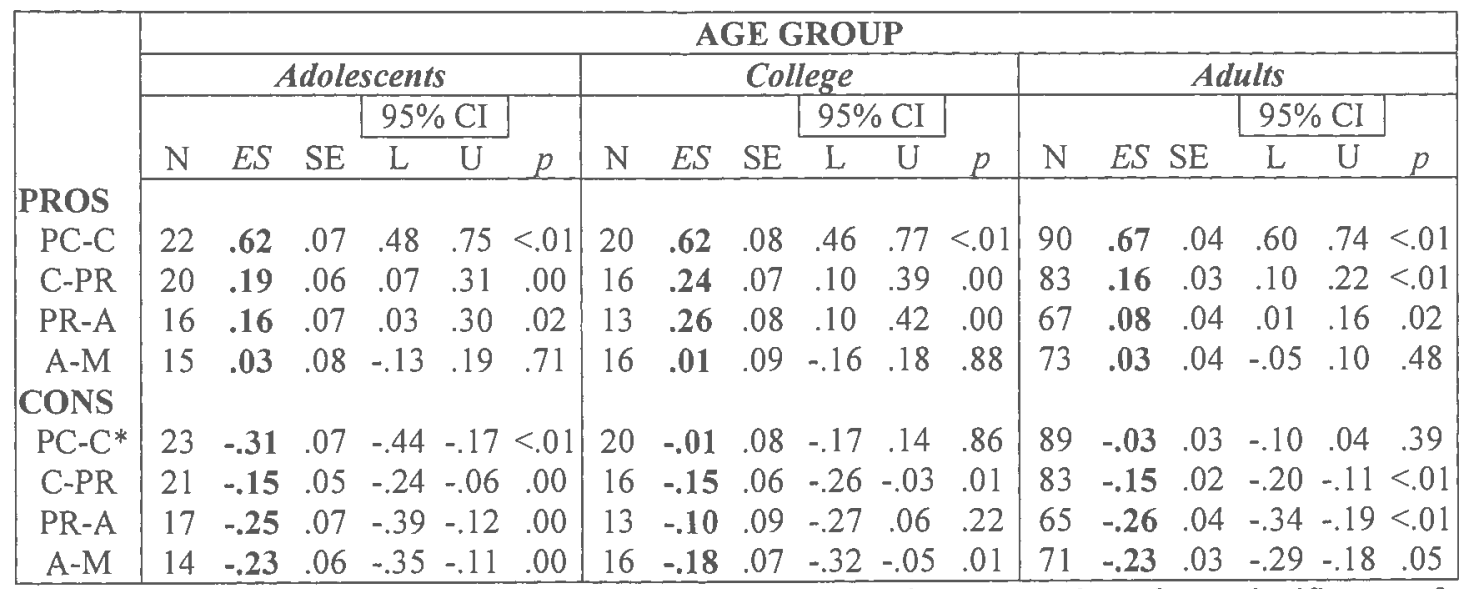

Note. $\mathrm{N}=$ sample size; $\mathrm{ES}=$ mean effect size; $\mathrm{L}=$ lower bound; $\mathrm{U}=$ upper bound; $p=$ significance of effect as compared to zero; * indicates overall significant differences between groups at $p=<.05$. 


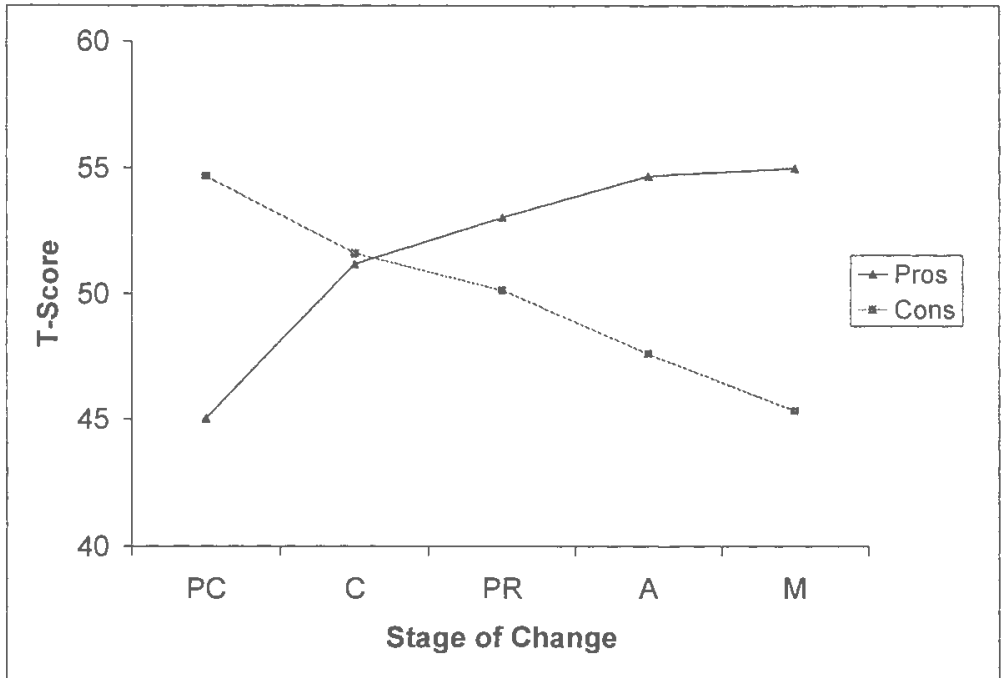

Figure 17. Composite Graphic for Adolescents.

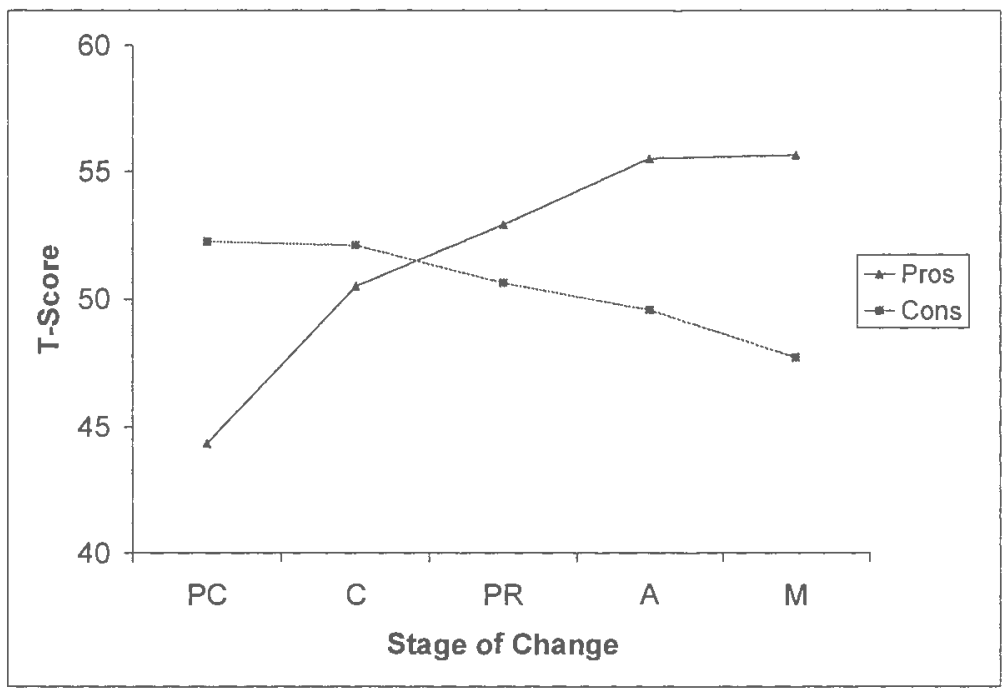

Figure 18. Composite Graphic for College Students. 


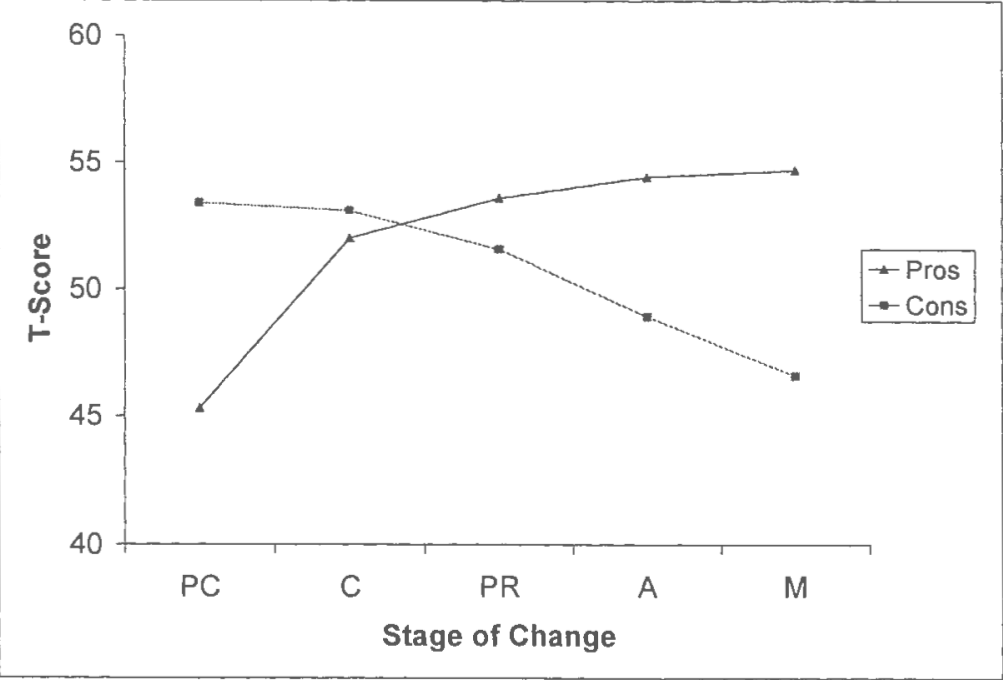

Figure 19. Composite Graphic for Adults.

\section{Cessation versus Acquisition}

Effect size means, sample sizes, standard error, $95 \%$ confidence intervals, and $p$-values for cessation and acquisition behaviors were computed (Table 23) and composite graphics were created (Figure 20 and Figure 21). Effect sizes for cessation and acquisition behaviors were compared across the four Stage transitions using random effects modeling. Acquisition behaviors were significantly greater than cessation behaviors for the Pros of Change in the transition from PC-C $(Q(d f=1)=$ $3.88, p=.05)$ and in the transition from PR-A $(Q(d f=1)=7.58, p=.01)$. No significant differences were found between acquisition and cessation behaviors for the Cons of Change. 
Means, Standard Error and Confidence Intervals for Cessation and Acquisition

\section{Behaviors}

\begin{tabular}{|c|c|c|c|c|c|c|c|c|c|c|c|c|}
\hline \multirow{4}{*}{ PROS } & \multicolumn{6}{|c|}{ ACQUISTION } & \multicolumn{6}{|c|}{ CESSATION } \\
\hline & \multirow[b]{2}{*}{$\mathrm{N}$} & \multirow[b]{2}{*}{$E S$} & \multirow[b]{2}{*}{ SE } & \multicolumn{2}{|c|}{$95 \% \mathrm{CI}$} & \multirow[b]{2}{*}{$p$} & \multirow[b]{2}{*}{$\mathrm{N}$} & \multirow[b]{2}{*}{$E S$} & \multirow[b]{2}{*}{ SE } & \multicolumn{2}{|c|}{$95 \% \mathrm{Cl}$} & \multirow[b]{2}{*}{$p$} \\
\hline & & & & $\mathrm{L}$ & $U$ & & & & & $\mathrm{~L}$ & $\mathrm{U}$ & \\
\hline & 90 & 70 & 04 & 62 & 77 & $<01$ & 46 & 58 & 05 & 48 & 67 & $<01$ \\
\hline C-PR & 81 & .17 & .03 & .11 & .23 & $<.01$ & 41 & .17 & .04 & .09 & 26 & .00 \\
\hline PR-A* & 72 & .17 & .03 & .10 & .24 & $<.01$ & 28 & -.01 & .06 & -.12 & .10 & .86 \\
\hline A-M & 80 & .05 & .04 & -.03 & .12 & .22 & 28 & -.07 & .06 & -.20 & .05 & .27 \\
\hline CONS & & & & & & & & & & & & \\
\hline PC-C & 89 & -.08 & .04 & -.15 & .00 & .04 & 47 & -.07 & .05 & -.17 & .03 & .15 \\
\hline C-PR & 81 & -.16 & .03 & -.20 & -.11 & $<.01$ & 43 & -.14 & .03 & -.20 & -.08 & $<.01$ \\
\hline PR-A & 72 & -.23 & .04 & -.31 & -.16 & $<.01$ & 27 & -.27 & .06 & -.39 & -.15 & $<.01$ \\
\hline A-M & 76 & -.21 & .03 & -.27 & -.16 & $<.01$ & 29 & -.28 & .05 & -.37 & -.19 & $<.01$ \\
\hline
\end{tabular}

Note. $\mathrm{N}=$ sample size; $E S=$ mean effect size; $\mathrm{L}=$ lower bound; $\mathrm{U}=$ upper bound; $p=$ significance of effect as compared to zero; * indicates overall significant differences between groups at $p=<.05$. 


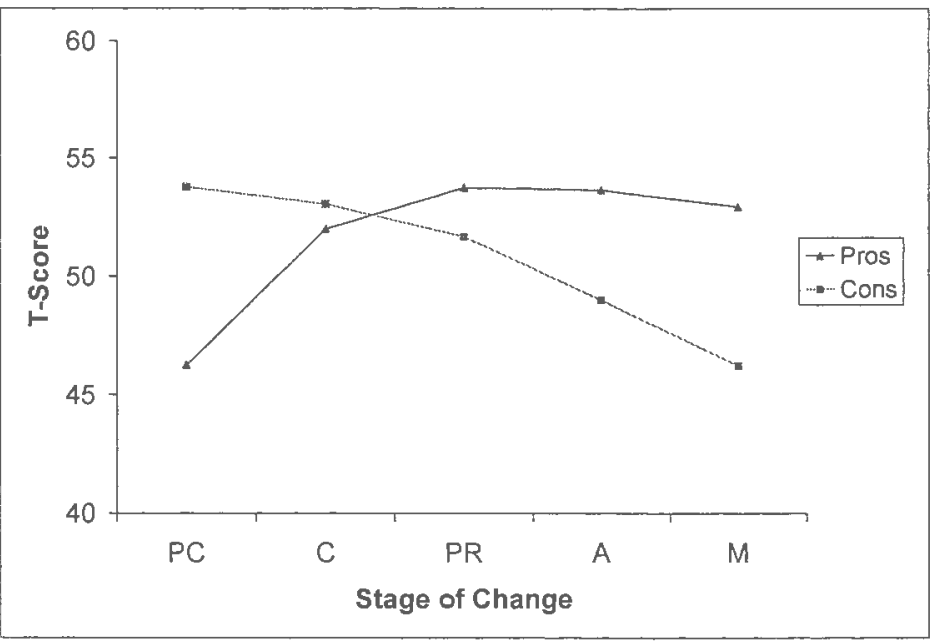

Figure 20. Composite Graphic for Cessation Behaviors.

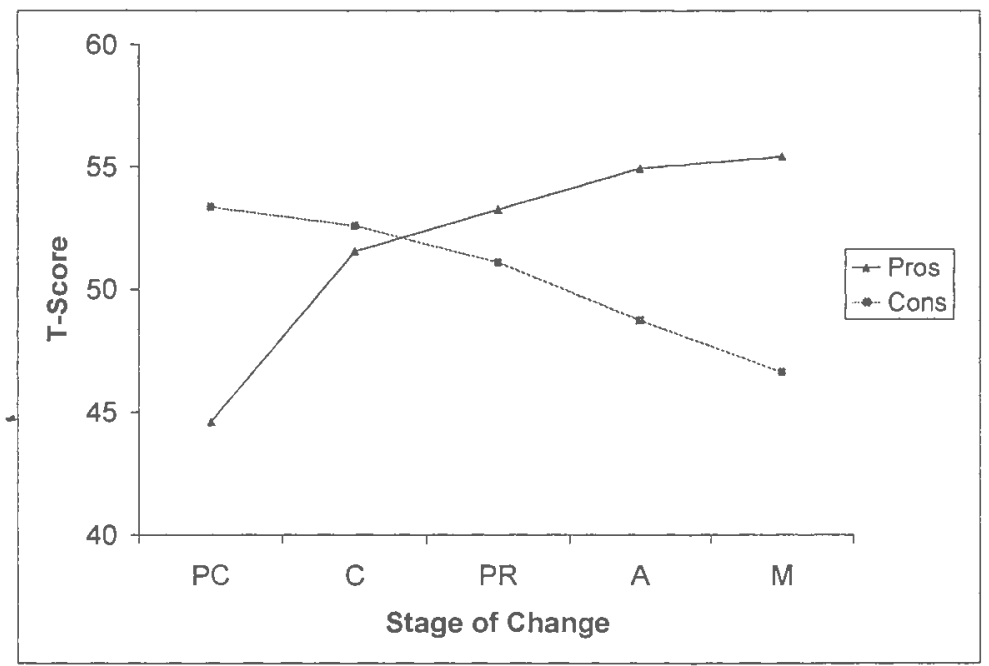

Figure 21. Composite Graphic for Acquisition Behaviors.

\section{Healthy versus Unhealthy}

Effect size means, sample sizes, standard error, 95\% confidence intervals, and $p$-values for health and unhealthy behaviors were computed (Table 24) and 
composite graphics were created (Figure 22 and Figure 23). Effect sizes for healthy

and unhealthy behaviors were compared across the four Stage transitions using

random effects modeling. Mean effect sizes for healthy behaviors were significantly greater than unhealthy behaviors for the Pros of Change in the transition from PR-A $(Q(d f=1)=9.08, p=.003)$ and from A-M $(Q(d f=1)=5.82, p=.007)$. No significant differences were found between healthy and unhealthy behaviors for the Cons of Change.

Table 24

Comparison of Healthy and Unhealthy Behaviors

\begin{tabular}{|c|c|c|c|c|c|c|c|c|c|c|c|c|}
\hline & \multicolumn{6}{|c|}{ HEALTHY } & \multicolumn{6}{|c|}{ UNHEALTHY } \\
\hline & \multirow[b]{2}{*}{$\mathrm{N}$} & \multirow[b]{2}{*}{$E S$} & \multirow[b]{2}{*}{ SE } & \multicolumn{2}{|c|}{$95 \% \mathrm{Cl}$} & \multirow[b]{2}{*}{$p$} & \multirow[b]{2}{*}{$\mathrm{N}$} & \multirow[b]{2}{*}{$E S$} & \multirow[b]{2}{*}{ SE } & \multicolumn{2}{|c|}{$95 \% \mathrm{Cl}$} & \multirow[b]{2}{*}{$p$} \\
\hline & & & & $\mathrm{L}$ & $\mathrm{U}$ & & & & & $\mathrm{L}$ & $\mathrm{U}$ & \\
\hline PROS & & & & & & & & & & & & \\
\hline PC-C & 72 & .71 & .04 & .63 & .79 & $<.01$ & 45 & .60 & .05 & .50 & .70 & $<.01$ \\
\hline C-PR & 66 & .18 & .03 & .11 & .25 & $<.01$ & 41 & .18 & .05 & .09 & .27 & .00 \\
\hline PR-A* & 61 & .17 & .04 & .10 & .24 & $<.01$ & 29 & -.04 & .06 & -.16 & .08 & .49 \\
\hline A-M* & 70 & .06 & .04 & -.01 & .14 & .09 & 28 & -.14 & .06 & -.27 & -.01 & .03 \\
\hline CONS & & & & & & & & & & & & \\
\hline PC-C & 72 & .00 & .04 & -.08 & .08 & .97 & 46 & -.11 & .05 & -.21 & -.01 & .03 \\
\hline C-PR & 65 & -.17 & .02 & -.21 & -.12 & $<.01$ & 43 & -.12 & .03 & -.18 & -.06 & $<.01$ \\
\hline PR-A & 60 & -.21 & .04 & -.28 & -.13 & $<.01$ & 28 & -.29 & .06 & -.41 & -.16 & $<.01$ \\
\hline A-M & 67 & -.22 & .03 & -.28 & -.17 & $<.01$ & 29 & -.25 & .05 & -.35 & -.15 & $<.01$ \\
\hline
\end{tabular}

Note. $\mathrm{N}=$ sample size; $E S=$ mean effect size; $\mathrm{L}=$ lower bound; $\mathrm{U}=$ upper bound; $p=$ significance of effect as compared to zero; $*$ indicates overall significant differences between groups at $p=<.05$. 


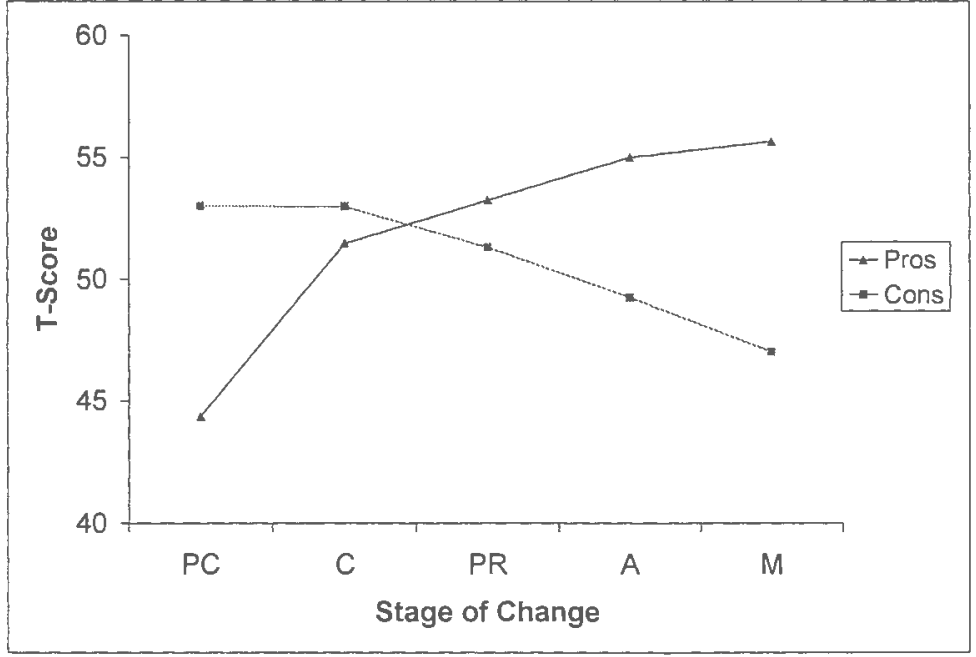

Figure 22. Composite Graphic for Studies Examining Healthy Behaviors.

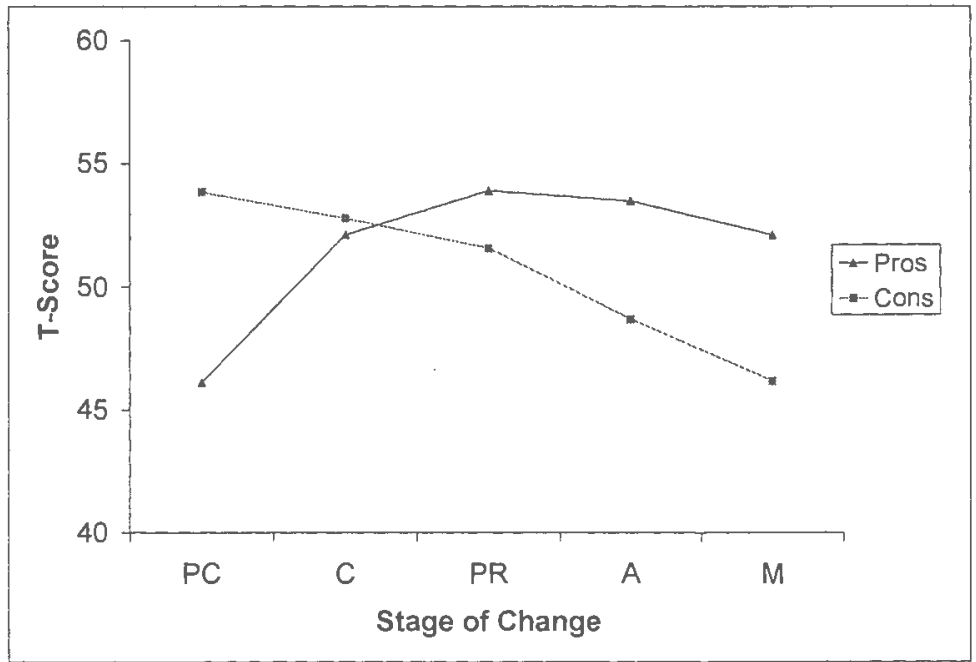

Figure 23. Composite Graphic for Studies Examining Unhealthy Behaviors.

\section{Response Format}

Effect size means, sample sizes, standard error, $95 \%$ confidence intervals, and $p$-values for cessation and acquisition behaviors were computed (Table 25) and 
composite graphics were created (Figure 24 and Figure 25). Effect sizes for

Decisional Balance measures using "How Important" and "Agree/Disagree" likert scales were compared across the four Stage transitions using random effects modeling. No significant differences were found between response formats for the Pros of Change. The mean effect sizes for the "Agree/Disagree" format were significantly greater than "How Important" format for the Cons of Change in the transition from PR-A $(Q(d f=1)=3.99, p=.046)$.

Table 25

Comparison of Response Formats

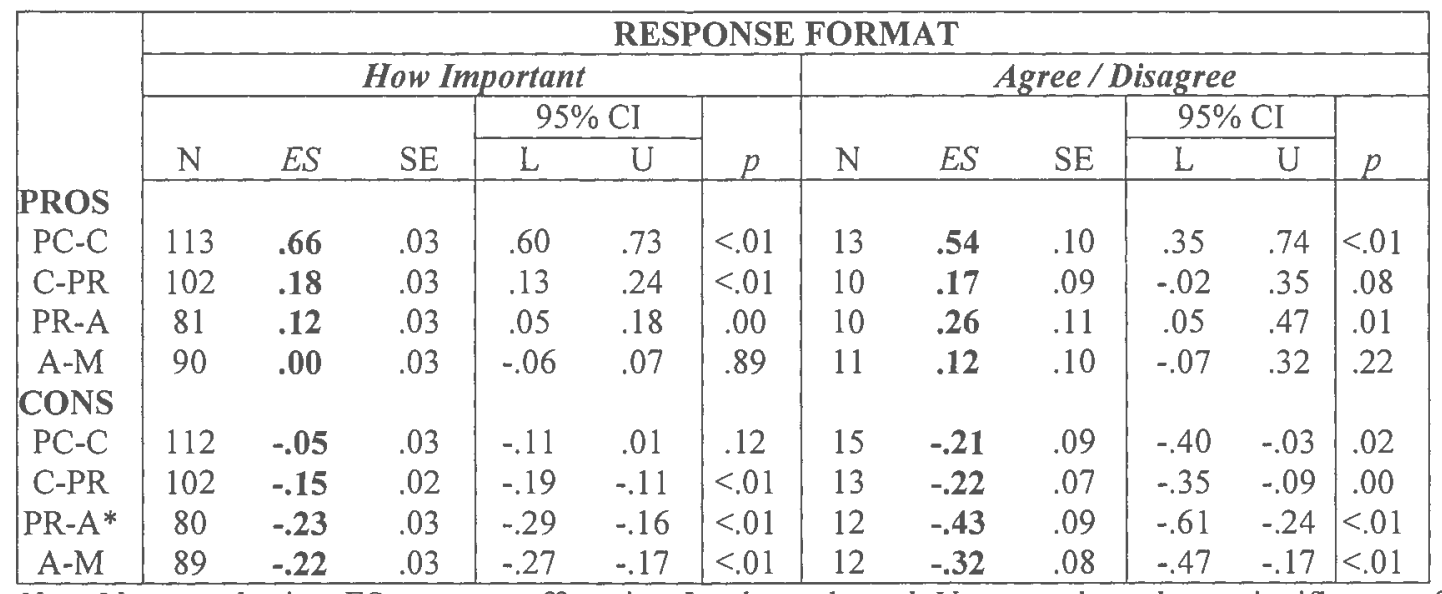

Note. $\mathrm{N}=$ sample size; $\mathrm{ES}=$ mean effect size; $\mathrm{L}=$ lower bound; $\mathrm{U}=$ upper bound; $p=$ significance of effect as compared to zero; ${ }^{*}$ indicates overall significant differences between groups at $p=<.05$. 


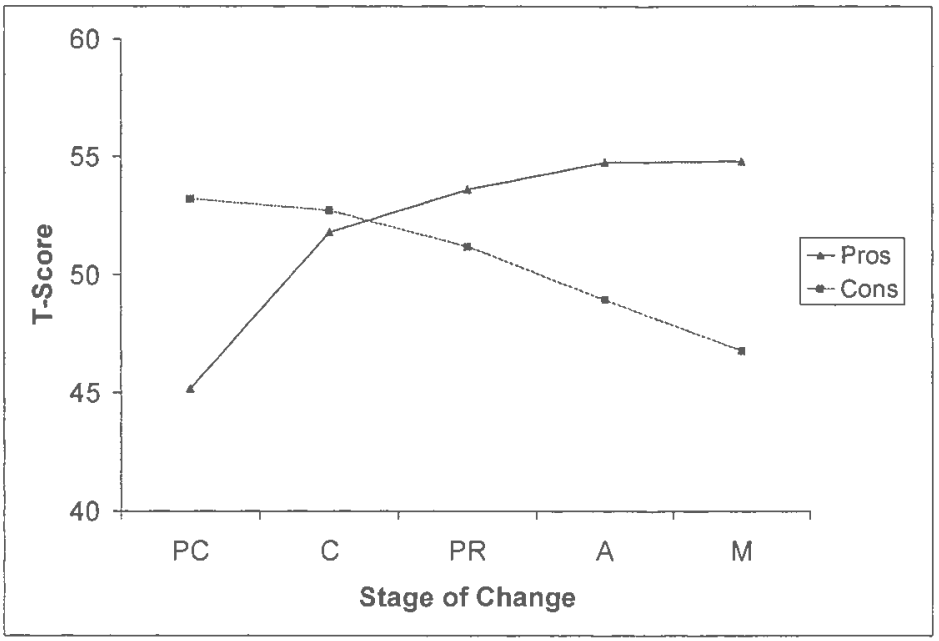

Figure 24. Composite Graphic for "How Important" Format.

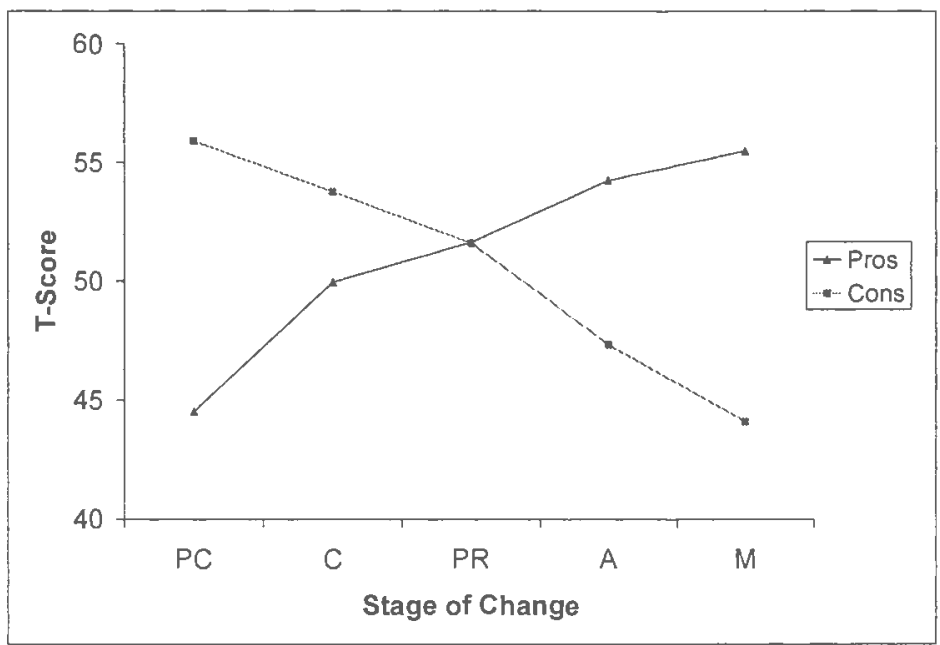

Figure 25. Composite Graphic for "Agree/Disagree" Format.

$$
\text { Country - US versus Non-US }
$$

Effect size means, sample sizes, standard error, $95 \%$ confidence intervals, and $p$-values for US and non-US studies were computed (Table 26) and composite 
graphics were created (Figure 26 and Figure 27). In order to determine potential differences between these sub-groups, an ANOVA analog using random effects modeling was performed. No significant differences were found for the Pros or Cons of Change across the Stage Transitions for studies conducted in the US or outside of the US.

Table 26

Comparison of Country

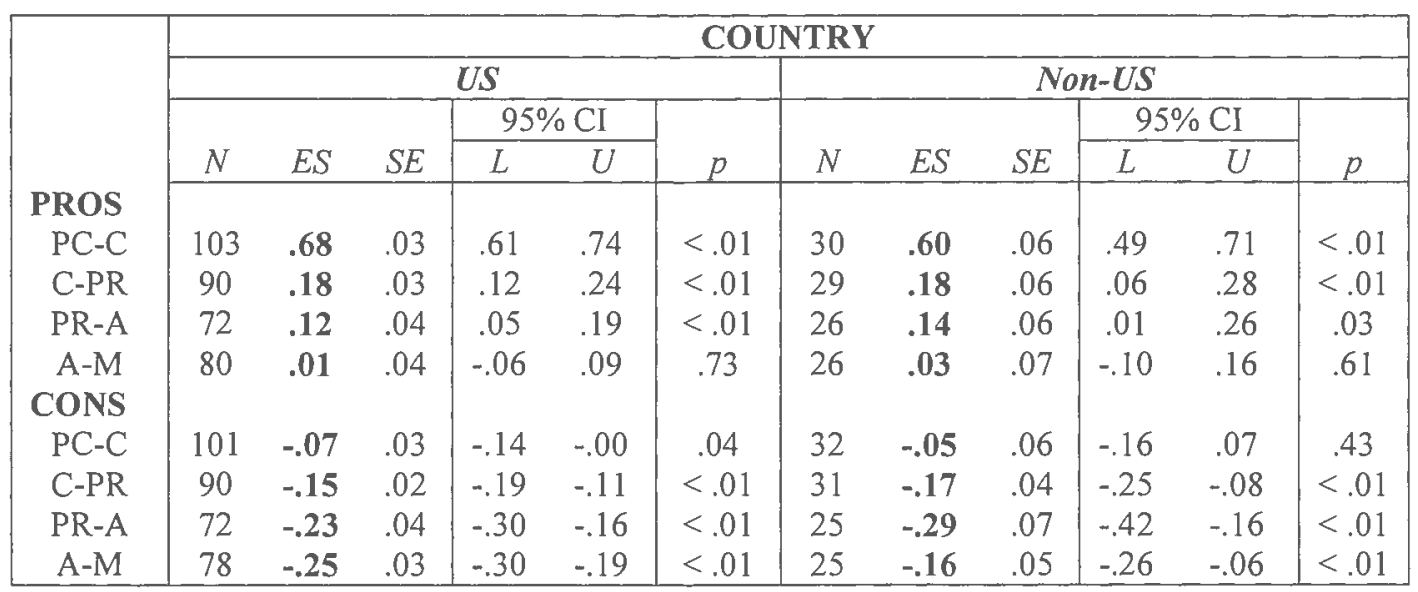

Note. $\mathrm{N}=$ sample size; $\mathrm{ES}=$ mean effect size; $\mathrm{L}=$ lower bound; $\mathrm{U}=$ upper bound; $p=$ significance of effect as compared to zero; ${ }^{*}$ indicates overall significant differences between groups at $p=<.05$. 


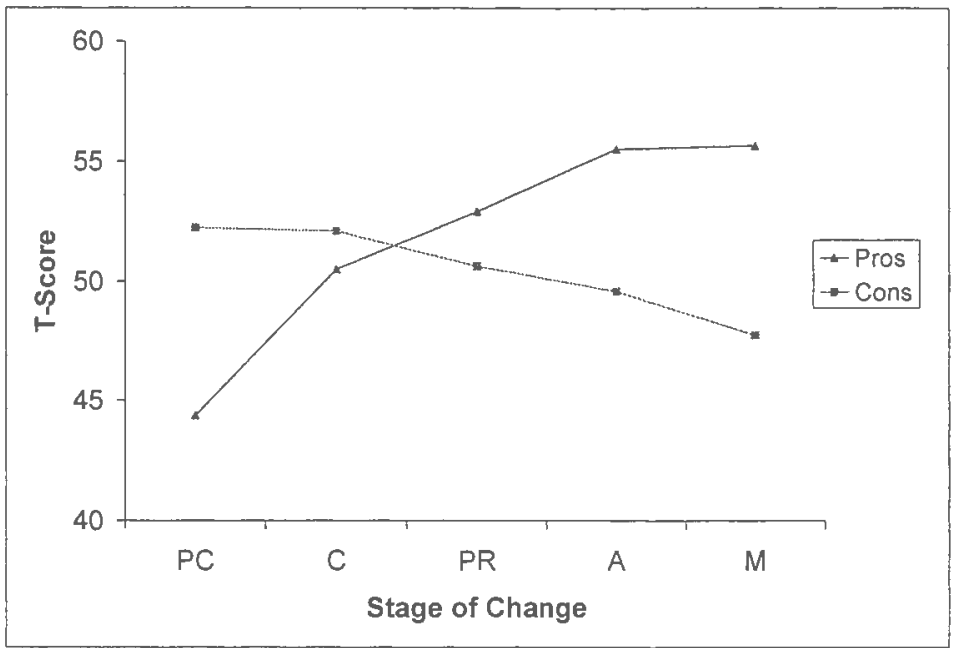

Figure 26. Composite Graphic for Studies Conducted in the US.

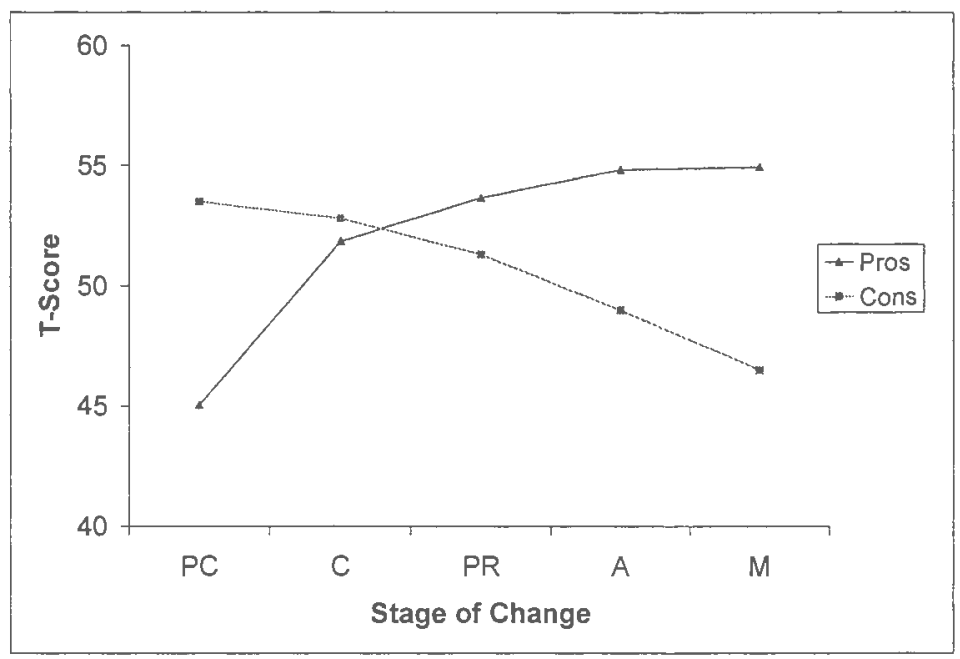

Figure 27. Composite Graphic for Studies Conducted Outside the US.

\section{Language - English versus Non-English}

Effect size means, sample sizes, standard error, $95 \%$ confidence intervals, and $p$-values for studies conducted in English and in languages other than English 
were computed (Table 27) and composite graphics were created (Figure 28 and

Figure 29). In order to determine potential differences between these sub-groups, an ANOVA analog using random effects modeling was performed. No significant differences were found for the Pros or Cons of Change across the Stage Transitions for studies conducted in English or a language other than English.

Table 27

Comparison of Language

\begin{tabular}{|c|c|c|c|c|c|c|c|c|c|c|c|c|}
\hline \multirow[b]{5}{*}{ PROS } & \multicolumn{12}{|c|}{ LANGUAGE } \\
\hline & \multicolumn{6}{|c|}{ English } & \multicolumn{6}{|c|}{ Non-English } \\
\hline & \multirow[b]{2}{*}{$N$} & \multirow[b]{2}{*}{$E S$} & \multirow[b]{2}{*}{$S E$} & \multicolumn{2}{|c|}{$95 \% \mathrm{CI}$} & \multirow[b]{2}{*}{$p$} & \multirow[b]{2}{*}{$N$} & \multirow[b]{2}{*}{$E S$} & \multirow[b]{2}{*}{$S E$} & \multicolumn{2}{|c|}{$95 \% \mathrm{CI}$} & \multirow[b]{2}{*}{$p$} \\
\hline & & & & $L$ & $U$ & & & & & $L$ & $U$ & \\
\hline & & & & & & & & & & & & \\
\hline PC-C & 108 & .67 & .03 & .60 & .73 & $<.01$ & 28 & .61 & .06 & .49 & .72 & $<.01$ \\
\hline C-PR & 96 & .18 & .03 & .12 & .24 & $<.01$ & 26 & .14 & .06 & .02 & .26 & .02 \\
\hline PR-A & 77 & .13 & .03 & .07 & .20 & $<.01$ & 23 & .06 & .07 & -.08 & .20 & .43 \\
\hline $\mathrm{A}-\mathrm{M}$ & 84 & .01 & .04 & -.06 & .08 & .78 & 24 & .04 & .07 & -.10 & .18 & .57 \\
\hline CONS & & & & & & & & & & & & \\
\hline PC-C & 107 & -.07 & .03 & -.14 & .00 & .05 & 29 & -.10 & .06 & -.22 & .02 & .11 \\
\hline C-PR & 96 & -.15 & .02 & -.19 & -.11 & $<.01$ & 28 & -.14 & .05 & -.23 & -.05 & $<.01$ \\
\hline PR-A & 77 & -.24 & .03 & -.30 & -.17 & $<.01$ & 22 & -.28 & .07 & -.42 & -.13 & $<.01$ \\
\hline A-M & 82 & -.24 & .03 & -.29 & -.18 & $<.01$ & 23 & -.20 & .06 & -.31 & -.10 & $<.01$ \\
\hline
\end{tabular}

Note. $\mathrm{N}=$ sample size; $\mathrm{ES}=$ mean effect size; $\mathrm{L}=$ lower bound; $\mathrm{U}=$ upper bound; $p=$ significance of effect as compared to zero; ${ }^{*}$ indicates overall significant differences between groups at $p=<.05$. 


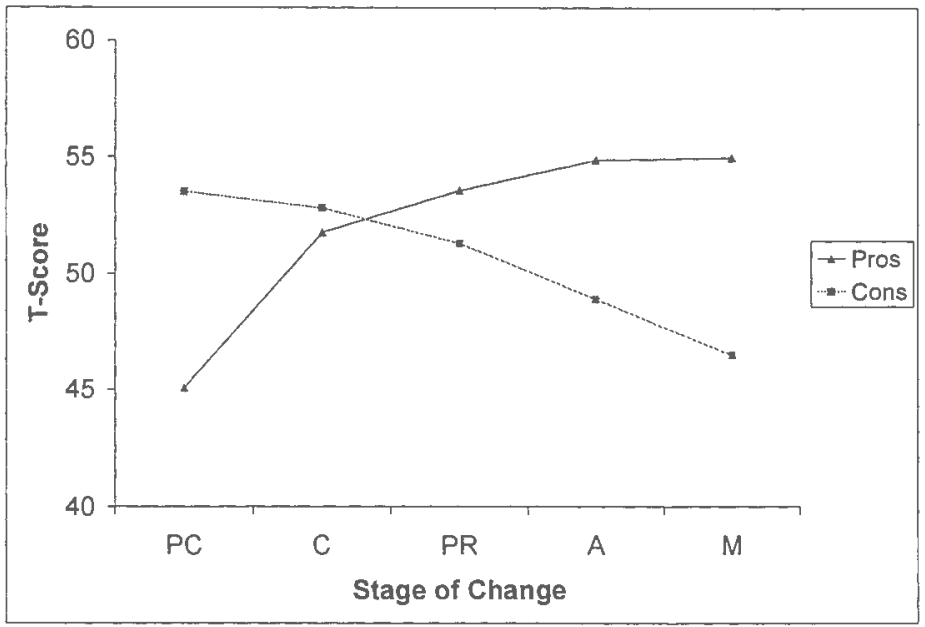

Figure 28. Composite Graphic for Studies Conducted in English.

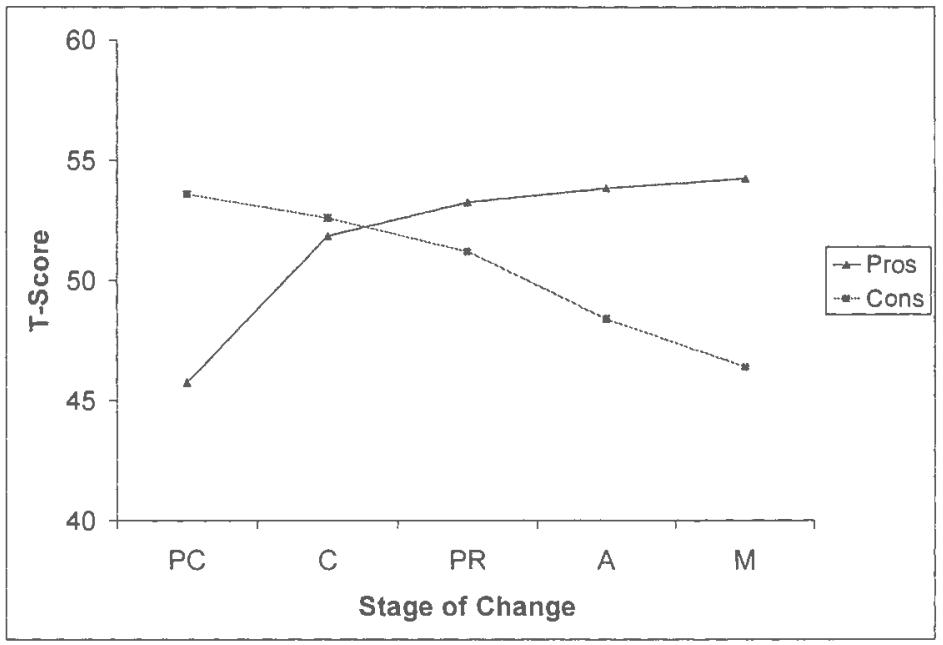

Figure 29. Composite Graphic for Studies Conducted in Languages Other than English.

Publication Status 
Effect size means, sample sizes, standard error, $95 \%$ confidence intervals, and $p$-values for peer-reviewed journals and non-peer-reviewed sources were computed (Table 28) and composite graphics were created (Figure 30 and Figure 31). Effect sizes for datasets that were from peer-reviewed journals and non-peerreviewed datasets were compared across the four Stage transitions using random effects modeling. No significant differences between effect sizes were found between data from peer-reviewed journals and non-peer-reviewed sources for the Pros of Change. Mean effect sizes for peer-reviewed journals were significantly greater than non-peer-reviewed sources for the Cons of Change in the transition from PR-A $(Q(d f=1)=4.10, p=.043)$.

Table 28

\section{Comparison of Publication Status}

\begin{tabular}{|c|c|c|c|c|c|c|c|c|c|c|c|c|}
\hline & \multicolumn{12}{|c|}{ PUBLICATION STATUS } \\
\hline & \multicolumn{6}{|c|}{ Peer-Reviewed } & \multicolumn{6}{|c|}{ Non-Peer-Reviewed } \\
\hline & \multirow[b]{2}{*}{$N$} & \multirow[b]{2}{*}{$E S$} & \multirow[b]{2}{*}{$S E$} & \multicolumn{2}{|c|}{$95 \% \mathrm{CI}$} & \multirow[b]{2}{*}{$p$} & \multirow[b]{2}{*}{$N$} & \multirow[b]{2}{*}{$E S$} & \multirow[b]{2}{*}{$S E$} & \multicolumn{2}{|c|}{$95 \% \mathrm{CI}$} & \multirow[b]{2}{*}{$p$} \\
\hline & & & & $L$ & $U$ & & & & & $L$ & $U$ & \\
\hline PROS & & & & & & & & & & & & \\
\hline PC-C & 74 & .69 & .04 & .61 & .76 & $<.01$ & 62 & .61 & .04 & .52 & .70 & $<.01$ \\
\hline C-PR & 68 & .14 & .03 & .08 & .21 & $<.01$ & 54 & .21 & .04 & .13 & .29 & $<.01$ \\
\hline PR-A & 53 & .13 & .04 & .05 & .21 & $<.01$ & 47 & .11 & .05 & .02 & .20 & .02 \\
\hline A-M & 53 & .03 & .05 & -.07 & .18 & .58 & 55 & .01 & .05 & -.08 & .10 & .86 \\
\hline CONS & & & & & & & & & & & & \\
\hline PC-C & 73 & -.12 & .04 & -.20 & -.04 & $<.01$ & .63 & -.03 & .04 & -.11 & .06 & .55 \\
\hline C-PR & 69 & -.16 & .02 & -.20 & -.11 & $<.01$ & 55 & -.13 & .03 & -.20 & -.07 & $<.01$ \\
\hline PR-A* & 53 & -.30 & .04 & -.38 & -.22 & $<.01$ & 46 & -.17 & .05 & -.26 & -.08 & $<.01$ \\
\hline A-M & 51 & -.20 & .04 & -.27 & -.13 & $<.01$ & 54 & -.25 & .03 & -.32 & -.19 & -.01 \\
\hline
\end{tabular}

Note. $\mathrm{N}=$ sample size; $\mathrm{ES}=$ mean effect size; $\mathrm{L}=$ lower bound; $\mathrm{U}=$ upper bound; $p=$ significance of effect as compared to zero; ${ }^{*}$ indicates overall significant differences between groups at $p=<.05$. 


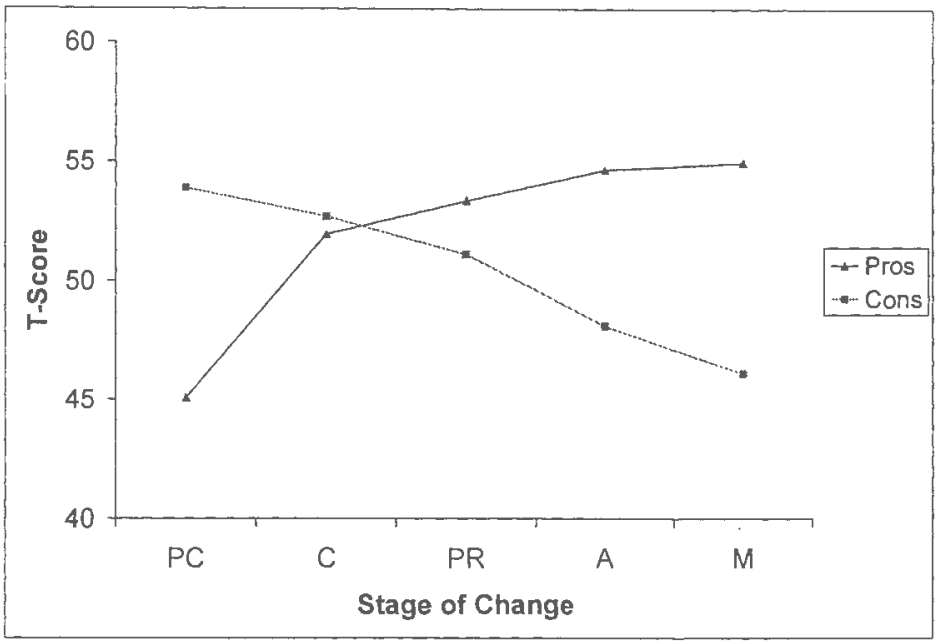

Figure 30. Composite Graphic for Peer-Reviewed Publications.

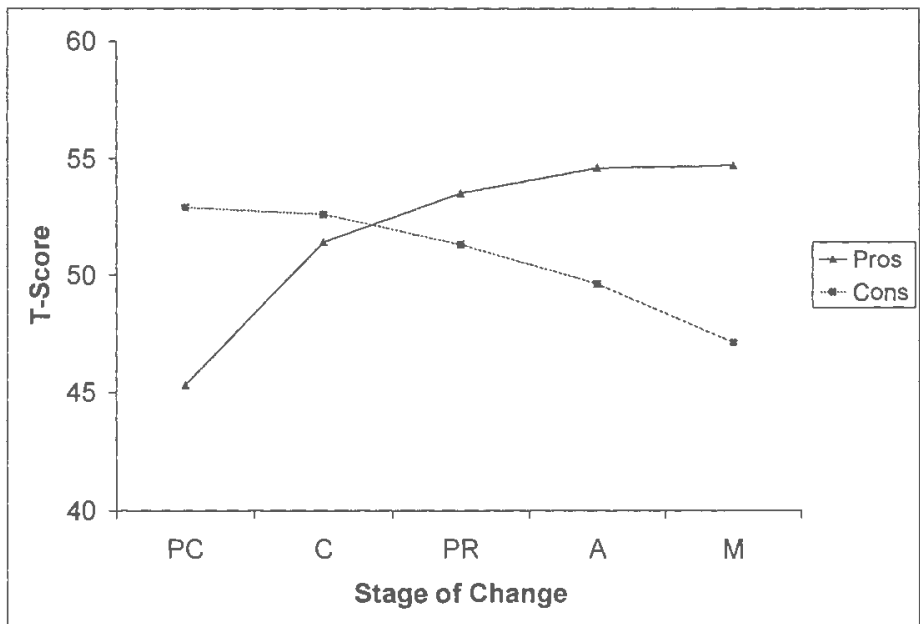

Figure 31. Composite Graphic for Non-Peer-Reviewed Datasets.

\section{Actual versus Reversed}

In some cases, the Pros and Cons are inversely defined, that is, the measures focus on the positive aspects of an unhealthy rather than of a healthy behavior (e.g., 
Borland \& Segan, 2000; Velicer et al., 1985; Rossi et al., 2001). For example, a study may examine the Pros of smoking (Velicer et al., 1985) rather than the Pros of quitting smoking (Keller et al., 1999). Actual and reversed refer to the way in which the data were used in the Pros and Cons analyses. Effect size means, sample sizes, standard error, $95 \%$ confidence intervals, and $p$-values for actual and reversed behaviors were computed (Table 29) and composite graphics were created (Figure 32 and Figure 33). Effect sizes for the behaviors with Pros and Cons reported in the "actual" direction and behaviors with the measures reversed were compared across the four Stage transitions using random effects modeling. For the Pros of Change in the transition from PR-A, the mean effect size for "actual" was significantly greater than the mean for the "reversed" measures $(Q(d f=1)=5.73, p=.02)$. For the Cons of Change, the "reversed" measures showed a larger decrease than the "actual" measures for the A-M transition.

Table 29

Comparison of Direction of Pros and Cons

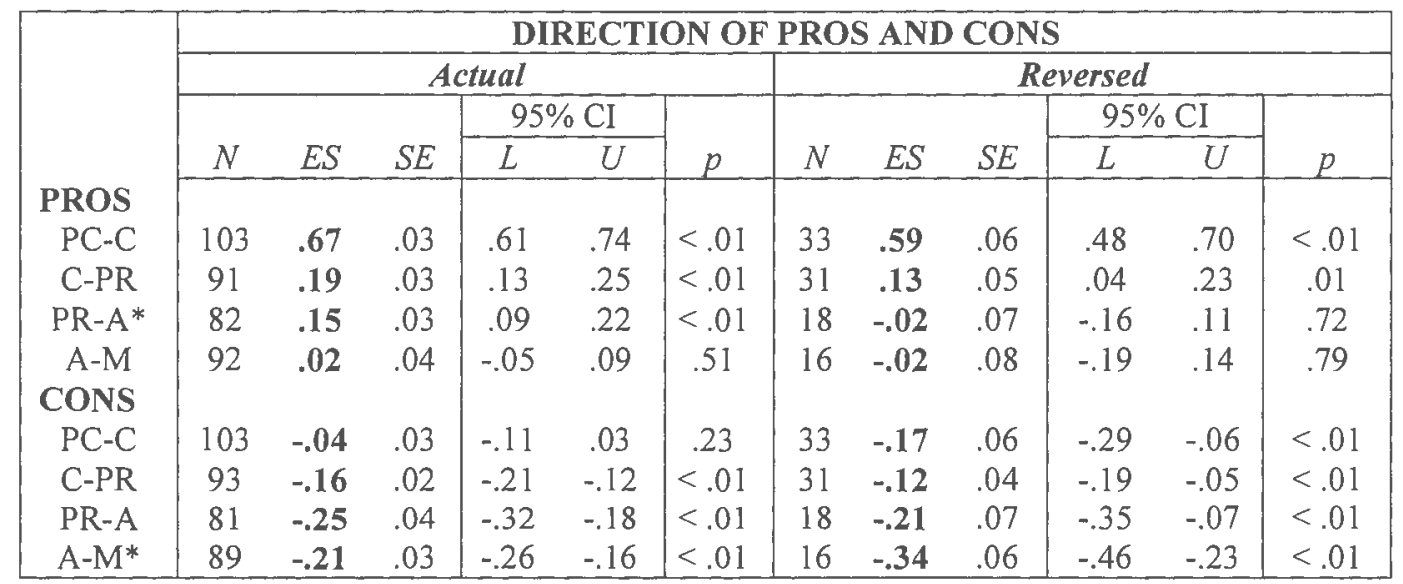

Note. $\mathrm{N}=$ sample size; $\mathrm{ES}=$ mean effect size; $\mathrm{L}=$ lower bound; $\mathrm{U}=$ upper bound; $p=$ significance of effect as compared to zero; * indicates overall significant differences between groups at $p=<.05$. 


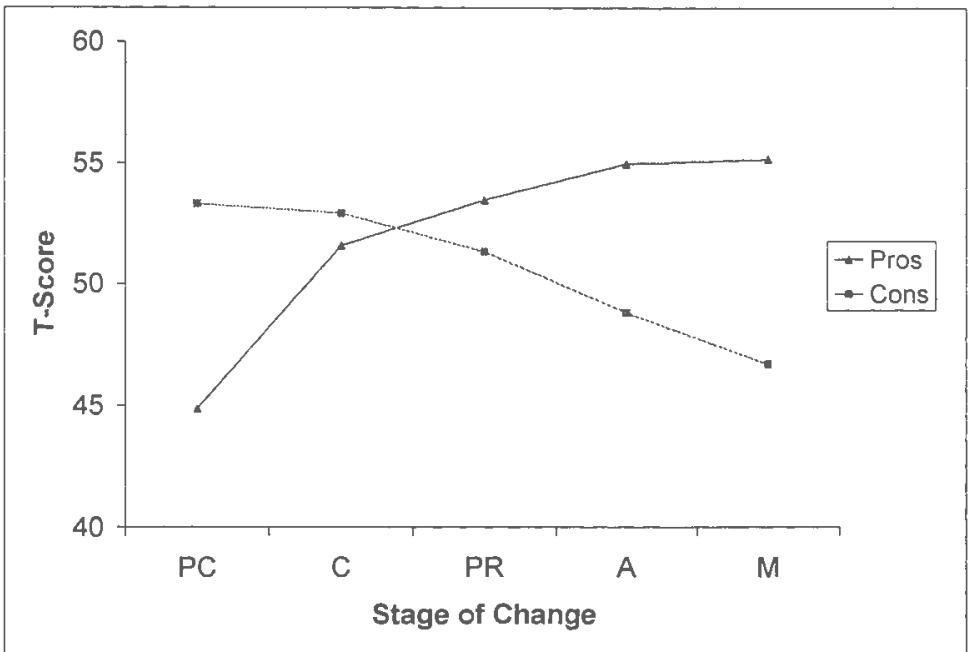

Figure 32. Composite Graphic for "Actual".

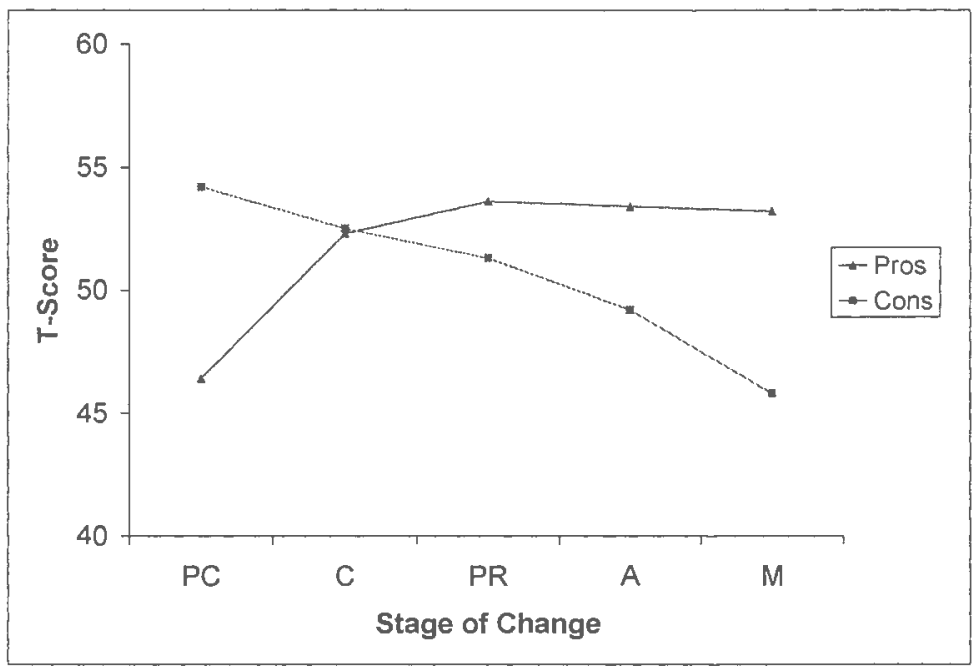

Figure 33. Composite Graphic for "Reversed".

Overview of Moderators

For descriptive and summary purposes the mean effect sizes for each of the primary moderator variables is listed in Table 30. 
Table 30

Overview of Effect Sizes by Moderator Variable

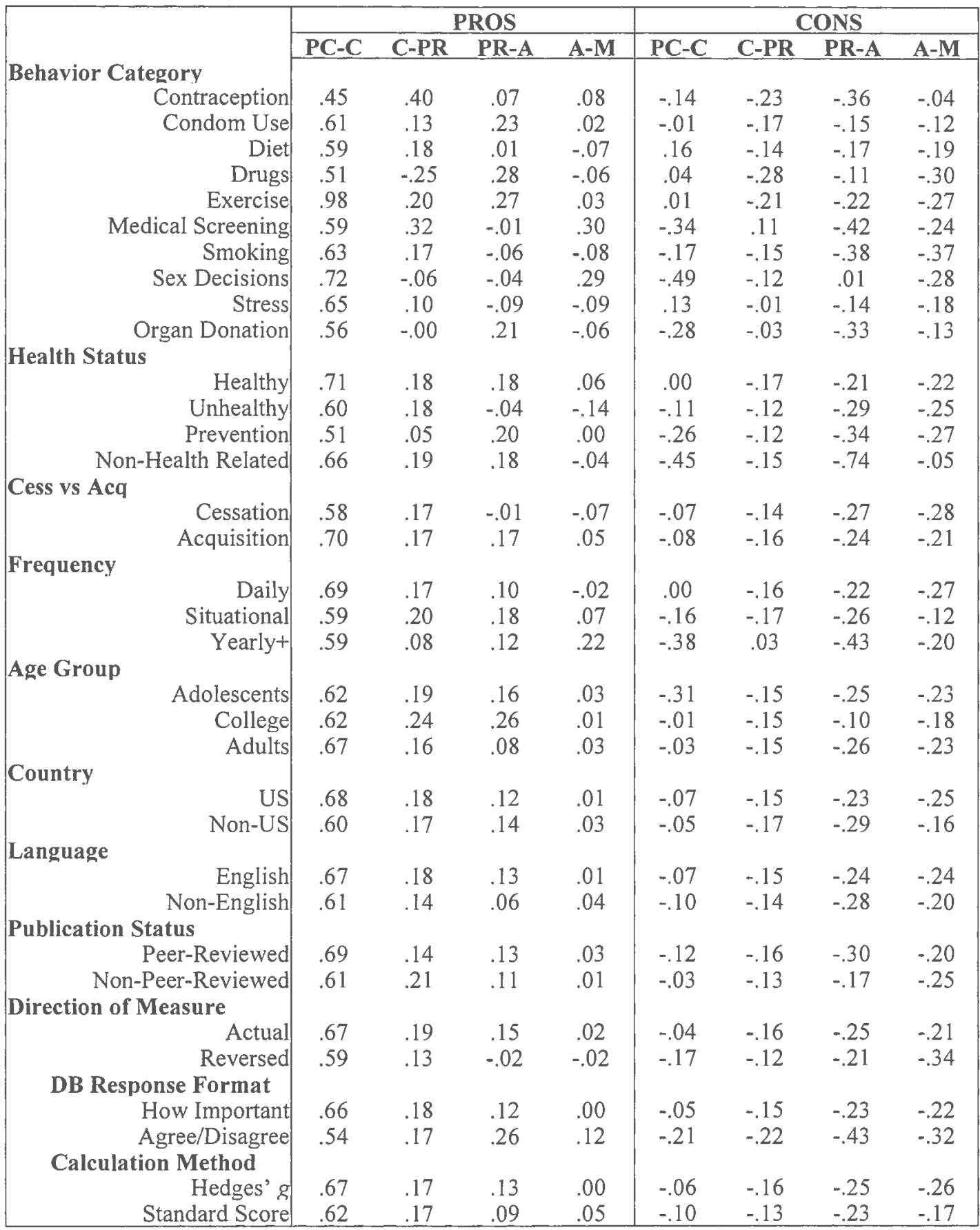




\section{Discussion}

In examining Decisional Balance and Stages of Change for 55 different behaviors across a variety of populations, several common characteristics were found. Structure, function and generalizability of the Stages of Change and Decisional Balance measures as well as the magnitude of effect and homogeneity for effect size distributions across Stage transitions for the Pros and Cons are discussed.

\section{Structure}

The internal validity of the two-factor structure of Pros and Cons of Change identified by Velicer et al. (1985) was maintained for $96 \%$ of the datasets, which is consistent with the $92 \%$ found by Prochaska et al. (1994) and $94 \%$ found by Hall and Rossi (2004). Although a large percentage of the studies support a much simpler configuration than posited by Janis and Mann (1977) as Prochaska et al. (1994) suggests, it should be noted it is unclear whether the measurement development in each study includes a sufficient variety of items to test this comparison effectively.

One hundred twelve datasets included alpha coefficients as a measure of internal consistency. For Pros the average coefficient alpha was $.82(S D=.09)$, with a range from .58 to .96 , whereas the average coefficient alpha for Cons was a bit lower at $.76(S D=.10)$ and ranged from .41 to .94 . The internal consistency measures found in this study overall are slightly lower with ranges wider than previous studies. In comparison, Prochaska (1994) found measures of internal consistency ranging from .75 to .95 and Hall and Rossi (2004) ranging from .41 to .95 , whereas the current study found an overall range of .41 to .96 . The similarity 
between the current study and the study by Hall and Rossi (2004) is somewhat surprising since the current study has nearly twice as many studies. It is likely that future studies will find internal consistencies well within this range.

\section{Function}

Consistent patterns were found for Pros and Cons; for instance, in nearly all studies the Cons of Changing outweighed the Pros of Changing during the Precontemplation Stage. Additionally, the majority of crossovers occur by the Action Stage. Although often thought of as a simple function, the crossovers in this study revealed possible complexities suggesting further examination is needed. For example, $17 \%$ of the datasets revealed multiple intersections of the Pros and Cons. Additionally, many of the studies have large numbers of participants in one Stage and not another and these unequal distributions of sample size across the Stages may "pull" on the crossover resulting in a less stable pattern. Preliminary examinations of the use of effect sizes to graph crossovers reveal that the use of this procedure could help resolve this problem, especially in the context of meta-analytic studies when relying on secondary data. Although not pursued in the present study, further exploration of the ability of the effect size graphic procedure to correct this problem is needed. In particular, a comparison study of various weighted transformations of crossover graphs with the procedure used in this study is warranted. It appears the use of weighting procedures may help elucidate the apparent cross-over biases. 
Random and fixed effects models were fit to the effect size distributions for the Pros and Cons of Change across the Stage transitions. Statistically, for each of these analyses on the total sample, the random effect variance component was found to be significant. The significant random effects components for each of the Stage transitions for the Pros and Cons support the use of the random effects model. Theoretically, due the large variation on populations across the 146 datasets, a random effects modeling approach was warranted. Another important distinction between fixed and random effects models is the overall inferential goal. A fixed effects model aims to make inferences regarding the particular observed parameters whereas the random effects model aims to make inferences regarding the distribution of effects (Hedges \& Vevea, 1998). Therefore, the random effects model will enable greater generalization beyond the observed studies, which is an important goal of this study.

The decision to use a fixed versus random effects model is an important one, and was so especially in the context of this study. Table 31 and Table 32 show the pattern of results for the primary moderators using fixed effects versus random effects modeling. Between-group heterogeneity is displayed for the major moderator categories (in bold) and within-group heterogeneity is displayed for the sub-groups (non-bolded text). It is clear that the pattern effects are distinct between the two models. A much larger percentage of heterogeneity is identified with the fixed effect model than the random effects model. Overall, this pattern of effects is as predicted. Increased standard error in the random effects model produces wider confidence 
intervals, therefore in the context of hypothesis-testing there is a greater likelihood of retaining the null with random effects relative to fixed effects. In sum, a random effects model is theoretically most consistent with the inferential goals for this study and was statistically supported. 
Patterns of Fixed and Random Effect Results across Moderator Variables for Pros

\begin{tabular}{|c|c|c|c|c|c|c|c|c|}
\hline & \multicolumn{8}{|c|}{ PROS } \\
\hline & \multicolumn{4}{|c|}{ Fixed Effects } & \multicolumn{4}{|c|}{ Random Effects } \\
\hline & PC-C & C-PR & PR-A & A-M & PC-C & C-PR & PR-A & A-M \\
\hline Behavior Category & + & + & + & + & + & & + & \\
\hline $\begin{array}{r}\text { Contraception } \\
\text { Condom Use }\end{array}$ & * & & & $*$ & & & & \\
\hline Diet & * & & * & * & & & & \\
\hline Drugs & & & & & & & & \\
\hline Exercise & * & * & * & * & & * & & \\
\hline Medical Screening & $*$ & & & & * & & & \\
\hline Smoking & * & $*$ & * & $*$ & & & $*$ & \\
\hline Sex Decisions & * & & * & * & * & & & \\
\hline $\begin{array}{r}\text { Stress } \\
\text { Organ Donation }\end{array}$ & & & & $*$ & & & & * \\
\hline Health Status & + & & + & + & & & + & + \\
\hline Healthy & * & * & * & * & & & & \\
\hline Unhealthy & * & * & * & * & & & & \\
\hline & * & * & & & & & & \\
\hline Prevention & & * & & - & & & & - \\
\hline Other & * & & & - & & & & - \\
\hline Cess vs Acq & + & & + & & + & & + & \\
\hline Acquisition & * & * & $*$ & * & & & & \\
\hline Cessation & $*$ & $*$ & * & * & & & $*$ & \\
\hline Frequency & & + & & + & & & & \\
\hline Daily & * & $*$ & * & * & & & & \\
\hline Situational & * & $*$ & & * & & & & \\
\hline Yearly+ & * & & & & & & & \\
\hline Age Group & & & + & + & & & & \\
\hline Adolescents & * & * & * & * & & & & \\
\hline College & * & * & & $*$ & & & & \\
\hline Adults & * & $*$ & * & * & & & & \\
\hline Country & + & & & & & & & \\
\hline US & * & * & * & * & & & & \\
\hline Non-US & * & & $*$ & * & & & & \\
\hline Language & + & & & & & & & \\
\hline English & * & * & * & * & & & & \\
\hline Non-English & * & * & & * & & & & \\
\hline Publication Status & + & & & & & & & \\
\hline Peer-Reviewed & * & * & * & * & & & & \\
\hline Non-Peer-Reviewed & $*$ & $*$ & & * & & & & \\
\hline Direction of Measure & + & & + & & & & + & \\
\hline Actual & * & * & $*$ & * & & & & \\
\hline Reversed & $*$ & $*$ & $*$ & * & & & * & \\
\hline DB Response Format & & & & + & & & & \\
\hline How Important & * & * & $*$ & * & & & & \\
\hline Agree/Disagree & $*$ & & & $*$ & & & & \\
\hline Calculation Method & & & & & & & & \\
\hline Hedges' g & * & * & * & * & & & & \\
\hline Standard Score & $*$ & $*$ & $*$ & * & & & & \\
\hline
\end{tabular}

Note. + indicates significant between group heterogeneity; $*$ indicates significant within group heterogeniety 
Patterns of Fixed and Random Effect Results across Moderator Variables for Cons

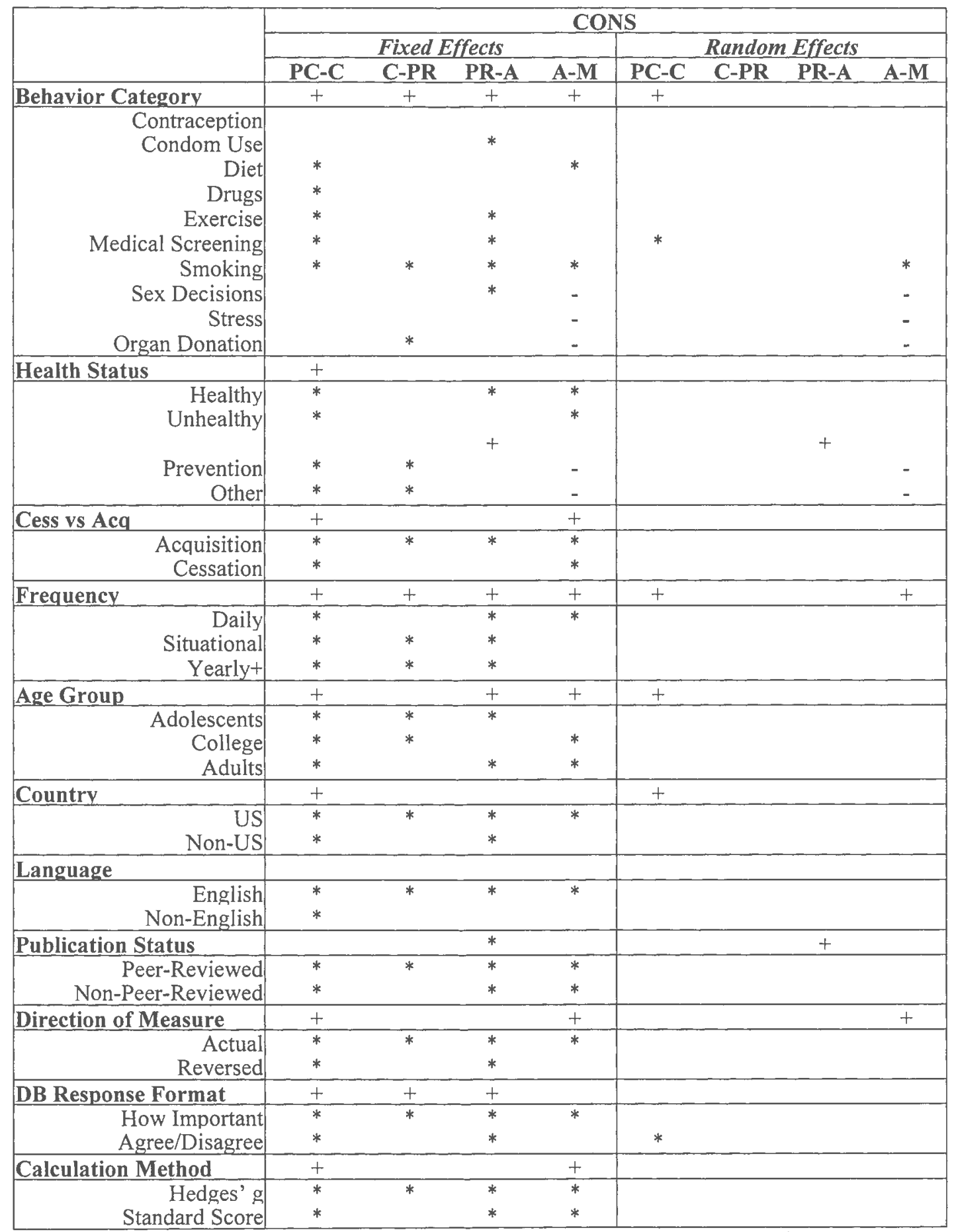

Note. + indicates significant between group heterogeneity; * indicates significant within group heterogeniety 
The largest effect size average was found for the Pros of Change, specifically in the transition between the Precontemplation and Contemplation Stages. The magnitude of this effect was .65 standard deviations. Additionally, smaller increases were seen in transitions from Contemplation to Preparation (Hedges' $g=.17$ ) and Preparation to Action $(g=.12)$, with essentially no effect found from Action to Maintenance $(g=.02)$. The Cons of change showed an inverse pattern across transitions, though with overall smaller effect sizes. Essentially no effect was found from Precontemplation to Contemplation $(g=-.08)$, with relatively small decreases in Cons from Contemplation to Preparation $(g=-.15)$, Preparation to Action $(g=-$ $.24)$, and Action to Maintenance $(g=-.23)$. These results suggest interventions may provide the greatest impact with maximum efficiency by placing the most emphasis on Pros during the Precontemplation Stage, followed by additional efforts during the Contemplation and Preparation Stages. In contrast, Cons are most likely to be useful during the Preparation Stage with continued emphasis, somewhat surprisingly, into the Action Stage.

The Strong and Weak Principles of Change have shown approximately 1 standard deviation increase in the Pros of Change as one progresses from Precontemplation to Action, whereas the Weak principle is approximately a $1 / 2$ standard deviation (Prochaska, 1994) or .62 SD (as found by Hall \& Rossi, 2004) decrease in the Cons of Change as one progresses from Precontemplation to Action). Since the Strong and Weak Principles measure the maximum increase and decrease of the Pros and Cons from Precontemplation to Action, rather than an absolute 
difference, these two principles are "biased". That is, the principles result in a potential over-estimation of the cumulative effect size across the three transitions PC-C, C-PR, and PR-A. Therefore it was predicted that $(\mathrm{PC}-\mathrm{C})+(\mathrm{C}-\mathrm{PR})+(\mathrm{PR}-\mathrm{A})<$ $1 S D$ (standard deviation) for Pros and (PC-C) + (C-PR) + (PR-A) <.62 SD for Cons. Based on the overall effect size estimates across the Stage transitions this prediction was not entirely supported. The total cumulative effect size for Pros $=.96$ and the cumulative effect for Cons $=.70$. Although the total for the Pros of Change was just slightly below $1 S D$ the Cons of Change was actually greater than .62 SD.

In general, use of effect sizes to assess the magnitude of Stage transitions can provide guidance in the development of tailored interventions by suggesting how much intervention resources should be allocated for each construct at each Stage transition. But most importantly, in order to best utilize effect size estimated for intervention purposes, detailed analyses of moderators of heterogeneity is essential for identifying where and when these effect sizes may vary.

\section{Heterogeniety}

As predicted, the overall effect size distributions for each of the Stage transitions for both the Pros and Cons were found to be heterogeneous. This warranted investigation of potential moderators of effect size distribution. Graphical and statistical approaches were used to explore moderators.

\section{Calculation Method}

No significant differences were found between the two primary calculation methods. This finding provides additional support for the effectiveness of the 
adjustment for calculation method developed in Chapter 5. The regression adjustment for calculation enabled $30 \%$ of the studies to be retained in this metaanalyses that otherwise would have been excluded. Overall, this increased the power of the study and allowed more thorough moderator analyses to be conducted.

\section{Target Behaviors and Behavior Categories}

The overall heterogeneity of the distribution (found for the effect sizes for both the Pros and Cons for each of the Stage transitions) signified that modifiers of magnitude were likely present across the datasets. The most apparent potential moderator of effect size was thought to be behavior.

Due to the large number of target behaviors represented by only one or two studies, analyses by target behaviors were limited. Additionally, an important consideration for the interpretation of magnitude across moderators, in relation to the small target behavior sample sizes, is the potential for differential impact of unequal representation of moderators. For example, in this meta-analysis 21 datasets investigated exercise, yet only one study examined calcium intake. Exercise studies were generally found to have greater magnitudes than other behaviors, specifically in the PC-C and PR-A transitions (see Figure 6), and therefore a disproportionate number of exercise studies in an alternate moderator analysis, such as publication status, could create an upward bias in a given subset for these transitions. Therefore unequal weighting of moderators such as behaviors may influence differences between behaviors on the results of a given dimension of the constructs.

Behavior categories were created to aggregate similar behaviors together to enhance the ability to conduct sub-group analyses. Preliminary descriptive analyses 
were performed on the ten behavior categories with more than 3 datasets. In general, the mean effect sizes for the behavior categories did not vary greatly from the overall means (for all 146 datasets) for the Pros and Cons of each Stage transition. Fifty percent of the mean effect sizes for the behavior categories varied less than .1 SD from their respective Stage transition mean. No mean effect size was found to be larger than $.42 S D$ from the given mean for the Stage transition. The largest difference in means between each of the behavior categories was $.65 \mathrm{SD}$. These relationships can be seen graphically in the forest plots above (see Figure 6 and Figure 7). The means and confidence intervals are plotted for each behavior. The axis for each transition is set at the overall mean for the study (i.e., mean for all 146 datasets).

Five behavior categories had sample sizes of greater than five. These five behaviors (condom use, diet, exercise, smoking, stress) were included in analog ANOVA analyses to assess differences among the behaviors. Significant differences between behavior categories were only found for the Pros of Change. These differences were in the transitions between PC-C and PR-A. Exercise was found to be significantly greater than each of the four other behavior categories for PC-C and significantly greater than smoking and diet in the PR-A transition. Interestingly, Hall and Rossi (2004) found exercise to have the largest magnitude of effect from Precontemplation to Action for the Pros of Change in comparison to other behavior categories examined (specifically diet, smoking, and condom use). It appears that instead of having larger effects across all stage transitions, the differences for exercise are in the PC-C and PR-A transitions. One important consideration is the 
potential difference in algorithms found in the exercise studies. Marcus et al. (1992) used a measure called the Contemplation Ladder. This measure was subsequently used by other researchers. Future studies should compare effect size differences between these two types of algorithms. Lastly, condom use was found to be significantly greater than smoking and stress in the transition from PR-A for Pros.

Within-group heterogeneity was assessed for each of the behavior categories to determine if variation within the categories existed. Of the 45 heterogeneity tests, only 2 were found to be significant: the distribution of effect sizes for the C-PR transition of exercise and the PR-A transition for smoking. This indicates that the overall behavior categories are homogeneous and likely few moderators are present within the behavior categories.

Although it is beyond the scope of this study to conduct full moderator analyses of each of these behaviors, a priori sub-groups analyses were conducted and variety of comparisons explored. Firstly, since the smoking behavior category was primarily made up smoking acquisition and smoking cessation, these two behaviors were compared. Smoking acquisition and cessation were in fact found to be different in the PR-A Stage transition. Interestingly, there were also differences found between these two behaviors in the PC-C Stage transition. In efforts to understand these differences, general differences between these behaviors were considered. For instance, the all smoking acquisition studies were conducted with adolescents. Since all smoking acquisition studies were all conducted with adolescent samples, comparisons between age groups for the smoking category were not possible. In the larger study context, when all studies were combined, no differences were found 
between age groups; therefore it is believed that age is not moderating this effect.

Clearly more research is needed to understand the influence on age since age is nested in these analyses.

Unlike smoking cessation, smoking acquisition is a prevention behavior. That is, by examining behavior change in smoking acquisition one is looking at "changing" a behavior that does not yet exist. Interestingly, when comparing the composite profile of smoking acquisition (Figure 34) with the composite profile of all the prevention behaviors (Figure 35) identified in this study, we can see a large difference between the Pros and Cons at Precontemplation with the Pros decreasing and the Cons increasing. A somewhat flat profile is seen between C-PR. Finally a flip flopped pattern for PR-A can be seen -- with the Pros strongly increasing and the Cons strongly decreasing. A similar pattern is also seen for behaviors that are infrequently performed ("yearly" behaviors) (Figure 36). The conceptual link between infrequent or "yearly" behaviors and acquisition or prevention behaviors is the overall minimal activity or engagement an individual has in the behavior of interest. This may have an influence on the relevance of the ContemplationPreparation process as currently defined by the model for such behaviors. 


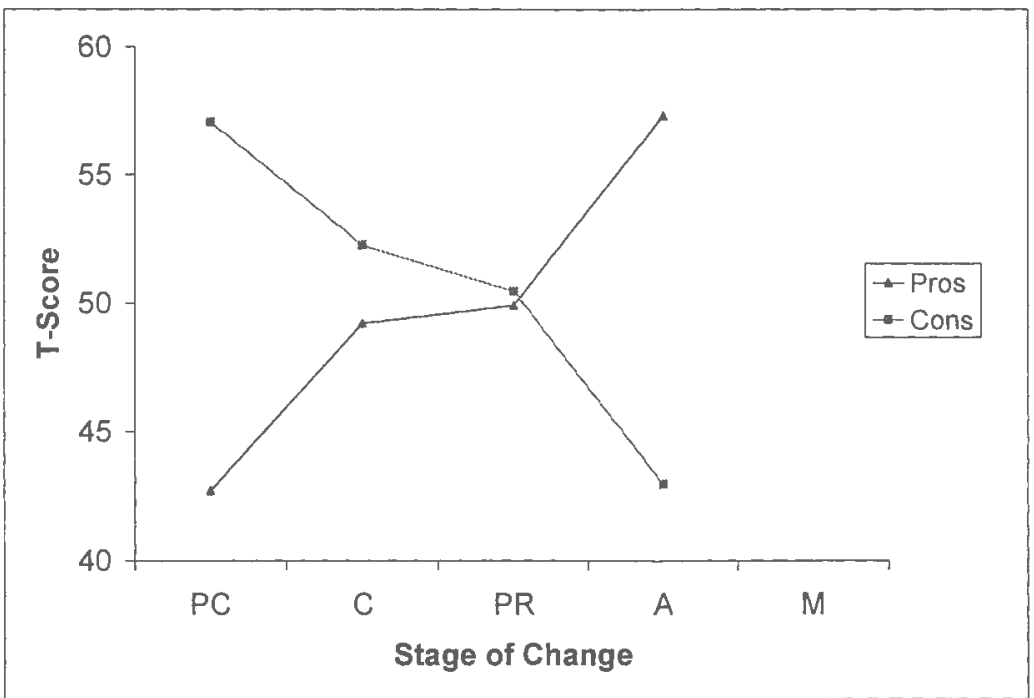

Figure 34. Composite Profile for Smoking Acquisition. 


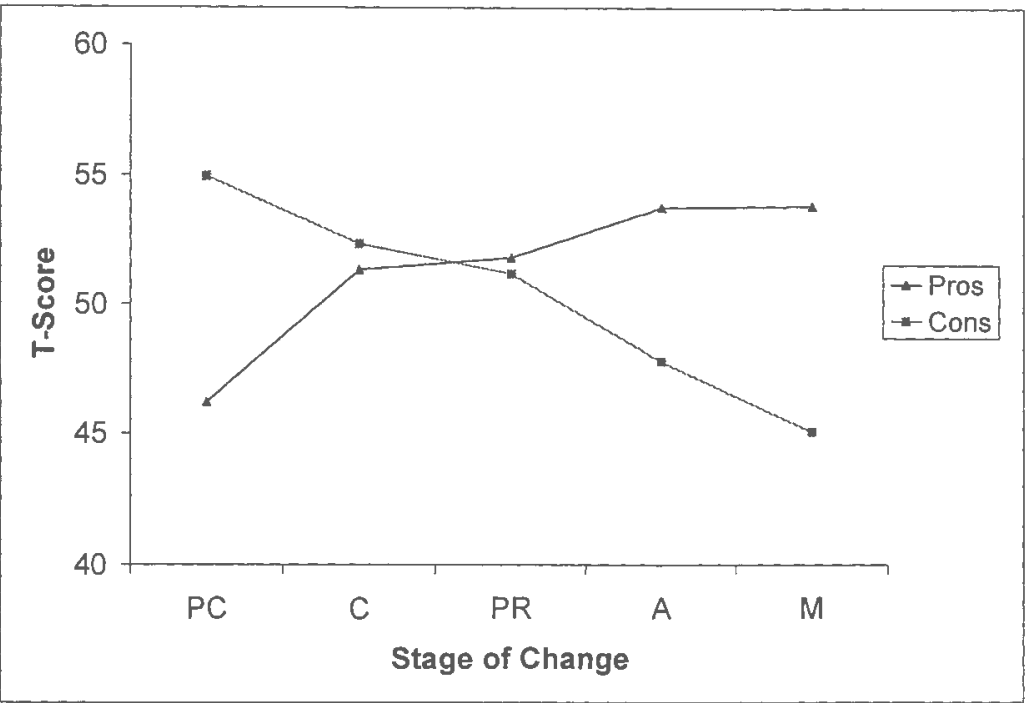

Figure 35. Composite Profile for Prevention Behaviors.

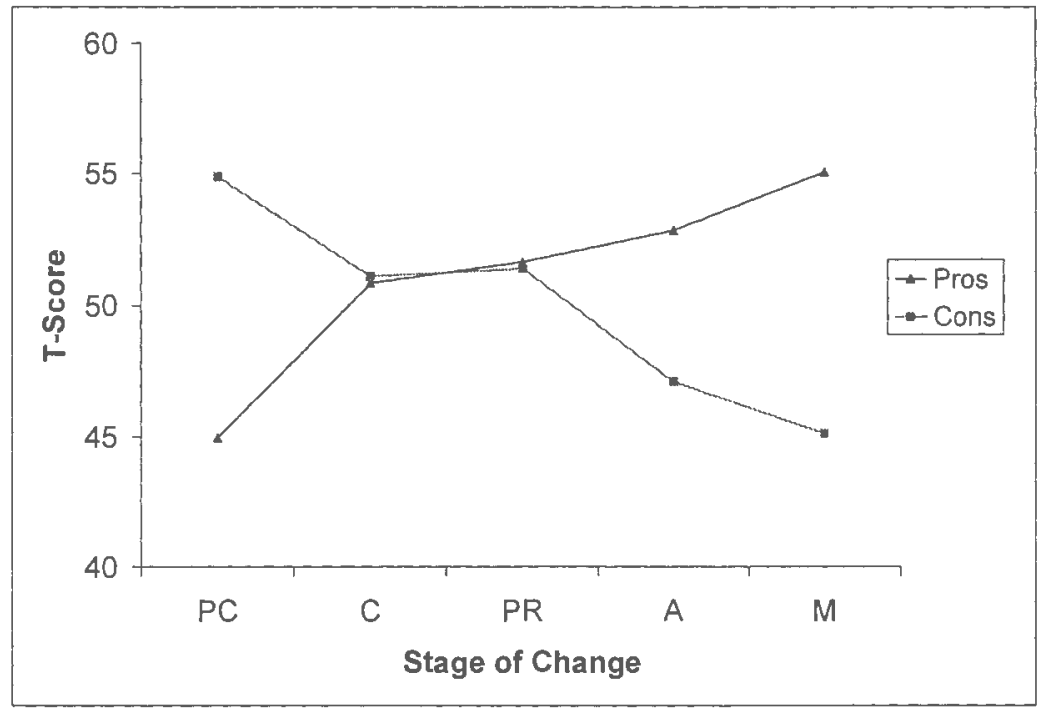

Figure 36. Composite Profile of "Yearly" behaviors.

More simply, the overall pattern of differences between smoking acquisition and smoking cessation were consistent with the findings of the differences between acquisition (e.g., exercise) and cessation (e.g., quitting drugs) behaviors across all the 
datasets. As with smoking, comparisons revealed significant differences for the PC$\mathrm{C}$ and PR-A transitions.

Smoking cessation was examined more closely in efforts to determine possible differences between the use of different Pros and Cons measures when examining smoking cessation. Some studies examine the Pros and Cons of Smoking whereas others examine the Pros and Cons of Quitting Smoking. This difference can be somewhat complicated. Firstly, in order to create conceptual consistency in the Pros and Cons with other behaviors, the Pros and Cons of Smoking are reversed such that the Pros of Smoking (which typically decrease across the stages) are depicted as the "Cons" and the Cons of Smoking are depicted as "Pros". The importance of this change in the context of averaging effect sizes across studies comes into play with the directionality of the parameter estimate. Effect sizes combined that are representing the opposite direction will wash each other out and in essence "average" zero. Additionally, graphically this reversal aids in visual comparisons across studies. But this reversal is certainly of theoretical interest and therefore has been examined within smoking cessation as well as across studies. Across all studies the comparison of effect sizes based on the "actual" direction of the Pros and Cons versus those Pros and Cons that have been "reversed" revealed significant differences for two Stage transitions (PR-A for Pros and A-M for Cons). For smoking, significant differences were found for the Cons of Change in the PC-C and A-M transitions, with smaller effect sizes for these transitions identified for the Cons of Quitting. 
Another a priori behavioral comparison included the behavior category of

condom use. Condom use was comprised of three primary sub-groups, condom use in general, for main partner, and for "other" partner. Condom use in general and condom use with main partner were found to be significantly greater than condom use with "other" for the C-PR transitions for Pros. Interestingly, this difference creates a composite profile for condom use with "other" that is similar to infrequently or "yearly" performed behaviors (as seen in Figure 36). In these profiles the Pros and Cons appear to have essentially the same $T$-scores and show essentially no change from C-PR. This similarity of pattern could be attributed to the fact that condom use with an "other" or secondary partner may be as infrequent as other "infrequent" behaviors.

The final a priori behavioral comparison was between the two diet subgroups, dietary fat reduction and fruit and vegetable consumption. No significant differences between these two groups were found.

In addition to the a priori behavioral comparisons and the moderators discussed above, several other moderators were explored. Velicer et al. (2000) illustrated that the relationship between the Stages and Decisional Balance for an unhealthy behavior is different than for a healthy behavior. That is the pattern for an unhealthy behavior was such that the Cons decreased across the Stages whereas the Pros displayed a curvilinear pattern, which paralleled the decline of the Cons in the later Stages. In contrast, healthy behaviors showed more of an X configuration, with the Pros continuing to increase across the Stages whereas the Cons decrease across the Stages. This finding was replicated in the current study. Unhealthy behaviors 
showed a significant decrease in the Pros of Change in the PR-A and A-M transition, producing the curvilinear pattern Velicer et al. (2000) describes.

Two response formats were used for the Pros and Cons measures in the majority of studies. Significant differences were only found for the Pros of Change in the PR-A. No significant differences were found for the Cons of Change. It is unclear what this difference indicates, but it would be warranted to explore these differences further. Unfortunately, the majority of the studies utilized the How Important format which may have influenced the degree of significance found between the two.

\section{Generalizability.}

Overall, the generalizability of the Transtheoretical Model across a variety of populations was supported with this large set of studies examining Stage of Change, Pros and Cons, and the relationship between them. A variety of moderators were explored but most notably, no significant differences in effect size distributions were found across the Stage transitions for country or language. These homogeneous findings lend important credence to the generalizability of the observed constructs. Although an attempt was made to examine demographic variables within studies of a particular behavior, these analyses were plagued by small sample sizes. Therefore as more studies emerge, it will be necessary to continue to look at the Transtheoretical constructs for particular demographic variables across behaviors. The populations varied greatly across studies and although many subject characteristics were examined as moderator variables, the investigation of some of these sample 
characteristics (e.g., gender) are best examined using longitudinal techniques and therefore interpretation should be made with caution. It is important that population characteristics continue to be examined to make a more definitive statement of generalizability.

Prochaska et al. (1994) originally found support for the strong generalizability of the TTM for Stage of Change and Pros and Cons across 12 behaviors. Hall and Rossi (2004) provided additional support for the generalizability of these constructs across 37 Behaviors. The current study continues to provide support for the generalizability of the model for a growing number of behaviors, 55 behaviors in total. Although there is strong support on the whole for the generalizability of the Transtheoretical Model and the integration of the Decisional Balance measures across the Stages of Change, there were some study characteristics that should be examined more closely to assess potential limitations of the model or necessary adaptations of the constructs. For instance, when averaged, behaviors identified as infrequent or "yearly" (e.g., mammography) seemed to show an overall lack of change between C-PR (see Figure 36) for both the Pros and Cons. In this case, the typical staging (e.g., time frame) for Contemplation and Preparation may not be sufficient. It will be important in the future to assess the relationships of other TTM constructs across the Stages for moderators such as frequency of behavior (i.e., "yearly") in order to establish if these relationships persist before making clear statements regarding generalizability. That is, if this pattern of essentially no change for the Pros and Cons from Contemplation to Preparation for behaviors that are performed "yearly" is also found for other constructs, such as Self-efficacy and 
Processes, this would give rise to the concern to the generalizability of

Contemplation and Preparation Stages for behaviors that infrequently occur. It is clear that further research is needed to more clearly identify such patterns.

Table 33 provides an overview of the between-group heterogeneity across the moderator variables. Although overall generalizability for the model was found, it is clear that a variety of characteristics can influence the degree of change in the Pros and Cons during a particular stage of the change process. These results provide a guide for where future studies can focus in order to establish more definitive relationships between variables and establish fine-grained analyses of these nuances.

Table 33

Between Groups Heterogeneity for Primary Moderators

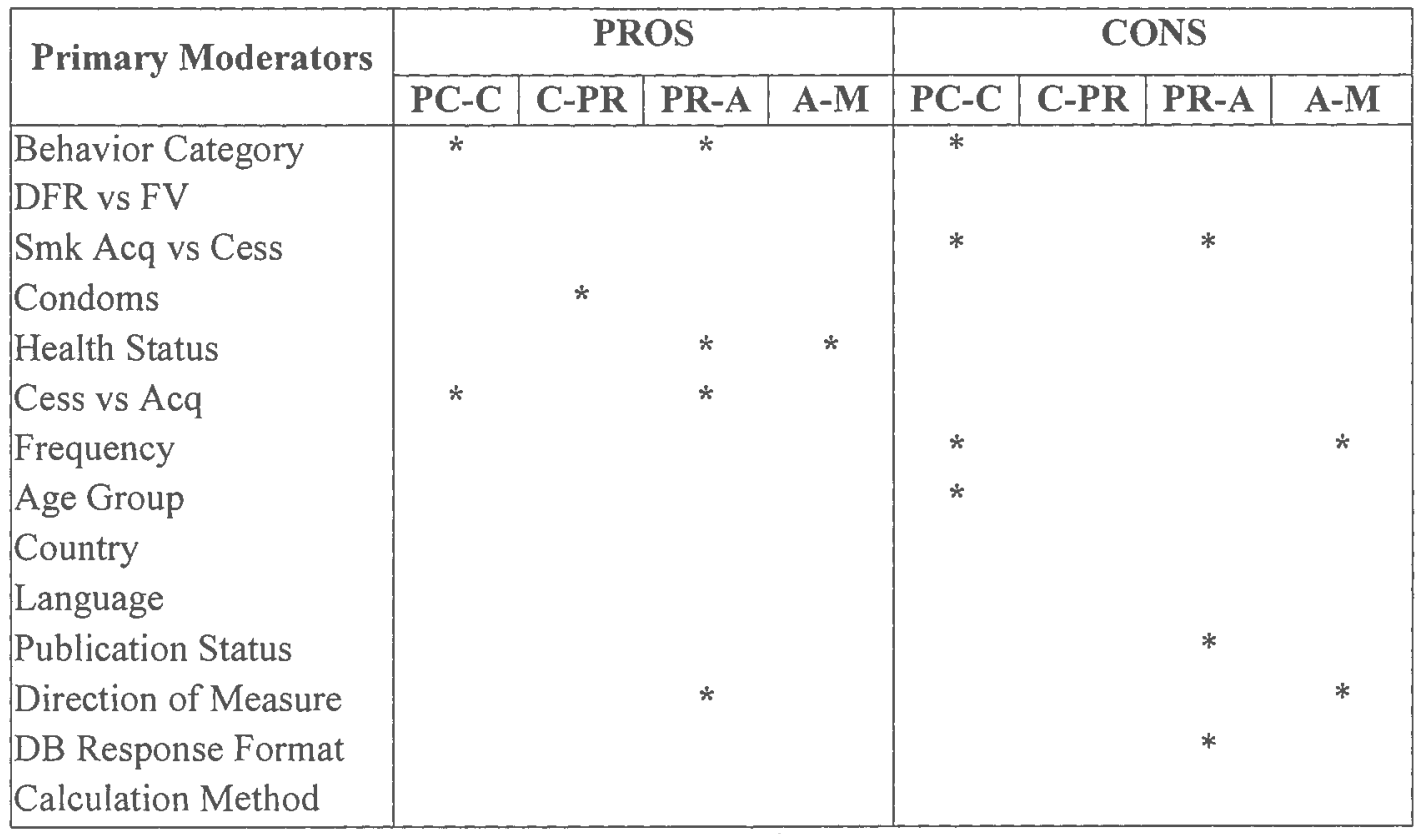

Note. Asterisks indicate significant between-group differences. 
In sum, the results suggest interventions may provide the greatest impact with maximum efficiency by placing the most emphasis on increasing the Pros during the Precontemplation Stage, followed by additional efforts during the Contemplation and Preparation Stages. In contrast, efforts to decrease the Cons are most likely to be useful during the Preparation Stage with continued emphasis into the Action Stage. Contrary to prediction, these results are only partly consistent with previous studies, which agrue that Decisional Balance is especially salient in the earlier Stages of Change (Redding et al., 1999; Velicer et al., 2000). Instead it appears that Pros of Change are more salient in the earlier Stages whereas the Cons of Change are more salient in the later Stages. Future studies examining moderator variables in greater detail can help more definitively determine the amount intervention resources should be allocated for each construct at each Stage transition in each behavior providing continued guidance in the development of tailored interventions.

Although a large number of studies overall were included in this metaanalysis, small sample sizes often precluded meaningful analyses of moderators. Continued efforts to gather studies to increase these sample sizes should be made. The primary analyses conducted in the study included ANOVA analogs to compare moderator subgroups. In addition to ANOVA analogs, one can employ the use of meta-regression to examine the association of effect among study characteristics. Meta-regression is a more sophisticated approach to assessing moderators when heterogeneity is observed (Song, Sheldon, Sutton, Abrams, Jones, 2001). Future studies should examine moderators using meta-regression in order to determine overall which moderators contribute to the variance in the effect size distributions. 
All datasets utilized cross-sectional data; therefore although the effect sizes

were exploring the increase and decreases across the Stage transitions between participants in various Stages, it cannot be assumed the same relationships will necessarily occur when examining the movement of participants from one Stage to another with longitudinal data. Therefore, it is important these results be compared to the examination of these relationships using longitudinal data.

\section{References}

References marked with an asterisk indicate studies included in the metaanalysis.

*Acton, G.S., Prochaska, J.J., Kaplan, A.S., Small, T., \& Hall, S.H. (2001).

Depression and stages of change for smoking in psychiatric outpatients. Addictive Behaviors, 26, 621-631.

*Ahijevych, K., \& Parsley, L.A. (1999). Smoke constituent exposure and stage of change in black and white women cigarette smokers. Addictive Behaviors, 24(1), $115-120$.

*Bane, C.M., Ruggiero, L., Dryfoos, J.M., \& Rossi, J.S. (1999). Development of a pregnancy tailored decisional balance measure for smoking cessation. Addictive Behaviors, 24(6), 795-9.

*Banikarim, C., Chacko, M.R., Wiemann, C.M., \& Smith, P.B. (2003). Gonorrhea and Chlamydia screening among young women: Stage of change, decisional balance, and self-efficacy. Journal of Adolescent Health, 32, 288-295.

*Basler, H., Jaekle, C., Keller, S., \& Baum, E. (1999). Selbstwirksamkeit, Entscheidungsbalance und die Motivation zu sportlicher Aktivitaet. [Self- 
efficacy, decisional balance and the motivation for physical activity]. Zeitschrift fuer Differentielle und Diagnostische Psychologie, 20(3), 203-216.

*Block, L.G., \& Keller P.A. (1998). Beyond protection motivation: An integrative theory of health appeals. Journal of Applied Social Psychology, 28(17), 15841608.

*Borland R., \& Segan C. (2000). Testing the Transtheoretical Model for smoking change: Victorian data. Australian Journal of Psychology, 52(2), 83-88.

Borenstein, M., \& Rothstein, H. (1999). Comprehensive meta-analysis: A computer program for research synthesis [Computer software manual]. Englewood, NJ: Biostat, Inc.

*Boudreaux, E., Carmack, C.L., Scarinci, I.C. \& Brantley, P. J. (1998). Predicting smoking stage of change among a sample of low socioeconomic status, primary care outpatients: Replication and extension using decisional balance and selfefficacy theories. International Journal of Behavioral Medicine, 5(2), 148-165.

*Buckworth, J., \& Wallace, L.S. (2002). Application of the transtheoretical model to physically active adults. Journal of Sports Medicine and Physical Fitness, 42, $360-367$.

*Callaghan, P., Eves, F.F., Norman, P., Chang, A.M., \& Lung, C.Y. (2002). Applying the transtheoretical model of change to exercise in young Chinese people. British Journal of Health Psychology, 7, 267-282.

${ }^{*}$ Chamot, E., Charvet, A.I., \& Perneger, T.V. (2001). Predicting stages of adoption of mammography screening in a general population. European Journal of Cancer, 37, 1869-1877. 
*DiClemente, C.C., Prochaska, J.O., Fairhurst, S.K., Velicer, W.F., Velasquez, M.M., \& Rossi, J.S. (1991). The process of smoking cessation: An analysis of Precontemplation, Contemplation and Preparation stages of change. Journal of Consulting and Clinical Psychology, 59, 295-304.

*Dijkstra, A., De Vries, H., \& Bakker, M. (1996). Pros and cons of quitting, selfefficacy, and the stages of change in smoking cessation. Journal of Consulting and Clinical Psychology, 64 (4), 758-763.

*Dijkstra, A., De Vries, H., Roijackers, J. \& Van Breukelen, G. (1998). Tailored interventions to communicate stage-matched information to smokers in different motivational stages. Journal of Consulting and Clinical Psychology, 3, 549-557.

*Dryfoos, J.M. (1996). Staging and decisional balance measures for two diabetes self-management behaviors. Unpublished master's thesis, University of Rhode Island, Kingston.

*Eaton, C., Goldstein, M.G., Guadagnoli, E., Niaura, R., McDonald, N., \& Dube, C. (1992). Development of a decisional balance measure of physician smoking cessation intervention. Manuscript submitted for publication.

*Evers, K.E., Johnson, J.L., Padula, J., Prochaska, J., \& Prochaska, J.O. (2003). Stress management measure development for transtheoretical constructs of decisional balance and self-efficacy. Manuscript in Preparation.

*Evers, K.E., Johnson, J.L., Prochaska, J.O., \& Prochaska, J.M. (Jan., 2001). Doncaster Project Measurement Development Report: Application of the Transtheoretical Model to Pregnancy Prevention. West Kingston, RI: Pro-Change Behavior Systems, Inc. 
*Evers, K.E., Johnson, J.L., Ward, R.M., Padula, J., Prochaska, J.O., \& Prochaska, J.M. (May 2001). European drugs initiative: Measurement development and data analysis report. West Kingston, RI: Pro-Change Behavior Systems.

*Fahrenwald, N.L., \& Walker, S. N. (2003). Application of the transtheoretical model of behavior change to the physical activity behavior of WIC mothers. Public Health Nursing, 20(4), 307-317.

*Fava, J.L., Velicer, W.F., \& Prochaska, J.O. (1995). Applying the transtheoretical model to a representative sample of smokers. Addictive Behaviors, 20(2), 189203.

*Frenn, M., Malin, S., \& Bansal, N.K. (2003). Stage-based interventions for low-fat diet with middle school students. Journal of Pediatric Nursing, 18(1), 36-45.

*Galvotti, C., Cabral, R., Lansky, A., Grimley, D., Riley, G., \& Prochaska, J. O. (1995). Validation of measures of condom and other contraceptive use among women at high risk for HIV infection and transmission: Stage of change, decisional balance, and self-efficacy. Health Psychology, 14, 570-578.

*Gazabon, S.A. (2000). An assessment of decisional balance, self efficacy, and condom stages of change among white and minority women. Unpublished doctoral dissertation, University of Rhode Island, Kingston.

${ }^{*}$ Giebel, M. (1999). Diagnostik der Motivation zur Verhaltensänderung kardiovaskulären Risikoverhaltens bei Bundeswehrsoldaten unter Berücksichtigung des transtheoretischen Stufenmodells der Verhaltensänderung nach Prochaska [Diagnostics of the motivation to change cardiovascular risk behaviors in professional soldiers based on Prochaska's Transtheoretical Model 
of Behavior Change]. Unpublished doctoral dissertation, University of Marburg, Marburg, Germany.

*Grimley, D.M., Prochaska, J.O., Velicer, W.F., \& Prochaska, G.E. (1995). Contraceptive and condom use adoption and Maintenance: A stage paradigm approach. Health Education Quarterly, 22(1), 20-35.

Hall, K.L., \& Rossi, J.S. (2003, August). Meta-Analysis of Three Health Behaviors: Stage Transitions and Decisional Balance. Poster presented at the 111th annual meeting of the American Psychological Association, Toronto, Canada.

Hall, K.L., \& Rossi, J.S. (2004). Structure, function, and effect size of decisional balance across the stages of change for 37 health behaviors: A meta-analytic study. Manuscript in preparation.

*Happel, L. (2002). Motivationale Aspekte des Ernährungsverhaltens: Eine Studie im Bereich des Obst-und Gemüseverzehr [Motivational aspects of dietary behavior: A study in the context of fruit and vegetable intake]. Unpublished thesis, Department of Psychology, Marburg, Germany.

Hedges, L.V. (1981). Distribution theory for Glass's estimator of effect size and related estimators. Journal of Educational Statistics, 6, 107-128.

Hedges, L. V. \& Pigott, T. D. (2001). The power of statistical tests in meta-analysis. Psychological Methods, 6, 203-217.

Hedges, L. V., \& Olkin, I. (1985). Statistical methods for meta-analysis. Orlando, FL: Academic Press.

Hedges, L.V. \& Vevea, J.L. (1998). Fixed-and random-effects models in metaanalysis. Psychological Methods, 3(4), 486-504. 
*Hempelmann, J. (2003). [Using the transtheoretical model to examine toothfriendly diets]. Unpublished raw data.

*Henderson, L. (1999). Applying the transtheoretical model to breast selfexamination. (Doctoral dissertation, University of Nebraska). Dissertation Abstracts International: Section B: The Sciences \& Engineering, 59(7-B), 3757.

*Herrick, A.B., Stone, W.J., \& Mettler, M.M. (1997). Stages of change, decisional balance, and self-efficacy across four health behaviors in a worksite environment. American Journal of Health Promotion, 12(1), 49-56.

*Herzog, T.A., Abrams, D.B., Emmons, K.M., Linnan, L.A., \& Shadel, W.G. (1999). Do processes of change predict smoking stage movements? A prospective analysis of the transtheoretical model. Health Psychology, 18(4), $369-375$.

*Hulton, L. J. (2001). Application of the transtheoretical model of change to adolescent decision-making. Issues in Comprehensive Pediatric Nursing. Special Issue, 24(2), 95-115.

Janis, I.L. (1959). Decisional conflicts: A theoretical analysis. Journal of Personality and Social Psychology, 31, 8-33-839.

Janis, I. L., \& Mann, L. (1977). Decision making: A psychological analysis of conflict, choice and commitment. New York: Free Press.

* Jeffries, S.K. (2001). The role of major and minor stressors in moderating the transtheoretical model of exercise behavior among predominantly low-income patients attending primary care clinics. (Doctoral dissertation, Louisiana State 
University). Dissertation Abstracts International: Section B: The Sciences \&

Engineering, 61(12-B), 6709.

*Johnson, J. L., Fava, J. L., Velicer, W. F., Monroe, A. D., \& Emmons, K. (2002).

Testing stage effects in an ethnically diverse sample. Addictive Behaviors, 27(4), 605-617.

*Johnson, J.L., Johnson, S.S., Wright, J.A, (2002a). Applying the transtheoretical model to calcium intake for Osteoporosis prevention [Abstract]. Annals of Behavioral Medicine, S24, 113.

*Johnson, J.L., Johnson, S.S., Wright, J.A, Prochaska, J.M., \& Prochaska, J.O. (2002b). Applying the transtheoretical model to bone density testing [Abstract]. Annals of Behavioral Medicine, S24, 113.

*Johnson, S.S., Wright, J.A., Johnson, J.L., Prochaska, J.O., \& Prochaska, J.M. (2002). Developing measures of transtheoretical model constructs for participating in decision-making about medications [Abstract]. Annals of Behavioral Medicine, S24, 154.

*Jordan, J.P. (2002). Development, validation, and factorial invariance of transtheoretical model measures for recovery from anorexia nervosa and bulimia nervosa. (Doctoral Dissertation, University of Rhode Island). Dissertation Abstracts International: Section B: The Sciences \& Engineering, 62 (9-B), 4267. *Jordan, P.J., Nigg, C.R., Norman, G.J., Rossi, J.S., \& Benisovich, S.V. (2002). Does the Transtheoretical Model need an additude adjustment? Integrating Attitude with Decisional Balance as predictors of Stage of Change for Exercise. Psychology of Sports and Exercise, 3, 65-83. 
*Katlin, E.N. (2001). Stages of change correlates for chemical abusers in out-patient treatment. (Doctoral dissertation, Temple University). Dissertation Abstracts International: Section B: The Sciences \& Engineering, 62(5-B), 2283.

*Keller, S. (1998). Zur Validität des Transtheoretischen Modells - Eine Untersuchung zur Veränderung des Ernährungsverhaltens [On the validity of the Transtheoretical Model - A study in the context of dietars behavior]. Unpublished doctoral dissertation, University of Marburg, Marburg, Germany.

* Keller, S., Giessel, H., Basler, H.-D. (2002). Flossing? No thank you!-Readiness to change oral hygiene behavior. International Journal of Behavioral Medicine, $31,56-67$.

*Keller, S., Herda, C., Ridder, K., \& Basler, H. (2001). Readiness to adopt adequate postural habits: An application of the transtheoretical model in the context of back pain prevention. Patient Education and Counseling, 42, 175-184.

*Keller, S., Kaluza, G., Baun, E., \& Basler, H.D. (2003). Stages of change, decisional balance and self-efficacy for stress management. Manuscript in Preparation.

*Keller, S., Kreis, J., \& Huck, C. (2001). Fünf am Tag? Motivationale und psychosoziale Aspekte des Obst-und Gemüseverzehrs [Five a day? Motivational and psychosocial aspects of fruit and vegetable consumption]. Zeitschrift für Gesundheitpsychologie, 9, 87-98.

*Keller, S., Nigg, C. R., Jaekle, C., Baum, E., \& Basler, H.D. (1999). Self-efficacy, decisional balance and the stages of change for smoking cessation in a German sample. Swiss Journal of Psychology - Schweizerische Zeitschrift fuer 
Psychologie - Revue Suisse de Psychologie. Special Issue: Smoking Behaviour, 58(2), 101-110.

*King, T.K., Marcus, B.H., Pinto, B.M., Emmons, K..M., \& Abrams, D.B. (1996). Cognitive-behavioral mediators of changing multiple behaviors: smoking and a sedentary lifestyle. Preventative Medicine, 25, 684-691.

*Kraft, P., Sutton, S.R., \& McCreath Reynolds, H. (1999). The transtheoretical model of behaviour change: Are the stages qualitatively different? Psychology and Health, 14, 433-450.

*Kremers, S.P.J., Mudde, A.N., \& DeVries, H. (2001). Subtypes within the precontemplation stage of adolescent smoking acquisition. Addictive Behaviors, $26,237-251$.

*Lauby, J. L., Semaan, S., Cohen, A., Leviton, L., Gielen, A., Pulley, L., Walls, C., \& O'Campo, P. (1998). Self-efficacy, decisional balance and stages of change for condom use among women at risk for HIV infection. Health Education Research, 13(3), 343-356.

*Lerner, C.F. (1990). The transtheoretical model of change: Self-change in adolescent deliquent behaviors. Unpublished master's thesis, University of Rhode Island, Kingston.

*Levesque, D. A., Prochaska, J. M., \& Prochaska, J.O. (1999). Stages of change and integrated service delivery. Consulting Psychology Journal: Practice \& Research, 5l(4), 226-241.

*Levesque, D.A, Redding, C.A., \& Prochaska, J.O. (1999, March). Stage of change and decisional balance for depression prevention among at-risk individuals. 
Paper presented at the annual meeting of the Society of Behavioral Medicine, San Diego, CA.

*Levesque, D.A. (1999). Violence desistance among battering men: Existing interventions and the application of the transtheoretical model of change. (Doctoral dissertation, University of Rhode Island). Dissertation Abstracts International: Section B: The Sciences \& Engineering, 59(8-B), 4449.

*Levesque, D.A., Dyment, S., \& Prochaska, J.M. (2000). Employee readiness for PeopleSoft at URI: Assessment results and recommendations. Kingston, RI: ProChange Behavior Systems.

Lewin, K. (1948). Resolving social conflicts: Selected papers on group dynamics. Gertrude W. Lewin (Ed.). New York: Harper.

Lipsey, M. W., \& Wilson, D. B. (2001). Practical meta-analysis. Thousand Oaks, CA: Sage.

*Lowry, C.R.S. (2000). Testing the transtheoretical model of change in young adult male smokeless tobacco users. (Doctoral dissertation, Texas Woman's University). Dissertation Abstracts International: Section B: The Sciences \& Engineering, 61(6-B), 2990.

*Ma J., Betts N.M., Horacek T., Georgiou C., White A., \& Nitzke S. (2002). The importance of decisional balance and self-efficacy in relation to stages of change for fruit and vegetable intakes by young adults. American Journal of Health Promotion, 16(3), 157-66.

*Maier, A. (2002). Eine Untersuchung zur Validatät des Transtheoretical Modells im Bereich sportlicher Aktivität [A study on the validity of the transtheoretical 
model in the area of physical activity]. Unpublished dissertation, Medical

Department, University of Marburg, Germany.

*Maleszka, M. (2002). Positive und negative strategien bei der veränderung des

Rauchverhaltens [Positive and negative processes of change for smoking

behavior]. Unpublished Thesis, Department of Psychology, Marburg, Germany.

*Manne S., Markowitz A., Winawer S., Meropol N.J., Haller D., Rakowski W.,

Babb J., \& Jandorf L. (2002). Correlates of colorectal cancer screening

compliance and stage of adoption among siblings of individuals with early onset

colorectal cancer. Health Psychology, 21(1), 3-15.

*Marcus, B.H., Pinto, B.M., Simkin, L.R., Audrain, J.E., \& Taylor, E.R. (1994).

Application of theoretical models to exercise behavior among employed women.

American Journal Health Promotion, 9(1), 49-55.

*Marcus, B.H., Rawkowski, W., \& Rossi, J.S. (1992). Assessing motivational readiness and decision making for exercise. Health Psychology, 11(4), 257-261.

*Mauriello, L.M. (2001). Applying the transtheoretical model of change to stress management among adolescents: development and validation of decisional balance and confidence measures. Unpublished master's thesis, University of Rhode Island, Kingston.

*Mettler, M.M., Stone, W.J., Herrick, A.B., \& Klein, D.A. (2000). Evaluation of a community-based physical activity campaign via the transtheoretical model. Health Promotion Practice, 1 (4), 351-359.

*Migneault, J.P., Pallonen, U.E., \& Velicer, W.F. (1997). Decisional balance and stage of change for adolescent drinking. Addictive Behavior, 22(3), 339-51. 
*Moll, H. (2001). [Using the transtheoretical model to examine physical activity].

Unpublished thesis, Department of Psychology, Marburg, Germany.

*Morrison-Beedy, D., \& Lewis, B.P. (2001). HIV prevention in single, urban, women: Condom-use readiness. Journal Obstetric, Gynecologic, and Neonatal Nursing, 30 (2), 148-156.

*Nigg, C.R., \& Courneya, K.S. (1998). Transtheoretical Model: examining adolescent exercise behavior. Journal of Adolescent Health, 22(3), 214-24.

*Nigg, C.R., Rossi, J.S., Norman, G.J., \& Benisovich, S.V. (1998). Decisional Balance Applied to Exercise Behavior. Manuscript submitted for publication.

*Noar, S. M. (2002). Unpublished raw data.

*Norman, G.J., Zabinski, M.F., Calfas, K.J., Sallis, J.F., \& Patrick, K. (2002).

Development of a decisional balance inventory for adolescent sedentary behavior [Abstract]. Annals of Behavioral Medicine,S 24, 217.

*O'Connell, D., \& Velicer, W.F. (1988). A decisional balance measure and the stages of change model for weight loss. The International Journal of the Addictions, 23(7), 729-50.

*Otake, K., \& Shimai, S. (2001). Adopting the stage model for smoking acquisition in Japanese adolescents. Journal of Health Psychology, 6(6), 629-643.

*Pallonen, U.E., Prochaska, J.O., Velicer, W.F., Prokhorov, A.V. \& Smith, N.F. (1997). Stages of acquisition and cessation for adolescent smoking: An empirical integration. Addictive Behaviors, 23(3), 303-324.

*Park, E.R., DePue, J.D., Goldstein, M.G., Niaura, R., Harlow, L.L., Willey, C., Rakowski, W., \& Prokhorov, A.V. (2003). Assessing the transtheoretical model 
of change constructs for physicians counseling smokers [Abstract]. Annals of Behavioral Medicine, S25 (2), 120-126.

*Perez, N.J. (2001). Transtheoretical Model comparisons on condom use in minority and non-minority adolescent females at two levels of risk for contracting HIV, STDS, and Cervical Cancer. Unpublished master's thesis, University of Rhode Island, Kingston.

Pigott, T.D. (2001). Methods for missing data for meta-analysis. Evaluation in the Health Professions, 24(3), 277-307.

*Plummer, B.A., Velicer, W.F., Redding, C.A., Prochaska, J.O., Rossi, J.S., Pallonen, U.E. \& Meier, K.S. (2001). Stage of change, decisional balance, and temptations for smoking: measurement and validation in a large, school-based population of adolescents. Addictive Behavior, 26(4), 551-71.

Prochaska, J. O. (1994). Strong and weak principles for progressing from precontemplation to action based on twelve problem behaviors. Health Psychology, 13, 47-51.

*Prochaska, J. O., Velicer, W. F., Rossi, J. S., Goldstein, M. G., Marcus, B. H., Rakowski, W., Fiore, C., Harlow, L. L., Redding, C. A., Rosenbloom, D., \& Rossi, S. R. (1994). Stages of change and decisional balance for 12 problem behaviors. Health Psychology, 13, 39-46.

*Prochaska, J.J., Rossi, J.S., Hall, S.M., Rosen, A.B., Tsoh, J.Y., Humfleet, G.L., \& Eisendrath, S.J. (2003). Depressed Smokers and Stage of Change: Implications for Cessation Interventions. Manuscript submitted for publication. 
*Rakowski, W., Dube, C.E., Marcus, B., Prochaska, J.O., Velicer, W.F., \& Abrams, D.B. (1992). Assessing elements of women's decisions about mammography. Health Psychology, 11(2), 111-118.

*Redding, C.A. (1990). The pros and cons of safer sex decision making: $A$ measurement model. Unpublished research competency, University of Rhode Island, Kingston.

*Redding, C.A. (1993). The transtheoretical model applied to safer sex behavior among univerity students: A cross-sectional investigation. (Doctoral dissertation, University of Rhode Island, 1993). Dissertation Abstracts International: Section B: The Sciences \& Engineering.

Redding, C.A., Rossi, J.S., Rossi, S.R., Prochaska, J.O., \& Velicer, W.F. (1999).

Health Behavior Models. In G.C. Hyner, K.W. Peterson, J.W. Travis, J.E.

Dewey, J.J. Foerster, \& E.M. Framer (Eds.), SPM Handbook of Health

Assessment Tools. Pittsburg, PA: The Society of Prospective Medicine \& The Institute for Health and Productivity Management. p. 83-93.

*Reed, G.R. (1996). Measuring stage of change for exercise. (Doctoral dissertation, University of Rhode Island). Dissertation Abstracts International: Section B: The Sciences \& Engineering, 56(9-B), 5220.

*Riley, T.A., \& Fava, J.L. (2003). Stress and transtheoretical indicators of stress management behaviors in HIV-positive women. Journal of Psychosomatic Research, 54, 245-252. 
*Riley, T.A., \& Weems, B.M. (2003). Health promoting behaviors, the transtheoretical model, and impoverished HIV-positive women. Paper presented at the meeting of the Midwest Nursing Research Society, Grand Rapids, MI.

*Riley, T.A., Toth, J.M., \& Fava, J.L. (2000). The transtheoretical model and stress management practices in women at risk for, or infected with, HIV. Journal of the Association of Nurses in AIDS Care, 11 (1), 67-77.

*Robbins, M.L., Evans, J.L., Kilgallen, C.E., \& Jacobbi, L.M. (1999).

Stages and decisional balance for organ donation in a representative sample [Abstract]. .Annals of Behavioral Medicine,S 21, 247.

*Robbins, M.L., Ganikos, M., Leino, E.V., Eaker, M.A, Eastwood, A., Webster, L., Bieterman, C. A. (2002). Stage-based intervention to increase intent for organ donation among college students [Abstract]. Annals of Behavioral Medicine, S 24, 138.

*Robbins, M.L., Kilgallen, C.E., Evans, J.L., Redding, C.A. \& Knott, E.J. (1999). Stages and decisional balance for becoming an organ donor [Abstract]. Annals of Behavioral Medicine, S 21, 177.

*Robbins, M.L., Levesque, D.A., Redding, C.A., Johnson, J.L., Prochaska, J.O., Rohr, M.S., Peters, T.G. (2001). Assessing family members' motivation readiness and decision making for consenting to cadaveric organ donation. Journal of Health Psychology, 6(5), 523-535.

*Robbins, M.L., Mattice, B., Knott, J.E. Individual and Campus-Wide Interventions to Increase Donation Intentions Among African American 
CollegeStudents (2001-2004). Health Resources and Services Administration, Division of Transplantation \#1H39OT00077.

Rosenthal, R. (1994). Parametric measures of effect size. In Cooper, H. \& Hedges L.V. (Eds.), The handbook of research synthesis (pp. 231-244). New York: Russell Sage Foundation.

*Rossi, J.S. (1990, August). Randon and ultraviolet light exposure: emerging cancer risk factors. In J.O. Prochaska (Chair), The stages of change: Extensions to new areas of behavior change. Symposium presented at the $98^{\text {th }}$ Annual Convention of the American Psychological Association, Boston, MA.

*Rossi, J.S., Martin, R.A., Redding, C.A., Rosenbloom, D., Rohsenow, D.J., \& Monti, P.M. (2001). Decisional balance and the stages of change for cocaine use. Manuscript submitted for publication.

Rossi, J. S., \& Redding, C. A. (2001). Structure and function of self-efficacy across the Stages of Change for 10 health behaviors. Annals of Behavioral Medicine, 23 (Supp1.), S094.

*Rossi, S.R., Greene, G.W., Rossi, J.S., Plummer, B.A., Benisovich, S.V., Keller, S., Velicer, W.F., Redding, C.A., Prochaska, J.O., Pallonen, U.E., \& Meier, K.S. (2001a). Validation of decisional balance and situational temptations measures for dietary fat reduction in a large school-based population of adolescents. Eating Behaviors, 2, 1-18.

*Rossi, S.R., Rossi, J.S., Greene, G.W., Fey-Yensan, N., Padula, C., Nigg, C., Owens, N.J. \& Clark, P. (2001b). Development and validation of a brief measure 
of decision-making for fruit and vegetable consumption in older adults.

Manuscript in Preparation.

*Rossi, S.R., Rossi, J.S., Greene, G.W., Ward, R.M., Maddock, J., Velicer, W.F. \& Prochaska, J.O. (2001c). Brief decisional balance and situational temptations measures for dietary fat reduction in a large population of adults. Manuscript in Preparation.

*Rossi, S.R., Rossi, J.S., Prochaska, J.O., \& Velicer, W.F. (1993, August). Measurement structure of a decisional balance questionaire for dietary fat reduction. Paper presented at the $101^{\text {st }}$ annual convention of the American Psychological Association, Toronto, Canada.

*Ruggiero, L. (2003). Validation of the transtheoretical model for glucose selftesting in a large sample of people with diabetes. Manuscript in Preparation.

*Ruggiero, L., Tsoh, J.Y., Everett, K., Fava, J.L., \& Guise, B.J. (2000). The transtheoretical model of smoking: Comparison of pregnant and nonpregnant smokers. Addictive Behaviors, 25 (2), 239-251.

*Sarkin, J.A., Johnson, S.S., Prochaska, J.O., \& Prochaska, J.M (2001). Applying the Transtheoretical Model to Regular Moderate Exercise in an Overweight Population: Validation of a Stages of Change Measure. Preventive Medicine, $33(5), 462-469$.

*Schmaling, K.B., Afari, N., \& Blume, A.W. (2000). Assessment of psychological factors associated with adherence to medication regimens among adult patients with asthma. Journal of Asthma, 37 (4), 335-343. 
*Schorling, J.B. (1995). The stages of change of rural African-American smokers. American Journal of Preventive Medicine, 11 (3), 170-177.

*Schulze, H. (n.d.). [Using the transtheoretical model to examine daily use of dental floss]. Unpublished raw data.

Schmidt, $F$. (1992). What do data really mean? Research findings, meta-analysis, and cumulative knowledge in psychology. American Psychologist, 47, 11731181.

*Schumann, A., Rumpf, H.J., Meyer, C., Hapke, U., \& John, U. (2003). Skalen zu Kernkonstruken des Transtheoretischen Modells (TTM) im Verhaltensbereich Tabakkonsum [Scales for transtheoretical model constructs (TTM) in the area of tobacco consumption]. Zeitschrift für Gesundheitpsychologie, 11(2), 57-66.

*Silverman, E.S. (1995). Evaluating the Stages of Behavior Change Model for use in diverse cultures: Hong Kong versus the United States. (Virginia Polytechnic Institute and State University, 1995). Dissertation Abstracts International: Section B: The Sciences \& Engineering, 56 (5-B), 2849.

*Snow, M.G., Prochaska, J.O., \& Rossi, J.S. (1992). Stages of change for smoking cessation among former problem drinkers: A cross-sectional analysis. Journal of Substance Abuse, 4, 107-116.

Song, F., Sheldon, T.A., Sutton, A.J., Abrams, K.R., \& Jones, D.R. (2001). Methods for exploring heterogeneity in meta-analysis. Evaluation \& The Health Professions, 24(2), 126-151. 
*Stark, M.J., Tesselaar, H.M., O'Connell, A.A., Person, B., Galavotti, C., Cohen, A., \& Walls, C. (1998). Psychosocial factors associated with the stages of change for condom use among women at risk for HIV and STDs: Implications for Intervention development. Journal of Consulting and Clinical Psychology, 66 (6), 967-978.

*Susenbeth, R. (2000). Anwendung des Transtheoretischen Modells gesundheitsfördernder Verhaltensänderung auf HIV-protektiven Kondomgebrauch [Application of the Transtheoretical Model on condom use for HIV prevention]. Unpublished doctoral dissertation, University of Marburg, Marburg, Germany.

Takkouche, B., Cadarso-Suarez, C., Spiegelman, D. (1999). Evaluation of old and new tests of heterogeneity in epidemiologic meta-analysis. American Journal of Epidemiology, 150, 206-15.

${ }^{*}$ Tseng, Y. (2001). Examining exercise determinants in elders residing in assisted living facilities: An application of the transtheoretical model. (Doctoral dissertation, University of Minnesota). Dissertation Abstracts International: Section B: The Sciences \& Engineering, 61(10-B), 5241.

*Tsoh, J.Y. (1993). Motivation and stages of change among drug addicts in drug abuse treatment programs. Unpublished master's thesis, University of Rhode Island, Kingston.

*Van Marter, D.F., Evers, K.E., Janet L. Johnson, Ph.D., Andrea L. Eastwood, B.S., Prochaska, J.M., \& Prochaska, J.O. (2003, March). Measure development of Transtheoretical Model constructs for bullying prevention 
in middle schools. Poster presented at the 24rd Annual Scientific Sessions of the Society of Behavioral Medicine, Salt Lake City, Utah.

*Velicer, W. F., DiClemente, C. C., Prochaska, J. O., \& Brandenberg, N. (1985). A decisional balance measure for assessing and predicting smoking status. Journal of Personality and Social Psychology, 48, 1279-1289.

Velicer, W.F., Prochaska, J.O., Fava, J.L., Rossi, J.S., Redding, C.A., Laforge, R.G., \& Robbins, M.L. (2000). Using the transtheoretical model for population-based approaches to health promotion and disease prevention. Homeostasis, 40, 174195.

*Wakui, S., Shimomitsu, T., Odagiri, Y., Inoue, S., Takamiya, T., \& Ohya, Y. (2002). Relation of the stages of change for exercise behaviors, self-efficacy, decisional-balance, and diet-related psycho-behavioral factors in young Japanese women. Journal of Sports Medicine and Physical Fitness, 42, 224-232.

*Whyte, S.R. (2003). Investigating stages of change and processes of change for dietary fat consumption on the World Wide Web. (Doctoral dissertation, University of Maryland). Dissertation Abstracts International: Section B: The Sciences \& Engineering, 63(8-B), 3947.

Weinstock, M. A., Rossi, J. S., Redding, C. A., \& Maddock, J. E. (2002). Randomized controlled community trial of the efficacy of a multi-component stage-matched intervention to increase sun protection among beachgoers. Preventive Medicine, 35, 584-592. 


\section{DECISIONAL BALANCE ACROSS STAGE TRANSITIONS}

\section{Introduction}

The primary goal of examining cross-sectional data is to use readily available or easily attainable data to explore theoretical relationships that explain a phenomenon across a temporal dimension. It is anticipated that cross-sectional investigations will provide a snap shot of what one might see if these same relationships were examined using more costly longitudinal data where individuals are actually followed over time. Ultimately, cross-sectional investigations are most valuable if they have the ability to provide the same or similar relationships as longitudinal investigations.

The Transtheoretical Model (TTM) is a theoretical model which explains behavior change through a variety of constructs which explain change across a temporal dimension. The central organizing construct of the TTM characterizes behavior change over time through five distinct Stages of Change: Precontemplation, Contemplation, Preparation, Action, and Maintenance. Two intermediate indicators of when these changes will occur are Decisional Balance (weighing of Pros and Cons) and Self-efficacy (Situational Confidence or Temptation). Additionally, the TTM explains behavior change strategies through ten Processes of Change.

A previous study examining the TTM (Prochaska, Velicer, Guadagnoli, Rossi \& DiClemente, 1991) has demonstrated the ability of longitudinal studies to validate 
cross-sectional results. To date, no study has investigated the ability of longitudinal data to validate cross-sectional results in the context of TTM Stage transitions.

Additionally, there are currently no established techniques for examining the effect sizes of Stage transitions in longitudinal data, therefore this study was treated as an exploratory examination of longitudinal datasets. Additionally, previously examined cross-sectional relationships will be compared to these longitudinal results.

\section{Stages of Change and Decisional Balance}

\section{Stages of Change}

In health psychology, the concept of change occurring in a series of stages has been examined in efforts to understand the temporal aspects of change in human nature or behavior. An example of the stage concept can be seen in Horn's (1976; Horn \& Waingrow, 1966) work with smoking behavior and cessation. Horn developed a four stage process of change investigating smoking behavior, which consisted of 1) contemplation of change; 2) the decision to change; 3 ) short-term change; and 4) long-term change. Although arising independently and in a different context, the Stages of Change as conceptualized by the TTM are similar to Horn's stages. Over the years, through its own evolution, the TTM ultimately identified five Stages (DiClemente, Prochaska, Fairhurst, Velicer, Velasquez, et al., 1991).

The algorithms for the five Stages of Change are specific for each behavior, but usually follow these general Stage concepts. Participants are considered to be in the Precontemplation Stage if they report an undesired status, that is, the presence of a problem behavior or the lack of a healthy one, and express no intention of changing 
in the next six months. Participants are considered in the Contemplation Stage if they intend to change in the next six months. Participants in the Preparation Stage plan to change in the next month and have begun to engage in target behaviors, but have not yet met particular criteria. Participants reach the Action Stage once they have met the given behavioral criteria. Lastly, if the participant has met the specified behavioral criteria for greater than six months, they have reached the Maintenance Stage.

\section{Decisional Balance}

The TTM originated by integrating theories of psychotherapy as well as incorporating constructs from alternative models. One of the most important and reliable TTM constructs, Decisional Balance, was inspired by Janis and Mann's (1977) conflict model of decision-making. Janis (1959) proposed a descriptive schema called a "balance sheet" of incentives. The four main categories of consideration for decisional conflicts are: a) utilitarian gains and loses for self; b) utilitarian gains and losses for significant others; c) self-approval or -disapproval; d) approval or disapproval by significant others. These four comparative categories of potential positive and negative incentives involve both instrumental effects of utilitarian objectives and nonutilitarian considerations such as issues of self-esteem and value-based determinations.

The development of the TTM Decisional Balance measure (Velicer, DiClemente, Prochaska, and Brandenburg, 1985) was based on the 8 factors (4 gains and 4 losses) of Janis and Mann (1977). The researchers constructed the scale to study the decision-making process across the Stages for smoking cessation. Instead 
of achieving an 8 factor-structure as anticipated, principal components analysis identified two orthogonal components. These two components were called the Pros and Cons of Smoking.

Following the original study by Velicer, the use of the construct began to expand, encompassing an array of behaviors such as exercise, condom use, and mammography screening. This early work culminated in a paper by Prochaska et al. (1994), which looked at patterns in Decisional Balance across Stages in 12 behaviors.

\section{Strong and Weak Principles}

Across twelve studies (Prochaska, 1994), mathematical relationships were found between the pros and cons of changing and progress across the early Stages into Action (Prochaska, 1994). The Strong Principle of Change states that PC $\rightarrow \mathrm{A}$ $\cong 1 S D \uparrow$ PROS: progress from Precontemplation to Action involves approximately one standard deviation increase in the Pros of Changing. The Weak Principle of Change states that $\mathrm{PC} \rightarrow \mathrm{A} \cong .5 S D \downarrow$ CONS: progress from Precontemplation to Action involves approximately $.5 S D$ decrease in the Cons of Changing.

In re-examination of the Strong and Weak Principles, the magnitude of the maximum increase in the Pros of Change was again found to be greater than the maximum decrease in the Cons of Change from Precontemplation to Action across 37 difference health behaviors (Hall \& Rossi, 2004). Consistent with Prochaska's (1994) Strong Principle, the average effect size for the pros was approximately one standard deviation $(d=1.05, S D=.45)$, almost identical to Prochaska's (1994) 
original finding ( $d=1.06, S D=.26)$. Hall and Rossi's (2004) findings also revealed that Prochaska's Weak Principle might not be so weak. That is, the average effect size for cons was stronger $(d=.62, S D=.38)$ than was found in the previous study $(d=.45, S D=.22)$ by Prochaska (1994), though clearly the cons remains weak relative to the pros. Practical implications of these principles are that the pros of changing must increase twice as much as the cons must decrease, suggesting that an intervention place twice as much emphasis on raising the benefits as on reducing the costs or barriers.

\section{Stage Transitions}

The Strong and Weak Principles are useful for understanding the amount of work generally needed to move from Precontemplation to Action. Although the principles have been important in conceptualizing and understanding the relationship between Decisional Balance and Stages of Change, the Strong and Weak Principles are essentially action-oriented when applied to interventions. By examining characteristics of the transition only from Precontemplation to Action, and using these to tailor interventions, one is potentially neglecting three Stages: Contemplation, Preparation and Maintenance. In contract, Stage transition analyses can help identify the most effective strategy for tailoring behavior change interventions. For instance, for a given behavior, if the effect size from Precontemplation to Contemplation for Pros is large but very small for Cons, Pros could be intervened on whereas Cons could be eliminated in the intervention for precontemplators. From Contemplation to Preparation, if both the Pros and Cons 
effect sizes were large, both should be emphasized at this transition. Following this strategy across each Stage transition would create the most efficacious and efficient application of the measure, maximizing the impact and minimizing the resource expenditure. Ultimately, careful and systematic investigation of the changes in these measures across Stage transitions by behavior can provide even more detailed evidence for exactly how to use these measures most efficiently in future Stagematched interventions.

It has generally been thought that Decisional Balance is especially salient in the earlier Stages of Change. To test this notion, the previous study (Chapter Six) examined 146 independent datasets across 55 health behaviors, generating and synthesizing over 2100 effect sizes. Datasets included nearly 90,000 participants across 18 countries utilizing measures in more than 10 languages. The largest effect size was found for the Pros (see Table 1), specifically in the earliest transition, Precontemplation to the Contemplation Stages (PC-C). Smaller increases were seen in following two Stage transitions, Contemplation-Preparation (C-PR) and Preparation-Action (PR-A), and essentially no effect found in the ActionMaintenance transition (A-M). The Cons showed an inverse pattern across transitions, though with overall smaller effect sizes. Essentially no effect was found for PC-C with relatively small decreases in Cons from C-PR, PR-A and A-M. These results are only partially consistent with the assumption that Decisional Balance is most salient in the earlier Stages of Change. It appears that the Pros are more salient in the earlier Stages whereas the Cons are more salient in the later Stages. 
Table 34

Magnitude of Stage Transitions for Pos and Cons of Change

\begin{tabular}{|c|c|c|c|c|c|c|c|c|c|c|}
\hline \multirow{3}{*}{$\begin{array}{c}\text { Stage } \\
\text { Transitions }\end{array}$} & \multicolumn{5}{|c|}{ PROS } & \multicolumn{5}{|c|}{ CONS } \\
\hline & \multirow[b]{2}{*}{$N$} & \multirow[b]{2}{*}{$E S$} & \multirow[b]{2}{*}{$S E$} & \multicolumn{2}{|c|}{$95 \% \mathrm{CI}$} & \multirow[b]{2}{*}{$N$} & \multirow[b]{2}{*}{$E S$} & \multirow[b]{2}{*}{$S E$} & \multicolumn{2}{|c|}{$95 \% \mathrm{CI}$} \\
\hline & & & & $\mathbf{L}$ & $\mathbf{U}$ & & & & $\mathbf{L}$ & $\mathrm{U}$ \\
\hline PC-C & 136 & .65 & .02 & .60 & .70 & 136 & -.08 & .02 & -.13 & -.04 \\
\hline C-PR & 122 & .17 & .02 & .12 & .22 & 124 & -.15 & .02 & -.19 & -.11 \\
\hline PR-A & 100 & .12 & .03 & .06 & .18 & 100 & -.24 & .03 & -.30 & -.18 \\
\hline A-M & 108 & .02 & .03 & -.05 & .08 & 105 & -.23 & .02 & -.28 & -.18 \\
\hline
\end{tabular}

Note. $N=$ sample size; $E S=$ mean effect size; $\mathrm{CI}=$ confidence interval; $\mathrm{L}=$ lower bound; $\mathrm{U}=$ upper bound

These results suggest interventions may provide the greatest impact with maximum efficiency by placing the most emphasis on Pros during the Precontemplation Stage, followed by additional efforts during the Contemplation and Preparation Stages. In contrast, Cons are most likely to be useful during the Preparation Stage with continued emphasis, somewhat surprisingly, into the Action Stage. In general, use of effect sizes to assess the magnitude of Stage transitions can provide guidance in the development of tailored interventions by suggesting how much intervention resources should be allocated for each construct at each Stage transition.

Figure 37 presents the remarkable pattern that was found from the integration of all of these data. Theoretically, this is one of the clearest and cleanest patterns of relationships that has been found in any single study or combination of studies to date. In the stable Stage of Precontemplation, the Cons of Changing are 0.8 S.D. higher than the Pros. The opposite is true in the stable Maintenance Stage where the 
Pros of Changing are 0.8 S.D. higher than the Cons. In the Contemplation Stage, which theoretically is characterized by intensive ambivalence about changing, the Pros and Cons of Changing are equal. The Pros begin to outweigh the Cons in the Preparation Stage. The Pros of Changing are $0.93 S D$ higher in the Maintenance Stage than in the Precontemplation Stage and there is a slight decrease in the Pros of Changing from Action to Maintenance. The Cons are $0.62 S D$ lower in Maintenance than in Precontemplation. Overall, the Pros were found to increase about twice as much as the Cons decrease.

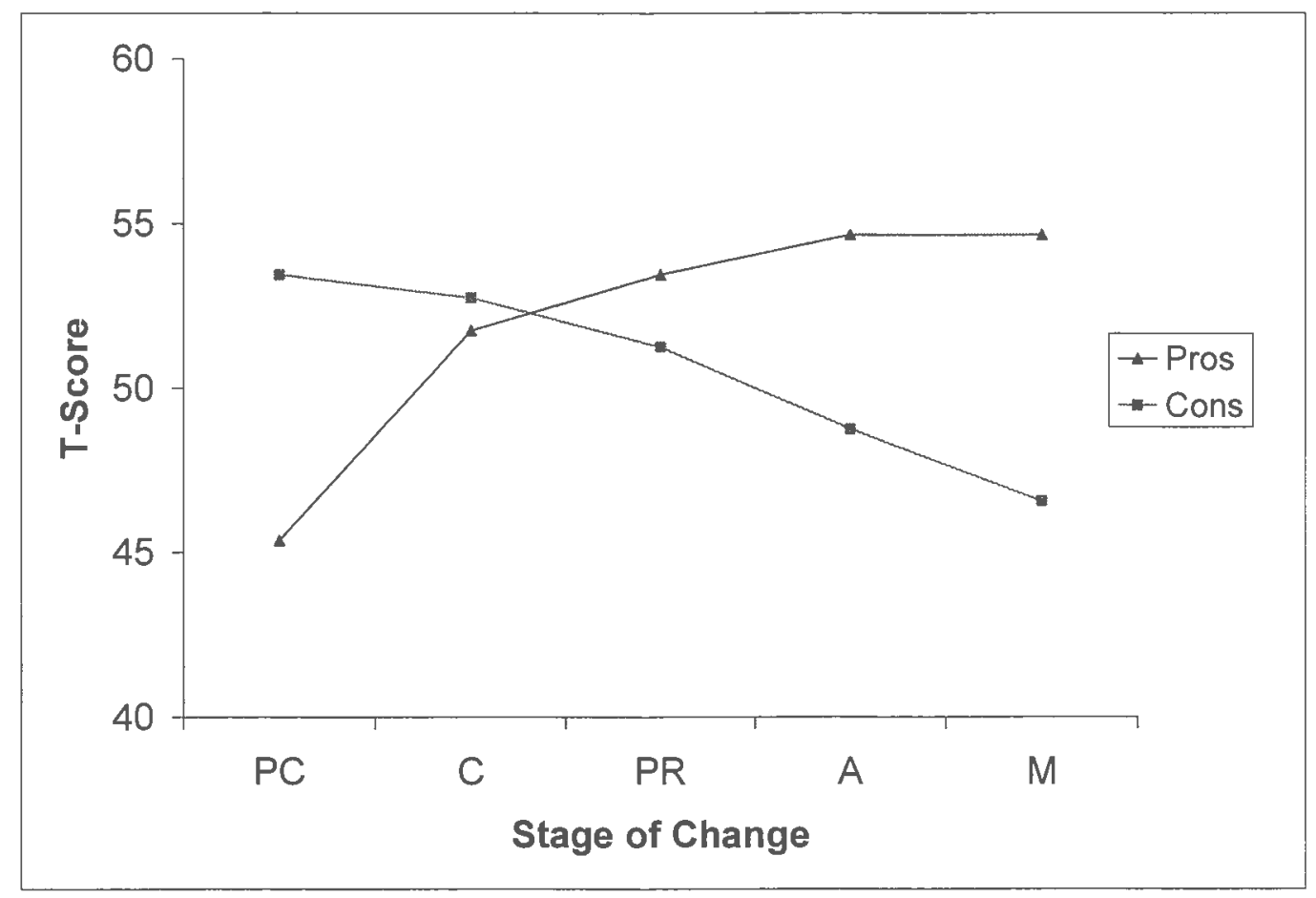

Figure 37. Composite Decisional Balance Profile across 55 Behaviors.

These remarkable relationships between the Pros and Cons and the Stages of Change have been examined cross-sectionally. The cross-sectional relationships are 
an essential part of this program of study and clearly provide important insights into the theoretical model and for its practical applications. In efforts to continue to expand this research, to broaden its generalizability and strengthen its potential for application, it is important to begin to explore these relationships in a longitudinal context. The current study aims to begin to establish methods for exploring these relationships and preliminarily compares cross-sectional data with longitudinal data in order to help establish how well cross-sectional data can predict longitudinal relationships in the context of Stage transitions.

\section{Research Hypotheses and Predictions}

Hypothesis 1. There is a relationship between the cross-sectional and longitudinal Stage transition effect sizes for Decisional Balance.

Prediction 1. A previous study (Prochaska et al., 1991) demonstrated that cross-sectional and longitudinal data examining patterns of change were comparable. It is predicted that the magnitudes of effect for the cross-sectional and longitudinal data will show similar patterns for Decisional Balance across Stage transitions.

Prediction 2. It is anticipated that the effect size index for the longitudinal data will produce larger effect sizes than the effect size index for independent groups (used for the cross-sectional data) due to the nature of dependency in the longitudinal data. Therefore it is predicted that the longitudinal Stage transition effect sizes will be larger that the cross-sectional Stage transition effect sizes. 


\section{Method}

\section{Procedure}

Since there are no established techniques for examining the effect size of Stage transitions in longitudinal data and there has not previously been a comparison of cross-sectional and longitudinal Stage transitions, therefore this study was approached as exploratory. This study cross-sequentially examined the longitudinal data and therefore should be considered a very preliminary investigation of longitudinal relationships.

Inclusion Criteria. Datasets must include Decisional Balance measures as well as at least two consecutive Stages of Change measured by an algorithm procedure. These datasets must also include at least 2 time points.

Datasets. Longitudinal datasets were acquired through researchers at the Cancer Prevention Research Center at the University of Rhode Island. The data were from three previously conducted studies. Two different health behaviors were examined: smoking cessation and sun protection. The participants of the three studies are as follows:

\section{A Population-based, Stage-based Expert System Intervention for Smoking}

Cessation (Prochaska, Velicer, Fava, Rossi, \& Tsoh, 2001): Random-digit dialing procedure was used to recruit a representative sample of smokers from Rhode Island. A total of 32, 456 calls were conducted, 14,266 participants were identified as eligible, and 12,109 agreed to complete a preliminary phone survey. Of these, 4209 were smokers and 7813 were non-smokers. A total of 4144 participants were 
included. These participants were randomly assigned to intervention or assessment only. The intervention group received Expert System materials in the mail, including a feedback report and stage-matched self-help manuals. The assessment only group was assessed in 6 month intervals. Participants in the intervention group received progress questionnaires at 3 and 6 months. Phone surveys were given for participants that did not respond via mail within 2 weeks. All participants were assessed by mail or phone at 12,18 and 24 months.

\section{Impact of Simultaneous Stage-matched Expert System Interventions for}

Smoking, High Fat Diet and Sun Exposure on a Population of Parents (Prochaska, Velicer, Rossi, Redding, Greene, Rossi, Sun, Fava, LaForge \& Plummer, in press): Schools participating in an on-going health promotion study provided a list of parents of $9^{\text {th }}$ graders. Initial screening identified 3507 potential households. A total of 2931 respondents were contacted by phone, with one parent recruited from each eligible household. Eligibility requirements necessitated the participants to be at risk for at least one of the three health risk behaviors (sun, smoking, and diet). Four hundred and seventy one participants were excluded for not meeting eligibility requirement, resulting in a baseline sample of 2460 participants. The 2460 participants were randomly assigned to the intervention or control groups. Participants in both groups were administered follow-up assessments at 12 and 24 months.

At baseline, 711 respondents were identified as smokers. Of the 711 smokers at baseline, 453 completed the 12 Month assessment and 409 completed the 24 Month assessment. For sun exposure, 1802 were identified as being at risk at 
baseline, 1318 completed the 12 Month assessment and 1242 completed the 24

Month assessment. For Diet, 1820 were identified as being at-risk at baseline, 1334

completed the 12 Month assessment and 1253 completed the 24 Month assessment.

Participants were randomly assigned to one of two groups, Intervention $(N=1209)$

or Assessment Only $(N=1251)$. Participants in both groups were administered

follow-up assessments at 12 and 24 months.

Randomized Controlled Community Trial of the Efficacy of Multi-Component

Stage-Matched Intervention to Increase Sun Protection among Beachgoers

(Weinstock, Redding, Rossi, Maddock, 2001): Seven coastal beaches in Rhode Island

were selected for the study. Participants were randomly assigned to intervention or

control conditions. Follow-up assessments were conducted for all study participants

at 2, 12 months, and 24 months after baseline by mail or telephone. Study retention

rates were $83 \%$ of baseline at 2 months $(N=1930), 70 \%$ of baseline at 12 months $(N$

$=1628) ;$ and $62 \%$ of baseline at 24 months $(N=1449)$. Participants completed a

survey on the beach with a trained interviewer. Ages of the participants ranged from

$16-65$, with an average age 33 years. The sample was primarily female $(60 \%)$, white $(94 \%)$, single (51\%) or married (40\%), with at least a high school education $(88 \%)$, and with a median income of $\$ 45,000-65,000$ per year.

\section{Analysis}

The longitudinal data was examined by behavior with treatment and control groups combined. Data was analyzed separately by behavior for each study sample. Firstly behaviors were examined separately by adjacent time points (e.g., baseline to 
6 months, 6 months to 12 months). Then the longitudinal data were combined across time points by behavior separately for each sample.

Stage Transition Membership. Stage transition membership was established by identifying subjects that moved from one Stage to the next from one time interval to the next. Figure 38 illustrates the Stage transition membership across the time points for participants who moved from Precontemplation to Contemplation. This procedure was repeated for each of the Stage transitions including, Contemplation to Preparation, Preparation to Action, and Action to Maintenance.

Once identified, the participants from each stage transition at each set of time points were grouped together to represent given Stage transitions (e.g., Precontemplation to Contemplation). A true longitudinal exploration would follow participants from Precontemplation to Contemplation to Preparation to Action and finally to Maintenance. Due to sample size issues, a cross-sequentially approach was taken to help increase the number of participants in each Stage transition. This resulted in different subjects in each of the transitions for each set time points. 

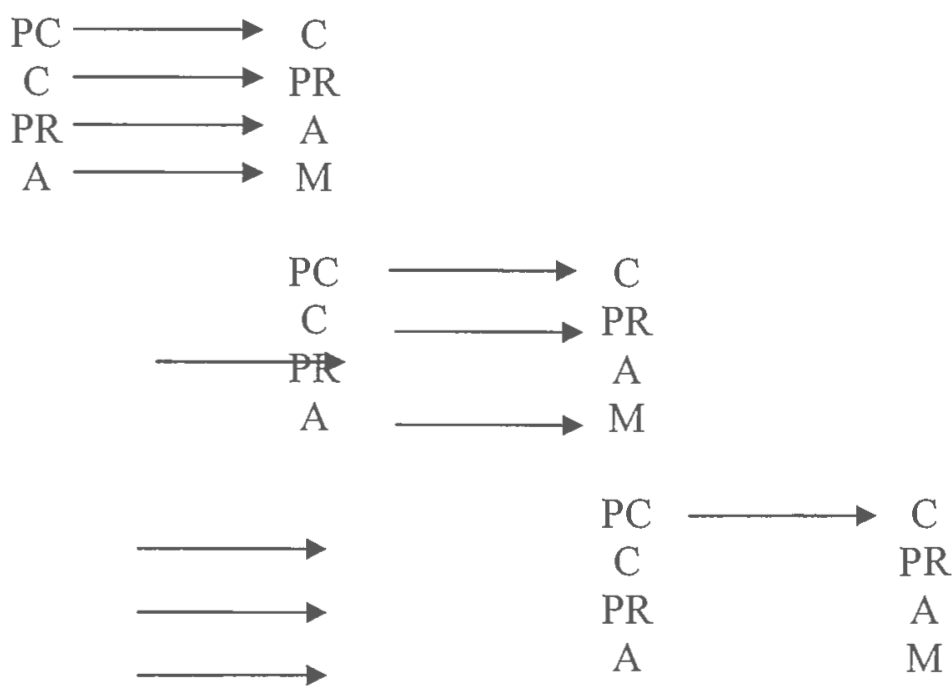

PR A

A $M$

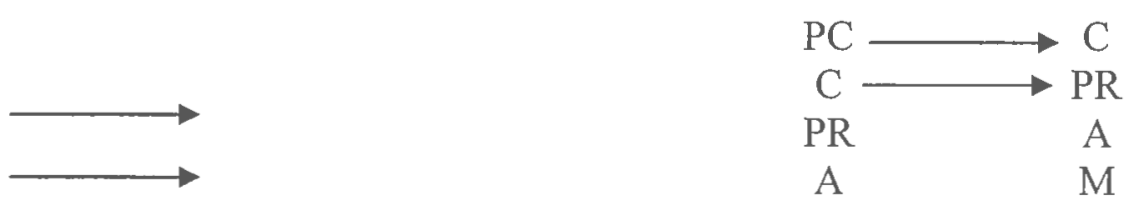

Note $. \mathrm{PC}=$ Precontemplation; $\mathrm{C}=$ Contemplation; $\mathrm{PR}=$ Preparation; $\mathrm{A}=$ Action; $\mathrm{M}=$ Maintenance Figure 38. Stage Transition Membership.

Magnitude. Effect sizes were calculated for each transition across time points. Effect sizes were examined using two main methods: 1) separately for each transition by time point; and 2) collapsed across time points. Magnitudes of effect were assessed using Hedges' $g$ with pooled variance of the standardized gain score:

$$
\begin{gathered}
\text { Hedges' } g=\frac{M_{1}-M_{2}}{S_{\text {pooled }}} \\
S_{\text {pooled }}=\frac{S_{\text {gain }}}{\sqrt{2(1-r)}}
\end{gathered}
$$


where $S_{\text {gain }}=$ the standard deviation of the mean of the difference between the two time points, and $\mathrm{r}=$ the correlation of the two means.

Finally, the cross-sectional and longitudinal Stage transition effect sizes for Pros and Cons will be descriptively compared.

T-score by Stage Graphs. In order to create graphical representations in the "classic" T-score by Stage format, as typically seen in the TTM body of literature, effect sizes were converted into "T-scores" and graphed by Stage. In order to illustrate the magnitude of effect across the Stages for the Pros and Cons, a starting point of 0 was chosen for the PC Stage for each set of data. Effect sizes were then incrementally added to this starting point for each Stage transition. Computing the mid-point of the effect sizes served to recenter the data. The midpoint was calculated by subtracting the difference between the lowest and highest effect sizes, dividing by two and adding this to the lowest effect size.

$$
\text { Midpoint }=\frac{\mathrm{T}_{\text {high }}-\mathrm{T}_{\text {low }}}{2}+\mathrm{T}_{\text {low }}
$$

This midpoint then functions as the T-score of 50 (once the score is converted as described below) and is subtracted from all Stage scores. This gives the number of standard deviations above and below the midpoint for each Stage. The scores are then converted to $\mathrm{T}$ scores by multiplying by 10 and adding 50 . Finally, these $\mathrm{T}-$ scores are plotted by Stage.

\section{Results}

Overview. Two behaviors were examined across three longitudinal datasets with a total of 8534 participants. Multiple time points were examined across the 
behaviors for a total of 17 sets of comparisons. A total of 136 effect sizes were computed using Hedge's $g$.

\section{Smoking Cessation - "Random Digit Dial" Sample}

Mean effect size estimates of the Pros and Cons for each of the Stage transitions for smoking cessation with the "random digit-dial" (RDD) sample across adjacent time points were calculated and are displayed in Table 35. Across the Stages from baseline to six months, small increases were seen from PC-C as well as C-PR for the Pros of Change. A larger magnitude is seen in the opposite direction (decrease) from PR-A. For the Cons of Change, essentially no change was seen in the PC-C and C-PR transitions, whereas nearly .6 SD decrease occurred in the transition form PR-A. Due to the fact that only participants in the pre-Action Stages (Precontemplation, Contemplation, and Preparation) were recruited into the original study no participants were available for the A-M transition for the baseline to 6month time points. A graphical depiction of these transitions can be seen in Figure 39.

As seen in the baseline to 6-month data, the PC-C transition for the Pros of Change a small increase in the Pros of Change was found in the 6- to 12-month time points. Essentially no change occurred it the transition from C-PR. A larger decrease was found for the PR-A transition and a smaller decrease was found from A-M. For the Cons of Change, as seen in the baseline to 6-month data, essentially no change was seen from PC-C. A small increase was seen from C-D. Greater decreases were then found from PR-A and A-M. These changes are illustrated in Figure 40. 
Slight increases in the Pros of Change were found for the transitions from PC-C and C-PR for time points 12 months to 18 months. Small decreases were then found for the transitions from PR-A and A-M. For the Cons of Change, a small decrease was seen in the PC-C followed by essentially no change for C-PR. A large decrease was found for the transition from PR-A and a small decrease from A-M. These magnitudes are graphed in Figure 41.

Lastly, for the Pros of Change between time points 18 months to 24 months a small increase was found for the transition from PC-C. Essentially no change occurred from C-PR, followed by a large decrease in the PR-A. Again, essentially no change was found for A-M. For the Cons of Change, essentially no change occurred in the PC-C transition. A small decrease was found for the transition from C-PR whereas the largest decrease was found in the D-A transition. Finally, a small decrease occurred in the A-M transition. These patterns are found in Figure 42. 
Mean Effect Sizes for Stage Transitions in the Random Digit Dial Sample across

Time Points for Smoking Cessation

\begin{tabular}{|c|ccccccccc|}
\hline & \multicolumn{8}{|c|}{ RDD Sample Smoking Cessation } \\
\cline { 2 - 9 } Stage & \multicolumn{7}{|c|}{ BL to 6 } & \multicolumn{6}{c|}{$\mathbf{6}$ to 12 } & \multicolumn{3}{c|}{$\mathbf{1 2}$ to 18 } & $\mathbf{1 8}$ to 24 \\
\cline { 2 - 9 } Transitions & $N$ & Mean & $N$ & Mean & $N$ & Mean & $N$ & Mean \\
\hline PROS & & & & & & & & \\
PC-C & 235 & $\mathbf{. 2 2}$ & 233 & $\mathbf{. 2 2}$ & 192 & $\mathbf{. 1 5}$ & 145 & $\mathbf{. 1 6}$ \\
C-PR & 187 & $\mathbf{. 2 5}$ & 151 & $\mathbf{. 0 8}$ & 115 & $\mathbf{. 1 5}$ & 115 & $\mathbf{- . 0 1}$ \\
PR-A & 65 & $\mathbf{- . 4 0}$ & 81 & $\mathbf{- . 5 7}$ & 54 & $\mathbf{- . 3 0}$ & 50 & $\mathbf{- . 5 4}$ \\
A-M & & - & 65 & $\mathbf{- . 2 5}$ & 118 & $\mathbf{- . 2 3}$ & 117 & $\mathbf{- . 0 4}$ \\
CONS & & & & & & & & \\
PC-C & 235 & $\mathbf{. 0 3}$ & 233 & $\mathbf{- . 0 4}$ & 192 & $\mathbf{- . 1 1}$ & 145 & $\mathbf{- . 0 5}$ \\
C-PR & 187 & $\mathbf{- . 0 8}$ & 151 & $\mathbf{. 1 3}$ & 115 & $\mathbf{. 0 4}$ & 115 & $\mathbf{- . 1 5}$ \\
PR-A & 65 & $\mathbf{- . 5 7}$ & 81 & $\mathbf{- . 4 6}$ & 54 & $\mathbf{- . 4 5}$ & 50 & $\mathbf{- . 9 0}$ \\
A-M & & - & 65 & $\mathbf{- . 5 5}$ & 118 & $\mathbf{. 1 1 7}$ & 117 & $\mathbf{- . 1 0}$ \\
\hline
\end{tabular}




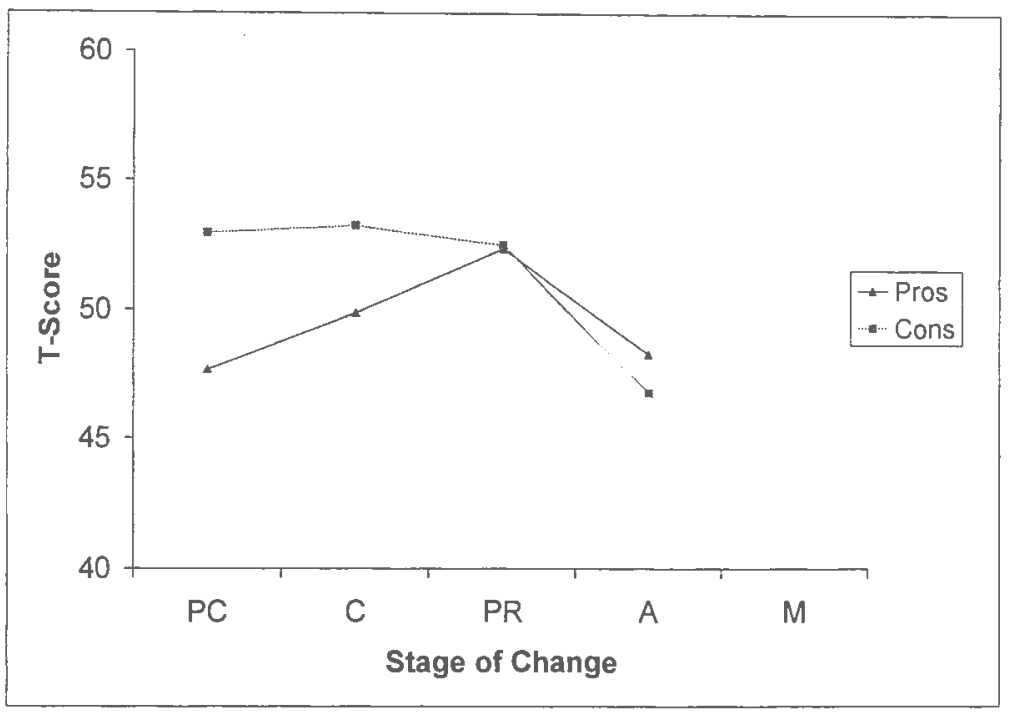

Figure 39. Graphic for Pros and Cons of Smoking from Baseline to 6 Months for the Random Digit Dial Sample.

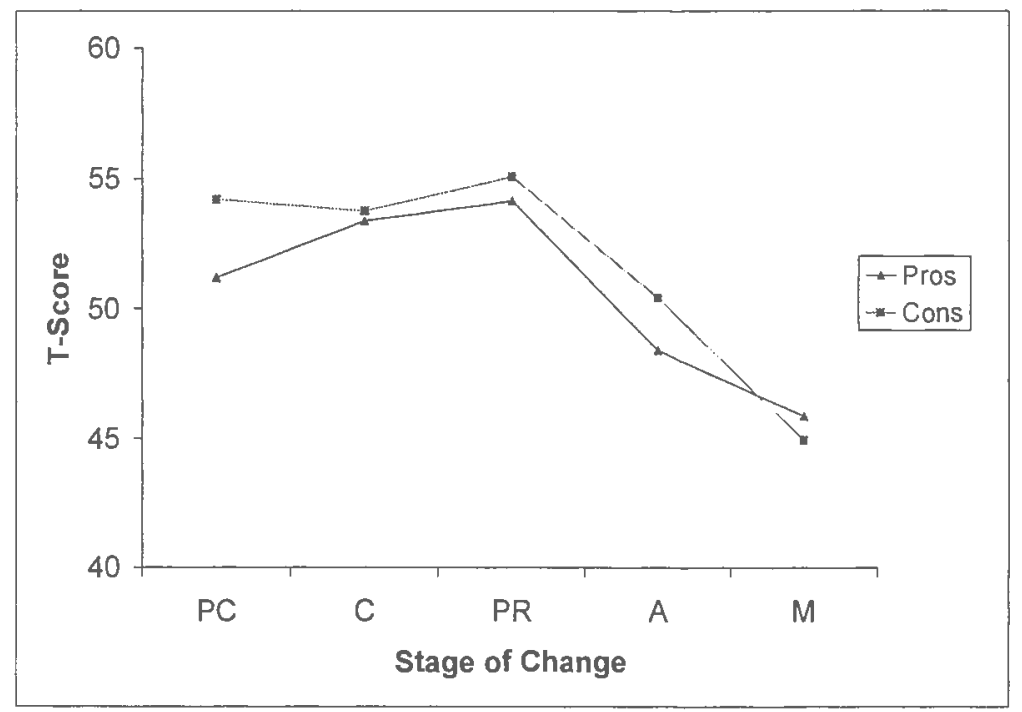

Figure 40. Graphic for Pros and Cons of Smoking from 6 to 12 Months for the Random Digit Dial Sample. 


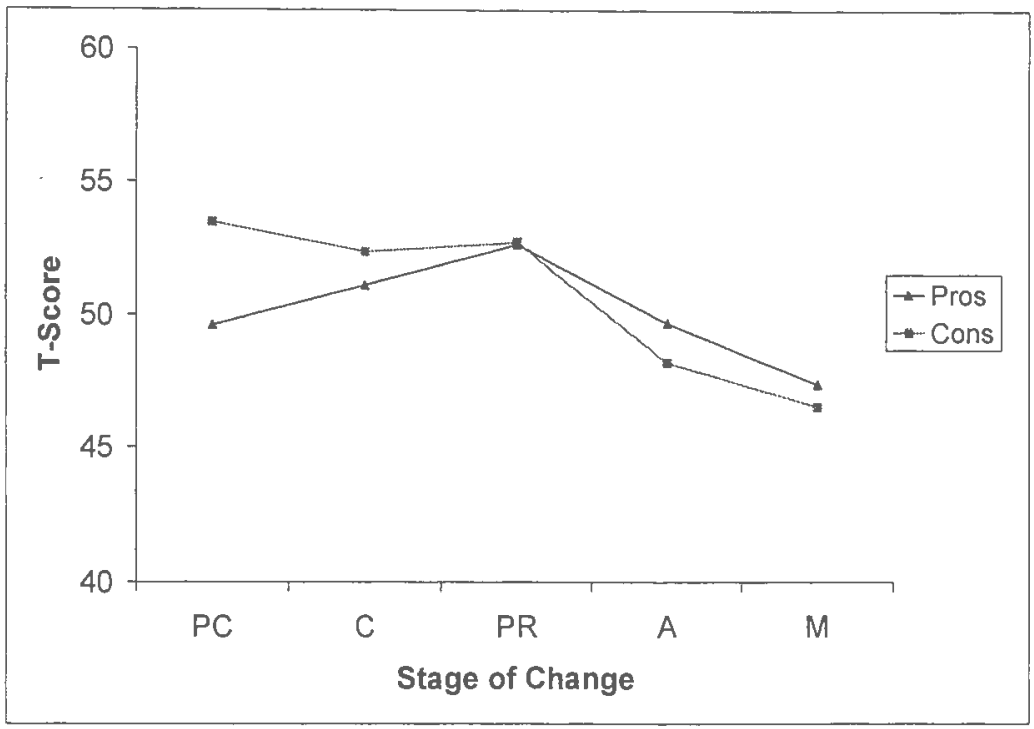

Figure 41. Graphic for Pros and Cons of Smoking from 12 to 18 Months for the Random Digit Dial Sample.

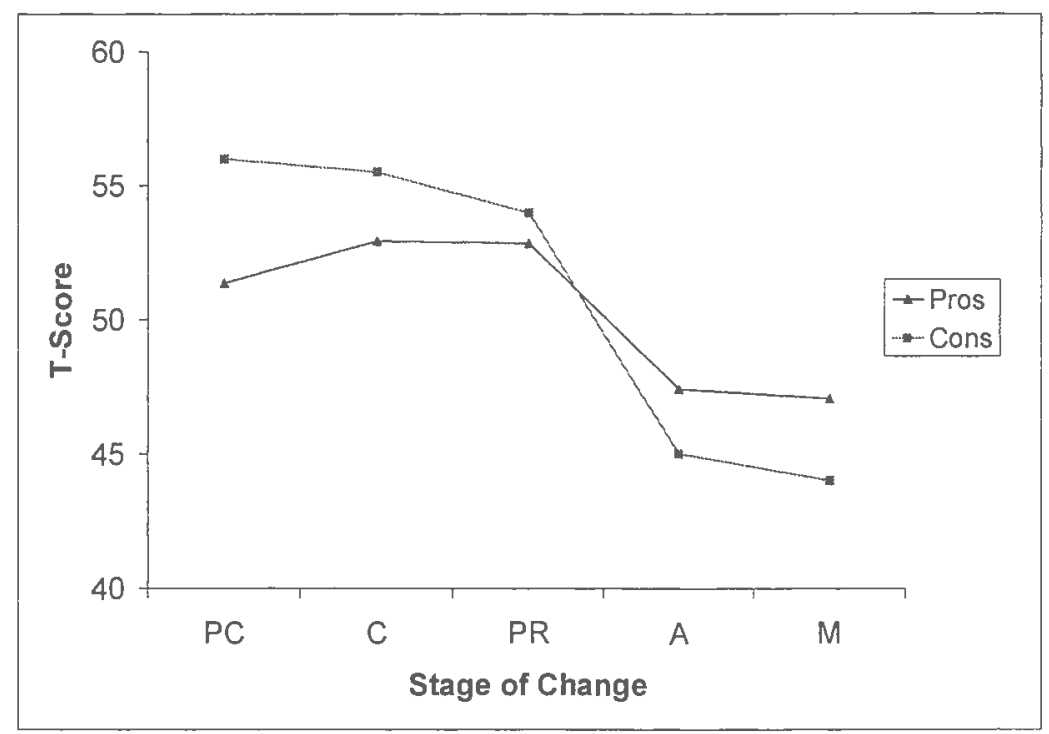

Figure 42. Graphic for Pros and Cons of Smoking from 18 to 24 Months for the Random Digit Dial Sample. 
Smoking Cessation - All Time Points Combined for the RDD Sample.

Participants in each of the four transition groups were combined across time points in order to increase sample size for each group. Once combined, mean effect sizes and $95 \%$ confidence intervals for each transition for the Pros and Cons were computed and displayed in Table 36. The largest increase for Pros was found for the PC-C transition followed by a slight increase in C-PR. The magnitude of effect for the transition from $\mathrm{PR}-\mathrm{A}$ showed a moderate decrease in effect and continued to decrease in A-M. Essentially no change was found in the transition from PC-C and C-PR for the Cons of Change followed by a large decrease from PR-A and a somewhat smaller decrease found from A-M. These transitions for Pros and Cons are graphically depicted in Figure 43.

Table 36

Mean Effect Sizes for Stage Transitions in the Random Digit Dial Sample with All Time Points Combined

\begin{tabular}{|c|cc|c|c|}
\hline \multirow{2}{*}{$\begin{array}{c}\text { Stage } \\
\text { Transitions }\end{array}$} & \multicolumn{4}{|c|}{ RDD Sample - Smoking } \\
\cline { 2 - 5 } & & & \multicolumn{2}{|c|}{$95 \%$ CI } \\
\cline { 2 - 5 } & $N$ & Mean & Lower & Upper \\
\hline PROS & & & & \\
PC-C & 805 & $\mathbf{. 1 9}$ & .01 & .37 \\
C-PR & 568 & $\mathbf{. 1 3}$ & -.05 & .31 \\
PR-A & 250 & $\mathbf{- . 4 6}$ & -.59 & -.33 \\
A-M & 299 & $\mathbf{- . 1 6}$ & -.27 & -.05 \\
CONS & & & & \\
PC-C & 805 & $\mathbf{- . 0 4}$ & -.13 & .05 \\
C-PR & 568 & $\mathbf{- . 0 1}$ & -.13 & .11 \\
PR-A & 250 & -.57 & -.70 & -.44 \\
A-M & 300 & $\mathbf{- . 2 1}$ & -.32 & -.10 \\
\hline
\end{tabular}




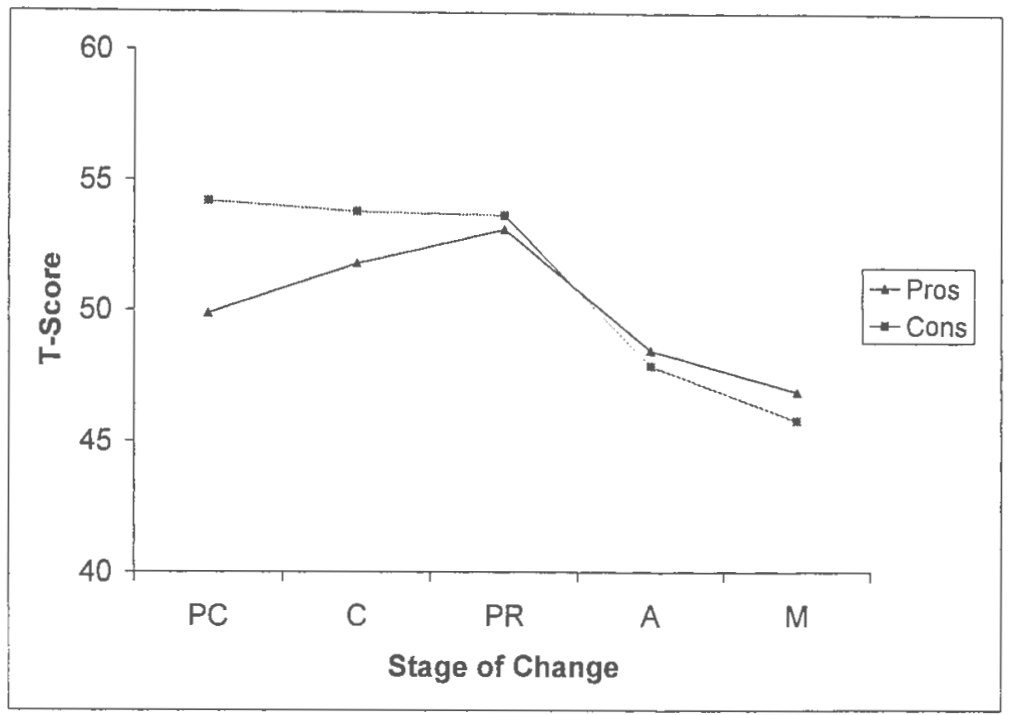

Figure 43. Graphic for Pros and Cons of Smoking for the Random Digit Dial Sample with all Time Points Combined.

\section{Smoking Cessation - "Parent" Sample.}

Effect sizes for each of the Stage transitions for the Pros and Cons of Change across the time points were calculated and are displayed in Table 37. Across the Stages from baseline to six months, a large increase was found in the PC-C with a smaller increase found for the C-PR transition, followed again by a large increase in the transition form PR-A. For the Cons of Change small increases were seen from PC-C as well as C-PR. A large decrease was found for the PR-A transition. As with the RDD sample, due to the fact that only participants in the pre-Action Stages (Precontemplation, Contemplation, and Preparation) were recruited into the original study no participants were available for the A-M transition for the baseline to 6month time points. A graphical depiction of these transitions can be seen in Figure 44. 
For time points 6 months to 12 months for the Pros of Change, essentially no change occurred from PC-C. A small increase was found from C-PR, followed by a large increase for the PR-A transition. Finally, a large decrease was seen for the A-M transition. For the Cons of Change, small decreases were found for the PC-C and CPR transitions, followed by a remarkabley large decrease in the PR-A transition. The transition from A-M displayed a small decrease. These changes are illustrated in Figure 45.

Small increases in the Pros of Change were found for the transitions from PC-C and C-PR for time points 12 months to 24 months. A remarkably large decrease was found for the transitions from PR-A, followed by a small decrease in A-M. For the Cons of Change, small increases were seen in the PC-C and C-PR. A moderate decrease was found for the transition from PR-A and essentially no change occurred in A-M. These magnitudes are illustrated in Figure 46. 
Table 37

Mean Effect Sizes for Stage Transitions in the Parent Sample across Time Points for

Smoking Cessation

\begin{tabular}{|c|c|c|c|c|c|c|}
\hline \multirow{3}{*}{$\begin{array}{c}\text { Stage } \\
\text { Transitions }\end{array}$} & \multicolumn{6}{|c|}{ Parent Sample - Smoking } \\
\hline & \multicolumn{3}{|c|}{ BL to 6} & \multicolumn{2}{|l|}{6 to 12} & 12 to 24 \\
\hline & $N$ & Mean & $N$ & Mean & $N$ & Mean \\
\hline \multicolumn{7}{|l|}{ PROS } \\
\hline PC-C & 25 & .40 & 12 & .03 & 19 & .38 \\
\hline C-PR & 18 & .21 & 10 & .23 & 16 & .22 \\
\hline PR-A & 5 & .53 & 3 & 1.01 & 10 & -1.10 \\
\hline A-M & & - & 5 & -.53 & 7 & -.22 \\
\hline \multicolumn{7}{|l|}{ CONS } \\
\hline $\mathrm{PC}-\mathrm{C}$ & 25 & .16 & 12 & -.31 & 19 & -.07 \\
\hline C-PR & 18 & .13 & 10 & -.18 & 16 & -.22 \\
\hline PR-A & 5 & -.86 & 3 & -1.51 & 10 & -.38 \\
\hline A-M & & - & & -.17 & & .00 \\
\hline
\end{tabular}




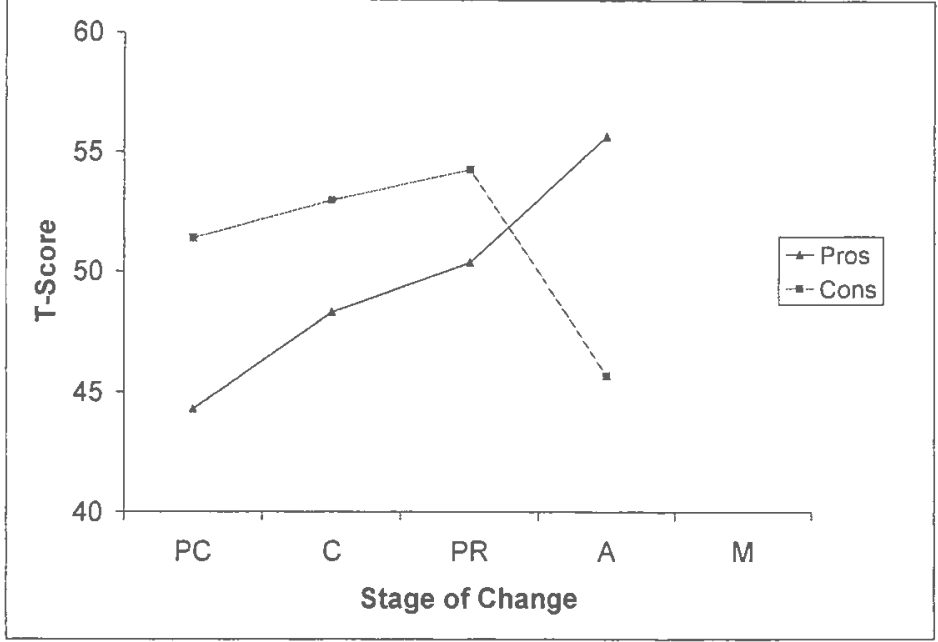

Figure 44. Graphic for Pros and Cons of Smoking from Baseline to 6 Months for the Parent Sample.

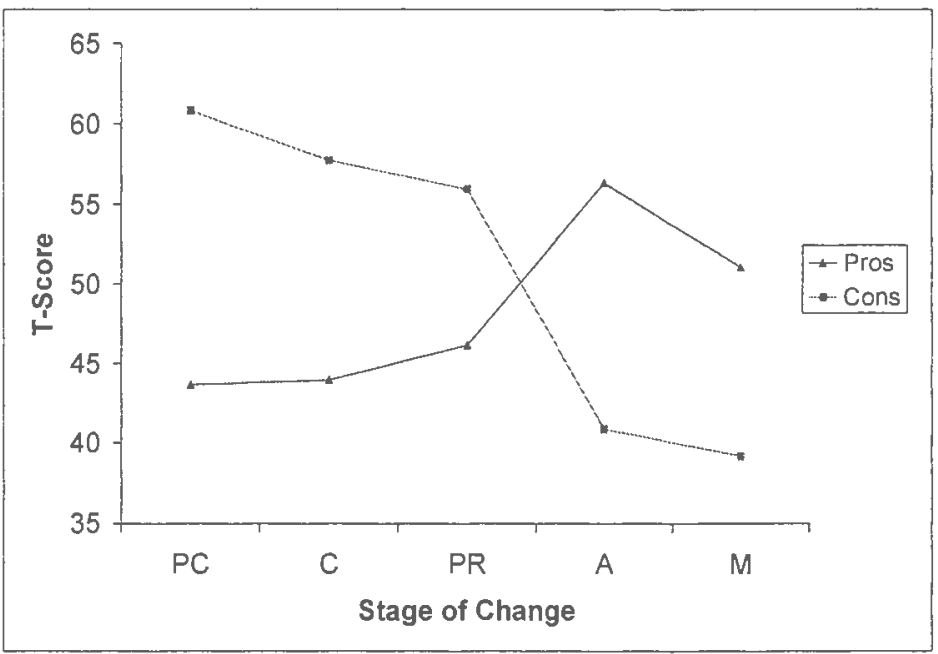

Figure 45. Graphic for Pros and Cons of Smoking from 6 to 12 Months for the Parent Sample. 


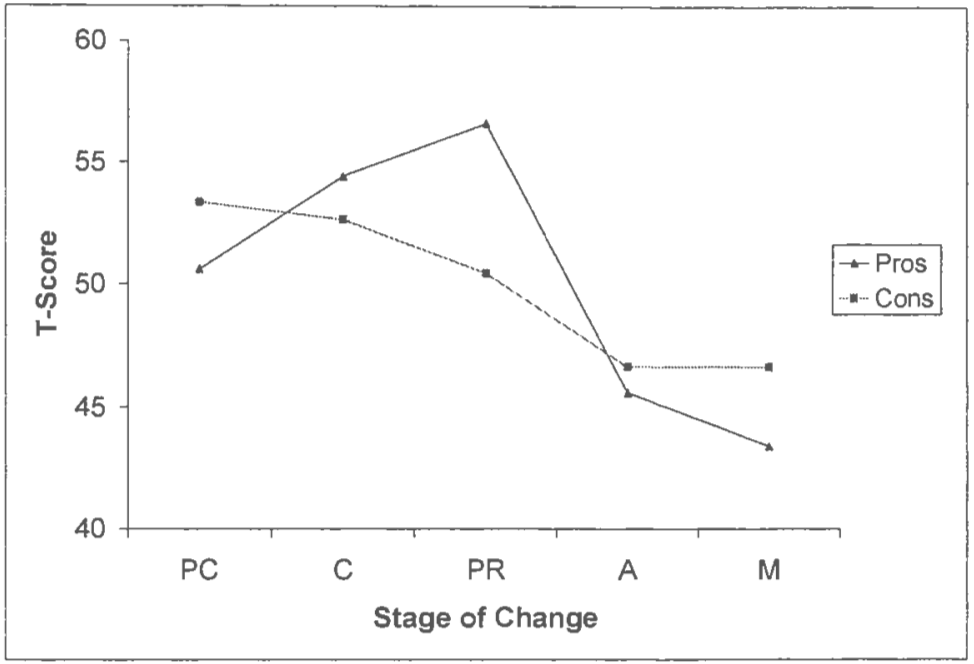

Figure 46. Graphic for Pros and Cons of Smoking Smoking from 12 to 24 Months for the Parent Sample.

Smoking Cessation - All Time Points Combined for Parent Sample

Participants in each of the four transition groups were combined across time points in order to increase sample size for each group. Once combined, mean effect sizes and $95 \%$ confidence intervals for each transition for the Pros and Cons were computed (Table 38). The largest increase for Pros was found for the PC-C transition followed by a small increase in C-PR. The magnitude of effect for the transition from decreased from PR-A and continued to decrease in A-M. Essentially no change was found in the transition from PC-C and C-PR for the Cons of Change followed by a large decrease from PR-A. Finally, a small increase was found from A-M. These transitions for Pros and Cons are graphically depicted in Figure 47. 
Table 38

Mean Effect Sizes for Stage Transitions in the Parent Sample for Smoking with All

Time Points Combined

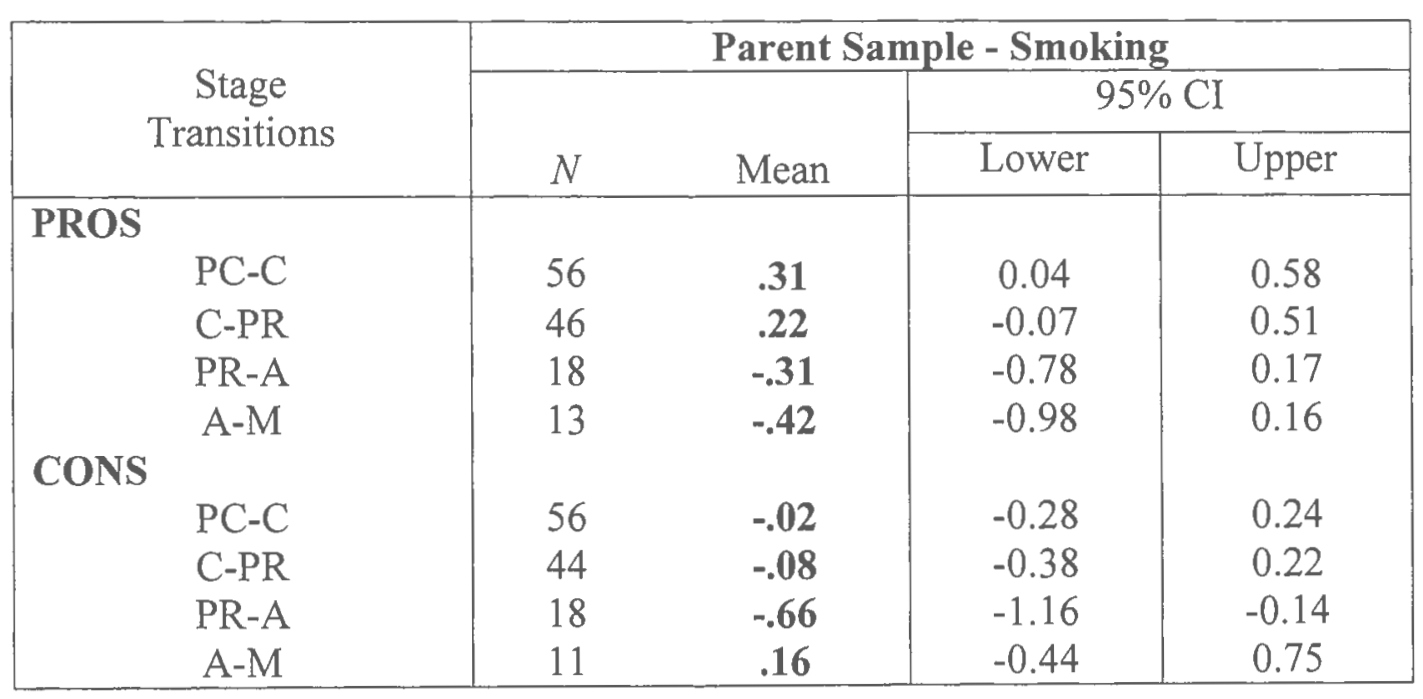




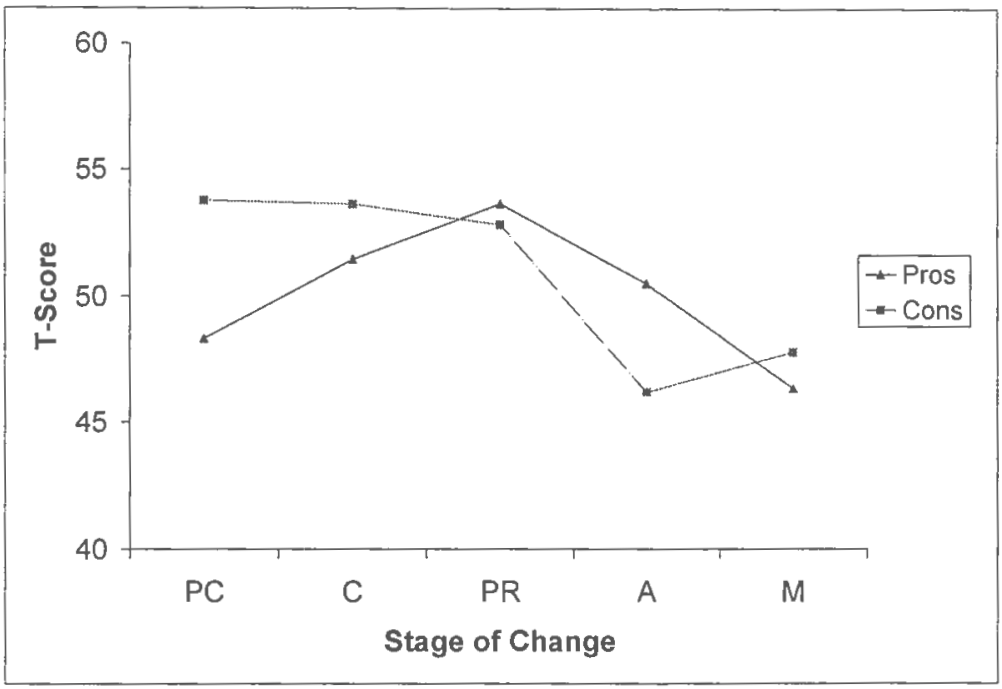

Figure 47. Graphic for Pros and Cons of Smoking for the Parent Sample with all Time Points Combined.

\section{Sun Protection - "Beach" Sample}

Effect sizes for each of the Stage transitions for the Pros and Cons of Change across the time points were calculated and are displayed in Table 39. Across the Stages from baseline to two months, essentially no change was seen from PC-C. A large increase was found for C-PR for the Pros of Change, with smaller increases from PR-A and A-M. For the Cons of Change, a small decrease was found in the PC$\mathrm{C}$ with essentially no change found for the C-PR transition. From PR-A a small increase was found followed by moderate decrease. A graphical depiction of these transitions can be seen in Figure 48.

The transitions for time points 2 months to 12 months for the Pros of Change showed a quite different pattern than baseline to 2 months, with a small increase in the PC-C transition followed by a small decrease in the Pros of Change. The last two 
Stages, PR-A and A-M showed essentially no change. In contrast a small increase for the Cons of Change was found for the PC-C transition. The Cons then decreased slightly in C-PR followed by a small increase in PR-A. Finally, essentially no change was seen from A-M. The magnitudes of effect for each of the transitions for Pros and Cons are illustrated in Figure 49.

In the final time points, 12 months to 24 months, for the Pros of Change a moderate increase was found for the PC-C transition. Due to a sample size of one, no effect size could be calculated for the transition from C-D. Essentially no change occurred in the Pros of Change for the transitions from A-M. A large decrease was found for the transitions from PC-C for the Cons of Change. Again, no data was available for the transition from C-PR. Finally, a small decrease was seen in the PRA followed by essentially no change for A-M. Due to missing data for transition from C-PR, these changes are not illustrated. 
Table 39

Mean Effect Sizes for Stage Transitions across Time Points in the Beach Study for

Sun Protection

\begin{tabular}{|c|c|c|c|c|c|c|}
\hline \multirow{3}{*}{$\begin{array}{c}\text { Stage } \\
\text { Transitions }\end{array}$} & \multicolumn{6}{|c|}{ Beach Study - Sun Protection } \\
\hline & \multicolumn{3}{|c|}{ BL to 2} & \multirow{2}{*}{$\frac{2 \text { to } 12}{\text { Mean }}$} & \multicolumn{2}{|r|}{12 to 24} \\
\hline & $N$ & Mean & $N$ & & $N$ & Mean \\
\hline \multicolumn{7}{|l|}{ Pros } \\
\hline $\mathrm{PC}-\mathrm{C}$ & 50 & .04 & 13 & .30 & 17 & .38 \\
\hline C-PR & 8 & .46 & 11 & -.13 & 1 & - \\
\hline PR-A & 16 & .15 & 18 & -.02 & 20 & .70 \\
\hline A-M & 22 & .18 & 40 & -.06 & 32 & .01 \\
\hline \multicolumn{7}{|l|}{ Cons } \\
\hline PC-C & 51 & -.18 & 13 & .20 & 17 & -.56 \\
\hline C-PR & 8 & -.07 & 11 & -.12 & 1 & - \\
\hline PR-A & 15 & .17 & 18 & .26 & 20 & .15 \\
\hline A-M & 22 & -.28 & 40 & .00 & 32 & .07 \\
\hline
\end{tabular}




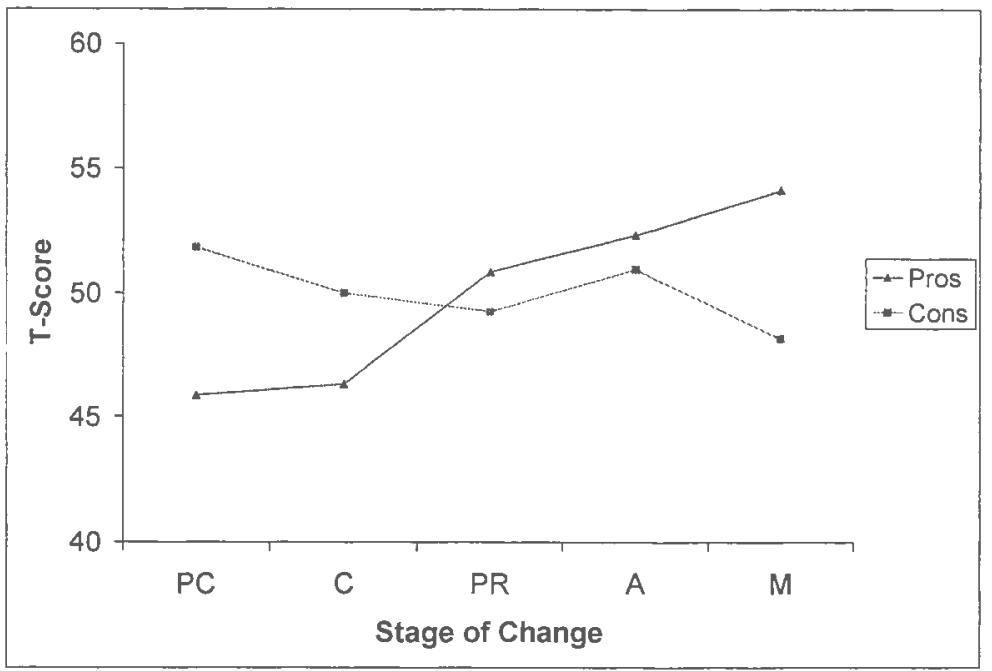

Figure 48. Graphic of Sun Protection for Baseline to 2 Months for the Beach Study.

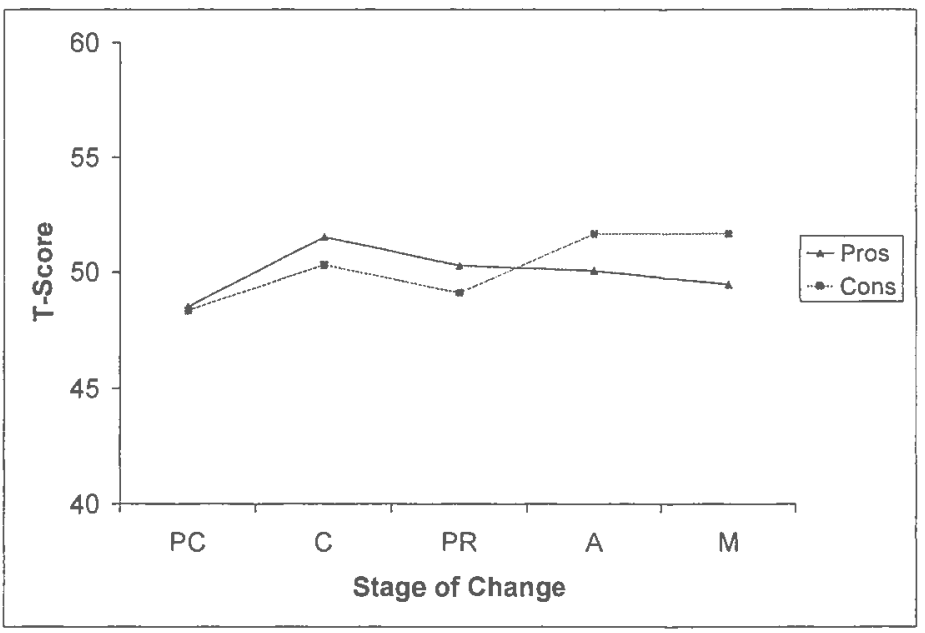

Figure 49. Graphic of Sun Protection for 2 to 12 Months for the Beach Study.

Sun Protection - All Time Points Combined for Beach Sample.

Participants in each of the four transition groups were combined across time points in order to increase sample size for each group. Once combined, mean effect sizes and 95\% confidence intervals for each transition for the Pros and Cons were 
computed (Table 40). Although it appears that small changes occurred for many of

the Stage transitions, confidence intervals indicate the only significant change

occurred for the Pros in the PR-A transition. This change was a small increase in

Pros for the PR-A transition. The magnitudes of effect for each of the transitions for

Pros and Cons are graphically depicted in Figure 50.

Table 40

Mean Effect Sizes for Stage Transitions in the Beach Sample with All Time Points

Combined

\begin{tabular}{|c|c|c|c|c|}
\hline \multirow{3}{*}{$\begin{array}{c}\text { Stage } \\
\text { Transitions }\end{array}$} & \multicolumn{4}{|c|}{ Beach Sample - Sun Protection } \\
\hline & \multirow[b]{2}{*}{$N$} & \multirow[b]{2}{*}{ Mean } & \multicolumn{2}{|c|}{$95 \% \mathrm{CI}$} \\
\hline & & & Lower & Upper \\
\hline \multicolumn{5}{|l|}{ PROS } \\
\hline PC-C & 80 & .15 & -0.07 & 0.37 \\
\hline C-PR & 20 & .14 & -0.30 & 0.58 \\
\hline PR-A & 53 & .29 & 0.01 & 0.56 \\
\hline A-M & 94 & .03 & -0.17 & 0.23 \\
\hline \multicolumn{5}{|l|}{ CONS } \\
\hline PC-C & 80 & -.19 & -0.41 & 0.03 \\
\hline C-PR & 20 & -.17 & -0.61 & 0.27 \\
\hline PR-A & 53 & .20 & -0.07 & 0.47 \\
\hline A-M & 94 & -.05 & -0.25 & 0.15 \\
\hline
\end{tabular}




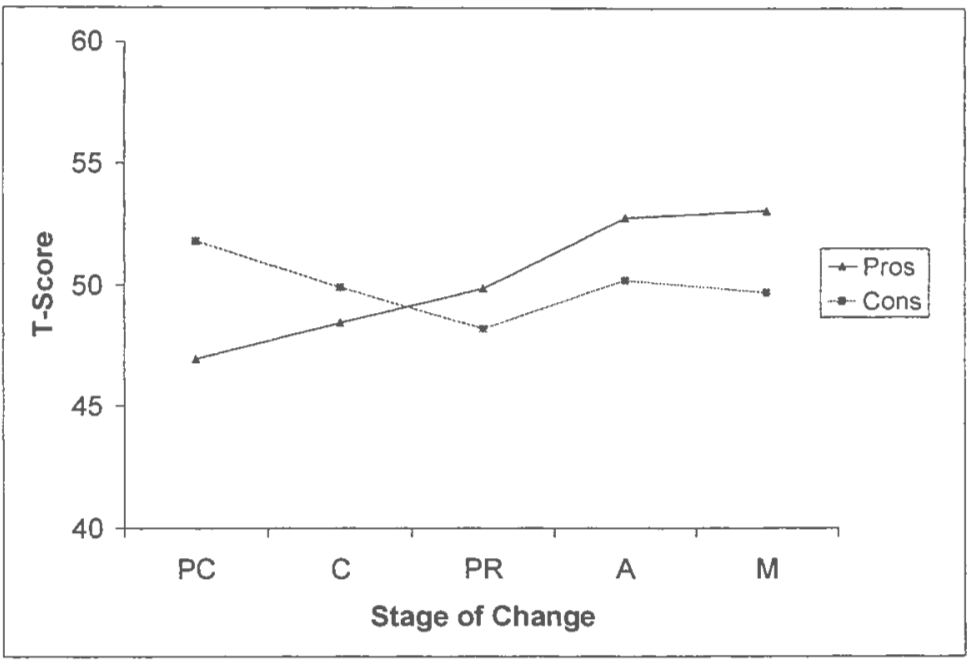

Figure 50. Composite Graphic for Sun Protection in the Beach Sample across All Time Points

\section{Sun Protection - Parent Sample}

Effect sizes for each of the Stage transitions for the Pros and Cons of Change across the time points were calculated and are displayed in Table 41. Across time points baseline to 6 months for the Pros of Change, a moderate decrease was found in the PC-C transition with essentially no change for the C-PR transition. Lastly, a slight increase occurred in the transition form PR-A. For the Pros of Change, small decreases were seen from PC-C whereas essentially no change occurred from C-PR. A small increase in magnitude of effect was found from PR-A. As mentioned previously, due to the fact that only participants in the pre-Action Stages (Precontemplation, Contemplation, and Preparation) were recruited into the original study no participants were available for the A-M transition for the baseline to 6- 
month time points. A graphical depiction of the magnitudes of effect for the Stage transitions can be seen in Figure 51.

Across time points 6 months to 12 months for the Pros of Change, a small increase was found in the PC-C transition, followed by a moderate increase in C-PR. Again a small increase was found for the Pros of Change from PR-A with an additional increase from A-M. For the Cons of Change, essentially no change was found from PC-C. The C-PR transition showed a small increase in the Cons of Change, followed again by essentially no change in PR-A. Finally, a decrease magnitude was found for the A-M transition from 6 months to 12 months. These changes are illustrated in Figure 52.

Across time points 12 months to 24 months for the Pros of Change, essentially no change was found in PC-C. Small increases were found for C-PR and PR-A, followed by a slight decrease from A-M. Essentially no change occurred in the Cons of Change were found for the transitions from PC-C and C-PR. A slight increase was found for the transition from PR-A with a slight decrease from A-M. These magnitudes are graphed in Figure 53. 
Table 41

Mean Effect Sizes for Stage Transitions in the Parent Sample across Time Points for

Sun Protection

\begin{tabular}{|c|c|c|c|c|c|c|}
\hline \multirow{3}{*}{$\begin{array}{c}\text { Stage } \\
\text { Transitions }\end{array}$} & \multicolumn{6}{|c|}{ Parent Sample - Sun Protection } \\
\hline & \multicolumn{3}{|c|}{ BL to 6} & \multicolumn{2}{|l|}{6 to 12} & \multirow{2}{*}{$\frac{12 \text { to } 24}{\text { Mean }}$} \\
\hline & $N$ & Mean & $N$ & Mean & $N$ & \\
\hline \multicolumn{7}{|l|}{ PROS } \\
\hline PC-C & 33 & -.36 & 27 & .18 & 34 & .06 \\
\hline C-PR & 47 & -.04 & 33 & .45 & 60 & .31 \\
\hline PR-A & 74 & .09 & 58 & .28 & 86 & .41 \\
\hline A-M & - & - & 51 & .11 & 102 & -.08 \\
\hline \multicolumn{7}{|l|}{ CONS } \\
\hline $\mathrm{PC}-\mathrm{C}$ & 33 & -.18 & 26 & .04 & 34 & .02 \\
\hline C-PR & 47 & -.00 & 33 & .11 & 60 & -.01 \\
\hline PR-A & 73 & .15 & 57 & .04 & 84 & .09 \\
\hline A-M & - & - & 51 & -.20 & 101 & -09 \\
\hline
\end{tabular}




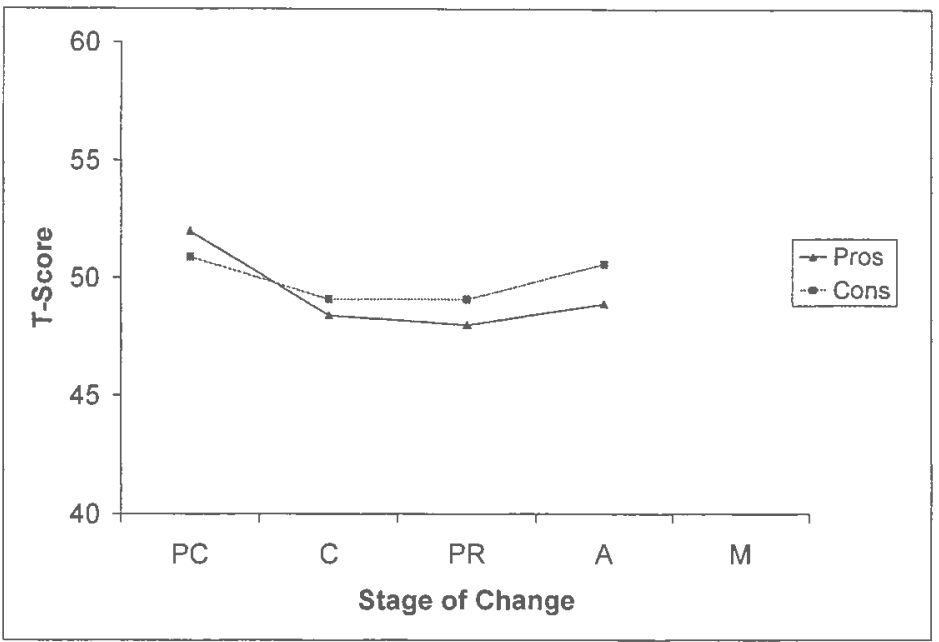

Figure 51. Graphic of Sun Protection for Baseline to 6 Months for the Parent Study

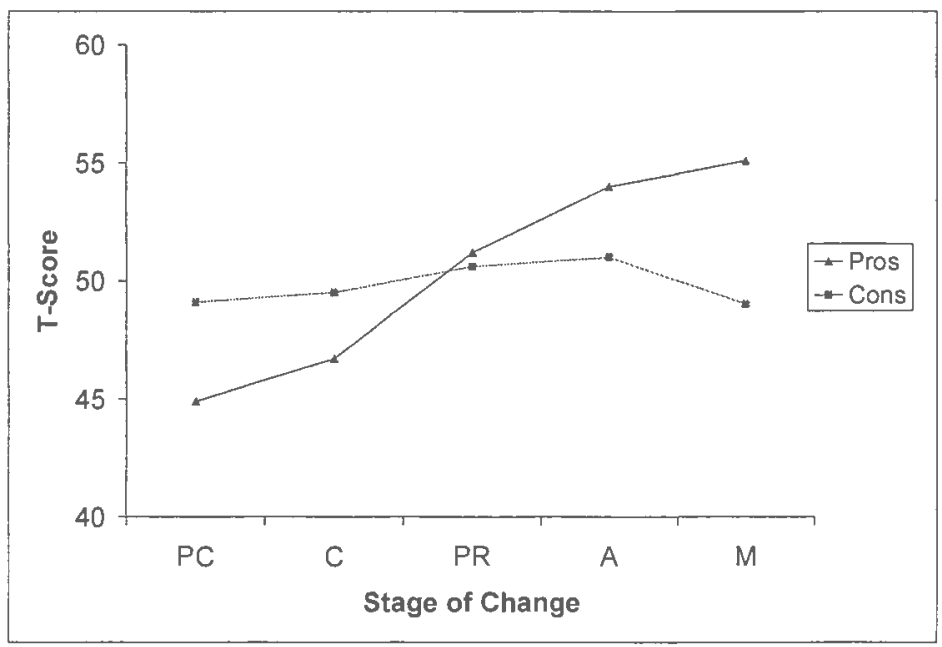

Figure 52. Graphic of Sun Protection for 6 to 12 Months for the Parent Study. 


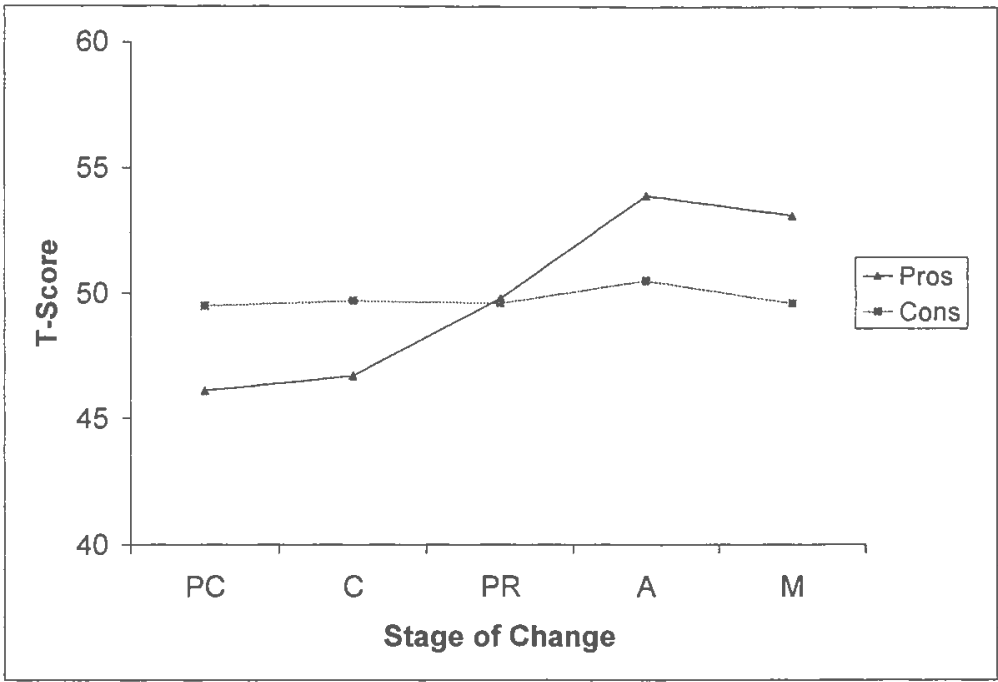

Figure 53. Graphic of Sun Protection for 12 to 24 Months for the Parent Study

Sun Protection - All Time Points Combined for Parent Sample.

Participants in each of the four transition groups were combined across time points in order to increase sample size for each group. Once combined, mean effect sizes and $95 \%$ confidence intervals for each transition for the Pros and Cons were computed and are displayed in Table 42. Essentially no change in the Pros was found for the PC-C transition. Small increases were found for the C-PR and PR-A transitions, with essentially no change in the A-M transitions. Essentially no change in the Cons of Change was found across all the Stage transitions. The magnitudes of effect for each of the transitions for Pros and Cons are graphically depicted in Figure 54. 
Mean Effect Sizes for Stage Transitions in the Parent Sample for Sun Protection with All Time Points Combined

\begin{tabular}{|c|c|c|c|c|}
\hline \multirow{3}{*}{$\begin{array}{c}\text { Stage } \\
\text { Transitions }\end{array}$} & \multicolumn{4}{|c|}{ Parent Sample - Sun Protection } \\
\hline & \multirow[b]{2}{*}{$N$} & \multirow[b]{2}{*}{ Mean } & \multicolumn{2}{|c|}{$95 \% \mathrm{CI}$} \\
\hline & & & Lower & Upper \\
\hline \multicolumn{5}{|l|}{$\overline{\text { PROS }}$} \\
\hline $\mathrm{PC}-\mathrm{C}$ & 93 & -.04 & -.24 & .16 \\
\hline C-PR & 139 & .23 & .06 & .40 \\
\hline PR-A & 214 & .26 & .12 & .40 \\
\hline A-M & 152 & -.01 & -.17 & .15 \\
\hline \multicolumn{5}{|l|}{ CONS } \\
\hline PC-C & 94 & -.05 & -.25 & .15 \\
\hline C-PR & 137 & .03 & -.14 & .20 \\
\hline PR-A & 216 & .09 & -.04 & .22 \\
\hline A-M & 153 & -.09 & -.25 & .07 \\
\hline
\end{tabular}




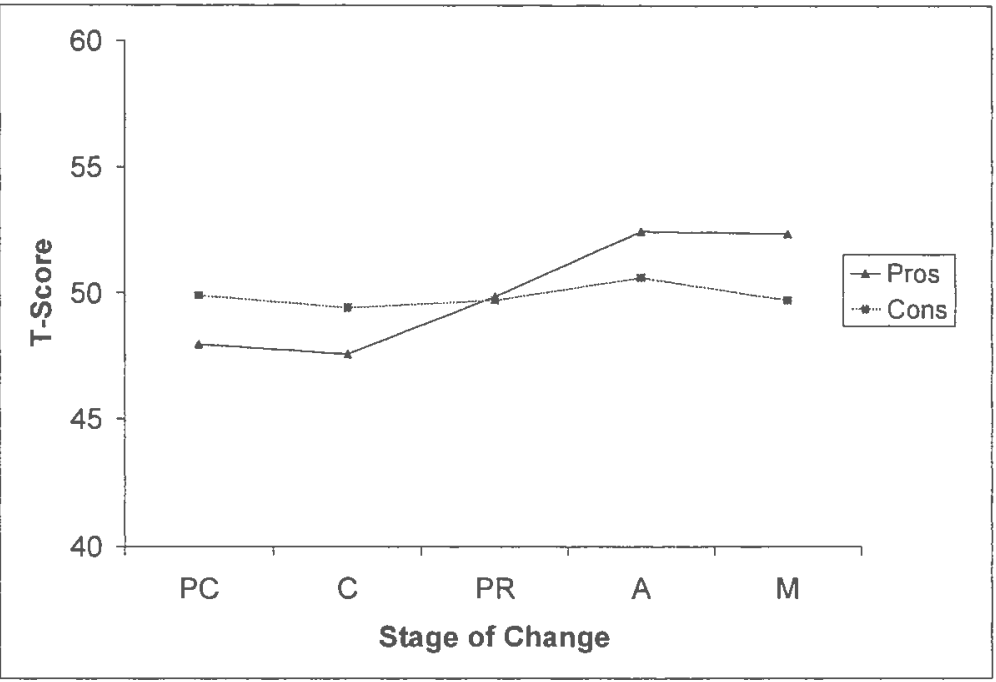

Figure 54. Composite Graphic for Sun Protection in the Parent Sample across All Time Points

\section{Discussion}

Two behaviors, sun protection and smoking cessation, were examined in this study. Two different samples were examined for each of the behaviors. Smoking cessation was examined in a sample of adults that were randomly contacted by phone using a random digit dial procedure as well as in a sample of parents that were identified in connection with a larger health behavior study with adolescents in schools. This same parent sample was analyzed separately for sun protection. Lastly, sun protection was examined in a sample of beach goers.

Overall, the sample sizes for each of the behaviors for the Pros and Cons across the Stage transitions were small. Quite small sample sizes were found for many of the separate time point analyses, especially for the Beach sample. Interpreting the results for each the behaviors for the individual the time points 
proved difficult, likely due to the small sample sizes. Clearer patterns began to emerge as sample sizes were increased by combining the Stage transitions across time points. Unfortunately, one consequence of combining the time points across the stages is the lose of independence in the data. When sets time points are examined separately - each participant, by design, can only be included in one of the four stage transitions for that set of time points. In contrast, when combining sets of time points (e.g., baseline to 6 months with 6 months to 12 months) participants may be included in more than one time point and therefore contribute more than one set of data. This type of dependency in the combined data was not accounted for in the crosssequential analyses that combined sets of time points. Therefore, although the combined data analyses have the advantage of larger sample size, the results should be interpreted with caution due to the dependency in the data. More research is needed to understand the impact of the dependency and methods developed to account for such dependency.

As predicted, the overall effect sizes appear larger for the longitudinal study 


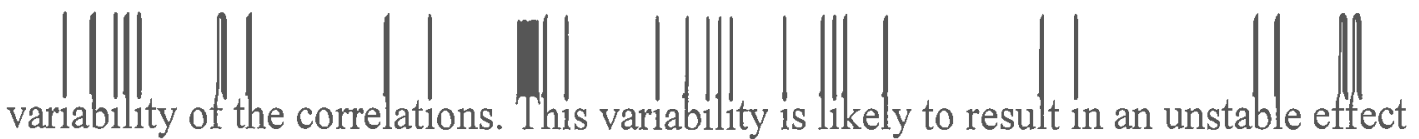
size measure, making it difficult to rely on the effect size patterns. Additionally the effect size measure used in the cross-sectional study is calculated using a different procedure. It is unclear how this difference effects the comparison of the two types of data. More research is essential in order to resolve these important issues.

Despite these caveats, composite profiles for each of the behaviors are somewhat consistent with the cross-sectional data. The cross-sectional profile of the Pros and Cons of Change for Smoking Cessation is shown in Figure 55. Nearly all nine of the sets of effects sizes for the Pros decrease in transitions from PR-A and AM. The primary difference across the longitudinal and cross-sectional profiles for smoking cessation is found in the large decrease later Stage transitions. In light of this pattern, it should be noted that on the whole participants in the Maintenance stages of these longitudinal datasets are recent Maintainers. Since no participants were in the Maintenance Stages at the beginning of the two smoking samples, these Maintainers have only maintained the given behavior for less than a year or two. In contrast, participants in the Maintenance stages of cross-sectional studies may have 


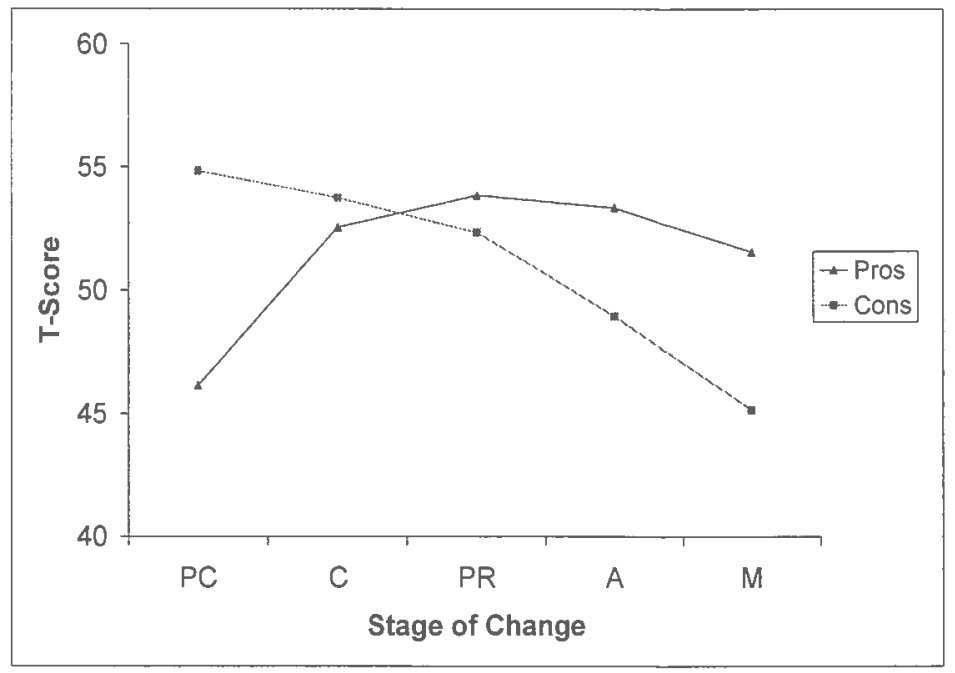

Figure 55. Cross-sectional Smoking Cessation Profile $(n=25)$.

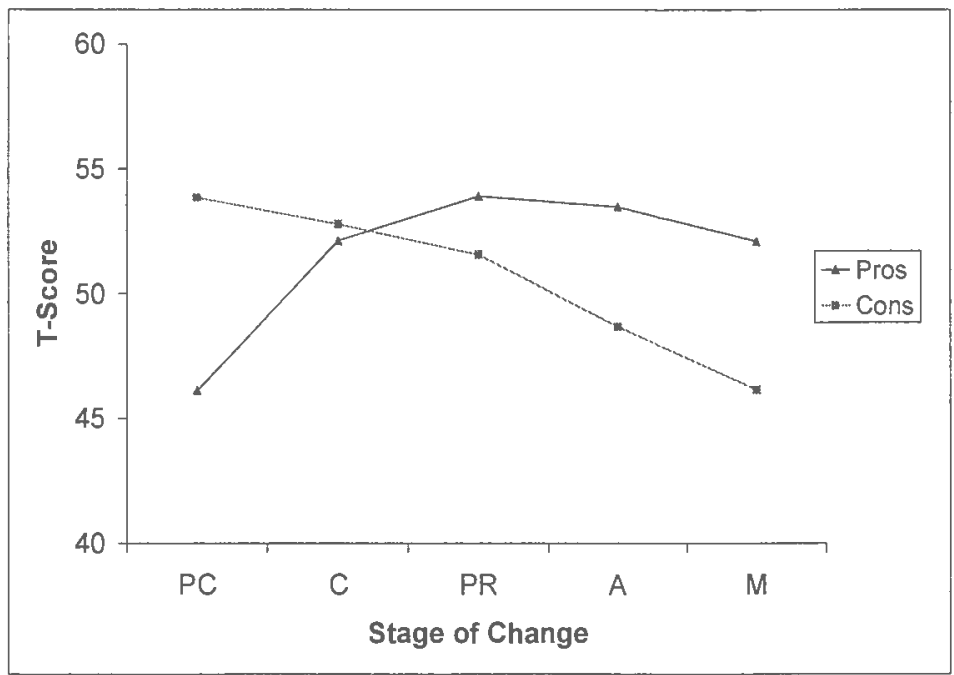

Figure 56. Cross-sectional Profile for Unhealthy Behaviors $(\mathrm{n}=45)$. 
The cross-sectional profile of the Pros and Cons of Sun Protection is shown in Figure 57. Unfortunately the cross-sectional profile for sun protection is represented by only one study. Although typically profiles represented by one dataset are somewhat unstable, a semi-consistent pattern can be seen between the crosssectional and longitudinal data. Five out of six longitudinal composite profiles for sun protection show an $\mathrm{S}$ shape across the stages for the Pros of change similar to that seen in the cross-sectional sun protection profile below. Additionally, similar to the cross-sectional data, the Cons of Change are somewhat flat across the changes with the largest decrease in the $\mathrm{PC}-\mathrm{C}$ transition. The reasonably flat profile indicates that only a small amount of change is occurring for the Cons of Sun Protection as individuals move from one Stage to the next.

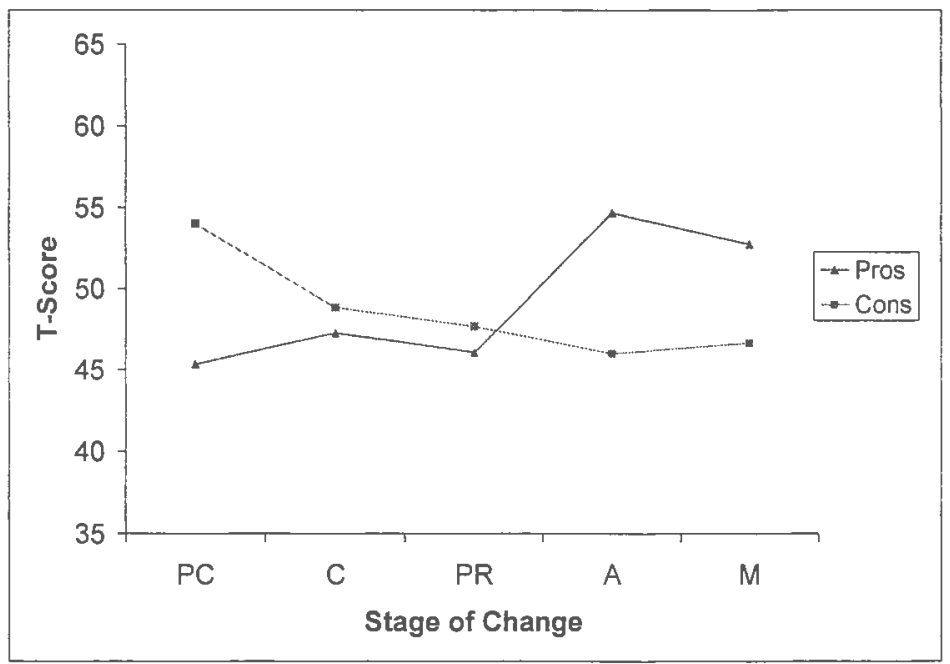

Figure 57. Cross-sectional Sun Protection Profile $(\mathrm{n}=1)$. 
This investigation serves as a very preliminary look into the comparison of cross-sectional and longitudinal data. These preliminary examinations found that overall the cross-sectional and longitudinal data were reasonably comparable, with the earlier stages looking more similar between the two than the later stages. This study is the first step to understanding the utility of cross-sectional data for representing longitudinal relationships across the Stages of Change, specifically for the Pros and Cons of Change. Looking ahead to more comprehensive examinations of longitudinal effect size comparisons of the TTM constructs across the Stages, a variety of limitations should be noted.

Firstly, the exploration of the current longitudinal datasets included the treatment and control groups combined. It would be of great interest to examine these groups separately. Participants in the control group moving from one Stage to the next, aside from the potential impact of assessment, in effect are changing naturalistically. Comparisons of the control groups with the treatment groups would begin to determine if those changing with the aid of an intervention change in a similar fashion to those changing naturalistically. One might ask, is behavior change facilitated by an intervention the same process for those who change naturalistically - just accelerated?

Another important issue is the time interval differences across time points. In the current exploration all adjacent time points were included to increase the number of transitions. This resulted in some effect sizes estimates representing a time interval of 2 months where as another represented 12 months. In the context of a larger study, differences in time intervals should be explored. 
The TTM utilizes the Pros and Cons of Change as an important part of the

theoretical model of behavior change. Cross-sectional and longitudinal explorations have shown Decisional Balance and the Stages of Change to be theoretically consistent and practically useful. The Pros and Cons are just one aspect of decision making. Literature spanning diverse disciplines such as psychology and economics has begun to amass a rich body of knowledge and theoretical ideas regarding decision making that would likely enrich the TTM. Future research should aim to expand the use of decision making in the context of the TTM of behavior change. Such an endeavor is likely to increase the effectiveness and utility of an already successful model.

\section{References}

DiClemente, C.C., Prochaska, J.O., Fairhurst, S.K., Velicer, W.F., Velasquez, M.M., \& Rossi, J.S. (1991). The process of smoking cessation: An analysis of precontemplation, contemplation and preparation stages of change. Journal of Consulting and Clinical Psychology, 59, 295-304.

Hall, K.L., \& Rossi, J.S. (2004). Structure, function, and effect size of decisional balance across the stages of change for 37 health behaviors: A meta-analytic study. Manuscript in preparation.

Horn, D. (1976). A model for the study of personal choice health behavior. International Journal of the Addictions, 19, 89-98.

Horn, D. \& Waingrow S. (1966). Some dimensions of a model for smoking behavior change. American Journal of Public Health, 56, 21. 
Janis, I.L. (1959). Decisional conflicts: A theoretical analysis. Journal of Personality and Social Psychology, 31, 8-33-839.

Janis, I. L., \& Mann, L. (1977). Decision making: A psychological analysis of conflict, choice and commitment. New York: Free Press.

Prochaska, J.O. (1994). Strong and weak principles for progressing from precontemplation to action on the basis of twelve problem behaviors. Health Psychology, 13(1), 47-51.

Prochaska, J. O., Velicer, W. F., Guadagnoli, E., Rossi, J. S. \& DiClemente, C. C. (1991). Patterns of change: A dynamic typology applied to smoking cessation. Multivariate Behavioral Research, 26, 83-107.

Prochaska, J.O., Velicer, W.F., Fava, J.L., Rossi, J.S., \& Tsoh, J.Y. (2001).

Evaluating a population-based recruitment approach and a stage-based expert system intervention for smoking cessation. Addictive Behaviors, 26, 583-602.

Prochaska, J. O., Velicer, W. F., Rossi, J. S., Goldstein, M. G., Marcus, B. H., Rakowski, W., Fiore, C., Harlow, L. L., Redding, C. A., Rosenbloom, D., \& Rossi, S. R. (1994). Stages of change and decisional balance for 12 problem behaviors. Health Psychology, 13, 39-46.

Prochaska, J.O., Velicer, W.F., Rossi, J.S., Redding, C., Greene, G., Rossi, S., Sun, X., Fava, J., Laforge, R., Plummer, B. (in press). Impact of Simultaneous Stagematched Expert System Interventions for Smoking, High Fat Diet and Sun Exposure on a Population of Parents. Health Psychology. 
Velicer, W. F., DiClemente, C. C., Prochaska, J. O., \& Brandenberg, N. (1985). A decisional balance measure for assessing and predicting smoking status. Journal of Personality and Social Psychology, 48, 1279-1289.

Velicer, W.F., Prochaska, J.O., Fava, J.L., Rossi, J.S., Redding, C.A., Laforge, R.G., \& Robbins, M.L. (2000). Using the transtheoretical model for population-based approaches to health promotion and disease prevention. Homeostasis, 40, 174195.

Weinstock, M. A., Rossi, J. S., Redding, C. A., \& Maddock, J. E. (2002). Randomized controlled community trial of the efficacy of a multi-component stage-matched intervention to increase sun protection among beachgoers. Preventive Medicine, 35, 584-592. 


\section{Appendix A}

Table A.1

Coding Manual

\begin{tabular}{|c|c|}
\hline Variable Name & Instructions / Description \\
\hline \multicolumn{2}{|l|}{ Study Descriptors } \\
\hline Study ID & $\begin{array}{l}\text { Assign a unique identification number to each study. Each } \\
\text { identification number is comprised of the first four letters of } \\
\text { the primary author's last name, the first letter of the primary } \\
\text { author's first name, the last two digits of the publication year, } \\
\text { and a three-letter behavior code. (See list behavior codes } \\
\text { below) If a report presents two independent studies (i.e., two } \\
\text { independent outcome studies with different participants or } \\
\text { different target behaviors) they must be entered } \\
\text { independently. }\end{array}$ \\
\hline Author & $\begin{array}{l}\text { List the last name of author for each dataset. For studies with } \\
\text { multiple authors, the primary author's last name is entered, } \\
\text { followed by the term "et al." }\end{array}$ \\
\hline Year & $\begin{array}{l}\text { Enter the 4-digit year of publication. For manuscripts in } \\
\text { preparation, enter year of draft. For unpublished datasets, } \\
\text { enter year as specified by author, if unspecified list year data } \\
\text { was obtained. }\end{array}$ \\
\hline $\begin{array}{l}\text { Recruitment } \\
\text { Setting }\end{array}$ & Enter the recuritment setting as described in the article/study. \\
\hline $\begin{array}{l}\text { Recruitment } \\
\text { Setting Category }\end{array}$ & $\begin{array}{l}\text { Code the Recruitment Setting in to one of the following } \\
\text { categories: } \\
1=\text { community-based } \\
2 \text { = health clinics } \\
3=\text { physicians offices } \\
4=\text { school } \\
5=\text { treatment centers }\end{array}$ \\
\hline
\end{tabular}




\begin{tabular}{|c|c|}
\hline & $\begin{array}{l}6=\text { university } \\
7=\text { work sites } \\
8=\text { other }\end{array}$ \\
\hline Country & List the country in which the study took place. \\
\hline Country Category & $\begin{array}{l}\text { Code the country into one of the following: } \\
1=\text { United States } \\
2=\text { Non- US }\end{array}$ \\
\hline Language & $\begin{array}{l}\text { Enter the language in which the study was conducted (i.e., the } \\
\text { language of the measures used). }\end{array}$ \\
\hline $\begin{array}{l}\text { Language } \\
\text { Category }\end{array}$ & $\begin{array}{l}\text { Code Language into the following categories: } \\
1=\text { English } \\
2=\text { Non-English }\end{array}$ \\
\hline Publication & $\begin{array}{l}\text { Enter the publication type and/or status of the study/data } \\
\text { being used. }\end{array}$ \\
\hline $\begin{array}{l}\text { Publication } \\
\text { Category }\end{array}$ & $\begin{array}{l}\text { Code Publication description into the following categories: } \\
1=\text { technical manual } \\
2=\text { dissertation } \\
3=\text { peer review journal } \\
4=\text { manuscript in prep } \\
5=\text { masters thesis } \\
6=\text { unpublished data } \\
7=\text { conference presentation } \\
8=\text { research competency } \\
9=\text { other }\end{array}$ \\
\hline $\begin{array}{l}\text { Recruitment } \\
\text { Method }\end{array}$ & Provide a brief description of the sample recruitment method. \\
\hline $\begin{array}{l}\text { Recruitment } \\
\text { Method Category }\end{array}$ & $\begin{array}{l}\text { Indicate whether the method for recruiting participants was } \\
\text { "Active" (e.g., telephoning people at home, approaching } \\
\text { people on the streets) or "Passive" (e.g., mailing out surveys, } \\
\text { advertising studies in the newspaper). } \\
1=\text { active } \\
2=\text { passive } \\
3=\text { active/passive } \\
4=\text { not reported }\end{array}$ \\
\hline $\begin{array}{l}\text { Classification of } \\
\text { Study }\end{array}$ & $\begin{array}{l}\text { Describe the type of study conducted (e.g., measurement } \\
\text { development, intervention) }\end{array}$ \\
\hline \multicolumn{2}{|c|}{ Behavior Descriptors } \\
\hline Target Behavior & $\begin{array}{l}\text { List the target behaviors examined in each dataset. (See list } \\
\text { of target behaviors for examples). The target behavior is the } \\
\text { specific behavior measured by the Stage algorithm }\end{array}$ \\
\hline
\end{tabular}




\begin{tabular}{|c|c|}
\hline $\begin{array}{l}\text { Behavior } \\
\text { Category }\end{array}$ & $\begin{array}{l}\text { Enter the primary behavior category on which each study is } \\
\text { focused (See list of Behavior Categories) }\end{array}$ \\
\hline $\begin{array}{l}\text { Cessation vs. } \\
\text { Acquisition }\end{array}$ & $\begin{array}{l}\text { Identify the target behavior according to whether it described } \\
\text { as ending a current behavior (cessation - e.g., quitting } \\
\text { smoking) or starting a new one (acquisition - e.g., bone } \\
\text { density testing). Code using the following: } \\
1 \text { = acquisition } \\
2=\text { cessation }\end{array}$ \\
\hline $\begin{array}{l}\text { Healthy, } \\
\text { Unhealthy, } \\
\text { Prevention or } \\
\text { Non-Health } \\
\text { related }\end{array}$ & $\begin{array}{l}\text { Code the target behavior according to whether the behavior is } \\
\text { rooted in a healthy (e.g., exercise) or unhealthy (e.g., binge } \\
\text { drinking). If a behavior does not examine a health related } \\
\text { behavior (administrative change) code accordingly. Code } \\
\text { using the following: } \\
1=\text { healthy } \\
2=\text { unhealthy } \\
3=\text { non-health related } \\
\text { Behaviors that examine prevention behaviors should be } \\
\text { coded separately. Prevention of a behavior typically involves } \\
\text { individuals whom are likely to be not engaging / exhibiting in } \\
\text { a particular behavior, but may or may not in the future. (e.g, } \\
\text { smoking acquisition, depression prevention). Code using the } \\
\text { following: } \\
4=\text { prevention }\end{array}$ \\
\hline Frequency & $\begin{array}{l}\text { Identify the frequency in which the behavior is generally } \\
\text { performed. A behavior is coded as daily/regularly if the } \\
\text { behavior is generally performed daily or multiple times a } \\
\text { week (e.g., flossing teeth, exercise). Situational describes the } \\
\text { frequency of a behavior for behaviors performed when the } \\
\text { situation arises (e.g., condom use). If a behavior is typically } \\
\text { performed one a year (e.g., STD screening) or less (e.g., } \\
\text { radon testing) it should be coded as yearly. Code into one of } \\
\text { the following categories: } \\
1=\text { daily/regularly } \\
2 \text { = situational } \\
3 \text { = yearly }+\end{array}$ \\
\hline \multicolumn{2}{|c|}{ Sample Descriptors } \\
\hline $\begin{array}{l}\text { Sample } \\
\text { Description }\end{array}$ & $\begin{array}{l}\text { Enter the population description as described in the } \\
\text { article/study. }\end{array}$ \\
\hline
\end{tabular}




\begin{tabular}{|l|l|}
\hline Percent Female & $\begin{array}{l}\text { Enter the percentage of female participants in each study as } \\
\text { stated in the article. If not reported specifically and } \\
\text { sufficient data is available, calculate percentage of females. }\end{array}$ \\
\hline Percent Male & $\begin{array}{l}\text { Enter the percentage of male participants in each study as } \\
\text { stated in the article. If not reported specifically and } \\
\text { sufficient data is available, calculate percentage of males. }\end{array}$ \\
\hline Mean Age & $\begin{array}{l}\text { Report the mean age for the entire sample as directly stated in } \\
\text { the article. If not reported specifically and sufficient data is } \\
\text { available, calculate the mean age. }\end{array}$ \\
\hline Age Group & $\begin{array}{l}\text { Enter the age group of the participants. This will be based on } \\
\text { the description the sample, as well as the setting of the study. } \\
\text { Code age group into one of the following categories: }\end{array}$ \\
& $\begin{array}{l}1=\text { adolescents } \\
2=\text { college } \\
3=\text { adults } \\
4=\text { mixed }\end{array}$ \\
\hline Educational Level & $\begin{array}{l}\text { Report the mean years of education based on the entire } \\
\text { sample. It should be entered exactly as it is reported in the } \\
\text { text. }\end{array}$ \\
\hline Sampling Method & $\begin{array}{l}\text { Enter the way in which the sample of participants in each } \\
\text { study was selected. } \\
1=\text { random } \\
2=\text { convenience } \\
3=\text { mixed } \\
4=\text { not reported }\end{array}$ \\
\hline
\end{tabular}

Stage Variables and Descriptors

\begin{tabular}{|c|c|}
\hline Cross-Over Stages & $\begin{array}{l}\text { Enter the Stages between which the pros and cons scores } \\
\text { graphically cross. This must be based on T-scores values. If t- } \\
\text { scores are not reported in a table or graph the cross-over } \\
\text { Stage can not be identified. } \\
1=\text { PC-C } \\
2=\text { C-PR } \\
3=\text { PR-A } \\
4=\text { A-M } \\
5=\text { C-A } \\
6=\text { multiple } \\
7=\text { raw/no graph } \\
8=\text { other (e.g., No Cross) } \\
9=\text { not available }\end{array}$ \\
\hline Inclusion of & Indicate whether or not Preparation Stage is included in the \\
\hline
\end{tabular}




\begin{tabular}{|c|c|}
\hline Preparation Stage & $\begin{array}{l}\text { final analysis for the study. } \\
1=\text { yes } \\
2=\text { no }\end{array}$ \\
\hline $\begin{array}{l}\text { Inclusion of } \\
\text { Maintenance } \\
\text { Stage }\end{array}$ & $\begin{array}{l}\text { Indicate whether or not Maintenance Stage is included in the } \\
\text { final analysis for the study } \\
1=\text { yes } \\
2=\text { no }\end{array}$ \\
\hline $\begin{array}{l}\text { Inclusion of } \\
\text { Cont/Prep Stage }\end{array}$ & $\begin{array}{l}\text { Indicate whether or not a combined } \\
\text { Contemplation/Preparation Stage is included in final analysis } \\
\text { for the study. } \\
1=\text { yes } \\
2=\text { no }\end{array}$ \\
\hline $\begin{array}{l}\text { Inclusion of } \\
\text { Action/Main } \\
\text { Stage }\end{array}$ & $\begin{array}{l}\text { Enter yes or no to indicate whether or not there is a combined } \\
\text { Action/Maintenance Stage included in final analysis for the } \\
\text { study. } \\
1=\text { yes } \\
2=\text { no }\end{array}$ \\
\hline $\begin{array}{l}\text { Stage Description } \\
\text { Code }\end{array}$ & $\begin{array}{l}\text { Indicate how the Staging descriptions were reported in the } \\
\text { text. Enter "TTM" if Stage descriptions were only included as } \\
\text { general TTM stage descriptions and indicate no target } \\
\text { behavior. Enter "SS" if Staging descriptions are study } \\
\text { specific, that is, if they are specific to the target behavior in } \\
\text { the given study. } \\
1=\text { TTM } \\
2=\text { SS } \\
3=\text { not reported }\end{array}$ \\
\hline $\begin{array}{l}\% \text { in } \\
\text { Precontemplation }\end{array}$ & $\begin{array}{l}\text { This category is defined as the percentage of participants who } \\
\text { were determined to be in the Precontemplation Stage of } \\
\text { Change for the given behavior in a study. It is reported } \\
\text { exactly as is done in the article. If the percentage of } \\
\text { participants in the Precontemplation Stage is not readily } \\
\text { available in the article, the percentage can be calculated by } \\
\text { dividing the number of participants in each Stage by the total } \\
\text { "N" of the study. If there are no Stage percentages in the } \\
\text { article, and there is not enough information to calculate } \\
\text { them, "NR" should be recorded in the database. }\end{array}$ \\
\hline $\begin{array}{l}\% \text { in } \\
\text { Contemplation }\end{array}$ & $\begin{array}{l}\text { This category is defined as the percentage of participants who } \\
\text { were determined to be in the Contemplation Stage of Change } \\
\text { for the given behavior in a study. It is reported exactly as is } \\
\text { done in the article. If the percentage of participants in the }\end{array}$ \\
\hline
\end{tabular}




\begin{tabular}{|c|c|}
\hline & $\begin{array}{l}\text { Contemplation Stage is not readily available in the article, the } \\
\text { percentage can be calculated by dividing the number of } \\
\text { participants in each Stage by the total "N" of the study. If } \\
\text { there are no Stage percentages in the article, and there is not } \\
\text { enough information to calculate them, "NR" should be } \\
\text { recorded in the database. }\end{array}$ \\
\hline$\%$ in Preparation & $\begin{array}{l}\text { This category is defined as the percentage of participants who } \\
\text { were determined to be in the Preparation Stage of Change for } \\
\text { the given behavior in a study. It is reported exactly as is done } \\
\text { in the article. If the percentage of participants in the } \\
\text { Preparation Stage is not readily available in the article, the } \\
\text { percentage can be calculated by dividing the number of } \\
\text { participants in each Stage by the total "N" of the study. If } \\
\text { there are no Stage percentages in the article, and there is not } \\
\text { enough information to calculate them, "NR" should be } \\
\text { recorded in the database. }\end{array}$ \\
\hline$\%$ in Action & $\begin{array}{l}\text { This category is defined as the percentage of participants who } \\
\text { were determined to be in the Action Stage of Change for the } \\
\text { given behavior in a study. It is reported exactly as is done in } \\
\text { the article. If the percentage of participants in the Action } \\
\text { Stage is not readily available in the article, the percentage can } \\
\text { be calculated by dividing the number of participants in each } \\
\text { Stage by the total "N" of the study. If there are no Stage } \\
\text { percentages in the article, and there is not enough } \\
\text { information to calculate them, "NR" should be recorded in } \\
\text { the database. }\end{array}$ \\
\hline$\%$ in Maintenance & $\begin{array}{l}\text { This category is defined as the percentage of participants who } \\
\text { were determined to be in the Maintenance Stage of Change } \\
\text { for the given behavior in a study. It is reported exactly as is } \\
\text { done in the article. If the percentage of participants in the } \\
\text { Maintenance Stage is not readily available in the article, the } \\
\text { percentage can be calculated by dividing the number of } \\
\text { participants in each Stage by the total "N" of the study. If } \\
\text { there are no Stage percentages in the article, and there is not } \\
\text { enough information to calculate them, "NR" should be } \\
\text { recorded in the database. }\end{array}$ \\
\hline$\% \mathrm{C} / \mathrm{D}$ & $\begin{array}{l}\text { This category is defined as the percentage of participants who } \\
\text { were determined to be in the combined } \\
\text { Contemplation/Preparation Stage of Change for the given } \\
\text { behavior in a study. It is reported exactly as is done in the } \\
\text { article. If the percentage of participants in the } \\
\text { Contemplation/Preparation Stage is not readily available in } \\
\text { the article, the percentage can be calculated by dividing the } \\
\text { number of participants in each Stage by the total "N" of the } \\
\text { study. If there is no Contemplation/Preparation Stage, there }\end{array}$ \\
\hline
\end{tabular}




\begin{tabular}{|c|c|}
\hline & $\begin{array}{l}\text { is no mention of Stage percentages in the article, or there is } \\
\text { not enough information to calculate them, "NR" should be } \\
\text { recorded in the database. }\end{array}$ \\
\hline$\% \mathrm{~A} / \mathrm{M}$ & $\begin{array}{l}\text { This category is defined as the percentage of participants who } \\
\text { were determined to be in the combined Action/Maintenance } \\
\text { Stage of Change for the given behavior in a study. It is } \\
\text { reported exactly as is done in the article. If the percentage of } \\
\text { participants in the Action/Maintenance Stage is not readily } \\
\text { available in the article, the percentage can be calculated by } \\
\text { dividing the number of participants in each Stage by the total } \\
\text { " } N \text { " of the study. }\end{array}$ \\
\hline $\begin{array}{l}\text { Are Stage } \\
\text { descriptions } \\
\text { General TTM } \\
\text { category } \\
\text { descriptions or } \\
\text { Study Specific? }\end{array}$ & 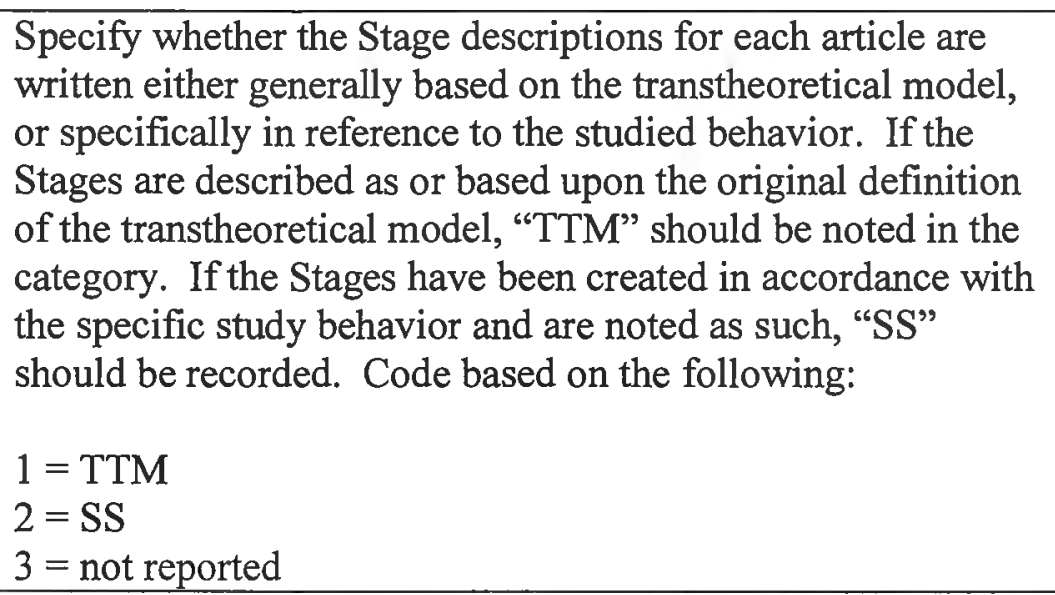 \\
\hline $\begin{array}{l}\text { Does study } \\
\text { provide staging } \\
\text { items? }\end{array}$ & $\begin{array}{l}\text { Indicate if the study provides the specific Stage items on } \\
\text { which the staging algorithms are based. }\end{array}$ \\
\hline $\begin{array}{l}\text { Stage items }=\text { Stage } \\
\text { category? }\end{array}$ & $\begin{array}{l}\text { Indicate if the Stage items have a one to one correspondence } \\
\text { to the Stage description. }\end{array}$ \\
\hline $\begin{array}{l}\text { PC; Stage } \\
\text { description }\end{array}$ & $\begin{array}{l}\text { Record the Stage description for Precontemplation exactly as } \\
\text { is written in the study. The Stages may have been geared } \\
\text { specifically toward the target behavior, or may appear in the } \\
\text { text as a general description of the transtheoretical model. } \\
\text { Either way, enter the exact description as it appears in the } \\
\text { database. }\end{array}$ \\
\hline $\begin{array}{l}\text { C; Stage } \\
\text { description }\end{array}$ & $\begin{array}{l}\text { Record the Stage description for Contemplation exactly as is } \\
\text { written in the study. The Stages may have been geared } \\
\text { specifically toward the target behavior, or may appear in the } \\
\text { text as a general description of the transtheoretical model. } \\
\text { Either way, enter the exact description as it appears in the } \\
\text { database. }\end{array}$ \\
\hline $\begin{array}{l}\text { PR; Stage } \\
\text { description }\end{array}$ & $\begin{array}{l}\text { Record the Stage description for Preparation exactly as is } \\
\text { written in the study. The Stages may have been geared } \\
\text { specifically toward the target behavior, or may appear in the } \\
\text { text as a general description of the transtheoretical model. }\end{array}$ \\
\hline
\end{tabular}




\begin{tabular}{|c|c|}
\hline & $\begin{array}{l}\text { Either way, enter the exact description as it appears in the } \\
\text { database. }\end{array}$ \\
\hline $\begin{array}{l}\text { A; Stage } \\
\text { description }\end{array}$ & $\begin{array}{l}\text { Record the Stage description for Action exactly as is written } \\
\text { in the study. The Stages may have been geared specifically } \\
\text { toward the target behavior, or may appear in the text as a } \\
\text { general description of the transtheoretical model. Either way, } \\
\text { the exact description appears in the database. }\end{array}$ \\
\hline $\begin{array}{l}\text { M; Stage } \\
\text { description }\end{array}$ & $\begin{array}{l}\text { Record the Stage description for Maintenance exactly as is } \\
\text { written in the study. The Stages may have been geared } \\
\text { specifically toward the target behavior, or may appear in the } \\
\text { text as a general description of the transtheoretical model. } \\
\text { Either way, enter the exact description as it appears in the } \\
\text { database. }\end{array}$ \\
\hline $\begin{array}{l}\text { Time Frame for } \\
\text { Precontemplation }\end{array}$ & $\begin{array}{l}1=6-\text { month time period } \\
2=\text { another time period } \\
3=\text { time period not } \\
\quad \text { specified } \\
4=\text { not reported }\end{array}$ \\
\hline $\begin{array}{l}\text { Time Frame for } \\
\text { Contemplation }\end{array}$ & $\begin{array}{l}1=6-\text { month time period } \\
2=\text { another time period } \\
3=\text { time period not } \\
\quad \text { specified } \\
4=\text { not reported }\end{array}$ \\
\hline $\begin{array}{l}\text { Time Frame for } \\
\text { Preparation }\end{array}$ & $\begin{array}{l}1=30 \text { day } / 1 \text { month time } \\
\quad \text { period } \\
2=\text { another time period } \\
3=\text { time period not specified } \\
4=\text { not reported } \\
5=\text { not used }\end{array}$ \\
\hline $\begin{array}{l}\text { Time Frame for } \\
\text { Action }\end{array}$ & $\begin{array}{l}1=\text { less than } 6 \text {-month time } \\
\text { period } \\
2=\text { another time period } \\
3=\text { time period not } \\
\\
\text { specified } \\
4=\text { not reported } \\
5=\text { Action/Maintenance }\end{array}$ \\
\hline $\begin{array}{l}\text { Time Frame for } \\
\text { Maintenance }\end{array}$ & $\begin{array}{l}1=\text { greater than } 6-\text { month } \\
\quad \text { time period } \\
2=\text { another time period } \\
3=\text { time period not } \\
\quad \text { specified } \\
4=\text { not reported } \\
5=\text { Action/Maintenance }\end{array}$ \\
\hline
\end{tabular}




\section{Decisional Balance Descriptors}

\begin{tabular}{|l|l|}
\hline $\begin{array}{l}\text { Description of } \\
\text { Pros and Cons }\end{array}$ & $\begin{array}{l}\text { Provide the exact description of the pros and cons (e.g. Pros } \\
\text { and Cons of Smoking or Pros and Cons of Quitting Smoking) }\end{array}$ \\
\hline Response Format & $\begin{array}{l}\text { Indicate the response format for the Decisional Balance } \\
\text { measure }\end{array}$ \\
\hline $\begin{array}{l}\text { Response Format } \\
\text { Category }\end{array}$ & $\begin{array}{l}\text { The two most typical response formats for Decisional } \\
\text { Balance are either a likert scale which asks participants to } \\
\text { indicate "how important" the given items are to them or to } \\
\text { the degree to which they agree or disagree with each of the } \\
\text { items. Code response format into one of the following } \\
\text { categories: }\end{array}$ \\
$\begin{array}{l}1=\text { "How Important" } \\
2=\text { "Agree / Disagree" } \\
3=\text { other }\end{array}$ \\
\hline $\begin{array}{l}\text { Number of Pros } \\
\text { Items }\end{array}$ & $\begin{array}{l}\text { Enter the number of pros indicated by the author that were } \\
\text { used in the final Decisional Balance measure. }\end{array}$ \\
\hline $\begin{array}{l}\text { Number of Cons } \\
\text { Items }\end{array}$ & $\begin{array}{l}\text { Enter the number of cons indicated by the author that were } \\
\text { used in the final Decisional Balance measure. }\end{array}$ \\
\hline Alpha Pros & $\begin{array}{l}\text { Enter the coefficient alpha for the Pros scale calculated } \\
\text { specifically for the study. Do not report alpha's that were } \\
\text { reported based on previous studies. }\end{array}$ \\
\hline Alpha Cons & $\begin{array}{l}\text { Enter the coefficient alpha for the Cons scale calculated } \\
\text { specifically for the study. Do not report alpha's that were } \\
\text { reported based on previous studies. }\end{array}$ \\
\hline
\end{tabular}

Note. Any data that can not be identified will be indicated in the database by "NR" (not reported).

\section{Behavior Code List}

\begin{tabular}{|c|c|}
\hline 1. $\mathrm{ADC}$ & Administrative change \\
\hline 2. AST & Asthma - Medication Management \\
\hline 3. ARF & Anorexia- recovery from \\
\hline 4. & \\
\hline 5. $\mathrm{BDA}$ & Binge drinking acquisition \\
\hline 6. $\mathrm{BDC}$ & Binge drinking cessation \\
\hline 7. $\mathrm{BDT}$ & Bone density testing \\
\hline 8. $\mathrm{BPA}$ & Back pain \\
\hline 9. $\mathrm{BRF}$ & Bulimia - recovery from \\
\hline
\end{tabular}




\begin{tabular}{|c|c|}
\hline 10. BSE & Breast Self-Examination \\
\hline 11. CAI & Calcium intake \\
\hline 12. CDS & Collaborative delivery service \\
\hline 13. CCS & Colorectal cancer screening \\
\hline 14. CCU & Cocaine use \\
\hline 15. CGE & Contraception-general \\
\hline 16. CMA & Condom use - main \\
\hline 17. CUA & Condom use - anal main \\
\hline 18. CUO & Condom use - other \\
\hline 19. CUS & Condom use \\
\hline 20. DBH & Delinquent behavior \\
\hline 21.DDU & Decreasing drug use \\
\hline 22. DFR & Dietary fat reduction \\
\hline 23. DIE & Diet \\
\hline 24. DMM & Participation in decision making for medication \\
\hline 25. DPV & Depression prevention \\
\hline 26. DRT & Drug treatment \\
\hline 27. DVI & Domestic violence \\
\hline 28. EAD & Eating disorders \\
\hline 29. EXE & Exercise \\
\hline 30. FRI & Fruit intake \\
\hline 31. FVC & Fruit and vegetable consumption \\
\hline 32. GLT & Glucose testing \\
\hline 33. HDI & Healthy diet \\
\hline 34. HRP & Hearing Protection \\
\hline 35. MAM & Mammography Screening \\
\hline 36. MEM & Medication management \\
\hline 37. ORD & Organ donation \\
\hline 38. ORH & Oral hygiene \\
\hline 39. PAS & Physician assisted smoker \\
\hline 40. QDR & Quitting drugs \\
\hline 41. RAT & Radon testing \\
\hline 42. SBH & Sedentary behavior \\
\hline 43. SDM & Sex decision making \\
\hline 44. SDN & Sex decision making (non virgins) \\
\hline 45. SDV & Sex decision making (virgins) \\
\hline 46. SMA & Smoking acquisition \\
\hline 47. SMC & Smoking cessation \\
\hline 48. SMG & Stress management \\
\hline 49. SPR & Sun protection / reduction \\
\hline 50. STR & Stress \\
\hline 51. SUU & Sunscreen use \\
\hline 52. SLT & Smokeless tobacco use \\
\hline 53. VIN & Vegetable intake \\
\hline
\end{tabular}


Appendix B

Table B.1

Stage Descriptions for Each Study 
Table B.1 (continued)

\begin{tabular}{|c|c|c|c|c|c|c|c|}
\hline $\begin{array}{c}\text { Source } \\
\text { (Year) } \\
\text { Target } \\
\text { Behavior }\end{array}$ & $\begin{array}{l}\text { Type of } \\
\text { Algorithm }\end{array}$ & \begin{tabular}{|} 
TTM \\
or \\
Study \\
Specific \\
(SS)
\end{tabular} & Precontemplation & Contemplation & Preparation & Action & Maintenance \\
\hline $\begin{array}{l}\text { Acton et al. } \\
(2001) \\
\text { Smoking } \\
\text { Cessation }\end{array}$ & \begin{tabular}{|l|}
-item \\
measure
\end{tabular} & SS & $\begin{array}{l}\text { Not seriously considering } \\
\text { quitting within the next } 6 \\
\text { months }\end{array}$ & $\begin{array}{l}\text { Seriously considering } \\
\text { quitting within the } \\
\text { next } 6 \text { months, however } \\
\text { they were not considering } \\
\text { quitting within the next } \\
30 \text { days, had not made a } \\
\text { quit attempt of } 24 \text { hours } \\
\text { in past year, or both }\end{array}$ & $\begin{array}{l}\text { Seriously considering } \\
\text { quitting in the next } 6 \\
\text { months and were } \\
\text { planning to quit within } \\
\text { the next } 30 \text { days, in } \\
\text { addition they made a } 24 \\
\text { hour quit attempt in past } \\
\text { year }\end{array}$ & $\begin{array}{l}\text { Has quit smoking for } \\
\text { under } 6 \\
\text { months }\end{array}$ & $\begin{array}{l}\text { Has quit smoking for } \\
\text { at least } 6 \text { months }\end{array}$ \\
\hline $\begin{array}{l}\text { Ahijevych et } \\
\text { al. } \\
(1999) \\
\text { Smoking } \\
\text { Cessation } \\
\end{array}$ & $\begin{array}{l}\text { 3-question } \\
\text { algorithm }\end{array}$ & \begin{tabular}{|l|} 
Not \\
reported
\end{tabular} & $\begin{array}{l}\text { Not intending to quit } \\
\text { smoking in the next six } \\
\text { months }\end{array}$ & $\begin{array}{l}\text { Intending to quit smoking } \\
\text { in the next six months but } \\
\text { not intending to quit } \\
\text { smoking in the next } \\
\text { month? }\end{array}$ & $\begin{array}{l}\text { Intending to quit smoking } \\
\text { in the next month and } \\
\text { tried to quit in the past } \\
\text { year }\end{array}$ & $\begin{array}{l}\text { Not } \\
\text { reported }\end{array}$ & $\begin{array}{l}\text { Not } \\
\text { reported }\end{array}$ \\
\hline $\begin{array}{l}\text { Bane et al. } \\
(1999) \\
\text { Smoking } \\
\text { Cessation }\end{array}$ & Not reported & SS & $\begin{array}{l}\text { Reported smoking at least } \\
\text { one cigarette per day } \\
\text { during the past } 7 \text { days } \\
\text { and not seriously } \\
\text { considering quitting } \\
\text { within the next } 6 \text { months }\end{array}$ & $\begin{array}{l}\text { Seriously considering } \\
\text { quitting smoking within } \\
\text { the next } 6 \text { months or } \\
\text { considering quitting } \\
\text { within the next } 30 \text { days, } \\
\text { but had not made any quit } \\
\text { attempts }\end{array}$ & $\begin{array}{l}\text { Planning to quit within } \\
\text { the next } 30 \text { days and had } \\
\text { made at least one } 24 \text {-hour } \\
\text { quit attempt in the past } \\
\text { year }\end{array}$ & $\begin{array}{l}\text { Quit within the past } 6 \\
\text { months }\end{array}$ & $\begin{array}{l}\text { Quit more than } 6 \text { months } \\
\text { before baseline }\end{array}$ \\
\hline $\begin{array}{l}\text { Banikarim et } \\
\text { al. } \\
(2003) \\
\\
\text { STD } \\
\text { Screening- } \\
\text { Change in } \\
\text { Partners }\end{array}$ & $\begin{array}{l}3 \text { separate } \\
\text { staging } \\
\text { algorithms }\end{array}$ & SS & $\begin{array}{l}\text { Women not ready to } \\
\text { obtain gonorrhea and } \\
\text { Chlamydia screening } \\
\text { every time they ever } \\
\text { changed partners or had } \\
\text { unprotected sex with their } \\
\text { current main partner or } \\
\text { current side partner }\end{array}$ & \begin{tabular}{|l|} 
Individuals thinking \\
about obtaining \\
gonorrhea and Chlamydia \\
screening every time they \\
ever changed partners or \\
had unprotected sex with \\
their current main partner \\
or current side partner
\end{tabular} & $\begin{array}{l}\text { Individuals planning to } \\
\text { obtain gonorrhea and } \\
\text { Chlamydia screening } \\
\text { every time they ever } \\
\text { changed partners or had } \\
\text { unprotected sex with their } \\
\text { current main partner or } \\
\text { current side partner }\end{array}$ & $\begin{array}{l}\text { Individuals who had been } \\
\text { obtaining gonorrhea and } \\
\text { Chlamydia screening for } \\
6 \text { months or less every } \\
\text { time they ever changed } \\
\text { partners or had } \\
\text { unprotected sex with their } \\
\text { current main partner or } \\
\text { current side partner }\end{array}$ & $\begin{array}{l}\text { Individuals who had been } \\
\text { receiving gonorrhea and } \\
\text { Chlamydia screening for } \\
\text { more than } 6 \text { months every } \\
\text { time they had ever change } \\
\text { partners or had unprotecte } \\
\text { sex with their current mair } \\
\text { partner or current side } \\
\text { partner }\end{array}$ \\
\hline
\end{tabular}


Table B.1 (continued)

\begin{tabular}{|c|c|c|c|c|c|c|c|}
\hline $\begin{array}{c}\text { Source } \\
\text { (Year) } \\
\text { Target } \\
\text { Behavior }\end{array}$ & $\begin{array}{c}\text { Type of } \\
\text { Algorithm }\end{array}$ & $\begin{array}{c}\text { TTM } \\
\text { or } \\
\text { Study } \\
\text { Specific } \\
\text { (SS) }\end{array}$ & Precontemplation & Contemplation & Preparation & Action & Maintenance \\
\hline $\begin{array}{l}\text { Banikarim et } \\
\text { al. } \\
(2003) \\
\text { STD } \\
\text { Screening- } \\
\text { Main }\end{array}$ & $\begin{array}{l}3 \text { separate } \\
\text { staging } \\
\text { algorithms }\end{array}$ & SS & $\begin{array}{l}\text { Women not ready to } \\
\text { obtain gonorrhea and } \\
\text { Chlamydia screening } \\
\text { every time they ever } \\
\text { changed partners or had } \\
\text { unprotected sex with their } \\
\text { current main partner or } \\
\text { current side partner }\end{array}$ & \begin{tabular}{|l|} 
Individuals thinking \\
about obtaining \\
gonorrhea and Chlamydia \\
screening every time they \\
ever changed partners or \\
had unprotected sex with \\
their current main partner \\
or current side partner
\end{tabular} & \begin{tabular}{|l|} 
Individuals planning to \\
obtain gonorrhea and \\
Chlamydia screening \\
every time they ever \\
changed partners or had \\
unprotected sex with their \\
current main partner or \\
current side partner
\end{tabular} & $\begin{array}{l}\text { Individuals who had been } \\
\text { obtaining gonorrhea and } \\
\text { Chlamydia screening for } \\
6 \text { months or less every } \\
\text { time they ever changed } \\
\text { partners or had } \\
\text { unprotected sex with their } \\
\text { current main partner or } \\
\text { current side partner }\end{array}$ & $\begin{array}{l}\text { Individuals who had been } \\
\text { receiving gonorrhea and } \\
\text { Chlamydia screening for } \\
\text { more than } 6 \text { months every } \\
\text { time they had ever changed } \\
\text { partners or had unprotecte } \\
\text { sex with their current main } \\
\text { partner or current side } \\
\text { partner }\end{array}$ \\
\hline $\begin{array}{l}\text { Basler et al. } \\
\text { (1999) } \\
\text { Exercise }\end{array}$ & $\begin{array}{l}\text { Single } \\
\text { question } 5 \\
\text { choice } \\
\text { response } \\
\text { format } \\
\end{array}$ & TTM & $\begin{array}{l}\text { No intention to change } \\
\text { behavior in the } \\
\text { foreseeable future, or } \\
\text { denial of need to change. }\end{array}$ & $\begin{array}{l}\text { Intention to change } \\
\text { within the next } 6 \text { months. }\end{array}$ & $\begin{array}{l}\text { Serious intention to } \\
\text { change in the next } 30 \\
\text { days. }\end{array}$ & $\begin{array}{l}\text { Initiation of overt } \\
\text { behavioral change. }\end{array}$ & $\begin{array}{l}\text { Sustaining behavioral } \\
\text { change for } 6 \text { months or } \\
\text { more. }\end{array}$ \\
\hline $\begin{array}{l}\text { Block et al. } \\
(1998) \\
\text { Condom Use }\end{array}$ & $\begin{array}{l}4 \text { question } \\
\text { algorithm w/ } \\
\text { process of } \\
\text { change self- } \\
\text { assessment } \\
\text { scale used as } \\
\text { criterion for } \\
\text { classification }\end{array}$ & TTM & $\begin{array}{l}\text { Often do not see or deny } \\
\text { the problem and have no } \\
\text { intention of changing } \\
\text { their behavior }\end{array}$ & $\begin{array}{l}\text { Acknowledge they have a } \\
\text { problem, but are not quite } \\
\text { ready to do anything } \\
\text { about it; may have } \\
\text { indefinite plans to take } \\
\text { action in the next } 6 \\
\text { months (in the next } 6 \\
\text { months) }\end{array}$ & $\begin{array}{l}\text { Plans to take action } \\
\text { within a definitive time- } \\
\text { frame }\end{array}$ & $\begin{array}{l}\text { Modifying their behavior } \\
\text { and environment (within } \\
\text { the past } 6 \text { months) }\end{array}$ & $\begin{array}{l}\text { The individual continues to } \\
\text { resist temptation and to } \\
\text { reinforce action }\end{array}$ \\
\hline $\begin{array}{l}\text { Borland et al. } \\
(2000) \\
\text { Smoking } \\
\text { Cessation }\end{array}$ & $\begin{array}{l}5 \text { questions } \\
\text { (unclear what } \\
\text { format) }\end{array}$ & TTM & $\begin{array}{l}\text { Not seriously } \\
\text { contemplating quitting in } \\
\text { the next } 6 \text { months }\end{array}$ & $\begin{array}{l}\text { Seriously contemplating } \\
\text { quitting smoking in the } \\
\text { next } 6 \text { months and not in } \\
\text { preparation }\end{array}$ & $\begin{array}{l}\text { Planning to quit in the } \\
\text { next } 30 \text { days and also } \\
\text { having made a quit } \\
\text { attempt of at least } 24 \\
\text { hours in the last year. }\end{array}$ & $\begin{array}{l}\text { Quit for less than } 6 \\
\text { months }\end{array}$ & $\begin{array}{l}\text { Quit for more than } 6 \\
\text { months }\end{array}$ \\
\hline $\begin{array}{l}\text { Boudreaux et } \\
\text { al. } \\
(1998) \\
\text { Smoking }\end{array}$ & $\begin{array}{l}\text { Single } \\
\text { question } 5 \\
\text { choice } \\
\text { response } \\
\text { format }\end{array}$ & $\overline{S S}$ & $\begin{array}{l}\text { Not thinking about } \\
\text { quitting smoking }\end{array}$ & $\begin{array}{l}\text { Thinking about quitting } \\
\text { smoking in the next } 6 \\
\text { months }\end{array}$ & $\begin{array}{l}\text { Planning to quit smoking } \\
\text { in the next } 30 \text { days and } \\
\text { already made a recent } \\
\text { quit attempt }\end{array}$ & $\begin{array}{l}\text { Continuous cessation for } \\
\text { greater than I day, but } \\
\text { less than } 6 \text { months }\end{array}$ & $\begin{array}{l}\text { Continuous cessation for } \\
\text { greater than } 6 \text { months }\end{array}$ \\
\hline
\end{tabular}


Table B.1 (continued)

\begin{tabular}{|c|c|c|c|c|c|c|c|}
\hline $\begin{array}{c}\begin{array}{c}\text { Source } \\
\text { (Year) }\end{array} \\
\text { Target } \\
\text { Behavior }\end{array}$ & $\begin{array}{l}\text { Type of } \\
\text { Algorithm }\end{array}$ & $\begin{array}{c}\text { TTM } \\
\text { or } \\
\text { Study } \\
\text { Specific } \\
\text { (SS) }\end{array}$ & Precontemplation & Contemplation & Preparation & Action & Maintenance \\
\hline \multicolumn{8}{|l|}{ Cessation } \\
\hline $\begin{array}{l}\text { Buckworth et } \\
\text { al. } \\
(2002) \\
\text { Exercise }\end{array}$ & $\begin{array}{l}\text { 5-item } \\
\text { algorithm } \\
\text { (True/False) }\end{array}$ & SS & Not reported & $\begin{array}{l}\text { I currently do not } \\
\text { exercise, but am thinking } \\
\text { of starting to exercise in } \\
\text { the next } 6 \text { months }\end{array}$ & $\begin{array}{l}\text { I currently exercise some, } \\
\text { but not regularly }\end{array}$ & $\begin{array}{l}\text { I currently exercise } \\
\text { regularly, but have only } \\
\text { begun doing so within the } \\
\text { last } 6 \text { months }\end{array}$ & $\begin{array}{l}\text { I currently exercise } \\
\text { regularly, but have only } \\
\text { begun doing so within the } \\
\text { last } 6 \text { months }\end{array}$ \\
\hline $\begin{array}{l}\text { Callaghan et } \\
\text { al. } \\
(2002) \\
\text { Exercise }\end{array}$ & $\begin{array}{l}\text { Contempla- } \\
\text { tion Ladder } \\
(11 \mathrm{pt})\end{array}$ & SS & $\begin{array}{l}\text { I do not currently } \\
\text { exercise, and I do not } \\
\text { intend to start exercising } \\
\text { in the next } 6 \text { months }\end{array}$ & $\begin{array}{l}\text { I currently do not } \\
\text { exercise, but I am } \\
\text { thinking about starting to } \\
\text { exercise in the next } 6 \\
\text { months }\end{array}$ & $\begin{array}{l}\text { I currently exercise some, } \\
\text { but not regularly }\end{array}$ & $\begin{array}{l}\text { I currently exercise } \\
\text { regularly and have been } \\
\text { exercising at the } \\
\text { recommended level for } 6 \\
\text { months or less }\end{array}$ & $\begin{array}{l}\text { I currently exercise } \\
\text { regularly and have done sc } \\
\text { for longer than } 6 \text { months }\end{array}$ \\
\hline $\begin{array}{l}\begin{array}{l}\text { Chamot et al. } \\
(2001)\end{array} \\
\text { Mammo- } \\
\text { graphy }\end{array}$ & $\begin{array}{l}\text { 4-item (6 } \\
\text { stages } \\
\text { including } \\
\text { Relapse Risk } \\
\text { and Relapse) } \\
\end{array}$ & SS & $\begin{array}{l}\text { Never had a screening } \\
\text { mammogram and no } \\
\text { intention of having one in } \\
\text { next six months }\end{array}$ & $\begin{array}{l}\text { No screening } \\
\text { mammogram in past } 2 \\
\text { years, but plans to have } \\
\text { one in the next } 6 \text { months }\end{array}$ & Not used & $\begin{array}{l}\text { no screening } \\
\text { mammogram 2-4 years } \\
\text { ago, with one in the past } \\
2 \text { years, intends to have } \\
\text { mammogram in future }\end{array}$ & $\begin{array}{l}\text { at least two mammograms } \\
\text { in last } 4 \text { years, one in last } \\
\text { two years, with plans to } \\
\text { have mammograms in the } \\
\text { future }\end{array}$ \\
\hline $\begin{array}{l}\text { DiClemente } \\
\text { et al. } \\
\text { (1991) } \\
\text { Smoking } \\
\text { Cessation }\end{array}$ & $\begin{array}{l}\text { Number of } \\
\text { quit attempts } \\
\text { and Yes/No } \\
\text { format }\end{array}$ & SS & $\begin{array}{l}\text { Not seriously considering } \\
\text { quitting within the next } 6 \\
\text { months }\end{array}$ & $\begin{array}{l}\text { Seriously considering } \\
\text { quitting within the next } 6 \\
\text { months, however they } \\
\text { were not considering } \\
\text { quitting within the next } \\
30 \text { days, had not made a } \\
\text { quit attempt of } 24 \text { hours } \\
\text { in past year, or both }\end{array}$ & $\begin{array}{l}\text { Seriously considering } \\
\text { quitting in the next } 6 \\
\text { months and were } \\
\text { planning to quit within } \\
\text { the next } 30 \text { days, in } \\
\text { addition they made a } 24 \\
\text { hour quit attempt in past } \\
\text { year }\end{array}$ & Not reported & Not reported \\
\hline
\end{tabular}


Table B.1 (continued)

\begin{tabular}{|c|c|c|c|c|c|c|c|}
\hline $\begin{array}{l}\text { Source } \\
\text { (Year) } \\
\text { Target } \\
\text { Behavior }\end{array}$ & $\begin{array}{l}\text { Type of } \\
\text { Algorithm }\end{array}$ & $\begin{array}{l}\text { TTM } \\
\text { or } \\
\text { Study } \\
\text { Specific } \\
\text { (SS) }\end{array}$ & Precontemplation & Contemplation & Preparation & Action & Maintenance \\
\hline \begin{tabular}{|l|} 
Dijkstra et al. \\
$(1996)$ \\
Smoking \\
Cessation
\end{tabular} & $\begin{array}{l}\text { Single } \\
\text { question } 5 \\
\text { choice } \\
\text { response } \\
\text { format }\end{array}$ & SS & $\begin{array}{l}\text { Has been smoking in the } \\
\text { past } 24 \mathrm{hr} \text { and is not } \\
\text { planning to quit within } \\
\text { the next } 6 \text { months }\end{array}$ & $\begin{array}{l}\text { Has been smoking in the } \\
\text { past } 24 \mathrm{hr} \text { and is planning } \\
\text { to quit within the next } 6 \\
\text { months but not yet within } \\
\text { the next month }\end{array}$ & $\begin{array}{l}\text { Has been smoking in the } \\
\text { past } 24 \mathrm{hr} \text { and is planning } \\
\text { to quit within the next } \\
\text { month }\end{array}$ & $\begin{array}{l}\text { Has not been smoking in } \\
\text { the past } 24 \text { hr but not } \\
\text { longer than } 6 \text { months }\end{array}$ & $\begin{array}{l}\text { Has not been smoking for } \\
\text { the past } 6 \text { months }\end{array}$ \\
\hline $\begin{array}{l}\text { Dijkstra et al. } \\
(1998) \\
\text { Smoking } \\
\text { Cessation }\end{array}$ & $\begin{array}{l}\text { Not reported; } \\
\text { Includes } \\
\text { immotives ( } 3 \\
\text { definitions } \\
\text { for } \\
\text { immotives) }\end{array}$ & SS & $\begin{array}{l}\text { Planning to quit within } \\
\text { the next year; Planning to } \\
\text { quit within the next } 5 \\
\text { years }\end{array}$ & $\begin{array}{l}\text { Planning to quit within } \\
\text { the next } 6 \text { months }\end{array}$ & $\begin{array}{l}\text { Planning to quit within } \\
\text { the next month }\end{array}$ & Not reported & Not reported \\
\hline $\begin{array}{l}\text { Dryfoos } \\
(1996) \\
\text { Glucose } \\
\text { Testing } \\
\end{array}$ & $\begin{array}{l}\text { Single } \\
\text { question } 5 \\
\text { choice } \\
\text { response } \\
\text { format } \\
\end{array}$ & SS & $\begin{array}{l}\text { Do you regularly follow } \\
\text { your glucose self-testing } \\
\text { plan? No, and I do not } \\
\text { intend to in the next } 6 \\
\text { months. }\end{array}$ & $\begin{array}{l}\text { Do you regularly follow } \\
\text { your glucose self-testing } \\
\text { plan? No, but I intend to } \\
\text { in the next } 6 \text { months. }\end{array}$ & $\begin{array}{l}\text { Do you regularly follow } \\
\text { your glucose self-testing } \\
\text { plan? No, but I intend to } \\
\text { in the next } 30 \text { days. }\end{array}$ & $\begin{array}{l}\text { Do you regularly follow } \\
\text { your glucose self-testing } \\
\text { plan? Yes, and I have } \\
\text { been, but for less than } 6 \\
\text { months. }\end{array}$ & $\begin{array}{l}\text { Do you regularly follow } \\
\text { your glucose self-testing } \\
\text { plan? Yes, and I have been } \\
\text { for more than } 6 \text { months. }\end{array}$ \\
\hline $\begin{array}{l}\text { Dryfoos } \\
(1996) \\
\text { Medication } \\
\text { Management }\end{array}$ & $\begin{array}{l}\text { Single } \\
\text { question } 5 \\
\text { choice } \\
\text { response } \\
\text { format }\end{array}$ & SS & $\begin{array}{l}\text { Do you regularly take } \\
\text { your insulin as you were } \\
\text { told to by your health } \\
\text { care provider? No, and I } \\
\text { do not intend to in the } \\
\text { next } 6 \text { months. }\end{array}$ & $\begin{array}{l}\text { Do you regularly take } \\
\text { your insulin as you were } \\
\text { told to by your health } \\
\text { care provider? No, but I } \\
\text { intend to in the next } 6 \\
\text { months. }\end{array}$ & $\begin{array}{l}\text { Do you regularly take } \\
\text { your insulin as you were } \\
\text { told to by your health } \\
\text { care provider? No, but I } \\
\text { intend to in the next } 30 \\
\text { days. }\end{array}$ & $\begin{array}{l}\text { Do you regularly take } \\
\text { your insulin as you were } \\
\text { told to by your health } \\
\text { care provider? Yes, and I } \\
\text { have been, but for less } \\
\text { than } 6 \text { months. }\end{array}$ & $\begin{array}{l}\text { Do you regularly take you } \\
\text { insulin as you were told to } \\
\text { by your health care } \\
\text { provider? Yes, and I have } \\
\text { been for more than } 6 \\
\text { months. }\end{array}$ \\
\hline $\begin{array}{l}\text { Eaton et al. } \\
\text { (1992) } \\
\text { Physicians } \\
\text { Assisting } \\
\text { Smoker }\end{array}$ & Not reported & $\begin{array}{l}\text { Not } \\
\text { reported }\end{array}$ & Not reported & Not reported & Not reported & Not reported & Not reported \\
\hline
\end{tabular}


Table B.1 (continued)

\begin{tabular}{|c|c|c|c|c|c|c|c|}
\hline $\begin{array}{l}\text { Source } \\
\text { (Year) } \\
\text { Target } \\
\text { Behavior }\end{array}$ & $\begin{array}{c}\text { Type of } \\
\text { Algorithm }\end{array}$ & $\begin{array}{c}\text { TTM } \\
\text { or } \\
\text { Study } \\
\text { Specific } \\
\text { (SS) }\end{array}$ & Precontemplation & Contemplation & Preparation & Action & Maintenance \\
\hline $\begin{array}{l}\text { Evers et al. } \\
\text { (2001) } \\
\text { Condom Use }\end{array}$ & $\begin{array}{l}\text { Single } \\
\text { question } 5 \\
\text { choice } \\
\text { response } \\
\text { format }\end{array}$ & SS & $\begin{array}{l}\text { Not intending to use } \\
\text { condoms in the next } 6 \\
\text { months }\end{array}$ & $\begin{array}{l}\text { Planning to use condoms } \\
\text { in the next } 6 \text { months }\end{array}$ & $\begin{array}{l}\text { Intending to use condoms } \\
\text { every time they have sex } \\
\text { in the next } 30 \text { days }\end{array}$ & $\begin{array}{l}\text { Have been using } \\
\text { condoms every time they } \\
\text { have sex for less than } 6 \\
\text { months }\end{array}$ & $\begin{array}{l}\text { Using condoms every time } \\
\text { they have sex for more } \\
\text { than } 6 \text { months }\end{array}$ \\
\hline $\begin{array}{l}\text { Evers et al. } \\
(2001) \\
\text { Waiting to } \\
\text { have sex }\end{array}$ & $\begin{array}{l}\text { Single } \\
\text { question } 5 \\
\text { choice } \\
\text { response } \\
\text { format }\end{array}$ & SS & $\begin{array}{l}\text { Not intending to have sex } \\
\text { in the next } 6 \text { months }\end{array}$ & $\begin{array}{l}\text { Planning to have sex in } \\
\text { the next } 6 \text { months }\end{array}$ & $\begin{array}{l}\text { Intending to have sex in } \\
\text { the next } 30 \text { days }\end{array}$ & Not reported & Not reported \\
\hline 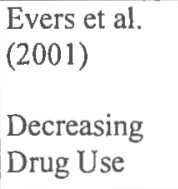 & Not reported & SS & $\begin{array}{l}\text { Not intending to stop } \\
\text { using the drug in the next } \\
6 \text { months }\end{array}$ & $\begin{array}{l}\text { Planning to stop using the } \\
\text { drug in the next } 6 \text { months }\end{array}$ & $\begin{array}{l}\text { Intending to stop using } \\
\text { the drug in the next } 30 \\
\text { days }\end{array}$ & $\begin{array}{l}\text { Have stopped using the } \\
\text { drug in the last } 6 \text { months }\end{array}$ & $\begin{array}{l}\text { Have stopped using the } \\
\text { drug more than } 6 \text { months } \\
\text { ago }\end{array}$ \\
\hline $\begin{array}{l}\text { Evers et al. } \\
(2003) \\
\text { Stress } \\
\text { Management }\end{array}$ & Not reported & $\begin{array}{l}\text { Not } \\
\text { reported }\end{array}$ & Not reported & Not reported & Not reported & Not reported & Not reported \\
\hline $\begin{array}{l}\text { Fahrenwald } \\
\text { et al. } \\
(2003) \\
\text { Exercise }\end{array}$ & $\begin{array}{l}\text { 5-question } \\
\text { (Yes/No) }\end{array}$ & $\mathrm{SS}$ & $\begin{array}{l}\text { Those not participating in } \\
\text { regular exercise activity } \\
\text { and not intending to } \\
\text { change in the next } 6 \\
\text { months }\end{array}$ & $\begin{array}{l}\text { Those not participating in } \\
\text { regular physical activity } \\
\text { but intending to do so in } \\
\text { the next } 6 \text { months }\end{array}$ & $\begin{array}{l}\text { Those not participating in } \\
\text { regular physical activity } \\
\text { but actively making small } \\
\text { changes to do so }\end{array}$ & $\begin{array}{l}\text { Those who have actively } \\
\text { engaged in regular } \\
\text { physical activity for less } \\
\text { than } 6 \text { months }\end{array}$ & $\begin{array}{l}\text { Those who have actively } \\
\text { engaged in regular physica } \\
\text { activity for } 6 \text { months or } \\
\text { more }\end{array}$ \\
\hline $\begin{array}{l}\text { Fava et al. } \\
\text { (1995) } \\
\text { Smoking } \\
\text { Cessation }\end{array}$ & Not reported & SS & $\begin{array}{l}\text { A smoker has no } \\
\text { intention to change } \\
\text { behavior in the next six } \\
\text { months }\end{array}$ & $\begin{array}{l}\text { A smoker is seriously } \\
\text { considering a change } \\
\text { within the next six } \\
\text { months }\end{array}$ & $\begin{array}{l}\text { A smoker is seriously } \\
\text { considering to quit within } \\
\text { the next } 30 \text { days and has } \\
\text { made at least one quit } \\
\text { attempt in the past year }\end{array}$ & $\begin{array}{l}\text { A former smoker has } \\
\text { been continuously quit } \\
\text { for less than } 6 \text { months }\end{array}$ & $\begin{array}{l}\text { A former smoker has been } \\
\text { continuously quit for more } \\
\text { than } 6 \text { months }\end{array}$ \\
\hline
\end{tabular}


Table B.1 (continued)

\begin{tabular}{|c|c|c|c|c|c|c|c|}
\hline $\begin{array}{c}\text { Source } \\
\text { (Year) } \\
\text { Target } \\
\text { Behavior }\end{array}$ & $\begin{array}{l}\text { Type of } \\
\text { Algorithm }\end{array}$ & \begin{tabular}{|c|} 
TTM \\
or \\
Study \\
Specific \\
(SS)
\end{tabular} & Precontemplation & Contemplation & Preparation & Action & Maintenance \\
\hline $\begin{array}{l}\text { Frenn et al. } \\
(2003) \\
\text { Dietary Fat } \\
\text { Reduction }\end{array}$ & $\begin{array}{l}\text { 7-item with } \\
\text { restaging } \\
\text { (per Rossi et } \\
\text { al. 1994) }\end{array}$ & $\begin{array}{l}\text { Not } \\
\text { reported }\end{array}$ & Not reported & Not reported & Not reported & Not reported & Not reported \\
\hline $\begin{array}{l}\text { Galavotti et } \\
\text { al. } \\
(1995) \\
\text { Contracep- } \\
\text { ion - General }\end{array}$ & $\begin{array}{l}2 \text { questions; } \\
7 \text {-pt scale }\end{array}$ & SS & $\begin{array}{l}\text { Neutral or negative } \\
\text { intention to start using a } \\
\text { contraceptive method } \\
\text { every time or all the time }\end{array}$ & $\begin{array}{l}\text { Positive intention (above } \\
\text { the neutral point) to start } \\
\text { using contraception every } \\
\text { time or all the time in the } \\
\text { next } 6 \text { months }\end{array}$ & $\begin{array}{l}\text { Positive intention to start } \\
\text { using contraception every } \\
\text { time or all the time in the } \\
\text { next } 30 \text { days and some } \\
\text { reported use (sometimes } \\
\text { or almost every } \\
\text { time/almost all the time) }\end{array}$ & $\begin{array}{l}\text { Use of a method every } \\
\text { time or all the time for } \\
\text { less than } 6 \text { months }\end{array}$ & $\begin{array}{l}\text { Use of a method every } \\
\text { time or all the time for } 6 \\
\text { months or longer }\end{array}$ \\
\hline $\begin{array}{l}\text { Galavotti et } \\
\text { al. } \\
(1995) \\
\text { Condom Use } \\
\text { - Main }\end{array}$ & $\begin{array}{l}3 \text { questions; } \\
\text { 7-pt scale }\end{array}$ & SS & $\begin{array}{l}\text { Neutral or negative } \\
\text { intention to start using a } \\
\text { contraceptive method all } \\
\text { the time or every time }\end{array}$ & $\begin{array}{l}\text { Positive intention (above } \\
\text { the neutral point) to start } \\
\text { using contraception all } \\
\text { the time or every time in } \\
\text { the next } 6 \text { months }\end{array}$ & $\begin{array}{l}\text { Positive intention to start } \\
\text { using contraception all } \\
\text { the time or every time in } \\
\text { the next } 30 \text { days and } \\
\text { some recorded use } \\
\text { (sometimes or almost } \\
\text { every time/almost all the } \\
\text { time) }\end{array}$ & $\begin{array}{l}\text { Use of a method all the } \\
\text { time or every time for } \\
\text { less than } 6 \text { months }\end{array}$ & $\begin{array}{l}\text { Use of a method all the } \\
\text { time or every time for six } \\
\text { months or longer }\end{array}$ \\
\hline $\begin{array}{l}\text { Galavotti et } \\
\text { al. } \\
(1995) \\
\text { Condom Use } \\
\text { - Other }\end{array}$ & $\begin{array}{l}4 \text { questions; } \\
\text { 7-pt scale }\end{array}$ & SS & $\begin{array}{l}\text { Neutral or negative } \\
\text { intention to start using a } \\
\text { contraceptive method all } \\
\text { the time or every time }\end{array}$ & $\begin{array}{l}\text { Positive intention (above } \\
\text { the neutral point) to start } \\
\text { using contraception all } \\
\text { the time or every time in } \\
\text { the next } 6 \text { months }\end{array}$ & $\begin{array}{l}\text { Positive intention to start } \\
\text { using contraception all } \\
\text { the time or every time in } \\
\text { the next } 30 \text { days and } \\
\text { some recorded use } \\
\text { (sometimes or almost } \\
\text { every time/almost all the } \\
\text { time) }\end{array}$ & $\begin{array}{l}\text { Use of a method all the } \\
\text { time or every time for } \\
\text { less than six months }\end{array}$ & $\begin{array}{l}\text { Use of a method all the } \\
\text { time or every time for six } \\
\text { months or longer }\end{array}$ \\
\hline
\end{tabular}


Table B.1 (continued)

\begin{tabular}{|c|c|c|c|c|c|c|c|}
\hline $\begin{array}{l}\text { Source } \\
\text { (Year) } \\
\text { Target } \\
\text { Behavior }\end{array}$ & $\begin{array}{l}\text { Type of } \\
\text { Algorithm }\end{array}$ & $\begin{array}{l}\text { TTM } \\
\text { or } \\
\text { Study } \\
\text { Specific } \\
\text { (SS) }\end{array}$ & Precontemplation & Contemplation & Preparation & Action & Maintenance \\
\hline $\begin{array}{l}\text { Gazabon } \\
(2000) \\
\text { Condom use }\end{array}$ & $\begin{array}{l}\text { 2-item } \\
\text { algorithm }\end{array}$ & SS & $\begin{array}{l}\text { Sometimes, not always, } \\
\text { or never use condoms and } \\
\text { do not plan to start using } \\
\text { condoms when they have } \\
\text { sex }\end{array}$ & $\begin{array}{l}\text { Sometimes, not always, } \\
\text { or never use condoms, } \\
\text { but plan to start using } \\
\text { condoms when having } \\
\text { sex within the next six } \\
\text { months }\end{array}$ & $\begin{array}{l}\text { Sometimes, not always, } \\
\text { or never use condoms but } \\
\text { plan to start using } \\
\text { condoms when having } \\
\text { sex within the next } 30 \\
\text { days }\end{array}$ & $\begin{array}{l}\text { Started always using } \\
\text { condoms either } 1 \text { month } \\
\text { or } 3-5 \text { months ago }\end{array}$ & $\begin{array}{l}\text { Started always using } \\
\text { condoms } 6 \text { months ago } \\
\text { or longer }\end{array}$ \\
\hline $\begin{array}{l}\text { Giebel } \\
\text { (1999) } \\
\text { Smoking } \\
\text { Cessation }\end{array}$ & \begin{tabular}{|l} 
Single \\
question 5 \\
choice \\
response \\
format \\
\end{tabular} & TTM & $\begin{array}{l}\text { No, and I am not thinking } \\
\text { about starting within the } \\
\text { next } 6 \text { months }\end{array}$ & $\begin{array}{l}\text { No, but I am planning to } \\
\text { start to always use them } \\
\text { within the next } 6 \text { months }\end{array}$ & $\begin{array}{l}\text { No, but I am planning to } \\
\text { start within the next } 30 \\
\text { days }\end{array}$ & $\begin{array}{l}\text { Yes, and I have been } \\
\text { doing so for } 6 \text { months or } \\
\text { less }\end{array}$ & $\begin{array}{l}\text { Yes, and I have been doing } \\
\text { so for } 6 \text { months or longer }\end{array}$ \\
\hline $\begin{array}{l}\text { Giebel } \\
(1999) \\
\text { Dietary Fat } \\
\text { Reduction }\end{array}$ & \begin{tabular}{|l} 
Single \\
question 5 \\
choice \\
response \\
format \\
\end{tabular} & TTM & $\begin{array}{l}\text { No, and I am not thinking } \\
\text { about starting within the } \\
\text { next } 6 \text { months }\end{array}$ & $\begin{array}{l}\text { No, but I am planning to } \\
\text { start to always use them } \\
\text { within the next } 6 \text { months }\end{array}$ & $\begin{array}{l}\text { No, but I am planning to } \\
\text { start within the next } 30 \\
\text { days }\end{array}$ & $\begin{array}{l}\text { Yes, and I have been } \\
\text { doing so for } 6 \text { months or } \\
\text { less }\end{array}$ & $\begin{array}{l}\text { Yes, and I have been doing } \\
\text { so for } 6 \text { months or longer }\end{array}$ \\
\hline $\begin{array}{l}\text { Giebel } \\
(1999) \\
\text { Exercise }\end{array}$ & \begin{tabular}{|l|} 
Single \\
question 5 \\
choice \\
response \\
format \\
\end{tabular} & $\overline{\text { TTM }}$ & $\begin{array}{l}\text { No, and I am not thinking } \\
\text { about starting within the } \\
\text { next } 6 \text { months }\end{array}$ & $\begin{array}{l}\text { No, but I am planning to } \\
\text { start to always use them } \\
\text { within the next } 6 \text { months }\end{array}$ & $\begin{array}{l}\text { No, but I am planning to } \\
\text { start within the next } 30 \\
\text { days }\end{array}$ & $\begin{array}{l}\text { Yes, and I have been } \\
\text { doing so for } 6 \text { months or } \\
\text { less }\end{array}$ & $\begin{array}{l}\text { Yes, and I have been doing } \\
\text { so for } 6 \text { months or longer }\end{array}$ \\
\hline $\begin{array}{l}\text { Giebel } \\
(1999) \\
\text { Stress } \\
\text { Manage- } \\
\text { ment }\end{array}$ & $\begin{array}{l}\text { Single } \\
\text { question } 5 \\
\text { choice } \\
\text { response } \\
\text { format }\end{array}$ & TTM & $\begin{array}{l}\text { No, and I am not thinking } \\
\text { about starting within the } \\
\text { next } 6 \text { months }\end{array}$ & $\begin{array}{l}\text { No, but I am planning to } \\
\text { start to always use them } \\
\text { within the next } 6 \text { months }\end{array}$ & $\begin{array}{l}\text { No, but I am planning to } \\
\text { start within the next } 30 \\
\text { days }\end{array}$ & $\begin{array}{l}\text { Yes, and I have been } \\
\text { doing so for } 6 \text { months or } \\
\text { less }\end{array}$ & $\begin{array}{l}\text { Yes, and I have been doing } \\
\text { so for } 6 \text { months or longer }\end{array}$ \\
\hline
\end{tabular}


Table B.1 (continued)

\begin{tabular}{|c|c|c|c|c|c|c|c|}
\hline $\begin{array}{c}\text { Source } \\
\text { (Year) } \\
\text { Target } \\
\text { Behavior }\end{array}$ & $\begin{array}{l}\text { Type of } \\
\text { Algorithm }\end{array}$ & \begin{tabular}{|} 
TTM \\
or \\
Study \\
Specific \\
(SS)
\end{tabular} & Precontemplation & Contemplation & Preparation & Action & Maintenance \\
\hline $\begin{array}{l}\text { Grimley et } \\
\text { al. } \\
(1995) \\
\text { Contracep- } \\
\text { tion - } \\
\text { General }\end{array}$ & $\begin{array}{l}\text { 4-item } \\
\text { staging }\end{array}$ & SS & $\begin{array}{l}\text { Not using contraception } \\
\text { and had no intention to } \\
\text { start doing so every time } \\
\text { in the next } 6 \text { months. }\end{array}$ & $\begin{array}{l}\text { Not currently using } \\
\text { contraception, but intend } \\
\text { to start doing so every } \\
\text { time in the next } 6 \text { months }\end{array}$ & $\begin{array}{l}\text { Intend to start using } \\
\text { contraception every time } \\
\text { within the next month } \\
\text { and are currently using } \\
\text { contraception almost } \\
\text { every time }\end{array}$ & $\begin{array}{l}\text { Have been using } \\
\text { contraception every time } \\
\text { for less than } 6 \text { months }\end{array}$ & $\begin{array}{l}\text { Have been using } \\
\text { contraception every time } \\
\text { for more than } 6 \text { months. }\end{array}$ \\
\hline $\begin{array}{l}\text { Grimley et } \\
\text { al. } \\
(1995) \\
\text { Condom } \\
\text { Use-Main }\end{array}$ & $\begin{array}{l}\text { 5-item } \\
\text { staging }\end{array}$ & $S \bar{S}$ & $\begin{array}{l}\text { Not using condoms with } \\
\text { their main partners and } \\
\text { had no intention to start } \\
\text { doing so every time in the } \\
\text { next } 6 \text { months. }\end{array}$ & $\begin{array}{l}\text { Not currently using } \\
\text { condoms with their main } \\
\text { partners, but intend to } \\
\text { start doing so every time } \\
\text { in the next } 6 \text { months }\end{array}$ & $\begin{array}{l}\text { Intend to start using } \\
\text { condoms every time } \\
\text { within the next month } \\
\text { and are currently using } \\
\text { condoms almost every } \\
\text { time with their main } \\
\text { partner }\end{array}$ & $\begin{array}{l}\text { Have been using } \\
\text { condoms every time for } \\
\text { less than } 6 \text { months }\end{array}$ & $\begin{array}{l}\text { Have been using condoms } \\
\text { with their main partners } \\
\text { every time for more than } 6 \\
\text { months. }\end{array}$ \\
\hline $\begin{array}{l}\text { Grimley et } \\
\text { al. } \\
(1995) \\
\text { Condom } \\
\text { Use-Other }\end{array}$ & $\begin{array}{l}5 \text {-item } \\
\text { staging }\end{array}$ & $\overline{\mathrm{SS}}$ & $\begin{array}{l}\text { Not using condoms with } \\
\text { their other partners and } \\
\text { had no intention to start } \\
\text { doing so every time in the } \\
\text { next } 6 \text { months. }\end{array}$ & $\begin{array}{l}\text { Not currently using } \\
\text { condoms with their other } \\
\text { partners, but intend to } \\
\text { start doing so every time } \\
\text { in the next } 6 \text { months }\end{array}$ & $\begin{array}{l}\text { Intend to start using } \\
\text { condoms every time } \\
\text { within the next month } \\
\text { and are currently using } \\
\text { condoms almost every } \\
\text { time with their other } \\
\text { partners }\end{array}$ & $\begin{array}{l}\text { Have been using } \\
\text { condoms every time for } \\
\text { less than } 6 \text { months }\end{array}$ & $\begin{array}{l}\text { Have been using condoms } \\
\text { with their other partners } \\
\text { every time for more than } 6 \\
\text { months. }\end{array}$ \\
\hline $\begin{array}{l}\text { Happel } \\
(2002) \\
\text { Fruit \& } \\
\text { Vegetable } \\
\text { Consumption } \\
\end{array}$ & $\begin{array}{l}\text { Single } \\
\text { question } 5 \\
\text { choice } \\
\text { response } \\
\text { format }\end{array}$ & TTM & $\begin{array}{l}\text { No, and I am not thinking } \\
\text { about starting within the } \\
\text { next } 6 \text { months }\end{array}$ & $\begin{array}{l}\text { No, but I am planning to } \\
\text { start to always use them } \\
\text { within the next } 6 \text { months }\end{array}$ & $\begin{array}{l}\text { No, but I am planning to } \\
\text { start within the next } 30 \\
\text { days }\end{array}$ & $\begin{array}{l}\text { Yes, and I have been } \\
\text { doing so for } 6 \text { months or } \\
\text { less }\end{array}$ & $\begin{array}{l}\text { Yes, and I have been doing } \\
\text { so for } 6 \text { months or longer }\end{array}$ \\
\hline $\begin{array}{l}\text { Hempel- } \\
\text { mann } \\
(2003) \\
\text { Tooth- } \\
\end{array}$ & $\begin{array}{l}\text { Single } \\
\text { question } 5 \\
\text { choice } \\
\text { response } \\
\text { format } \\
\end{array}$ & TTM & $\begin{array}{l}\text { No, and } 1 \text { am not thinking } \\
\text { about starting within the } \\
\text { next } 6 \text { months }\end{array}$ & $\begin{array}{l}\text { No, but I am planning to } \\
\text { start to always use them } \\
\text { within the next } 6 \text { months }\end{array}$ & $\begin{array}{l}\text { No, but I am planning to } \\
\text { start within the next } 30 \\
\text { days }\end{array}$ & $\begin{array}{l}\text { Yes, and I have been } \\
\text { doing so for } 6 \text { months or } \\
\text { less }\end{array}$ & $\begin{array}{l}\text { Yes, and I have been doing } \\
\text { so for } 6 \text { months or longer }\end{array}$ \\
\hline
\end{tabular}


Table B.1 (continued)

\begin{tabular}{|c|c|c|c|c|c|c|c|}
\hline $\begin{array}{c}\text { Source } \\
\text { (Year) } \\
\text { Target } \\
\text { Behavior }\end{array}$ & $\begin{array}{l}\text { Type of } \\
\text { Algorithm }\end{array}$ & $\begin{array}{c}\text { TTM } \\
\text { or } \\
\text { Study } \\
\text { Specific } \\
\text { (SS) }\end{array}$ & Precontemplation & Contemplation & Preparation & Action & Maintenance \\
\hline \multicolumn{8}{|l|}{ friendly Diet } \\
\hline $\begin{array}{l}\text { Henderson } \\
\text { (1998) } \\
\text { Breast Self- } \\
\text { Examination }\end{array}$ & $\begin{array}{l}\text { 5-item } \\
\text { algorithm } \\
\text { (Yes/No) }\end{array}$ & SS & $\begin{array}{l}\text { Not currently performing } \\
\text { monthly BSEs; no plan } \\
\text { for performing BSEs }\end{array}$ & $\begin{array}{l}\text { Not currently performing } \\
\text { monthly BSEs; planning } \\
\text { one within the next six } \\
\text { months }\end{array}$ & $\begin{array}{l}\text { Not currently performing } \\
\text { monthly BSEs; planning } \\
\text { one in the next } 30 \text { days }\end{array}$ & $\begin{array}{l}\text { Performed a monthly } \\
\text { BSE for less than six } \\
\text { consecutive months }\end{array}$ & $\begin{array}{l}\text { Performed BSEs each } \\
\text { month for at least the past } \\
12 \text { months }\end{array}$ \\
\hline $\begin{array}{l}\text { Herrick et al. } \\
\text { (1997) } \\
\text { Dietary Fat } \\
\text { Reduction }\end{array}$ & $\begin{array}{l}\text { Single } \\
\text { question } 5 \\
\text { choice } \\
\text { response } \\
\text { format }\end{array}$ & TTM & $\begin{array}{l}\text { No intention to change } \\
\text { behavior in the next six } \\
\text { months }\end{array}$ & $\begin{array}{l}\text { Intention to change } \\
\text { within the next } 6 \text { months. }\end{array}$ & $\begin{array}{l}\text { Small and/or inconsistent } \\
\text { changes }\end{array}$ & $\begin{array}{l}\text { Actively engaging in the } \\
\text { new behavior for less } \\
\text { than six months }\end{array}$ & $\begin{array}{l}\text { Sustained behavior change } \\
\text { for more than six months }\end{array}$ \\
\hline $\begin{array}{l}\text { Herrick et al. } \\
(1997) \\
\text { Exercise }\end{array}$ & $\begin{array}{l}\text { Single } \\
\text { question } 5 \\
\text { choice } \\
\text { response } \\
\text { format }\end{array}$ & TTM & $\begin{array}{l}\text { No intention to change } \\
\text { behavior in the next six } \\
\text { months }\end{array}$ & $\begin{array}{l}\text { Intention to change } \\
\text { within the next } 6 \text { months. }\end{array}$ & $\begin{array}{l}\text { Small and/or inconsistent } \\
\text { changes }\end{array}$ & $\begin{array}{l}\text { Actively engaging in the } \\
\text { new behavior for less } \\
\text { than six months }\end{array}$ & $\begin{array}{l}\text { Sustained behavior change } \\
\text { for more than six months }\end{array}$ \\
\hline $\begin{array}{l}\text { Herrick et al. } \\
\text { (1997) } \\
\text { Smoking } \\
\text { Cessation } \\
\end{array}$ & $\begin{array}{l}\text { Single } \\
\text { question } 5 \\
\text { choice } \\
\text { response } \\
\text { format }\end{array}$ & TTM & $\begin{array}{l}\text { No intention to change } \\
\text { behavior in the next six } \\
\text { months }\end{array}$ & $\begin{array}{l}\text { lntention to change } \\
\text { within the next } 6 \text { months. }\end{array}$ & $\begin{array}{l}\text { Small and/or inconsistent } \\
\text { changes }\end{array}$ & $\begin{array}{l}\text { Actively engaging in the } \\
\text { new behavior for less } \\
\text { than six months }\end{array}$ & $\begin{array}{l}\text { Sustained behavior change } \\
\text { for more than six months }\end{array}$ \\
\hline $\begin{array}{l}\text { Herrick et al. } \\
\text { (1997) } \\
\text { Sun } \\
\text { Protection/Re } \\
\text { duction }\end{array}$ & $\begin{array}{l}\text { Single } \\
\text { question } 5 \\
\text { choice } \\
\text { response } \\
\text { format }\end{array}$ & TTM & $\begin{array}{l}\text { No intention to change } \\
\text { behavior in the next six } \\
\text { months }\end{array}$ & $\begin{array}{l}\text { Intention to change } \\
\text { within the next } 6 \text { months. }\end{array}$ & $\begin{array}{l}\text { Small and/or inconsistent } \\
\text { changes }\end{array}$ & $\begin{array}{l}\text { Actively engaging in the } \\
\text { new behavior for less } \\
\text { than six months }\end{array}$ & $\begin{array}{l}\text { Sustained behavior change } \\
\text { for more than six months }\end{array}$ \\
\hline
\end{tabular}


Table B.1 (continued)

\begin{tabular}{|c|c|c|c|c|c|c|c|}
\hline $\begin{array}{c}\text { Source } \\
\text { (Year) } \\
\text { Target } \\
\text { Behavior }\end{array}$ & $\begin{array}{l}\text { Type of } \\
\text { Algorithm }\end{array}$ & $\begin{array}{c}\text { TTM } \\
\text { or } \\
\text { Study } \\
\text { Specific } \\
\text { (SS) }\end{array}$ & Precontemplation & Contemplation & Preparation & Action & Maintenance \\
\hline $\begin{array}{l}\text { Herzog et al. } \\
(1999) \\
\text { Smoking } \\
\text { Cessation }\end{array}$ & $\begin{array}{l}5 \text {-item } \\
\text { measure } \\
\text { (format } \\
\text { unclear; } \\
\text { DiClemente, } \\
\text { 199I) }\end{array}$ & SS & $\begin{array}{l}\text { Not seriously thinking } \\
\text { about quitting smoking in } \\
\text { the next } 6 \text { months }\end{array}$ & $\begin{array}{l}\text { Seriously thinking about } \\
\text { quitting in the next } 6 \\
\text { months and were not } \\
\text { seriously thinking about } \\
\text { quitting in the next } 30 \\
\text { days and had not made at } \\
\text { least one } 24 \text { hour quit } \\
\text { attempt in the past year, } \\
\text { or both }\end{array}$ & $\begin{array}{l}\text { Seriously thinking about } \\
\text { quitting within the next } \\
30 \text { days and had at least } \\
\text { one } 24 \text { hour quit attempt } \\
\text { in the past year }\end{array}$ & $\begin{array}{l}\text { The first } 6 \text { months of } \\
\text { smoking abstinence }\end{array}$ & $\begin{array}{l}\text { Abstinence beyond } 6 \\
\text { months }\end{array}$ \\
\hline \begin{tabular}{|l|} 
Hulton \\
$(2001)$ \\
\\
Sex Decision \\
Making/ \\
Abstinence \\
(Non \\
Virgins) \\
\end{tabular} & 9-item & SS & $\begin{array}{l}\text { Has had sexual } \\
\text { intercourse and is not } \\
\text { thinking about stopping }\end{array}$ & $\begin{array}{l}\text { Has had sexual } \\
\text { intercourse and doesn't } \\
\text { want to stop, but may } \\
\text { stop in the future }\end{array}$ & $\begin{array}{l}\text { Has had sexual } \\
\text { intercourse but is } \\
\text { planning to stop }\end{array}$ & $\begin{array}{l}\text { Has had sexual } \\
\text { intercourse, but has made } \\
\text { attempts to stop in the } \\
\text { past } 6 \text { months }\end{array}$ & $\begin{array}{l}\text { Has had sexual intercourse } \\
\text { but has now stopped for } \\
\text { more than } 6 \text { months }\end{array}$ \\
\hline \begin{tabular}{|l|} 
Hulton \\
$(2001)$ \\
\\
Sex Decision \\
Making/ \\
Abstinence \\
(Virgins) \\
\end{tabular} & 9-item & SS & $\begin{array}{l}\text { Has not had sexual } \\
\text { intercourse and is not } \\
\text { considering learning } \\
\text { abstinence skills }\end{array}$ & $\begin{array}{l}\text { Has not had sexual } \\
\text { intercourse but is } \\
\text { thinking about needing } \\
\text { skills to remain abstinent }\end{array}$ & \begin{tabular}{|l|} 
Has not had sexual \\
intercourse and is making \\
plans to learn skills to \\
remain abstinent
\end{tabular} & $\begin{array}{l}\text { Has not had sexual } \\
\text { intercourse and is } \\
\text { currently using skills to } \\
\text { stay abstinent until } \\
\text { marriage }\end{array}$ & Not reported \\
\hline \begin{tabular}{|l} 
Jeffries \\
$(2000)$ \\
Exercise
\end{tabular} & \begin{tabular}{|l|} 
Single \\
question 5 \\
choice \\
response \\
format
\end{tabular} & SS & $\begin{array}{l}\text { No, I do not currently } \\
\text { engage in regular exercise } \\
\text { for } 20 \text { minutes or longer } \\
\text { for } 3 \text { times or more per } \\
\text { week, and I do not intend } \\
\text { to in the next } 6 \text { months }\end{array}$ & $\begin{array}{l}\text { No, I do not currently } \\
\text { engage in regular exercise } \\
\text { for } 20 \text { minutes or longer } \\
\text { for } 3 \text { times or more per } \\
\text { week, but I intend to in } \\
\text { the next } 6 \text { months }\end{array}$ & $\begin{array}{l}\text { No, I do not currently } \\
\text { engage in regular exercise } \\
\text { for } 20 \text { minutes or longer } \\
\text { for } 3 \text { times or more per } \\
\text { week, but I intend to in } \\
\text { the next } 30 \text { days }\end{array}$ & $\begin{array}{l}\text { Yes, I currently engage in } \\
\text { regular exercise for } 20 \\
\text { minutes or longer for } 3 \\
\text { times or more per week, } \\
\text { and have been for less } \\
\text { than } 6 \text { months }\end{array}$ & $\begin{array}{l}\text { Yes, I currently engage in } \\
\text { regular exercise for } 20 \\
\text { minutes or longer for } 3 \\
\text { times or more per week, } \\
\text { and have been for more } \\
\text { than } 6 \text { months }\end{array}$ \\
\hline
\end{tabular}


Table B.1 (continued)

\begin{tabular}{|c|c|c|c|c|c|c|c|}
\hline $\begin{array}{c}\text { Source } \\
\text { (Year) } \\
\text { Target } \\
\text { Behavior }\end{array}$ & $\begin{array}{l}\text { Type of } \\
\text { Algorithm }\end{array}$ & $\begin{array}{l}\text { TTM } \\
\text { or } \\
\text { Study } \\
\text { Specific } \\
\text { (SS) }\end{array}$ & Precontemplation & Contemplation & Preparation & Action & Maintenance \\
\hline $\begin{array}{l}\text { Johnson, J.L. } \\
\text { et al. } \\
(2002) \\
\text { Bone Density } \\
\text { Testing }\end{array}$ & $\begin{array}{l}\text { Single } \\
\text { question } 5 \\
\text { choice } \\
\text { response } \\
\text { format }\end{array}$ & SS & $\begin{array}{l}\text { Have you had a BDT in } \\
\text { the past three years? No, } \\
\text { and I do not intend to in } \\
\text { the next } 12 \text { months }\end{array}$ & $\begin{array}{l}\text { Have you had a BDT in } \\
\text { the past three years? No, } \\
\text { but I have one scheduled } \\
\text { for the next } 12 \text { months }\end{array}$ & $\begin{array}{l}\text { Have you had a BDT in } \\
\text { the past three years? Yes, } \\
\text { I have had one and intend } \\
\text { to have another one } \\
\text { within the next } 3 \text { years }\end{array}$ & $\begin{array}{l}\text { Have you have a BDT in } \\
\text { the past three years? Yes, } \\
\text { I have had more than one } \\
\text { and intend to have } \\
\text { another within the next } \\
\text { three years }\end{array}$ & $\begin{array}{l}\text { Have you had a BDT in th } \\
\text { past three years? Yes, I } \\
\text { have had one, but I do not } \\
\text { intend to have another one } \\
\text { within the next three years }\end{array}$ \\
\hline $\begin{array}{l}\text { Johnson, J.L. } \\
\text { et al. } \\
(2002) \\
\text { Calcium } \\
\text { Intake }\end{array}$ & $\begin{array}{l}\text { Single } \\
\text { question } 5 \\
\text { choice } \\
\text { response } \\
\text { format }\end{array}$ & SS & $\begin{array}{l}\text { Adequate calcium intake } \\
\text { for postmeno-pausal } \\
\text { women (1200- } \\
1500 \mathrm{md} / \text { day) through } \\
\text { some combination of } \\
\text { food and supplement: } \\
\text { "No, and I don't intend to } \\
\text { in the next six months" }\end{array}$ & $\begin{array}{l}\text { Adequate calcium intake } \\
\text { for postmeno-pausal } \\
\text { women (1200- } \\
1500 \mathrm{md} / \text { day) through } \\
\text { some combination of } \\
\text { food and supplement: } \\
\text { "No, but I intend to in } \\
\text { the next six months" }\end{array}$ & $\begin{array}{l}\text { Adequate calcium intake } \\
\text { for postmeno-pausal } \\
\text { women ( } 1200- \\
1500 \mathrm{md} / \text { day) through } \\
\text { some combination of } \\
\text { food and supplement: } \\
\text { "No, but I intend to in the } \\
\text { next } 30 \text { days" }\end{array}$ & $\begin{array}{l}\text { Adequate calcium intake } \\
\text { for postmeno-pausal } \\
\text { women ( } 1200- \\
\text { I500md/day) through } \\
\text { some combination of } \\
\text { food and supplement: } \\
\text { "Yes, I have been but for } \\
\text { less than six months" }\end{array}$ & $\begin{array}{l}\text { Adequate calcium intake } \\
\text { for postmeno-pausal } \\
\text { women (1200- } \\
1500 \mathrm{md} / \text { day) through som } \\
\text { combination of food and } \\
\text { supplement: "Yes, I have } \\
\text { been for more than six } \\
\text { months" }\end{array}$ \\
\hline $\begin{array}{l}\text { Johnson, S.S. } \\
\text { et al. } \\
(2002) \\
\text { Participat-ing } \\
\text { in Decision } \\
\text { Making for } \\
\text { Medication }\end{array}$ & $\begin{array}{l}\text { Single } \\
\text { question } 5 \\
\text { choice } \\
\text { response } \\
\text { format }\end{array}$ & SS & $\begin{array}{l}\text { Working w/your Dr. to } \\
\text { decide if you need } \\
\text { medication and which } \\
\text { medication is right for } \\
\text { you and discussing w/ } \\
\text { your Dr. any concerns } \\
\text { you have about taking } \\
\text { medications: "No, and I } \\
\text { don't intend to in the next } \\
\text { six months" }\end{array}$ & $\begin{array}{l}\text { Working w/your Dr. to } \\
\text { decide if you need } \\
\text { medication and which } \\
\text { medication is right for } \\
\text { you and discussing w/ } \\
\text { your Dr. any concerns } \\
\text { you have about taking } \\
\text { medications: "No, but I } \\
\text { intend to in the next six } \\
\text { months" }\end{array}$ & $\begin{array}{l}\text { Working w/your Dr. to } \\
\text { decide if you need } \\
\text { medication and which } \\
\text { medication is right for } \\
\text { you and discussing w/ } \\
\text { your Dr. any concerns } \\
\text { you have about taking } \\
\text { medications: "No, but I } \\
\text { intend to in the next } 30 \\
\text { days" }\end{array}$ & $\begin{array}{l}\text { Working w/your Dr. to } \\
\text { decide if you need } \\
\text { medication and which } \\
\text { medication is right for } \\
\text { you and discussing w/ } \\
\text { your Dr. any concerns } \\
\text { you have about taking } \\
\text { medications: "Yes, I } \\
\text { have been but for less } \\
\text { than six months" }\end{array}$ & $\begin{array}{l}\text { Working w/your Dr. to } \\
\text { decide if you need } \\
\text { medication and which } \\
\text { medication is right for you } \\
\text { and discussing w/ your Dr. } \\
\text { any concerns you have } \\
\text { about taking medications: } \\
\text { "Yes, I have been for more } \\
\text { than six months" }\end{array}$ \\
\hline $\begin{array}{l}\text { Johnson et al. } \\
(2002) \\
\text { Smoking } \\
\text { Cessation }\end{array}$ & Not reported & SS & $\begin{array}{l}\text { Smoking and not } \\
\text { intending to quit smoking } \\
\text { within the next } 6 \text { months }\end{array}$ & $\begin{array}{l}\text { Smoking, but are } \\
\text { considering quitting } \\
\text { smoking in the next } 6 \\
\text { months }\end{array}$ & $\begin{array}{l}\text { Smoking, but planning to } \\
\text { quit smoking in the next } \\
30 \text { days, and have made a } \\
\text { previous quit attempt }\end{array}$ & $\begin{array}{l}\text { Have quit smoking in the } \\
\text { past } 6 \text { months }\end{array}$ & Have quit smoking \\
\hline
\end{tabular}


Table B.1 (continued)

\begin{tabular}{|c|c|c|c|c|c|c|c|}
\hline $\begin{array}{l}\text { Source } \\
\text { (Year) } \\
\text { Target } \\
\text { Behavior }\end{array}$ & $\begin{array}{c}\text { Type of } \\
\text { Algorithm }\end{array}$ & $\begin{array}{l}\text { TTM } \\
\text { or } \\
\text { Study } \\
\text { Specific } \\
\text { (SS) }\end{array}$ & Precontemplation & Contemplation & Preparation & Action & Maintenance \\
\hline $\begin{array}{l}\text { Jordan } \\
(2001) \\
\text { Anorexia- } \\
\text { Recovery } \\
\text { From } \\
\end{array}$ & $\begin{array}{l}\text { Single } \\
\text { question } 5 \\
\text { choice } \\
\text { response } \\
\text { format }\end{array}$ & SS & $\begin{array}{l}\text { Not thinking about and } \\
\text { not planning to recover } \\
\text { from anorexia/bulimia in } \\
\text { the next } 6 \text { months }\end{array}$ & $\begin{array}{l}\text { Thinking about trying to } \\
\text { recover in the next } 6 \\
\text { months }\end{array}$ & $\begin{array}{l}\text { Intended to start trying to } \\
\text { recover in the next } 30 \\
\text { days }\end{array}$ & $\begin{array}{l}\text { Had been actively } \\
\text { recovering from their } \\
\text { eating disorder for fewer } \\
\text { than } 6 \text { months }\end{array}$ & $\begin{array}{l}\text { Had been in active } \\
\text { recovery for more than } 6 \\
\text { months }\end{array}$ \\
\hline $\begin{array}{l}\text { Jordan } \\
(2001) \\
\text { Bulimia- } \\
\text { Recovery } \\
\text { From }\end{array}$ & $\begin{array}{l}\text { Single } \\
\text { question 5- } \\
\text { choice } \\
\text { response } \\
\text { format }\end{array}$ & SS & $\begin{array}{l}\text { Not thinking about and } \\
\text { not planning to recover } \\
\text { from anorexia/ bulimia in } \\
\text { the next } 6 \text { months }\end{array}$ & $\begin{array}{l}\text { Thinking about trying to } \\
\text { recover in the next } 6 \\
\text { months }\end{array}$ & $\begin{array}{l}\text { Intended to start trying to } \\
\text { recover in the next } 30 \\
\text { days }\end{array}$ & $\begin{array}{l}\text { Had been actively } \\
\text { recovering from their } \\
\text { eating disorder for fewer } \\
\text { than } 6 \text { months }\end{array}$ & $\begin{array}{l}\text { Had been in active } \\
\text { recovery for more than } 6 \\
\text { months }\end{array}$ \\
\hline $\begin{array}{l}\text { Jordon et al. } \\
(2002) \\
\text { Exercise }\end{array}$ & $\begin{array}{l}\text { 5-question } \\
\text { (Yes/No) }\end{array}$ & SS & $\begin{array}{l}\text { Participants who did not } \\
\text { exercise regularly and } \\
\text { had no intention to begin } \\
\text { in the next } 6 \text { months }\end{array}$ & $\begin{array}{l}\text { Those who did not } \\
\text { exercise regularly, but } \\
\text { intended to start within } \\
\text { the next } 6 \text { months, not the } \\
\text { next } 30 \text { days }\end{array}$ & $\begin{array}{l}\text { Those who did not } \\
\text { exercise regularly, but } \\
\text { intended to start in the } \\
\text { next } 30 \text { days }\end{array}$ & $\begin{array}{l}\text { Participants who did } \\
\text { exercise regularly, but } \\
\text { had not done so for a } \\
\text { minimum of six months }\end{array}$ & $\begin{array}{l}\text { Those who had been } \\
\text { exercising regularly for } \\
\text { more than six months }\end{array}$ \\
\hline $\begin{array}{l}\text { Katlin } \\
(2001) \\
\text { Drug } \\
\text { treatment }\end{array}$ & $\begin{array}{l}\text { Single } \\
\text { question } 5 \\
\text { choice } \\
\text { response } \\
\text { format }\end{array}$ & $\overline{\mathrm{SS}}$ & $\begin{array}{l}\text { No intention to stop } \\
\text { alcohol or drug use. }\end{array}$ & $\begin{array}{l}\text { Plans to stop alcohol or } \\
\text { drug use in the next } 6 \\
\text { months. }\end{array}$ & $\begin{array}{l}\text { Plans to stop alcohol or } \\
\text { drug use in the next } \\
\text { month and has made one } \\
\text { quit attempt in the past } \\
\text { year (lasting } 24 \text { hours or } \\
\text { longer). }\end{array}$ & $\begin{array}{l}\text { Has completely stopped } \\
\text { using alcohol or drugs } \\
\text { less than } 6 \text { months ago. }\end{array}$ & $\begin{array}{l}\text { Has completely stopped } \\
\text { using alcohol or drugs } \\
\text { more than } 6 \text { months ago. }\end{array}$ \\
\hline $\begin{array}{l}\text { Keller et al. } \\
(2000) \\
\text { Back Pain }\end{array}$ & $\begin{array}{l}\text { 5-item w/ } \\
\text { restaging in } \\
\text { Cont.; Prep } \\
\text { restaged into } \\
\text { Cont. if no } \\
\text { previous } \\
\text { attempt to } \\
\text { change }\end{array}$ & $\overline{\mathrm{SS}}$ & $\begin{array}{l}\text { I do not intend to change } \\
\text { my behavior }\end{array}$ & $\begin{array}{l}\text { I intend to change my } \\
\text { behavior in the next } 6 \\
\text { months }\end{array}$ & $\begin{array}{l}\text { I intend to change my } \\
\text { behavior in the next } 30 \\
\text { days (and in the last } 6 \\
\text { months, have made steps } \\
\text { to actively deal with the } \\
\text { topic of good body } \\
\text { posture, like reading a } \\
\text { book or watching a TV } \\
\text { program about it }\end{array}$ & $\begin{array}{l}\text { I have kept a good body } \\
\text { posture for less than } 6 \\
\text { months }\end{array}$ & $\begin{array}{l}\text { I have kept a good body } \\
\text { posture for more than } 6 \\
\text { months }\end{array}$ \\
\hline
\end{tabular}


Table B.1 (continued)

\begin{tabular}{|c|c|c|c|c|c|c|c|}
\hline $\begin{array}{c}\text { Source } \\
\text { (Year) } \\
\text { Target } \\
\text { Behavior }\end{array}$ & $\begin{array}{l}\text { Type of } \\
\text { Algorithm }\end{array}$ & \begin{tabular}{|c} 
TTM \\
or \\
Study \\
Specific \\
(SS)
\end{tabular} & Precontemplation & Contemplation & Preparation & Action & Maintenance \\
\hline \begin{tabular}{|l|}
$\begin{array}{l}\text { Keller et al. } \\
(200 \mathrm{I})\end{array}$ \\
Fruit and \\
Vegetable \\
Consumption \\
\end{tabular} & $\begin{array}{l}\text { Single } \\
\text { question } 5 \\
\text { choice } \\
\text { response } \\
\text { format }\end{array}$ & TTM & $\begin{array}{l}\text { No, and I am not thinking } \\
\text { about starting within the } \\
\text { next } 6 \text { months }\end{array}$ & $\begin{array}{l}\text { No, but I am planning to } \\
\text { start to always use them } \\
\text { within the next } 6 \text { months }\end{array}$ & $\begin{array}{l}\text { No, but I am planning to } \\
\text { start within the next } 30 \\
\text { days }\end{array}$ & Action/ Maintenance & Action/ Maintenance \\
\hline \begin{tabular}{|l|}
$\begin{array}{l}\text { Keller et al. } \\
(2002)\end{array}$ \\
Oral Hygiene
\end{tabular} & $\begin{array}{l}\text { Single } \\
\text { question 5- } \\
\text { choice } \\
\text { response } \\
\text { format }\end{array}$ & TTM & $\begin{array}{l}\text { No, and I am not thinking } \\
\text { about starting within the } \\
\text { next } 6 \text { months }\end{array}$ & $\begin{array}{l}\text { No, but I am planning to } \\
\text { start to always use them } \\
\text { within the next } 6 \text { months }\end{array}$ & $\begin{array}{l}\text { No, but I am planning to } \\
\text { start within the next } 30 \\
\text { days }\end{array}$ & $\begin{array}{l}\text { Yes, and I have been } \\
\text { doing so for } 6 \text { months or } \\
\text { less }\end{array}$ & $\begin{array}{l}\text { Yes, and I have been doin } \\
\text { so for } 6 \text { months or longer }\end{array}$ \\
\hline $\begin{array}{l}\text { Keller et al. } \\
(2003) \\
\text { Stress } \\
\text { Manage- } \\
\text { ment } \\
\end{array}$ & \begin{tabular}{|l|} 
Single \\
question 5 \\
choice \\
response \\
format
\end{tabular} & TTM & $\begin{array}{l}\text { No, and I am not thinking } \\
\text { about starting within the } \\
\text { next } 6 \text { months }\end{array}$ & $\begin{array}{l}\text { No, but I am planning to } \\
\text { start to always use them } \\
\text { within the next } 6 \text { months }\end{array}$ & $\begin{array}{l}\text { No, but I am planning to } \\
\text { start within the next } 30 \\
\text { days }\end{array}$ & $\begin{array}{l}\text { Yes, and I have been } \\
\text { doing so for } 6 \text { months or } \\
\text { less }\end{array}$ & $\begin{array}{l}\text { Yes, and I have been doing } \\
\text { so for } 6 \text { months or longer }\end{array}$ \\
\hline $\begin{array}{l}\begin{array}{l}\text { Keller et al. } \\
(1998)\end{array} \\
\text { Healthy Diet }\end{array}$ & $\begin{array}{l}\text { Single } \\
\text { question } 5 \\
\text { choice } \\
\text { response } \\
\text { format } \\
\end{array}$ & TTM & $\begin{array}{l}\text { No, and I am not thinking } \\
\text { about starting within the } \\
\text { next } 6 \text { months }\end{array}$ & $\begin{array}{l}\text { No, but I am planning to } \\
\text { start to always use them } \\
\text { within the next } 6 \text { months }\end{array}$ & $\begin{array}{l}\text { No, but I am planning to } \\
\text { start within the next } 30 \\
\text { days }\end{array}$ & $\begin{array}{l}\text { Yes, and I have been } \\
\text { doing so for } 6 \text { months or } \\
\text { less }\end{array}$ & $\begin{array}{l}\text { Yes, and I have been doin } \\
\text { so for } 6 \text { months or longer }\end{array}$ \\
\hline $\begin{array}{l}\text { Keller et al. } \\
(1999) \\
\text { Smoking } \\
\text { Cessation }\end{array}$ & \begin{tabular}{|l|} 
Single \\
question 5 \\
choice \\
response \\
format
\end{tabular} & SS & \begin{tabular}{|l|} 
Are you currently \\
smoking cigarettes? Yes, \\
and I do not intend to quit
\end{tabular} & $\begin{array}{l}\text { Are you currently } \\
\text { smoking cigarettes? Yes, } \\
\text { but I intend to quit in the } \\
\text { next } 6 \text { months }\end{array}$ & $\begin{array}{l}\text { Are you currently } \\
\text { smoking cigarettes? Yes, } \\
\text { but I intend to quit in the } \\
\text { next } 30 \text { days (Restage PR } \\
\text { to C if zero for "How } \\
\text { often in the last } 6 \text { months } \\
\text { have you deliberately } \\
\text { tried not to smoke for at } \\
\text { least } 24 \text { hours?") }\end{array}$ & \begin{tabular}{|l|} 
Are you currently \\
smoking cigarettes? No, I \\
quit in the last 6 months
\end{tabular} & $\begin{array}{l}\text { Are you currently smoking } \\
\text { cigarettes? No, I quit more } \\
\text { than } 6 \text { months ago }\end{array}$ \\
\hline
\end{tabular}


Table B.1 (continued)

\begin{tabular}{|c|c|c|c|c|c|c|c|}
\hline $\begin{array}{c}\text { Source } \\
\text { (Year) } \\
\text { Target } \\
\text { Behavior }\end{array}$ & $\begin{array}{l}\text { Type of } \\
\text { Algorithm }\end{array}$ & \begin{tabular}{|l} 
TTM \\
or \\
Study \\
Specific \\
(SS)
\end{tabular} & Precontemplation & Contemplation & Preparation & Action & Maintenance \\
\hline $\begin{array}{l}\text { King et al. } \\
(1996) \\
\text { Exercise }\end{array}$ & $\begin{array}{l}\text { 4-item } \\
\text { algorithm }\end{array}$ & SS & $\begin{array}{l}\text { Has not engaged in } \\
\text { exercise with no intention } \\
\text { of exercising in the next } 6 \\
\text { months }\end{array}$ & $\begin{array}{l}\text { Has not engaged in } \\
\text { exercise and is thinking } \\
\text { about exercising in the } \\
\text { next } 6 \text { months }\end{array}$ & $\begin{array}{l}\text { Has participated in } \\
\text { occasional exercise }\end{array}$ & Action/Maintenance & Action/ Maintenance \\
\hline $\begin{array}{l}\text { King et al. } \\
(1996) \\
\text { Smoking } \\
\text { Cessation }\end{array}$ & $\begin{array}{l}\text { 4-item } \\
\text { algorithm }\end{array}$ & $\overline{S S}$ & $\begin{array}{l}\text { Not considering changing } \\
\text { their smoking behavior }\end{array}$ & $\begin{array}{l}\text { Considering changing } \\
\text { smoking behavior }\end{array}$ & $\begin{array}{l}\text { Recently taken steps to } \\
\text { reduce or quit smoking }\end{array}$ & $\begin{array}{l}\text { Currently sticking to a } \\
\text { smoking cessation plan }\end{array}$ & Not reported \\
\hline $\begin{array}{l}\text { Kraft et al. } \\
\text { (1999) } \\
\text { Smoking } \\
\text { Cessation }\end{array}$ & Not reported & SS & $\begin{array}{l}\text { Participants were } \\
\text { smoking and had no } \\
\text { intention to quit within } \\
\text { the next } 6 \text { months }\end{array}$ & $\begin{array}{l}\text { Participants who were } \\
\text { smoking but seriously } \\
\text { considering quitting } \\
\text { within the next } 6 \text { months }\end{array}$ & $\begin{array}{l}\text { Participants who were } \\
\text { smoking but who were } \\
\text { seriously considering } \\
\text { quitting within the next } \\
30 \text { days and had made at } \\
\text { least one quit attempt } \\
\text { during the past year }\end{array}$ & Not reported & Not reported \\
\hline $\begin{array}{l}\text { Kremers et } \\
\text { al. } \\
(2001) \\
\text { Smoking } \\
\text { Acquisition }\end{array}$ & $\begin{array}{l}\text { 3-Precontem- } \\
\text { plation stages }\end{array}$ & SS & $\begin{array}{l}3 \text { Precontem-plation } \\
\text { Stages }\end{array}$ & $\begin{array}{l}\text { Never smoked but there } \\
\text { is an interest in acquiring } \\
\text { behavior in next six } \\
\text { months }\end{array}$ & $\begin{array}{l}\text { Never smoked but there } \\
\text { is an interest in acquiring } \\
\text { behavior in next month }\end{array}$ & $\begin{array}{l}\text { Smoked cigarettes } \\
\text { regularly, less than } 6 \\
\text { months }\end{array}$ & Not reported \\
\hline \begin{tabular}{|l|} 
Lauby et al. \\
$(1998)$ \\
Contraceptio \\
$n-$ General \\
\end{tabular} & Not reported & SS & $\begin{array}{l}\text { No intention of using } \\
\text { birth control or condoms } \\
\text { in next } 6 \text { months }\end{array}$ & $\begin{array}{l}\text { Intends to consistently } \\
\text { use birth control or } \\
\text { condoms in next } 6 \\
\text { months }\end{array}$ & $\begin{array}{l}\text { Intends to start using } \\
\text { birth control or condoms } \\
\text { consistently in the next } \\
\text { month }\end{array}$ & $\begin{array}{l}\text { Used birth control or } \\
\text { condoms consistently for } \\
\text { less than } 6 \text { months }\end{array}$ & $\begin{array}{l}\text { Used birth control or } \\
\text { condoms consistently for } \\
\text { the last } 6 \text { months }\end{array}$ \\
\hline
\end{tabular}


Table B.1 (continued)

\begin{tabular}{|c|c|c|c|c|c|c|c|}
\hline $\begin{array}{c}\text { Source } \\
\text { (Year) } \\
\text { Target } \\
\text { Behavior }\end{array}$ & $\begin{array}{l}\text { Type of } \\
\text { Algorithm }\end{array}$ & \begin{tabular}{|} 
TTM \\
or \\
Study \\
Specific \\
(SS)
\end{tabular} & Precontemplation & Contemplation & Preparation & Action & Maintenance \\
\hline $\begin{array}{l}\text { Lauby et al. } \\
(1998) \\
\text { Condom Use } \\
\text { - Main }\end{array}$ & Not reported & SS & $\begin{array}{l}\text { No intentions of } \\
\text { consistently using birth } \\
\text { control or condoms in the } \\
\text { next } 6 \text { months }\end{array}$ & $\begin{array}{l}\text { Intended to consistently } \\
\text { use birth control or } \\
\text { condoms in the next } 6 \\
\text { months, but had not yet } \\
\text { made a commitment to do } \\
\text { so }\end{array}$ & $\begin{array}{l}\text { Had intentions to start } \\
\text { using birth control or } \\
\text { condoms consistently in } \\
\text { the next month and had } \\
\text { used them inconsistently } \\
\text { in the past } 6 \text { months }\end{array}$ & $\begin{array}{l}\text { Had used birth control or } \\
\text { condoms consistently for } \\
\text { less than } 6 \text { months }\end{array}$ & $\begin{array}{l}\text { Had used birth control or } \\
\text { condoms consistently for } \\
\text { months or more }\end{array}$ \\
\hline $\begin{array}{l}\text { Lauby et al. } \\
(1998) \\
\text { Condom Use } \\
- \text { Other }\end{array}$ & Not reported & SS & $\begin{array}{l}\text { No intentions of } \\
\text { consistently using birth } \\
\text { control or condoms in the } \\
\text { next } 6 \text { months }\end{array}$ & $\begin{array}{l}\text { Intended to consistently } \\
\text { use birth control or } \\
\text { condoms in the next } 6 \\
\text { months, but had not yet } \\
\text { made a commitment to do } \\
\text { so }\end{array}$ & $\begin{array}{l}\text { Had intentions to start } \\
\text { using birth control or } \\
\text { condoms consistently in } \\
\text { the next month and had } \\
\text { used them inconsistently } \\
\text { in the past } 6 \text { months }\end{array}$ & $\begin{array}{l}\text { Had used birth control or } \\
\text { condoms consistently for } \\
\text { less than } 6 \text { months }\end{array}$ & $\begin{array}{l}\text { Had used birth control or } \\
\text { condoms consistently for } \\
\text { months or more }\end{array}$ \\
\hline \begin{tabular}{|l|} 
Lerner \\
$(1990)$ \\
Delinquent \\
Behavior
\end{tabular} & $\begin{array}{l}\text { 4-item (4 } \\
\text { stages); } \\
\text { single-choice } \\
\text { format }\end{array}$ & SS & $\begin{array}{l}\text { I don't need to change to } \\
\text { stay out of trouble }\end{array}$ & $\begin{array}{l}\text { I think I need to change } \\
\text { to stay out of trouble, but } \\
\text { I haven't done anything } \\
\text { yet }\end{array}$ & Not used & $\begin{array}{l}\text { I know I need to change } \\
\text { to stay out of trouble, and } \\
\text { I am doing something } \\
\text { about it right now }\end{array}$ & $\begin{array}{l}\text { I have already made the } \\
\text { changes I need to stay out } \\
\text { of trouble }\end{array}$ \\
\hline $\begin{array}{l}\text { Levesque et } \\
\text { al. } \\
(2000) \\
\\
\text { Administra- } \\
\text { tive Change }\end{array}$ & Not reported & SS & $\begin{array}{l}\text { Not intending to get } \\
\text { involved in the next } 6 \\
\text { months }\end{array}$ & $\begin{array}{l}\text { Intending to get involved } \\
\text { in the next } 6 \text { months }\end{array}$ & $\begin{array}{l}\text { Intending to get involved } \\
\text { in the next } 30 \text { days }\end{array}$ & $\begin{array}{l}\text { Involved, but for less } \\
\text { than } 6 \text { months }\end{array}$ & $\begin{array}{l}\text { Involved for more than } 6 \\
\text { months }\end{array}$ \\
\hline \begin{tabular}{|l} 
Levesque \\
(I998) \\
Domestic \\
Violence
\end{tabular} & $\begin{array}{l}\text { 3-component } \\
\text { staging } \\
\text { algorithm } \\
\text { (intentional, } \\
\text { cogntive, } \\
\text { behavioral) } \\
\end{array}$ & SS & $\begin{array}{l}\text { Considered relationship } \\
\text { fine the way it is or only } \\
\text { planned to change in the } \\
\text { distant future; and/or not } \\
\text { considering to put an end } \\
\text { to their violent behavior }\end{array}$ & $\begin{array}{l}\text { Seriously considering } \\
\text { ending their violent } \\
\text { behavior in next } 6 \text { months }\end{array}$ & $\begin{array}{l}\text { Seriously considering } \\
\text { ending their violent } \\
\text { behavior in next } 30 \text { days }\end{array}$ & $\begin{array}{l}\text { Currently doing } \\
\text { something to end violent } \\
\text { behavior }\end{array}$ & Not reported \\
\hline
\end{tabular}


Table B.1 (continued)

\begin{tabular}{|c|c|c|c|c|c|c|c|}
\hline $\begin{array}{c}\text { Source } \\
\text { (Year) } \\
\text { Target } \\
\text { Behavior }\end{array}$ & $\begin{array}{l}\text { Type of } \\
\text { Algorithm }\end{array}$ & $\begin{array}{l}\text { TTM } \\
\text { or } \\
\text { Study } \\
\text { Specific } \\
\text { (SS) }\end{array}$ & Precontemplation & Contemplation & Preparation & Action & Maintenance \\
\hline $\begin{array}{l}\text { Levesque et } \\
\text { al. } \\
\text { (1999) } \\
\text { Collabora- } \\
\text { tive Delivery } \\
\text { Service }\end{array}$ & $\begin{array}{l}\text { Single } \\
\text { question } 5 \\
\text { choice } \\
\text { response } \\
\text { format }\end{array}$ & SS & $\begin{array}{l}\text { No intention of getting } \\
\text { involved in Collaborative } \\
\text { Service Delivery in the } \\
\text { next } 6 \text { months }\end{array}$ & $\begin{array}{l}\text { Intended to get involved } \\
\text { in Collaborative Service } \\
\text { Delivery in the next } 6 \\
\text { months }\end{array}$ & $\begin{array}{l}\text { Intended to get involved } \\
\text { in Collaborative Service } \\
\text { Delivery in the next } 30 \\
\text { days }\end{array}$ & $\begin{array}{l}\text { Been involved in } \\
\text { Collaborative Service } \\
\text { Delivery, but for less than } \\
6 \text { months }\end{array}$ & $\begin{array}{l}\text { Been involved in } \\
\text { Collaborative Service } \\
\text { Delivery } 6 \text { months or } \\
\text { longer }\end{array}$ \\
\hline $\begin{array}{l}\text { Levesque et } \\
\text { al. } \\
\text { (I999) } \\
\text { Depression } \\
\text { Prevention }\end{array}$ & $\begin{array}{l}\text { Single } \\
\text { question } 5 \\
\text { choice } \\
\text { response } \\
\text { format }\end{array}$ & SS & $\begin{array}{l}\text { Does not consistently use } \\
\text { strategies to prevent } \\
\text { depression, and does not } \\
\text { intend to within the next } \\
6 \text { months }\end{array}$ & $\begin{array}{l}\text { Does not consistently use } \\
\text { strategies to prevent } \\
\text { depression, but intends to } \\
\text { within the next } 6 \text { months }\end{array}$ & $\begin{array}{l}\text { Does not consistently use } \\
\text { strategies to prevent } \\
\text { depression, but intends to } \\
\text { within the next } 30 \text { days }\end{array}$ & $\begin{array}{l}\text { Does consistently use } \\
\text { strategies to prevent } \\
\text { depression, but for less } \\
\text { than } 6 \text { months }\end{array}$ & $\begin{array}{l}\text { Does consistently use } \\
\text { strategies to prevent } \\
\text { depression, and has for } \\
\text { more than } 6 \text { months }\end{array}$ \\
\hline $\begin{array}{l}\text { Lowry } \\
(2000) \\
\text { Smokeless } \\
\text { Tobacco Use }\end{array}$ & Missing & Missing & Missing & Missing & Missing & Missing & Missing \\
\hline $\begin{array}{l}\text { Maier } \\
(2002) \\
\text { Exercise }\end{array}$ & $\begin{array}{l}\text { Single } \\
\text { question } 5 \\
\text { choice } \\
\text { response } \\
\text { format } \\
\end{array}$ & TTM & $\begin{array}{l}\text { No, and I am not thinking } \\
\text { about starting within the } \\
\text { next } 6 \text { months }\end{array}$ & $\begin{array}{l}\text { No, but I am planning to } \\
\text { start to always use them } \\
\text { within the next } 6 \text { months }\end{array}$ & $\begin{array}{l}\text { No, but I am planning to } \\
\text { start within the next } 30 \\
\text { days }\end{array}$ & $\begin{array}{l}\text { Yes, and I have been } \\
\text { doing so for } 6 \text { months or } \\
\text { less }\end{array}$ & $\begin{array}{l}\text { Yes, and I have been doing } \\
\text { so for } 6 \text { months or longer }\end{array}$ \\
\hline $\begin{array}{l}\text { Ma et al. } \\
(2002) \\
\text { Fruit Intake }\end{array}$ & $\begin{array}{l}\text { 3-item } \\
\text { algorithm }\end{array}$ & SS & $\begin{array}{l}\text { No intention to eat more } \\
\text { in the next } 6 \text { months }\end{array}$ & $\begin{array}{l}\text { Intending to increase } \\
\text { intake in the next } 6 \\
\text { months }\end{array}$ & $\begin{array}{l}\text { Intending to increase } \\
\text { intake in the next } 30 \text { days }\end{array}$ & $\begin{array}{l}\text { Has consumed } 2 \text { or more } \\
\text { servings per day, for less } \\
\text { than } 6 \text { months }\end{array}$ & $\begin{array}{l}\text { Has consumed } 2 \text { or more } \\
\text { servings per day for more } \\
\text { than } 6 \text { months }\end{array}$ \\
\hline $\begin{array}{l}\text { Ma et al. } \\
(2002) \\
\text { Vegetable } \\
\text { Intake }\end{array}$ & $\begin{array}{l}\text { 3-item } \\
\text { algorithm }\end{array}$ & SS & $\begin{array}{l}\text { No intention to eat more } \\
\text { in the next } 6 \text { months }\end{array}$ & $\begin{array}{l}\text { Intending to increase } \\
\text { intake in the next } 6 \\
\text { months }\end{array}$ & $\begin{array}{l}\text { Intending to increase } \\
\text { intake in the next } 30 \text { days }\end{array}$ & $\begin{array}{l}\text { Has consumed } 2 \text { or more } \\
\text { servings per day, for less } \\
\text { than } 6 \text { months }\end{array}$ & $\begin{array}{l}\text { Has consumed } 2 \text { or more } \\
\text { servings per day for more } \\
\text { than } 6 \text { months }\end{array}$ \\
\hline
\end{tabular}


Table B.1 (continued)

\begin{tabular}{|c|c|c|c|c|c|c|c|}
\hline $\begin{array}{c}\text { Source } \\
\text { (Year) } \\
\text { Target } \\
\text { Behavior }\end{array}$ & $\begin{array}{c}\text { Type of } \\
\text { Algorithm }\end{array}$ & \begin{tabular}{|} 
TTM \\
or \\
Study \\
Specific \\
(SS)
\end{tabular} & Precontemplation & Contemplation & Preparation & Action & Maintenance \\
\hline $\begin{array}{l}\text { Maleszka } \\
(2002) \\
\text { Smoking } \\
\text { Cessation }\end{array}$ & $\begin{array}{l}\text { Single } \\
\text { question } 5 \\
\text { choice } \\
\text { response } \\
\text { format } \\
\end{array}$ & TTM & $\begin{array}{l}\text { No, and I am not thinking } \\
\text { about starting within the } \\
\text { next } 6 \text { months }\end{array}$ & $\begin{array}{l}\text { No, but I am planning to } \\
\text { start to always use them } \\
\text { within the next } 6 \text { months }\end{array}$ & $\begin{array}{l}\text { No, but I am planning to } \\
\text { start within the next } 30 \\
\text { days }\end{array}$ & $\begin{array}{l}\text { Yes, and I have been } \\
\text { doing so for } 6 \text { months or } \\
\text { less }\end{array}$ & $\begin{array}{l}\text { Yes, and I have been doing } \\
\text { so for } 6 \text { months or longer }\end{array}$ \\
\hline $\begin{array}{l}\text { Manne et al. } \\
(2002) \\
\text { Colorectal } \\
\text { Cancer } \\
\text { Screening }\end{array}$ & Not reported & SS & $\begin{array}{l}\text { Has not had screening } \\
\text { and has no plan for any in } \\
\text { the next year }\end{array}$ & $\begin{array}{l}\text { (Relapse/Risk for } \\
\text { Relapse) Has had one or } \\
\text { more screening tests in } \\
\text { the past year but is now } \\
\text { off schedule and does not } \\
\text { plan to have a screening } \\
\text { test in the next year or is } \\
\text { presently on schedule but } \\
\text { does not plan to have a } \\
\text { test that would place } \\
\text { them on schedule in the } \\
\text { next year }\end{array}$ & $\begin{array}{l}\text { (Contemplation) Has not } \\
\text { had a prior screening test } \\
\text { but intends to have one in } \\
\text { the next year or is off } \\
\text { schedule after having } \\
\text { prior screening but } \\
\text { intends to have one in the } \\
\text { next year }\end{array}$ & $\begin{array}{l}\text { Has had a colonoscopy } \\
\text { on schedule and plans on } \\
\text { having another in a time- } \\
\text { frame that would keep the } \\
\text { person on schedule; or } \\
\text { has had an FOBT and a } \\
\text { sigmoidoscopy on } \\
\text { schedule and plans to } \\
\text { have both tests in the } \\
\text { time period that would } \\
\text { keep the person on } \\
\text { schedule }\end{array}$ & $\begin{array}{l}\text { Has had two or more } \\
\text { colonoscopies on a regular } \\
\text { schedule and plans to have } \\
\text { another on schedule; or ha } \\
\text { had two or more FOBTs } \\
\text { and two or more sigmoid- } \\
\text { oscopies on a regular } \\
\text { schedule and plans to have } \\
\text { both tests in the time } \\
\text { period that would keep the } \\
\text { person on schedule }\end{array}$ \\
\hline $\begin{array}{l}\text { Marcus et al. } \\
\text { (1992) } \\
\text { Exercise }\end{array}$ & $\begin{array}{l}\text { Contempla- } \\
\text { tion Ladder } \\
(11 \mathrm{pt})\end{array}$ & SS & $\begin{array}{l}\text { I currently do not } \\
\text { exercise and do not } \\
\text { intend to start exercising } \\
\text { in the next } 6 \text { months }\end{array}$ & $\begin{array}{l}\text { I currently do not } \\
\text { exercise, but am thinking } \\
\text { of starting to exercise in } \\
\text { the next } 6 \text { months }\end{array}$ & $\begin{array}{l}\text { I currently exercise some, } \\
\text { but not regularly }\end{array}$ & $\begin{array}{l}\text { I currently exercise } \\
\text { regularly, but have only } \\
\text { begun doing so within the } \\
\text { last } 6 \text { months }\end{array}$ & $\begin{array}{l}\text { I currently exercise } \\
\text { regularly, but have only } \\
\text { begun doing so within the } \\
\text { last } 6 \text { months }\end{array}$ \\
\hline $\begin{array}{l}\text { Marcus et al. } \\
\text { (1994) } \\
\text { Exercise }\end{array}$ & $\begin{array}{l}\text { Contempla- } \\
\text { tion Ladder } \\
(11 \mathrm{pt})\end{array}$ & SS & $\begin{array}{l}\text { I currently do not } \\
\text { exercise and do not } \\
\text { intend to start exercising } \\
\text { in the next } 6 \text { months }\end{array}$ & $\begin{array}{l}\text { I currently do not } \\
\text { exercise, but am thinking } \\
\text { of starting to exercise in } \\
\text { the next } 6 \text { months }\end{array}$ & $\begin{array}{l}\text { I currently exercise some, } \\
\text { but not regularly }\end{array}$ & $\begin{array}{l}\text { I currently exercise } \\
\text { regularly, but have only } \\
\text { begun doing so within the } \\
\text { last } 6 \text { months }\end{array}$ & $\begin{array}{l}\text { I currently exercise } \\
\text { regularly and have done so } \\
\text { for longer than } 6 \text { months }\end{array}$ \\
\hline $\begin{array}{l}\text { Mauriello } \\
(2001) \\
\text { Stress } \\
\text { Manage- } \\
\text { ment }\end{array}$ & $\begin{array}{l}\text { 4-item } \\
\text { staging }\end{array}$ & SS & $\begin{array}{l}\text { Not intend to effectively } \\
\text { practice stress } \\
\text { management in the next } 6 \\
\text { months }\end{array}$ & $\begin{array}{l}\text { Intend to effectively } \\
\text { practice stress } \\
\text { management in the next } 6 \\
\text { months }\end{array}$ & $\begin{array}{l}\text { Intend to effectively } \\
\text { practice stress } \\
\text { management in the next } \\
30 \text { days }\end{array}$ & $\begin{array}{l}\text { Effectively practicing } \\
\text { stress management for } \\
\text { less than } 6 \text { months }\end{array}$ & $\begin{array}{l}\text { Effectively practicing } \\
\text { stress management for } \\
\text { more than } 6 \text { months }\end{array}$ \\
\hline
\end{tabular}


Table B.1 (continued)

\begin{tabular}{|c|c|c|c|c|c|c|c|}
\hline $\begin{array}{l}\text { Source } \\
\text { (Year) } \\
\text { Target } \\
\text { Behavior }\end{array}$ & $\begin{array}{c}\text { Type of } \\
\text { Algorithm }\end{array}$ & $\begin{array}{l}\text { TTM } \\
\text { or } \\
\text { Study } \\
\text { Specific } \\
\text { (SS) }\end{array}$ & Precontemplation & Contemplation & Preparation & Action & Maintenance \\
\hline $\begin{array}{l}\text { Mettler et al. } \\
(2000) \\
\text { Exercise }\end{array}$ & $\begin{array}{l}\text { Contempla- } \\
\text { tion Ladder } \\
(11 \mathrm{pt})\end{array}$ & SS & $\begin{array}{l}\text { I currently do not } \\
\text { exercise and do not } \\
\text { intend to start exercising } \\
\text { in the next } 6 \text { months }\end{array}$ & $\begin{array}{l}\text { I currently do not } \\
\text { exercise, but am thinking } \\
\text { of starting to exercise in } \\
\text { the next } 6 \text { months }\end{array}$ & $\begin{array}{l}\text { I currently exercise some, } \\
\text { but not regularly }\end{array}$ & $\begin{array}{l}\text { I currently exercise } \\
\text { regularly, but have only } \\
\text { begun doing so within the } \\
\text { last } 6 \text { months }\end{array}$ & $\begin{array}{l}\text { I currently exercise } \\
\text { regularly and have done so } \\
\text { for longer than } 6 \text { months }\end{array}$ \\
\hline $\begin{array}{l}\text { Migneault et } \\
\text { al. } \\
\text { (1997) } \\
\text { Binge } \\
\text { Drinking } \\
\text { Acquisition }\end{array}$ & $\begin{array}{l}\text { 5-question } \\
\text { (Yes/No) }\end{array}$ & TTM & Not intending to change & $\begin{array}{l}\text { Considering changing } \\
\text { their behavior in the next } \\
\text { six months }\end{array}$ & $\begin{array}{l}\text { Getting ready to change } \\
\text { in the next } 30 \text { days }\end{array}$ & $\begin{array}{l}\text { Having recently changed } \\
\text { the behavior }\end{array}$ & $\begin{array}{l}\text { Sustaining the change over } \\
\text { time }\end{array}$ \\
\hline $\begin{array}{l}\text { Migneault et } \\
\text { al. } \\
\text { (1997) } \\
\text { Binge } \\
\text { Drinking } \\
\text { Cessation }\end{array}$ & $\begin{array}{l}5 \text {-question } \\
\text { (Yes/No) }\end{array}$ & TTM & Not intending to change & $\begin{array}{l}\text { Considering changing } \\
\text { their behavior in the next } \\
\text { six months }\end{array}$ & $\begin{array}{l}\text { Getting ready to change } \\
\text { in the next } 30 \text { days }\end{array}$ & $\begin{array}{l}\text { Having recently changed } \\
\text { the behavior }\end{array}$ & $\begin{array}{l}\text { Sustaining the change over } \\
\text { time }\end{array}$ \\
\hline $\begin{array}{l}\text { Moll } \\
(2001) \\
\text { Physical } \\
\text { activity }\end{array}$ & $\begin{array}{l}\text { Single } \\
\text { question } 5 \\
\text { choice } \\
\text { response } \\
\text { format } \\
\end{array}$ & TTM & $\begin{array}{l}\text { No, and I am not thinking } \\
\text { about starting within the } \\
\text { next } 6 \text { months }\end{array}$ & $\begin{array}{l}\text { No, but I am planning to } \\
\text { start to always use them } \\
\text { within the next } 6 \text { months }\end{array}$ & $\begin{array}{l}\text { No, but I am planning to } \\
\text { start within the next } 30 \\
\text { days }\end{array}$ & $\begin{array}{l}\text { Yes, and I have been } \\
\text { doing so for } 6 \text { months or } \\
\text { less }\end{array}$ & $\begin{array}{l}\text { Yes, and I have been doing } \\
\text { so for } 6 \text { months or longer }\end{array}$ \\
\hline $\begin{array}{l}\text { Morrison- } \\
\text { Beedy et al. } \\
(2001) \\
\text { Condom } \\
\text { Use-Main }\end{array}$ & $\begin{array}{l}\text { 4-item } \\
\text { staging }\end{array}$ & SS & $\begin{array}{l}\text { Never use condoms when } \\
\text { having sex, and do not } \\
\text { plan on starting to use } \\
\text { condoms every time } \\
\text { when having sex in the } \\
\text { next } 6 \text { months }\end{array}$ & $\begin{array}{l}\text { Never or almost never } \\
\text { use condoms when } \\
\text { having sex, but plan to } \\
\text { start using condoms } \\
\text { every time when having } \\
\text { sex in the next } 6 \text { months }\end{array}$ & $\begin{array}{l}\text { May use condoms } \\
\text { sometimes when having } \\
\text { sex, and plans on using } \\
\text { condoms within the next } \\
30 \text { days }\end{array}$ & $\begin{array}{l}\text { Uses condoms every time } \\
\text { when having sex, and has } \\
\text { been doing so for less } \\
\text { than } 6 \text { months }\end{array}$ & $\begin{array}{l}\text { Uses condoms every time } \\
\text { when having sex, and has } \\
\text { been doing so for more } \\
\text { than } 6 \text { months }\end{array}$ \\
\hline
\end{tabular}


Table B.1 (continued)

\begin{tabular}{|c|c|c|c|c|c|c|c|}
\hline $\begin{array}{c}\text { Source } \\
\text { (Year) } \\
\text { Target } \\
\text { Behavior }\end{array}$ & $\begin{array}{l}\text { Type of } \\
\text { Algorithm }\end{array}$ & \begin{tabular}{|} 
TTM \\
or \\
Study \\
Specific \\
(SS)
\end{tabular} & Precontemplation & Contemplation & Preparation & Action & Maintenance \\
\hline $\begin{array}{l}\text { Morrison- } \\
\text { Beedy et al. } \\
(2001) \\
\text { Condom } \\
\text { Use-Other }\end{array}$ & $\begin{array}{l}\text { 4-item } \\
\text { staging }\end{array}$ & SS & $\begin{array}{l}\text { Never use condoms when } \\
\text { having sex, and do not } \\
\text { plan on starting to use } \\
\text { condoms every time } \\
\text { when having sex in the } \\
\text { next } 6 \text { months }\end{array}$ & $\begin{array}{l}\text { Never or almost never } \\
\text { use condoms when } \\
\text { having sex, but plan to } \\
\text { start using condoms } \\
\text { every time when having } \\
\text { sex in the next } 6 \text { months }\end{array}$ & $\begin{array}{l}\text { May use condoms } \\
\text { sometimes when having } \\
\text { sex, and plans on using } \\
\text { condoms within the next } \\
30 \text { days }\end{array}$ & $\begin{array}{l}\text { Uses condoms every time } \\
\text { when having sex, and has } \\
\text { been doing so for less } \\
\text { than } 6 \text { months }\end{array}$ & $\begin{array}{l}\text { Uses condoms every time } \\
\text { when having sex, and has } \\
\text { been doing so for more } \\
\text { than } 6 \text { months }\end{array}$ \\
\hline $\begin{array}{l}\text { Nigg et al. } \\
(1998) \\
\text { Exercise }\end{array}$ & $\begin{array}{l}5 \text {-item } \\
\text { algorithm } \\
(\text { Yes/No) }\end{array}$ & SS & $\begin{array}{l}\text { Does not exercise } \\
\text { regularly and has no } \\
\text { intention of beginning in } \\
\text { the next six months }\end{array}$ & $\begin{array}{l}\text { Does not exercise } \\
\text { regularly but intends to } \\
\text { start within the next six } \\
\text { months, but not } 30 \text { days }\end{array}$ & $\begin{array}{l}\text { Does not exercise } \\
\text { regularly but intends to } \\
\text { start in next } 30 \text { days }\end{array}$ & $\begin{array}{l}\text { Exercises regularly but } \\
\text { not for the last six months }\end{array}$ & $\begin{array}{l}\text { Exercised regularly for } \\
\text { more than six months }\end{array}$ \\
\hline $\begin{array}{l}\text { Nigg et al. } \\
(1998) \\
\text { Exercise }\end{array}$ & $\begin{array}{l}5 \text {-item } \\
\text { algorithm } \\
(\text { Yes/No) }\end{array}$ & SS & $\begin{array}{l}\text { Does not exercise } \\
\text { regularly and has no } \\
\text { intention of beginning in } \\
\text { the next six months }\end{array}$ & $\begin{array}{l}\text { Does not exercise } \\
\text { regularly but intends to } \\
\text { start within the next six } \\
\text { months, but not } 30 \text { days }\end{array}$ & $\begin{array}{l}\text { Does not exercise } \\
\text { regularly but intends to } \\
\text { start in next } 30 \text { days }\end{array}$ & $\begin{array}{l}\text { Exercises regularly but } \\
\text { not for the last six months }\end{array}$ & $\begin{array}{l}\text { Exercised regularly for } \\
\text { more than six months }\end{array}$ \\
\hline \begin{tabular}{|l|} 
Nigg \& \\
Courneya \\
$(1998)$ \\
Exercise \\
\end{tabular} & $\begin{array}{l}\text { 5-item } \\
\text { algorithm }\end{array}$ & SS & $\begin{array}{l}\text { I currently do not engage } \\
\text { in exercise in my leisure } \\
\text { time and I am not } \\
\text { intending to start }\end{array}$ & $\begin{array}{l}\text { I currently do not engage } \\
\text { in exercise in my leisure } \\
\text { time but I intend to start } \\
\text { within the next } 6 \text { months }\end{array}$ & $\begin{array}{l}1 \text { intend to exercise } \\
\text { regularly within the next } \\
30 \text { days }\end{array}$ & $\begin{array}{l}\text { I currently do engage in } \\
\text { regular exercise in my } \\
\text { leisure time, but have } \\
\text { only begun to do so } \\
\text { within the last } 6 \text { months }\end{array}$ & $\begin{array}{l}1 \text { currently do engage in } \\
\text { regular exercise in my } \\
\text { leisure time and } 1 \text { have } \\
\text { done so for longer than } 6 \\
\text { months }\end{array}$ \\
\hline \begin{tabular}{|l|} 
Noar et al. \\
$(1998)$ \\
Condom Use \\
- Vaginal Sex
\end{tabular} & $\begin{array}{l}\text { 4-question } \\
\text { algorithm }\end{array}$ & SS & $\begin{array}{l}\text { No intention to use } \\
\text { condoms consistently in } \\
\text { the next } 6 \text { months }\end{array}$ & $\begin{array}{l}\text { Intentions to use } \\
\text { condoms consistently in } \\
\text { the next } 6 \text { months }\end{array}$ & $\begin{array}{l}\text { Intentions to use } \\
\text { condoms consistently in } \\
\text { the next } 30 \text { days, and } \\
\text { were currently using } \\
\text { condoms almost every } \\
\text { time they had sex }\end{array}$ & $\begin{array}{l}\text { Consistently used } \\
\text { condoms for less than } 6 \\
\text { months }\end{array}$ & $\begin{array}{l}\text { Consistently used condom } \\
\text { for } 6 \text { months or more }\end{array}$ \\
\hline $\begin{array}{l}\text { Norman et al. } \\
(2002) \\
\text { Sedentary } \\
\text { Behavior } \\
\end{array}$ & Not reported & $\begin{array}{l}\text { Not } \\
\text { reported }\end{array}$ & Not reported & Not reported & Not reported & Not reported & Not reported \\
\hline
\end{tabular}


Table B.1 (continued)

\begin{tabular}{|c|c|c|c|c|c|c|c|}
\hline $\begin{array}{c}\text { Source } \\
\text { (Year) } \\
\text { Target } \\
\text { Behavior }\end{array}$ & $\begin{array}{c}\text { Type of } \\
\text { Algorithm }\end{array}$ & \begin{tabular}{|c|} 
TTM \\
or \\
Study \\
Specific \\
(SS)
\end{tabular} & Precontemplation & Contemplation & Preparation & Action & Maintenance \\
\hline $\begin{array}{l}\text { Norman et al. } \\
(2002) \\
\text { Sedentary } \\
\text { Behavior } \\
\end{array}$ & Not reported & \begin{tabular}{|l|} 
Not \\
reported
\end{tabular} & Not reported & Not reported & Not reported & Not reported & Not reported \\
\hline \begin{tabular}{|l|}
$O^{\prime}$ Connell \& \\
Velicer \\
$(1988)$ \\
Weight Loss \\
\end{tabular} & \begin{tabular}{|l|} 
6-item \\
algorithm
\end{tabular} & SS & $\begin{array}{l}\text { Minimum of } 5 \mathrm{lb} \text {. } \\
\text { overweight and no } \\
\text { thought of or action to } \\
\text { lose weight }\end{array}$ & \begin{tabular}{|l} 
Acknowledgement of \\
minimum $10 \mathrm{lb}$ weight \\
loss goal, but without \\
consistently or \\
successfully taking action
\end{tabular} & Not used & $\begin{array}{l}\text { Recent success at losing } \\
10 \mathrm{lb} \text { or more and } \\
\text { continued action to lose } \\
\text { additional weight }\end{array}$ & $\begin{array}{l}\text { Minimum } 10 \mathrm{lb} \text { loss in the } \\
\text { past and current action } \\
\text { being successfully taken to } \\
\text { combat regaining weight }\end{array}$ \\
\hline $\begin{array}{l}\text { Otake \& } \\
\text { Shimai } \\
(2001) \\
\\
\text { Smoking } \\
\text { Acquisition }\end{array}$ & $\begin{array}{l}\text { 4-item } \\
\text { algorithm } \\
\text { (Yes/No) }\end{array}$ & SS & $\begin{array}{l}\text { No interest in acquiring } \\
\text { smoking behavior or } \\
\text { intention of doing so in } \\
\text { the future }\end{array}$ & $\begin{array}{l}\text { Never smoked but there } \\
\text { is an interest in acquiring } \\
\text { behavior }\end{array}$ & $\begin{array}{l}\text { Smoked in last year with } \\
\text { a current interest in } \\
\text { smoking }\end{array}$ & $\begin{array}{l}\text { Smoking within the past } \\
\text { month and exhibiting } \\
\text { different dimensions of } \\
\text { cigarette smoking }\end{array}$ & Not reported \\
\hline \begin{tabular}{|l|} 
Otake \& \\
Shimai \\
$(2001)$ \\
Smoking \\
Acquisition \\
\end{tabular} & $\begin{array}{l}\text {-item } \\
\text { algorithm } \\
\text { (Yes/No) }\end{array}$ & SS & $\begin{array}{l}\text { No interest in acquiring } \\
\text { smoking behavior or } \\
\text { intention of doing so in } \\
\text { the future }\end{array}$ & $\begin{array}{l}\text { Never smoked but there } \\
\text { is an interest in acquiring } \\
\text { behavior }\end{array}$ & $\begin{array}{l}\text { Smoked in last year with } \\
\text { a current interest in } \\
\text { smoking }\end{array}$ & $\begin{array}{l}\text { Smoking within the past } \\
\text { month and exhibiting } \\
\text { different dimensions of } \\
\text { cigarette smoking }\end{array}$ & Not reported \\
\hline $\begin{array}{l}\text { Pallonen et } \\
\text { al. } \\
(1998) \\
\text { Smoking } \\
\text { Acquisition }\end{array}$ & \begin{tabular}{|l} 
Current \\
smoking \\
status then 2- \\
item \\
algorithm \\
with Yes/No \\
responses
\end{tabular} & SS & $\begin{array}{l}\text { No interest in acquiring } \\
\text { smoking behavior or } \\
\text { intention of doing so in } \\
\text { the next six months }\end{array}$ & $\begin{array}{l}\text { Never smoked but there } \\
\text { is an interest in acquiring } \\
\text { behavior in next six } \\
\text { months }\end{array}$ & $\begin{array}{l}\text { Never smoked but there } \\
\text { is an interest in acquiring } \\
\text { behavior in next month }\end{array}$ & $\begin{array}{l}\text { Smoked cigarettes } \\
\text { regularly, less than } 6 \\
\text { months }\end{array}$ & Not reported \\
\hline
\end{tabular}


Table B.1 (continued)

\begin{tabular}{|c|c|c|c|c|c|c|c|}
\hline $\begin{array}{l}\text { Source } \\
\text { (Year) } \\
\text { Target } \\
\text { Behavior }\end{array}$ & $\begin{array}{l}\text { Type of } \\
\text { Algorithm }\end{array}$ & $\begin{array}{c}\text { TTM } \\
\text { or } \\
\text { Study } \\
\text { Specific } \\
\text { (SS) }\end{array}$ & Precontemplation & Contemplation & Preparation & Action & Maintenance \\
\hline $\begin{array}{l}\text { Pallonen et } \\
\text { al. } \\
\text { (1998) } \\
\text { Smoking } \\
\text { Cessation }\end{array}$ & \begin{tabular}{|l|} 
Current \\
smoking \\
status then 5. \\
item \\
algorithm \\
with Yes/No \\
responses \\
\end{tabular} & SS & $\begin{array}{l}\text { Smokers who have } \\
\text { smoked for more than six } \\
\text { months with no } \\
\text { immediate plans to quit }\end{array}$ & $\begin{array}{l}\text { Smokers who have } \\
\text { smoked for more than six } \\
\text { months without history of } \\
\text { serious quit attempts and } \\
\text { are thinking about } \\
\text { quitting }\end{array}$ & $\begin{array}{l}\text { Smoked for more than six } \\
\text { months with at least one } \\
\text { serious quitting attempt } \\
\text { and is thinking about } \\
\text { quitting within the next } \\
30 \text { days }\end{array}$ & $\begin{array}{l}\text { Has quit smoking within } \\
\text { the last six months }\end{array}$ & $\begin{array}{l}\text { Has quit smoking for more } \\
\text { than six months }\end{array}$ \\
\hline $\begin{array}{l}\text { Park et al. } \\
(2003) \\
\text { Physicians } \\
\text { Counseling } \\
\text { Smokers }\end{array}$ & $\begin{array}{l}\text { 2-question } \\
\text { algorithm }\end{array}$ & SS & $\begin{array}{l}\text { Not yet active in } \\
\text { providing SCIs to more } \\
\text { than } 80 \% \text { of their patients } \\
\text { who smoked and were } \\
\text { not considering doing so } \\
\text { in the next } 6 \text { months }\end{array}$ & $\begin{array}{l}\text { Considering adopting } \\
\text { SCIs for more than } 80 \% \\
\text { of their patients who } \\
\text { smoked within the next } 6 \\
\text { months }\end{array}$ & $\begin{array}{l}\text { Planning to adopt SCIs } \\
\text { for more than } 80 \% \text { of } \\
\text { their patients in the next } \\
\text { month }\end{array}$ & $\begin{array}{l}\text { Had been providing SCIs, } \\
\text { for less than } 6 \text { months, to } \\
\text { more than } 80 \% \text { of their } \\
\text { patients who smoked }\end{array}$ & $\begin{array}{l}\text { Had been providing SCIs } \\
\text { to more than } 80 \% \text { of their } \\
\text { patients who smoked, and } \\
\text { had been doing so for mor } \\
\text { than } 6 \text { months }\end{array}$ \\
\hline $\begin{array}{l}\text { Perez } \\
(2001) \\
\text { Condom Use }\end{array}$ & \begin{tabular}{|l} 
Single \\
question 5 \\
choice \\
response \\
format \\
\end{tabular} & SS & $\begin{array}{l}\text { No intention of } \\
\text { consistently using } \\
\text { condoms in the next } 6 \\
\text { months }\end{array}$ & $\begin{array}{l}\text { Thinking of consistently } \\
\text { using condoms in the } \\
\text { next } 6 \text { months }\end{array}$ & $\begin{array}{l}\text { Planning to consistently } \\
\text { use condoms in the next } \\
30 \text { days }\end{array}$ & $\begin{array}{l}\text { Have been consistently } \\
\text { using condoms for less } \\
\text { than } 6 \text { months }\end{array}$ & $\begin{array}{l}\text { Have been consistently } \\
\text { using condoms for more } \\
\text { than } 6 \text { months }\end{array}$ \\
\hline $\begin{array}{l}\text { Plummer et } \\
\text { al. } \\
(2001) \\
\text { Smoking } \\
\text { Acquisition }\end{array}$ & $\begin{array}{l}\text { Past and } \\
\text { Current } \\
\text { smoking } \\
\text { status then } 3 \\
\text { item Yes/No } \\
\text { response } \\
\end{array}$ & SS & $\begin{array}{l}\text { No interest in acquiring } \\
\text { smoking behavior or } \\
\text { intention of doing so in } \\
\text { the next six months }\end{array}$ & $\begin{array}{l}\text { Never smoked but there } \\
\text { is an interest in acquiring } \\
\text { behavior in next six } \\
\text { months }\end{array}$ & $\begin{array}{l}\text { Never smoked but there } \\
\text { is an interest in acquiring } \\
\text { behavior in next month }\end{array}$ & Not reported & Not reported \\
\hline $\begin{array}{l}\text { Plummer et } \\
\text { al. } \\
(2001) \\
\text { Smoking } \\
\text { Cessation }\end{array}$ & $\begin{array}{l}\text { Past and } \\
\text { Current } \\
\text { smoking } \\
\text { status then } 3 \\
\text { item Yes/No } \\
\text { response } \\
\end{array}$ & SS & $\begin{array}{l}\text { Smokers who have } \\
\text { smoked for more than six } \\
\text { months with no } \\
\text { immediate plans to quit }\end{array}$ & $\begin{array}{l}\text { Smokers who have } \\
\text { smoked for more than six } \\
\text { months without history of } \\
\text { serious quit attempts and } \\
\text { are thinking about } \\
\text { quitting }\end{array}$ & $\begin{array}{l}\text { Smoked for more than six } \\
\text { months with at least one } \\
\text { serious quitting attempt } \\
\text { and is thinking about } \\
\text { quitting within the next } \\
30 \text { days }\end{array}$ & $\begin{array}{l}\text { Has quit smoking within } \\
\text { the last six months }\end{array}$ & $\begin{array}{l}\text { Has quit smoking for more } \\
\text { than six months }\end{array}$ \\
\hline
\end{tabular}


Table B.1 (continued)

\begin{tabular}{|c|c|c|c|c|c|c|c|}
\hline $\begin{array}{c}\text { Source } \\
\text { (Year) } \\
\text { Target } \\
\text { Behavior }\end{array}$ & $\begin{array}{l}\text { Type of } \\
\text { Algorithm }\end{array}$ & \begin{tabular}{|l} 
TTM \\
or \\
Study \\
Specific \\
(SS)
\end{tabular} & Precontemplation & Contemplation & Preparation & Action & Maintenance \\
\hline $\begin{array}{l}\text { Prochaska, JJ } \\
\text { et al. } \\
\text { (2003) } \\
\text { Smoking } \\
\text { cessation }\end{array}$ & \begin{tabular}{|l|}
-item \\
measure \\
(format \\
unclear; \\
DiClemente, \\
1991 ) \\
\end{tabular} & SS & $\begin{array}{l}\text { Not seriously considering } \\
\text { quitting within the next } 6 \\
\text { months }\end{array}$ & $\begin{array}{l}\text { Seriously considering } \\
\text { quitting within the next } 6 \\
\text { months, however they } \\
\text { were not considering } \\
\text { quitting within the next } \\
30 \text { days, had not made a } \\
\text { quit attempt of } 24 \text { hours } \\
\text { in past year, or both }\end{array}$ & $\begin{array}{l}\text { Seriously considering } \\
\text { quitting in the next } 6 \\
\text { months and were } \\
\text { planning to quit within } \\
\text { the next } 30 \text { days, in } \\
\text { addition they made a } 24 \\
\text { hour quit attempt in past } \\
\text { year }\end{array}$ & Not reported & Not reported \\
\hline $\begin{array}{l}\text { Prochaska et } \\
\text { al. } \\
(1994) \\
\text { Condom Use } \\
\text {-Anal Main } \\
\end{array}$ & \begin{tabular}{|l|} 
4- or 5- item \\
algorithm
\end{tabular} & TTM & $\begin{array}{l}\text { Reported the undesired } \\
\text { status and did not intend } \\
\text { to change in the next } 6 \\
\text { months }\end{array}$ & $\begin{array}{l}\text { Intended to change in the } \\
\text { next } 6 \text { months }\end{array}$ & Not used & $\begin{array}{l}\text { Had reached a particular } \\
\text { criterion within the past } 6 \\
\text { months }\end{array}$ & $\begin{array}{l}\text { Had reached criterion mor } \\
\text { than } 6 \text { months prior to the } \\
\text { study }\end{array}$ \\
\hline $\begin{array}{l}\text { Rakowski et } \\
\text { al. } \\
\text { (1992) } \\
\text { Mammo- } \\
\text { graphy }\end{array}$ & \begin{tabular}{|l|}
4 -item(6 \\
stages \\
including \\
Relapse Risk \\
and Relapse)
\end{tabular} & SS & $\begin{array}{l}\text { No prior mammogram } \\
\text { and no plan for one in the } \\
\text { coming year }\end{array}$ & $\begin{array}{l}\text { No prior mammogram } \\
\text { but planning for one in } \\
\text { the next year, or one with } \\
\text { no plans for the next year }\end{array}$ & Not used & $\begin{array}{l}\text { One prior mammogram } \\
\text { and planning for one in } \\
\text { the next year }\end{array}$ & $\begin{array}{l}\text { More than one prior } \\
\text { mammogram and planning } \\
\text { for one in the next year }\end{array}$ \\
\hline $\begin{array}{l}\text { Redding } \\
(1990) \\
\text { Safer Sex } \\
\text { Decision } \\
\text { Making }\end{array}$ & Not reported & TTM & $\begin{array}{l}\text { Not considering changing } \\
\text { or denying the need for it }\end{array}$ & $\begin{array}{l}\text { Seriously thinking about } \\
\text { changing }\end{array}$ & Not used & Action/ Maintenance & Action/ Maintenance \\
\hline $\begin{array}{l}\text { Redding } \\
(1993) \\
\text { Safer Sex } \\
\text { Decision } \\
\text { Making } \\
\end{array}$ & $\begin{array}{l}\text { 4-item } \\
\text { algorithm }\end{array}$ & SS & $\begin{array}{l}\text { Participants who } \\
\text { indicated that they had } \\
\text { not changed their sexual } \\
\text { behavior in response to } \\
\text { AIDS and were not } \\
\text { considering doing so }\end{array}$ & $\begin{array}{l}\text { Participants who } \\
\text { indicated that they were } \\
\text { considering changing } \\
\text { their sexual behavior }\end{array}$ & Not used & $\begin{array}{l}\text { Participants who } \\
\text { indicated that they had } \\
\text { changed their sexual } \\
\text { behavior in response to } \\
\text { AlDS with consistency, } \\
\text { but for less than } 6 \text { months }\end{array}$ & $\begin{array}{l}\text { Participants who indicated } \\
\text { that they had changed their } \\
\text { sexual behavior in } \\
\text { response to AIDS with } \\
\text { consistency, but for } 6 \\
\text { months or more }\end{array}$ \\
\hline
\end{tabular}


Table B.1 (continued)

\begin{tabular}{|c|c|c|c|c|c|c|c|}
\hline $\begin{array}{c}\text { Source } \\
\text { (Year) } \\
\text { Target } \\
\text { Behavior }\end{array}$ & $\begin{array}{c}\text { Type of } \\
\text { Algorithm }\end{array}$ & $\begin{array}{c}\text { TTM } \\
\text { or } \\
\text { Study } \\
\text { Specific } \\
\text { (SS) }\end{array}$ & Precontemplation & Contemplation & Preparation & Action & Maintenance \\
\hline $\begin{array}{l}\text { Reed } \\
(1995) \\
\text { Exercise }\end{array}$ & \begin{tabular}{|l|} 
Single \\
question 5 \\
choice \\
response \\
format \\
\end{tabular} & SS & $\begin{array}{l}\text { No intention to start } \\
\text { exercising in the next } 6 \\
\text { months }\end{array}$ & $\begin{array}{l}\text { Those not participating in } \\
\text { regular physical activity } \\
\text { but intending to do so in } \\
\text { the next } 6 \text { months }\end{array}$ & $\begin{array}{l}\text { Currently exercise, not } \\
\text { regularly }\end{array}$ & $\begin{array}{l}\text { Exercise regularly, for } \\
\text { less than } 6 \text { months }\end{array}$ & $\begin{array}{l}\text { Exercise regularly, for } \\
\text { more than } 6 \text { months }\end{array}$ \\
\hline $\begin{array}{l}\text { Riley et al. } \\
(2000) \\
\text { Stress } \\
\text { Manage- } \\
\text { ment }\end{array}$ & $\begin{array}{l}\text { Single } \\
\text { question } 5 \\
\text { choice } \\
\text { response } \\
\text { format }\end{array}$ & SS & $\begin{array}{l}\text { Have you attempted to } \\
\text { manage stress in your } \\
\text { daily life? No, and I don't } \\
\text { intend to for the next } 6 \\
\text { month }\end{array}$ & $\begin{array}{l}\text { Have you attempted to } \\
\text { manage stress in your } \\
\text { daily life? No, but I } \\
\text { intend to in the next } 6 \\
\text { month }\end{array}$ & $\begin{array}{l}\text { Have you attempted to } \\
\text { manage stress in your } \\
\text { daily life? No, but I am } \\
\text { planning to change in the } \\
\text { next } 30 \text { days, and have } \\
\text { perhaps made small steps } \\
\text { toward that change }\end{array}$ & $\begin{array}{l}\text { Have you attempted to } \\
\text { manage stress in your } \\
\text { daily life? Yes, but have } \\
\text { been doing so for a } \\
\text { period of less than } 6 \\
\text { months }\end{array}$ & $\begin{array}{l}\text { Have you attempted to } \\
\text { manage stress in your dail } \\
\text { life? Yes, and I am taking } \\
\text { steps to sustain the change } \\
\text { during a 6-month time } \\
\text { frame and the prevention } \\
\text { of relapse }\end{array}$ \\
\hline $\begin{array}{l}\text { Riley et al. } \\
(2003) \\
\text { Stress } \\
\text { Manage- } \\
\text { ment }\end{array}$ & $\begin{array}{l}\text { Single } \\
\text { question } 5 \\
\text { choice } \\
\text { response } \\
\text { format }\end{array}$ & SS & $\begin{array}{l}\text { Have you attempted to } \\
\text { manage stress in your } \\
\text { daily life? No, and I don't } \\
\text { intend to for the next } 6 \\
\text { month }\end{array}$ & $\begin{array}{l}\text { Have you attempted to } \\
\text { manage stress in your } \\
\text { daily life? No, but I } \\
\text { intend to in the next } 6 \\
\text { month }\end{array}$ & $\begin{array}{l}\text { Have you attempted to } \\
\text { manage stress in your } \\
\text { daily life? No, but I am } \\
\text { planning to change in the } \\
\text { next } 30 \text { days, and have } \\
\text { perhaps made small steps } \\
\text { toward that change }\end{array}$ & $\begin{array}{l}\text { Have you attempted to } \\
\text { manage stress in your } \\
\text { daily life? Yes, but have } \\
\text { been doing so for a } \\
\text { period of less than } 6 \\
\text { months }\end{array}$ & $\begin{array}{l}\text { Have you attempted to } \\
\text { manage stress in your dail } \\
\text { life? Yes, and I am taking } \\
\text { steps to sustain the change } \\
\text { during a 6-month time } \\
\text { frame and the prevention } \\
\text { of relapse }\end{array}$ \\
\hline $\begin{array}{l}\text { Riley et al. } \\
(2003) \\
\text { Stress } \\
\text { Manage- } \\
\text { ment }\end{array}$ & $\begin{array}{l}\text { Single } \\
\text { question } 5 \\
\text { choice } \\
\text { response } \\
\text { format }\end{array}$ & SS & $\begin{array}{l}\text { Have you attempted to } \\
\text { manage stress in your } \\
\text { daily life? No, and I don't } \\
\text { intend to for the next } 6 \\
\text { month }\end{array}$ & $\begin{array}{l}\text { Have you attempted to } \\
\text { manage stress in your } \\
\text { daily life? No, but I } \\
\text { intend to in the next } 6 \\
\text { month }\end{array}$ & $\begin{array}{l}\text { Have you attempted to } \\
\text { manage stress in your } \\
\text { daily life? No, but I am } \\
\text { planning to change in the } \\
\text { next } 30 \text { days, and have } \\
\text { perhaps made small steps } \\
\text { toward that change }\end{array}$ & $\begin{array}{l}\text { Have you attempted to } \\
\text { manage stress in your } \\
\text { daily life? Yes, but have } \\
\text { been doing so for a } \\
\text { period of less than } 6 \\
\text { months }\end{array}$ & $\mid \begin{array}{l}\text { Have you attempted to } \\
\text { manage stress in your dail } \\
\text { life? Yes, and I am taking } \\
\text { steps to sustain the change } \\
\text { during a 6-month time } \\
\text { frame and the prevention } \\
\text { of relapse }\end{array}$ \\
\hline
\end{tabular}


Table B.1 (continued)

\begin{tabular}{|c|c|c|c|c|c|c|c|}
\hline $\begin{array}{c}\text { Source } \\
\text { (Year) } \\
\text { Target } \\
\text { Behavior }\end{array}$ & $\begin{array}{l}\text { Type of } \\
\text { Algorithm }\end{array}$ & $\begin{array}{l}\text { TTM } \\
\text { or } \\
\text { Study } \\
\text { Specific } \\
\text { (SS) }\end{array}$ & Precontemplation & Contemplation & Preparation & Action & Maintenance \\
\hline $\begin{array}{l}\text { Robbins et } \\
\text { al. } \\
(2001) \\
\\
\text { Organ } \\
\text { Donation }\end{array}$ & \begin{tabular}{|l|} 
Not reported \\
(Marcus et \\
al., 1992; \\
Velicer et al., \\
1985)
\end{tabular} & SS & $\begin{array}{l}\text { I was opposed to organ } \\
\text { donation for [name]; or I } \\
\text { was not considering } \\
\text { organ donation as an } \\
\text { option for [name] }\end{array}$ & $\begin{array}{l}\text { I was considering organ } \\
\text { donation as an option for } \\
\text { [name] but was not yet } \\
\text { ready to make that } \\
\text { decision }\end{array}$ & $\begin{array}{l}\text { I was considering organ } \\
\text { donation as an option for } \\
\text { [name] and needed more } \\
\text { information on the } \\
\text { process to go ahead }\end{array}$ & $\begin{array}{l}\text { I had already decided to } \\
\text { donate [name's] and only } \\
\text { needed to move the } \\
\text { donation process along }\end{array}$ & Not reported \\
\hline $\begin{array}{l}\text { Robbins et } \\
\text { al. } \\
(1999) \\
\\
\text { Organ } \\
\text { Donation }\end{array}$ & $\begin{array}{l}5 \text {-item } \\
\text { algorithm } \\
\text { with } \\
\text { restaging } \\
\text { based on } \\
\text { behavioral } \\
\text { intention } \\
\end{array}$ & SS & $\begin{array}{l}\text { Did not intend to become } \\
\text { an organ donor in the } \\
\text { next } 6 \text { months }\end{array}$ & $\begin{array}{l}\text { Intended to become an } \\
\text { organ donor in the next } 6 \\
\text { months }\end{array}$ & $\begin{array}{l}\text { Intended to become an } \\
\text { organ donor in the next } \\
30 \text { days }\end{array}$ & $\begin{array}{l}\text { Had been an organ donor } \\
\text { for less than six months }\end{array}$ & $\begin{array}{l}\text { Had been a donor for more } \\
\text { than six months }\end{array}$ \\
\hline $\begin{array}{l}\text { Robbins et } \\
\text { al. } \\
(2002) \\
\\
\text { Organ } \\
\text { Donation }\end{array}$ & $\begin{array}{l}\text { MuItiple } \\
\text { question } \\
\text { format }\end{array}$ & SS & $\begin{array}{l}\text { Not thinking about } \\
\text { making the decision to } \\
\text { become an organ donor in } \\
\text { the next } 6 \text { months }\end{array}$ & $\begin{array}{l}\text { Planning on making the } \\
\text { decision to become an } \\
\text { organ donor in next } 6 \\
\text { months or next } 30 \text { days }\end{array}$ & $\begin{array}{l}\text { Made the decision to } \\
\text { become an organ donor, } \\
\text { but still have to document } \\
\text { or tell their family }\end{array}$ & Action/Maintenance & Action/Maintenance \\
\hline $\begin{array}{l}\text { Robbins et } \\
\text { al. } \\
(1999) \\
\text { Organ } \\
\text { Donation }\end{array}$ & \begin{tabular}{|l}
5 -item \\
algorithm \\
with \\
restaging \\
based on \\
behavioral \\
intention \\
\end{tabular} & SS & $\begin{array}{l}\text { Did not intend to become } \\
\text { an organ donor in the } \\
\text { next } 6 \text { months }\end{array}$ & $\begin{array}{l}\text { Intended to become an } \\
\text { organ donor in the next } 6 \\
\text { months }\end{array}$ & $\begin{array}{l}\text { Intended to become an } \\
\text { organ donor in the next } \\
30 \text { days }\end{array}$ & $\begin{array}{l}\text { Had been an organ donor } \\
\text { for less than six months }\end{array}$ & $\begin{array}{l}\text { Had been a donor for more } \\
\text { than six months }\end{array}$ \\
\hline \begin{tabular}{|l} 
Robbins et \\
al. \\
$(2002)$ \\
Organ \\
\end{tabular} & \begin{tabular}{|l}
5 -item \\
algorithm \\
with \\
restaging \\
based on \\
\end{tabular} & SS & $\begin{array}{l}\text { Did not intend to become } \\
\text { an organ donor in the } \\
\text { next } 6 \text { months }\end{array}$ & $\begin{array}{l}\text { Intended to become an } \\
\text { organ donor in the next } 6 \\
\text { months }\end{array}$ & $\begin{array}{l}\text { Intended to become an } \\
\text { organ donor in the next } \\
30 \text { days }\end{array}$ & $\begin{array}{l}\text { Had been an organ donor } \\
\text { for less than six months }\end{array}$ & $\begin{array}{l}\text { Had been a donor for more } \\
\text { than six months }\end{array}$ \\
\hline
\end{tabular}


Table B.1 (continued)

\begin{tabular}{|c|c|c|c|c|c|c|c|}
\hline $\begin{array}{c}\text { Source } \\
\text { (Year) } \\
\text { Target } \\
\text { Behavior }\end{array}$ & $\begin{array}{l}\text { Type of } \\
\text { Algorithm }\end{array}$ & \begin{tabular}{|c} 
TTM \\
or \\
Study \\
Specific \\
(SS)
\end{tabular} & Precontemplation & Contemplation & Preparation & Action & Maintenance \\
\hline Donation & $\begin{array}{l}\text { behavioral } \\
\text { intention }\end{array}$ & & & & & & \\
\hline \begin{tabular}{|l|} 
Rossi \\
$(1990)$ \\
Radon \\
Testing
\end{tabular} & Not reported & $\begin{array}{l}\text { Not } \\
\text { reported }\end{array}$ & Not reported & Not reported & Not reported & Not reported & Not reported \\
\hline $\begin{array}{l}\text { Rossi } \\
(1990) \\
\\
\text { Sunscreen } \\
\text { Use } \\
\end{array}$ & $\begin{array}{l}\text { 4- or 5-item } \\
\text { algorithm }\end{array}$ & TTM & $\begin{array}{l}\text { Do not currently use } \\
\text { sunscreen and do not } \\
\text { intend to change in the } \\
\text { next } 6 \text { months }\end{array}$ & $\begin{array}{l}\text { Do not currently use } \\
\text { sunscreen, but intend to } \\
\text { change in the next } 6 \\
\text { months }\end{array}$ & $\begin{array}{l}\text { Planning to use sunscreen } \\
\text { in the next month or had } \\
\text { made some changes but } \\
\text { were not at a particular } \\
\text { criterion } \\
\end{array}$ & $\begin{array}{l}\text { Began using sunscreen } \\
\text { within the past } 6 \text { months }\end{array}$ & $\begin{array}{l}\text { Have used sunscreen for } \\
\text { more than } 6 \text { months prior } \\
\text { to the study }\end{array}$ \\
\hline $\begin{array}{l}\text { Rossi et al. } \\
(2001) \\
\text { Cocaine Use }\end{array}$ & Not reported & SS & $\begin{array}{l}\text { Not planning to quit } \\
\text { using cocaine in the next } \\
6 \text { months }\end{array}$ & $\begin{array}{l}\text { Planning to quit cocaine } \\
\text { use within the next } 6 \\
\text { months }\end{array}$ & Not used & $\begin{array}{l}\text { Stopped using cocaine } \\
\text { within the past } 6 \text { months }\end{array}$ & $\begin{array}{l}\text { No use of cocaine for } 6 \\
\text { months or more }\end{array}$ \\
\hline $\begin{array}{l}\text { Rossi, SR et } \\
\text { al. } \\
(2001) \\
\text { Dietary Fat } \\
\text { Reduction } \\
\end{array}$ & \begin{tabular}{|l}
3 step \\
algorithm \\
(Green \& \\
Rossi 1998)
\end{tabular} & TTM & $\begin{array}{l}\text { Not ready and thus have } \\
\text { no intention of changing } \\
\text { behavior }\end{array}$ & $\begin{array}{l}\text { Seriously considering } \\
\text { changing behavior in the } \\
\text { next } 6 \text { months }\end{array}$ & $\begin{array}{l}\text { Not only ready to change, } \\
\text { but intend to do so } \\
\text { sometime in the } \\
\text { immediate future }\end{array}$ & $\begin{array}{l}\text { Recently changed their } \\
\text { behavior, typically for } 6 \\
\text { months or less }\end{array}$ & $\begin{array}{l}\text { Have sustained the } \\
\text { behavioral change, usually } \\
\text { for more than } 6 \text { months }\end{array}$ \\
\hline \begin{tabular}{|l} 
Rossi, SR et \\
al. \\
$(2001)$ \\
Dietary Fat \\
Reduction \\
\end{tabular} & \begin{tabular}{|l}
3 step \\
algorithm \\
(Green \& \\
Rossi 1998)
\end{tabular} & TTM & $\begin{array}{l}\text { Individuals who are not } \\
\text { ready and thus have no } \\
\text { intention of changing } \\
\text { behavior }\end{array}$ & $\begin{array}{l}\text { Individuals who are } \\
\text { seriously considering } \\
\text { changing behavior in the } \\
\text { next six months }\end{array}$ & $\begin{array}{l}\text { Not only ready to change, } \\
\text { but intend to do so } \\
\text { sometime in the } \\
\text { immediate future }\end{array}$ & $\begin{array}{l}\text { Recently changed their } \\
\text { behavior, typically for } 6 \\
\text { months or less }\end{array}$ & $\begin{array}{l}\text { Have sustained the } \\
\text { behavioral change, usually } \\
\text { for more than } 6 \text { months }\end{array}$ \\
\hline
\end{tabular}


Table B.1 (continued)

\begin{tabular}{|c|c|c|c|c|c|c|c|}
\hline $\begin{array}{c}\text { Source } \\
\text { (Year) } \\
\text { Target } \\
\text { Behavior }\end{array}$ & $\begin{array}{l}\text { Type of } \\
\text { Algorithm }\end{array}$ & $\begin{array}{c}\text { TTM } \\
\text { or } \\
\text { Study } \\
\text { Specific } \\
\text { (SS) }\end{array}$ & Precontemplation & Contemplation & Preparation & Action & Maintenance \\
\hline \begin{tabular}{|l|} 
Rossi, SR et \\
al. \\
$(2001)$ \\
Fruit \& \\
Vegetable \\
Consumption \\
\end{tabular} & $\begin{array}{l}\text { Two-step, 4- } \\
\text { question } \\
\text { algorithm }\end{array}$ & SS & $\begin{array}{l}\text { Individuals who are not } \\
\text { ready to eat five fruits } \\
\text { and vegetables a day and } \\
\text { have no intentions of } \\
\text { doing so in the next six } \\
\text { months }\end{array}$ & $\begin{array}{l}\text { Thinking about eat five } \\
\text { fruits and vegetables a } \\
\text { day and intend to do so in } \\
\text { the next six months }\end{array}$ & $\begin{array}{l}\text { Ready to eat five fruits } \\
\text { and vegetables a day and } \\
\text { intend to do so in the next } \\
\text { thirty days }\end{array}$ & $\begin{array}{l}\text { Have recently begun to } \\
\text { eat five fruits and } \\
\text { vegetables a day, but } \\
\text { have been doing so for } \\
\text { six months or less }\end{array}$ & $\begin{array}{l}\text { Have sustained } \\
\text { consumption of five fruits } \\
\text { and vegetables daily for } \\
\text { more than six months }\end{array}$ \\
\hline \begin{tabular}{|l|} 
Rossi, SR et \\
al. \\
$(1993)$ \\
Dietary Fat \\
Reduction \\
\end{tabular} & Not reported & \begin{tabular}{l|} 
Not \\
reported
\end{tabular} & Not reported & Not reported & Not used & Not reported & Not reported \\
\hline $\begin{array}{l}\text { Ruggiero et } \\
\text { al. } \\
(2000) \\
\text { Smoking } \\
\text { Cessation }\end{array}$ & $\begin{array}{l}\text { Single } \\
\text { question } 5 \\
\text { choice } \\
\text { response } \\
\text { format }\end{array}$ & SS & $\begin{array}{l}\text { No intention to quit } \\
\text { smoking in the next } 6 \\
\text { months or during } \\
\text { pregnancy }\end{array}$ & $\begin{array}{l}\text { Seriously considering } \\
\text { quitting in the next } 6 \\
\text { months or during } \\
\text { pregnancy }\end{array}$ & $\begin{array}{l}\text { Considering quitting in } \\
\text { the next } 30 \text { days, has } \\
\text { attempted to quit in the } \\
\text { last } 24 \text { hours during } \\
\text { current pregnancy or the } \\
\text { past year }\end{array}$ & Not reported & Not reported \\
\hline $\begin{array}{l}\text { Ruggiero et } \\
\text { al. } \\
(2003) \\
\text { Glucose } \\
\text { Testing }\end{array}$ & Not reported & TTM & $\begin{array}{l}\text { Not intending to change } \\
\text { in the foreseeable future }\end{array}$ & $\begin{array}{l}\text { Seriously intending to } \\
\text { take action in the } \\
\text { foreseeable future }\end{array}$ & $\begin{array}{l}\text { Seriously intending to } \\
\text { take action in the } \\
\text { immediate future }\end{array}$ & $\begin{array}{l}\text { Recently reached the goal } \\
\text { behavior }\end{array}$ & $\begin{array}{l}\text { Continued the goal } \\
\text { behavior for at least } 6 \\
\text { months }\end{array}$ \\
\hline $\begin{array}{l}\text { Sarkin et al. } \\
(2001) \\
\text { Exercise }\end{array}$ & $\begin{array}{l}\text { Single } \\
\text { question } 5 \\
\text { choice } \\
\text { response } \\
\text { format } \\
\end{array}$ & SS & $\begin{array}{l}\text { Does not exercise and has } \\
\text { no intention to do so in } \\
\text { the next } 6 \text { months }\end{array}$ & $\begin{array}{l}\text { Does not exercise but } \\
\text { intends to do so in next } 6 \\
\text { months }\end{array}$ & $\begin{array}{l}\text { Does not exercise but } \\
\text { intends to in next } 30 \text { days }\end{array}$ & $\begin{array}{l}\text { Does exercise, but for } \\
\text { less than the last } 6 \\
\text { months }\end{array}$ & $\begin{array}{l}\text { Does exercise and has bee } \\
\text { for more than the last } 6 \\
\text { months }\end{array}$ \\
\hline
\end{tabular}


Table B.1 (continued)

\begin{tabular}{|c|c|c|c|c|c|c|c|}
\hline $\begin{array}{c}\text { Source } \\
\text { (Year) } \\
\text { Target } \\
\text { Behavior }\end{array}$ & $\begin{array}{l}\text { Type of } \\
\text { Algorithm }\end{array}$ & $\begin{array}{l}\text { TTM } \\
\text { or } \\
\text { Study } \\
\text { Specific } \\
\text { (SS) }\end{array}$ & Precontemplation & Contemplation & Preparation & Action & Maintenance \\
\hline $\begin{array}{l}\text { Schorling } \\
\text { (1995) }\end{array}$ & Not reported & $\mathrm{SS}$ & $\begin{array}{l}\text { Did not plan to quit } \\
\text { smoking in the next } 6 \\
\text { months. }\end{array}$ & $\begin{array}{l}\text { Planned to quit smoking } \\
\text { in the next } 6 \text { months, but } \\
\text { not in the next } 30 \text { days or } \\
\text { planned to quit in the } \\
\text { next } 30 \text { days, but had not } \\
\text { made a serious cessation } \\
\text { attempt. }\end{array}$ & $\begin{array}{l}\text { Planned to quit in the } \\
\text { next } 30 \text { days, and has had } \\
\text { a serious cessation } \\
\text { attempt. }\end{array}$ & Not reported & Not reported \\
\hline $\begin{array}{l}\text { Smoking } \\
\text { cessation }\end{array}$ & Not reported & SS & $\begin{array}{l}\text { Did not plan to quit } \\
\text { smoking in the next } 6 \\
\text { months. }\end{array}$ & $\begin{array}{l}\text { Planned to quit smoking } \\
\text { in the next } 6 \text { months, but } \\
\text { not in the next } 30 \text { days or } \\
\text { planned to quit in the } \\
\text { next } 30 \text { days, but had not } \\
\text { made a serious cessation } \\
\text { attempt. }\end{array}$ & $\begin{array}{l}\text { Planned to quit in the } \\
\text { next } 30 \text { days, and has had } \\
\text { a serious cessation } \\
\text { attempt. }\end{array}$ & Not reported & Not reported \\
\hline $\begin{array}{l}\text { Schumann et } \\
\text { al. } \\
(2003) \\
\text { Smoking } \\
\text { Cessation } \\
\end{array}$ & $\begin{array}{l}4 \text {-item } \\
\text { algorithm }\end{array}$ & SS & $\begin{array}{l}\text { Not intending to quit } \\
\text { smoking within the next } 6 \\
\text { months }\end{array}$ & $\begin{array}{l}\text { Intending to quit smoking } \\
\text { within the next } 6 \text { months }\end{array}$ & $\begin{array}{l}\text { Intending to quit smoking } \\
\text { within the next } 4 \text { weeks } \\
\text { and reporting a serious } \\
\text { quit attempt in the past } \\
\text { year }\end{array}$ & $\begin{array}{l}\text { Reporting abstinence } \\
\text { from smoking for less } \\
\text { than } 6 \text { months }\end{array}$ & $\begin{array}{l}\text { Reporting abstinence from } \\
\text { smoking for more than } 6 \\
\text { months }\end{array}$ \\
\hline $\begin{array}{l}\text { Shulze } \\
(2003) \\
\text { Daily Use of } \\
\text { Dental Floss } \\
\end{array}$ & \begin{tabular}{|l|} 
Single \\
question 5 \\
choice \\
response \\
format \\
\end{tabular} & $\begin{array}{l}\text { Not } \\
\text { reported }\end{array}$ & $\begin{array}{l}\text { No, and I am not thinking } \\
\text { about starting within the } \\
\text { next } 6 \text { months }\end{array}$ & $\begin{array}{l}\text { No, but I am planning to } \\
\text { start to always use them } \\
\text { within the next } 6 \text { months }\end{array}$ & $\begin{array}{l}\text { No, but I am planning to } \\
\text { start within the next } 30 \\
\text { days }\end{array}$ & $\begin{array}{l}\text { Yes, and I have been } \\
\text { doing so for } 6 \text { months or } \\
\text { less }\end{array}$ & $\begin{array}{l}\text { Yes, and I have been doin } \\
\text { so for } 6 \text { months or longer }\end{array}$ \\
\hline $\begin{array}{l}\text { Silverman } \\
\text { (1995) } \\
\text { Condom Use }\end{array}$ & $\begin{array}{l}\text { Single } \\
\text { question } 5 \\
\text { choice } \\
\text { response } \\
\text { format }\end{array}$ & SS & $\begin{array}{l}\text { No, (I do not always use } \\
\text { condoms when I have } \\
\text { sex) and I am not } \\
\text { thinking about starting } \\
\text { within the next } 6 \text { months }\end{array}$ & $\begin{array}{l}\text { No, (I do not always use } \\
\text { condoms when I have } \\
\text { sex) but I am planning to } \\
\text { start to always use them } \\
\text { within the next } 6 \text { months }\end{array}$ & $\begin{array}{l}\text { No, (I do not always use } \\
\text { condoms when I have } \\
\text { sex) but I am planning to } \\
\text { start within the next } 30 \\
\text { days }\end{array}$ & $\begin{array}{l}\text { Yes, (I do not always use } \\
\text { condoms when I have } \\
\text { sex) and I have been } \\
\text { doing so for } 6 \text { months or } \\
\text { less }\end{array}$ & $\begin{array}{l}\text { Yes, (I do not always use } \\
\text { condoms when I have sex) } \\
\text { and I have been doing so } \\
\text { for } 6 \text { months or longer }\end{array}$ \\
\hline
\end{tabular}


Table B.1 (continued)

\begin{tabular}{|c|c|c|c|c|c|c|c|}
\hline $\begin{array}{c}\text { Source } \\
\text { (Year) } \\
\text { Target } \\
\text { Behavior }\end{array}$ & $\begin{array}{l}\text { Type of } \\
\text { Algorithm }\end{array}$ & $\begin{array}{l}\text { TTM } \\
\text { or } \\
\text { Study } \\
\text { Specific } \\
\text { (SS) }\end{array}$ & Precontemplation & Contemplation & Preparation & Action & Maintenance \\
\hline \begin{tabular}{|l|}
$\begin{array}{l}\text { Silverman } \\
(1995)\end{array}$ \\
Condom Use
\end{tabular} & $\begin{array}{l}\text { Single } \\
\text { question } 5 \\
\text { choice } \\
\text { response } \\
\text { format }\end{array}$ & SS & $\begin{array}{l}\text { No, (I do not always use } \\
\text { condoms when I have } \\
\text { sex) and I am not } \\
\text { thinking about starting } \\
\text { within the next } 6 \text { months }\end{array}$ & $\begin{array}{l}\text { No, (I do not always use } \\
\text { condoms when I have } \\
\text { sex) but I am planning to } \\
\text { start to always use them } \\
\text { within the next } 6 \text { months }\end{array}$ & $\begin{array}{l}\text { No, (I do not always use } \\
\text { condoms when I have } \\
\text { sex) but I am planning to } \\
\text { start within the next } 30 \\
\text { days }\end{array}$ & $\begin{array}{l}\text { Yes, (I do not always use } \\
\text { condoms when I have } \\
\text { sex) and I have been } \\
\text { doing so for } 6 \text { months or } \\
\text { less }\end{array}$ & $\begin{array}{l}\text { Yes, (I do not always use } \\
\text { condoms when I have sex) } \\
\text { and I have been doing so } \\
\text { for } 6 \text { months or longer }\end{array}$ \\
\hline $\begin{array}{l}\text { Snow et al. } \\
(1992) \\
\text { Smoking } \\
\text { Cessation }\end{array}$ & $\begin{array}{l}\text { 3-item } \\
\text { algorithm }\end{array}$ & SS & $\begin{array}{l}\text { Not seriously considering } \\
\text { quitting smoking in the } \\
\text { next } 6 \text { months }\end{array}$ & $\begin{array}{l}\text { Intending to quit within } \\
\text { the next } 6 \text { months }\end{array}$ & $\begin{array}{l}\text { Intention to quit within } \\
\text { the next } 30 \text { days }\end{array}$ & Not reported & Not reported \\
\hline $\begin{array}{l}\text { Stark et al. } \\
(1998) \\
\text { Condom use- } \\
\text { Main }\end{array}$ & $\begin{array}{l}\text { 3-question } \\
\text { algorithm }\end{array}$ & SS & $\begin{array}{l}\text { Neither used condoms } \\
\text { consistently nor intended } \\
\text { to use them consistently } \\
\text { within } 6 \text { months }\end{array}$ & $\begin{array}{l}\text { Intended consistent } \\
\text { condom use within the } \\
\text { next } 6 \text { months }\end{array}$ & $\begin{array}{l}\text { Were not using condoms } \\
\text { consistently, but intended } \\
\text { to do so within the next } \\
\text { month }\end{array}$ & $\begin{array}{l}\text { Using condoms } \\
\text { consistently, but for } 6 \\
\text { months or less }\end{array}$ & $\begin{array}{l}\text { Reported using condoms } \\
\text { consistently (every time } \\
\text { they had intercourse) for } \\
\text { more than } 6 \text { months }\end{array}$ \\
\hline $\begin{array}{l}\text { Stark et al. } \\
\text { (1998) } \\
\text { Condom } \\
\text { Use-Other } \\
\end{array}$ & $\begin{array}{l}\text { 3-question } \\
\text { algorithm }\end{array}$ & SS & \begin{tabular}{|l|} 
Neither used condoms \\
consistently nor intended \\
to use them consistently \\
within 6 months
\end{tabular} & $\begin{array}{l}\text { Intended consistent } \\
\text { condom use within the } \\
\text { next } 6 \text { months }\end{array}$ & $\begin{array}{l}\text { Were not using condoms } \\
\text { consistently, but intended } \\
\text { to do so within the next } \\
\text { month }\end{array}$ & $\begin{array}{l}\text { Using condoms } \\
\text { consistently, but for } 6 \\
\text { months or less }\end{array}$ & $\begin{array}{l}\text { Reported using condoms } \\
\text { consistently (every time } \\
\text { they had intercourse) for } \\
\text { more than } 6 \text { months }\end{array}$ \\
\hline $\begin{array}{l}\text { Susenbeth } \\
(1999) \\
\text { Condom Use }\end{array}$ & $\begin{array}{l}\text { Single } \\
\text { question } 5 \\
\text { choice } \\
\text { response } \\
\text { format }\end{array}$ & TTM & $\begin{array}{l}\text { No, and I am not thinking } \\
\text { about starting within the } \\
\text { next } 6 \text { months }\end{array}$ & $\begin{array}{l}\text { No, but I am planning to } \\
\text { start to always use them } \\
\text { within the next } 6 \text { months }\end{array}$ & $\begin{array}{l}\text { No, but I am planning to } \\
\text { start within the next } 30 \\
\text { days }\end{array}$ & $\begin{array}{l}\text { Yes, and I have been } \\
\text { doing so for } 6 \text { months or } \\
\text { less }\end{array}$ & $\begin{array}{l}\text { Yes, and I have been doing } \\
\text { so for } 6 \text { months or longer }\end{array}$ \\
\hline $\begin{array}{l}\text { Tseng } \\
(2000) \\
\text { Exercise }\end{array}$ & $\begin{array}{l}\text { Single } \\
\text { question } 5 \\
\text { choice } \\
\text { response }\end{array}$ & SS & $\begin{array}{l}\text { Currently do not exercise } \\
\text { and do not intend to start } \\
\text { exercise in the next } 6 \\
\text { months }\end{array}$ & $\begin{array}{l}\text { Currently do not exercise, } \\
\text { but am thinking about } \\
\text { starting to exercise in the } \\
\text { next } 6 \text { months }\end{array}$ & $\begin{array}{l}\text { Currently exercise some, } \\
\text { but not regularly }\end{array}$ & $\begin{array}{l}\text { Currently exercise } \\
\text { regularly but have only } \\
\text { begun doing so within the } \\
\text { last } 6 \text { months }\end{array}$ & $\begin{array}{l}\text { Currently exercise } \\
\text { regularly and have done so } \\
\text { for longer than } 6 \text { months }\end{array}$ \\
\hline
\end{tabular}


Table B.1 (continued)

\begin{tabular}{|c|c|c|c|c|c|c|c|}
\hline $\begin{array}{c}\text { Source } \\
\text { (Year) } \\
\text { Target } \\
\text { Behavior }\end{array}$ & $\begin{array}{c}\text { Type of } \\
\text { Algorithm }\end{array}$ & $\begin{array}{c}\text { TTM } \\
\text { or } \\
\text { Study } \\
\text { Specific } \\
\text { (SS) }\end{array}$ & Precontemplation & Contemplation & Preparation & Action & Maintenance \\
\hline & \begin{tabular}{|l|} 
format \\
(Marcus \& \\
Simkin 1993)
\end{tabular} & & & & & & \\
\hline $\begin{array}{l}\text { Tsoh } \\
\text { (1993) } \\
\text { Quitting } \\
\text { Drugs } \\
\end{array}$ & $\begin{array}{l}\text { Single } \\
\text { question } 5 \\
\text { choice } \\
\text { response } \\
\text { format } \\
\end{array}$ & SS & $\begin{array}{l}\text { I do not intend to stay off } \\
\text { drugs completely in the } \\
\text { next } 6 \text { months }\end{array}$ & $\begin{array}{l}\text { I intend to stay off drugs } \\
\text { completely in the next six } \\
\text { months but not in the } \\
\text { next } 30 \text { days }\end{array}$ & $\begin{array}{l}1 \text { intend to stay off drugs } \\
\text { completely in the next } 30 \\
\text { days }\end{array}$ & $\begin{array}{l}\text { I have stayed off any } \\
\text { drug use for less than } 6 \\
\text { months }\end{array}$ & $\begin{array}{l}\text { I have stayed off any drug } \\
\text { use for more than } 6 \text { month }\end{array}$ \\
\hline $\begin{array}{l}\text { Van Marter } \\
\text { et al. } \\
(2003) \\
\text { Bullying } \\
\text { Prevention }\end{array}$ & \begin{tabular}{|l|} 
Single \\
question 5 \\
choice \\
response \\
format (w/ 6- \\
item \\
behavior \\
identfier) \\
\end{tabular} & SS & $\begin{array}{l}\text { Involved in at least one } \\
\text { role related to bullying } \\
\text { and not intending to } \\
\text { change their behavior in } \\
\text { the next } 6 \text { months }\end{array}$ & $\begin{array}{l}\text { Involved in at least one } \\
\text { role related to bullying } \\
\text { and intending to change } \\
\text { their behavior in the next } \\
6 \text { months }\end{array}$ & $\begin{array}{l}\text { Involved in at least one } \\
\text { role related to bullying } \\
\text { and intending to change } \\
\text { in the next } 30 \text { days }\end{array}$ & $\begin{array}{l}\text { Not involved in any of } \\
\text { the three roles of bullying }\end{array}$ & $\begin{array}{l}\text { Not involved in any of the } \\
\text { three roles of bullying }\end{array}$ \\
\hline $\begin{array}{l}\text { Velicer et al. } \\
(1985) \\
\text { Smoking } \\
\text { Cessation } \\
\end{array}$ & Not reported & SS & $\begin{array}{l}\text { No intention of quitting } \\
\text { in the near future }\end{array}$ & $\begin{array}{l}\text { Currently smoking and } \\
\text { intending to quit in the } \\
\text { next year }\end{array}$ & $\begin{array}{l}\text { Currently smoking, but } \\
\text { had quit for a period of at } \\
\text { least } 24 \text { hours within the } \\
\text { last } 6 \text { months }\end{array}$ & $\begin{array}{l}\text { Currently not smoking, } \\
\text { but had smoked within } \\
\text { the last } 6 \text { months }\end{array}$ & $\begin{array}{l}\text { Currently not smoking, an } \\
\text { had not smoked for at leas } \\
6 \text { months }\end{array}$ \\
\hline $\begin{array}{l}\text { Wakui et al. } \\
(2002) \\
\text { Exercise }\end{array}$ & $\begin{array}{l}\text { Single } \\
\text { question } 5 \\
\text { choice } \\
\text { response } \\
\text { format } \\
\end{array}$ & $\overline{\mathrm{SS}}$ & $\begin{array}{l}\text { "I presently do not } \\
\text { exercise and do not plan } \\
\text { to start exercising in the } \\
\text { next six months" }\end{array}$ & $\begin{array}{l}\text { "I presently do not } \\
\text { exercise but I have been } \\
\text { thinking about starting to } \\
\text { exercise within the next } \\
\text { six months" }\end{array}$ & $\begin{array}{l}\text { "I presently get some } \\
\text { exercise, but not } \\
\text { regularly" }\end{array}$ & $\begin{array}{l}\text { "I presently exercise on a } \\
\text { regular basis, but I began } \\
\text { only within the past six } \\
\text { months" }\end{array}$ & $\begin{array}{l}\text { "I presently get some } \\
\text { exercise and have been } \\
\text { exercising regularly for } \\
\text { longer than six months" }\end{array}$ \\
\hline
\end{tabular}


Table B.1 (continued)

\begin{tabular}{|c|c|c|c|c|c|c|c|}
\hline $\begin{array}{c}\text { Source } \\
\text { (Year) } \\
\\
\text { Target } \\
\text { Behavior }\end{array}$ & $\begin{array}{l}\text { Type of } \\
\text { Algorithm }\end{array}$ & \begin{tabular}{|c} 
TTM \\
or \\
Study \\
Specific \\
(SS)
\end{tabular} & Precontemplation & Contemplation & Preparation & Action & Maintenance \\
\hline $\begin{array}{l}\text { Whyte } \\
(2002) \\
\\
\text { Dietary Fat } \\
\text { Reduction }\end{array}$ & $\begin{array}{l}\text { Multiple } \\
\text { question } \\
\text { format (5 } \\
\text { questions; } \\
\text { Curry et al., } \\
1992)\end{array}$ & SS & $\begin{array}{l}\text { Not currently limiting } \\
\text { dietary fat intake and } \\
\text { have not thought about } \\
\text { doing so in the past } \\
\text { month }\end{array}$ & $\begin{array}{l}\text { Not currently limiting } \\
\text { dietary fat intake, but } \\
\text { individuals have thought } \\
\text { about doing so in the past } \\
\text { month and are either } \\
\text { mildly confident or not at } \\
\text { all confident about } \\
\text { making changes in the } \\
\text { next month }\end{array}$ & $\begin{array}{l}\text { Not currently limiting } \\
\text { dietary fat intake, but } \\
\text { individuals have thought } \\
\text { about doing so in the past } \\
\text { month and are somewhat } \\
\text { or very confident that } \\
\text { they will make some of } \\
\text { these changes in the next } \\
\text { month }\end{array}$ & $\begin{array}{l}\text { Limiting dietary fat } \\
\text { intake for } 6 \text { months or } \\
\text { less }\end{array}$ & $\begin{array}{l}\text { Limiting dietary fat intake } \\
\text { for longer than } 6 \text { months }\end{array}$ \\
\hline $\begin{array}{l}\text { Wright et al. } \\
(2002) \\
\\
\text { Weight } \\
\text { Bearing } \\
\text { Exercise }\end{array}$ & $\begin{array}{l}\text { Single } \\
\text { question } 5 \\
\text { choice } \\
\text { response } \\
\text { format }\end{array}$ & SS & $\begin{array}{l}\text { Engaging in any activity } \\
\text { done on the feet with the } \\
\text { bones supporting the } \\
\text { body's weight for at least } \\
30 \text { min, a day, and at least } \\
\text { three times per week: } \\
\text { "No, and I don't intend to } \\
\text { in the next six months" }\end{array}$ & $\begin{array}{l}\text { Engaging in any activity } \\
\text { done on the feet with the } \\
\text { bones supporting the } \\
\text { body's weight for at least } \\
30 \text { min, a day, and at least } \\
\text { three times per week: } \\
\text { "No, but I intend to in } \\
\text { the next six months" }\end{array}$ & $\begin{array}{l}\text { Engaging in any activity } \\
\text { done on the feet with the } \\
\text { bones supporting the } \\
\text { body's weight for at least } \\
30 \text { min, a day, and at least } \\
\text { three times per week: } \\
\text { "No, but I intend to in the } \\
\text { next } 30 \text { days" }\end{array}$ & $\begin{array}{l}\text { Engaging in any activity } \\
\text { done on the feet with the } \\
\text { bones supporting the } \\
\text { body's weight for at least } \\
30 \text { min, a day, and at least } \\
\text { three times per week: } \\
\text { "Yes, I have been but for } \\
\text { less than six months" }\end{array}$ & $\begin{array}{l}\text { Engaging in any activity } \\
\text { done on the feet with the } \\
\text { bones supporting the } \\
\text { body's weight for at least } \\
30 \text { min, a day, and at least } \\
\text { three times per week: } \\
\text { "Yes, I have been for more } \\
\text { than six months" }\end{array}$ \\
\hline
\end{tabular}


Table B.2

Supplemental Matrix of Coded Variables 
Table B.2 (continued)

\begin{tabular}{|c|c|c|c|c|c|c|c|c|c|c|c|}
\hline $\begin{array}{l}\text { Source } \\
\text { (Year) }\end{array}$ & \begin{tabular}{|c|} 
N/ \\
data \\
-set \\
\end{tabular} & \begin{tabular}{|c|}
$\begin{array}{c}\text { Description } \\
\text { of Pros and } \\
\text { Cons }\end{array}$ \\
\end{tabular} & $\begin{array}{c}\text { Direction } \\
\text { of Pros } \\
\text { and Cons }\end{array}$ & $\begin{array}{c}\text { Calcula- } \\
\text { tion } \\
\text { Method }\end{array}$ & $\begin{array}{c}\text { Scale } \\
\text { Instruct }\end{array}$ & Cess/ Acq & \begin{tabular}{|c|} 
Publica- \\
tion \\
Status
\end{tabular} & $\begin{array}{l}\text { Lan- } \\
\text { guage }\end{array}$ & $\begin{array}{l}\text { Health } \\
\text { Status }\end{array}$ & $\begin{array}{c}\text { Fre- } \\
\text { quency }\end{array}$ & $\begin{array}{l}\text { Cross- } \\
\text { over }\end{array}$ \\
\hline $\begin{array}{l}\text { Acton et al. } \\
\text { (2001) }\end{array}$ & 205 & $\begin{array}{l}\text { Pros and } \\
\text { Cons of } \\
\text { Smoking }\end{array}$ & Reversed & Hedge's & NR & Cessation & \begin{tabular}{|c|} 
Peer- \\
Review \\
Journal
\end{tabular} & English & Unhealthy & Daily & NA \\
\hline $\begin{array}{l}\text { Ahijevych et } \\
\text { al. (1999) }\end{array}$ & 95 & $\begin{array}{l}\text { Pros and } \\
\text { Cons of } \\
\text { Smoking }\end{array}$ & Reversed & Hedge's & $\begin{array}{c}\text { How } \\
\text { Important }\end{array}$ & Cessation & \begin{tabular}{|c|} 
Peer- \\
Review \\
Journal \\
\end{tabular} & English & Unhealthy & Daily & NA \\
\hline $\begin{array}{c}\text { Bane et al. } \\
\text { (1999) }\end{array}$ & 281 & $\begin{array}{l}\text { Pros and } \\
\text { Cons of } \\
\text { Smoking }\end{array}$ & Reversed & $\begin{array}{l}\text { Standard } \\
\text { Score }\end{array}$ & $\begin{array}{c}\text { How } \\
\text { Important }\end{array}$ & Cessation & $\begin{array}{c}\text { Peer- } \\
\text { Review } \\
\text { Journal }\end{array}$ & English & Unhealthy & Daily & PC-C \\
\hline $\begin{array}{c}\text { Banikarim et } \\
\text { al. (2003) }\end{array}$ & 135 & $\begin{array}{c}\text { Pros and } \\
\text { Cons of STD } \\
\text { Screening }\end{array}$ & Actual & $\begin{array}{l}\text { Standard } \\
\text { Score }\end{array}$ & $\begin{array}{c}\text { How } \\
\text { Important }\end{array}$ & Acquisition & \begin{tabular}{|c|} 
Peer- \\
Review \\
Journal
\end{tabular} & English & Healthy & Situational & PR-A \\
\hline $\begin{array}{c}\text { Banikarim et } \\
\text { al. }(2003)\end{array}$ & 201 & $\begin{array}{c}\text { Pros and } \\
\text { Cons of STD } \\
\text { Screening }\end{array}$ & Actual & $\begin{array}{c}\text { Standard } \\
\text { Score }\end{array}$ & $\begin{array}{c}\text { How } \\
\text { Important }\end{array}$ & Acquisition & \begin{tabular}{|c|} 
Peer- \\
Review \\
Journal
\end{tabular} & English & Healthy & Yearly + & Multiple \\
\hline $\begin{array}{l}\text { Basler et al. } \\
\quad(1999)\end{array}$ & 451 & $\begin{array}{l}\text { Pros and } \\
\text { Cons of } \\
\text { Exercise }\end{array}$ & Actual & Hedge's & $\begin{array}{c}\text { How } \\
\text { Important }\end{array}$ & Acquisition & \begin{tabular}{|c|} 
Peer- \\
Review \\
Journal \\
\end{tabular} & $\begin{array}{l}\text { Non- } \\
\text { English }\end{array}$ & Healthy & Daily & PR-A \\
\hline $\begin{array}{c}\text { Block et al. } \\
\quad(1998)\end{array}$ & 127 & $\begin{array}{c}\text { Pros and } \\
\text { Cons of } \\
\text { Condom Use }\end{array}$ & Actual & Hedge's & $\begin{array}{c}\text { How } \\
\text { Important }\end{array}$ & Acquisition & \begin{tabular}{|c|} 
Non- \\
Peer- \\
Review \\
Journal
\end{tabular} & English & Healthy & Situational & NA \\
\hline $\begin{array}{c}\text { Borland et al. } \\
\quad(2000)\end{array}$ & 304 & $\begin{array}{l}\text { Pros and } \\
\text { Cons of } \\
\text { Smoking }\end{array}$ & Reversed & Hedge's & NR & Cessation & $\begin{array}{c}\text { Peer- } \\
\text { Review } \\
\text { Journal }\end{array}$ & English & Unhealthy & Daily & NA \\
\hline
\end{tabular}


Table B.2 (continued)

\begin{tabular}{|c|c|c|c|c|c|c|c|c|c|c|c|}
\hline $\begin{array}{l}\text { Source } \\
\text { (Year) }\end{array}$ & \begin{tabular}{|c|} 
N/ \\
data \\
-set \\
\end{tabular} & $\begin{array}{c}\text { Description } \\
\text { of Pros and } \\
\text { Cons } \\
\end{array}$ & $\begin{array}{c}\text { Direction } \\
\text { of Pros } \\
\text { and Cons } \\
\end{array}$ & $\begin{array}{l}\text { Calcula- } \\
\text { tion } \\
\text { Method } \\
\end{array}$ & $\begin{array}{c}\text { Scale } \\
\text { Instruct }\end{array}$ & Cess/ Acq & $\begin{array}{c}\text { Publica- } \\
\text { tion } \\
\text { Status }\end{array}$ & $\begin{array}{l}\text { Lan- } \\
\text { guage }\end{array}$ & $\begin{array}{l}\text { Health } \\
\text { Status }\end{array}$ & $\begin{array}{c}\text { Fre- } \\
\text { quency }\end{array}$ & $\begin{array}{c}\text { Cross- } \\
\text { over }\end{array}$ \\
\hline $\begin{array}{l}\text { Boudreaux et } \\
\text { al. (1998) }\end{array}$ & 554 & $\begin{array}{l}\text { Pros and } \\
\text { Cons of } \\
\text { Smoking }\end{array}$ & Reversed & Hedge's & $\begin{array}{c}\text { How } \\
\text { Important }\end{array}$ & Cessation & $\begin{array}{l}\text { Peer- } \\
\text { Review } \\
\text { Journal }\end{array}$ & English & Unhealthy & Daily & C-PR \\
\hline $\begin{array}{l}\text { Buckworth et } \\
\text { al. }(2002)\end{array}$ & 57 & $\begin{array}{l}\text { Pros and } \\
\text { Cons of } \\
\text { Exercise }\end{array}$ & Actual & Hedge's & $\begin{array}{c}\text { How } \\
\text { Important }\end{array}$ & Acquisition & $\begin{array}{c}\text { Peer- } \\
\text { Review } \\
\text { Journal }\end{array}$ & English & Healthy & Daily & NA \\
\hline $\begin{array}{c}\text { Callaghan et } \\
\text { al. }(2002)\end{array}$ & 298 & $\begin{array}{l}\text { Pros and } \\
\text { Cons of } \\
\text { Exercise }\end{array}$ & Actual & Hedge's & $\begin{array}{c}\text { How } \\
\text { Important }\end{array}$ & Acquisition & $\begin{array}{c}\text { Peer- } \\
\text { Review } \\
\text { Journal }\end{array}$ & $\begin{array}{l}\text { Non- } \\
\text { English }\end{array}$ & Healthy & Daily & NA \\
\hline $\begin{array}{l}\text { Chamot et al. } \\
\text { (2001) }\end{array}$ & 885 & $\begin{array}{c}\text { Pros and } \\
\text { Cons of } \\
\text { mammograp } \\
\text { hy screening }\end{array}$ & Actual & $\begin{array}{l}\text { Standard } \\
\text { Score }\end{array}$ & $\begin{array}{c}\text { Agree / } \\
\text { Disagree }\end{array}$ & Acquisition & $\begin{array}{l}\text { Peer- } \\
\text { Review } \\
\text { Journal }\end{array}$ & $\begin{array}{l}\text { Non- } \\
\text { English }\end{array}$ & Healthy & Yearly + & PC-C \\
\hline $\begin{array}{l}\text { DiClemente } \\
\text { et al. (1991) }\end{array}$ & 1466 & $\begin{array}{l}\text { Pros and } \\
\text { Cons of } \\
\text { Smoking }\end{array}$ & Reversed & Hedge's & $\begin{array}{c}\text { Agree / } \\
\text { Disagree }\end{array}$ & Cessation & $\begin{array}{l}\text { Peer- } \\
\text { Review } \\
\text { Journal }\end{array}$ & English & Unhealthy & Daily & C-PR \\
\hline $\begin{array}{l}\text { Dijkstra et al. } \\
\quad(1996)\end{array}$ & 275 & $\begin{array}{l}\text { Pros and } \\
\text { Cons of } \\
\text { Quitting } \\
\text { Smoking }\end{array}$ & Actual & Hedge's & Other & Cessation & $\begin{array}{l}\text { Peer- } \\
\text { Review } \\
\text { Journal }\end{array}$ & $\begin{array}{l}\text { Non- } \\
\text { English }\end{array}$ & Unhealthy & Daily & C-PR \\
\hline $\begin{array}{l}\text { Dijkstra et al. } \\
\text { (1998) }\end{array}$ & 1540 & $\begin{array}{l}\text { Pros and } \\
\text { cons of } \\
\text { quiting } \\
\text { smoking }\end{array}$ & Actual & $\begin{array}{c}\text { Standard } \\
\text { Score }\end{array}$ & Other & Cessation & $\begin{array}{c}\text { Peer- } \\
\text { Review } \\
\text { Journal }\end{array}$ & $\begin{array}{l}\text { Non- } \\
\text { English }\end{array}$ & Unhealthy & Daily & $\mathrm{OK}$ \\
\hline
\end{tabular}


Table B.2 (continued)

\begin{tabular}{|c|c|c|c|c|c|c|c|c|c|c|c|}
\hline $\begin{array}{l}\text { Source } \\
\text { (Year) }\end{array}$ & \begin{tabular}{|c|}
$\mathrm{N} /$ \\
data \\
-set
\end{tabular} & $\begin{array}{c}\text { Description } \\
\text { of Pros and } \\
\text { Cons }\end{array}$ & $\begin{array}{l}\text { Direction } \\
\text { of Pros } \\
\text { and Cons }\end{array}$ & $\begin{array}{l}\text { Calcula- } \\
\text { tion } \\
\text { Method }\end{array}$ & $\begin{array}{c}\text { Scale } \\
\text { Instruct }\end{array}$ & Cess/ Acq & \begin{tabular}{|c} 
Publica- \\
tion \\
Status \\
\end{tabular} & $\begin{array}{l}\text { Lan- } \\
\text { guage }\end{array}$ & $\begin{array}{l}\text { Health } \\
\text { Status }\end{array}$ & $\begin{array}{c}\text { Fre- } \\
\text { quency }\end{array}$ & $\begin{array}{c}\text { Cross- } \\
\text { over }\end{array}$ \\
\hline $\begin{array}{c}\text { Dryfoos } \\
\text { (1996) }\end{array}$ & 338 & $\begin{array}{l}\text { Pros and } \\
\text { Cons of } \\
\text { Glucose } \\
\text { Testing }\end{array}$ & Actual & Hedge's & $\begin{array}{c}\text { How } \\
\text { Important }\end{array}$ & Acquisition & $\begin{array}{c}\text { Non- } \\
\text { Peer- } \\
\text { Review } \\
\text { Journal }\end{array}$ & English & Healthy & Daily & PR-A \\
\hline $\begin{array}{c}\text { Dryfoos } \\
(1996)\end{array}$ & 1048 & $\begin{array}{c}\text { Pros and } \\
\text { Cons of } \\
\text { Medication }\end{array}$ & Actual & Hedge's & $\begin{array}{c}\text { How } \\
\text { Important }\end{array}$ & Acquisition & $\begin{array}{l}\text { Non- } \\
\text { Peer- } \\
\text { Review } \\
\text { Journal }\end{array}$ & English & Healthy & Daily & A-M \\
\hline $\begin{array}{l}\text { Eaton et al. } \\
\text { (1992) }\end{array}$ & 165 & $\begin{array}{l}\text { Pros and } \\
\text { Cons of } \\
\text { Physicians } \\
\text { Assisting } \\
\text { Smokers }\end{array}$ & Actual & Hedge's & $\begin{array}{c}\text { How } \\
\text { Important }\end{array}$ & Acquisition & $\begin{array}{l}\text { Non- } \\
\text { Peer- } \\
\text { Review } \\
\text { Journal }\end{array}$ & English & Other & Situational & PC-C \\
\hline $\begin{array}{c}\text { Evers et al. } \\
\text { (2001) }\end{array}$ & 270 & $\begin{array}{c}\text { Pros and } \\
\text { Cons of } \\
\text { Condom Use }\end{array}$ & Actual & $\begin{array}{l}\text { Standard } \\
\text { Score }\end{array}$ & $\begin{array}{c}\text { How } \\
\text { Important }\end{array}$ & Acquisition & \begin{tabular}{|c|} 
Non- \\
Peer- \\
Review \\
Journal
\end{tabular} & English & Healthy & Situational & PR-A \\
\hline $\begin{array}{l}\text { Evers et al. } \\
\text { (2001) }\end{array}$ & 293 & $\begin{array}{c}\text { Pros and } \\
\text { Cons of } \\
\text { Cessation of } \\
\text { Drug Use }\end{array}$ & Actual & Hedge's & $\begin{array}{c}\text { How } \\
\text { Important }\end{array}$ & Cessation & \begin{tabular}{|c|} 
Non- \\
Peer- \\
Review \\
Journal
\end{tabular} & $\begin{array}{c}\text { Non- } \\
\text { English }\end{array}$ & Unhealthy & Daily & Multiple \\
\hline $\begin{array}{c}\text { Evers et al. } \\
\text { (2001) }\end{array}$ & 602 & $\begin{array}{l}\text { Pros and } \\
\text { Cons of } \\
\text { Waiting to } \\
\text { Have Sex }\end{array}$ & Reversed & $\begin{array}{c}\text { Standard } \\
\text { Score }\end{array}$ & $\begin{array}{c}\text { How } \\
\text { Important }\end{array}$ & Acquisition & $\begin{array}{l}\text { Non- } \\
\text { Peer- } \\
\text { Review } \\
\text { Journal }\end{array}$ & English & Healthy & Situational & PC-C \\
\hline
\end{tabular}


Table B.2 (continued)

\begin{tabular}{|c|c|c|c|c|c|c|c|c|c|c|c|}
\hline $\begin{array}{l}\text { Source } \\
\text { (Year) }\end{array}$ & \begin{tabular}{|c|}
$\mathrm{N} /$ \\
data \\
-set \\
\end{tabular} & $\begin{array}{c}\text { Description } \\
\text { of Pros and } \\
\text { Cons }\end{array}$ & $\begin{array}{l}\text { Direction } \\
\text { of Pros } \\
\text { and Cons }\end{array}$ & $\begin{array}{c}\text { Calcula- } \\
\text { tion } \\
\text { Method }\end{array}$ & $\begin{array}{c}\text { Scale } \\
\text { Instruct }\end{array}$ & Cess/ Acq & \begin{tabular}{|c|} 
Publica- \\
tion \\
Status \\
\end{tabular} & $\begin{array}{l}\text { Lan- } \\
\text { guage }\end{array}$ & $\begin{array}{l}\text { Health } \\
\text { Status }\end{array}$ & $\begin{array}{c}\text { Fre- } \\
\text { quency }\end{array}$ & $\begin{array}{l}\text { Cross- } \\
\text { over }\end{array}$ \\
\hline $\begin{array}{c}\text { Evers et al. } \\
(2003)\end{array}$ & 241 & $\begin{array}{l}\text { Pros and } \\
\text { Cons of } \\
\text { Managing } \\
\text { Stress }\end{array}$ & Actual & Hedge's & NR & Acquisition & $\begin{array}{l}\text { Non- } \\
\text { Peer- } \\
\text { Review } \\
\text { Journal }\end{array}$ & English & Unhealthy & Daily & PC-C \\
\hline $\begin{array}{l}\text { Fahrenwald et } \\
\text { al. (2003) }\end{array}$ & 30 & $\begin{array}{l}\text { Pros and } \\
\text { Cons of } \\
\text { Exercise }\end{array}$ & Actual & Hedge's & $\begin{array}{c}\text { Agree / } \\
\text { Disagree }\end{array}$ & Acquisition & $\begin{array}{l}\text { Peer- } \\
\text { Review } \\
\text { Journal }\end{array}$ & English & Healthy & Daily & C-PR \\
\hline $\begin{array}{l}\text { Fava et al. } \\
\text { (1995) }\end{array}$ & 4144 & $\begin{array}{l}\text { Pros and } \\
\text { Cons of } \\
\text { Smoking }\end{array}$ & Reversed & Hedge's & $\begin{array}{c}\text { How } \\
\text { Important }\end{array}$ & Cessation & $\begin{array}{c}\text { Peer- } \\
\text { Review } \\
\text { Journal }\end{array}$ & English & Unhealthy & Daily & PC-C \\
\hline $\begin{array}{l}\text { Frenn et al. } \\
\quad(2003)\end{array}$ & 74 & $\begin{array}{c}\text { Pros and } \\
\text { Cons of } \\
\text { Eating a Diet } \\
\text { w/30\% or } \\
\text { fewer } \\
\text { calories from } \\
\text { fat }\end{array}$ & Actual & Hedge's & NR & Cessation & $\begin{array}{l}\text { Peer- } \\
\text { Review } \\
\text { Journal }\end{array}$ & English & Healthy & Daily & C-PR \\
\hline $\begin{array}{c}\text { Galavotti et } \\
\text { al. (1995) }\end{array}$ & 233 & $\begin{array}{c}\text { Pros and } \\
\text { Cons of } \\
\text { General } \\
\text { Contraceptio } \\
\mathbf{n} \\
\end{array}$ & Actual & $\begin{array}{c}\text { Standard } \\
\text { Score }\end{array}$ & $\begin{array}{c}\text { How } \\
\text { Important }\end{array}$ & Acquisition & $\begin{array}{l}\text { Peer- } \\
\text { Review } \\
\text { Journal }\end{array}$ & English & Healthy & Situational & C-PR \\
\hline $\begin{array}{l}\text { Galavotti et } \\
\text { al. (1995) }\end{array}$ & 233 & $\begin{array}{c}\text { Pros and } \\
\text { Cons of } \\
\text { Condom Use } \\
\text { with Main } \\
\text { Partner } \\
\end{array}$ & Actual & $\begin{array}{c}\text { Standard } \\
\text { Score }\end{array}$ & $\begin{array}{c}\text { How } \\
\text { Important }\end{array}$ & Acquisition & $\begin{array}{l}\text { Peer- } \\
\text { Review } \\
\text { Journal }\end{array}$ & English & Healthy & Situational & C-PR \\
\hline
\end{tabular}


Table B.2 (continued)

\begin{tabular}{|c|c|c|c|c|c|c|c|c|c|c|c|}
\hline $\begin{array}{l}\text { Source } \\
\text { (Year) }\end{array}$ & $\begin{array}{c}\text { N/ } \\
\text { data } \\
\text {-set }\end{array}$ & $\begin{array}{c}\text { Description } \\
\text { of Pros and } \\
\text { Cons }\end{array}$ & $\begin{array}{l}\text { Direction } \\
\text { of Pros } \\
\text { and Cons }\end{array}$ & $\begin{array}{c}\text { Calcula- } \\
\text { tion } \\
\text { Method } \\
\end{array}$ & $\begin{array}{c}\text { Scale } \\
\text { Instruct }\end{array}$ & Cess/ Acq & $\begin{array}{c}\text { Publica- } \\
\text { tion } \\
\text { Status } \\
\end{array}$ & $\begin{array}{l}\text { Lan- } \\
\text { guage }\end{array}$ & $\begin{array}{l}\text { Health } \\
\text { Status }\end{array}$ & $\begin{array}{c}\text { Fre- } \\
\text { quency }\end{array}$ & $\begin{array}{l}\text { Cross- } \\
\text { over }\end{array}$ \\
\hline $\begin{array}{l}\text { Galavotti et } \\
\text { al. (1995) }\end{array}$ & 122 & $\begin{array}{c}\text { Pros and } \\
\text { Cons of } \\
\text { Condom Use } \\
\text { with Other } \\
\text { Partners }\end{array}$ & Actual & $\begin{array}{c}\text { Standard } \\
\text { Score }\end{array}$ & $\begin{array}{c}\text { How } \\
\text { Important }\end{array}$ & Acquisition & $\begin{array}{c}\text { Peer- } \\
\text { Review } \\
\text { Journal }\end{array}$ & English & Healthy & Situational & Multiple \\
\hline $\begin{array}{c}\text { Gazabon } \\
(2000)\end{array}$ & 340 & $\begin{array}{l}\text { Pros and } \\
\text { cons of using } \\
\text { condoms }\end{array}$ & Actual & Hedge's & $\begin{array}{c}\text { How } \\
\text { Important }\end{array}$ & Acquisition & $\begin{array}{c}\text { Non- } \\
\text { Peer- } \\
\text { Review } \\
\text { Journal }\end{array}$ & English & Healthy & Situational & C-PR \\
\hline $\begin{array}{l}\text { Giebel } \\
(1999)\end{array}$ & 235 & $\begin{array}{l}\text { Pros and } \\
\text { Cons of } \\
\text { Quitting } \\
\text { Smoking }\end{array}$ & Actual & Hedge's & $\begin{array}{c}\text { How } \\
\text { Important }\end{array}$ & Cessation & $\begin{array}{c}\text { Non- } \\
\text { Peer- } \\
\text { Review } \\
\text { Journal }\end{array}$ & $\begin{array}{l}\text { Non- } \\
\text { English }\end{array}$ & Unhealthy & Daily & Multiple \\
\hline $\begin{array}{l}\text { Giebel } \\
\text { (1999) }\end{array}$ & 361 & $\begin{array}{c}\text { Pros and } \\
\text { Cons of } \\
\text { Healthy Diet }\end{array}$ & Actual & Hedge's & $\begin{array}{c}\text { How } \\
\text { Important }\end{array}$ & Cessation & $\begin{array}{c}\text { Non- } \\
\text { Peer- } \\
\text { Review } \\
\text { Journal }\end{array}$ & $\begin{array}{l}\text { Non- } \\
\text { English }\end{array}$ & Healthy & Daily & PC-C \\
\hline $\begin{array}{l}\text { Giebel } \\
(1999)\end{array}$ & 361 & $\begin{array}{l}\text { Pros and } \\
\text { Cons of } \\
\text { Exercising }\end{array}$ & Actual & Hedge's & $\begin{array}{c}\text { How } \\
\text { Important }\end{array}$ & Acquisition & $\begin{array}{c}\text { Non- } \\
\text { Peer- } \\
\text { Review } \\
\text { Journal }\end{array}$ & $\begin{array}{l}\text { Non- } \\
\text { English }\end{array}$ & Healthy & Daily & A-M \\
\hline $\begin{array}{l}\text { Giebel } \\
(1999)\end{array}$ & 244 & $\begin{array}{l}\text { Pros and } \\
\text { Cons of } \\
\text { Stress } \\
\text { Reduction }\end{array}$ & Actual & Hedge's & $\begin{array}{c}\text { How } \\
\text { Important }\end{array}$ & Acquisition & $\begin{array}{l}\text { Non- } \\
\text { Peer- } \\
\text { Review } \\
\text { Journal }\end{array}$ & $\begin{array}{l}\text { Non- } \\
\text { English }\end{array}$ & Unhealthy & Daily & PR-A \\
\hline
\end{tabular}


Table B.2 (continued)

\begin{tabular}{|c|c|c|c|c|c|c|c|c|c|c|c|}
\hline $\begin{array}{l}\text { Source } \\
\text { (Year) }\end{array}$ & \begin{tabular}{|c|}
$\mathrm{N} /$ \\
data \\
-set \\
\end{tabular} & $\begin{array}{c}\text { Description } \\
\text { of Pros and } \\
\text { Cons } \\
\end{array}$ & $\begin{array}{c}\text { Direction } \\
\text { of Pros } \\
\text { and Cons }\end{array}$ & $\begin{array}{l}\text { Calcula- } \\
\text { tion } \\
\text { Method } \\
\end{array}$ & $\begin{array}{c}\text { Scale } \\
\text { Instruct }\end{array}$ & Cess/ Acq & $\begin{array}{l}\text { Publica- } \\
\text { tion } \\
\text { Status } \\
\end{array}$ & $\begin{array}{l}\text { Lan- } \\
\text { guage }\end{array}$ & $\begin{array}{l}\text { Health } \\
\text { Status }\end{array}$ & $\begin{array}{c}\text { Fre- } \\
\text { quency }\end{array}$ & $\begin{array}{l}\text { Cross- } \\
\text { over }\end{array}$ \\
\hline $\begin{array}{c}\text { Grimley et al. } \\
\text { (1995) }\end{array}$ & 244 & $\begin{array}{c}\text { Pros and } \\
\text { Cons of } \\
\text { General } \\
\text { Contraceptio } \\
n\end{array}$ & Actual & Hedge's & $\begin{array}{c}\text { How } \\
\text { Important }\end{array}$ & Acquisition & $\begin{array}{c}\text { Peer- } \\
\text { Review } \\
\text { Journal }\end{array}$ & English & Healthy & Situational & PR-A \\
\hline $\begin{array}{c}\text { Grimley et al. } \\
\text { (1995) }\end{array}$ & 78 & $\begin{array}{l}\text { Pros and } \\
\text { Cons of } \\
\text { Condom Use } \\
\text { with Main } \\
\text { Partner }\end{array}$ & Actual & Hedge's & $\begin{array}{c}\text { How } \\
\text { Important }\end{array}$ & Acquisition & $\begin{array}{c}\text { Peer- } \\
\text { Review } \\
\text { Journal }\end{array}$ & English & Healthy & Situational & C-PR \\
\hline $\begin{array}{c}\text { Grimley et al. } \\
\text { (1995) }\end{array}$ & 134 & $\begin{array}{c}\text { Pros and } \\
\text { Cons of } \\
\text { Condom Use } \\
\text { with Other } \\
\text { Partners }\end{array}$ & Actual & Hedge's & $\begin{array}{c}\text { How } \\
\text { Important }\end{array}$ & Acquisition & $\begin{array}{c}\text { Peer- } \\
\text { Review } \\
\text { Journal }\end{array}$ & English & Healthy & Situational & C-PR \\
\hline $\begin{array}{l}\text { Happel } \\
(2002)\end{array}$ & 255 & $\begin{array}{c}\text { Pros and } \\
\text { Cons of Fruit } \\
\text { and } \\
\text { Vegetable } \\
\text { Consumption }\end{array}$ & Actual & Hedge's & $\begin{array}{c}\text { How } \\
\text { Important }\end{array}$ & Acquisition & $\begin{array}{l}\text { Non- } \\
\text { Peer- } \\
\text { Review } \\
\text { Journal }\end{array}$ & $\begin{array}{l}\text { Non- } \\
\text { English }\end{array}$ & Healthy & Daily & C-PR \\
\hline $\begin{array}{l}\text { Hempelmann } \\
\text { (2003) }\end{array}$ & 448 & $\begin{array}{l}\text { Pros and } \\
\text { Cons of } \\
\text { Tooth- } \\
\text { Friendly Diet }\end{array}$ & Actual & Hedge's & $\begin{array}{c}\text { How } \\
\text { Important }\end{array}$ & Acquisition & $\begin{array}{c}\text { Non- } \\
\text { Peer- } \\
\text { Review } \\
\text { Journal }\end{array}$ & $\begin{array}{l}\text { Non- } \\
\text { English }\end{array}$ & Healthy & Daily & PR-A \\
\hline
\end{tabular}


Table B.2 (continued)

\begin{tabular}{|c|c|c|c|c|c|c|c|c|c|c|c|}
\hline $\begin{array}{l}\text { Source } \\
\text { (Year) }\end{array}$ & $\begin{array}{c}\text { N/ } \\
\text { data } \\
\text {-set } \\
\end{array}$ & $\begin{array}{c}\text { Description } \\
\text { of Pros and } \\
\text { Cons }\end{array}$ & $\begin{array}{c}\text { Direction } \\
\text { of Pros } \\
\text { and Cons }\end{array}$ & $\begin{array}{l}\text { Calcula- } \\
\text { tion } \\
\text { Method }\end{array}$ & $\begin{array}{c}\text { Scale } \\
\text { Instruct }\end{array}$ & Cess/ Acq & $\begin{array}{l}\text { Publica- } \\
\text { tion } \\
\text { Status }\end{array}$ & $\begin{array}{l}\text { Lan- } \\
\text { guage }\end{array}$ & $\begin{array}{l}\text { Health } \\
\text { Status }\end{array}$ & $\begin{array}{c}\text { Fre- } \\
\text { quency }\end{array}$ & $\begin{array}{l}\text { Cross- } \\
\text { over }\end{array}$ \\
\hline $\begin{array}{l}\text { Henderson } \\
\text { (1999) }\end{array}$ & 214 & $\begin{array}{c}\text { Pros and } \\
\text { Cons of } \\
\text { Breast Self- } \\
\text { Examination }\end{array}$ & Actual & Hedge's & $\begin{array}{c}\text { Agree / } \\
\text { Disagree }\end{array}$ & Acquisition & $\begin{array}{l}\text { Non- } \\
\text { Peer- } \\
\text { Review } \\
\text { Journal }\end{array}$ & English & Healthy & Daily & PR \\
\hline $\begin{array}{l}\text { Herrick et al. } \\
\text { (1997) }\end{array}$ & 392 & $\begin{array}{c}\text { Pros and } \\
\text { Cons of } \\
\text { Reducing } \\
\text { Dietary Fat } \\
\text { Consumption }\end{array}$ & Reversed & Hedge's & $\begin{array}{c}\text { Agree / } \\
\text { Disagree }\end{array}$ & Cessation & $\begin{array}{c}\text { Peer- } \\
\text { Review } \\
\text { Journal }\end{array}$ & English & Healthy & Daily & PR-A \\
\hline $\begin{array}{l}\text { Herrick et al. } \\
\quad(1997)\end{array}$ & 389 & $\begin{array}{l}\text { Pros and } \\
\text { Cons of } \\
\text { Exercise }\end{array}$ & Actual & Hedge's & $\begin{array}{c}\text { Agree / } \\
\text { Disagree }\end{array}$ & Acquisition & $\begin{array}{c}\text { Peer- } \\
\text { Review } \\
\text { Journal }\end{array}$ & English & Healthy & Daily & PR-A \\
\hline $\begin{array}{l}\text { Herrick et al. } \\
\quad(1997)\end{array}$ & 393 & $\begin{array}{l}\text { Pros and } \\
\text { Cons of } \\
\text { Smoking }\end{array}$ & Reversed & Hedge's & $\begin{array}{l}\text { Agree / } \\
\text { Disagree }\end{array}$ & Cessation & $\begin{array}{c}\text { Peer- } \\
\text { Review } \\
\text { Journal }\end{array}$ & English & Unhealthy & Daily & PR-A \\
\hline $\begin{array}{l}\text { Herrick et al. } \\
\quad(1997)\end{array}$ & 392 & $\begin{array}{l}\text { Pros and } \\
\text { Cons for } \\
\text { Protection } \\
\text { from Sun } \\
\text { Exposure }\end{array}$ & Actual & Hedge's & $\begin{array}{c}\text { Agree / } \\
\text { Disagree }\end{array}$ & Acquisition & $\begin{array}{c}\text { Peer- } \\
\text { Review } \\
\text { Journal }\end{array}$ & English & Healthy & Situational & PR-A \\
\hline $\begin{array}{l}\text { Herzog et al. } \\
\quad(1999)\end{array}$ & 1390 & $\begin{array}{l}\text { Pros and } \\
\text { Cons of } \\
\text { Smoking }\end{array}$ & Reversed & Hedge's & $\begin{array}{c}\text { How } \\
\text { Important }\end{array}$ & Cessation & $\begin{array}{c}\text { Peer- } \\
\text { Review } \\
\text { Journal }\end{array}$ & English & Unhealthy & Daily & NA \\
\hline $\begin{array}{l}\text { Hulton } \\
(2001)\end{array}$ & 103 & $\begin{array}{c}\text { Pros and } \\
\text { Cons of } \\
\text { Abstinence }\end{array}$ & Reversed & Hedge's & NR & Acquisition & $\begin{array}{c}\text { Peer- } \\
\text { Review } \\
\text { Journal }\end{array}$ & English & Healthy & Situational & $\begin{array}{l}\text { No } \\
\text { Cross }\end{array}$ \\
\hline
\end{tabular}


Table B.2 (continued)

\begin{tabular}{|c|c|c|c|c|c|c|c|c|c|c|c|}
\hline $\begin{array}{l}\text { Source } \\
\text { (Year) }\end{array}$ & $\begin{array}{c}\mathrm{N} / \\
\text { data } \\
\text {-set }\end{array}$ & $\begin{array}{c}\text { Description } \\
\text { of Pros and } \\
\text { Cons } \\
\end{array}$ & $\begin{array}{c}\text { Direction } \\
\text { of Pros } \\
\text { and Cons }\end{array}$ & $\begin{array}{c}\text { Calcula- } \\
\text { tion } \\
\text { Method }\end{array}$ & $\begin{array}{c}\text { Scale } \\
\text { Instruct }\end{array}$ & Cess/ Acq & $\begin{array}{c}\text { Publica- } \\
\text { tion } \\
\text { Status }\end{array}$ & $\begin{array}{c}\text { Lan- } \\
\text { guage }\end{array}$ & $\begin{array}{l}\text { Health } \\
\text { Status }\end{array}$ & $\begin{array}{l}\text { Fre- } \\
\text { quency }\end{array}$ & $\begin{array}{l}\text { Cross- } \\
\text { over }\end{array}$ \\
\hline $\begin{array}{l}\text { Hulton } \\
(2001)\end{array}$ & 622 & $\begin{array}{c}\text { Pros and } \\
\text { Cons of } \\
\text { Abstinence }\end{array}$ & Reversed & Hedge's & NR & Acquisition & $\begin{array}{c}\text { Peer- } \\
\text { Review } \\
\text { Journal }\end{array}$ & English & Healthy & Situational & PC-C \\
\hline $\begin{array}{c}\text { Jeffries } \\
(2001)\end{array}$ & 537 & $\begin{array}{l}\text { Pros and } \\
\text { Cons of } \\
\text { Exercising }\end{array}$ & Actual & $\begin{array}{c}\text { Standard } \\
\text { Score }\end{array}$ & $\begin{array}{c}\text { How } \\
\text { Important }\end{array}$ & Acquisition & $\begin{array}{l}\text { Non- } \\
\text { Peer- } \\
\text { Review } \\
\text { Journal }\end{array}$ & English & Healthy & Daily & PC-C \\
\hline $\begin{array}{l}\text { Johnson, J.L. } \\
\text { et al. }(2002)\end{array}$ & 465 & $\begin{array}{c}\text { Pros and } \\
\text { Cons of } \\
\text { Bone } \\
\text { Density } \\
\text { Testing }\end{array}$ & Actual & $\begin{array}{l}\text { Standard } \\
\text { Score }\end{array}$ & $\begin{array}{c}\text { How } \\
\text { Important }\end{array}$ & Acquisition & $\begin{array}{l}\text { Non- } \\
\text { Peer- } \\
\text { Review } \\
\text { Journal }\end{array}$ & English & Healthy & Daily & C-PR \\
\hline $\begin{array}{c}\text { Johnson, J.L. } \\
\text { et al. (2002) }\end{array}$ & 419 & $\begin{array}{c}\text { Pros and } \\
\text { Cons of } \\
\text { Calcium } \\
\text { Intake }\end{array}$ & Actual & $\begin{array}{c}\text { Standard } \\
\text { Score }\end{array}$ & $\begin{array}{c}\text { How } \\
\text { Important }\end{array}$ & Acquisition & $\begin{array}{l}\text { Non- } \\
\text { Peer- } \\
\text { Review } \\
\text { Journal }\end{array}$ & English & Healthy & Daily & Multiple \\
\hline $\begin{array}{c}\text { Johnson, S.S. } \\
\text { et al. }(2002)\end{array}$ & 185 & $\begin{array}{c}\text { Pros and } \\
\text { Cons of } \\
\text { Participating } \\
\text { in Decision } \\
\text { Making for } \\
\text { Medication }\end{array}$ & Actual & $\begin{array}{c}\text { Standard } \\
\text { Score }\end{array}$ & $\begin{array}{c}\text { How } \\
\text { Important }\end{array}$ & Acquisition & $\begin{array}{l}\text { Non- } \\
\text { Peer- } \\
\text { Review } \\
\text { Journal }\end{array}$ & English & Healthy & Situational & $\mathrm{PC}-\mathrm{C}$ \\
\hline $\begin{array}{l}\text { Johnson, S.S } \\
\text { et al. }(2002)\end{array}$ & 296 & $\begin{array}{l}\text { Pros and } \\
\text { Cons of } \\
\text { Smoking }\end{array}$ & Reversed & Hedge's & $\begin{array}{c}\text { How } \\
\text { Important }\end{array}$ & Cessation & $\begin{array}{c}\text { Peer- } \\
\text { Review } \\
\text { Journal }\end{array}$ & English & Unhealthy & Daily & PC-C \\
\hline
\end{tabular}


Table B.2 (continued)

\begin{tabular}{|c|c|c|c|c|c|c|c|c|c|c|c|}
\hline $\begin{array}{l}\text { Source } \\
\text { (Year) }\end{array}$ & $\begin{array}{c}\text { N/ } \\
\text { data } \\
\text {-set } \\
\end{array}$ & $\begin{array}{c}\text { Description } \\
\text { of Pros and } \\
\text { Cons } \\
\end{array}$ & $\begin{array}{c}\text { Direction } \\
\text { of Pros } \\
\text { and Cons } \\
\end{array}$ & $\begin{array}{l}\text { Calcula- } \\
\text { tion } \\
\text { Method } \\
\end{array}$ & $\begin{array}{c}\text { Scale } \\
\text { Instruct }\end{array}$ & Cess/ Acq & $\begin{array}{c}\text { Publica- } \\
\text { tion } \\
\text { Status } \\
\end{array}$ & $\begin{array}{l}\text { Lan- } \\
\text { guage }\end{array}$ & $\begin{array}{l}\text { Health } \\
\text { Status }\end{array}$ & $\begin{array}{c}\text { Fre- } \\
\text { quency }\end{array}$ & $\begin{array}{l}\text { Cross- } \\
\text { over }\end{array}$ \\
\hline $\begin{array}{l}\text { Jordan } \\
(2002)\end{array}$ & 276 & $\begin{array}{c}\text { Pros and } \\
\text { Cons of } \\
\text { Recovering } \\
\text { from } \\
\text { Anorexia }\end{array}$ & Actual & Hedge's & $\begin{array}{c}\text { How } \\
\text { Important }\end{array}$ & Cessation & $\begin{array}{l}\text { Non- } \\
\text { Peer- } \\
\text { Review } \\
\text { Journal }\end{array}$ & English & Unhealthy & Daily & C-PR \\
\hline $\begin{array}{l}\text { Jordan } \\
(2002)\end{array}$ & 148 & $\begin{array}{c}\text { Pros and } \\
\text { Cons of } \\
\text { Recovering } \\
\text { from } \\
\text { Bulemia }\end{array}$ & Actual & Hedge's & $\begin{array}{c}\text { How } \\
\text { Important }\end{array}$ & Cessation & $\begin{array}{l}\text { Non- } \\
\text { Peer- } \\
\text { Review } \\
\text { Journal }\end{array}$ & English & Unhealthy & Daily & Multiple \\
\hline $\begin{array}{l}\text { Jordon et al. } \\
\qquad(2002)\end{array}$ & 223 & $\begin{array}{l}\text { Pros and } \\
\text { Cons of } \\
\text { Exercise }\end{array}$ & Actual & Hedge's & $\begin{array}{c}\text { How } \\
\text { Important }\end{array}$ & Acquisition & $\begin{array}{c}\text { Peer- } \\
\text { Review } \\
\text { Journal }\end{array}$ & English & Healthy & Daily & C-PR \\
\hline $\begin{array}{l}\text { Katlin } \\
(2001)\end{array}$ & 114 & $\begin{array}{c}\text { Pros and } \\
\text { Cons of } \\
\text { Using } \\
\text { Drugs/Alcoh } \\
\text { ol }\end{array}$ & Reversed & $\begin{array}{l}\text { Standard } \\
\text { Score }\end{array}$ & $\begin{array}{c}\text { How } \\
\text { Important }\end{array}$ & Cessation & $\begin{array}{l}\text { Non- } \\
\text { Peer- } \\
\text { Review } \\
\text { Journal }\end{array}$ & English & Unhealthy & Daily & A-M \\
\hline $\begin{array}{l}\text { Keller et al. } \\
\quad(2001)\end{array}$ & 149 & $\begin{array}{c}\text { Pros and } \\
\text { Cons of } \\
\text { Keeping } \\
\text { Body Posture }\end{array}$ & Actual & Hedge's & $\begin{array}{c}\text { How } \\
\text { Important }\end{array}$ & Acquisition & $\begin{array}{l}\text { Peer- } \\
\text { Review } \\
\text { Journal }\end{array}$ & $\begin{array}{l}\text { Non- } \\
\text { English }\end{array}$ & Healthy & Daily & PR-A \\
\hline $\begin{array}{l}\text { Keller et al. } \\
\quad(2001)\end{array}$ & 282 & $\begin{array}{c}\text { Pros and } \\
\text { Cons of Fruit } \\
\text { and } \\
\text { Vegetable } \\
\text { Consumption }\end{array}$ & Actual & Hedge's & $\begin{array}{c}\text { How } \\
\text { Important }\end{array}$ & Acquisition & $\begin{array}{c}\text { Peer- } \\
\text { Review } \\
\text { Journal }\end{array}$ & $\begin{array}{l}\text { Non- } \\
\text { English }\end{array}$ & Healthy & Daily & PC-C \\
\hline
\end{tabular}


Table B.2 (continued)

\begin{tabular}{|c|c|c|c|c|c|c|c|c|c|c|c|}
\hline $\begin{array}{l}\text { Source } \\
\text { (Year) }\end{array}$ & $\begin{array}{c}\text { N/ } \\
\text { data } \\
\text {-set }\end{array}$ & $\begin{array}{c}\text { Description } \\
\text { of Pros and } \\
\text { Cons }\end{array}$ & $\begin{array}{l}\text { Direction } \\
\text { of Pros } \\
\text { and Cons }\end{array}$ & $\begin{array}{l}\text { Calcula- } \\
\text { tion } \\
\text { Method }\end{array}$ & $\begin{array}{c}\text { Scale } \\
\text { Instruct }\end{array}$ & Cess/Acq & \begin{tabular}{|l|} 
Publica- \\
tion \\
Status \\
\end{tabular} & $\begin{array}{l}\text { Lan- } \\
\text { guage }\end{array}$ & $\begin{array}{l}\text { Health } \\
\text { Status }\end{array}$ & $\begin{array}{c}\text { Frem } \\
\text { quency }\end{array}$ & $\begin{array}{c}\text { Cross- } \\
\text { over }\end{array}$ \\
\hline $\begin{array}{c}\text { Keller et al. } \\
\text { (2002) }\end{array}$ & 276 & $\begin{array}{c}\text { Pros and } \\
\text { Cons of Oral } \\
\text { Hygiene }\end{array}$ & Actual & Hedge's & $\begin{array}{c}\text { How } \\
\text { Important }\end{array}$ & Acquisition & $\begin{array}{c}\text { Non- } \\
\text { Peer- } \\
\text { Review } \\
\text { Journal }\end{array}$ & $\begin{array}{l}\text { Non- } \\
\text { English }\end{array}$ & Healthy & Daily & C-PR \\
\hline $\begin{array}{l}\text { Keller et al. } \\
\qquad(2003)\end{array}$ & 665 & $\begin{array}{c}\text { Pros and } \\
\text { Cons of } \\
\text { Stress } \\
\text { Management }\end{array}$ & Actual & Hedge's & $\begin{array}{c}\text { How } \\
\text { Important }\end{array}$ & Acquisition & $\begin{array}{c}\text { Non- } \\
\text { Peer- } \\
\text { Review } \\
\text { Journal }\end{array}$ & $\begin{array}{l}\text { Non- } \\
\text { English }\end{array}$ & Unhealthy & Daily & PC-C \\
\hline $\begin{array}{l}\text { Keller } \\
(1998)\end{array}$ & 405 & $\begin{array}{l}\text { Pros and } \\
\text { Cons of } \\
\text { Healthy } \\
\text { Eating }\end{array}$ & Actual & Hedge's & $\begin{array}{c}\text { How } \\
\text { Important }\end{array}$ & Acquisition & $\begin{array}{c}\text { Non- } \\
\text { Peer- } \\
\text { Review } \\
\text { Journal }\end{array}$ & $\begin{array}{l}\text { Non- } \\
\text { English }\end{array}$ & Healthy & Daily & PC-C \\
\hline $\begin{array}{l}\text { Keller } \\
(1998)\end{array}$ & 407 & $\begin{array}{c}\text { Pros and } \\
\text { Cons of } \\
\text { Healthy } \\
\text { Eating }\end{array}$ & Actual & Hedge's & $\begin{array}{c}\text { How } \\
\text { Important }\end{array}$ & Acquisition & \begin{tabular}{|c|} 
Non- \\
Peer- \\
Review \\
Journal \\
\end{tabular} & $\begin{array}{l}\text { Non- } \\
\text { English }\end{array}$ & Healthy & Daily & $\begin{array}{l}\text { No } \\
\text { Cross }\end{array}$ \\
\hline $\begin{array}{c}\text { Keller et al. } \\
\text { (1999) }\end{array}$ & 401 & $\begin{array}{l}\text { Pros and } \\
\text { Cons of } \\
\text { Quitting } \\
\text { Smoking }\end{array}$ & Actual & Hedge's & $\begin{array}{c}\text { How } \\
\text { Important }\end{array}$ & Cessation & $\begin{array}{c}\text { Peer- } \\
\text { Review } \\
\text { Journal }\end{array}$ & $\begin{array}{l}\text { Non- } \\
\text { English }\end{array}$ & Unhealthy & Daily & C-PR \\
\hline $\begin{array}{c}\text { King et al. } \\
\text { (1996) }\end{array}$ & 332 & $\begin{array}{l}\text { Pros and } \\
\text { Cons of } \\
\text { Exercise }\end{array}$ & Actual & Hedge's & $\begin{array}{c}\text { How } \\
\text { Important }\end{array}$ & Acquisition & $\begin{array}{c}\text { Peer- } \\
\text { Review } \\
\text { Journal }\end{array}$ & English & Healthy & Daily & Multiple \\
\hline $\begin{array}{l}\text { King et al. } \\
\text { (1996) }\end{array}$ & 296 & $\begin{array}{l}\text { Pros and } \\
\text { Cons of } \\
\text { Smoking }\end{array}$ & Reversed & Hedge's & $\begin{array}{c}\text { How } \\
\text { Important }\end{array}$ & Cessation & $\begin{array}{c}\text { Peer- } \\
\text { Review } \\
\text { Journal }\end{array}$ & English & Unhealthy & Daily & PC-C \\
\hline
\end{tabular}


Table B.2 (continued)

\begin{tabular}{|c|c|c|c|c|c|c|c|c|c|c|c|}
\hline $\begin{array}{l}\text { Source } \\
\text { (Year) }\end{array}$ & $\begin{array}{c}\text { N/ } \\
\text { data } \\
\text {-set } \\
\end{array}$ & $\begin{array}{c}\text { Description } \\
\text { of Pros and } \\
\text { Cons }\end{array}$ & $\begin{array}{c}\text { Direction } \\
\text { of Pros } \\
\text { and Cons } \\
\end{array}$ & $\begin{array}{c}\text { Calcula- } \\
\text { tion } \\
\text { Method } \\
\end{array}$ & $\begin{array}{c}\text { Scale } \\
\text { Instruct }\end{array}$ & Cess/ Acq & $\begin{array}{c}\text { Publica- } \\
\text { tion } \\
\text { Status } \\
\end{array}$ & $\begin{array}{l}\text { Lan- } \\
\text { guage }\end{array}$ & $\begin{array}{l}\text { Health } \\
\text { Status }\end{array}$ & $\begin{array}{c}\text { Fre- } \\
\text { quency }\end{array}$ & $\begin{array}{c}\text { Cross- } \\
\text { over }\end{array}$ \\
\hline $\begin{array}{l}\text { Kraft et al. } \\
\text { (1999) }\end{array}$ & 421 & $\begin{array}{l}\text { Pros and } \\
\text { Cons of } \\
\text { Smoking }\end{array}$ & Reversed & $\begin{array}{c}\text { Standard } \\
\text { Score }\end{array}$ & $\begin{array}{c}\text { How } \\
\text { Important }\end{array}$ & Cessation & $\begin{array}{c}\text { Peer- } \\
\text { Review } \\
\text { Journal }\end{array}$ & $\begin{array}{l}\text { Non- } \\
\text { English }\end{array}$ & Unhealthy & Daily & PC-C \\
\hline $\begin{array}{l}\text { Kremers et al. } \\
(2001)\end{array}$ & $\begin{array}{c}2153 \\
5\end{array}$ & $\begin{array}{l}\text { Pros and } \\
\text { Cons of } \\
\text { Smoking }\end{array}$ & Actual & $\begin{array}{c}\text { Standard } \\
\text { Score }\end{array}$ & NR & Acquisition & $\begin{array}{c}\text { Peer- } \\
\text { Review } \\
\text { Journal } \\
\end{array}$ & $\begin{array}{l}\text { Non- } \\
\text { English }\end{array}$ & Prevention & Situational & $\mathrm{OK}$ \\
\hline $\begin{array}{c}\text { Lauby et al. } \\
\text { (1998) }\end{array}$ & 2361 & $\begin{array}{c}\text { Pros and } \\
\text { Cons of } \\
\text { General } \\
\text { Contraceptio } \\
\mathbf{n} \\
\end{array}$ & Actual & $\begin{array}{c}\text { Standard } \\
\text { Score }\end{array}$ & $\begin{array}{c}\text { How } \\
\text { Important }\end{array}$ & Acquisition & $\begin{array}{c}\text { Peer- } \\
\text { Review } \\
\text { Journal }\end{array}$ & English & Healthy & Situational & C-PR \\
\hline $\begin{array}{l}\text { Lauby et al. } \\
\text { (1998) }\end{array}$ & 2925 & $\begin{array}{c}\text { Pros and } \\
\text { Cons of } \\
\text { Condom Use } \\
\text { with Main } \\
\text { Partner }\end{array}$ & Actual & $\begin{array}{c}\text { Standard } \\
\text { Score }\end{array}$ & $\begin{array}{c}\text { How } \\
\text { Important }\end{array}$ & Acquisition & $\begin{array}{c}\text { Peer- } \\
\text { Review } \\
\text { Journal }\end{array}$ & English & Healthy & Situational & PC-C \\
\hline $\begin{array}{l}\text { Lauby et al. } \\
\text { (1998) }\end{array}$ & 1213 & $\begin{array}{c}\text { Pros and } \\
\text { Cons of } \\
\text { Condom Use } \\
\text { with Other } \\
\text { Partners }\end{array}$ & Actual & $\begin{array}{c}\text { Standard } \\
\text { Score }\end{array}$ & $\begin{array}{c}\text { How } \\
\text { Important }\end{array}$ & Acquisition & $\begin{array}{c}\text { Peer- } \\
\text { Review } \\
\text { Journal }\end{array}$ & English & Healthy & Situational & PR-A \\
\hline $\begin{array}{l}\text { Lerner } \\
(1990)\end{array}$ & 159 & $\begin{array}{c}\text { Pros and } \\
\text { Cons of } \\
\text { Changing } \\
\text { Delinquent } \\
\text { Behavior }\end{array}$ & Actual & Hedge's & $\begin{array}{c}\text { How } \\
\text { Important }\end{array}$ & Cessation & $\begin{array}{c}\text { Non- } \\
\text { Peer- } \\
\text { Review } \\
\text { Journal }\end{array}$ & English & Other & Situational & C-A \\
\hline
\end{tabular}


Table B.2 (continued)

\begin{tabular}{|c|c|c|c|c|c|c|c|c|c|c|c|}
\hline $\begin{array}{l}\text { Source } \\
\text { (Year) }\end{array}$ & $\begin{array}{c}\text { N/ } \\
\text { data } \\
\text {-set }\end{array}$ & $\begin{array}{c}\text { Description } \\
\text { of Pros and } \\
\text { Cons }\end{array}$ & $\begin{array}{c}\text { Direction } \\
\text { of Pros } \\
\text { and Cons }\end{array}$ & $\begin{array}{l}\text { Calcula- } \\
\text { tion } \\
\text { Method }\end{array}$ & $\begin{array}{c}\text { Scale } \\
\text { Instruct }\end{array}$ & Cess/ Acq & $\begin{array}{l}\text { Publica- } \\
\text { tion } \\
\text { Status } \\
\end{array}$ & $\begin{array}{l}\text { Lan- } \\
\text { guage }\end{array}$ & $\begin{array}{l}\text { Health } \\
\text { Status }\end{array}$ & $\begin{array}{c}\text { Fre- } \\
\text { quency }\end{array}$ & $\begin{array}{l}\text { Cross- } \\
\text { over }\end{array}$ \\
\hline $\begin{array}{l}\text { Levesque et } \\
\text { al. }(2000)\end{array}$ & 134 & $\begin{array}{l}\text { Pros and } \\
\text { Cons of } \\
\text { getting } \\
\text { involved in } \\
\text { the change- } \\
\text { over to } \\
\text { PeopleSoft }\end{array}$ & Actual & Hedge's & $\begin{array}{c}\text { How } \\
\text { Important }\end{array}$ & Acquisition & $\begin{array}{l}\text { Non- } \\
\text { Peer- } \\
\text { Review } \\
\text { Journal }\end{array}$ & English & Other & Situational & $\begin{array}{l}\text { PC- } \\
\text { C/PR }\end{array}$ \\
\hline $\begin{array}{l}\text { Levesque } \\
\text { (1999) }\end{array}$ & 256 & $\begin{array}{l}\text { Pros and } \\
\text { Cons of } \\
\text { Ending } \\
\text { Violence }\end{array}$ & Actual & Hedge's & $\begin{array}{c}\text { Agree / } \\
\text { Disagree }\end{array}$ & Cessation & $\begin{array}{c}\text { Non- } \\
\text { Peer- } \\
\text { Review } \\
\text { Journal }\end{array}$ & English & Other & Situational & Multiple \\
\hline $\begin{array}{l}\text { Levesque et } \\
\text { al. (1999) }\end{array}$ & 31 & \begin{tabular}{|c|} 
Pros and \\
Cons of \\
Collaborative \\
Service \\
Delivery
\end{tabular} & Actual & $\begin{array}{l}\text { Standard } \\
\text { Score }\end{array}$ & $\begin{array}{c}\text { How } \\
\text { Important }\end{array}$ & Acquisition & $\begin{array}{c}\text { Peer- } \\
\text { Review } \\
\text { Journal }\end{array}$ & English & Other & Situational & C-A \\
\hline $\begin{array}{l}\text { Levesque et } \\
\text { al. (1999) }\end{array}$ & 181 & $\begin{array}{l}\text { Pros and } \\
\text { Cons of } \\
\text { Using } \\
\text { Depression } \\
\text { Prevention } \\
\text { Strategies }\end{array}$ & Actual & $\begin{array}{l}\text { Standard } \\
\text { Score }\end{array}$ & $\begin{array}{c}\text { How } \\
\text { Important }\end{array}$ & Acquisition & $\begin{array}{l}\text { Non- } \\
\text { Peer- } \\
\text { Review } \\
\text { Journal }\end{array}$ & English & Other & Daily & Multiple \\
\hline $\begin{array}{l}\text { Lowry } \\
(2000)\end{array}$ & 99 & $\begin{array}{l}\text { Pros and } \\
\text { Cons of } \\
\text { Using } \\
\text { Smokeless } \\
\text { Tobacco }\end{array}$ & Reversed & Hedge's & $\begin{array}{c}\text { How } \\
\text { Important }\end{array}$ & Cessation & $\begin{array}{l}\text { Non- } \\
\text { Peer- } \\
\text { Review } \\
\text { Journal }\end{array}$ & English & Unhealthy & Daily & NA \\
\hline
\end{tabular}


Table B.2 (continued)

\begin{tabular}{|c|c|c|c|c|c|c|c|c|c|c|c|}
\hline $\begin{array}{l}\text { Source } \\
\text { (Year) }\end{array}$ & $\begin{array}{c}\text { N/ } \\
\text { data } \\
\text {-set }\end{array}$ & $\begin{array}{c}\text { Description } \\
\text { of Pros and } \\
\text { Cons }\end{array}$ & $\begin{array}{c}\text { Direction } \\
\text { of Pros } \\
\text { and Cons }\end{array}$ & $\begin{array}{l}\text { Calcula- } \\
\text { tion } \\
\text { Method }\end{array}$ & $\begin{array}{c}\text { Scale } \\
\text { Instruct }\end{array}$ & Cess/ Acq & \begin{tabular}{|c} 
Publica- \\
tion \\
Status
\end{tabular} & $\begin{array}{l}\text { Lan- } \\
\text { guage }\end{array}$ & $\begin{array}{l}\text { Health } \\
\text { Status }\end{array}$ & $\begin{array}{c}\text { Fre- } \\
\text { quency }\end{array}$ & $\begin{array}{l}\text { Cross- } \\
\text { over }\end{array}$ \\
\hline $\begin{array}{l}\text { Maier } \\
(2002)\end{array}$ & 251 & $\begin{array}{l}\text { Pros and } \\
\text { Cons of } \\
\text { Exercise }\end{array}$ & Actual & Hedge's & $\begin{array}{c}\text { How } \\
\text { Important }\end{array}$ & Acquisition & \begin{tabular}{|c|} 
Non- \\
Peer- \\
Review \\
Journal
\end{tabular} & $\begin{array}{l}\text { Non- } \\
\text { English }\end{array}$ & Healthy & Daily & PR-A \\
\hline $\begin{array}{c}\text { Ma et al. } \\
(2002)\end{array}$ & 1545 & \begin{tabular}{|c|} 
Pros and \\
Cons of Fruit \\
Intake \\
\end{tabular} & Actual & $\begin{array}{l}\text { Standard } \\
\text { Score }\end{array}$ & $\begin{array}{c}\text { How } \\
\text { Important }\end{array}$ & Acquisition & $\begin{array}{c}\text { Peer- } \\
\text { Review } \\
\text { Journal }\end{array}$ & English & Healthy & Daily & C-PR \\
\hline $\begin{array}{c}\text { Ma et al. } \\
(2002)\end{array}$ & 1545 & $\begin{array}{l}\text { Pros and } \\
\text { Cons of } \\
\text { Vegetable } \\
\text { Intake }\end{array}$ & Actual & $\begin{array}{c}\text { Standard } \\
\text { Score }\end{array}$ & $\begin{array}{c}\text { How } \\
\text { Important }\end{array}$ & Acquisition & $\begin{array}{l}\text { Peer- } \\
\text { Review } \\
\text { Journal }\end{array}$ & English & Healthy & Daily & C-PR \\
\hline $\begin{array}{c}\text { Maleszka } \\
(2002)\end{array}$ & 216 & $\begin{array}{l}\text { Pros and } \\
\text { Cons of } \\
\text { Quitting } \\
\text { Smoking }\end{array}$ & Actual & Hedge's & $\begin{array}{c}\text { How } \\
\text { Important }\end{array}$ & Cessation & \begin{tabular}{|c|} 
Non- \\
Peer- \\
Review \\
Journal
\end{tabular} & $\begin{array}{l}\text { Non- } \\
\text { English }\end{array}$ & Unhealthy & Daily & C-PR \\
\hline $\begin{array}{c}\text { Manne et al. } \\
(2002)\end{array}$ & 480 & $\begin{array}{l}\text { Pros and } \\
\text { Cons of } \\
\text { Colorectal } \\
\text { Cancer } \\
\text { Screening }\end{array}$ & Actual & Hedge's & $\begin{array}{c}\text { Agree / } \\
\text { Disagree }\end{array}$ & Acquisition & $\begin{array}{c}\text { Peer- } \\
\text { Review } \\
\text { Journal }\end{array}$ & English & Healthy & Yearly + & OK \\
\hline $\begin{array}{l}\text { Marcus et al. } \\
\text { (1992) }\end{array}$ & 717 & $\begin{array}{l}\text { Pros and } \\
\text { Cons of } \\
\text { Exercise }\end{array}$ & Actual & Hedge's & $\begin{array}{c}\text { How } \\
\text { Important }\end{array}$ & Acquisition & $\begin{array}{l}\text { Peer- } \\
\text { Review } \\
\text { Journal }\end{array}$ & English & Healthy & Daily & C-PR \\
\hline $\begin{array}{l}\text { Marcus et al. } \\
\quad(1994)\end{array}$ & 431 & $\begin{array}{l}\text { Pros and } \\
\text { Cons of } \\
\text { Exercise }\end{array}$ & Actual & Hedge's & $\begin{array}{c}\text { How } \\
\text { Important }\end{array}$ & Acquisition & $\begin{array}{c}\text { Peer- } \\
\text { Review } \\
\text { Journal }\end{array}$ & English & Healthy & Daily & PR-A \\
\hline
\end{tabular}


Table B.2 (continued)

\begin{tabular}{|c|c|c|c|c|c|c|c|c|c|c|c|}
\hline $\begin{array}{l}\text { Source } \\
\text { (Year) }\end{array}$ & $\begin{array}{c}\text { N/ } \\
\text { data } \\
\text {-set }\end{array}$ & $\begin{array}{c}\text { Description } \\
\text { of Pros and } \\
\text { Cons }\end{array}$ & $\begin{array}{c}\text { Direction } \\
\text { of Pros } \\
\text { and Cons }\end{array}$ & $\begin{array}{c}\text { Calcula- } \\
\text { tion } \\
\text { Method }\end{array}$ & $\begin{array}{c}\text { Scale } \\
\text { Instruct }\end{array}$ & Cess/ Acq & $\begin{array}{c}\text { Publica- } \\
\text { tion } \\
\text { Status }\end{array}$ & $\begin{array}{c}\text { Lan- } \\
\text { guage }\end{array}$ & $\begin{array}{l}\text { Health } \\
\text { Status }\end{array}$ & $\begin{array}{c}\text { Fre- } \\
\text { quency }\end{array}$ & $\begin{array}{l}\text { Cross- } \\
\text { over }\end{array}$ \\
\hline $\begin{array}{l}\text { Mauriello } \\
(2001)\end{array}$ & 294 & $\begin{array}{l}\text { Pros and } \\
\text { Cons of } \\
\text { Managing } \\
\text { Stress }\end{array}$ & Actual & Hedge's & $\begin{array}{c}\text { How } \\
\text { Important }\end{array}$ & Acquisition & $\begin{array}{c}\text { Non- } \\
\text { Peer- } \\
\text { Review } \\
\text { Journal }\end{array}$ & English & Unhealthy & Daily & PC-C \\
\hline $\begin{array}{l}\text { Mettler et al. } \\
\qquad(2000)\end{array}$ & 690 & $\begin{array}{l}\text { Pros and } \\
\text { Cons of } \\
\text { Exercise }\end{array}$ & Actual & Hedge's & $\begin{array}{c}\text { How } \\
\text { Important }\end{array}$ & Acquisition & $\begin{array}{c}\text { Peer- } \\
\text { Review } \\
\text { Journal }\end{array}$ & English & Healthy & Daily & NA \\
\hline $\begin{array}{l}\text { Migneault et } \\
\text { al. (1997) }\end{array}$ & 421 & $\begin{array}{c}\text { Pros and } \\
\text { Cons of } \\
\text { Alcohol Use }\end{array}$ & Actual & Hedge's & $\begin{array}{c}\text { How } \\
\text { Important }\end{array}$ & Acquisition & $\begin{array}{c}\text { Peer- } \\
\text { Review } \\
\text { Journal }\end{array}$ & English & Prevention & Situational & $\mathrm{PC}-\mathrm{C}$ \\
\hline $\begin{array}{l}\text { Migneault et } \\
\text { al. (1997) }\end{array}$ & 255 & $\begin{array}{c}\text { Pros and } \\
\text { Cons of } \\
\text { Alcohol Use }\end{array}$ & Reversed & Hedge's & $\begin{array}{c}\text { How } \\
\text { Important }\end{array}$ & Cessation & $\begin{array}{c}\text { Peer- } \\
\text { Review } \\
\text { Journal }\end{array}$ & English & Unhealthy & Situational & PR-A \\
\hline $\begin{array}{l}\text { Moll } \\
(2001)\end{array}$ & 287 & $\begin{array}{l}\text { Pros and } \\
\text { Cons of } \\
\text { Exercise }\end{array}$ & Actual & Hedge's & $\begin{array}{c}\text { How } \\
\text { Important }\end{array}$ & Acquisition & $\begin{array}{c}\text { Non- } \\
\text { Peer- } \\
\text { Review } \\
\text { Journal }\end{array}$ & $\begin{array}{l}\text { Non- } \\
\text { English }\end{array}$ & Healthy & Daily & C-PR \\
\hline $\begin{array}{l}\text { Morrison- } \\
\text { Beedy et al. } \\
\quad(2001)\end{array}$ & 254 & $\begin{array}{c}\text { Pros and } \\
\text { Cons of } \\
\text { Condom Use } \\
\text { with Main } \\
\text { Partners } \\
\end{array}$ & Actual & $\begin{array}{c}\text { Standard } \\
\text { Score }\end{array}$ & $\begin{array}{c}\text { How } \\
\text { Important }\end{array}$ & Acquisition & $\begin{array}{c}\text { Peer- } \\
\text { Review } \\
\text { Journal }\end{array}$ & English & Healthy & Situational & NA \\
\hline $\begin{array}{l}\text { Morrison- } \\
\text { Beedy et al. } \\
\quad(2001)\end{array}$ & 110 & $\begin{array}{c}\text { Pros and } \\
\text { Cons of } \\
\text { Condom Use } \\
\text { With Other } \\
\text { Partners }\end{array}$ & Actual & $\begin{array}{c}\text { Standard } \\
\text { Score }\end{array}$ & $\begin{array}{c}\text { How } \\
\text { Important }\end{array}$ & Acquisition & $\begin{array}{c}\text { Peer- } \\
\text { Review } \\
\text { Journal }\end{array}$ & English & Healthy & Situational & NA \\
\hline
\end{tabular}


Table B.2 (continued)

\begin{tabular}{|c|c|c|c|c|c|c|c|c|c|c|c|}
\hline $\begin{array}{c}\text { Source } \\
\text { (Year) }\end{array}$ & $\begin{array}{c}\mathrm{N} / \\
\text { data } \\
\text {-set }\end{array}$ & $\begin{array}{c}\text { Description } \\
\text { of Pros and } \\
\text { Cons }\end{array}$ & $\begin{array}{c}\text { Direction } \\
\text { of Pros } \\
\text { and Cons }\end{array}$ & $\begin{array}{l}\text { Calcula- } \\
\text { tion } \\
\text { Method }\end{array}$ & $\begin{array}{c}\text { Scale } \\
\text { Instruct }\end{array}$ & Cess/Acq & \begin{tabular}{|c} 
Publica- \\
tion \\
Status
\end{tabular} & $\begin{array}{c}\text { Lan- } \\
\text { guage }\end{array}$ & $\begin{array}{l}\text { Health } \\
\text { Status }\end{array}$ & $\begin{array}{c}\text { Fre- } \\
\text { quency }\end{array}$ & $\begin{array}{l}\text { Cross- } \\
\text { over }\end{array}$ \\
\hline $\begin{array}{l}\text { Nigg et al. } \\
\text { (1998) }\end{array}$ & 240 & $\begin{array}{l}\text { Pros and } \\
\text { Cons of } \\
\text { Exercise }\end{array}$ & Actual & $\begin{array}{l}\text { Standard } \\
\text { Score }\end{array}$ & $\begin{array}{c}\text { How } \\
\text { Important }\end{array}$ & Acquisition & $\begin{array}{c}\text { Non- } \\
\text { Peer- } \\
\text { Review } \\
\text { Journal }\end{array}$ & English & Healthy & Daily & C-PR \\
\hline $\begin{array}{l}\text { Nigg et al. } \\
\text { (1998) }\end{array}$ & 346 & $\begin{array}{l}\text { Pros and } \\
\text { Cons of } \\
\text { Exercise }\end{array}$ & Actual & $\begin{array}{l}\text { Standard } \\
\text { Score }\end{array}$ & $\begin{array}{c}\text { How } \\
\text { Important }\end{array}$ & Acquisition & $\begin{array}{c}\text { Non- } \\
\text { Peer- } \\
\text { Review } \\
\text { Journal }\end{array}$ & English & Healthy & Daily & PR-A \\
\hline $\begin{array}{c}\text { Nigg \& } \\
\text { Courneya } \\
(1998)\end{array}$ & 819 & $\begin{array}{l}\text { Pros and } \\
\text { Cons of } \\
\text { Exercise }\end{array}$ & Actual & Hedge's & $\begin{array}{c}\text { How } \\
\text { Important }\end{array}$ & Acquisition & $\begin{array}{c}\text { Peer- } \\
\text { Review } \\
\text { Journal }\end{array}$ & English & Healthy & Daily & PR-A \\
\hline $\begin{array}{c}\text { Noar et al } \\
(1998)\end{array}$ & 168 & $\begin{array}{c}\text { Pros and } \\
\text { Cons of } \\
\text { Condom Use }\end{array}$ & Actual & Hedge's & $\begin{array}{c}\text { How } \\
\text { Important }\end{array}$ & Acquisition & $\begin{array}{c}\text { Non- } \\
\text { Peer- } \\
\text { Review } \\
\text { Journal }\end{array}$ & English & Healthy & Situational & C-PR \\
\hline $\begin{array}{c}\text { Norman et al. } \\
\qquad(2002)\end{array}$ & 178 & $\begin{array}{c}\text { Pros and } \\
\text { Cons of } \\
\text { sedentary } \\
\text { behavior }\end{array}$ & Actual & $\begin{array}{l}\text { Standard } \\
\text { Score }\end{array}$ & $\begin{array}{c}\text { How } \\
\text { Important }\end{array}$ & Cessation & $\begin{array}{c}\text { Non- } \\
\text { Peer- } \\
\text { Review } \\
\text { Journal }\end{array}$ & English & Unhealthy & Daily & C-PR \\
\hline $\begin{array}{c}\text { Norman et al. } \\
\qquad(2002)\end{array}$ & 254 & $\begin{array}{c}\text { Pros and } \\
\text { Cons of } \\
\text { sedentary } \\
\text { behavior }\end{array}$ & Actual & $\begin{array}{l}\text { Standard } \\
\text { Score }\end{array}$ & $\begin{array}{c}\text { How } \\
\text { Important }\end{array}$ & Cessation & $\begin{array}{c}\text { Non- } \\
\text { Peer- } \\
\text { Review } \\
\text { Journal }\end{array}$ & English & Unhealthy & Daily & C-PR \\
\hline $\begin{array}{c}\text { O'Connell \& } \\
\text { Velicer } \\
(1988)\end{array}$ & 123 & $\begin{array}{c}\text { Pros and } \\
\text { Cons of } \\
\text { Weight Loss }\end{array}$ & Actual & Hedge's & $\begin{array}{c}\text { How } \\
\text { Important }\end{array}$ & Cessation & $\begin{array}{c}\text { Peer- } \\
\text { Review } \\
\text { Journal }\end{array}$ & English & Unhealthy & Daily & PC-C \\
\hline
\end{tabular}


Table B.2 (continued)

\begin{tabular}{|c|c|c|c|c|c|c|c|c|c|c|c|}
\hline $\begin{array}{c}\text { Source } \\
\text { (Year) }\end{array}$ & \begin{tabular}{|c|} 
N/ \\
data \\
-set
\end{tabular} & $\begin{array}{c}\text { Description } \\
\text { of Pros and } \\
\text { Cons }\end{array}$ & $\begin{array}{l}\text { Direction } \\
\text { of Pros } \\
\text { and Cons }\end{array}$ & \begin{tabular}{|l|} 
Calcula- \\
tion \\
Method \\
\end{tabular} & $\begin{array}{l}\text { Scale } \\
\text { Instruct }\end{array}$ & Cess/ Acq & $\begin{array}{l}\text { Publica- } \\
\text { tion } \\
\text { Status }\end{array}$ & $\begin{array}{l}\text { Lan- } \\
\text { guage }\end{array}$ & $\begin{array}{l}\text { Health } \\
\text { Status }\end{array}$ & $\begin{array}{c}\text { Fre- } \\
\text { quency }\end{array}$ & $\begin{array}{l}\text { Cross- } \\
\text { over }\end{array}$ \\
\hline $\begin{array}{l}\text { Otake \& } \\
\text { Shimai } \\
(2001)\end{array}$ & 556 & $\begin{array}{l}\text { Pros and } \\
\text { Cons of } \\
\text { Smoking }\end{array}$ & Actual & $\begin{array}{c}\text { Standard } \\
\text { Score }\end{array}$ & $\begin{array}{c}\text { How } \\
\text { Important }\end{array}$ & Acquisition & $\begin{array}{c}\text { Peer- } \\
\text { Review } \\
\text { Journal }\end{array}$ & $\begin{array}{l}\text { Non- } \\
\text { English }\end{array}$ & Prevention & Situational & $\mathrm{PC}-\mathrm{C}$ \\
\hline $\begin{array}{c}\text { Otake \& } \\
\text { Shimai } \\
(2001) \\
\end{array}$ & 1002 & $\begin{array}{l}\text { Pros and } \\
\text { Cons of } \\
\text { Smoking }\end{array}$ & Actual & $\begin{array}{c}\text { Standard } \\
\text { Score }\end{array}$ & $\begin{array}{c}\text { How } \\
\text { Important }\end{array}$ & Acquisition & $\begin{array}{c}\text { Peer- } \\
\text { Review } \\
\text { Journal } \\
\end{array}$ & $\begin{array}{l}\text { Non- } \\
\text { English }\end{array}$ & Prevention & Situational & PC-C \\
\hline $\begin{array}{l}\text { Pallonen et al. } \\
\qquad(1998)\end{array}$ & 505 & $\begin{array}{l}\text { Pros and } \\
\text { Cons of } \\
\text { Smoking }\end{array}$ & Actual & Hedge's & $\begin{array}{c}\text { Agree / } \\
\text { Disagree }\end{array}$ & Acquisition & $\begin{array}{c}\text { Peer- } \\
\text { Review } \\
\text { Journal }\end{array}$ & English & Prevention & Situational & NA \\
\hline $\begin{array}{l}\text { Pallonen et al. } \\
\qquad(1998)\end{array}$ & 254 & $\begin{array}{l}\text { Pros and } \\
\text { Cons of } \\
\text { Smoking }\end{array}$ & Reversed & Hedge's & $\begin{array}{c}\text { Agree / } \\
\text { Disagree }\end{array}$ & Cessation & $\begin{array}{c}\text { Peer- } \\
\text { Review } \\
\text { Journal }\end{array}$ & English & Unhealthy & Daily & NA \\
\hline $\begin{array}{l}\text { Park et al. } \\
\quad(2003)\end{array}$ & 259 & $\begin{array}{l}\text { Pros and } \\
\text { Cons of } \\
\text { Providing } \\
\text { Smoking } \\
\text { Cessation } \\
\text { Interventions }\end{array}$ & Actual & Hedge's & $\begin{array}{c}\text { How } \\
\text { Important }\end{array}$ & Acquisition & $\begin{array}{c}\text { Peer- } \\
\text { Review } \\
\text { Journal }\end{array}$ & English & Other & Situational & C-PR \\
\hline $\begin{array}{l}\text { Perez } \\
(2001)\end{array}$ & 828 & $\begin{array}{c}\text { Pros and } \\
\text { Cons of } \\
\text { Condom Use }\end{array}$ & Actual & Hedge's & $\begin{array}{c}\text { How } \\
\text { Important }\end{array}$ & Acquisition & $\begin{array}{c}\text { Non- } \\
\text { Peer- } \\
\text { Review } \\
\text { Journal }\end{array}$ & English & Healthy & Situational & C-PR \\
\hline $\begin{array}{l}\text { Plummer et } \\
\text { al. (2001) }\end{array}$ & 2010 & $\begin{array}{l}\text { Pros and } \\
\text { Cons of } \\
\text { Smoking }\end{array}$ & Actual & Hedge's & $\begin{array}{c}\text { How } \\
\text { Important }\end{array}$ & Acquisition & $\begin{array}{c}\text { Peer- } \\
\text { Review } \\
\text { Journal }\end{array}$ & English & Prevention & Situational & NA \\
\hline
\end{tabular}


Table B.2 (continued)

\begin{tabular}{|c|c|c|c|c|c|c|c|c|c|c|c|}
\hline $\begin{array}{l}\text { Source } \\
\text { (Year) }\end{array}$ & $\begin{array}{c}\text { N/ } \\
\text { data } \\
\text {-set } \\
\end{array}$ & $\begin{array}{c}\text { Description } \\
\text { of Pros and } \\
\text { Cons } \\
\end{array}$ & $\begin{array}{c}\text { Direction } \\
\text { of Pros } \\
\text { and Cons }\end{array}$ & \begin{tabular}{|c|} 
Calcula- \\
tion \\
Method \\
\end{tabular} & $\begin{array}{c}\text { Scale } \\
\text { Instruct }\end{array}$ & Cess/ Acq & \begin{tabular}{|c|} 
Publica- \\
tion \\
Status \\
\end{tabular} & $\begin{array}{l}\text { Lan- } \\
\text { guage }\end{array}$ & $\begin{array}{l}\text { Health } \\
\text { Status }\end{array}$ & $\begin{array}{c}\text { Fre- } \\
\text { quency }\end{array}$ & $\begin{array}{c}\text { Cross- } \\
\text { over }\end{array}$ \\
\hline $\begin{array}{l}\text { Plummer et } \\
\text { al. (2001) }\end{array}$ & 798 & $\begin{array}{c}\text { Pros and } \\
\text { Cons of } \\
\text { Smoking }\end{array}$ & Reversed & Hedge's & $\begin{array}{c}\text { How } \\
\text { Important }\end{array}$ & Cessation & \begin{tabular}{|c|} 
Peer- \\
Review \\
Journal \\
\end{tabular} & English & Unhealthy & Daily & Multiple \\
\hline $\begin{array}{c}\text { Prochaska, JJ } \\
\text { et al. }(2003)\end{array}$ & 322 & $\begin{array}{l}\text { Pros and } \\
\text { Cons of } \\
\text { Smoking }\end{array}$ & Reversed & Hedge's & $\begin{array}{c}\text { How } \\
\text { Important }\end{array}$ & Cessation & \begin{tabular}{|c|} 
Non- \\
Peer- \\
Review \\
Journal \\
\end{tabular} & English & Unhealthy & Daily & C-PR \\
\hline $\begin{array}{c}\text { Prochaska et } \\
\text { al. (1994) }\end{array}$ & 325 & $\begin{array}{c}\text { Pros and } \\
\text { Cons of } \\
\text { Condom Use } \\
\text { for Anal Sex } \\
\text { w/Main } \\
\text { Partner }\end{array}$ & Actual & $\begin{array}{c}\text { Standard } \\
\text { Score }\end{array}$ & $\begin{array}{c}\text { How } \\
\text { Important }\end{array}$ & Acquisition & $\begin{array}{c}\text { Peer- } \\
\text { Review } \\
\text { Journal }\end{array}$ & English & Healthy & Situational & $\mathrm{PC}-\mathrm{C}$ \\
\hline $\begin{array}{l}\text { Rakowski et } \\
\text { al. (1992) }\end{array}$ & 141 & $\begin{array}{c}\text { Pros and } \\
\text { Cons of } \\
\text { Obtaining a } \\
\text { Mamogram }\end{array}$ & Actual & Hedge's & $\begin{array}{c}\text { Agree / } \\
\text { Disagree }\end{array}$ & Acquisition & $\begin{array}{c}\text { Peer- } \\
\text { Review } \\
\text { Journal }\end{array}$ & English & Healthy & Yearly + & $\mathrm{C}-\mathrm{A}$ \\
\hline $\begin{array}{l}\text { Redding } \\
(1990)\end{array}$ & 213 & $\begin{array}{l}\text { Pros and } \\
\text { Cons of } \\
\text { Safer Sex }\end{array}$ & Actual & $\begin{array}{c}\text { Standard } \\
\text { Score }\end{array}$ & $\begin{array}{c}\text { How } \\
\text { Important }\end{array}$ & Acquisition & \begin{tabular}{|c|} 
Non- \\
Peer- \\
Review \\
Journal
\end{tabular} & English & Healthy & Situational & $\mathrm{PC}-\mathrm{C}$ \\
\hline $\begin{array}{l}\text { Redding } \\
\text { (1993) }\end{array}$ & 305 & $\begin{array}{l}\text { Pros and } \\
\text { Cons of } \\
\text { Safer Sex }\end{array}$ & Actual & Hedge's & $\begin{array}{c}\text { How } \\
\text { Important }\end{array}$ & Acquisition & $\begin{array}{l}\text { Non- } \\
\text { Peer- } \\
\text { Review } \\
\text { Journal }\end{array}$ & English & Healthy & Situational & C-A \\
\hline
\end{tabular}


Table B.2 (continued)

\begin{tabular}{|c|c|c|c|c|c|c|c|c|c|c|c|}
\hline $\begin{array}{l}\text { Source } \\
\text { (Year) }\end{array}$ & $\begin{array}{c}\mathrm{N} / \\
\text { data } \\
\text {-set }\end{array}$ & $\begin{array}{c}\text { Description } \\
\text { of Pros and } \\
\text { Cons }\end{array}$ & $\begin{array}{l}\text { Direction } \\
\text { of Pros } \\
\text { and Cons }\end{array}$ & $\begin{array}{c}\text { Calcula- } \\
\text { tion } \\
\text { Method }\end{array}$ & $\begin{array}{c}\text { Scale } \\
\text { Instruct }\end{array}$ & Cess/ Acq & $\begin{array}{c}\text { Publica- } \\
\text { tion } \\
\text { Status }\end{array}$ & $\begin{array}{l}\text { Lan- } \\
\text { guage }\end{array}$ & $\begin{array}{l}\text { Health } \\
\text { Status }\end{array}$ & $\begin{array}{c}\text { Fre- } \\
\text { quency }\end{array}$ & $\begin{array}{l}\text { Cross- } \\
\text { over }\end{array}$ \\
\hline $\begin{array}{l}\text { Reed } \\
(1996)\end{array}$ & 271 & $\begin{array}{l}\text { Pros and } \\
\text { Cons of } \\
\text { Exercise }\end{array}$ & Actual & Hedge's & $\begin{array}{c}\text { How } \\
\text { Important }\end{array}$ & Acquisition & $\begin{array}{c}\text { Non- } \\
\text { Peer- } \\
\text { Review } \\
\text { Journal }\end{array}$ & English & Healthy & Daily & C-PR \\
\hline $\begin{array}{l}\text { Riley et al. } \\
\quad(2000)\end{array}$ & 19 & $\begin{array}{l}\text { Pros and } \\
\text { Cons of } \\
\text { Managing } \\
\text { Stress } \\
\end{array}$ & Actual & Hedge's & $\begin{array}{c}\text { How } \\
\text { Important }\end{array}$ & Acquisition & $\begin{array}{c}\text { Peer- } \\
\text { Review } \\
\text { Journal }\end{array}$ & English & Unhealthy & Daily & Multiple \\
\hline $\begin{array}{l}\text { Riley et al. } \\
\quad(2003)\end{array}$ & 42 & $\begin{array}{l}\text { Pros and } \\
\text { Cons of } \\
\text { Managing } \\
\text { Stress }\end{array}$ & Actual & Hedge's & $\begin{array}{c}\text { How } \\
\text { Important }\end{array}$ & Acquisition & $\begin{array}{c}\text { Non- } \\
\text { Peer- } \\
\text { Review } \\
\text { Journal }\end{array}$ & English & Unhealthy & Daily & Multiple \\
\hline $\begin{array}{l}\text { Riley et al. } \\
\quad(2003)\end{array}$ & 126 & $\begin{array}{l}\text { Pros and } \\
\text { Cons of } \\
\text { Managing } \\
\text { Stress }\end{array}$ & Actual & Hedge's & $\begin{array}{c}\text { How } \\
\text { Important }\end{array}$ & Acquisition & $\begin{array}{c}\text { Peer- } \\
\text { Review } \\
\text { Journal }\end{array}$ & English & Unhealthy & Daily & Multiple \\
\hline $\begin{array}{c}\text { Robbins et al. } \\
(2001)\end{array}$ & 163 & $\begin{array}{l}\text { Pros and } \\
\text { Cons of } \\
\text { Organ } \\
\text { Donation }\end{array}$ & Actual & Hedge's & $\begin{array}{c}\text { How } \\
\text { Important }\end{array}$ & Acquisition & $\begin{array}{c}\text { Peer- } \\
\text { Review } \\
\text { Journal }\end{array}$ & English & Other & Situational & C-PR \\
\hline $\begin{array}{l}\text { Robbins } \\
\text { (1999) }\end{array}$ & 280 & $\begin{array}{l}\text { Pros and } \\
\text { Cons of } \\
\text { Organ } \\
\text { Donation } \\
\end{array}$ & Actual & Hedge's & $\begin{array}{c}\text { How } \\
\text { Important }\end{array}$ & Acquisition & $\begin{array}{c}\text { Non- } \\
\text { Peer- } \\
\text { Review } \\
\text { Journal } \\
\end{array}$ & English & Other & Yearly + & C-PR \\
\hline
\end{tabular}


Table B.2 (continued)

\begin{tabular}{|c|c|c|c|c|c|c|c|c|c|c|c|}
\hline $\begin{array}{l}\text { Source } \\
\text { (Year) }\end{array}$ & $\begin{array}{c}\text { N/ } \\
\text { data } \\
\text {-set }\end{array}$ & $\begin{array}{c}\text { Description } \\
\text { of Pros and } \\
\text { Cons }\end{array}$ & $\begin{array}{l}\text { Direction } \\
\text { of Pros } \\
\text { and Cons }\end{array}$ & $\begin{array}{c}\text { Calcula- } \\
\text { tion } \\
\text { Method }\end{array}$ & $\begin{array}{c}\text { Scale } \\
\text { Instruct }\end{array}$ & Cess/Acq & $\begin{array}{c}\text { Publica- } \\
\text { tion } \\
\text { Status }\end{array}$ & $\begin{array}{l}\text { Lan- } \\
\text { guage }\end{array}$ & $\begin{array}{l}\text { Health } \\
\text { Status }\end{array}$ & $\begin{array}{c}\text { Fre- } \\
\text { quency }\end{array}$ & $\begin{array}{l}\text { Cross- } \\
\text { over }\end{array}$ \\
\hline $\begin{array}{l}\text { Robbins } \\
\text { (2002) }\end{array}$ & 333 & $\begin{array}{l}\text { Pros and } \\
\text { Cons of } \\
\text { Organ } \\
\text { Donation }\end{array}$ & Actual & Hedge's & $\begin{array}{c}\text { How } \\
\text { Important }\end{array}$ & Acquisition & $\begin{array}{c}\text { Non- } \\
\text { Peer- } \\
\text { Review } \\
\text { Journal }\end{array}$ & English & Other & Yearly + & C-PR \\
\hline $\begin{array}{l}\text { Robbins } \\
\text { (1999) }\end{array}$ & 475 & $\begin{array}{l}\text { Pros and } \\
\text { Cons of } \\
\text { Organ } \\
\text { Donation }\end{array}$ & Actual & Hedge's & $\begin{array}{c}\text { How } \\
\text { Important }\end{array}$ & Acquisition & $\begin{array}{c}\text { Non- } \\
\text { Peer- } \\
\text { Review } \\
\text { Journal }\end{array}$ & English & Other & Yearly + & C-PR \\
\hline $\begin{array}{l}\text { Robbins } \\
(2002)\end{array}$ & 2233 & $\begin{array}{l}\text { Pros and } \\
\text { Cons of } \\
\text { Organ } \\
\text { Donation }\end{array}$ & Actual & Hedge's & $\begin{array}{c}\text { How } \\
\text { Important }\end{array}$ & Acquisition & $\begin{array}{c}\text { Non- } \\
\text { Peer- } \\
\text { Review } \\
\text { Journal }\end{array}$ & English & Other & Yearly + & Multiple \\
\hline $\begin{array}{l}\text { Rossi } \\
(1990)\end{array}$ & 698 & $\begin{array}{l}\text { Pros and } \\
\text { Cons of } \\
\text { Testing for } \\
\text { Radon }\end{array}$ & Actual & $\begin{array}{c}\text { Standard } \\
\text { Score }\end{array}$ & $\begin{array}{c}\text { How } \\
\text { Important }\end{array}$ & Acquisition & $\begin{array}{c}\text { Non- } \\
\text { Peer- } \\
\text { Review } \\
\text { Journal }\end{array}$ & English & Other & Yearly + & PC-C \\
\hline $\begin{array}{c}\text { Rossi } \\
(1990)\end{array}$ & 136 & $\begin{array}{l}\text { Pros and } \\
\text { Cons for } \\
\text { Using } \\
\text { Sunscreens }\end{array}$ & Actual & $\begin{array}{c}\text { Standard } \\
\text { Score }\end{array}$ & $\begin{array}{c}\text { How } \\
\text { Important }\end{array}$ & Acquisition & $\begin{array}{c}\text { Non- } \\
\text { Peer- } \\
\text { Review } \\
\text { Journal }\end{array}$ & English & Healthy & Situational & C-PR \\
\hline $\begin{array}{l}\text { Rossi et al. } \\
\text { (2001) }\end{array}$ & 298 & $\begin{array}{c}\text { Pros and } \\
\text { Cons of } \\
\text { Cocaine Use }\end{array}$ & Reversed & Hedge's & $\begin{array}{c}\text { How } \\
\text { Important }\end{array}$ & Cessation & $\begin{array}{c}\text { Non- } \\
\text { Peer- } \\
\text { Review } \\
\text { Journal }\end{array}$ & English & Unhealthy & Daily & Multiple \\
\hline
\end{tabular}


Table B.2 (continued)

\begin{tabular}{|c|c|c|c|c|c|c|c|c|c|c|c|}
\hline $\begin{array}{c}\text { Source } \\
\text { (Year) }\end{array}$ & $\begin{array}{c}\mathrm{N} / \\
\text { data } \\
\text {-set } \\
\end{array}$ & $\begin{array}{c}\text { Description } \\
\text { of Pros and } \\
\text { Cons } \\
\end{array}$ & $\begin{array}{c}\text { Direction } \\
\text { of Pros } \\
\text { and Cons }\end{array}$ & \begin{tabular}{|c|} 
Calcula- \\
tion \\
Method \\
\end{tabular} & $\begin{array}{c}\text { Scale } \\
\text { Instruct }\end{array}$ & Cess/ Acq & \begin{tabular}{|c|} 
Publica- \\
tion \\
Status \\
\end{tabular} & $\begin{array}{l}\text { Lan- } \\
\text { guage }\end{array}$ & $\begin{array}{l}\text { Health } \\
\text { Status }\end{array}$ & $\begin{array}{c}\text { Fre- } \\
\text { quency }\end{array}$ & $\begin{array}{c}\text { Cross- } \\
\text { over }\end{array}$ \\
\hline $\begin{array}{l}\text { Rossi. SR et } \\
\text { al. (2001) }\end{array}$ & 2639 & $\begin{array}{c}\text { Pros and } \\
\text { Cons of } \\
\text { Reducing } \\
\text { Dietary Fat } \\
\text { Consumption }\end{array}$ & Reversed & Hedge's & $\begin{array}{c}\text { How } \\
\text { Important }\end{array}$ & Cessation & $\begin{array}{c}\text { Peer- } \\
\text { Review } \\
\text { Journal }\end{array}$ & English & Healthy & Daily & C-PR \\
\hline $\begin{array}{l}\text { Rossi, SR et } \\
\text { al. }(2001)\end{array}$ & 1204 & $\begin{array}{c}\text { Pros and } \\
\text { Cons of } \\
\text { Reducing } \\
\text { Dietary Fat } \\
\text { Consumption }\end{array}$ & Reversed & Hedge's & $\begin{array}{c}\text { How } \\
\text { Important }\end{array}$ & Cessation & $\begin{array}{l}\text { Non- } \\
\text { Peer- } \\
\text { Review } \\
\text { Journal }\end{array}$ & English & Healthy & Daily & Multiple \\
\hline $\begin{array}{l}\text { Rossi, SR et } \\
\text { al. }(2001)\end{array}$ & 353 & $\begin{array}{c}\text { Pros and } \\
\text { Cons of Fruit } \\
\text { and } \\
\text { Vegetable } \\
\text { Consumption } \\
\end{array}$ & Actual & Hedge's & $\begin{array}{c}\text { How } \\
\text { Important }\end{array}$ & Acquisition & $\begin{array}{l}\text { Non- } \\
\text { Peer- } \\
\text { Review } \\
\text { Journal }\end{array}$ & English & Healthy & Daily & C-PR \\
\hline $\begin{array}{l}\text { Rossi, SR et } \\
\text { al. (1993) }\end{array}$ & 196 & $\begin{array}{c}\text { Pros and } \\
\text { Cons of } \\
\text { Reducing } \\
\text { Dietary Fat } \\
\text { Consumption } \\
\end{array}$ & Reversed & Hedge's & $\begin{array}{c}\text { How } \\
\text { Important }\end{array}$ & Cessation & $\begin{array}{l}\text { Non- } \\
\text { Peer- } \\
\text { Review } \\
\text { Journal }\end{array}$ & English & Healthy & Daily & C-A \\
\hline $\begin{array}{l}\text { Ruggiero et } \\
\text { al. (2000) }\end{array}$ & 206 & $\begin{array}{l}\text { Pros and } \\
\text { Cons of } \\
\text { Smoking }\end{array}$ & Reversed & $\begin{array}{c}\text { Standard } \\
\text { Score }\end{array}$ & $\begin{array}{c}\text { How } \\
\text { Important }\end{array}$ & Cessation & $\begin{array}{c}\text { Peer- } \\
\text { Review } \\
\text { Journal }\end{array}$ & English & Unhealthy & Daily & $\mathrm{PC}-\mathrm{C}$ \\
\hline $\begin{array}{c}\text { Ruggiero et } \\
\text { al. (2003) }\end{array}$ & 668 & $\begin{array}{c}\text { Pros and } \\
\text { Cons of } \\
\text { Glucose } \\
\text { Testing }\end{array}$ & Actual & Hedge's & $\begin{array}{c}\text { How } \\
\text { Important }\end{array}$ & Acquisition & $\begin{array}{l}\text { Non- } \\
\text { Peer- } \\
\text { Review } \\
\text { Journal }\end{array}$ & English & Healthy & Daily & NA \\
\hline
\end{tabular}


Table B.2 (continued)

\begin{tabular}{|c|c|c|c|c|c|c|c|c|c|c|c|}
\hline $\begin{array}{l}\text { Source } \\
\text { (Year) }\end{array}$ & $\begin{array}{c}\text { N/ } \\
\text { data } \\
\text {-set }\end{array}$ & $\begin{array}{c}\text { Description } \\
\text { of Pros and } \\
\text { Cons }\end{array}$ & $\begin{array}{c}\text { Direction } \\
\text { of Pros } \\
\text { and Cons }\end{array}$ & $\begin{array}{l}\text { Calcula- } \\
\text { tion } \\
\text { Method }\end{array}$ & $\begin{array}{c}\text { Scale } \\
\text { Instruct }\end{array}$ & Cess/ Acq & $\begin{array}{c}\text { Publica- } \\
\text { tion } \\
\text { Status } \\
\end{array}$ & $\begin{array}{l}\text { Lan- } \\
\text { guage }\end{array}$ & $\begin{array}{l}\text { Health } \\
\text { Status }\end{array}$ & $\begin{array}{l}\text { Fre- } \\
\text { quency }\end{array}$ & $\begin{array}{l}\text { Cross- } \\
\text { over }\end{array}$ \\
\hline $\begin{array}{l}\text { Sarkin et al. } \\
\quad(2001)\end{array}$ & 670 & $\begin{array}{l}\text { Pros and } \\
\text { Cons of } \\
\text { Exercise }\end{array}$ & Actual & Hedge's & $\begin{array}{c}\text { How } \\
\text { Important }\end{array}$ & Acquisition & \begin{tabular}{c|} 
Peer- \\
Review \\
Journal \\
\end{tabular} & English & Healthy & Daily & C-PR \\
\hline $\begin{array}{l}\text { Schmaling et } \\
\text { al. }(2000)\end{array}$ & 53 & $\begin{array}{l}\text { Pros and } \\
\text { cons of } \\
\text { taking } \\
\text { asthma } \\
\text { medication } \\
\text { as } \\
\text { prescribed. }\end{array}$ & Actual & $\begin{array}{c}\text { Standard } \\
\text { Score }\end{array}$ & $\begin{array}{c}\text { Agree / } \\
\text { Disagree }\end{array}$ & Acquisition & $\begin{array}{c}\text { Peer- } \\
\text { Review } \\
\text { Journal }\end{array}$ & English & Healthy & Daily & PR-A \\
\hline $\begin{array}{l}\text { Schorling } \\
(1995)\end{array}$ & 243 & $\begin{array}{l}\text { Pros and } \\
\text { Cons of } \\
\text { Smoking }\end{array}$ & Reversed & Hedge's & $\begin{array}{c}\text { How } \\
\text { Important }\end{array}$ & Cessation & $\begin{array}{c}\text { Peer- } \\
\text { Review } \\
\text { Journal } \\
\end{array}$ & English & Unhealthy & Daily & $\mathrm{PC}-\mathrm{C}$ \\
\hline $\begin{array}{l}\text { Schorling } \\
\text { (1995) }\end{array}$ & 292 & $\begin{array}{l}\text { Pros and } \\
\text { Cons of } \\
\text { Smoking }\end{array}$ & Reversed & Hedge's & $\begin{array}{c}\text { How } \\
\text { Important }\end{array}$ & Cessation & $\begin{array}{c}\text { Peer- } \\
\text { Review } \\
\text { Journal }\end{array}$ & English & Unhealthy & Daily & $\mathrm{PC}-\mathrm{C}$ \\
\hline $\begin{array}{c}\text { Schumann et } \\
\text { al. }(2003)\end{array}$ & 877 & $\begin{array}{l}\text { Pros and } \\
\text { Cons of } \\
\text { Quitting } \\
\text { Smoking }\end{array}$ & Actual & Hedge's & $\begin{array}{c}\text { How } \\
\text { Important }\end{array}$ & Cessation & $\begin{array}{l}\text { Peer- } \\
\text { Review } \\
\text { Journal }\end{array}$ & $\begin{array}{l}\text { Non- } \\
\text { English }\end{array}$ & Unhealthy & Daily & NA \\
\hline $\begin{array}{l}\text { Shulze } \\
\text { (2003) }\end{array}$ & 453 & $\begin{array}{c}\text { Pros and } \\
\text { Cons of } \\
\text { Using Dental } \\
\text { Floss }\end{array}$ & Actual & Hedge's & $\begin{array}{c}\text { How } \\
\text { Important }\end{array}$ & Acquisition & \begin{tabular}{|c|} 
Non- \\
Peer- \\
Review \\
Journal \\
\end{tabular} & $\begin{array}{l}\text { Non- } \\
\text { English }\end{array}$ & Healthy & Daily & Multiple \\
\hline
\end{tabular}


Table B.2 (continued)

\begin{tabular}{|c|c|c|c|c|c|c|c|c|c|c|c|}
\hline $\begin{array}{c}\text { Source } \\
\text { (Year) }\end{array}$ & $\begin{array}{c}\text { N/ } \\
\text { data } \\
\text {-set }\end{array}$ & $\begin{array}{c}\text { Description } \\
\text { of Pros and } \\
\text { Cons }\end{array}$ & $\begin{array}{l}\text { Direction } \\
\text { of Pros } \\
\text { and Cons }\end{array}$ & $\begin{array}{l}\text { Calcula- } \\
\text { tion } \\
\text { Method }\end{array}$ & $\begin{array}{c}\text { Scale } \\
\text { Instruct }\end{array}$ & Cess/ Acq & $\begin{array}{l}\text { Publica- } \\
\text { tion } \\
\text { Status }\end{array}$ & $\begin{array}{c}\text { Lan- } \\
\text { guage }\end{array}$ & $\begin{array}{l}\text { Health } \\
\text { Status }\end{array}$ & $\begin{array}{c}\text { Fre- } \\
\text { quency }\end{array}$ & $\begin{array}{c}\text { Cross- } \\
\text { over }\end{array}$ \\
\hline $\begin{array}{l}\text { Silverman } \\
(1995)\end{array}$ & 223 & $\begin{array}{c}\text { Pros and } \\
\text { Cons of } \\
\text { Condom Use }\end{array}$ & Actual & Hedge's & $\begin{array}{c}\text { How } \\
\text { Important }\end{array}$ & Acquisition & $\begin{array}{c}\text { Non- } \\
\text { Peer- } \\
\text { Review } \\
\text { Journal }\end{array}$ & $\begin{array}{l}\text { Non- } \\
\text { English }\end{array}$ & Healthy & Situational & Multiple \\
\hline $\begin{array}{l}\text { Silverman } \\
(1995)\end{array}$ & 230 & $\begin{array}{c}\text { Pros and } \\
\text { Cons of } \\
\text { Condom Use }\end{array}$ & Actual & Hedge's & $\begin{array}{c}\text { How } \\
\text { Important }\end{array}$ & Acquisition & $\begin{array}{l}\text { Non- } \\
\text { Peer- } \\
\text { Review } \\
\text { Journal }\end{array}$ & English & Healthy & Situational & Multiple \\
\hline $\begin{array}{c}\text { Snow et al. } \\
(1992)\end{array}$ & 191 & $\begin{array}{l}\text { Pros and } \\
\text { Cons of } \\
\text { Smoking }\end{array}$ & Reversed & $\begin{array}{c}\text { Standard } \\
\text { Score }\end{array}$ & $\begin{array}{c}\text { How } \\
\text { Important }\end{array}$ & Cessation & $\begin{array}{c}\text { Peer- } \\
\text { Review } \\
\text { Journal }\end{array}$ & English & Unhealthy & Daily & $\mathrm{PC}-\mathrm{C}$ \\
\hline $\begin{array}{l}\text { Stark et al. } \\
\text { (1998) }\end{array}$ & 754 & $\begin{array}{l}\text { Pros and of } \\
\text { Condom Use }\end{array}$ & Actual & Hedge's & Other & Acquisition & $\begin{array}{l}\text { Peer- } \\
\text { Review } \\
\text { Journal }\end{array}$ & English & Healthy & Situational & NA \\
\hline $\begin{array}{l}\text { Stark et al. } \\
\text { (1998) }\end{array}$ & 822 & $\begin{array}{c}\text { Pros of } \\
\text { Condom Use }\end{array}$ & Actual & Hedge's & Other & Acquisition & $\begin{array}{c}\text { Peer- } \\
\text { Review } \\
\text { Journal }\end{array}$ & English & Healthy & Situational & NA \\
\hline $\begin{array}{l}\text { Susenbeth } \\
(2000)\end{array}$ & 96 & $\begin{array}{c}\text { Pros and } \\
\text { Cons of } \\
\text { Condom Use }\end{array}$ & Actual & Hedge's & $\begin{array}{c}\text { How } \\
\text { Important }\end{array}$ & Acquisition & $\begin{array}{c}\text { Non- } \\
\text { Peer- } \\
\text { Review } \\
\text { Journal }\end{array}$ & $\begin{array}{l}\text { Non- } \\
\text { English }\end{array}$ & Healthy & Situational & Multiple \\
\hline $\begin{array}{l}\text { Tseng } \\
(2001)\end{array}$ & 154 & $\begin{array}{l}\text { Pros and } \\
\text { Cons of } \\
\text { Exercise }\end{array}$ & Actual & Hedge's & $\begin{array}{c}\text { How } \\
\text { Important }\end{array}$ & Acquisition & $\begin{array}{c}\text { Non- } \\
\text { Peer- } \\
\text { Review } \\
\text { Journal }\end{array}$ & English & Healthy & Daily & PR-A \\
\hline
\end{tabular}


Table B. 2 (continued)

\begin{tabular}{|c|c|c|c|c|c|c|c|c|c|c|c|}
\hline $\begin{array}{l}\text { Source } \\
\text { (Year) }\end{array}$ & $\begin{array}{c}\mathrm{N} / \\
\text { data } \\
\text {-set }\end{array}$ & $\begin{array}{c}\text { Description } \\
\text { of Pros and } \\
\text { Cons }\end{array}$ & $\begin{array}{c}\text { Direction } \\
\text { of Pros } \\
\text { and Cons }\end{array}$ & $\begin{array}{c}\text { Calcula- } \\
\text { tion } \\
\text { Method }\end{array}$ & $\begin{array}{c}\text { Scale } \\
\text { Instruct }\end{array}$ & Cess/ Acq & $\begin{array}{c}\text { Publica- } \\
\text { tion } \\
\text { Status } \\
\end{array}$ & $\begin{array}{l}\text { Lan- } \\
\text { guage }\end{array}$ & $\begin{array}{l}\text { Health } \\
\text { Status }\end{array}$ & $\begin{array}{l}\text { Fre- } \\
\text { quency }\end{array}$ & $\begin{array}{l}\text { Cross- } \\
\text { over }\end{array}$ \\
\hline $\begin{array}{c}\text { Tsoh } \\
\text { (1993) }\end{array}$ & 169 & $\begin{array}{l}\text { Pros and } \\
\text { Cons of } \\
\text { Qutitting } \\
\text { Drugs }\end{array}$ & Actual & Hedge's & $\begin{array}{c}\text { How } \\
\text { Important }\end{array}$ & Cessation & $\begin{array}{c}\text { Non- } \\
\text { Peer- } \\
\text { Review } \\
\text { Journal }\end{array}$ & English & Healthy & Daily & C-PR \\
\hline $\begin{array}{l}\text { Van Marter et } \\
\text { al. }(2003)\end{array}$ & 473 & $\begin{array}{l}\text { Pros and } \\
\text { Cons of } \\
\text { treating self } \\
\text { and others } \\
\text { with respect }\end{array}$ & Actual & Hedge's & $\begin{array}{c}\text { How } \\
\text { Important }\end{array}$ & Cessation & $\begin{array}{c}\text { Non- } \\
\text { Peer- } \\
\text { Review } \\
\text { Journal }\end{array}$ & English & Other & Situational & C-PR \\
\hline $\begin{array}{l}\text { Velicer et al. } \\
\qquad(1985)\end{array}$ & 930 & $\begin{array}{l}\text { Pros and } \\
\text { Cons of } \\
\text { Smoking } \\
\end{array}$ & Reversed & Hedge's & $\begin{array}{c}\text { How } \\
\text { Important }\end{array}$ & Cessation & $\begin{array}{c}\text { Peer- } \\
\text { Review } \\
\text { Journal } \\
\end{array}$ & English & Unhealthy & Daily & Multiple \\
\hline $\begin{array}{l}\text { Wakui et al. } \\
(2002)\end{array}$ & 450 & $\begin{array}{l}\text { Pros and } \\
\text { Cons of } \\
\text { Exercise }\end{array}$ & Actual & Hedge's & $\begin{array}{c}\text { How } \\
\text { Important }\end{array}$ & Acquisition & $\begin{array}{c}\text { Peer- } \\
\text { Review } \\
\text { Journal } \\
\end{array}$ & $\begin{array}{l}\text { Non- } \\
\text { English }\end{array}$ & Healthy & Daily & PC-C \\
\hline Whyte (2003) & 416 & $\begin{array}{l}\text { Pros and } \\
\text { Cons of } \\
\text { Healthy } \\
\text { Eating }\end{array}$ & Actual & $\begin{array}{c}\text { Standard } \\
\text { Score }\end{array}$ & $\begin{array}{c}\text { Agree / } \\
\text { Disagree }\end{array}$ & Cessation & $\begin{array}{c}\text { Non- } \\
\text { Peer- } \\
\text { Review } \\
\text { Journal }\end{array}$ & English & Healthy & Daily & PR-A \\
\hline $\begin{array}{c}\text { Wright et al. } \\
(2002)\end{array}$ & 250 & $\begin{array}{l}\text { Pros and } \\
\text { Cons of } \\
\text { Weight- } \\
\text { Bearing } \\
\text { Exercise }\end{array}$ & Actual & $\begin{array}{c}\text { Standard } \\
\text { Score }\end{array}$ & $\begin{array}{c}\text { How } \\
\text { Important }\end{array}$ & Acquisition & $\begin{array}{c}\text { Non- } \\
\text { Peer- } \\
\text { Review } \\
\text { Journal }\end{array}$ & English & Healthy & Daily & PR-A \\
\hline
\end{tabular}




\section{Appendix C}

Figure C.1. Composite Pros and Cons Graphs for 33 Target Behaviors.

Note. Only target behaviors with data for all four transitions are included.

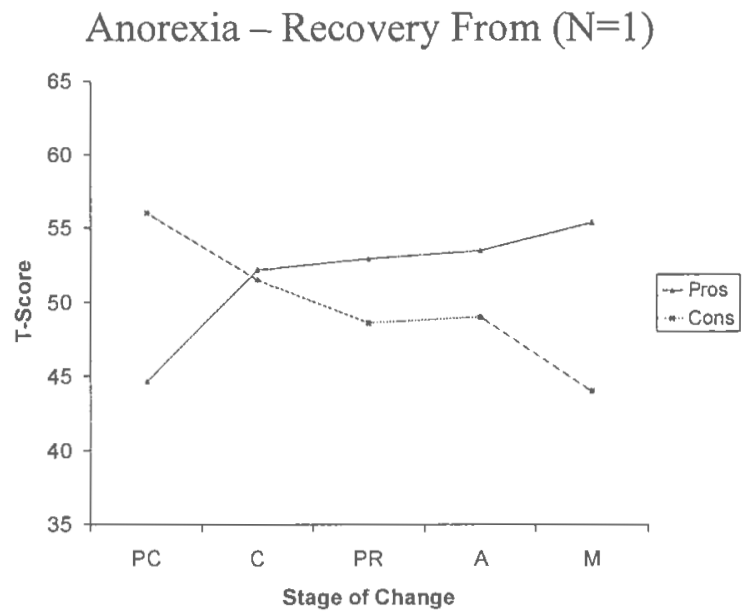

Back Pain /Keeping Body Posture $(\mathrm{N}=1)$

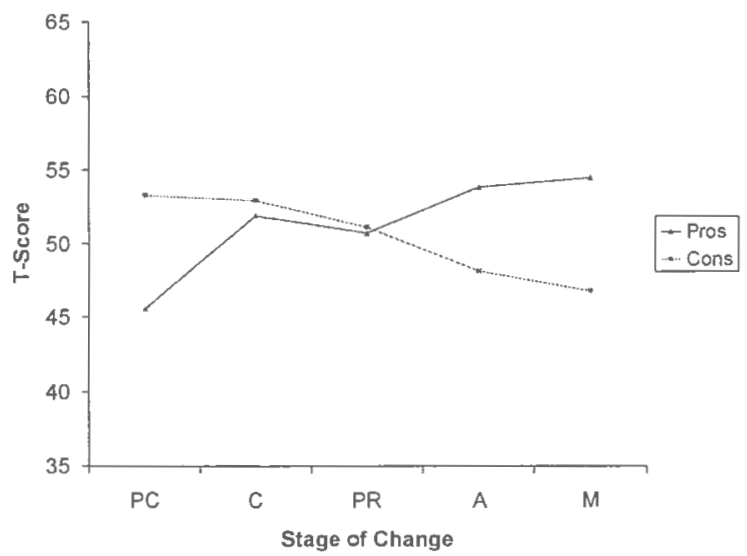


Binge Drinking Cessation $(\mathrm{N}=1)$

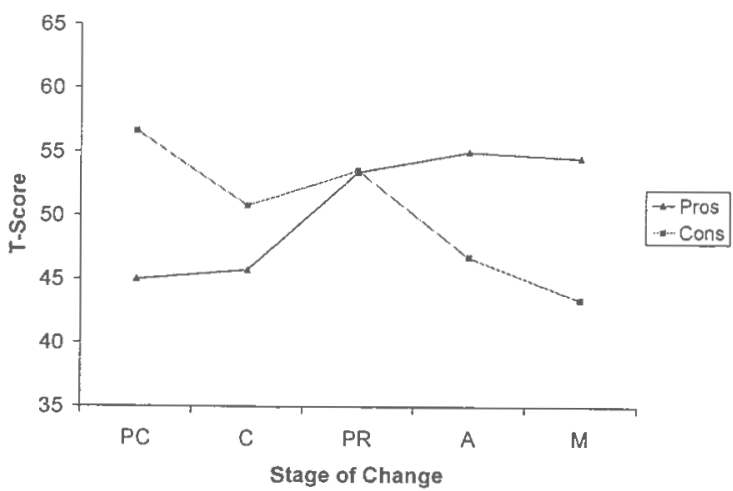

Bone Density Testing $(\mathrm{N}=1)$

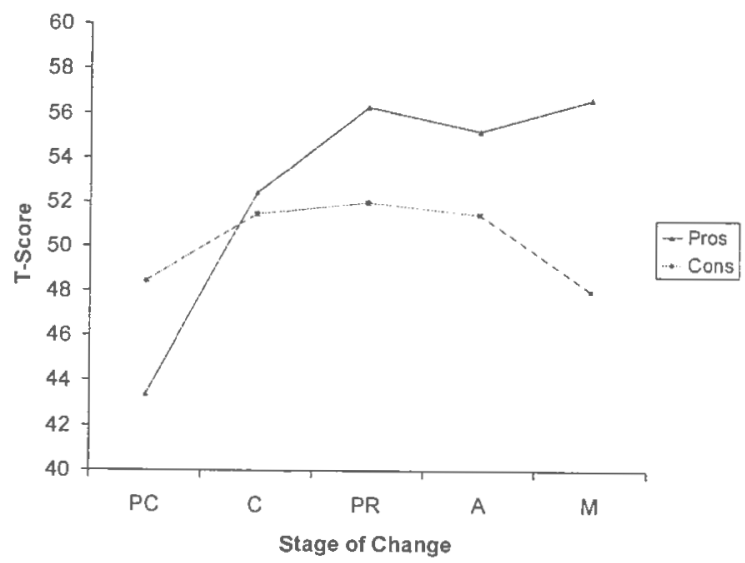

Breast Self-Examination $(\mathrm{N}=1)$

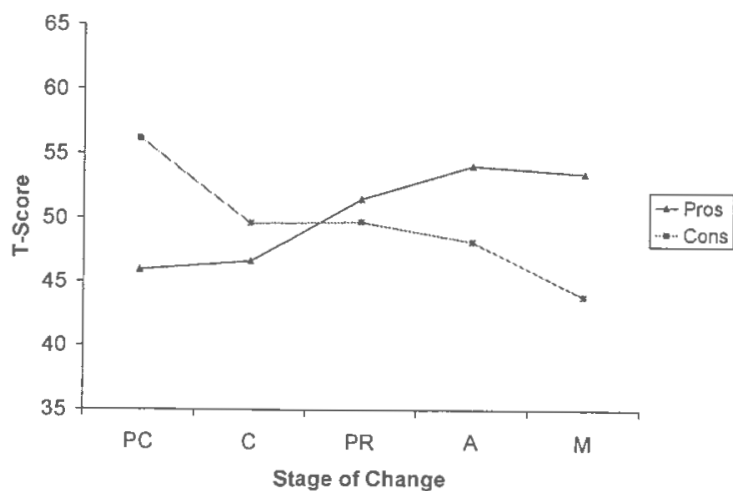


Bulimia - Recovery From $(\mathrm{N}=1)$

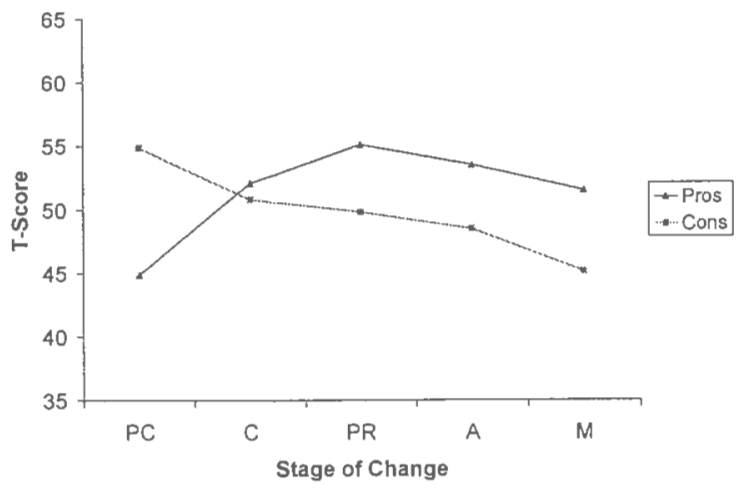

Calcium Intake $(\mathrm{N}=1)$

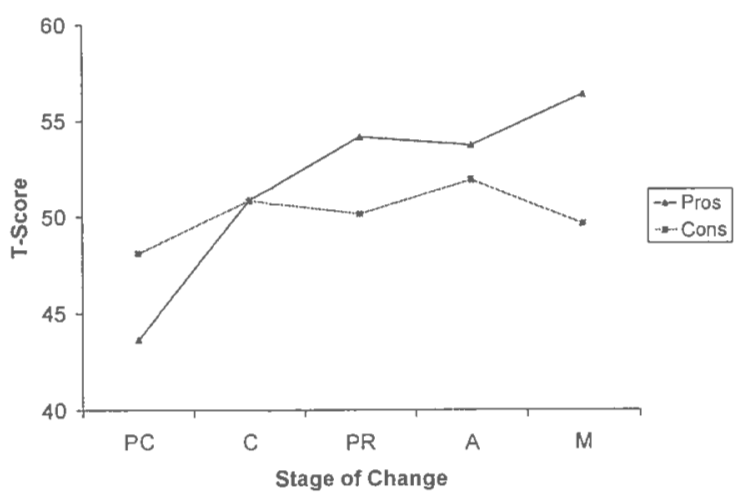

Condom Use - General (N=5)

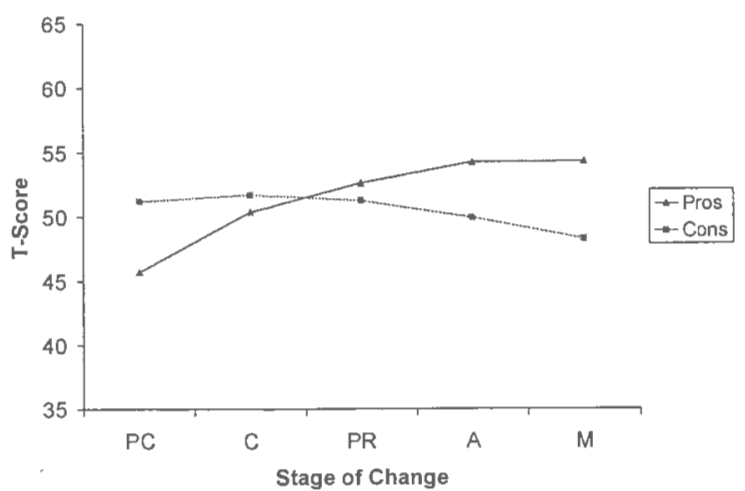


Condom Use with Main Partner $(\mathrm{N}=5)$

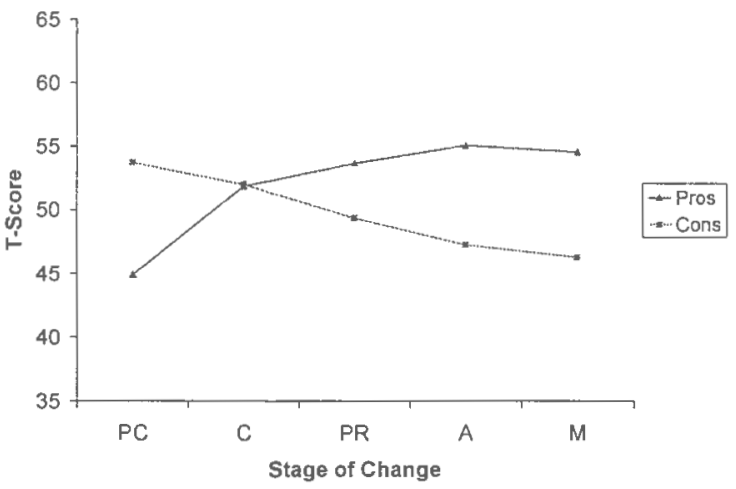

Condom Use with Other Partners $(\mathrm{N}=5)$

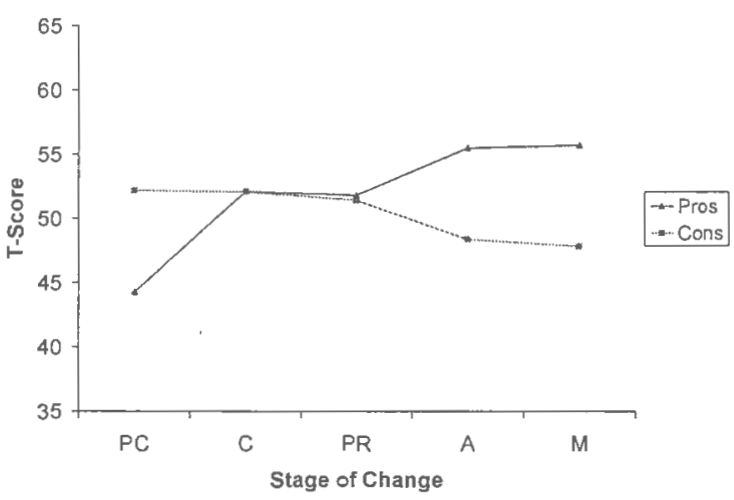

Condom Use - Vaginal Sex (N=1)

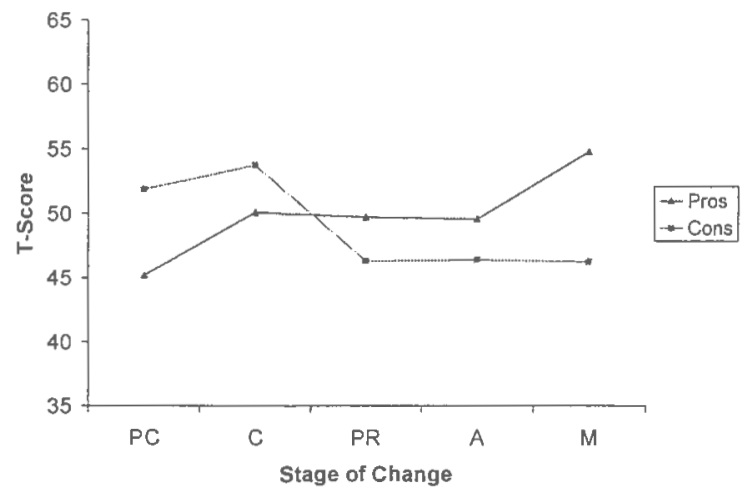


Contraception - General $(\mathrm{N}=3)$

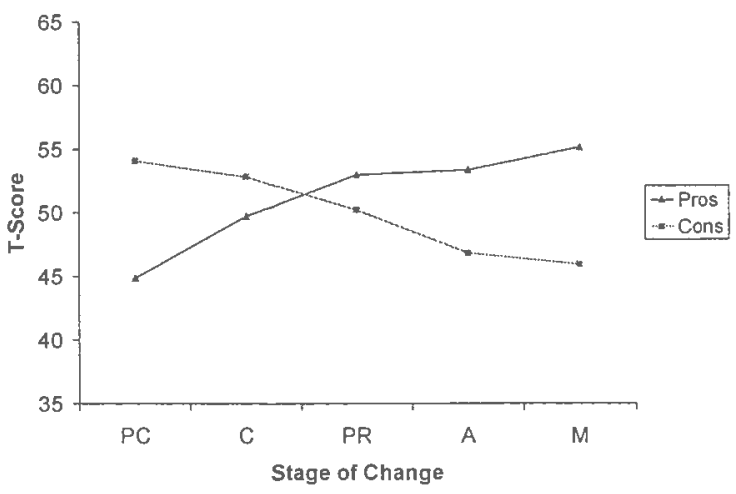

Daily Use of Dental Floss (N=1)

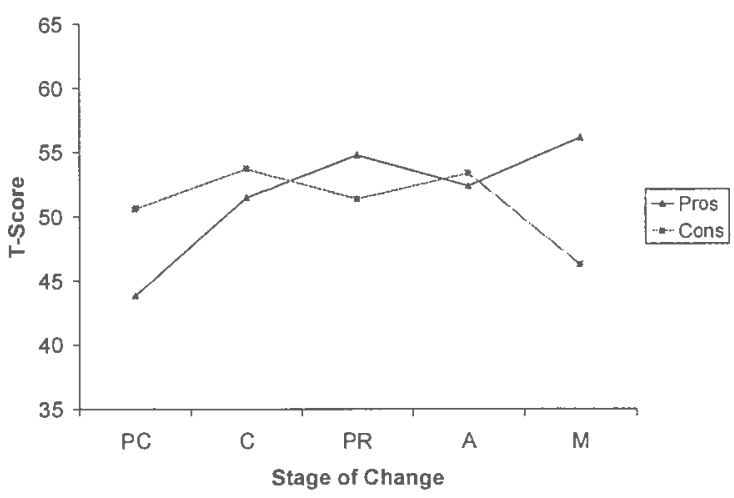

Decreasing Drug Use $(\mathbb{N}=1)$

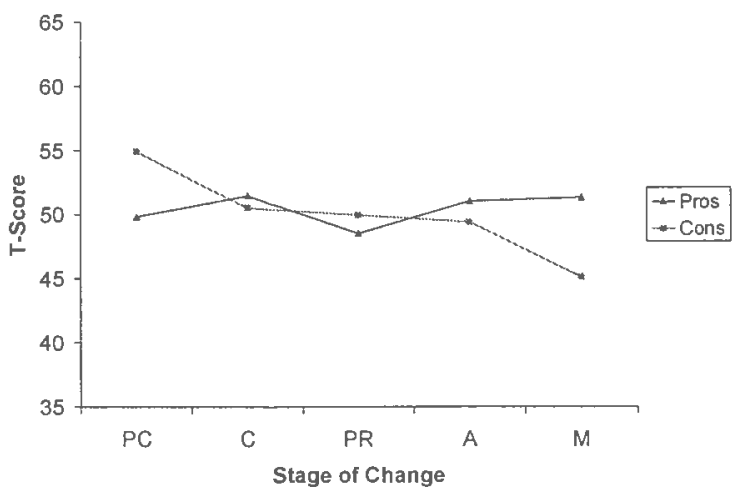


Depression Prevention $(\mathrm{N}=1)$

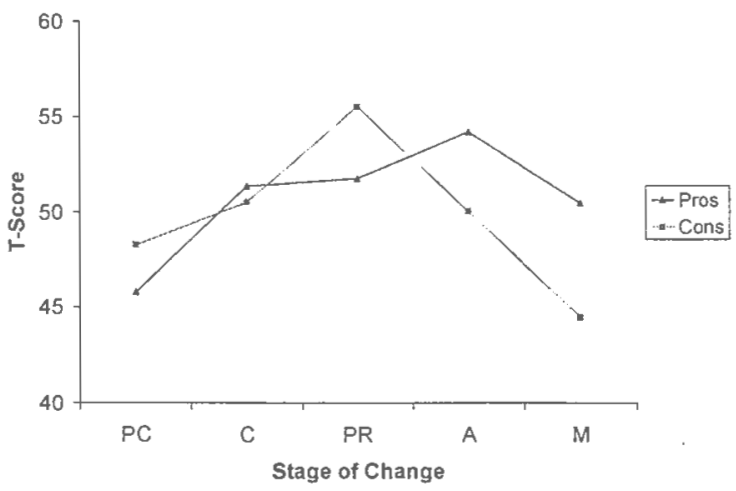

Dietary Fat Reduction ( $\mathrm{N}=7$ )

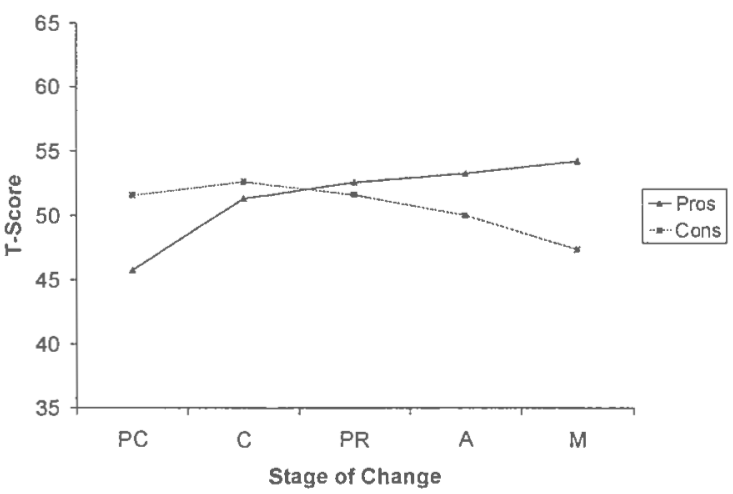

Drug Treatment $(\mathrm{N}=1)$

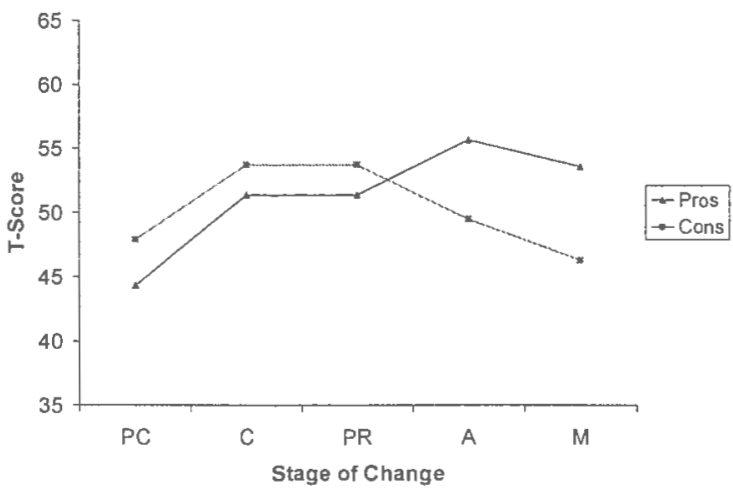


Exercise $(\mathrm{N}=20)$

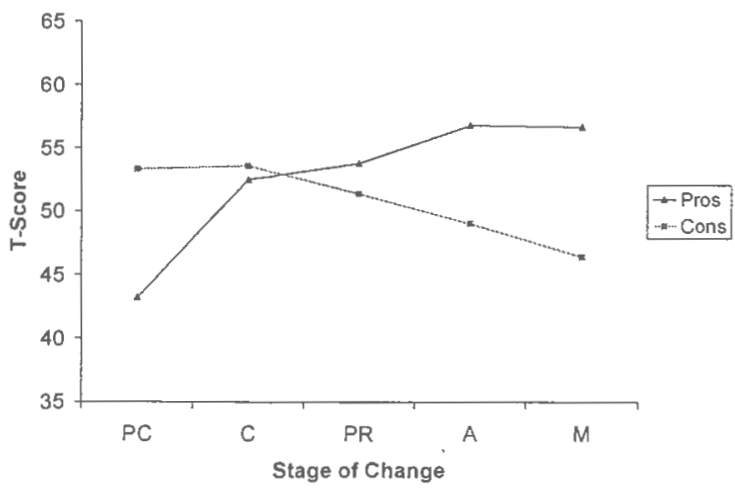

Fruit \& Vegetable Consumption $(\mathrm{N}=2)$

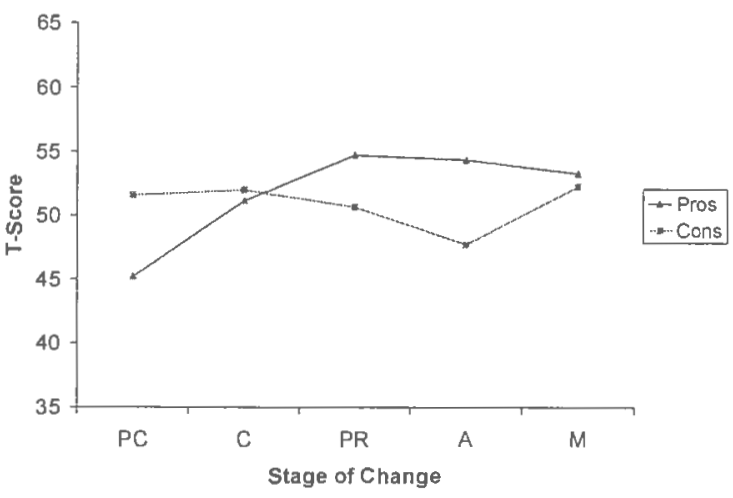

Fruit Intake $(\mathrm{N}=1)$

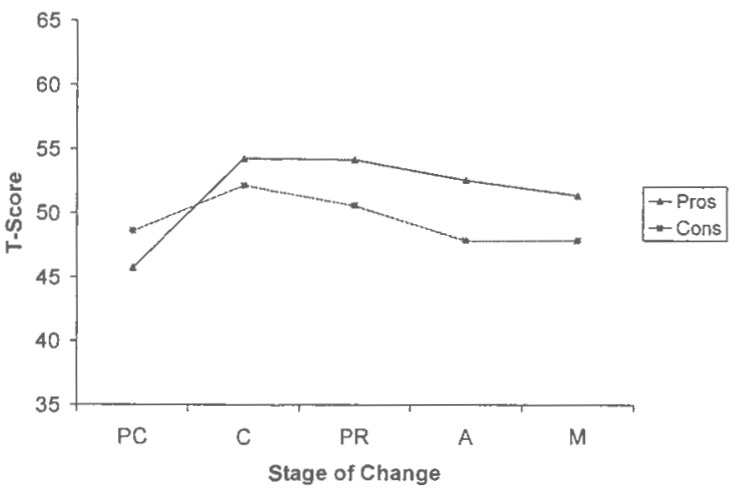


Glucose Testing $(\mathrm{N}=2)$

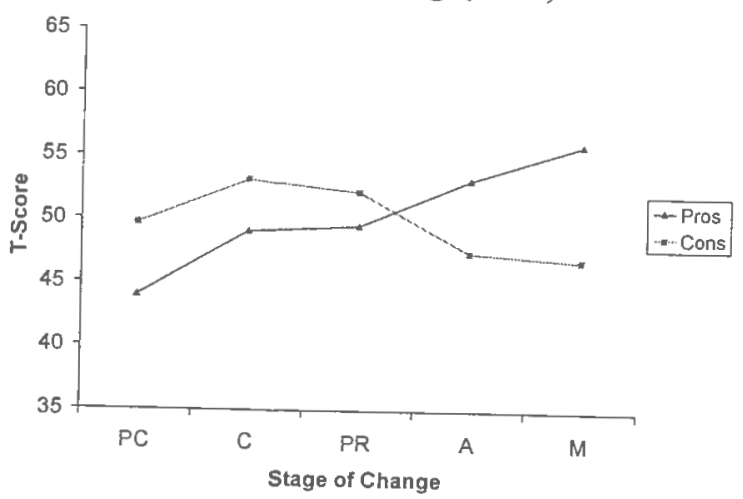

Healthy Diet $(\mathrm{N}=2)$

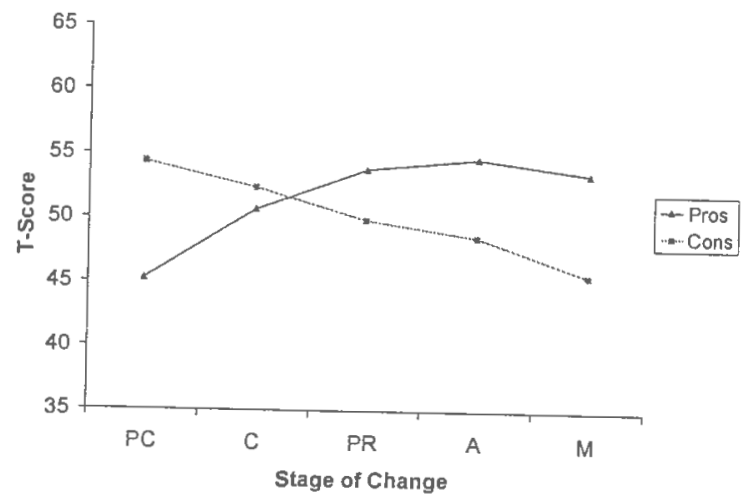

Oral Hygiene $(\mathrm{N}=1)$

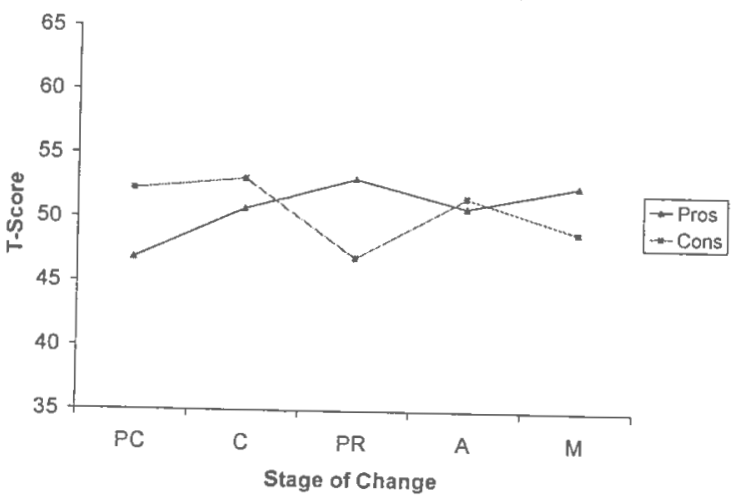




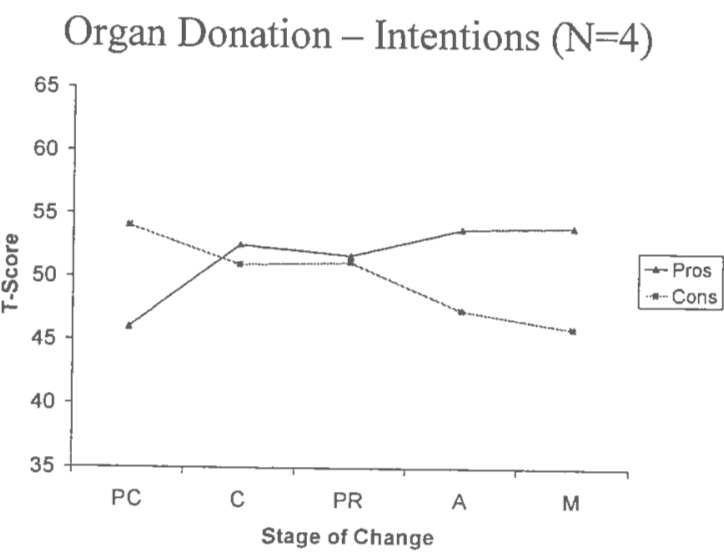

Participating in Decision Making for Medication $(\mathrm{N}=1)$

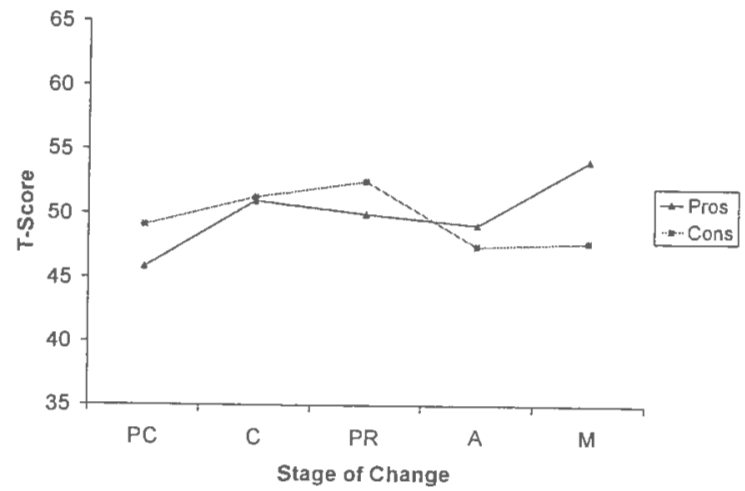

Physicians Assisting Smokers $(\mathrm{N}=1)$

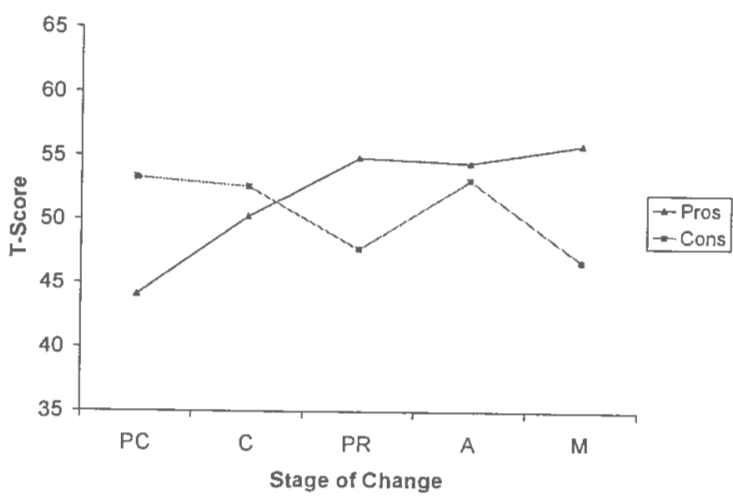


Sedentary Behavior $(\mathrm{N}=2)$

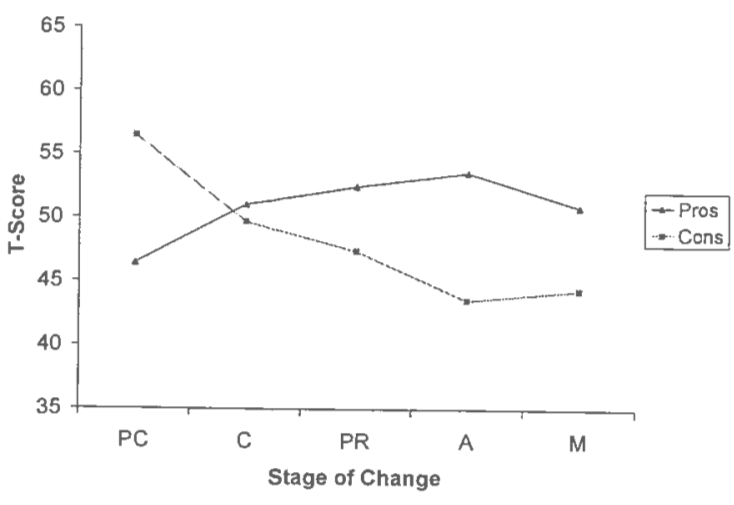

\section{Smoking Cessation $(\mathrm{N}=24)$}

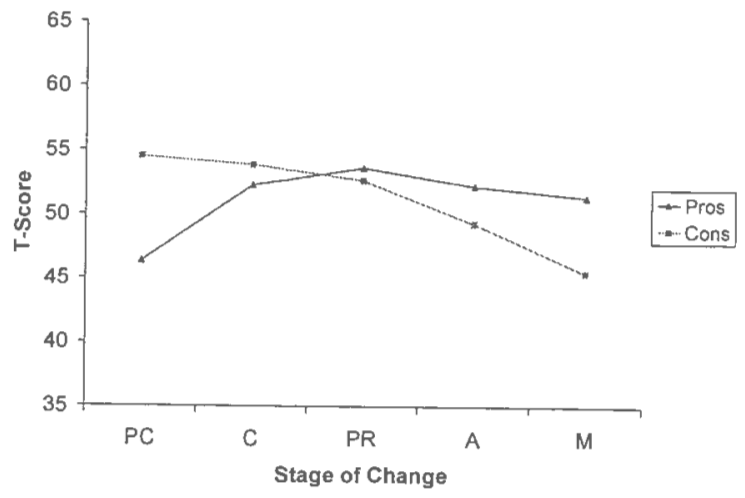

STD Screening $(\mathrm{N}=2)$

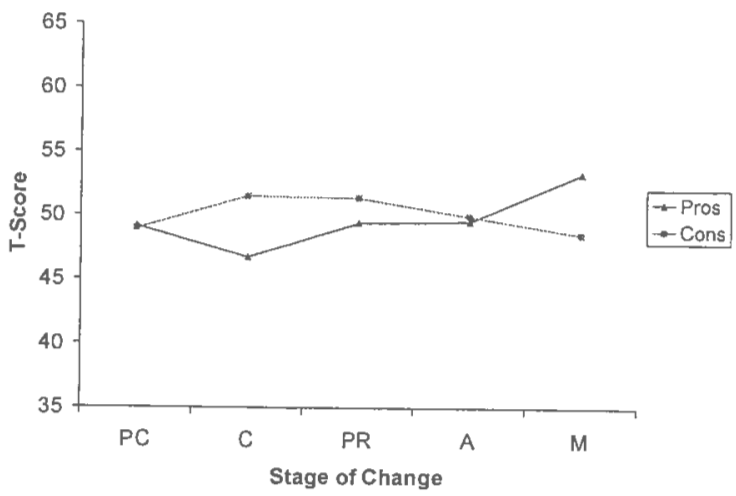


Stress Management $(\mathrm{N}=7$ )
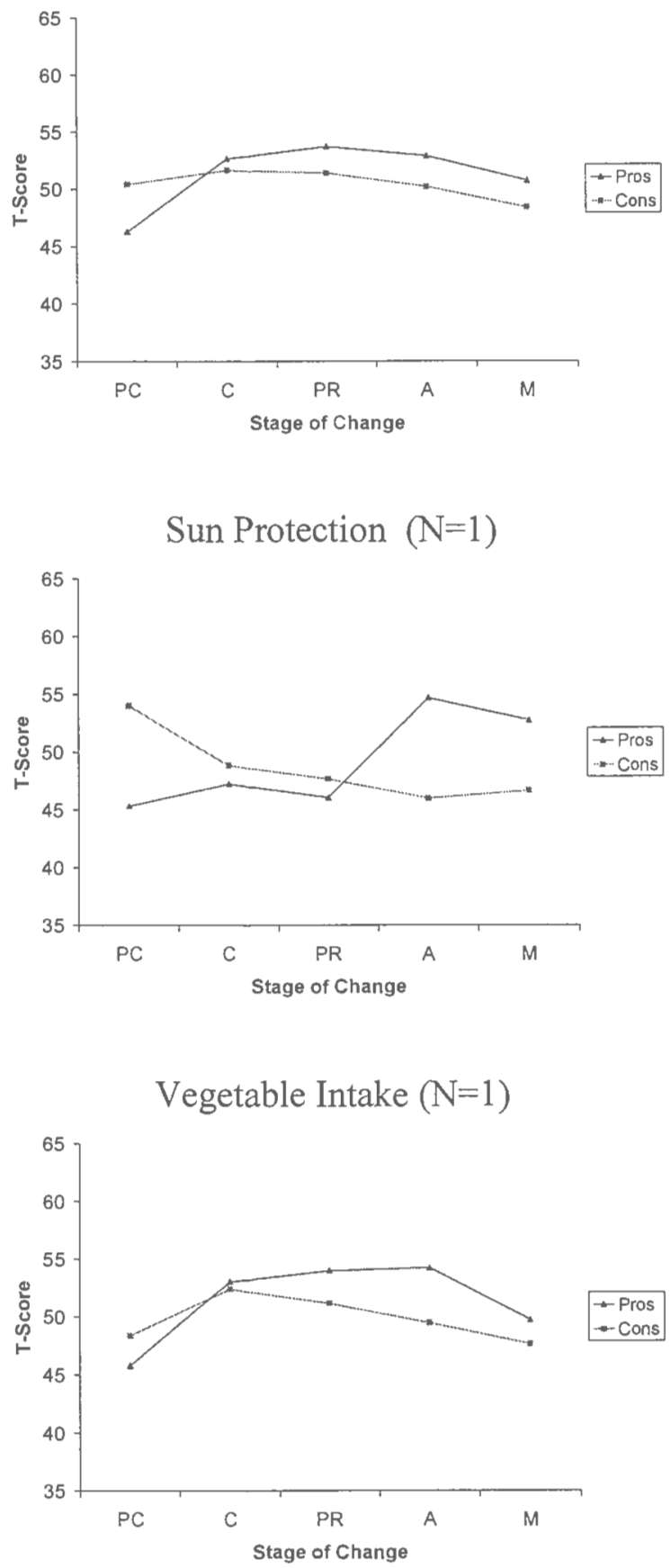
Weight Bearing Exercise $(\mathrm{N}=1)$

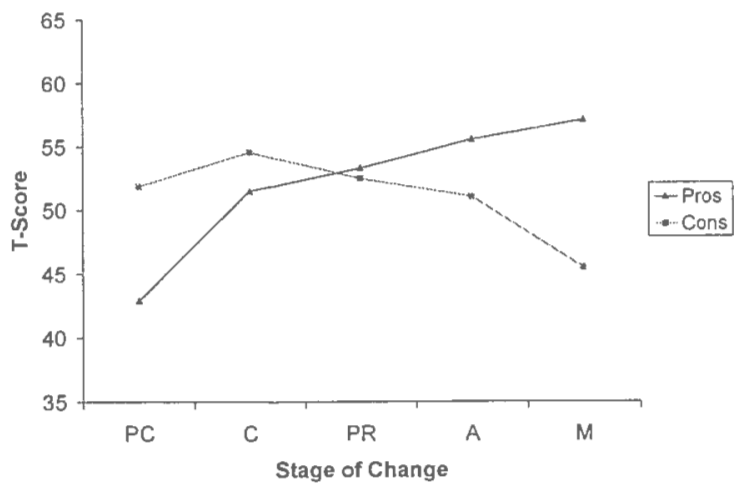


Table D.1

Behavior Categories with Associated Target Behaviors

\begin{tabular}{|c|c|}
\hline Behavior Category & Target Behaviors \\
\hline Alcohol & $\begin{array}{l}\text { Binge Drinking Acquisition } \\
\text { Binge Drinking Cessation }\end{array}$ \\
\hline Contraception & $\begin{array}{l}\text { Birth Control } \\
\text { General Contraception }\end{array}$ \\
\hline Condom Use & $\begin{array}{l}\text { Condom Use - General } \\
\text { Condom Use - Main Partner } \\
\text { Condom Use - Other Partner(s) } \\
\text { Condom Use - Anal Sex w/ Main Partner } \\
\text { Condom Use - Vaginal Sex }\end{array}$ \\
\hline Delinquent Behavior & $\begin{array}{l}\text { Delinquent Behavior } \\
\text { Bullying }\end{array}$ \\
\hline Diabetes & $\begin{array}{l}\text { Glucose Testing } \\
\text { Medication Management }\end{array}$ \\
\hline Diet & $\begin{array}{l}\text { Dietary Fat Reduction } \\
\text { Fruit Intake } \\
\text { Fruit and Vegetable Consumption } \\
\text { Healthy Diet } \\
\text { Vegetable Intake }\end{array}$ \\
\hline Drugs & $\begin{array}{l}\text { Cocaine Use } \\
\text { Decreasing Drug Use } \\
\text { Drug Treatment }\end{array}$ \\
\hline & Quitting Drugs \\
\hline Eating Disorders & $\begin{array}{l}\text { Recovery From Anorexia } \\
\text { Recovery From Bulimia }\end{array}$ \\
\hline Exercise & Exercise Regularly \\
\hline
\end{tabular}


Weight Bearing Exercise

Medical Screening

Bone Density Testing

Cancer Screening

Colorectal Cancer Screening

Mammography

STD Screening

Oral Hygiene

Daily Use of Dental Floss

Tooth-Friendly Diet

Osteoporosis

Calcium Intake

Sun

Sun Protection /Reduction

Sunscreen Use

Smoking

Smoking Acquisition

Smoking Cessation

Smokeless Tobacco Use

Sex Decisions

Safer Sex Decision Making

Sex Decision Making / Abstinence

Stress

Stress Management

Stress Reduction

Other Behaviors:

Administrative Change

Back Pain/Keeping Body Posture

Breast Self-Examination

Collaborative Delivery Service

Depression Prevention

Domestic Violence

Organ Donation

Participating in Decision Making for Medication Management

Physicians Assisting Smokers 
Radon Testing

Sedentary Behavior

Weight Loss 


\section{BIBLIOGRAPHY}

Acton, G.S., Prochaska, J.J., Kaplan, A.S., Small, T., \& Hall, S.H. (2001).

Depression and stages of change for smoking in psychiatric outpatients. Addictive Behaviors, 26, 621-631.

Ahijevych, K., \& Parsley, L.A. (1999). Smoke constituent exposure and stage of change in black and white women cigarette smokers. Addictive Behaviors, 24(1), 115-120.

Armstrong, C.A., Sallis, J.F., Hovell, M.F., and Hofstetter, C.R. (1993). Stages of change, self-efficacy, and the adoption of vigorous exercise: A prospective analysis. Journal of Sport and Exercise Psychology, 15, 390-402.

Aveyard, P., Cheng, K. K., Almond, J., et al. (1999). Cluster randomized controlled trial of an expert system based on the transtheoretical ("stages of change") model for smoking prevention and cessation in schools. British Medical Journal, 319, 948-953.

Bandura, A. (1977). Self-efficacy: Toward a unifying theory of behavior change. Bandura, A. (1982). Self-efficacy mechanism in human agency. American Psychologist, 37, 122-147.

Bane, C.M., Ruggiero, L., Dryfoos, J.M., \& Rossi, J.S. (1999). Development of a pregnancy tailored decisional balance measure for smoking cessation. Addictive Behaviors, 24(6), 795-9.

Banikarim, C., Chacko, M.R., Wiemann, C.M., \& Smith, P.B. (2003). Gonorrhea and Chlamydia screening among young women: Stage of change, decisional balance, and self-efficacy. Journal of Adolescent Health, 32, 288-295. 
Basler, H., Jaekle, C., Keller, S., \& Baum, E. (1999). Selbstwirksamkeit, Entscheidungsbalance und die Motivation zu sportlicher Aktivitaet. [Selfefficacy, deciosional balance and the motivation for physical activity]. Zeitschrift fuer Differentielle und Diagnostische Psychologie, 20(3), 203-216.

Beal, D. J., Corey, D. M., \& Dunlap, W. P. (2002). On the bias of Huffcutt and Arthur's (1995) procedure for identifying outliers in meta-analysis of correlations. Journal of Applied Psychology, 87, 583-589.

Beck, A.T., Steer, R.A., \& Brown, G.K. (1996). Beck depression inventory manual $\left(2^{\text {nd }}\right.$ ed.). San Antonio: Psychological Corporation.

Benowitz, N.L., Jacob, P., III, Ahijevych, K., Jarvis, M. J., Hall, S., LeHouezec, J., Hansson, A., Lichtenstein, E., Henningfield, J., Tsoh, J., Hurt, R.D., \& Velicer, W.F. (2002). Biochemical verification of tobacco use and cessation. Nicotine and Tobacco Research, 4, 149-159.

Bentler, P.M. (1980). Multivariate analysis with latent variables: Causal modeling. Annual Review of Psychology, 31, 419-456.

Bernstein, S.M., \& Stoduto, G. (1999). Adding a choice-based program for tobacco smoking to an abstinence-based addiction treatment program. Journal of Substance Abuse Treatment, 17, 167-173.

Biener L., \& Abrams D.D. (1991). The Contemplation Ladder: Validation of a measure of readiness to consider smoking cessation. Health Psychology, 10, $360-365$. 
Block, L.G., \& Keller P.A. (1998). Beyond protection motivation: An integrative theory of health appeals. Journal of Applied Social Psychology, 28(17), 15841608.

Bluthenthal R.N., Gogineni A., Longshore D., \& Stein M. (2001). Factors associated with readiness to change drug use among needle-exchange users. Drug and Alcohol Dependence, 62(3), 225-30.

Borenstein, M., \& Rothstein, H. (1999). Comprehensive meta-analysis: A computer program for research synthesis [Computer software manual]. Englewood, NJ: Biostat, Inc.

Borland R., \& Segan C. (2000). Testing the Transtheoretical Model for smoking change: Victorian data. Australian Journal of Psychology, 52(2), 83-88.

Boudreaux, E., Carmack, C.L., Scarinci, I.C. \& Brantley, P. J. (1998). Predicting smoking stage of change among a sample of low socioeconomic status, primary care outpatients: Replication and extension using decisional balance and selfefficacy theories. International Journal of Behavioral Medicine, 5(2), 148-165.

Buckworth, J., \& Wallace, L.S. (2002). Application of the transtheoretical model to physically active adults. Journal of Sports Medicine and Physical Fitness, 42, 360-367.

Burkholder, G.J., \& Harlow, L.L. (2003). An illustration of a longitudinal crosslagged design for larger structural equation models. Structural Equation Modeling, 10, 465-486. 
Callaghan, P., Eves, F.F., Norman, P., Chang, A.M., \& Lung, C.Y. (2002). Applying the transtheoretical model of change to exercise in young Chinese people. British Journal of Health Psychology, 7, 267-282.

Canga, N., DeIrala, J., Vara, E. et al. (2000). Intervention study for smoking cessation in diabetic patients. Diabetes Care, 23, 1455-1460.

Chamot, E., Charvet, A.I., \& Perneger, T.V. (2001). Predicting stages of adoption of mammography screening in a general population. European Journal of Cancer, $37,1869-1877$.

Cohen, J. (1977). Statistical power analysis for the behavioral sciences, $\left(2^{\text {nd }}\right.$ ed.). New York: Academic Press.

Cohen, J. (1988). Statistical power analysis for the behavioral and social sciences ( $2^{\text {nd }}$ ed.). Hillsdale, NJ: Lawrence Erlbaum.

Cohen, J. (1990). Things I have learned (so far). American Psychologist, 45, $1304-1312$.

Cohen, J. (1994). The earth is round $(p<.05)$. American Psychologist, 49, 997-1003.

Coleman-Wallace, D., Lee, J. W., Montgomery, et al. (1999). Evaluation of developmentally appropriate programs for adolescent tobacco cessation. Journal of Social Health, 69, 314-319.

Cook, T.D., Cooper, H., Cordray, D.S., Hartmann, H., Hedges, L.V., Light, R.J., Louis, T.A., Mostseller, F. (1992). Meta-Analysis for Explanation. New York: Russell Sage Foundation. 
Cooper, H.M. (1989). Integrating research: A guide for literature reviews (2nd ed.).

Newbury Park, CA: Sage.

Cooper, H.M., \& Hedges, L.V. (1994). Research synthesis as a scientific enterprise.

New York: Russell Sage Foundation.

Colten, M.E., \& Janis, I.L. (1982). Effects of self-disclosure and the decisional balance-sheet procedure. In I.L. Janis (Ed.), Counseling on personal decisions: Theory and research on helping short-term relationships (pp. 159-171). New Haven, CT: Yale University Press.

Cumming, G., \& Finch, S. (2001). A primer on the understanding, use, and calculation of confidence intervals that are based on central and noncentral distributions. Educational and Psychological Measurement, 61, 532-574.

DiClemente, C. C. (1986). Self-efficacy and the addictive behaviors. Journal of Social \& Clinical Psychology, 4(3), 302-315.

DiClemente C.C. \& Hughs, S.O. (1990). Stages of change profiles in outpatient alcoholism treatment. Journal of Substance Abuse, 2, 217-235.

DiClemente, C.C., Prochaska, J.O., Fairhurst, S.K., Velicer, W.F., Velasquez, M.M., \& Rossi, J.S. (1991). The process of smoking cessation: An analysis of precontemplation, contemplation and preparation stages of change. Journal of Consulting and Clinical Psychology, 59, 295-304.

Dijkstra, A. \& De Vries, H. (2000). Clusters of precontempating smokers defined by the perception of the pros, cons, and self-efficacy. Addictive Behaviors, 25, 373385. 
Dijkstra, A., De Vries, H., \& Bakker, M. (1996). Pros and cons of quitting, selfefficacy, and the stages of change in smoking cessation. Journal of Consulting and Clinical Psychology, 64 (4), 758-763.

Dijkstra, A., De Vries, H. \& Roijackers, M. A. (1999). Targeting smokers with low readiness to change with tailored and nontailored self-help materials. Preventive Medicine, 28, 203-211.

Dijkstra, A., De Vries, H., Roijackers, J. \& Van Breukelen, G. (1998). Tailored interventions to communicate stage-matched information to smokers in different motivational stages. Journal of Consulting and Clinical Psychology, 3, 549557.

Dryfoos, J.M. (1996). Staging and decisional balance measures for two diabetes self-management behaviors. Unpublished master's thesis, University of Rhode Island, Kingston.

Duval S.J., \& Tweedie R.L. (2000). Trim and fill: A simple funnel plot-based method of testing and adjusting for publication bias in meta-analysis. Biometrics, 56, 276-284.

Eaton, C., Goldstein, M.G., Guadagnoli, E., Niaura, R., McDonald, N., \& Dube, C. (1992). Development of a decisional balance measure of physician smoking cessation intervention. Manuscript submitted for publication.

Egger M, Davey Smith G, Schneider M, Minder C. (1997). Bias in meta-analysis detected by a simple graphical test. British Medical Journal, 315, 629-34. 
Etter, J.F., Perneger, T.V., \& Ronchi, A. (1997). Distributions of smokers by stage: international comparison and association with smoking prevalence. Preventive Medicine, 26, 580-85.

Evers, K.E. (1999) Quantitative methods for assessing change: Application to condom use. (Doctoral dissertation, University of Rhode Island). Dissertation Abstracts International: Section B: The Sciences \& Engineering, 59(8-B), 4448.

Evers, K. E., \& Harlow, L. L. (1997, August). Longitudinal transtheoretical model of naturalistic change for condom use. Paper presented at the $105^{\text {th }}$ Annual Convention of the American Psychological Association, Chicago, IL.

Evers, K.E., Johnson, J.L., Padula, J., Prochaska, J., \& Prochaska, J.O. (2003). Stress management measure development for transtheoretical constructs of decisional balance and self-efficacy. Manuscript in preparation.

Evers, K.E., Johnson, J.L., Prochaska, J.O., \& Prochaska, J.M. (Jan., 2001). Doncaster Project Measurement Development Report: Application of the Transtheoretical Model to Pregnancy Prevention. West Kingston, RI: Pro-Change Behavior Systems, Inc.

Evers, K.E., Johnson, J.L., Ward, R.M., Padula, J., Prochaska, J.O., \& Prochaska, J.M. (May 2001). European drugs initiative: Measurement development and data analysis report. West Kingston, RI: Pro-Change Behavior Systems.

Fabrigar, L. R., Wegener, D. T., MacCallum, R. C., \& Strahan, E. J. (1999). Evaluating the use of exploratory factor analysis in psychological research. Psychological Methods, 4, 272-299. 
Fagerstrom, K. L., Heatherton, T. F., Kozlowski, L. T. (1992). Nicotine addiction and its assessment. Ear Nose and Throat Journal, 69(11), 763-767.

Fahrenwald, N.L., \& Walker, S. N. (2003). Application of the transtheoretical model of behavior change to the physical activity behavior of WIC mothers. Public Health Nursing, 20(4), 307-317.

Fan, X. (2003). Power of latent growth modeling for detecting group differences in linear growth trajectory parameters. Structural Equation Modeling, 10, 380-400.

Fava, J.L., Velicer, W.F., \& Prochaska, J.O. (1995). Applying the transtheoretical model to a representative sample of smokers. Addictive Behaviors, 20(2), 189203.

Fidler, F., \& Thompson, B. (2001). Computing correct confidence intervals for ANOVA fixed- and random-effects effect sizes. Educational and Psychological Measurement, 61, 575-604.

Fisher, R.A. (1932). Statistical methods for research workers ( $4^{\text {th }}$ ed.). London: Oliver and Boyd.

Fitzgerald, T. E., \& Prochaska, J. O. (1990). Nonprogressing profiles in smoking cessation: What keeps people refractory to self-change? Journal of Substance Abuse, 2, 87-105.

Frenn, M., Malin, S., \& Bansal, N.K. (2003). Stage-based interventions for low-fat diet with middle school students. Journal of Pediatric Nursing, 18(1), 36-45. Galvotti, C., Cabral, R., Lansky, A., Grimley, D., Riley, G., \& Prochaska, J. O. (1995). Validation of measures of condom and other contraceptive use among 
women at high risk for HIV infection and transmission: Stage of change, decisional balance, and self-efficacy. Health Psychology, 14, 570-578.

Gazabon, S.A. (2000). An assessment of decisional balance, self efficacy, and condom stages of change among white and minority women. Unpublished doctoral dissertation, University of Rhode Island, Kingston.

Giebel, M. (1999). Diagnostik der Motivation zur Verhaltensänderung kardiovaskulären Risikoverhaltens bei Bundeswehrsoldaten unter Berücksichtigung des transtheoretischen Stufenmodells der Verhaltensänderung nach Prochaska [Diagnostics of the motivation to change cardiovascular risk behaviors in professional soldiers based on Prochaska's Transtheoretical Model of Behavior Change]. Unpublished doctoral dissertation, University of Marburg, Marburg, Germany.

Glass G.V. (1976). Primary, secondary and meta-analysis of research. Educational Researcher 10, 3-8.

Glesser L.J., \& Olkin I. (1994). Stochastically dependent effect sizes. In: Cooper H, Hedges LV, eds. The handbook of research synthesis. New York: Russell Sage Foundation, 339-56.

Glesser LJ, \& Olkin I. (1996). Models for estimating the number of unpublished studies. Statistics in Medicine, 15, 2493-2507.

Godin, G., \& Shephard, R. J. (1985, April). Gender differences in perceived physical self-efficacy among older individuals. Perceptual \& Motor Skills, 60(2), 599-602.

Goldberg, D.N., Hoffman, A.M., Farinha, M.F., Marder, D.C., Tinson-Mitchem, L. Burton, D., \& Smith, E.G. (1994). Physician delivery of smoking-cessation 
advice based on the stages-of-change model. American Journal of Preventive Medicine, 10(5), 267-74.

Goldstein, M. G., DePue, J. D., Monroe, A. D., Monroe, A..D., Lessne, C. W.,

Rakowski, W., Prokhorov, A., Niaura, R., \& Dube, C. E. (1998). A populationbased survey of physician smoking cessation counseling practices. Preventive Medicine, 27(5), 720-729.

Goldstein, M.G., Pinto, B.M., Marcus, B.H., Lynn, H., Jette, A.M., Rakowski, W., McDermott, S., DePue, J.D., Milan, F.B., Dube, C.E., \& Tennstedt, S. (1999) Physician-based physical activity counseling for middle-aged and older adults: A randomized trial [Abstract]. Annals of Behavioral Medicine, S21, 40-47. Grimley, D.M., Prochaska, J.O., Velicer, W.F., \& Prochaska, G.E. (1995). Contraceptive and condom use adoption and maintenance: A stage paradigm approach. Health Education Quarterly, 22(1), 20-35.

Hall, K.L., \& Rossi, J.S. (2002). Meta-analysis of the structure, function, and effect size of decisional balance across the stages of change for 25 health behaviors [Abstract]. Annals of Behavioral Medicine, 24, S051.

Hall, K. L., \& Rossi, J. S. (2003). Informing interventions: A meta-analysis of the magnitude of effect in decisional balance stage transitions across 43 health behaviors [Abstract]. Annals of Behavioral Medicine, S25, 180.

Hall, K.L., \& Rossi, J.S. (2003, August). Meta-Analysis of Three Health Behaviors: Stage Transitions and Decisional Balance. Poster presented at the 111 th annual meeting of the American Psychological Association, Toronto, Canada. 
Hall, K.L., \& Rossi, J.S. (2004). A meta-analysis of the magnitude of effect in selfefficacy across adjacent stage transitions for 24 health behaviors: informing interventions [Abstract]. Annals of Behavioral Medicine, 26, S123.

Hall, K.L., \& Rossi, J.S. (2004). Structure, function, and effect size of decisional balance across the stages of change for 37 health behaviors: A meta-analytic study. Manuscript in preparation.

Hall, K.L., \& Rossi, J.S. (2004). Determining effect size for meta-analysis: A comparison of alternative procedures. Manuscript in preparation.

Happel, L. (2002). Motivationale Aspekte des Ernährungsverhaltens: Eine Studie im Bereich des Obst-und Gemüseverzehr [Motivational aspects of dietary behavior: A study in the context of fruit and vegetable intake]. Unpublished thesis, Department of Psychology, Marburg, Germany.

Hancock, G.R., Kuo, W.-L., \& Lawrence, F.R. (2001). An illustration of secondorder latent growth models. Structural Equation Modeling, 8, 470-489.

Harlow, L. L., Mulaik, S. A., \& Steiger, J. H. (Eds.) (1997). What if there were no significance tests? Hillsdale, NJ: Lawrence Erlbaum.

Heather, N., Rollnick, S., \& Bell, A. (1993). Predictive validity of the Readiness to Change Questionnaire. Addiction, 88(12), 1667-77.

Hedges, L.V. (1981). Distribution theory for Glass's estimator of effect size and related estimators. Journal of Educational Statistics, 6, 107-128.

Hedges, L. V., \& Olkin, I. (1985). Statistical methods for meta-analysis. Orlando, FL: Academic Press. 
Hedges, L. V. \& Pigott, T. D. (2001). The power of statistical tests in meta-analysis.

Psychological Methods, 6, 203-217.

Hedges, L.V. \& Vevea, J.L. (1998). Fixed-and random-effects models in metaanalysis. Psychological Methods, 3(4), 486-504.

Hempelmann, J. (2003). [Using the transtheoretical model to examine tooth-friendly diets]. Unpublished raw data.

Henderson, L. (1999). Applying the transtheoretical model to breast selfexamination. (Doctoral dissertation, University of Nebraska). Dissertation Abstracts International: Section B: The Sciences \& Engineering, 59(7-B), 3757. Herrick, A.B., Stone, W.J., \& Mettler, M.M. (1997). Stages of change, decisional balance, and self-efficacy across four health behaviors in a worksite environment. American Journal of Health Promotion, 12(1), 49-56.

Herzog, T.A., Abrams, D.B., Emmons, K.M., Linnan, L.A., \& Shadel, W.G. (1999). Do processes of change predict smoking stage movements? A prospective analysis of the transtheoretical model. Health Psychology, 18(4), 369-375.

Horn, D. (1976). A model for the study of personal choice health behavior. International Journal of the Addictions, 19, 89-98.

Horn, D. \& Waingrow S. (1966). Some dimensions of a model for smoking behavior change. American Journal of Public Health, 56, 21.

Horowitz, S. M. (2003). Applying the transtheoretical model to pregnancy and STD prevention: a review of the literature. American Journal of Health Promotion, $17(5), 304-328$ 
Horwath, C. C. (1999). Applying the transtheoretical model to eating behaviour change: Challenges and opportunities. Nutrition Research Reviews, 12, 281317.

Hoyt, M. F., \& Janis, I.L. (1975). Increasing adherence to a stressful decision via a motivational balance-sheet procedure: A field experiment. Journal of Personality and Social Psychology, 31, 833-839.

Huffcutt, A.I. \& Arthur, W. (1995). Development of a new outlier statistic for metaanalytic data. Journal of Applied Psychology, 80 (2) 327-334.

Hulton, L. J. (2001). Application of the transtheoretical model of change to adolescent decision-making. Issues in Comprehensive Pediatric Nursing. Special Issue, 24(2), 95-115.

Hunter, J., \& Schmidt, F. (1990). Methods of meta-analysis: Correcting error and bias in research findings. Beverly Hills CA: Sage.

Iyengar S., \& Greenhouse, J.B. (1988). Selection models and the file drawer problem. Statistical Sciences, 3, 109-35.

Janis, I.L. (1959). Decisional conflicts: A theoretical analysis. Journal of Personality and Social Psychology, 31, 8-33-839.

Janis, I.L. (1968). Pilot studies on new procedures for improving the quality of decision making. In Yale studies in attitudes and decisions. New Haven, CT: Yale University.

Janis, I. L., \& Mann, L. (1977). Decision making: A psychological analysis of conflict, choice and commitment. New York: Free Press. 
Jeffries, S.K. (2001). The role of major and minor stressors in moderating the transtheoretical model of exercise behavior among predominantly low-income patients attending primary care clinics. (Doctoral dissertation, Louisiana State University). Dissertation Abstracts International: Section B: The Sciences \& Engineering, 61(12-B), 6709.

Johnson, J.L., Johnson, S.S., Wright, J.A, (2002a). Applying the transtheoretical model to calcium intake for Osteoporosis prevention [Abstract]. Annals of Behavioral Medicine, S24, 113.

Johnson, J.L., Johnson, S.S., Wright, J.A, Prochaska, J.M., \& Prochaska, J.O. (2002b). Applying the transtheoretical model to bone density testing [Abstract]. Annals of Behavioral Medicine, S24, 113.

Johnson, J. L., \& Rossi, J. S. (2002). Quantitative structural integration of the transtheoretical model [Abstract]. Annals of Behavioral Medicine, S 24, 122.

Johnson, J. L., Fava, J. L., Velicer, W. F., Monroe, A. D., \& Emmons, K. (2002). Testing stage effects in an ethnically diverse sample. Addictive Behaviors, 27(4), 605-617.

Johnson, S.S., Sarkin, J.A., \& Prochaska, J.O. (2001). Women's health: The application of the Transtheoretical Model of Behavior Change to the Prevention \& Management of Osteoporosis. Technical Report submitted to Optum, Inc. and Lilly Pharmaceutical.

Johnson, S. S., Sarkin, J. A., Prochaska, J. M., \& Prochaska, J. O. (2001, April). Stage Matched Multiple Behaivor Weight Management Intervention for Adults 
with Diabetes: From Science to Practice. Paper presented at the $22^{\text {nd }}$ Annual Meeting of the Society of Behavioral Medicine, Seattle, WA.

Johnson, S.S., Wright, J.A., Johnson, J.L., Prochaska, J.O., \& Prochaska, J.M. (2002). Developing measures of transtheoretical model constructs for participating in decision-making about medications [Abstract]. Annals of Behavioral Medicine, S24, 154.

Jones, J., Edwards, L., Vallis, M. T., Ruggiero, L., Rossi, S., Rossi, J. S., Greene, G., Prochaska, J. O., and Zinman, B. (in press). Changes in diabetes self-care behaviors make a difference to glycemic control: The Diabetes Stages of Change (DiSC) study. Health Education.

Jordan, J.P. (2002). Development, validation, and factorial invariance of transtheoretical model measures for recovery from anorexia nervosa and bulimia nervosa. (Doctoral Dissertation, University of Rhode Island). Dissertation Abstracts International: Section B: The Sciences \& Engineering, 62 (9-B), 4267.

Jordan, P.J., Nigg, C.R., Norman, G.J., Rossi, J.S., \& Benisovich, S.V. (2002). Does the Transtheoretical Model need an additude adjustment? Integrating Attitude with Decisional Balance as predictors of Stage of Change for Exercise. Psychology of Sports and Exercise, 3, 65-83.

Jordan, P.J., Rossi, J.S., \& Redding, C.A. (2003). Using factorial invariance to test measurement models in transtheoretical model constructs applied to eating disorders. Manuscript submitted for publication. 
Katlin, E.N. (2001). Stages of change correlates for chemical abusers in out-patient treatment. (Doctoral dissertation, Temple University). Dissertation Abstracts International: Section B: The Sciences \& Engineering, 62(5-B), 2283.

Keller, S. (1998). Zur Validität des Transtheoretischen Modells - Eine Untersuchung zur Veränderung des Ernährungsverhaltens [On the validity of the Transtheoretical Model - A study in the context of dietars behavior]. Unpublished doctoral dissertation, University of Marburg, Marburg, Germany. Keller, S., Giessel, H., Basler, H.-D. (2002). Flossing? No thank you!-Readiness to change oral hygiene behavior. International Journal of Behavioral Medicine, $31,56-67$.

Keller, S., Herda, C., Ridder, K., \& Basler, H. (2001). Readiness to adopt adequate postural habits: An application of the transtheoretical model in the context of back pain prevention. Patient Education and Counseling, 42, 175-184.

Keller, S., Kaluza, G., Baun, E., \& Basler, H.D. (2003). Stages of change, decisional balance and self-efficacy for stress management. Manuscript in preparation.

Keller, S., Kreis, J., \& Huck, C. (2001). Fünf am Tag? Motivationale und psychosoziale Aspekte des Obst-und Gemüseverzehrs [Five a day? Motivational and psychosocial aspects of fruit and vegetable consumption]. Zeitschrift für Gesundheitpsychologie, 9, 87-98.

Keller, S., Nigg, C. R., Jaekle, C., Baum, E., \& Basler, H.D. (1999). Self-efficacy, decisional balance and the stages of change for smoking cessation in a German sample. Swiss Journal of Psychology - Schweizerische Zeitschrift fuer 
Psychologie - Revue Suisse de Psychologie. Special Issue: Smoking Behaviour, 58(2), 101-110.

King, T.K., Marcus, B.H., Pinto, B.M., Emmons, K..M., \& Abrams, D.B. (1996).

Cognitive-behavioral mediators of changing multiple behaviors: smoking and a sedentary lifestyle. Preventative Medicine, 25, 684-691.

Kraft, P., Sutton, S.R., \& McCreath Reynolds, H. (1999). The transtheoretical model of behaviour change: Are the stages qualitatively different? Psychology and Health, 14, 433-450.

Krebs, P. (2003). Composition and comparison of stage effects for smoking cessation, dietary fat reduction, and sun protection. Unpublished Masters Thesis, University of Rhode Island, Kingston.

Kremers, S.P.J., Mudde, A.N., \& DeVries, H. (2001). Subtypes within the precontemplation stage of adolescent smoking acquisition. Addictive Behaviors, 26, 237-251.

Laforge, R.G. et al. (2003). Enhancing brief tailored college alcohol interventions. Unpublished NIAAA grant proposal.

Lancaster, T., Dobbie, W., Vos, K., et al. (1999). Randomized trial of nurse-assisted strategies for smoking cessation in primary care. British Journal General Practice, 49, 267-274.

Lauby, J. L., Semaan, S., Cohen, A., Leviton, L., Gielen, A., Pulley, L., Walls, C., \& O'Campo, P. (1998). Self-efficacy, decisional balance and stages of change for condom use among women at risk for HIV infection. Health Education Research, 13(3), 343-356. 
Lauver, D.R., Settersten, L., Kane, J.H., \& Henriques, J.B.(2003). Tailored messages, external barriers, and women's utilization of professional breast cancer screening over time. Cancer, 97(11), 2724-35.

Leed-Kelly, A., Russell, K. S., Bobo, J. K. (1996). Feasibility of smoking cessation counseling by phone with alcohol treatment center graduates. Journal of Substance Abuse Treatment, 13, 203-210.

Lennox, A. S., Bain, N., Groves, J., et al. (1998). Stages of Change training for opportunistic smoking intervention by the primary health care team. Part I: randomized controlled trial of the effect of training on patient smoking outcomes and health professional behaviour as recalled by patients. Health Education Journal, 57, 140-149.

Lerner, C.F. (1990). The transtheoretical model of change: Self-change in adolescent deliquent behaviors. Unpublished master's thesis, University of Rhode Island, Kingston.

Levesque, D.A. (1999). Violence desistance among battering men: Existing interventions and the application of the transtheoretical model of change. (Doctoral dissertation, University of Rhode Island). Dissertation Abstracts International: Section B: The Sciences \& Engineering, 59(8-B), 4449.

Levesque, D.A., Dyment, S., \& Prochaska, J.M. (2000). Employee readiness for PeopleSoft at URI: Assessment results and recommendations. Kingston, RI: ProChange Behavior Systems. 
Levesque, D. A., Prochaska, J. M., \& Prochaska, J.O. (1999). Stages of change and integrated service delivery. Consulting Psychology Journal: Practice \& Research, 51(4), 226-241.

Levesque, D.A, Redding, C.A., \& Prochaska, J.O. (1999, March). Stage of change and decisional balance for depression prevention among at-risk individuals. Paper presented at the annual meeting of the Society of Behavioral Medicine, San Diego, CA.

Lewin, K. (1948). Resolving social conflicts: Selected papers on group dynamics. Gertrude W. Lewin (Ed.). New York: Harper.

Lipsey, M. W., \& Wilson, D. B. (2001). Practical meta-analysis. Thousand Oaks, CA: Sage.

Littell, J.H., \& Girvin, H. (2002). Stages of change: A critique. Behavior Modification, 26(2), 223-273.

Little, T.D., Schnabel, K.U., \& Baumert, J. (2000). Modeling longitudinal and multilevel data: Practical issues, applied approaches, and specific examples. Mahwah, NJ: Lawrence Erlbaum.

Lowry, C.R.S. (2000). Testing the transtheoretical model of change in young adult male smokeless tobacco users. (Doctoral dissertation, Texas Woman's University). Dissertation Abstracts International: Section B: The Sciences \& Engineering, 61(6-B), 2990.

Ma, J., Betts, N..M., Horacek T., Georgiou C., White A., \& Nitzke S. (2002). The importance of decisional balance and self-efficacy in relation to stages of change 
for fruit and vegetable intakes by young adults. American Journal of Health Promotion, 16(3), 157-66.

Maddock, J., Redding, C., Rossi, J.S., \& Weinstock, M. (2003). Development and Validation of an Appearance Motivation Attitudes Scale for Sun Protection. Manuscript Submitted for Publication.

Maier, A. (2002). Eine Untersuchung zur Validatät des Transtheoretical Modells im Bereich sportlicher Aktivität [A study on the validity of the transtheoretical model in the area of physical activity]. Unpublished dissertation, Medical Department, University of Marburg, Germany.

Maleszka, M. (2002). Positive und negative strategien bei der veränderung des Rauchverhaltens [Positive and negative processes of change for smoking behavior]. Unpublished Thesis, Department of Psychology, Marburg, Germany. Manne S., Markowitz A., Winawer S., Meropol N.J., Haller D., Rakowski W., Babb J., \& Jandorf L. (2002). Correlates of colorectal cancer screening compliance and stage of adoption among siblings of individuals with early onset colorectal cancer. Health Psychology, 21(1), 3-15.

Marcus, B. H., Eaton, C. A., Rossi, J. S., \& Harlow, L. L. (1994). Self-efficacy, decision-making and stages of change: An integrative model of physical exercise. Journal of Applied Social Psychology, 24, 489-508.

Marcus, B.H., Pinto, B.M., Simkin, L.R., Audrain, J.E., \& Taylor, E.R. (1994). Application of theoretical models to exercise behavior among employed women. American Journal Health Promotion, 9(1), 49-55. 
Marcus, B.H., Rawkowski, W., \& Rossi, J.S. (1992). Assessing motivational readiness and decision making for exercise. Health Psychology, 11(4), 257-261.

Marcus, B. H., Simkin, L. R., Rossi, J. S., \& Pinto, B. M. (1996). Longitudinal shifts in employees' stages and processes of exercise behavior change. American Journal of Health Promotion, 10, 195-200.

Marsh, K.L., Johnson, B.T., Carey, M.P. (2001). Conducting meta-analyses of HIV prevention literatures from a theory-testing perspective. Evaluation \& The Health Professions, 24(3):255-76.

Marshall, S. J., \& Biddle, S. J. (2001). The transtheoretical model of behavior change: a meta-analysis of applications to physical activity and exercise. Annals of Behavioral Medicine, 23(4), 229-246.

Mauriello, L.M. (2001). Applying the transtheoretical model of change to stress management among adolescents: development and validation of decisional balance and confidence measures. Unpublished master's thesis, University of Rhode Island, Kingston.

McArdle, J.J., \& Bell, R.Q. (2000). An introduction to latent growth models for developmental data analysis. In T.D. Little, K.U. Schnabel, \& J. Baumert (Eds.)., Modeling longitudinal and multilevel data: Practical issues, applied approaches, and specific examples (pp. 69-107). Mahwah, NJ: Lawrence Erlbaum.

McAuley L, Pham B, Tugwell P, Moher D. (2000). Does the inclusion of grey literature influence estimates of intervention effectiveness reported in metaanalyses? Lancet, 7 (356), 1228-31. 
McConnaughy, E.A., DiClemente, C.C., Proschaska, J.O., Velicer, W.F.(1983).

Stages of change in psychotherapy: Measurement and sample profiles.

Psychotherapy: Theory, Research and Practice, 20, 368-375.

McConnaughy, E.A., DiClemente, C.C., Proschaska, J.O., Velicer, W.F.(1989).

Stages of change in psychotherapy: a follow up report. Psychotherapy: Theory, Research and Practice, 26, 494-503.

Mettler, M.M., Stone, W.J., Herrick, A.B., \& Klein, D.A. (2000). Evaluation of a community-based physical activity campaign via the transtheoretical model. Health Promotion Practice, 1 (4), 351-359.

Meyer, C., Rumpf, H.J., Schumann, A., Hapke, U., \& John, U. (2003). Intentionally reduced smoking among untreated general population smokers: Prevalence, stability, prediction of smoking behaviour change and differences between subjects choosing either reduction or abstinence. Addiction, 98 (8), 1101-1110.

Migneault, J.P., Pallonen, U.E., \& Velicer, W.F. (1997). Decisional balance and stage of change for adolescent drinking. Addictive Behavior, 22(3), 339-51.

Moll, H. (2001). [Using the transtheoretical model to examine physical activity]. Unpublished thesis, Department of Psychology, Marburg, Germany.,

Morisky, D. E., Green, L. W., \& Levine, D. M. (1986). Concurrent and predictive validity of self-reported measure of medication adherence. Medical Care, 24(1), $67-74$.

Morrison-Beedy, D., \& Lewis, B.P. (2001). HIV prevention in single, urban, women: Condom-use readiness. Journal Obstetric, Gynecologic, and Neonatal Nursing, 30 (2), 148-156. 
Negotia, U. N. (1985). Expert systems and fuzzy systems. Menlo Park, CA:

Benjamin.

Nigg, C.R., Burbank, P., Padula, C., Dufresne, R., Rossi, J. S., Velicer, W. F., Laforge, R. G. \& Prochaska, J. O. (1999). Stages of change across ten health risk behaviors for older adults. The Gerontologist, 39, 473-482.

Nigg, C.R., \& Courneya, K.S. (1998). Transtheoretical Model: examining adolescent exercise behavior. Journal of Adolescent Health, 22(3), 214-24.

Nigg, C.R., Rossi, J.S., Norman, G.J., \& Benisovich, S.V. (1998). Decisional Balance Applied to Exercise Behavior. Manuscript submitted for publication.

Noar, S. M. (2002). Unpublished raw data.

Noar, S.M., Morokoff, P. J., \& Redding, C.A. (2001). An examination of transtheoretical predictors of condom use in late-adolescent heterosexual men. Journal of Applied Biobehavioral Research, 6(1), 1-26.

Norcross, J. C., Prochaska, J. O., \& Hambrecht, M. (1985). The Levels of Attribution and Change (LAC) Scale: Development and measurement. Cognitive Therapy and Research, 9, 631-649.

Norman, G.J., Zabinski, M.F., Calfas, K.J., Sallis, J.F., \& Patrick, K. (2002). Development of a decisional balance inventory for adolescent sedentary behavior [Abstract]. Annals of Behavioral Medicine,S 24, 217.

O'Connell, D., \& Velicer, W.F. (1988). A decisional balance measure and the stages of change model for weight loss. The International Journal of the Addictions, 23(7), 729-50. 
Orwin, R.G. (1983). A fail-safe N for effect size in meta-analysis. Journal of Educational Statistics, 8, 157-159.

Otake, K., \& Shimai, S. (2001). Adopting the stage model for smoking acquisition in Japanese adolescents. Journal of Health Psychology, 6(6), 629-643.

Pallonen, U.E. (1998). Transtheoretical measures for adolescent and adult smokers: similarities and differences. Preventative Medicine, 27, A29-A38.

Pallonen, U. E., Leskinen, L., Prochaska, J. O., Willey, C. J., Kaariainen, R., \& Salonen, J. T. (1994). A 2-year self-help smoking cessation manual intervention among middle-aged Finnish men: An application of the Transtheoretical Model. Preventive Medicine, 23, 507-514.

Pallonen, U.E., Prochaska, J.O., Velicer, W.F., Prokhorov, A.V. \& Smith, N.F. (1997). Stages of acquisition and cessation for adolescent smoking: An empirical integration. Addictive Behaviors, 23(3), 303-324.

Pallonen, U. E., Velicer, W. F., Prochaska, J. O., Rossi, J. S., Bellis, J. M., Tsoh, J. Y., Migneault, J. P., Smith, N. F., \& Prokhorov, A. V. (1998). Computer-based smoking cessation interventions in adolescents: Description, feasibility, and sixmonth follow-up findings. Substance Use and Misuse, 33, 935-965.

Park, E.R., DePue, J.D., Goldstein, M.G., Niaura, R., Harlow, L.L., Willey, C., Rakowski, W., \& Prokhorov, A.V. (2003). Assessing the transtheoretical model of change constructs for physicians counseling smokers [Abstract]. Annals of Behavioral Medicine, S25 (2), 120-126.

Pearson, K. (1933). On a method of determining whether a sample of given size $n$ supposed to have been drawn from a parent population having a known 
probability integral has probably been drawn at random. Biometrika 25,370 410.

Perez, N.J. (2001). Transtheoretical Model comparisons on condom use in minority and non-minority adolescent females at two levels of risk for contracting HIV, STDS, and Cervical Cancer. Unpublished master's thesis, University of Rhode Island, Kingston.

Perz, C.A. (1995). Validation and exploration of the transtheoretical model with union painters. (Doctoral dissertation, University of Houston). Dissertation Abstracts International: Section B: The Sciences \& Engineering, 56(2-B), 1118. Pham, B., Platt, R., McAuley, L., Sampson, M., Klassen, T.P., Moher, D. (2001). Detecting and Minimizing the Impact of for Publication Bias: An Empirical study of Methods. Evaluation and the Health Professions, 24(2), 109-125.

Pigott, T.D. (2001). Methods for missing data for meta-analysis. Evaluation in the Health Professions, 24(3), 277-307.

Plummer, B.A., Velicer, W.F., Redding, C.A., Prochaska, J.O., Rossi, J.S., Pallonen, U.E. \& Meier, K.S. (2001). Stage of change, decisional balance, and temptations for smoking: measurement and validation in a large, school-based population of adolescents. Addictive Behavior, 26(4), 551-71.

Povey, R., Conner, M., Sparks, P., James, R., \& Shepherd, R. (2000). Application of the Theory of Planned Behaviour to two dietary behaviours: Roles of perceived control and self-efficacy. British Journal of Health Psychology, 5(2), 121-139. 
Prochaska, J.J., Rossi, J.S., Hall, S.M., Rosen, A.B., Tsoh, J.Y., Humfleet, G.L., \& Eisendrath, S.J. (2003). Depressed Smokers and Stage of Change: Implications for Cessation Interventions. Manuscript submitted for publication.

Prochaska, J.O. (1994). Strong and weak principles for progressing from precontemplation to action on the basis of twelve problem behaviors. Health Psychology, 13(1), 47-51.

Prochaska, J.O., \& DiClemente, C.C. (1982). Transtheoretical therapy: Toward a more integrative model of change. Psychotherapy, Theory, Research and Practice, 19, 276-288.

Prochaska, J.O., \& DiClemente, C.C. (1983). Stages and processes of self-change in smoking: Toward and integration model of change. Journal of Consulting and Clinical Psychology, 5, 390-395.

Prochaska, J.O., \&DiClemente, C.C. (1998). Comments, criteria, and creating better models: In response to Davidson. In W.R. Miller \& N. Heather (Eds.), Treating addictive behaviors ( $2^{\text {nd }}$ ed., pp 39-45). New York: Plenum.

Prochaska, J. O., DiClemente, C. C., Velicer, W. F., \& Rossi, J. S. (1993).

Standardized, individualized, interactive, and personalized self-help programs for smoking cessation. Health Psychology, 12, 399-405.

Prochaska, J. O., DiClemente, C. C., Velicer, W. F., Ginpil, S., \& Norcross, J. C. (1985). Predicting change in smoking status for self-changers. Addictive Behaviors, 10,395-406.

Prochaska, J. O., Norcross, J. C., Fowler, J. L., Follick, M. J., \& Abrams, D.B. (1992). Attendance and outcome in a work site weight control program: 
Processes and stages of change as process and predictor variables. Addictive Behaviors, 17, 35-45.

Prochaska, J. O., Velicer, W. F., DiClemente, C. C., \& Fava, J. L. (1988). Measuring processes of change: Applications to the cessation of smoking. Journal of Consulting \& Clinical Psychology, 56, 520-528.

Prochaska, J.O., Velicer, W.F., Fava, J.L., Rossi, J.S., \& Tsoh, J.Y. (2001).

Evaluating a population-based recruitment approach and a stage-based expert system intervention for smoking cessation. Addictive Behaviors, 26, 583-602.

Prochaska, J. O., Velicer, W. F., Fava, J. L., Ruggiero, L., Laforge, R. G., Rossi, J. S., Johnson, S. S., \& Lee, P. A. (2001). Counselor and stimulus control enhancements of a stage-matched expert system intervention for smokers in a managed care setting. Preventive Medicine, 32, 23-32.

Prochaska, J. O., Velicer, W. F., Guadagnoli, E., Rossi, J. S. \& DiClemente, C. C. (1991). Patterns of change: A dynamic typology applied to smoking cessation. Multivariate Behavioral Research, 26, 83-107.

Prochaska, J. O., Velicer, W. F., Rossi, J. S., Goldstein, M. G., Marcus, B. H., Rakowski, W., Fiore, C., Harlow, L. L., Redding, C. A., Rosenbloom, D., \& Rossi, S. R. (1994). Stages of change and decisional balance for 12 problem behaviors. Health Psychology, 13, 39-46.

Prochaska, J.O., Velicer, W.F., Rossi, J.S., Redding, C., Greene, G., Rossi, S., Sun, X., Fava, J., Laforge, R., Plummer, B. (in press). Impact of Simultaneous Stagematched Expert System Interventions for Smoking, High Fat Diet and Sun Exposure on a Population of Parents. Health Psychology. 
Quinlan, K. B., McCaul, K. D. (2000). Matched and mis-matched interventions with young adult smokers: testing a stage theory. Health Psychology, 9, 165171.

Rakowski, W., Dube, C.E., Marcus, B., Prochaska, J.O., Velicer, W.F., \& Abrams, D.B. (1992). Assessing elements of women's decisions about mammography. Health Psychology, 11(2), 111-118.

Redding, C.A. (1990). The pros and cons of safer sex decision making: A measurement model. Unpublished research competency, University of Rhode Island, Kingston.

Redding, C.A. (1993). The transtheoretical model applied to safer sex behavior among univerity students: A cross-sectional investigation. (Doctoral dissertation, University of Rhode Island, 1993). Dissertation Abstracts International: Section B: The Sciences \& Engineering.

Redding, C.A., Prochaska, J.O., Pallonen, U.E., Rossi, J.S., Velicer, W.F., Rossi, S.R., Greene, G.W., Meier, K.S., Evers, K.E., Plummer, B.A., Maddock, J.E. (1999). Transtheoretical individualized multimedia expert systems targeting adolescents' health behaviors. Cognitive \& Behavioral Practice, 6(2), 144-153. Redding, C. A., Rossi, J. S., Rossi, S. R., Velicer, W. F., \& Prochaksa, J. O. (1999). Health behavior models. In G. C. Hyner, K. W. Peterson, J. W. Travis, J. E. Dewey, J. J. Foerster, \& E. M. Framer (Eds.), SPM handbook of health assessment tools (pp. 83-93). Pittsburgh, PA: The Society of Prospective Medicine \& The Institute for Health and Productivity Management. 
Reed, G.R. (1996). Measuring stage of change for exercise. (Doctoral dissertation, University of Rhode Island). Dissertation Abstracts International: Section B: The Sciences \& Engineering, 56(9-B), 5220.

Reed, G.R., Velicer, W.F., Prochaska, J.O., Rossi, J.R , \& Marcus, B.F. (1997). What makes a good staging algorithm: Examples from regular exercise. American Journal of Health Promotion, 12, 57-66.

Riemsma, R. P., Pattenden, J., Bridle., C, Sowden, A. J., Mather, L., Watt, I. S., \& Walker, A. (2003). Systematic review of the effectiveness of stage based interventions to promote smoking cessation. British Medical Journal, 326, 1175-1177.

Riley, T.A., \& Fava, J.L. (2003). Stress and transtheoretical indicators of stress management behaviors in HIV-positive women. Journal of Psychosomatic Research, 54, 245-252.

Riley, T.A., Toth, J.M., \& Fava, J.L. (2000). The transtheoretical model and stress management practices in women at risk for, or infected with, HIV. Journal of the Association of Nurses in AIDS Care, 11 (1), 67-77.

Riley, T.A., \& Weems, B.M. (2003). Health promoting behaviors, the transtheoretical model, and impoverished HIV-positive women. Paper presented at the meeting of the Midwest Nursing Research Society, Grand Rapids, MI. Robbins, M.L., Evans, J.L., Kilgallen, C.E., \& Jacobbi, L.M. (1999). Stages and decisional balance for organ donation in a representative sample [Abstract]. Annals of Behavioral Medicine, 21, S247. 
Robbins, M.L., Ganikos, M., Leino, E.V., Eaker, M.A, Eastwood, A.,

Webster, L., Bieterman, C. A. (2002). Stage-based intervention to increase intent for organ donation among college students [Abstract]. Annals of Behavioral Medicine, 24, S138.

Robbins, M.L., Kilgallen, C.E., Evans, J.L., Redding, C.A. \& Knott, E.J. (1999). Stages and decisional balance for becoming an organ donor[Abstract]. Annals of Behavioral Medicine, 21, S177.

Robbins, M.L., Levesque, D.A., Redding, C.A., Johnson, J.L., Prochaska, J.O., Rohr, M.S., Peters, T.G. (2001). Assessing family members' motivation readiness and decision making for consenting to cadaveric organ donation. Journal of Health Psychology, 6(5), 523-535.

Robbins, M.L., Mattice, B., Knott, J.E. Individual and Campus-Wide Interventions to Increase Donation Intentions Among African American CollegeStudents (2001-2004). Health Resources and Services Administration, Division of Transplantation \#1H390T00077.

Rohren, C. L., Croghan, I. T., Hurt, R. D. et al. (1994). Predicting smoking cessation outcome in a medical center from stage of readiness: contemplation vs. action. Preventive Medicine, 23, 335-344.

Rosenthal, R. (1991). Meta-analytic procedures for social research. Thousand Oaks, CA: Sage.

Rosenthal, R. (1979). The "file drawer problem" and tolerance for null results. Psychological Bulletin, 86, 638-641. 
Rosenthal, R. (1984). Meta-analytic procedures for social research. Beverly Hilss, CA: Sage.

Rosenthal, R. (1994). Parametric measures of effect size. In Cooper, H. \& Hedges L.V. (Eds.), The handbook of research synthesis (pp. 231-244). New York: Russell Sage Foundation.

Rosenthal, R. (1995). Writing meta-analytic reviews. Psychological Bulletin, 118, 183-192.

Rossi, J. S. (1990). Statistical power of psychological research: What have we gained in 20 years? Journal of Consulting and Clinical Psychology, 58, 646-656.

Rossi, J.S. (1990, August). Randon and ultraviolet light exposure: emerging cancer risk factors. In J.O. Prochaska (Chair), The stages of change: Extensions to new areas of behavior change. Symposium presented at the $98^{\text {th }}$ Annual Convention of the American Psychological Association, Boston, MA.

Rossi, J. S. (1997). A case study in the failure of psychology as a cumulative science: The spontaneous recovery of verbal learning. In L. L. Harlow, S. A. Mulaik, \& J. H. Steiger (Eds.), What if there were no significance tests? (pp. 175-197). Hillsdale, NJ: Lawrence Erlbaum.

Rossi, J. S. (1999). Cumulative knowledge in health and behavioral science: Lessons from quantum physics [Abstract]. Annals of Behavioral Medicine, 21, S084.

Rossi, J. S. (2001). Contrast effect size analysis and theory testing: An example using the transtheoretical model [Abstract]. Annals of Behavioral Medicine, 23, S156. 
Rossi, J.S., Martin, R.A., Redding, C.A., Rosenbloom, D., Rohsenow, D.J., \& Monti, P.M. (2001). Decisional balance and the stages of change for cocaine use. Manuscript submitted for publication.

Rossi, J. S., \& Redding, C. A. (2001). Structure and function of self-efficacy across the stages of change for 10 health behaviors [Abstract]. Annals of Behavioral Medicine, 23, S94.

Rossi, J.S. (1992, August). Common processes of change across nine problem behaviors. Paper presented at the $100^{\text {th }}$ annual convention of the American Psychological Association, Washington, DC.

Rossi, J.S. (2003, September). Comparison of the use of significance testing and effect sizes in theory-based health promotion research. Paper presented at the 43rd annual meeting of the Society for Multivariate Experimental Psychology, Keystone, CO.

Rossi, J.S., Benisovich, S.V., Norman, G.J., \& Nigg, C.R. (2004). Development of a hierarchical multidimensional measure of exercise self-efficacy. Manuscript submitted for publication.

Rossi, S.R., Greene, G.W., Rossi, J.S., Plummer, B.A., Benisovich, S.V., Keller, S., Velicer, W.F., Redding, C.A., Prochaska, J.O., Pallonen, U.E., \& Meier, K.S. (2001). Validation of decisional balance and situational temptations measures for dietary fat reduction in a large school-based population of adolescents. Eating Behaviors, 2, 1-18.

Rossi, J.S., Prochaska, J.O., \& DiClemente, C.C. (1988). Processes of change in heavy and light smokers. Journal of Substance Abuse, 1, 1-9. 
Rossi, S.R., Rossi, J.S., Greene, G.W., Fey-Yensan, N., Padula, C., Nigg, C., Owens, N.J. \& Clark, P. (2001a). Development and validation of a brief measure of decision-making for fruit and vegetable consumption in older adults. Manuscript in preparation.

Rossi, S.R., Rossi, J.S., Greene, G.W., Ward, R.M., Maddock, J., Velicer, W.F. \& Prochaska, J.O. (2001b). Brief decisional balance and situational temptations measures for dietary fat reduction in a large population of adults. Manuscript in preparation.

Rossi, S.R., Rossi, J.S., Prochaska, J.O., \& Velicer, W.F. (1993, August). Measurement structure of a decisional balance questionaire for dietary fat reduction. Paper presented at the $101^{\text {st }}$ annual convention of the American Psychological Association, Toronto, Canada.

Ruggiero, L. (2003). Validation of the transtheoretical model for glucose self-testing in a large sample of people with diabetes. Manuscript in preparation.

Ruggiero, L., Tsoh, J.Y., Everett, K., Fava, J.L., \& Guise, B.J. (2000). The transtheoretical model of smoking: Comparison of pregnant and nonpregnant smokers. Addictive Behaviors, 25 (2), 239-251.

Sarkin, J.A., Johnson, S.S., Prochaska, J.O., \& Prochaska, J.M (2001). Applying the Transtheoretical Model to Regular Moderate Exercise in an Overweight Population: Validation of a Stages of Change Measure. Preventive Medicine, $33(5), 462-469$. 
Schmaling, K.B., Afari, N., \& Blume, A.W. (2000). Assessment of psychological factors associated with adherence to medication regimens among adult patients with asthma. Journal of Asthma, 37 (4), 335-343.

Schmidt, F. (1992). What do data really mean? Research findings, meta-analysis, and cumulative knowledge in psychology. American Psychologist, 47, 1173-1181.

Schorling, J.B. (1995). The stages of change of rural African-American smokers. American Journal of Preventive Medicine, 11 (3), 170-177.

Schulze, H. (n.d.). [Using the transtheoretical model to examine daily use of dental floss]. Unpublished raw data.

Shumann, A., Rumpf, H.J., Meyer, C., Hapke, U., \& John, U. (2003). Skalen zu Kernkonstruken des Transtheoretischen Modells (TTM) im Verhaltensbereich Tabakkonsum [Scales for transtheoretical model constructs (TTM) in the area of tobacco consumption]. Zeitschrift für Gesundheitpsychologie, 11(2), 57-66.

Shepard, M. F., \& Campbell, J. A. (1992). The Abusive Behavior Inventory: A measure of psychological and physical abuse. Journal of Interpersonal Violence, 7, 291-305.

Silverman, E.S. (1995). Evaluating the Stages of Behavior Change Model for use in diverse cultures: Hong Kong versus the United States. (Virginia Polytechnic Institute and State University, 1995). Dissertation Abstracts International: Section B: The Sciences \& Engineering, 56 (5-B), 2849.

Snow, M.G., Prochaska, J.O., \& Rossi, J.S. (1992). Stages of change for smoking cessation among former problem drinkers: A cross-sectional analysis. Journal of Substance Abuse, 4, 107-116. 
Song, F., Sheldon, T.A., Sutton, A.J., Abrams, K.R., \& Jones, D.R. (2001). Methods for exploring heterogeneity in meta-analysis. Evaluation \& The Health Professions, 24(2), 126-151.

Spencer, L., Pagell, F., Hellion, M. E., \& Abrams, D. B. (2002). Applying the Transtheoretical model to tobacco cessation and prevention: A review of the literature. American Journal of Health Promotion, 17(1), 7-71.

Stark, M.J., Tesselaar, H.M., O’Connell, A.A., Person, B., Galavotti, C., Cohen, A., \& Walls, C. (1998). Psychosocial factors associated with the stages of change for condom use among women at risk for HIV and STDs: Implications for Intervention development. Journal of Consulting and Clinical Psychology, 66 (6), 967-978.

Stepnowsky, C. \& Moore, P. (2003). Nasal CPAP treatment for obstructive sleep apnea: Developing a new perspective on dosing strategies and compliance. Journal of Psychosomatic Research, 54(6): 599-605

Steptoe, A., Kerry, S. Rink, E. et al. (2001). The impact of behavioral counseling on stage of change in fat intake, physical activity, and cigarette smoking in adults at increased risk of coronary heart disease. American Journal of Public Health, $91,265-269$.

Sterne JAC, Gavaghan D, Egger ME. (2000). Publication and related bias in metaanalysis: power of statistical tests and prevalence in the literature. Journal of Clinical Epidemiology, 53, 1119-1129.

Susenbeth, R. (2000). Anwendung des Transtheoretischen Modells gesundheitsfördernder Verhaltensänderung auf HIV-protektiven 
Kondomgebrauch [Application of the Transtheoretical Model on condom use for HIV prevention]. Unpublished doctoral dissertation, University of Marburg, Marburg, Germany.

Sutton, S. (1996). Can 'stages of change' provide guidance in the treatment of addictions? A critical examination of Prochaska and DiClemente's model. In Edward, G. \& Dare, C. (Eds.), Psychotherapy, Psychological Treatments and the Addictions, 189-205. Cambridge: Cambridge University Press.

Takkouche, B., Cadarso-Suarez, C., Spiegelman, D. (1999). Evaluation of old and new tests of heterogeneity in epidemiologic meta-analysis. American Journal of Epidemiology, 150, 206-15.

Thompson, B. (2002, April). What future quantitative social science research could look like: Confidence intervals for effect sizes. Educational Researcher, 25-32.

Tobler, N. (1994). Meta-analytical issues for prevention intervention research. In L.M. Collins \& L.A. Seitz (Eds.), Advances in data analysis for prevention intervention research (pp. 342-403). NIDA Research Monograph 142. Rockville, MD: U.S. Department of Health and Human Services.

Tseng, Y. (2001). Examining exercise determinants in elders residing in assisted living facilities: An application of the transtheoretical model. (Doctoral dissertation, University of Minnesota). Dissertation Abstracts International: Section B: The Sciences \& Engineering, 61(10-B), 5241.

Tsoh, J.Y. (1993). Motivation and stages of change among drug addicts in drug abuse treatment programs. Unpublished master's thesis, University of Rhode Island, Kingston. 
U.S. Public Health Service. (1990). The Health Benefits of Smoking Cessation. A report of the surgeon general, 469-515. (DHHS Publication No. CDC 90-416.) Washington, DC: Department of Health and Human Services.

Valanis, B., Lichtenstein, E., Mullooly, J. P., Labuhn, K., Brody, K., Severson, H. H., \& Stevens, N. (2001). Maternal smoking cessation and relapse prevention during health care visits. American Journal of Preventive Medicine, 20(1), 1-8. Van Marter, D.F., Evers, K.E., Janet L. Johnson, Ph.D., Andrea L. Eastwood, B.S., Prochaska, J.M., \& Prochaska, J.O. (2003, March). Measure development of Transtheoretical Model constructs for bullying prevention in middle schools. Poster presented at the 24rd Annual Scientific Sessions of the Society of Behavioral Medicine, Salt Lake City, Utah.

Velicer, W.F., Cumming, G., Rossi, J.S., Fava, J.L., Prochaska, J.O., \& Johnson, J. (2003). Theory testing using quantitative predictions of effect size. Psychological Methods. Manuscript submitted for publication.

Velicer, W. F., DiClemente, C. C., Prochaska, J. O., \& Brandenberg, N. (1985). A decisional balance measure for assessing and predicting smoking status. Journal of Personality and Social Psychology, 48, 1279-1289.

Velicer, W. F., DiClemente, C. C., Rossi, J. S., \& Prochaska, J. O. (1990). Relapse situations and self-efficacy: An integrative model. Addictive Behaviors, 15, 271-283.

Velicer, W. F., Fava, J. L., Prochaska, J. O., Abrams, D. B., Emmons, K. M., \& Pierce, J. P. (1995). Distribution of smokers by stage in three representative samples. Preventive Medicine, 24, 401-411. 
Velicer, W.F., Martin, R.A., \& Collins, L.M. (1996). Latent transition analysis for longitudinal data. Addiction, 91(Suppl.), S197-S209.

Velicer, W. F., Norman, G. J., Fava, J. L., \& Prochaska, J. O. (1999). Testing 40 predictions from the Transtheoretical Model. Addictive Behaviors, 24, 455-469. Velicer, W. F., Prochaska, J. O., Bellis, J. M., DiClemente, C. C., Rossi, J. S., Fava, J. L., \& Steiger, J. H. (1993). An expert system intervention for smoking cessation. Addictive Behaviors, 18, 269-290.

Velicer, W. F., \& Prochaska, J. O. (1999). An expert system intervention for smoking cessation. Patient Education and Counseling, 36, 119-129.

Velicer, W. F, Prochaska, J. O., Fava, J. L., Laforge, R. G., \& Rossi, J. S. (1999). Interactive versus non-interactive interventions and dose-response relationships for stage matched smoking cessation programs in a managed care setting. Health Psychology, 18, 21-28.

Velicer, W.F., Prochaska, J.O., Fava, J.L., Norman, G.J., \& Redding, C.A. (1998). Smoking cessation and stress management: Applications of the transtheoretical model of behavior change. Homeostasis in Health \& Disease, 38(5-6), 216-233. Velicer, W.F., Prochaska, J.O., Fava, J.L., Rossi, J.S., Redding, C.A., Laforge, R.G., \& Robbins, M.L. (2000). Using the transtheoretical model for population-based approaches to health promotion and disease prevention. Homeostasis, 40, 174 195.

Velicer, W.F., Prochaska, J.O., Redding, C., Rossi, J.S., Sun, X., Rossi, S., Greene, G., Fava, J., Laforge, R., Goldstein, M., Rakowski, W., DePue, J., Ehrlich, B. 
(2003). Simultaneous Stage-matched Expert System Interventions for Four Risk

Factors in a Population of Primary Care Patients. Unpublished Manuscript.

Velicer, W. F., Rossi, J. S., Prochaska, J. O., \& DiClemente, C. C. (1996). A

criterion measurement model for health behavior change. Addictive Behaviors, $21,555-584$.

Wakui, S., Shimomitsu, T., Odagiri, Y., Inoue, S., Takamiya, T., \& Ohya, Y. (2002). Relation of the stages of change for exercise behaviors, self-efficacy, decisionalbalance, and diet-related psycho-behavioral factors in young Japanese women. Journal of Sports Medicine and Physical Fitness, 42, 224-232.

Wang, W. D. (1994). Feasibility and effectiveness of stages-of-change model in cigarette smoking cessation counseling. Journal of Formosa Medical Association, 93, 752-757.

Ward, R.M., Velicer, W.F., Rossi, J.S., Fava, J.L., \& Prochaska, J.O. (in press). Factorial invariance and internal consistency for the decisional balance inventory - short form. Addictive Behaviors.

Weinstein, N. D., Rothman, A. J., \& Sutton, S. R. (1998). Stage theories of health behavior: Conceptual and methodological issues. Health Psychology, 17(3), 290-299.

Weinstock, M. A., Rossi, J. S., Redding, C. A., \& Maddock, J. E. (2002). Randomized controlled community trial of the efficacy of a multi-component stage-matched intervention to increase sun protection among beachgoers. Preventive Medicine, 35, 584-592. 
Whyte, S.R. (2003). Investigating stages of change and processes of change for

dietary fat consumption on the World Wide Web. (Doctoral dissertation, University of Maryland). Dissertation Abstracts International: Section B: The Sciences \& Engineering, 63(8-B), 3947.

Wilcox, N.S., Prochaska, J.O., Velicer, W.F., \& DiClemente, C.C. (1985). Subject characteristics as predictors of self-change in smoking. Addictive Behaviors, 10 , $407-412$.

Wilkinson, L. and the Task Force on Statistical Inference (1999). Statistical methods in psychology journals: Guidelines and explanations. American Psychologist, $54,594-604$.

Wright, J.A, Johnson, S.S., Johnson, J.L., Prochaska, J.M., \& Prochaska, J.O. (2002a). Weight-Bearing Exercise Stage Of Change Measure For Osteoporosis Interventions [Abstract]. Annals of Behavioral Medicine, 24, S134.

Wyse, J., Mercer, T., Ashford, B., Buxton, K., \& Gleeson, N. (1995). Evidence for the validity and utility of the stages of exercise behavior change scale in young adults. Health Education Research, 10, 365-377.

Zwick, W. R. \& Velicer, W. F. (1986). Comparison of five rules for determining the number of components to retain. Psychological Bulletin, 99, 432-442. 